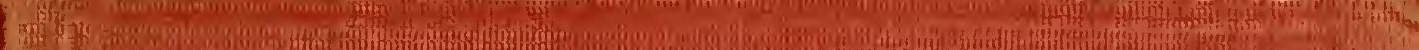

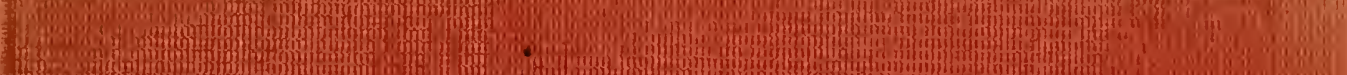
1) 319

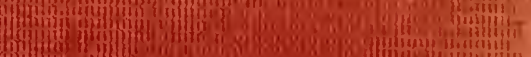

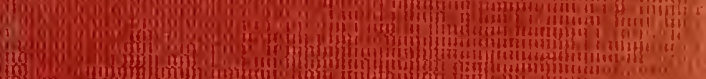

(1)

\title{
WS GENETICS
}

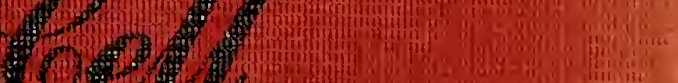
Wis

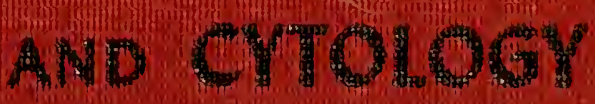

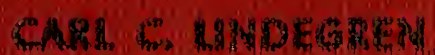

G.

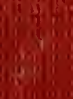

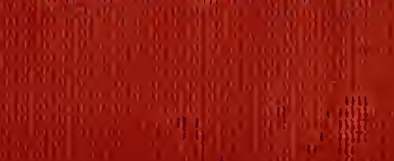

Jiow

A

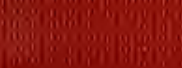

2.8.8.

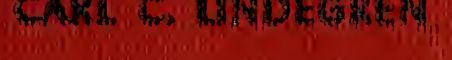

bisinge:

(1) 
1,

$\frac{1}{3}$ 


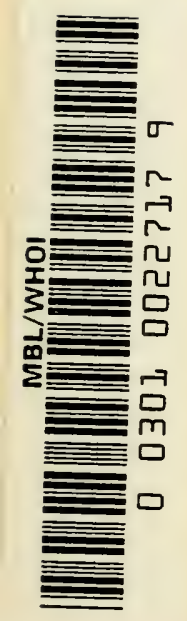





\section{THE YEAST CELL,}

Its Genetics and Cytology 


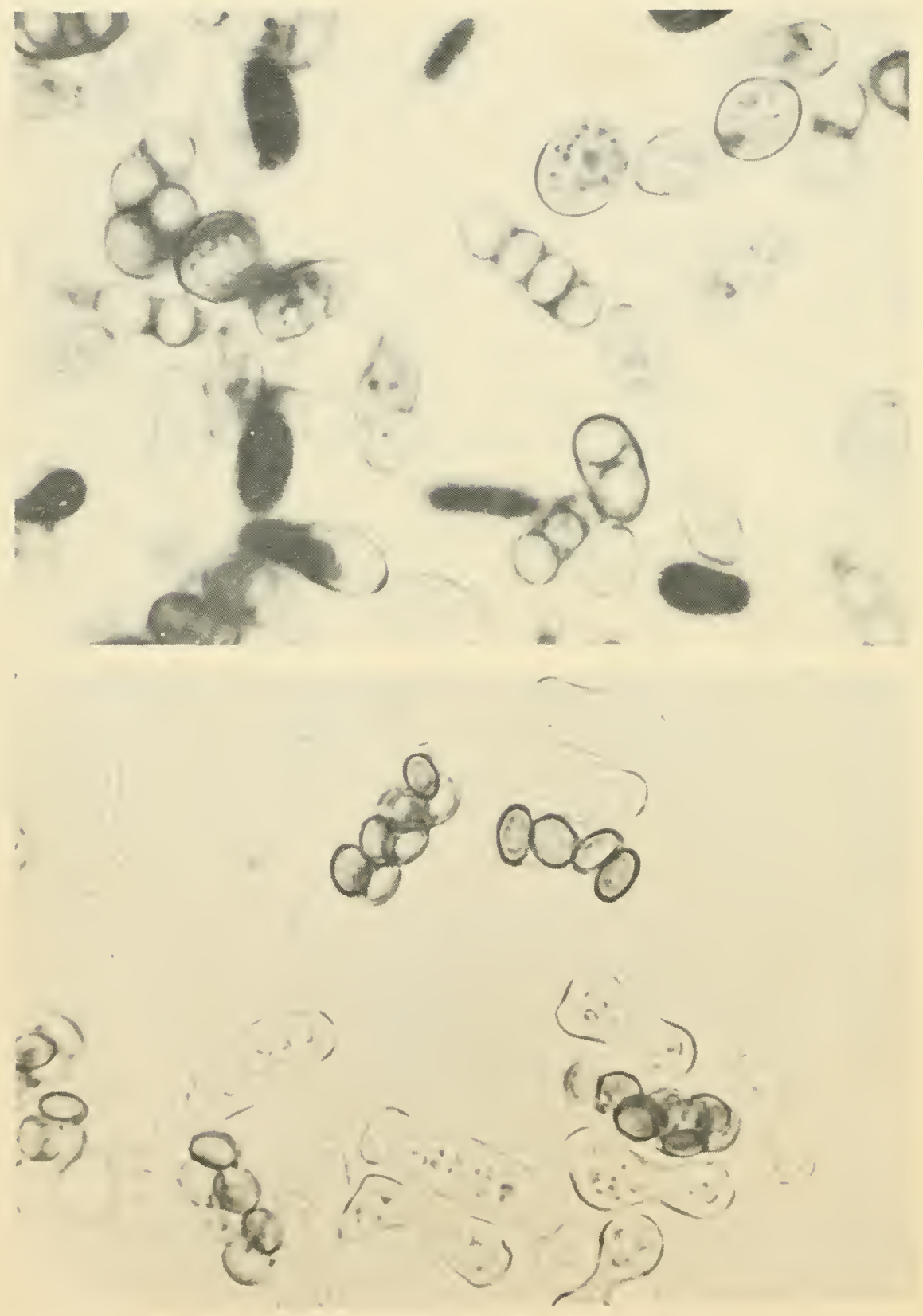

Spores of Saccharomyces Cerevisiae and Schizosaccharomyces Octosporus 


\section{THE YEAST CELL,}

\section{Its Genetics and Cytology}

by

CARL C. LINDEGREN

Southern Illinois University

Carbondale, Illinois

FIRST EDITION

1949

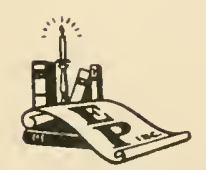

EDUCATIONAL PUBLISHERS, Incorporated 
Copyright, 1949

by

CARL C. LINDEGREN

Al] Rights Reserved 
This book is dedicated to my beloved wife in grateful recognition of the major role that she has played in its creation. By virtue of her impatient and energetic initiative, which demands immediate translation of thought into experiment, and her unflagging enthusiasm, which has always proved equal to the discouraging task of testing each new alternative that experiment implacably imposes, she has succeeded in the extraordinarily difficult task of constructing the chromosome maps of Saccharomyces. This has graduated Saccharomyces from a promising organism for experimental breeding to a full-fledged membership in the Drosophila-maize-Neurospora hierarchy. 



\section{PREFACE}

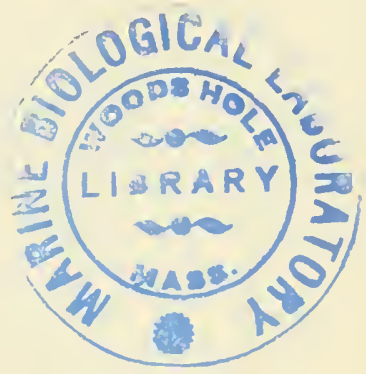

Study of the yeast cell began with the announcement of the cell theory by Schleiden and Schwann. The difficulties encountered in interpreting the numerous observations were only partly due to technical obstacles. Modern concepts of cellular organization have been as helpful in interpreting the yeast cell's structure as the modern knowledge of nutrition has been helpful in understanding its metabolism. We have finally been able to show that the yeast cell is a conventional cell with chromosomes, nucleolus, heterochromatin, centrosomes, and mitochondria.

Because yeast is an article of trade and has been available to biochemists in considerable quantities as a source of living protoplasm, modern biochemistry is based largely on the study of enzymes isolated from yeasts. Winge and Laustsen showed that yeasts were also acceptable genetical material and our own work has considerably extended the knowledge of yeast genetics; we have constructed the first chromosome maps of yeasts and have developed new concepts of the nature of the hereditary apparatus which have been confirmed by tetrad analysis of hybrids with well marked chromosomes.

This volume reviews the genetical and cytological work thus far achieved in our laboratory during the last eight years by Mrs. Lindegren and me and our assistants, first at Washington University, St. Louis, Missouri, and more recently at Southern Illinois University, Carbondale, Illinois, and includes discussions of work carried on at the same time by others associated with us, notably Dr. Folke Skoog, Dr. S. Hestrin, Dr. S. Velick, Dr. Michael Doudoroff, Dr. Sol Spiegelman, Dr. J. M. Wiame, Dr. J. O. Lampen, Dr. E. L. Tatum, Dr. Caroline Raut, Dr. J. S. Rafalko, and Mrs. Margaret Rafalko. It was first organized in a series of lectures given at the University of Washington, Seattle, in the spring of 1947 while I held the Walker Ames Professorship of Botany there.

Our work has been made possible by the continued and generous support of our laboratory by Anheuser-Busch, Inc., for which we are deeply grateful. Subsidiary grants have been received from the U. S. Public Health Service, the American Cancer Society, Washington University, Southern Illinois University, and the Ameri can Philosophical Society. The support from Anheuser-Busch, Inc. is in line with the honorable tradition of breweries which have generously supported so many investigations in pure science. The 
Carlsberg Brewery supported Hansen, the inventor of the pureculture technique; Sorenson, the inventor of $\mathrm{pH}$; Kjeldahl, whose ni trogen determination is a byword; and Winge, who brought yeast genetics into being. Joule determined the mechanical equivalence of heat in a brewery laboratory. Modern statistics are based largely on the work of Student, an employee of the Guiness Breweries in Dublin. Beijerinck, whom many consider the most penetrating student of the biology of yeasts of the last century, was largely supported by a brewery in Delft; and his successor, Kluyver, from whose laboratory have come some of the very important advances in enzymology and zymology has lived up to the standards set by his illustrious predecessor. Henneberg's work was also supported primarily by German breweries and we are indebted to him for the discovery of food yeast, a source that may one day provide much of the world's proteins and vitamins. Finally, Pasteur's germ theory of disease has its origin in his demonstration that fermentation is caused by microorganisms. He developed this concept of disease in the course of his successful attempt to discover the cause of spoilage of beer in the French breweries. It is safe to say that very few industries have contributed as much to modern biological thought as the brewing industry.

In the tetrad analysis of heterozygous yeasts, we have encountered many cases of apparent non-Mendelian heredity. There have been three phases in the interpretation of these phenomena. At first, the interpretation was on a classical Mendelian theory assuming that different alleles produced the same phenotype. When this view was shown to be inadequate, the data wer interpreted by assuming that the cytoplasm contained autonomous entities produced by gene action. This interpretation was also rejected, and at present genes are assumed to respond adaptively to the environment, rather than assuming that autonomous cytoplasmic entities are produced. These changes have resulted in the reinterpretation of many of the previously published experimental data. In this volume all data are interpreted accorcing to the current view and the history of changes in point of view is not discussed unless it seems essential.

Our data on non-Mendelian inheritance indicate that the current views concerning the stability of the gene and the regular segregation of alleles are in need of revision. Some readers may interpret this as support of Lysenko's theories of heredity. This is by no means the case. Lysenko's views are based on (1) belief in Lamarck's theory of the inheritance of acquired characters and (2) the view that genes and chromosomes have no control over heredity. I have shown that the gene may be readily affected by the environment, but my data indicate that heredity and phenotype are both under genic control and that non-genic influences are practically non-esixtent. Current editorial criticism of Lysenko consists largely of attacks on the tenability of his scientific views. I believe this attitude is 
deplorable. Opposition to Lysenko should not be based on the criticism of his scientific views, weak and naive though they are, but on the fact that he has achieved his prestige by conniving at the murder of his scientific adversaries rather than by argument and discussion and that he acts as an arbitrary dictator over other biologists, suppressing freedom of discussion and research.

In addition to acknowledging the assistance of those who have helped directly with the present volume, I would like to express my thanks and affection for my teachers whose influence, I hope, is apparent in my treatment of the problems of genetics and cytology. Principal among them are T. H. Morgan, Calvin Bridges, B. O. Dodge and A. H. Sturtevant. To them, and a host of others, I am sincerely grateful.

I am grateful to Dr. Jack Schultz for his helpful criticism of the manuscript.

Finally, my gratitude to Mr. James D. Veron for his continued interest and constant encouragement.

Carbondale, Illinois

November 5, 1948

C. C. L.

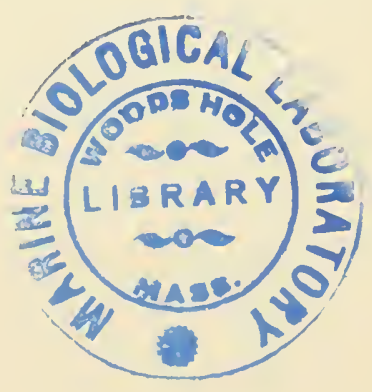





\section{CONTENTS}

Chapter

Page

1 Some Important Yeasts and Their Life Cycles . . 1-1

Criteria for Distinguishing Haplophase and Diplophase ......... . 1-8

2 Cultivated Yeasts ............ 2-1

Origin of Cultivated Yeasts....... . 2-1

Top and Bottom Yeasts . . . . . . . . . 2-2

Frohberg and Saaz Type Bottom Fermenters 2-3

Bakers' Yeasts . . . . . . . . . . 2-3

Food Yeasts............. 2-3

3 Sporulation ............. 3-1

Presporulation Medium........ 3- 3-

Media and Methods .......... 3-3

Free Cell Formation ........... 3-4

Unreduced One-Spored Asci . . . . . . . 3-4

Sporulation of Legitimate and Illegitimate

Diploids ............ 3-6

4 Spore Formation in the Colony ......... 4-1

5 Staining Techniques............ 5-1

Dead Cell Stain ... . . . . . . 5 5-1

Lugol's Solution. . . . . . . . . . 5-2

Aceto-orcein and Aceto-carmine . . . . 5-2

Metaphosphate (Volutin) Stain . . . . . . 5-5

Alkali-Toluidine Blue Stain. . . . . . . 5-5

Acid Fast Stain for Yeast Spores . . . . . 5-5

Delafield Spore Stain . . . . . . . 5-7

Weak Killers . . . . . . . . . . 5-7

Conventional Cytological Techniques. . . . 5-8

Materials and Methods . . . . . . . 5-9

Procedures and Schedules ...... . 5-9

6 Cytology................ 6- 6- . .

The Fungal Nucleus. . . . . . . 6 6-1 
The Division of the Centrosome . . . . . 6-5

The Heterochromatin. . . . . . . 6-7

Mechanism of Budding . . . . . . 6-7

The Mitochondria. . . . . . . . . 6 6-10

Mitosis ............ 6- 6-18

Demonstration of Nucleolus, Chromosomes, and Centrosome by Standard Cytological Techniques .......... 6- 6-23

Volutin (Metaphosphate) . . . . . . 6-29

The Transfer of Volutin from Chromosomes to Nucleolus . . . . . . . . . 6-30

"Abnormal" Distribution of Volutin in the Yeast Cell .......... 6 6-38

Volutin and Ribosenucleoprotein Synthesis . 6-39

The Possible Role of Volutin (Metaphosphate) and Nucleic Acids in Conferring Specificity to Cellular Enzymes . . . . 6-40

7 Storage of Reserves in the Cell ....... 7-1

Glycogen ............ 7- 7-2

Stainable Glycogen . . . . . . . . 7-3

Mitochondria ............ 7-5

The Feulgen-Positive Heterochromatin in the Centrosome. . . . . . . 7-6

8 The Induction of Dormancy and the Conditions for Reactivation .......... 8-1

Dormancy. . . . . . . . . 8-1

Storage of Glycogen under Different Conditions ............. 8-2

Dormant Vegetative Cells ........ 8-2

Formation of Fat and Mitochondria . . . 8 8-4

9 Techniques for Microdissection and Preservation of Cultures. ............ 9- 9-1

The Dissecting Needle ........ 9-1

The de Fonbrune Pneumatic Micromanipulator

The Moist Chamber . . . . . . . 9-5

Dissection ........... . . . 9-6

Drying Cultures .......... 9- 9-9

10 Hybridization............. 10-1

Mating Types........... 10-2 
Selection versus Hybridization. . . . . 10-6 Span of Life Cycle . . . . . . . . . 10-6

Hybridization Procedures for the Practical Yeast Worker. . . . . . . 10-7

Hybridization in Unfavorable Media . . . 10-7

11 Genetical Characters. ........... 11-1

Terminal Characters. . . . . . . . 11-1

Rough Colonies. . . . . . . . . . 11-4

Quantitative Characters ........ 11-5

Lethal Genes . . . . . . . . . . 11-6

Biochemical and Physiological Characters . 11-6

Fermentative Ability. . . . . . . 11-7

The Fermentation Test . . . . . . . 11-7

Galactose-Melibiose Combinations .. . 11-8

Tests for the Presence of Galactose in

Fermentation Tubes after Ten Days. . . 11-14

Raffinose ............. 11-14

Production of $\mathrm{CO}_{2}$ When Different Sugars

are Used in the Fermentation Tubes. . . 11-15

Alpha Glucosides.......... 11-15

Vitamin, Amino Acid, and Nucleic Acid

Component Deficiencies . . . . . 11-16

Mating Type Specificity. . . . . . . . 11-17

Suppressors ........... 11-17

Plus and Minus Modifiers . . . . . . 11-18

12 A Comparison of Sexual Reproduction in Yeasts and Other Microorganisms. . . . . . . 12-1

Mucors ............. 12-2

Schizomycetes ........... 12-2

Hymenomycetes ... . . . . . . 12-3

Paramecium .......... 12- 12

Smuts . . . . . . . . . . . . 12-8

Rusts . . . . . . . . . . 12-10

Neurospora. . . . . . . . . 12-12

Venturia ................ 12-15

Glomerella . . . . . . . . . . 12-16

Penicillium and Aspergillus ...... 12-17

Cross-Fertilization in Saccharomyces and

Other Organisms .......... 12-18

Self-sterility alleles ....... 12-18

Sexual Dimorphism ....... 12-18

Plus-Minus Factors ....... 12-18 
Neurospora . . . . . . . . . 12-18

Hymenomycetes ......... . 12-19

Paramecium ........... 12-19

The Adaptability of Yeasts for Uniting.

Biochemistry and Experimental Cytology 12-19

The Necessity for Direct Tetrad Analyses . 12-20

13 Segregation, Variation and Recombination. ... 13-1

Segregation of Mating Type Alleles . . . . . 13-1

Segregation of Colonial Types . . . . . . 13-1

Variation and Colonial Types ..... . 13-5

Variations in Relation to Life . . . . . . 13-6

Recombination .......... 13-7

Transformation of Saccharomyces into

Torulopsis . . . . . . 13-8

The Instability of the Mating Types on Subculture ........... 13-8

14 Variation in Growth and Fermentative Capacity. 14-1

The "D" Family ............. 14-1

Variation in Growth and Gas Production. . . 14=5

Gas Evolved Versus Growth . . . . . . 14-6

Range of Variability ......... 14-13

15 Depletion Mutations .......... . 15-1

Relation of Pink Color to Adenine and

Methionine Synthesis . . . . . . 15-1

False Mutations . . . . . . . . . . 15-4

Depletion Mutations . . . . . . . 15-4

16 Adaptation in a Yeast Unable to Utilize Glucose 16-1

Adaptation to Glucose Utilization .... . 16-2

Adaptation to Glucose on Lactate Medium . . 16-10

Adaptation on Standard Peptone Glucose

Broth. .............. 16-10

Crosses and Backcrosses with Strain No. $97 \quad 16-10$

The Mechanism of Adaptation . . . . . 16-10

17 Back-Mutation and Progressive Mutation . . . 17-1

Adaptive Fermentation of Galactose without

Formation of New Cells . . . . . . 17-1

Adaptation by "Back-Mutation" followed by

Selection ............ 17-1

Progressive Mutation . . . . . . . 17-4

The Origin of Lactose Fermenters . . . . 17-5

Unequal Crossing Over and Evolution . . . 17-5

A Hybrid between S. Cerevisiae and S.

Fragilis

$17-8$ 
18 Development of Fertile Breeding Stocks. . . . 18-1

Fermentative Characters ....... 18-1

Vitamin Deficiencies. . . . . . . 18-3

Haploid Non-Fermenters from a Weak Fermenter by a Strong Fermenter Hybrid. . 18-5

19 Regular Mendelian Inheritance. . . . . . . . 19-1

A Gene-Controlled Adaptive Enzyme ... . 19-1

The Origin of a Nonfermenter of Galactose . 19-1

Tetrad Analysis of an $\alpha$ G/ag Hybrid . . . . 19-1

Mendelian Inheritance of Genes Affecting

Fermentative and Vitamin-Synthesizing

Ability ............ 19-4

20 Irregular Ratios ............. 20-1

Non-Mendelian Inheritance of Ability to Ferment Galactose . . . . . . . . 20-1

Non-Mendelian Inheritance of Ability to

Ferment Melibiose........ 20-4

Irregular Ratios in Other Organisms . . . 20-6

The "Good Gene" Concept . . . . . . . . 20-7

Graded Impairment of Gene Function . . . 20-8

21 The Effect of the Medium on Apparent VitaminSynthesizing Deficiencies ........ 21-1

A Comparison of Two Synthetic Media. . . 21-1

The "Vitamin" Pedigree ......... 21-3

Hybrids between Yeasts Supposedly Differ-

ent in Vitamin-Synthesizing Ability . . . 21-4

Vitamin-Synthesizing Ability of Supposedly

Deficient Yeasts in a Natural Medium. . 21-4

Continuous Observation of Growth . . . . . 21-4

The Inadequacy of the Criterion, Equal

Increments of Growth for Equal Incre-

ments of Vitamin. . . . . . . 21-6

The Effect of Multiple Deficiencies of the

B Vitamins .......... 21-7

The Relation between Viability of Cells

and Inability to Grow on Deficient

Medium ........... 21-8

22 The Separate Functions of Recessive and

Dominant Alleles in Adaptation to Pantothenate

Deficiency ............. 22-1 
The Effect of the Environment on Adaptation to a Deficient Medium . . . . . . 22-1

Slow Adaptation to the Absence of Pantothenate by a Pantothenateless

Mendelian Recessive . . . . 22-4

Adaptation to the Absence of Pantothenate by a Regularly Segregating, Slowly

Adapting Culture . . . . . . . . 22-9

Demonstrations of the Genic Nature of the Adaptation . . . . . . . . . . 22-12

Synthesis of Pantothenate by Gene B . . . 22-16

Alternate Route of Synthesis . . . . . . 22-16

Mutation in the Presence of Pantothenate . . 22-18 Growth of the Recessive Culture in the

Absence of Pantothenate. . . . . . 22-22

Mutation in the Absence of Pantothenate . 22-24

Randomness of Mutation in the Absence of

Pantothenate . . . . . . . . . . 22-27

Competition Between Adapted and Unadapted

Cells in Limiting Concentrations of

Pantothenate........... 22-31

The Functions of Recessive and Dominant

Alleles in Adaptation . . . . . . . 22-36

Conclusions ... . . . . . . 22-38

23 The Balanced Lethal - Saccharomycodes . . . 23-1

The Accumulation of Lethal Genes . . . 23-5

Reversion of $n$ to N. . . . . . . 23-6

24 Chromosome Maps............ 24-1

Tetrad Analysis and Centromere Linkage. . 24-1

25 Proof of Interchromosomal Transfer of Hereditary Material by Tetrad Analysis . . . . . 25-1

Conversion of Recessives to Dominants in the Heterozygote

26 Irregular Segregations as Supporting Evidence for Gene Conversion . . . . . . . . . 26-1

The Origin of Nonfermenters of Maltose . . 26-9

Genetical Analysis of Transformed ME

Alleles ........... 26-9

Tests for Cytoplasmic Effects by Dr.

Doudoroff and our Staff. . . . . . 26-13

Conclusions from Dr. Doudoroff's Experiments. 
Genetical Analysis of the Four Haplophases

from a Transformed Triply Heterozygous Ascus . . . . . . . . . . . 26-26

Backcross to Fermenter G ME MA . . . . . 26-27

Proof that the Multiple Factor Hypothesis

Cannot Explain the Irregular Ratios. .

$26-28$

The Significance of Conversion in the Long-

Term Adaptation to Galactose by

Saccharomyces . . . . . 26-32

Evidence for Mutation of $g$ to $G \ldots 26-33$

High $g$ to $G$ Mutation Rate Among the

Descendents of $g_{S}$ Parents . . . . 26-34

Non-Mendelian Segregations . . . . 26-35

Hybrids of $\mathrm{g}$ By $\mathrm{g}_{\mathrm{S}}$ Clones ...... 26-35

Evidence in Well-Marked Hybrids that Con-

versions Occur in the Heterozygote . . 26-39

27 Examples of Apparent Cytoplasmic Inheritance . 27-1

Heterosis and "Running Out" of Hybrid

Vigor ............. 27-2

The Effect of Nuclear Division without Cytoplasmic Division........ . 27-4

Adaptation without Detectable Change of

Genome . . . . . . . . 27-6

Haplophase Variability vs. Diplophase

Stability. . . . . . . . . . 27-8

The Plasmagene Hypothesis . . . . . . 27-9

A. The Paracrinkle Virus . . . . . . 27-9

B. The Kappa Substance . . . . . . 27-11

C. Antigenic Plasmagenes in Paramecium . . . . . . . . . . 27-12

D. Adaptive Enzyme Formation ... . 27-12

Gene-Dosage and Gene-Product . . . . . . 27-15

Dauermodifikationen and Depletion Muta-

tions .............. 27-15

"Nongenic" Modifications ....... 27-15

Cytogenes. . . . . . . . . . 27-16

Haplophase Variability and Diplophase

Stability in terms of the Plasmagene

Hypothesis .......... 27-17

28 The Bearing of the Data from Yeast Genetics on the Current Concept of the Gene . . . . 28-1

Gene -to-Gene Transfer of Gene Component. 28-4

The Tripartite Nature of the Gene. . . . 28-4

Degradation of the Gene . . . . . 28-4 
Mendelism as a Special Case of the General Theory. ........ 28-4

Nutrition and the Integrity of the Genome . 28-5

Linear Differentiation Along the Chromosome ............... $28-6$

The Linear Chromosome as the Seat of

Orderly Reáctions ....... 28-7

Linear Differentiation and Position Effect. 28-8 


\section{Chapter 1}

\section{SOME IMPORTANT YEASTS AND THEIR LIFE CYCLES}

Yeast is a general term for any growth that appears in a fermenting fluid. Whenever grape juice is collected, the growth that accumulates and settles on the bottom is yeast; the film that forms on the top of a pickle barrel is also yeast. The term does not have any botanical connotation; it merely describes a sediment or a film, containing a great variety of bacteria and filamentous fungi along with true yeasts. True yeasts are non-filamentous, single celled microorganisms that generally grow in sugary solutions; many of them produce spores. Their non-filamentous nature distinguishes them from the other fungi; their larger size distinguishes them from bacteria. There are two genera that are very easily differentiated; (1) Saccharomyces and (2) Schizosaccharomyces.

The cells of Saccharomyces cerevisiae exist in both haplophase and diplophase (fig. 1-1). We found that this species possesses two mating types in the haplophase, and that haploid cells of different mating type copulate to produce diploid cells. Kruis and Satava (1918) in Prague, and Winge (1935) in Copenhagen showed that the standard vegetative cells of $S$. cerevisiae are diploid, produced by copulation of two spores or gametes derived from spores. The diploid nuclei undergo reduction at spore formation to produce four haploid ascospores. The large, ellipsoidal vegetative yeast cell is produced by the fusion of two round haploid gametes derived from ascospores. Winge established the basic facts of this life cycle by a classical series of observations on the germination of ascospores and fusion of haploid cells. When spores of Saccharomyces are planted in culture medium each spore germinates to produce a cluster of round haploid cells which undergo a period of vegetative growth of variable length. There may be an extended haplophase before copulation begins and cell fusion reconstitutes the diplophase.

Schizosaccharomyces (fig. 1-2) was originally described by Beijerinck (1894). This organism has long, cylindrical cells which become asci (spore sacs) usually containing elght haploid spores each. The spores often fuse in pairs on germination to produce a long diploid cell. The Schizosaccharomyces described by Beijerinck (1898) produced nonsporulating variants rather readily, suggesting that Schizosaccharomyces may have a lengthy haplophase like Saccharomyces. Further investigation of this interesting possibility may yield profitable results.

Saccharomyces increases in numbers by budding; Schizosac- 


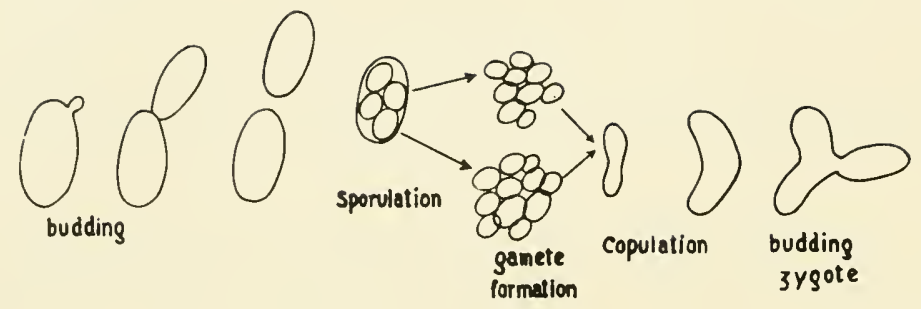

Fig. 1-1 Life Cycle of Saccharomyces.

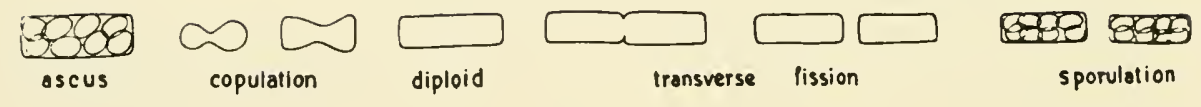

Fig. 1-2 Life Cycle of Schizosaccharomyces.

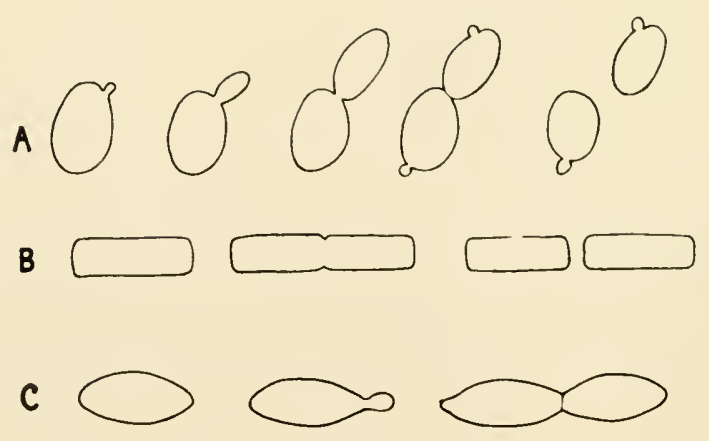

Fig. $1-3$ (A) Budding (B) Transverse Fission (C) Terminal Budding. 
Table $1-1$

General Relationships of Three Genera

\begin{tabular}{|c|c|c|}
\hline Saccharomyces & Saccharomycodes & $\begin{array}{c}\text { Schizosaccharo- } \\
\text { myces }\end{array}$ \\
\hline 1. Budding & $\begin{array}{l}\text { 1. Terminal } \\
\text { budding }\end{array}$ & $\begin{array}{c}\text { 1. Transverse } \\
\text { fission }\end{array}$ \\
\hline 2. $30^{\circ} \mathrm{C}$. optimum & 2. $\quad---$ & 2. $37^{\circ} \mathrm{C}$. optimum \\
\hline 3. Waxy spore & 3. Waxy spore & 3. Starchy spore \\
\hline $\begin{array}{l}\text { 4. Store glycogen; } \\
\text { no starch }\end{array}$ & $\begin{array}{l}\text { 4. Store gly- } \\
\text { cogen; no } \\
\text { starch }\end{array}$ & $\begin{array}{l}\text { 4. Store starch; } \\
\text { no glycogen; } \\
\text { no wax }\end{array}$ \\
\hline 5. 4-spored & $\begin{array}{l}\text { 5. } 2 \text { pairs of } \\
\text { spores per } \\
\text { ascus }\end{array}$ & $\begin{array}{l}\text { 5. 4- and } \\
\text { 8-spored }\end{array}$ \\
\hline $\begin{array}{l}\text { 6. Long haplo- } \\
\text { phase }\end{array}$ & 6. No haplophase & $\begin{array}{l}\text { 6. Long haplo- } \\
\text { phase }\end{array}$ \\
\hline 7. Cannot use & --- & 7. Use dextrin \\
\hline
\end{tabular}

charomyces by transverse fission (fig. 1-3). Schizosaccharomyces is a tropical yeast with optimum growth at $37^{\circ} \mathrm{C}$.; Saccharomyces has an optimum at $30^{\circ} \mathrm{C}$. Native tropical beers are usually made with Schizosaccharomyces and temperate zone beers are made with Saccharomyces. Saccharomyces spores have a waxy cover and for that reason are "acid fast." This means that when they are stained with a strong dye they retain the stain after the stained cells have been washed with a dilute acid. Schizosaccharomyces spores are starchy. They stain blue-black with iodine (Schizosaccharomyces Frontispiece) and are not acid fast. They have no waxy coating and they contain starch. This distinguishes them from the Saccharomyces which do not store starch. No Saccharomyces can utilize dextrin but Schizosaccharomyces possesses the ability. This is important industrially for much of the carbohydrates in some mashes are dextrins and therefore unavailable to the customary yeasts; a yeast capable of utilizing dextrins might be very useful in making alcohol. The Schizosaccharomyces are generally weak fermenters, 
usually incapable of acting on galactose. This genus does not store glycogen. Saccharomyces are generally 4-spored. The Schizosaccharomyces are 4-or 8-spored.

Saccharomycodes (fig. 1-4) has a unique characteristic. The cells multiply by terminal budding (fig. 1-3) which amounts to a compromise between the budding and binary fission. The cells are lemon-shaped with buds on each end and with a cross wall between bud and mother cell. The bud in the Saccharomyces is never terminated by a cross wall, it simply increases in size and is cut off by a very narrow constriction. The spores of Saccharomycodes are not starchy but turn yellow with iodine solution and are acid fast.

There is no specific orientation of spores in either Saccharomyces or Schizosaccharomyces. In Saccharomycodes each cell becomes an ascus containing two pairs of spores (one pair at each end). Each pair of spores copulates just as the cell germinates and again produces the regular diploid cell. There is no opportunity for haplophase multiplication. This means that there is no natural haplophase in Saccharomycodes.

The different species of Saccharomyces differ principally in their ability to ferment different sugars. The sugars dextrose, mannose, fructose, galactose, sucrose, maltose, lactose, melibiose, and raffinose are generally used in a study of this type. Most of the different species of Saccharomyces are distinguishable from one another by their action on these sugars. The commonest is S. cerevisiae (the Saccharomyces of beer). It ferments all these sugars except lactose and melibiose, and ferments only one-third of raffinose.

Raffinose is a tri-saccharide with the following constitution:

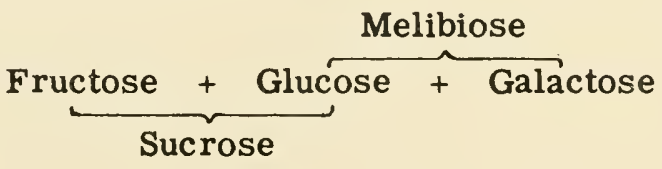

Any yeast which can hydrolyze sucrose should be able to ferment one-third of raffinose leaving one molecule of melibiose. The diagnostic fraction is the melibiose. S. carlsbergensis ferments raffinose completely and therefore ferments melibiose also. S. cerevisiae ferments one-third of raffinose; it does not ferment melibiose but can ferment sucrose. These species are differentiated by their ability to ferment melibiose. We have made hybrids between them and have found that this difference is due to a single gene.

Saccharomyces lactis alpha is able to ferment lactose but is not able to ferment maltose. Kluvyer established the rule that there are no Saccharomyces which can ferment both lactose and maltose. $\mathrm{S}$. lactis probably has its origin in contaminations in milk, it may be relatively recent in origin and its origin may be associated with 


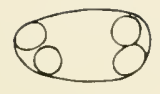

ascus

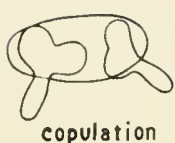

copulation

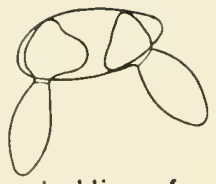

budding of<smiles>C1CCC2CCCCC2C1</smiles>

terminal budding
$(82$

sporulation

Fig. 1-4 Life Cycle of Saccharomycodes.
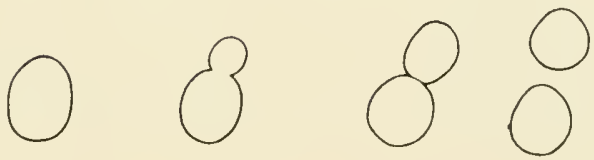

Fig. $1-5$ Life Cycle of Torulopsis.

0

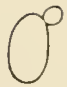

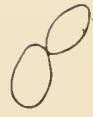

budding
$00 \Omega$

copulation

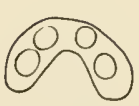

sporulation

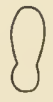

spore germination

Fig. 1-6 Life Cycle of Zygosaccharomyces (Diplophase Only in $Z_{y g o t e}$ ).

$1-5$ 
Table $1-2$

Permentative Ablitities of Various Yeasts

\begin{tabular}{|c|c|c|c|c|c|c|c|c|}
\hline & & 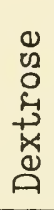 & 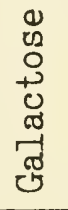 & 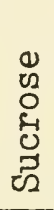 & $\begin{array}{l}0 \\
0 \\
0 \\
+ \\
+ \\
\stackrel{\infty}{\infty}\end{array}$ & 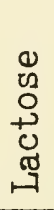 & 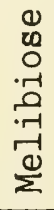 & 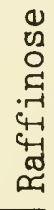 \\
\hline \multirow[t]{12}{*}{ Saccharomyces } & cerevisiae & + & + & + & + & - & - & $\frac{1}{3}$ \\
\hline & carlsbergensis & + & + & + & + & - & + & + \\
\hline & chodati & + & + & - & + & - & - & - \\
\hline & lactis alpha & + & + & + & - & + & - & $\frac{1}{3}$ \\
\hline & microellipsoideus & + & + & + & - & - & + & + \\
\hline & bayanus & + & - & + & + & - & - & $\frac{1}{3}$ \\
\hline & globosus & + & + & - & + & - & - & - \\
\hline & fragilis & + & + & + & - & + & - & $\frac{1}{3}$ \\
\hline & pastorianus & + & + & + & + & - & + & + \\
\hline & lactis beta* & + & + & + & + & - & - & $\frac{1}{3}$ \\
\hline & validus & + & + & + & + & - & + & + \\
\hline & turbidans & + & + & + & + & - & - & $\frac{1}{3}$ \\
\hline \multicolumn{2}{|c|}{ Zygosaccharomyces barkeri } & + & - & + & - & - & - & $\frac{1}{3}$ \\
\hline & marxianus & + & - & + & - & - & - & $\frac{1}{3}$ \\
\hline & bailii & $t_{i}$ & - & - & - & - & - & - \\
\hline \multicolumn{2}{|c|}{ Torulopsis utilis } & + & - & + & - & - & - & $\frac{1}{3}$ \\
\hline \multicolumn{2}{|c|}{ aeria } & - & - & - & - & - & - & - \\
\hline \multicolumn{2}{|c|}{ molischiana } & + & - & - & - & - & - & - \\
\hline \multicolumn{2}{|c|}{ Saccharomycodes ludwigii } & + & - & + & - & - & - & $\frac{1}{3}$ \\
\hline \multirow{3}{*}{\multicolumn{2}{|c|}{$\begin{array}{c}\text { Schizosaccharomyces octosporus } \\
\text { pombe }\end{array}$}} & + & - & - & + & - & - & $\frac{1}{3}$ \\
\hline & & + & - & + & + & - & - & $\frac{1}{3}$ \\
\hline & & + & - & + & + & - & $\frac{1}{2}$ & $\frac{2}{3}$ \\
\hline
\end{tabular}

*Peptonizes milk 
the collection of milk by humans. As far as I know, there are no common yeasts existing in the udder, and yeasts are not generally associated with milk in the body. S. lactis beta does not ferment lactose but digests milk proteins; it may have a similar recent origin but depends principally on the protein rather than the carbohydrate component of the milk for its nutrition.

Saccharomyces cerevisiae and $\mathrm{S}$. carlsbergensis may be very ancient. They are adapted to grow in the natural sugary solutions which contain enough acid to inhibit most bacteria and enable the yeasts to get started before other microorganisms. They are found on dates or figs, apricots, grapes, apples, and pears, or any other kind of spoiled fruit.

Saccharomyces, Schizosaccharomyces and Saccharomycodes are "perfect" yeasts. They are able to produce ascospores and, therefore, have a complete life cycle, haploid to diploid.

There are many "imperfect" yeasts, so-called because they do not have a complete sexual cycle. Torulopsis, Cryptococcus, and Torula (fig. 1-5) are synonyms for a genus which produces vegetative cells that never sporulate. Torulospora, a very similar genus, sporulates but is otherwise close to Torulopsis. These strains are generally distinguishable from the perfect Saccharomyces. The specific names are convenient means of classifying these organisms, but the classification of imperfect fungi has limited phylogenetical significance.

Torulopsis is adapted to grow best in a well-aerated medium. Most species of this genus ferment only a few sugars; some even fail to ferment glucose. The famous Torulopsis utilis was discovered by Henneberg (1926) as a contaminant of baking yeast plants in Germany. Henneberg realized this new yeast provided a very rapid means of synthesizing proteins from ammonia and sugar.

Another organism derived from the haplophase of Saccharomyces is Zygosaccharomyces (fig. 1-6). It produces cells resembling rather closely the haplophase of Saccharomyces. Under proper conditions the vegetative cells of Zygosaccharomyces (which are obviously haploid) copulate with each other to produce the diplophase; zygotes and spores are produced immediately in the dumbbell shaped ascus. Some species are adapted to grow in very hypertonic sugar solutions. They are important industrially as contaminants in honey, which may contain as much as 65 per cent sugar. Winge and Laustsen (1939) produced hybrids between Zygosaccharomyces and Saccharomyces by spore to spore matings; this significant fact shows the close relationship between these genera.

There are a large number of minor Saccharomyces which can be used as sources cf genetical characteristics: Saccharomyces bayanus is an interesting species; instead of elliptical cells, like $\mathrm{S}$. cerevisiae, it has cylindrical cells. This character segregates 
in a Mendelian manner in S. cerevisiae by S. bayanus hybrids. Saccharomyces bayanus does not ferment galactose but otherwise resembles S. cerevisiae in fermentative ability. Saccharomyces pastorianus is responsible for bitter taste, disagreeable odor, and turbidity in beer. It is a bottom fermenting yeast producing cylindrical cells. Saccharomyces validus is a top fermenting yeast with long cylindrical thread-shaped cells producing turbidity in beer. Saccharomyces turbidans grows well over a wide temperature range producing elliptical cells and a very turbid broth. It is also undesirable in beer because of the turbidity.

\section{CRITERIA FOR DISTINGUISHING HAPLOPHASE AND DIPLOPHASE}

Winge (1935) distinguished clearly between haplophase and diplophase yeast cultures, and we have corroborated his observation, adding the concept that genes controlling mating type specificity segregate at meiosis to produce two kinds of haplophases. I shall summarize all the arguments, Winge's reinforced by ours, for distinguishing haplophase and diplophase. Over four-fifths of the cultures which one encounters are easily characterized by microscopic examination. They are either obviously haploid or diploid, as shown simply by size, shape and aggregation of the cells. The reasons for classifying them are as follows:

a. The large vegetative cell, which we call "legitimate diploids" (fig. 1-7), produce viable 4-spored asci. These spores germinate to produce smaller, usually round, cells which we call "haploid." The latter multiply vegetatively, generally maintaining their specific cell-shape and size.

b. Two of these smaller cells may fuse to produce a zygote which on budding produces a large "diploid" cell capable of vegetative multiplication. While the large cell is undergoing vegetative reproduction, it retains its characteristic ellipsoidal shape and size. Under certain conditions this diploid cell can be induced to sporulate. Spores from it in turn produce haploids and the process can be repeated indefinitely.

c. The large cells which we recognize as diploids are extraordinarily stable in their genetical characteristics when they are grown under conditions in which sporulation does not occur. Transferring the cultures every forty-eight hours in broth is generally sufficient to maintain the vegetative diplophase. Colonies produced by plating out are not sectored; the plates do not show colonial variants. However, when haplophase (single ascospore) cultures of any age are plated out, a variety of colonial variants appears on the plate or the giant colonies are sectored. These facts are consistent with the view that the large cells are diploid, thus minimizing the num- 
ber of variants which may appear, while in the haplophase most variants become apparent and are easily discovered.

d. When the diploid cells sporulate to produce haploid cells, tetrad analysis of the 4 spores has proved that a reduction division occurs. Hybrids heterozygous for as many as 12 alleles have been analyzed, all of which segregated regularly in the ascus.

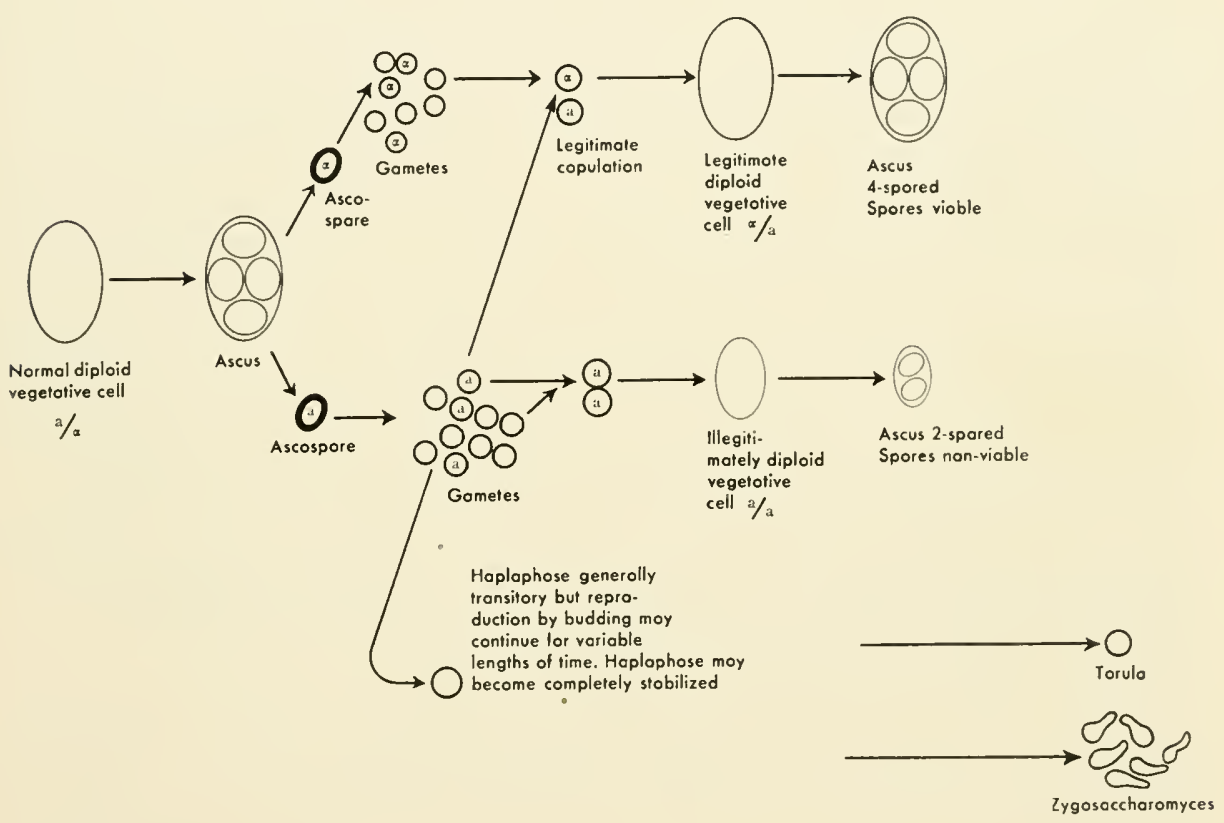

Fig. $1_{-7}$ Legitimate and Illegitimate Diploids.

Extensive pedigrees of many generations are available for numerous characters, and four linkage groups have been demonstrated.

These facts prove conclusively that the terms haplophase and diplophase can be used as definitely in speaking of yeasts as of organisms in which the cytological evidence is more complete. It is necessary to transfer diploid cultures frequently if they are capable of producing spores in order to maintain the original characteristics of the cultures. If sporulation occurs, new genotypes are segregated and variability appears. In this connection it is interesting to observe that industrial yeasts are usually grown in broth, and transfers are made by pipetting a large proportion of the sediment from the original culture into the transfer. This technique minimizes the possibility of variation. 


\section{Chapter 2}

\section{CULTIVATED YEASTS}

\section{ORIGIN OF CULTIVATED YEASTS}

Most domesticated plants differ markedly from their natural ancestors. Domesticated plants and animals are specialized, usually at the expense of characters which make for survival under conditions of natural competition. Corn seeds are accidentally sown by the hundreds of thousands, but corn plants are practically never found in any place except a cultivated field, for this domesticated plant is incapable of competing with wild plants under natural conditions. Wild plants are in genetical balance with the environment. Domestication has selected specialized variants in which the characters essential to competition in the natural state have been diminished in potency. Wild yeasts are adapted to grow in sugary, sour fluids, such as are found in nature in ripe bursted fruits, like grapes, apricots, and figs, in the nectar of flowers, and other places where sugary, slightly acid solutions are available. They obtain energy largely by the fermentation of sugar, and produce as byproducts of growth carbon dioxide and alcohol.

These two by-products have been the basis for their domestication. Yeasts are used to make bread, because of their ability to lighten the loaf by filling it with bubbles resulting from the production of carbon dioxide; and the alcohol produced by the fermentation of sugars led to their use in brewing. Yeasts are also adapted to the synthesis of inexpensive protein which may become an important source of human food. They are able to synthesize B-vitamins, of which they are the richest natural source. Their cultivation under modern mass production factory methods may make the production of some sources of human food partially independent of weather and climate.

There are a variety of genetical processes by which cultivated yeasts may have originated from wild yeasts. Improvement of yeasts has often been achieved by critical study of a great variety of cultures followed by selection of the cultures showing desirable characters. The early workers recognized that the variability of yeast cultures made it possible to obtain a great variety of yeasts from one original culture, but they found that cultures with desirabie characters were sometimes relatively variable. Our ability to recognize the variable and stable phases in the life cycle of yeasts enables us to control the stability of our derived stocks. 


\section{TOP AND BOTTOM YEASTS}

Cultivated yeasts include (1) bottom and top fermenting beer yeasts, (2) distillery yeasts, (3) baking yeasts, (4) wine yeasts, (5) food yeasts including vitamin-, protein-, or fat-producing forms. Some yeasts belonging to the genera Torulopsis, Rhodotorula, and Mycoderma, are nonfermenters, but most yeasts are fermenters. In top fermenting beer yeasts, distillery yeasts, or baking yeasts the yeast is suspended in the culture broth and finds its way into the foam. Bottom fermenting wine and beer yeasts produce a heavy sediment of yeast at the bottom of the container and a clear supernatant fluid. Anomolous yeasts (pickle barrel yeasts) form a membrane over the nutrient solution after 24 hours, being neither top nor bottom fermenters, in fact, they are generally nonfermenters as their growth in the direct contact with the air would suggest. Yeasts not belonging to the anomolous group may form a membrane after several weeks. Typical top fermenting yeasts ferment with a yeasty foam, sporulate abundantly, and ferment only one-third of the raffinose molecule. The transformation of a bottom fermenting beer yeast into a typical top fermenting bakers' yeast (and the reverse) has been reported frequently. Top fermenting yeasts produce "stormy" fermentations with rapid growth at a relatively high optimum temperature (300) as compared with a typical bottom fermenter. Most top fermenters characteristically ferment only one third of the raffinose molecule; they are unable to ferment melibiose. When shaken with water they produce a milky fluid while an agglutinated bottom fermenter cannot be dispersed. They grow more rapidly and are much more vigorous and generally more viable than bottom fermenters.

A typical bottom fermenter may have an optimum temperature of around $25^{\circ} \mathrm{C}$. to $26^{\circ} \mathrm{C}$., but may ferment in beer vats at a much lower temperature $\left(5^{\circ} \mathrm{C}\right.$. to $8^{\circ} \mathrm{C}$.). The cells are characteristically clumped and the supernatant fluid is clear and when shaken up the clumped cells do not form an even suspension but the clumps settle out quickly. Small amounts of alcohol are produced by the inefficient fermentation and a pleasant aroma is characteristic. Bottom fermenters usually produce a membrane covering the nutrient broth sooner than top fermenters, possibly because of their general habit of coherent growth. The original bottom fermenting yeasts were capable of fermenting melibiose, 1.e., of fermenting raffinose completely, but it seems unlikely that this is a characteristic of all bottom fermenters. Bottom fermenting beer yeasts are relatively weak fermenters, producing small amounts of alcohol. 


\section{FROHBERG AND SAAZ TYPE BOTTOM FERMENTERS}

Bottom beer yeasts are again subdivided into vigorous and weak fermenters. The vigorous fermenters are called Frohberg yeasts; the weak fermenters are Saaz yeasts. There are many types of the Frohberg yeasts, but only a few Saaz yeasts are known. Saaz yeasts are generally low in vigor and therefore not desirable for most purposes. Frohberg yeasts have long ellipsoidal cells and are obviously diploid. Saaz cells are probable haploid and are much more variable with occasional giant cells. Frohberg yeasts form few dauer cells; Saaz cells are less viable than Frohberg. The optimum temperature for both yeasts is $25^{\circ}$ to $26^{\circ}$; the maximum is $35^{\circ}$ for Frohberg and $33^{\circ}$ for Saaz. Saaz yeast is especially sensitive to alcohol.

Many haplophase yeasts resemble the characteristic bottom fermenting yeasts in being autoagglutinable and weakly fermentative, while most diplophase yeasts are top fermenters.

\section{BAKERS' YEASTS}

Distillery and bakers' yeasts are top fermenters. There are no clear cut differences between top fermenting beer yeasts and distillery and bakers' yeast, but they are not generally interchangeable. A good bakers' yeast has the following characteristics: (1) keeping quality, it does not autolyze nor lose viability excessively when held in a cake for a few days at room temperature; (2) baking strength, it causes dough to rise quickly; (3) high yield, it transforms the maximum amount of nutrients in the fermentors into yeast in the shortest possible time.

\section{FOOD YEASTS}

Torulopsis utilis is widely used as a food yeast, but other standard variants of Saccharomyces have also been used for this purpose. Torulopsis utilis was originally selected by Henneberg as a food yeast when he observed that it was capable of crowding out bakers' yeast in plants engaged in the production of baking yeast. Torulopsis is not a fermenter and compressed bakers' yeast containing a large proportion of this organism causes the dough to rise very slowly. Complaints from bakers resulted in the original isolation of the Torulopsis from the abnormally slow yeasts. Bakers' yeast is grown in a heavily aerated nutrient medium. Generally speaking, Saccharomyces is adapted to relatively anaerobic conditions and heavy inoculations are necessary in industrial practice to insure the predominance of Saccharomyces over chance inoculation by the more aerobic Torulopsis. When the need for protein became acute in Germany during the first World War, Henneberg proposed that pure cultures of Torulopsis be used as a source. 
Torulopsis is adapted to grow in well-aerated nutrients. Bakers' yeast is grown in heavily aerated broth to prevent the transformation of sugar into alcohol and carbon dioxide. Unless it is aerated Saccharomyces lies on the bottom of the culture tank and changes the sugar into glycogen and alcohol but produces little yeast. Torulopsis does not store much glycogen and transforms sugar and ammonia into protein with greater efficiency than Saccharmoyces because it is not a fermenter.

The skill that Henneberg showed in taking an industrially useless menace and transforming it into a source of protein may have had very serious repercussions for us because the Germans might not have been able to continue fighting nearly so long if they hadn't had this source of nutrient. During the Second World War, they produced about one hundred times as much dried yeast as we did. Their population was notably free from B-vitamin deficiencies at the end of the war. 


\section{Chapter 3}

\section{SPORULATION}

\section{PRESPORULATION MEDIUM}

Sporulation in yeasts was probably first studied by deSeynes (1868), who reported that round spores found "in the surface of the water" germinated to produce elongated cells in a mixture of wine and water, but that these elongated cells produced spores again when transferred to a more dilute medium. Rees (1869) found that when yeast was planted on the cut surface of various vegetables (cooked or raw), growth continued until the fourth day, when budding stopped. On the fifth day the vacuoles in the cell disappeared and the protoplasm became coarsely granular. Spore formation occurred regularly on the sixth day. Spores also appeared on the sixth day if fresh yeast from beer vats or wine must was transferred to the cut surface of carrot or potato. Rees did not find spores in old lagering vats nor in compressed yeast, but washed yeast from these sources placed in a beaker in a layer approximately $4 \mathrm{~mm}$. thick and protected from dust sporulated abundantly in about three weeks.

Engel (1872) devised the classic method of inducing yeasts to sporulate by transferring the cells from a nutrient broth to plaster of Paris blocks. Hansen (1883) used this method successfully. Graham and Hastings (1941) have improved the technique considerably by making the gypsum slants in test tubes, thus reducing the danger of contamination. Beijernick (1898) obtained yeast spores by trans ferring the suspensions to slants made from agar that had been fermented and subsequently washed. He preferred this method to the gypsum block, probably because less contamination was encountered.

Hansen and Beijerinck considered it essential that the yeast cells be well nourished before transferring them to the aerating surface, but they did not specify any nutrients aside from beer wort or grape must. They believed that sporulation occurred when the "well-nourished" cells were deprived of all nutrients, and Hansen's blocks and Beijerinck's purified agar were flooded with distilled water. They agreed that starvation conditions and an abundance of oxygen were essential.

Gorodkowa (1908) developed an agar containing 1 per cent peptone and $\mathbf{0 . 2 5}$ per cent glucose. On this medium sporulation occurred after 3-4 days. She showed that on a similar medium containing 5 per cent glucose no spores were formed, indicating that the "star- 
vation" conditions essential to sporulation involved primarily the sugars in the substrate rather than other components. Welten (1914) challenged the view that starvation was essential to sporulation. He found that on prune extract agar, yeasts sporulated well. He even doubted the necessity for oxygen, since in his experiments colonies imbedded in the agar sporulated as well as those on the surface. He found that yeasts grown in pear extract or beer wort did not sporulate so well as those grown on prune extract agar, but sporulation occurred when the washed yeast grown on prune extract was placed on glass plates, filter paper, or sterile washed agar slopes, if a drop of prune extract were added. If no prune extract were added, no spores were produced. Gorodkowa's and Welten's work proved that starvation alone is not the complete explanation of the phenonenon. Welten showed further that acidity of the medium in which sporulation occurred was important, no spores occurring in an alkaline milieu. Welten also found that more spores were produced in concentrated than in dilute prune extract, also that a small amount of $\mathrm{MgSO}_{4}$ aided sporulation. Young cells were not essential; those 3-4 days old sporulated better than those 1-2 days old.

Baltatu (1939) found that spores were produced by mycodermas in grape juice but usually germinated immediately, so that they could be detected only by periodic examination of the culture. The addition of very small amounts of acetic acid and grape juice to the water in which the gypsum blocks were placed increased sporulation.

Mrak, Phaff and Douglas (1942) discovered that many yeast sporulated well on slopes of an agar medium containing a mixture of vegetable (cucumber, beet, potato and carrot) extracts. This is clearly a medium containing only a small amount of sugar, which is probably soon exhausted to about the concentration of Gorodkowa's.

Nickerson and Thimann (1942) found that conjugation and sporulation occurred more abundantly in a Zygosaccharomyces when many dead cells were present and postulated some stimulating substance derived from the dead cells. Maneval (1942) found that sporulation occurred in Saccharomyces on the outside of the compressed yeast cakes. Nickerson and Thimann also discovered that an extract from Aspergillus increased conjugation and sporulation, and later (1943) suggested that riboflavin and sodium glutarate were the substances in the extract responsible for the stimulation.

The general conclusions from these data are that a specific presporulation nutrient is essential to abundant sporulation. If this nutrient is satisfactory and the sugar content of the medium low enough, sporulation may occur even on the agar slant, as in Gorodkowa's or Mrak's media. Generally, the yeast should be removed from the substrate, especially if its sugar content is high, and placed on gypsum. The water saturating the gypsum slant should be acid and 
should contain by-products of yeast growth and possibly some sub stances produced by the death and disintegration of yeast cells.

Genetical analysis requires an abundance of large viable 4 spored asci, and an investigation of the conditions controlling sporulation in Saccharomyces cerevisiae was made. The objective was to develop an optimal medium for the production of spores, and especially to reduce the time required to obtain them. Grape juice was found a satisfactory presporulation nutrient for some yeasts but was ineffective with others. Gorodkowa's medium and carrot agar were ineffective generally. Yeasts vary extremely in their capacity for sporulation, and it was essential to develop a medium that would activate even the poorest sporulating cultures. The study of presporulation nutrition was extended and an extremely effective combination of nutrients developed. There seems to be a general correlation between the size of the ascospores and their vigor, and media which produce the larger spores were favored. Since the larger asci are easier to dissect, this characteristic has a twofold advantage. Immature spores are usually nonacidfast, and components of the media which reduce the number of such spores to a minimum have been selected. Throughout this work counts were made or percentages of sporulation estimated on the slides after staining, but different numbers of strains as well as different strains were tested on the various media and changes in technique developed as the work progressed. Sporulation was more abundant directly on the slants of simple potato agar than on most of the media tested, but this medium was not used because the asci were small and predominantly 2 -spored, even when cultures genetically capable of producing 4-spored asci were used. Cells grown on a substrate containing sugar became much larger than those grown on a vegetable slant. Sporulation does not occur on a rich sugar medium, and therefore transfer to gypsum is required. However, since this extra step results in the production of larger spores, the advantage more than counterbalances the extra work.

\section{MEDIA AND METHODS}

The following presporulation medium was finally chosen:

Beet (leaves) extract (100 gms. per $100 \mathrm{cc}$. boiling water). $10 \mathrm{cc}$. Beet (roots) extract (100 gms. per $100 \mathrm{cc}$. boiling water) . $20 \mathrm{cc}$. Apricot juice (canned). . . . . . . . . . 35 cc. Grape juice (bottled) . . . . . . . . . $16.5 \mathrm{cc}$. Yeast (dried) ................... 2 gm. Glycerin. $2.5 \mathrm{cc}$. 
Water was added to give a final volume of $100 \mathrm{cc}$. The mixture was steamed for 10 minutes and tubed. Tubes were sterilized at $15 \mathrm{lb}$. for 20 minutes and slanted. Some strains of yeast produce spores directly on the slants if allowed to grow for a few weeks. However, if spores are needed sooner, transfer to gypsum is necessary.

Engel's gypsum block method was replaced by the method of Graham and Hastings. A mixture of plaster of Paris and water (100 gm. of each) was poured into test tubes and solidified in a slanting position. These slants were dried at $50^{\circ} \mathrm{C}$. for 24 hours and autoclaved.

TECHNIQUE: About $1 \mathrm{cc}$. of sterilized water is poured over a 3 -day growth of yeast on the presporulation agar and allowed to stand 10 minutes, then a thick suspension is made by stirring the yeast cells around in this supernatant water, using the side of the pipette. The yeast suspension is taken up in the pipette and poured over the upper part of the gypsum slant. About $3 \mathrm{cc}$. of sterile water containing enough acetic acid to bring the $\mathrm{pH}$ down to 4 is pipetted into the lower half of the gypsum slant. The inoculated gypsum slants are incubated $1-2$ days at $23^{\circ} \mathrm{C}$. Spores may appear in 12 hours.

\section{FREE CELL FORMATION}

One of the unusual characteristics of sporulation is the phenomenon which Harper called "free cell formation." It is the only case in which a cell is cut out of cytoplasm leaving a residuum of cytoplasm. In this respect it is markedly different from cleavage which divides a mass of multinucleated cytoplasm into mononucleate cells without leaving any residual cytoplasm. When ascospores of the higher ascomycetes are cut out of cytoplasm the process is apparently under the control of the centriole which sends out small fibres resembling an umbrella frame. These eventually lead to the separation of the spore cytoplasm from the epiplasm, and a wall is finally laid down around the spore cytoplasm.

\section{UNREDUCED ONE-SPORED ASCI}

When the sporulating cells are removed from the gypsum slant the diploid cells which have been transformed into asci differ in the number of spores which they contain. Asci containing 1, 2, 3, or 4 spores can be found. It is generally assumed that in sporulation four original nuclel are produced after meiosis and that when fewer than four spores are found one or more of the original nuclei degenerated preceding spore formation. If this view were correct the 1spored asci would result from a reduction division followed by the degeneration of 3 nuclei. The following experiments indicate that some 1 -spored asci are produced by enclosing the intact, unreduced 
nucleus of a diploid cell in a spore wall. We have studied the viability of the spores produced by our, apparently illegitimate, Rstrain culture in some detail. The 1-spored asci germinate directly to produce diploid cells while the spores from asci containing more than 1 spore are either non-viable or germinate to produce the characteristic small haploid cells. The viable colonies obtained from $1-, 2-, 3-$, and 4-spored asci of the $\mathrm{R}$-strain are shown in the following tabulation:

\section{Type of Ascus}

1 -spored
2 -spored
3 -spored
4 -spored
Number Tested

16

47

4

3
Viable Colonies

10

2

1

0

Two-spored asci are generally more abundant than either 1-, $3-$, or 4-spored asci. In fact, on some media, notably on potato slants, 2-spored asci are produced almost exclusively. Cultures which would normally produce a considerable number of 4-spored

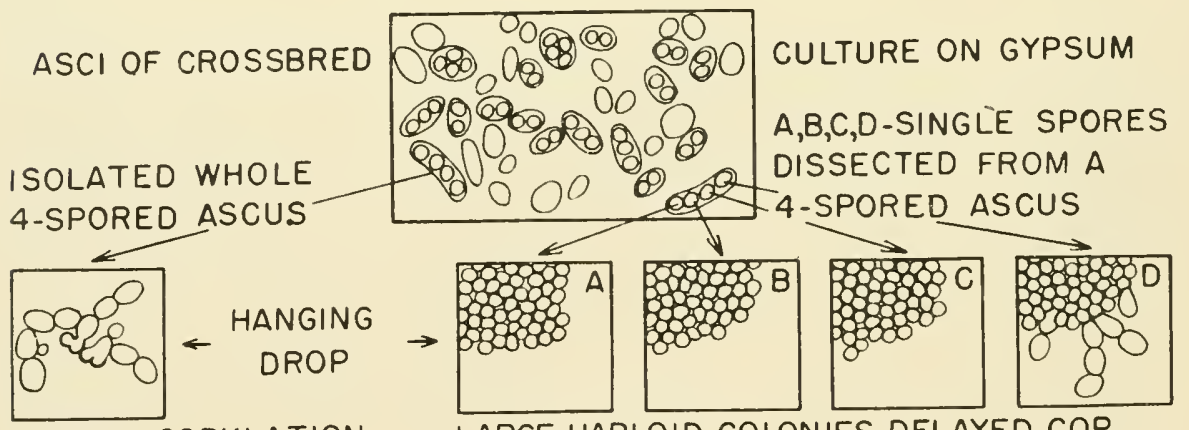

NORMAL COPULATION

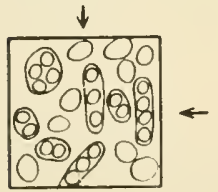
ON
GYPSUM
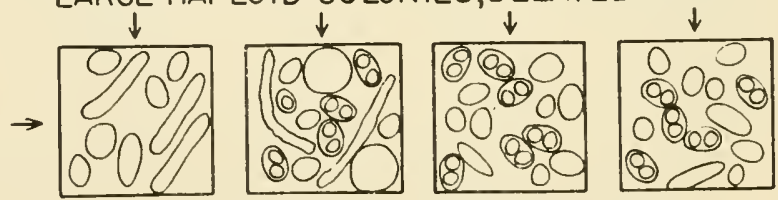

NON-VIABLE, 2-SPORED ASCI
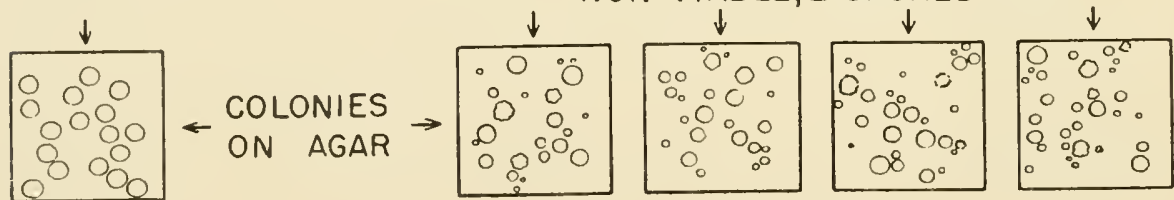

UNIFORM

VARIABLE

Fig. 3-1 Diagram Showing the Differences between Cultures Originating from Intact 4-spored Asci and from Single Ascospores. 
asci will produce only 2-spored forms on potato. It has been difficult to understand why this should occur. The first explanation which comes to mind is that a lethal gene has been segregated; however, it is not reasonable for a lethal gamete to exist as a free cell. It seems probable, however, that a gene, having no effect on the free living state, may act as a spore lethal. We have recently encountered an example. One of our haplophase cultures mutated to produce colonies with a brownish tint. These brown colonies proved capable of copulating and hybrids between them and one of our standard stocks were produced. The hybrids produced 4-spored asci, and a number of them were dissected. Only 2 viable spores were obtained from each ascus. This suggests that the brown mutant, although capable of growing in the haplophase, was incapable of producing a viable spore and therefore acted as a spore lethal.

\section{SPORULATION OF LEGITIMATE AND ILLEGITIMATE DIPLOIDS}

The original concept of heterothallism in yeast (Lindegren and Lindegren, 1943) was based on the following argument. Intact 4 spored asci and single ascospores were isolated from a vigorously sporulating diplophase. The colonies which each produced were tested for their ability to produce spores. Each culture produced by an intact 4 -spored ascus developed into a large round colony containing large ellipsoidal cells; all were capable of producing a large percentage of 4-spored asci (Fig. 3-1). On the other hand, when 4-spored asci from the original culture were dissected and the four single spores grown separately, each culture produced small rough colonies, and when these cultures sporulated the asci were usually 2-spored and were relatively non-viable. 


\section{Chapter 4}

\section{SPORE FORMATION IN THE COLONY}

Knowledge of life cycles and variations in microorganisms has developed almost exclusively from the study of isolated individual cells, without consideration of the structural organization of the colony. Legroux and Magrou (1920) studied the structure of colonies of Vibrio cholerae and of a number of other bacteria. They discovered that rod-shaped variants of the Vibrio appear in perpendicularly arranged packets on the exterior of the colonies, held together by a transparent substance apparently exuded from the cells. They assumed that this substance gave the rough colonies their rigidity. A special stain was used:

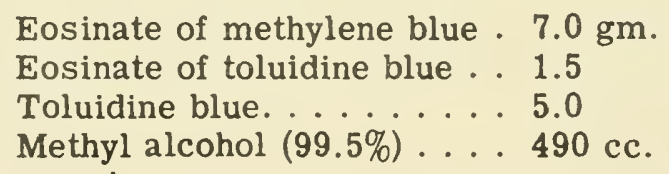

The rod-shaped organisms in the outer layer of the colony were made up of a violet central region and an azure exterior. The central region underwent divisions into two or four rounded violet particles, often of unequal size. This phenomenon was found in Vibrio, in the typhoid bacillus, in the diphtheria bacillus, and in the tuber cle bacillus. Kahn and Nonidez (1933), apparently unacquainted with the work of Legroux and Magrou, confirmed their findings in a study of the tubercle bacillus but concluded that the outer layer was the younger part of the colony. The work of Legroux and Magrou proves this view untenable.

Pisova (1930) found that when a yeast colony is grown on agar, a pseudo-mycelial growth of long fibrous cells penetrates the agar, especially at a high sugar concentration. After a few days or weeks, the surface cells begin to autolyze, continuing until an outer layer of autolyzed cells is formed.

Lindegren and Hamilton (1944) grew yeast colonies on maltextract yeast agar, and cut out portions of the agar containing colonies which were then dropped into Flemming's solution. After 24 hours the killing fluid was washed out in tap water, and the material was imbedded in paraffin. Sections were made and stained with a variety of dyes. Direct smears were also made by cutting the fresh colony in half vertically with a razor and pressing the exposed section gently against the slide. The cells were fixed on the slide by heat and stained. 
Fig. 4-1 shows a section of the yeast colony. The outer layer of autolyzed cells stains very lightly, and the inner central mass of vegetative cells with their dense protoplasts is much darker. A pseudo-mycelium of yeast cells penetrates the agar and is thickest and deepest at the edges of the colony, apparently where oxygen is most abundant. The thin peripheral film of cells at the edge of the colony spreads over the surface of the agar. Fig. 4-2 shows details at the ends of the section, with cells penetrating the agar. At the points of origin of penetration the cells are very abundant. This may be due to the channelling of the substrate nutrient into these regions along the cracks made by the pseudo-mycelium.

Figs. 4-3 and 4-4 illustrate the autolyzed layer containing the asci. Autolysis apparently occurs early in the history of the colony, at least before competition reduces cell size. The autolyzed layer contains the only asci found, suggesting that autolysis supplies essential nutrients on which sporulation depends.

Fig. 4-5 shows the central vegetative cells, many of which are extremely small, indicating that cell division continued after the nutrients became less readily available and competition resulted in a decrease in cell size. Fig. 4-6 shows the pseudo-myceluim produced in the agar in higher magnification.

In some of the contact smears the autolyzed cells were not so shrunken as those obtained by the paraffin method. The walls seemed relatively intact, but there were no stainable cell contents. The "ghost" cells were larger than the densely stained cells in the vegetative section of the colony. These autolyzed cells, which apparently served as sources of nutrients for the sporogenous cells, have a parallel in the paraphyses found in Pyrenomycetes and Discomycetes which also act as nurse cells. In some regions of the autolyzed layer small clusters of round, apparently haploid cells were found, suggesting that some spores may germinate in the layer. New genetic rearrangements could result from such spore germination and subsequent copulation. These experiments suggested that yeast autolysate is an essential nutrient for sporulation and led to the addition of 2 per cent dried brewers' yeast to our pre-sporulation medium, with excellent results.

The striking parallelism between the structure of yeast colonies and those of bacteria, as shown by Legroux and Magrou, suggests that the life cycles may be similarly parallel. Yeasts possess a nuclear mechanism, and the vegetative cells undergo meiosis and sporulation in the outer layer of the colony. In bacterial colonies a similar division of the cell contents into two or four bodies occurs in the corresponding layer. The analogy suggests strongly that the granulation of the bacterial cell is the result of a reduction division similar to that known to occur in yeasts. Badian (1933) and Lindegren (1941) have shown that bacteria undergo a series of 


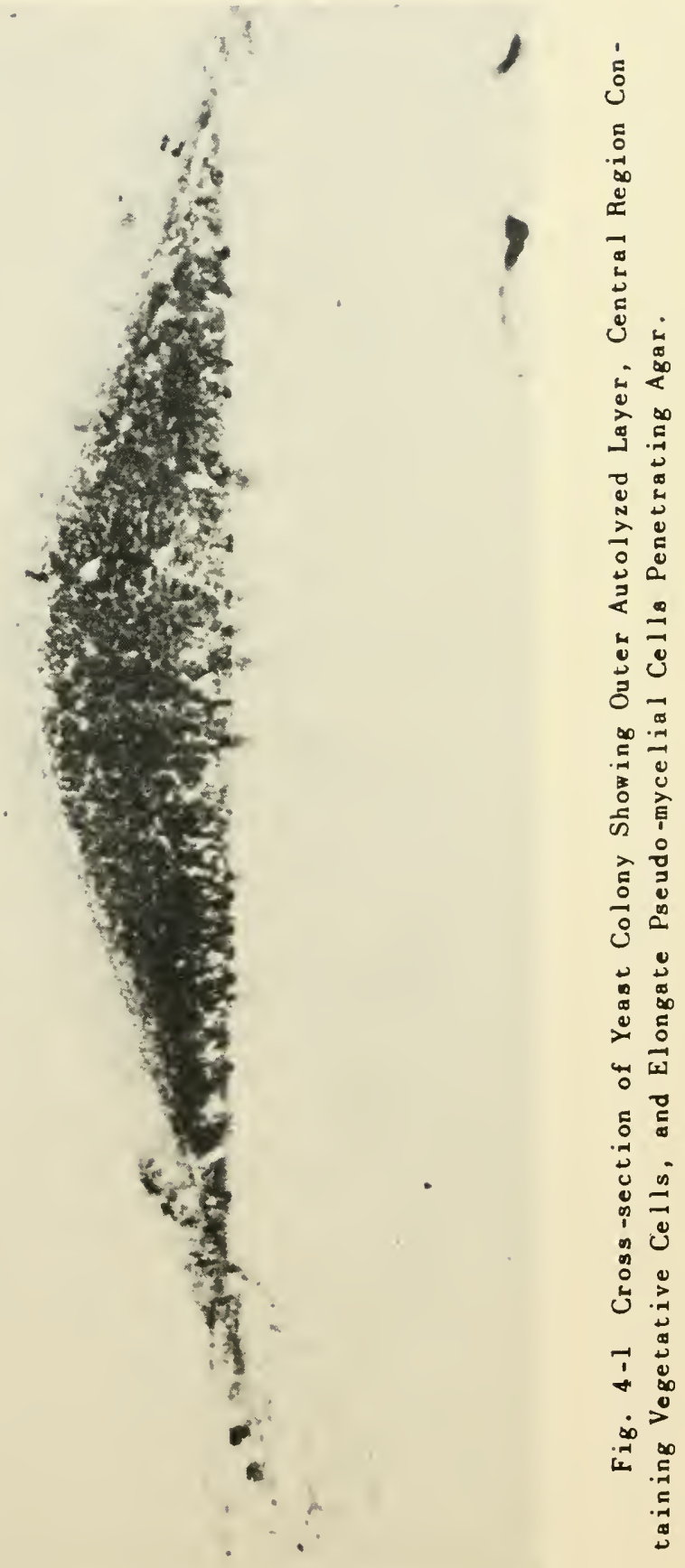

4-3 


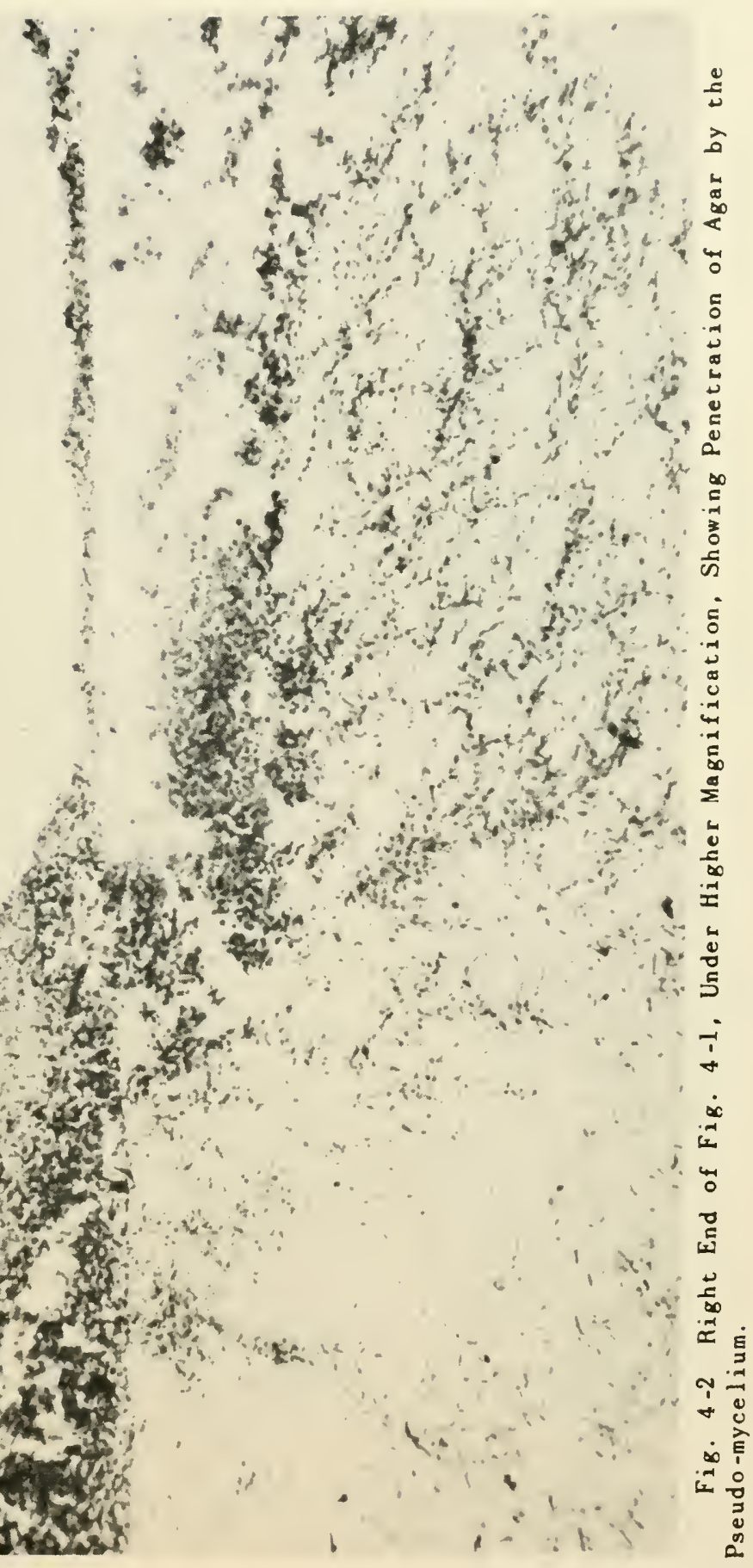




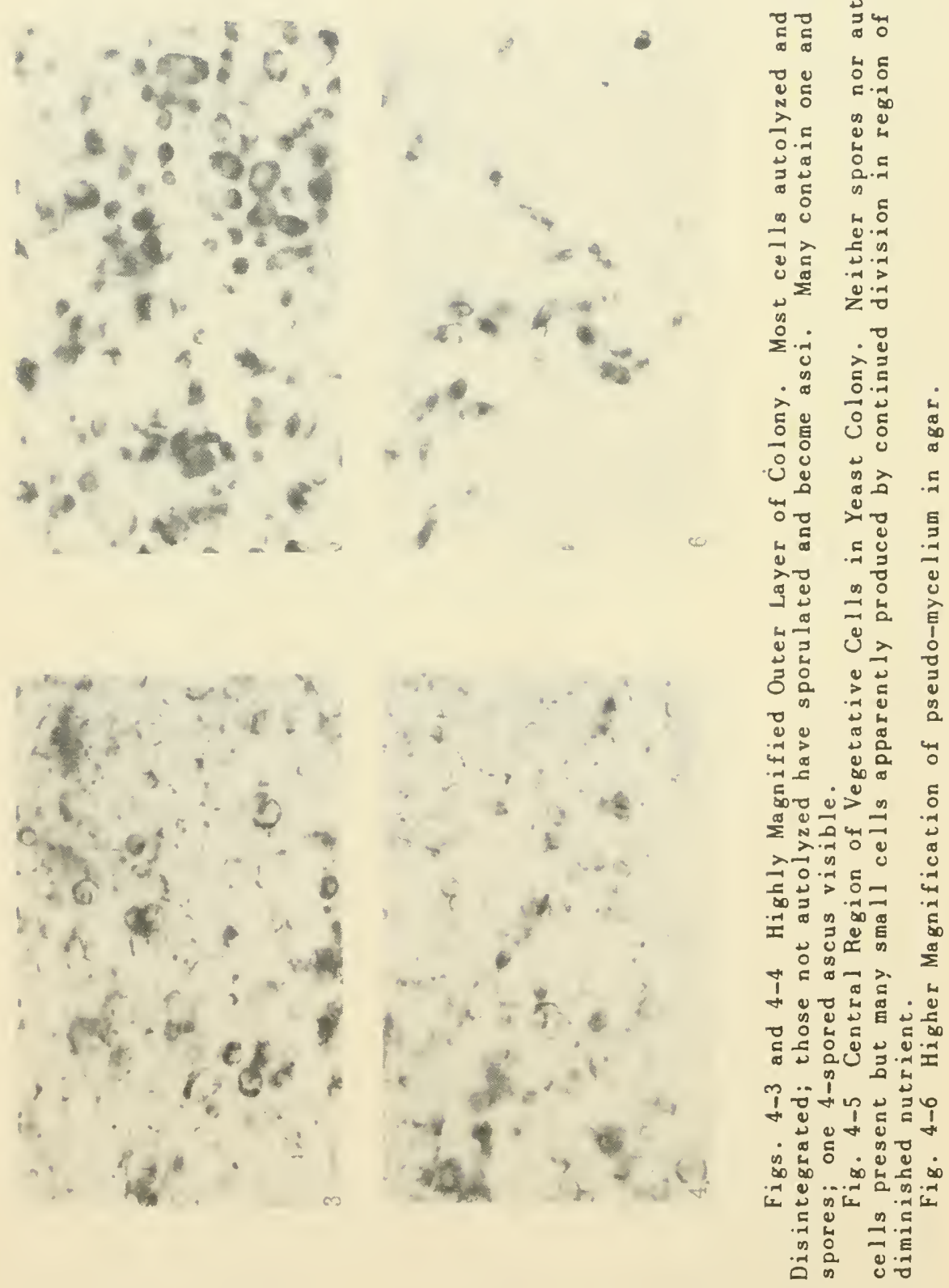


changes comparable with those observed in the reduction divisions of higher organisms.

It is important to point out that mycologists have found it extra ordinarily difficult to germinate the spores of most fungi. Many are peculiarly adapted to germinate only when exceptionally favorable conditions for their growth occur like Neurospora which requires heat treatment, or a chemical (Emerson, 1948) produced by heating. In this instance the relation is clear for Neurospora grows best in nature after a fire has cleared away the green vegetation and left it without competition. Therefore, the morphological demonstration of spores like that made by Legroux and Magrou is sufficient to establish their existence, whether or not they can be germinated.

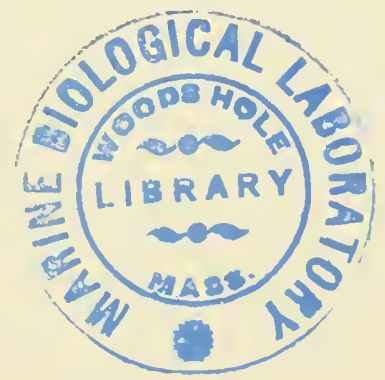




\section{Chapter 5}

\section{STAINING TECHNIQUES}

The phase-difference microscope has proved extremely valuable in yeast cytology. This instrument increases slight contrasts immensely, making lightly stained substances appear deep black. If the cytoplasm is lightly stained it will also appear opaque; this makes the phase difference microscope useless when the cytoplasm is colored even faintly. It is necessary to shift from the ordinary light to the phase-difference microscope as the occasion requires.

An essential part of the equipment for yeast cytology is a small centrifuge. A dilute suspension of cells can be concentrated in thirty seconds, the supernate discarded, and the sediment used for observation, thus insuring an adequate amount of material on the slide. Wet mounts are preferred because there is so much shrinkage of the cell in a dry mount that much detail is lost. One of the first es sentials in yeast cytology is that the cells must never be allowed to dry, for this invariably produces artifacts. The wet mounts are pressed out using blotting paper (small pads of "bibulous" paper can be purchased) and then sealed with melted paraffin. Cover glasses $18 \mathrm{~mm}$. square make sealing easier because they leave a wide margin at the edge of the slide.

\section{DEAD CELL STAIN}

Yeast should first be observed in the dead cell stain, methylene blue, diluted 1-10.000, at pH 5.5. It stains the dead cells blue and stains living cells only slightly. The number of dead cells in a culture is quite variable and judicious use of the dead cell stain makes it possible to avoid staining dead cells with their abundant artifacts on the assumption that they are alive. There is one important precaution: When a yeast cell contains an abundance of stored glycogen the cytoplasm does not take the dead cell stain. In these cells some cytoplasm and the nuclear vacuole are crowded to one side or one end of the cell and when the cells are presumably dead these stain deeply with the dead cell stain suggesting that the cell, which is otherwise completely unstained, may actually be dead. The slight coloration of the living cells by the dead cell stain is usually concentrated in the mitochondria which appear black with the phasedifference lens (fig. 5-1) but are much more difficult to see under the ordinary light microscope.

In applying methylene blue it is important to remember that this dye is decolorized when reduced. Since yeasts contain con- 
siderable amounts of reducing substances, the dye is decolorized if a large excess of yeast is used and the dead cells cannot be detected. For this reason only a small loop of relatively dilute yeast cells is placed in a large drop of the stain. The dead cells can be detected without the stain by their characteristic disintegretation, their coagulated cytoplasm, and the fact that the vacuole is irregularly wrinkled rather than round or lobed if visible. The phasedifference microscope can be used to advantage in observing these cells. In addition to revealing the number of dead cells, the stain usually gives slightly more color to the cytoplasm of the living cell and helps to show the shape and condition of the vacuole.

\section{LUGOL'S SOLUTION}

Lugol's stain is valuable in studying yeast for it reveals the presence of glycogen in the cells. The standard formula for Lugol's solution is:

\section{$5 \mathrm{gm}$. I \\ $10 \mathrm{gm} . \mathrm{KI}$ \\ 100 cc. $\mathrm{H}_{2} \mathrm{O}$}

This is generally too strong and causes considerable shrinkage of the cells. Lugol's can be diluted 1 to 100 and yeast cells can be sus pended in it, centrifuged to concentrate them and mounted in the same liquid. The concentration of the stain may be varied by the operator depending on conditions and on what he is looking for. The preparations must be observed with the standard light microscope since the stain makes the cells too opaque to show details under the phase-difference microscope. Either a blue 45 or a green 58 Wratten filter, or both together, are very helpful. Gylcogen stains reddish brown with iodine and appears black with these filters.

The centrosome in old cells is made visible by Lugol's if the cytoplasm is not too dense; it stains yellow brown and has the appearance of being surrounded by a membrane, fig. 5-2. The vacuole may be either a single round body or a lobed structure with numerous interconnections.

Lugol's makes the vacuole visible in cells in which it is surrounded by glycogen, and therefore concealed, before applying the stain. Cells without glycogen stain golden yellow.

The nucleolus often appears in cells stained by Lugol's.

In preparations fixed with formalin, the chromosomes often stain well with iodine.

\section{ACETO-ORCEIN AND ACETO-CARMINE}

These dyes are only effective when the cytoplasm is not too dense. (fig. 5-3). If much protein is present in the cytoplasm it 


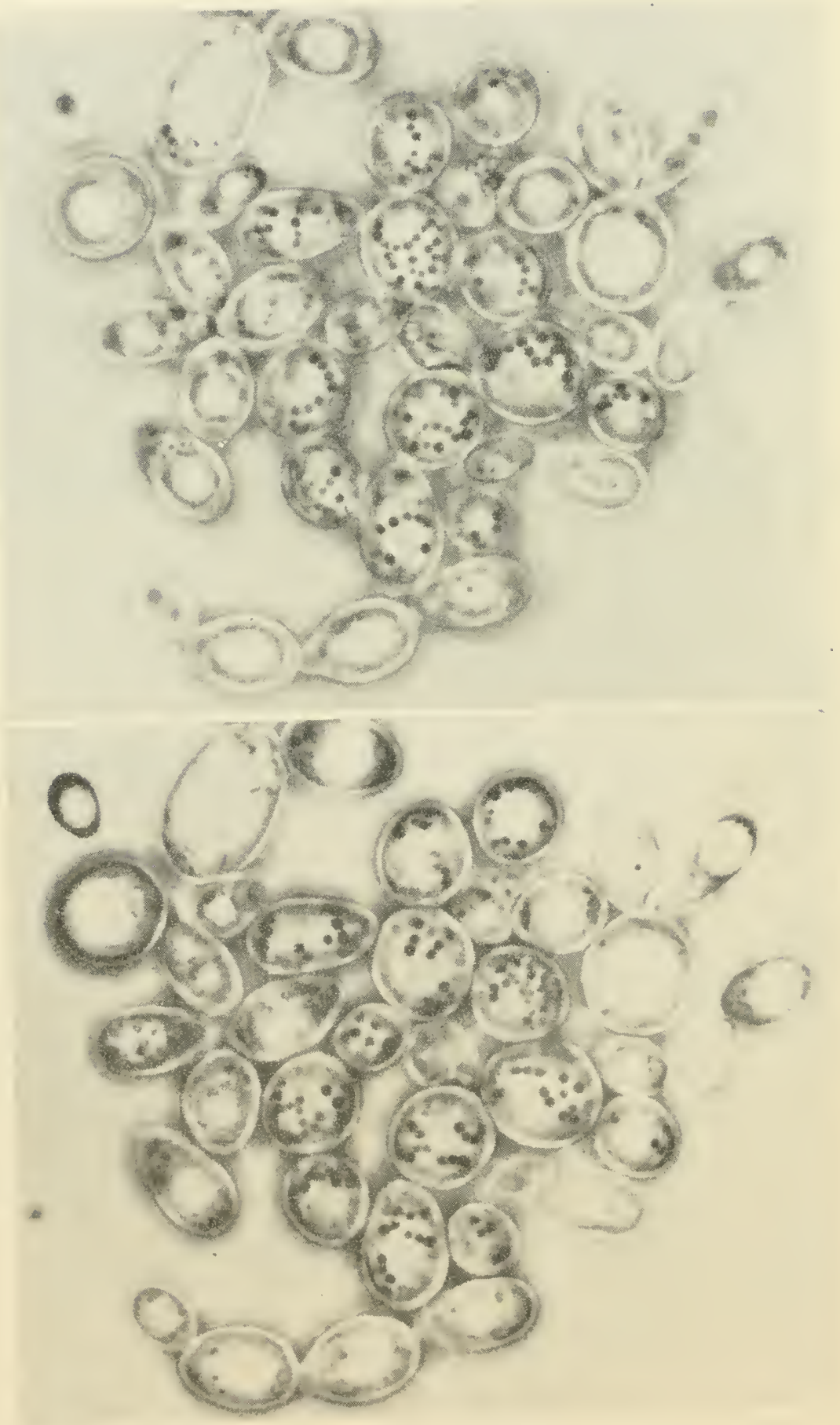




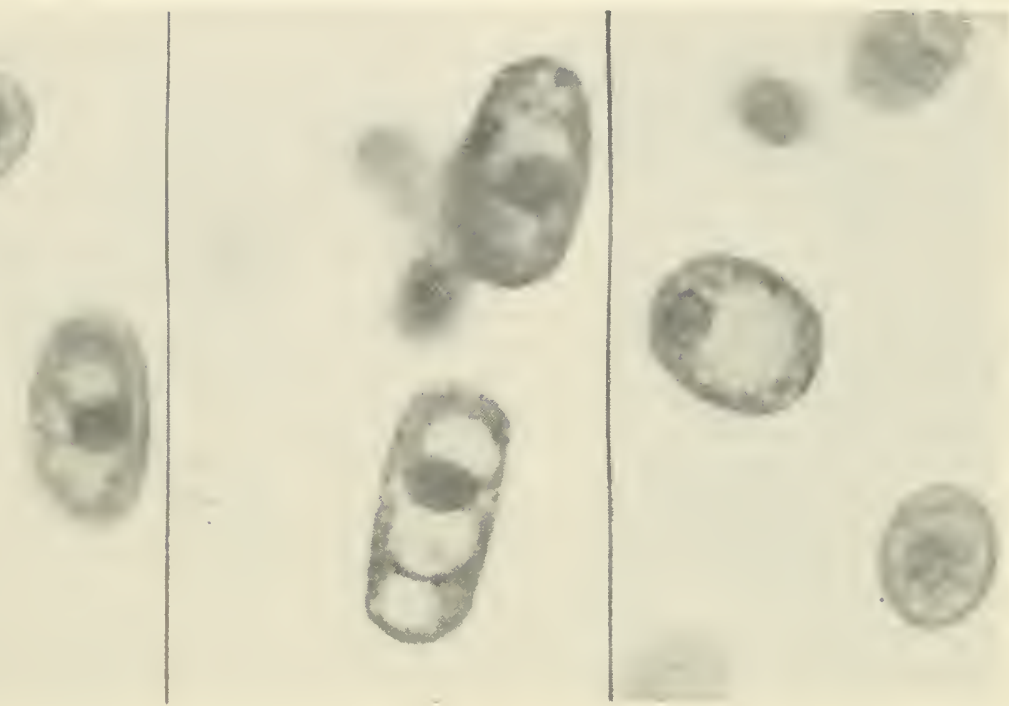

Fig. 5-2. Yeast Cells Stained with Lugol's Solution Showing the Centrosome Attached to the Vacuole. The vacuole is lobed but the centrosome maintains an attachment with all lobes.

Fig. 5-1 Two Different Optical Sections through a Cluster of Yeast Ciells Which Have Just Begun to Bud. These cells were photographed with the Spencer phase-difference microscope. In the resting cells in the center of the cluster, the mitochondria are very clear, with sharply delimited borders. Fewer and smaller mitochondria appear in the budding cells at the edges of the cluster, showing that the granules become dispersed in the cytoplasm of an activated cell.

With the Spencer phase-difference lens the mitochondria are viaible either stained or unstained and it has been possible to corroborate the findings of Caspersson and Brandt regarding their appearance in resting cells and their disappearance in active cells. This lens has given us a marked advantage over other workers, such as Wager and Peniston, and Guilliermond, who used ordinary light microscopes, and whose work was done before Caspersson and Brandt had worked out the cyclical appearance and disappearance of the mitochondria. Since many stains dissolve or obscure the mitochondria, differences of opinion concerning their position or presence are understandable. They might easily be confused with volutin if large numbers are present, because the volutin stain, as devised by Meyer, requires destaining and this is always erratic through the yeast cell wall. 
becomes overstained and the centrosome is concealed. If protein is not too abundant, the centrosome stains well with aceto-orcein or aceto-carmine, and these stains also reveal its structure. To see this structure the ordinary light microscope should be used.

\section{METAPHOSPHATE (VOLUTIN) STAIN}

I have devised a specific stain for volutin which contains $4 \mathrm{cc}$. saturated aqueous toluidine blue, $20 \mathrm{cc}$. commercial formalin adjusted to $\mathrm{pH}$ of 2.5 with about $0.6 \mathrm{cc}$. of glacial acetic acid. Volutin (metaphosphate) stains red with this stain while basophilic proteins stain light blue. A large loop of the sediment from a centrifuge tube is introduced into 10 drops of the dye in a $4 \times 1 / 2$ inch test tube and allowed to stand about 20 minutes. It may stand over-night without harm. The suspension is centrifuged and the cells mounted in the same fluid under an $18 \mathrm{~mm}$. cover slip, blotted, and sealed with paraffin. The glacial acetic acid in the stain prevents complete overstaining of the cytoplasmic ribose nucleoprotein. The preparations must be observed with an ordinary light microscope, since the light blue cytoplasm makes the cell opaque in phase-difference microscopy.

\section{ALKALI-TOLUIDINE BLUE STAIN}

Cells are centrifuged and allowed to stand for ten minutes in two per cent sodium hydroxide, which probably dissolves out much of the ribose nucleoprotein but leaves most of the desoxyribose nucleoprotein. The cells are centrifuged and washed with water and then brought down again. They are mounted in a 1 to 100 dilution of saturated aqueous toluidine blue. No color appears in the cytoplasm, but the heterochromatin stands out clearly differentiated from the colorless cytoplasm. The fine desoxyribose nucleoprotein filaments which form the chromosomes and which are clearly revealed by Rafalko's modified Feulgen stain are also visible in some preparations, although probably they usually disintegrate due to the sodium hydroxide treatment. This procedure works very well with nondividing yeast cells. When yeast cells are dividing rapidly they contain very large amounts of ribose nucleoprotein and overstain very readily; it may be helpful to follow the alkaline washing with a 10minute wash of 1 per cent sulfuric acid before applying the stain. When this is done the the heterochromatin will stain with dilute toluidine blue.

\section{ACID FAST STAIN FOR YEAST SPORES}

The asci are smeared on the slide from the gypsum slope. The smear is fixed by heat and placed in a coplin jar of carbol fuchsin for 20 minutes or longer. Destaining is accomplished by washing rap- 

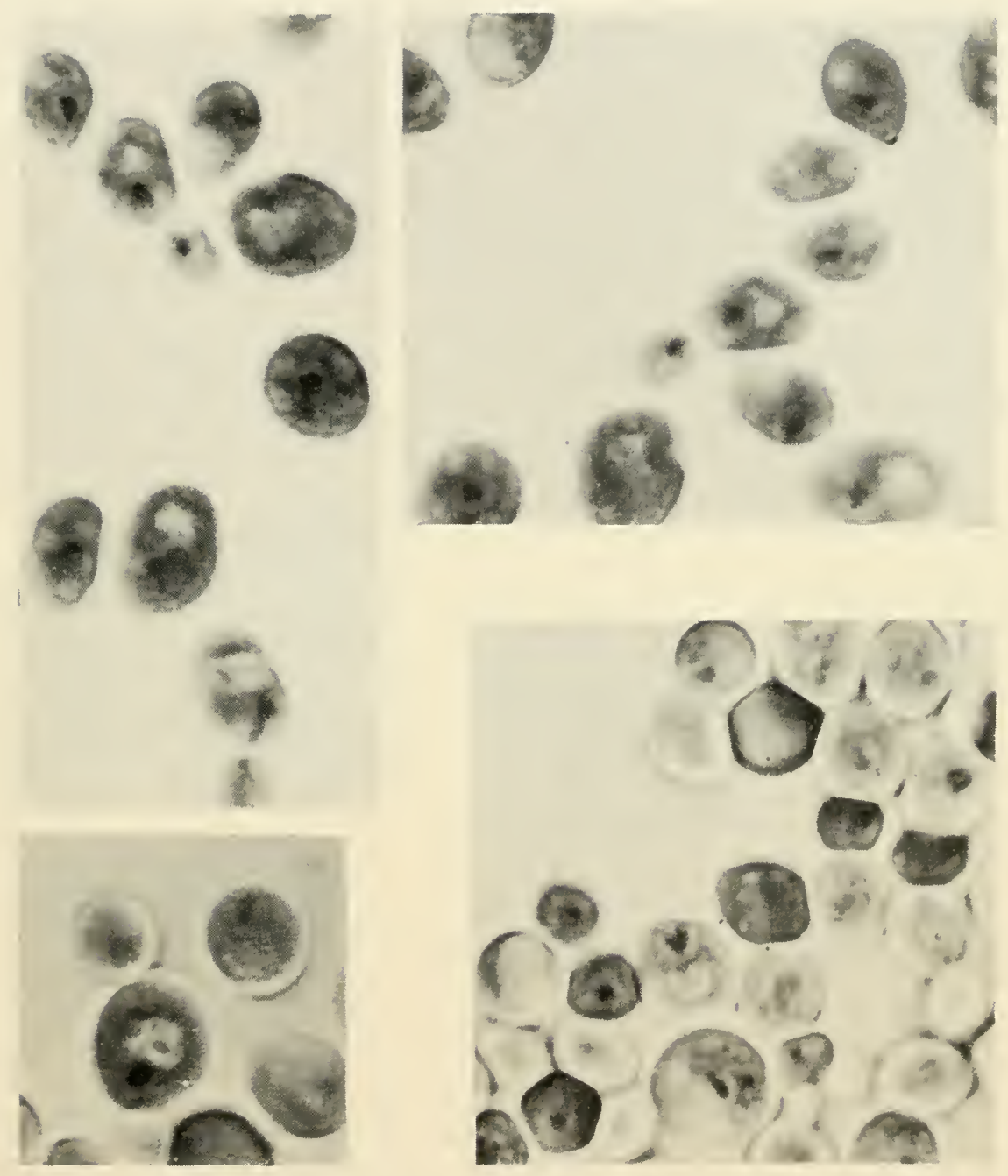

Fig. 5-3 Photographs of Aceto-orcein Preparations Showing that there are Two Heterochromatic Structures in Each Cell, that they are not in the Nuclear Vacuole, and that they Divide by Pulling out and Dividing Transversely During Budding. 
idly in 30 per cent acetic acid while holding the slide with a forceps. The slide in not rinsed in water before destaining, and this prevents the formation of the precipitate that usually occurs if it is transferred directly to water from carbol fuchsin. Two or three rapid swishes through the acetic acid should immediately be followed by a rinse in water. If the slide is held too long in the acid, or not rinsed quickly enough in water, it may be completely destained. The success of this method depends on critical control of destaining. After the water rinse, methylene blue is used as a counterstain. This procedure stains the spores red and the vegetative cells blue because the waxes or lipoids in the spore retain the carbol fuchsin which is washed out of the vegetative cells since they contain no wax. The blue counterstain is incapable of staining the waxy spores so it does not cover the red stain.

\section{DELAFIELD SPORE STAIN}

A tiny drop of cell suspension containing spores is placed on a slide, a small drop of Delafield's haematoxylin added and covered with a coverglass. This makes a preparation which dries out very slowly, because the Delafield's contains glycerin (Frontispiece, S. cerevisiae). The cell walls of the vegetative cells and spores may be clearly defined. Some internal structures can be seen if 7 parts of Delafield's is mixed with 3 parts of acetic acid, using the same procedure.

\section{WEAK KULLERS}

The conventional cytological procedure of killing the cell with concentrated solutions containing formaldehyde, acetic acid, osmic acid, corrosive sublimate, picric acid, or chromic acid causes the yeast cell to shrink to about one-fifth its normal size. It also causes the precipitation of the cytoplasm and thus introduces an artifact of some importance in addition to reducing the size of structures which are already near the limit of visibility of the microscope. The necessity for high concentration of a toxic substance to kill cells quickly to prevent necrotic changes from occurring is not so serious in yeast cells as it is with animal tissues tor the distance which killers must penetrate is only from 2 to 5 microns. I have used various dilute cytoplasmic or enzyme killers such as sodium fluoride or sodium azide with considerable success. Pretreatment with these substances in concentrations of about 0.5 per cent renders the cell permeable to dyes which are otherwise unable to enter the cell. Without pretreatment concentrated toluidine blue is unable to enter the cell but after pretreatment it enters the cell rapidiy. The dye is diluted to prevent overstaining. Aqueous saturated toluidine blue is diluted from 1 to 200 up to 1 to 800 and the cells are immedi- 
ately centrifuged, mounted in the dye and sealed. There is no shrinkage of the cell and the chromosomes are readily stained.

Sodium fluoride or azide can be mixed with toluidine blue without precipitating the dye and mixtures of various concentrations have been used successfully. This makes staining of the chromosomes possible in a single operation. This method does not stain the centrosome. The use of delicate cytoplasmic poisons which inactivate the metabolic enzymes instead of grossly precipitating the cytoplasm may be effective in the cytological examination of larger organisms.

\section{CONVENTIONAL CYTOLOGICAL TECHNIQUES}

Dr. J. S. Rafalko (1945) devised a modified Feulgen stain with which the yeast chromosomes were successfully stained for the first time. The procedure is as follows:

The leuco basic fuchsin is prepared by gentle bubbling of sulphur dioxide gas from a small aperture in glass tubing into $1 / 2 \%$ solution of basic fuchsin until decolorization occurs (in about 10-30 minutes). The solution is then ready for use. Distilled water is similarly saturated for the sulphurous acid rinsing bath and may be stored for many weeks in a tightly-corked flask. The sulphur dioxide can be produced easily by a simple flask-and-funnel generator using sodium bisulphite and dilute sulphuric acid.

The following schedule gave satisfactory results for smear preparations of yeasts.

1. Fixation, usually 2 to 20 minutes.

2. Washing in tap water, minimum time according to fixative used (not over 20 minutes).

3. Distilled water, 2 minutes.

4. Normal $\mathrm{HCl}$ at room temperature, 2 minutes.

5. Normal $\mathrm{HCl}$ at $60^{\circ} \mathrm{C}$., 8 to 10 minutes.

6. Normal $\mathrm{HCl}$ at room temperature, rinse.

7. Distilled water, rinse.

8. Sulphurous acid bath, 2 minutes.

9. Leuco basic fuchsin, $1 \frac{1}{2}$ to 2 hours.

10. Sulphurous acid bath, for sufficient time to remove the free unreacted leuco basic fuchsin (usually two or three 1-minute changes).

11. Tap water, 10 to 15 minutes.

12. Dehydration through alcohol, and counterstaining in $95 \%$ alcohol tinted with fast green.

In collaboration with Mrs. Margaret Rafalko (Lindegren and Rafalko, 1949), the use of the standard killers such as PFA, Navashin, corrosive sublimate, Flemming's chrome-aceto-formalin and others 
followed by Rafalko's Feulgen, Heidenhain, acid fuchsin-methyl green, pyronin-methyl green have been studied.

\section{MATERIALS AND METHODS}

The yeast used was grown at room temperature on agar slants in test tubes. When slides were to be made, a sample of yeast was transferred from a 24-hour slant growth to a flask of liquid medium and incubated at $30^{\circ} \mathrm{C}$. on a shaker for a period of from one to six hours. After centrifuging a sample from the flask, cover-slip smears were made without the use of albumin and were fixed by putting them into a Petri dish of fixative. Throughout the staining process, the material was handled in this way until mounted on a slide. At no time was the material allowed to dry.

The fixatives most commonly used were Navashin, Flemming, Carnoy, Bouin, mercuric chloride and osmic acid vapors. Best results were obtained with Navashin and Carnoy. Staining procedures employed included the Feulgen reaction, acid fuchsin-methyl green, pyronin-methyl green, safranin-fast green, crystal violet-methyl blue, Heidenhain's hematoxylin, Giemsa and Sudan IV.

\section{PROCEDURES AND SCHEDULES}

It might be well to note here that, with the possible exceptions of the Feulgen reagents and Heidenhain's, it is best to employ fixatives and stains that have been prepared immediately prior to their use. This is especially true of fixatives, if best results are to be obtained. In all the schedules given below, methyl alcohol and toluene were used in preference to ethyl alcohol and xylol because the former are smaller molecules and therefore penetrate faster and more evenly.

1. Feulgen-fast green. The method of Rafalko (1945) was used. This schedule is given on p. 5-8.

Results: Chromosomes - red-red

Centrosome - purple-red

Nucleolus - pale green

Cytoplasm - pale green

2. Acid fuchsin-methyl green. In separate bottles a $0.1 \%$ aqueous solution of acid fuchsin and a $0.1 \%$ aqueous solution of methyl green. When ready to use, two parts of the acid fuchsin were mixed with 4-5 parts of methyl green. The standard procedure calls for acidulating every $50 \mathrm{ml}$. of the acid fuchsin with one drop of a $10 \%$ solution of acetic acid just prior to mixing the two dyes. Since that made no difference in the staining results, that step was gene rally omitted. 
a. Fixation, 8-10 minutes.

b. Tap water wash, 10-15 minutes.

c. Distilled water, 2 minutes.

d. Acid fuchsin-methyl green mixture, 25-30 minutes.

e. Direct transfer to $95 \%$ alcohol until green stain ceases to leave smears in clouds.

f. Rapid dehydration in absolute alcohol.

g. Clear, two 2-5 minute changes in toluene and mount.

Results: Chromosomes - dark green

Centrosome - red

Nucleolus - pale green

Cytoplasm - pink

3. Pyronin $Y$ - methyl green.

Prepare the staining solution:

$\begin{array}{lr}\text { Methyl green } & 0.50 \mathrm{gm} \text {. } \\ \text { Pyronin Y } & 0.05 \mathrm{gm} \text {. } \\ \text { Alcohol } & 5.00 \mathrm{ml} \text {. } \\ \text { Glycerine } & 10.00 \mathrm{ml} \text {. } \\ 0.5 \% \text { carbolized } \mathrm{H}_{2} \mathrm{O} \text { to } 100 \mathrm{ml} \text {. }\end{array}$

Follow the schedule given above for acid fuchsin-methyl green, using this stain mixture 15-20 minutes.

Results: Chromosomes - dark green

Centrosome - pale green

Nucleolus - pale green

Cytoplasm - pink

4. Safranin 0 - fast green.

a. Fixation, 8-10 minutes.

b. Tap water wash, 10-15 minutes.

c. Distilled water, 2 minutes.

d. Stain in $1 \%$ aqueous safranin $0,1 / 2-1$ hour.

e. Distilled water, rinse.

f. 50\% alcohol, 2 minutes.

g. Fast green in 95\% alcohol (enough fast green to color the alcohol faintly), 3-5 minutes.

h. Absolute alcohol, rinse.

i. Clear, two 2-5 minute changes in toluene, mount.

Results: Chromosomes - red

Centrosome - red

Nucleolus - pale green

Cytoplasm - pale green

5. Crystal violet-anilin blue. This stain combination used after the I and KI mordant proved an excellent one for showing the paired chromosomes.

a. Fixation, 8-10 minutes.

b. Tap water wash, 10-15 minutes.

c. Pass through 50\%, 70\% alcohols, 2 minutes each. 
d. Mordant in iodine solution (1\% iodine plus $1 \% \mathrm{KI}$ in $80 \%$ alcohol), 20 minutes.

e. Distilled water, rinse.

f. Stain in $1 \%$ aqueous crystal violet that has been brought to $a$ boil and filtered, 20 minutes.

g. Tap $\mathrm{H}_{2} \mathrm{O}$, rinse.

h. Distllled $\mathrm{H}_{2} \mathrm{O}$, rinse.

1. $70 \%$ alcohol, 2 minutes.

f. Anilin blue in $95 \%$ alcohol (enough to color alcohol faintly), 2-5 minutes.

k. Dehydrate in absolute alcohol, clear, toluene, mount.

Results: Chromosomes - deep purple

Centrosome - pale blue

Nucleolus - pale blue

Cytoplasm - pale blue

6. Heidenhain's hematoxylin.

a. Fixation, 8-10 minutes.

b. Tap $\mathrm{H}_{2} \mathrm{O}$, wash, 10-15 minutes.

c. Mordant in fresh $4 \%$ ferric alum, 3-4 hours.

d. Tap water wash, 5-10 minutes.

e. Distilled water, rinse.

f. Stain in hematoxylin, 4 hours.

g. Tap water, rinse thoroughly.

h. Destain in $2 \%$ aqueous ferric alum or in picric acid, which is more painstaking but gives good results.

i. Tap water wash, 20 minutes.

j. Dehydrate in $70 \%, 95 \%$ alcohols, clear, 2 changes of toluene, mount in neutral damar.

Results: Chromosomes - black Centrosome - blue-black Nucleolus - blue-black Cytoplasm - gray or colorless.

7. Giemsa.

a. Fixation, 8-10 minutes.

b. Tap water wash, 10 minutes.

c. Wash in $0.5 \%$ solution of sodium thiosulfate, 10 minutes.

d. Distilled water, 3-5 minutes.

e. Stain in 1-20 Giemsa-distilled $\mathrm{H}_{2} \mathrm{O}$ solution, 15-40 minutes.

f. Distilled water, rinse.

g. Dehydrate and clear in following acetone-toluene steps, checking progress with microscope: (a) pure acetone 95 parts, toluene 5 parts; (b) acetone 70 parts, toluene 30 parts; (c) acetone 30 parts, toluene 70 parts, (d) pure toluene; then mount in balsam. 
Results: Chromosomes - purple-red

Centrosome -

Nucleolus - pale blue

Cytoplasm - pale blue

8. Sudan IV. Use a saturated solution of Sudan IV in $70 \%$ alcohol which has been standing 24 hours or overnight before using.

a. Fixation; Navashin 5 minutes or formalin vapor $10 \mathrm{~min}$ utes.

b. Tap $\mathrm{H}_{2} \mathrm{O}$ wash, 10-15 minutes.

c. 50\% alcohol, 1-2 minutes.

d. Stain in Sudan IV in $70 \% 45$ minutes.

e. $70 \%$ alcohol, rinse.

f. Distilled water wash, 2 minutes.

g. Aqueous solution of fast green, 2-3 minutes.

h. Distllled $\mathrm{H}_{2} \mathrm{O}$, rinse.

1. Mount in Karo syrup (blue label) and ring with gold sizing.

Results: Chromosomes, sometimes nucleolus, as well as cytoplasmic bodies take on the stain. 


\section{Chapter 6}

\section{CYTOLOGY}

\section{THE FUNGAL NUCLEUS}

The fungal nucleus is a distinctive structure and our study of the yeast cell has revealed that it shares the salient features of other fungal nuclei. These characteristics were first clearly established by Harper in his classical work on Phyllactinia. He showed that the chromosomes are polarized to a densely chromatic central body located on the periphery of the conspicuous nuclear vacuole, giving the nucleus a characteristically eccentric appearance. Each chromosome is attached to the centriole; each mitosis is preceded by the division of the centriole and the spindle forms between the two daughter centrioles. The nuclear membrane remains intact throughout mitosis and the spindle is intranuclear as it is in all fungi. The daughter centrioles merely move to opposite ends of the nuclear vacuole. Harper proposed that the spindle fibers also remained intact throughout the cell cycle and that the division of the centriole was followed by the division of the spindle fibers and finally by division of the chromosomes. Harper's interesting and revolutionary views on the origin of spindle fibers have not been reinvestigated although some fungi with large prophase nuclei might be exceptionally favorable cytological material. The nucleolus was a conspicuous structure in the fungal nuclei described by Harper, and it is clear from his study that the characteristic eccentric nature of the fungal nucleus with a large densely staining particle in or on the nuclear vacuole may arise either from the presence of a large nucleolus or from a large centriole but that critical analysis is necessary to make the proper distinction between these two structures in small cells.

Dodge, and Lindegren and Rumann made critical studies of the nucleus of Neurospora and discovered that it also is a characteristically eccentric nucleus with polarized chromosomes and a conspicuous structure which Dodge called the "beak" to which the chromosomes are polarized. This large umbrella handle-shaped, deeply staining structure is the source of the "astral rays" which originate from it to cut the spore. out of the cytoplasm in the manner characteristic of the ascomycetes. Dodge did not call this structure the centriole and he may have been properly cautious in avoiding a sharply limiting term because it seems too large to be 
homologous to the smaller standard centrioles found in invertebrate eggs. The present analysis of the yeast nucleus suggests that the similar structure in the yeast cell carries the heterochromatin in addition to the centriole and we have called the entire complex the "heterochromatinic centrosome." This may also be the case in Neurospora, although no Feulgen stains of the Neurospora nucleus have been reported. The extensive genetical study of this organism in recent years has not been accompanied by equally intensive study of the nuclear apparatus; the recent work of $\mathrm{McClintock}$ has been concerned with the enumeration and description of the chromosomes rather than with the other problems of nuclear structure.

Wager and Peniston's description of the yeast nucleus correspond in many respects to Harper's description of the nucleus of Phyllactinia. They called the deeply staining eccentric structure attached to the nuclear vacuole the "nucleolus"; we have identified this structure as the "heterochromatinic centrosome." They showed, and we have confirmed this finding, that the chromosomes are often polarized to it in the manner characteristic of most fungal nuclei.

Our observations agree rather well with those of Wager and Peniston (1910). Fig. 6-1 which summarizes their findings, is reproduced herewith.

Table 6-1 lists the designations which they gave the different organelles along with the name applied to the same structures by Guilliermond, Janssens and Leblanc, and Lindegren. Wager and Peniston's interpretation was limited by contemporary concepts of cell structure, but their drawings reveal an organization easily understandable in terms of modern concepts of the nucleus.

Janssens, who discovered chiasmata, and Beijerinck agreed with Wager and Peniston in characterizing the large vacuole in the yeast cell as the nucleus. However, Guilliermond, in a polemic with Wager and Peniston ridiculed the significance of their analysis and insisted that only the deeply staining eccentric structure attached to the vacuole was the nucleus. There was a general acceptance of Guilliermond's interpretation because this eccentric structure (which we call the "heterochromatinic centrosome") is easy to demonstrate by the crudest cytological methods and because it has been found to be Feulgen-positive by a number of workers including Margolena, Badian, and more recently Delameter. The chromosomes themselves, however, are extraordinarily delicate and difficult to make visible with most ordinary techniques, and when the cell is killed with Carnoy or other substances containing large amounts of acetic acid, the nuclear vacuole usually dis integrates and only the densely staining centrosome is found in the cell. In fact, cytologists like Kater and Henneberg, who also demonstrated the same chromatinic threads in the vacuole as those 
seen by Wager and Penistun interpreted their observations according to the dominart views of Guilliermond, probably because of his great prestige, calling the eccentric structure attached to the nuclear vacuole the nucleus. They described the polarized chromatinic threads which they found attached to it, but failed to recognize

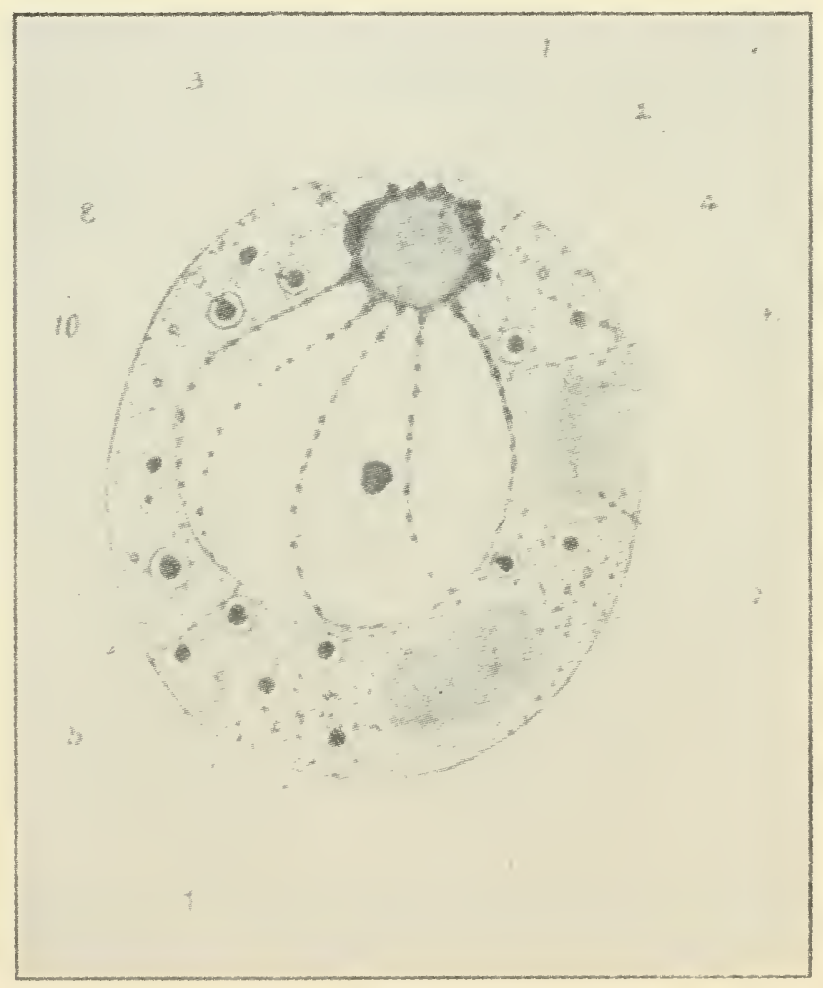

Fig. 6-1 A Drawing Copied from Wager and Peniston, Showing their Interpretation of the Structure of the Yeast Cell. See Table 6-10.

their true significance. In fact, Kater concluded that the threads were outside the nuclear yacuole and confused them with the mitochondria which are visible outside the nuclear vacuole in the unstained material. However, the mitochondria are disintegrated by the fixatives which he used and were therefore not present in his stained preparation. Conversely, the chromosomes which are invisible in unstained material only become visible aiter staining of 
Table 6-1

NAMES OF ORGANELLES IN FIGURE BY DIFFERENT AUTHORS

\begin{tabular}{|c|c|c|c|}
\hline $\begin{array}{l}\text { Wager and } \\
\text { Peniston }\end{array}$ & Guilliermond & $\begin{array}{c}\text { Janssens } \\
\text { and } \\
\text { Leblanc }\end{array}$ & Lindegren \\
\hline $\begin{array}{l}\text { 1. Nucleolus } \\
\text { 2. Peripheral } \\
\text { layer of } \\
\text { chromatin }\end{array}$ & Nucleus & & $\begin{array}{l}\text { Centrosome } \\
\text { Heterochromatin }\end{array}$ \\
\hline $\begin{array}{l}\text { 3. Chromatin } \\
\text { patch on } \\
\text { one side } \\
\text { of nucleo- } \\
\text { Ius }\end{array}$ & & & Centrioles \\
\hline $\begin{array}{l}\text { 4. Nuclear } \\
\text { vacuole }\end{array}$ & Vacuole & Nucleus & $\begin{array}{l}\text { Nuclear } \\
\text { vacuole }\end{array}$ \\
\hline $\begin{array}{l}\text { 5. Central } \\
\text { volutin } \\
\text { granule } \\
\text { in the } \\
\text { vacuole }\end{array}$ & & Nucleolus & Nucleolus \\
\hline $\begin{array}{l}\text { 6. Chromatin } \\
\text { network }\end{array}$ & & & Chromosomes \\
\hline $\begin{array}{l}\text { 7. Granules of } \\
\text { fatty sub- } \\
\text { stance }\end{array}$ & & & \\
\hline $\begin{array}{l}\text { 8. Volutin } \\
\text { granules }\end{array}$ & & & Mitochondria \\
\hline $\begin{array}{l}\text { 9. Glycogen } \\
\text { vacuoles }\end{array}$ & & & Glycogen \\
\hline $\begin{array}{l}\text { 10. Delicate } \\
\text { suspending } \\
\text { threads for } \\
\text { the central } \\
\text { volutin } \\
\text { granule }\end{array}$ & & & Chromosomes \\
\hline
\end{tabular}


the fixed cells. It seems probable, the refore, that Kater observed mitochondria in the living cell and destroyed them by fixation; he subsequently stained the chromosomes in the killed material and identified the visible chromosomes in these preparations with the mitochondria which he had seen in living preparations. If he had employed the Feulgen stain, he would have avoided this error, for it reveals the chromatinic threads as Feulgen-positive structures.

Lindegren described the "chromatinic threads" which Wager and Peniston observed in the nuclear vacuole as chromosomes because of their characteristic pairing and their characteristic chromosomal morphology. Rafalko subsequently devised a modified Feulgen stain with which he stained chromosomes in Protozoa that had previously been refractory to the Feulgen technique. He revealed that both the "chromatinic threads," which Wager and Peniston had demonstrated in the nuclear vacuole, and the centrosome were Feulgen-positive. Previous to his work, only the centrosome had taken the stain and earlier workers had insisted that this, therefore, was the true nucleus. Since his announcement, the new stain has become widely used, and several unpublished reports of its superiority in other material than yeast have reached us. Mirsky, Preer, and others, in personal communications, have indicated their success in staining Feulgen-positive structures with Rafalko's more sensitive Feulgen method.

Previous workers have generally disagreed as to which structures in the yeast cell are chromosomes, centrosomes, or nucleoli; the eccentric structure attached to the nuclear vacuole has been called the "nucleus" by Guilliermond, the "nucleolus" by Wager and Peniston, and the "centrosome" by Lindegren. The structure which we consider the true nucleolus has only recently been demonstrated by Rafalko to resemble a protozoan endosome for it is found within the nuclear vacuole, is Feulgen negative, may contain plastin bodies and is surrounded by a clear area which often has chromosomes attached to it. We have concluded that the yeast cell contains a nucleus composed of a hemispherical centrosome and nuclear vacuole; the former contains the centroplasm, the heterochromatin, and a central clear area; the latter the chromosomes and the nucleolus; the cytoplasm contains the mitochondria.

\section{THE DIVISION OF THE CENTROSOME}

Badian (1937) (fig. 6-2), found that the centrosome (which he called the "nucleus") contains two structures which take the Feulgen stain. Badian developed an effective stain; he killed the cells with osmic vapors, stained with methylene blue and destained with eosin. He followed the centrosome in mitosis and meiosis in $\mathrm{S}$. cerevisiae 
and found that it contained two bodies which he called the "chromosomes." He stated that each cell contained two "chromosomes" which divided by longitudinal splitting. He stated that the haploid "chromosomes" fuse end to end to form the diplophase, rather than associating to form a pair of chromosomes according to the usual method. The life cycle described by Badian is shown in fig. 6-2, copied from his paper.

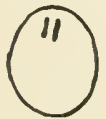

a

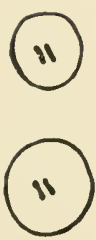

h

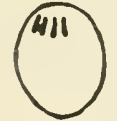

b

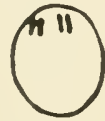

C

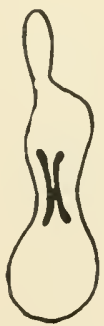

j

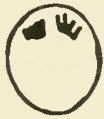

d

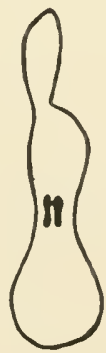

$k$

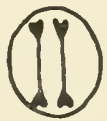

e

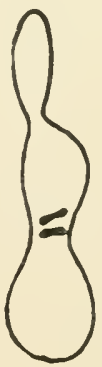

1

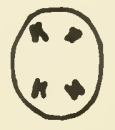

$f$

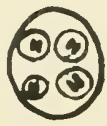

0

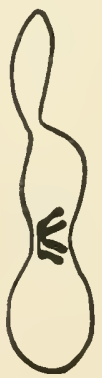

m

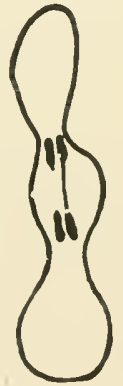

n

Fig. 6-2 Yeast Life Cycle as Described by Badian. This Figure is Copied from his Paper Showing Spore Formation from the Diploid Cell (a-g), and the Fusion of Two Haploid Cells to Reconstitute the diploid $(h, i, j)$. However, it is not Clear Whether these Bodies are the Chromosomes, as Badian Thought, or the Heterochromatin Carried.in the Centrosome.

The centrosome contains two rod-shaped bodies which stain well with Feulgen and aceto-orcein (fig. 5-3). They may be the bodies described by Badian as "chromosomes" which he states fuse end to end to produce the diplophase. However, the vacuoles also fuse following copulation. Harper showed that fusion of the nuclei in Phyllactinia is initiated by fusion of the centrioles, followed by fusion of the nuclear vacuoles and synapsis of the chromosomes. 
Badian's failure to locate the vacuole makes it difficult to interpret his work, although it seems probable that he is describing the heterochromatin rather than the chromosomes.

Levan (1946) has recently shown that the structure of the centrosome is more complex than was originally proposed by Badian.

However, he agrees with Badian (and disagrees with me) in calling it the "nucleus." Badian's drawings of the centrosome reveal it as a bipartite body; Levan found it contained a maximum of ten bodies which he called "chromosomes." Renaud (fig. 6-3) also discovered the centriole in the centrosome, which I have not yet seen by my techniques. Levan used the Feulgen technique, while Renaud's preparations were made with iron hemotoxylin. I have been able to confirm Renaud's concept of the linear arrangement of the particlee of chromatin in the centrosome by alkali-toluidine blue stain. The findings on the division of the centrosome are very similar to those of Renaud. Renaud has shown two clearly vísible small centrioles which precede the chromatin in the division of the structure which I have called the centrosome.

\section{THE HETEROCHROMATIN}

The structure of the centrosome is clearly shown by the alkali-toluidine blue stain (fig. 6-4). This stain reveals the structure which Levan, Badian, and Renaud described as chromosomes. It usually stains structures in the centrosome only. As many as 20 or more small particles can be seen in each centrosome and careful focusing shows that they are connected by threads. In some cells only 2 dense rods are found suggesting that long threads have coiled down to form the rods. A bridge connects two cells in division showing that this is the same material as that shown to divide by transverse fission in the cells stained with aceto-orcein (fig. 5-3).

I have identified this densely staining, Feulgen positive material as heterochromatin since it divides by transverse division and therefore could not compose the chromosomes. Furthermore, Rafalko has succeeded in staining the chromosomes within the nuclear vacuole with the Feulgen stain and I have described their mitosis so it is no longer necessary to insist that this Feulgen positive material is chromosomal in character.

\section{MECHANISM OF BUDDING}

Budding is a unique method of cell division; I have shown (1945, fig. $6-5$ ) that it is the direct result of the extension of a tube from the vacuole to a point on the cell wall where a very tiny protuber ance is formed on the outer surface of the cell into which the vacuole-tube passes and in which it enlarges to form the bud-vacuole. 
When a yeast cell buds the chromosomes in the nuclear vacuole and the centrosome divide together. The first step is the formation of a long slender tube leading from the vacuole to the periphery of the cell. This phenonenon is best observed in cells containing enough glycogen so that the iodine stain delimits the vacuole and its tube as a clear space in the surrounding reddish brown cytoplasm. Observation is facilitated by the use of a Wratten 45 filter which converts the reddish brown color of the cyto-
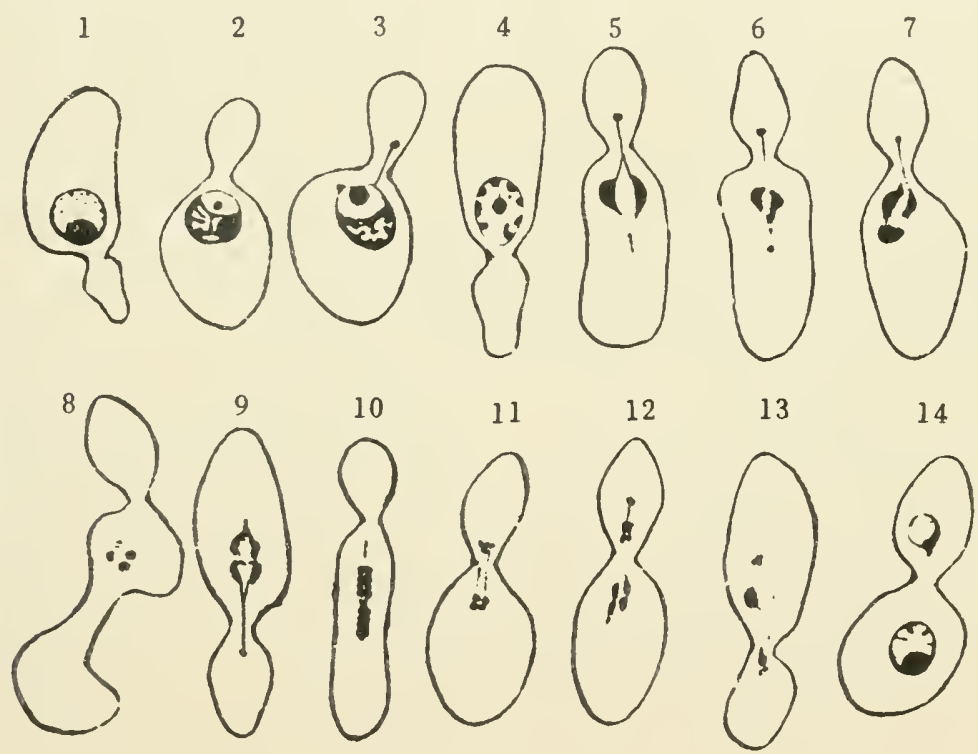

Fig. 6-3 A Copy of Renaud's Drawing of the Division of the Centrosome in the Yeast Cell Showing the Existence, especially in Figs. 3 and 6, of the Centriole. This Division is Concurrent with the Division of the Chromosomes but, for the Reasons Stated in the Text, I Have Concluded that what Renaud Has Shown is the Distribution of Heterochromatin Between Mother and Daughter Cell rather than a Nuclear Division.

plasm to blue-black and reduces the chromatic aberration of the lens system. The canal from the vacuole may begin any place on the surface of the vacuole, but usually appears at a point on the vacuole near the centrosome. The bud is produced where the process from the vacuole meets the cell wall; when the canal emerges at the side of the vacuole, distal to the centrosome, the long, slender process extends all the way from the most distant part of the cell through the cytoplasm and finally produces the bud near the 

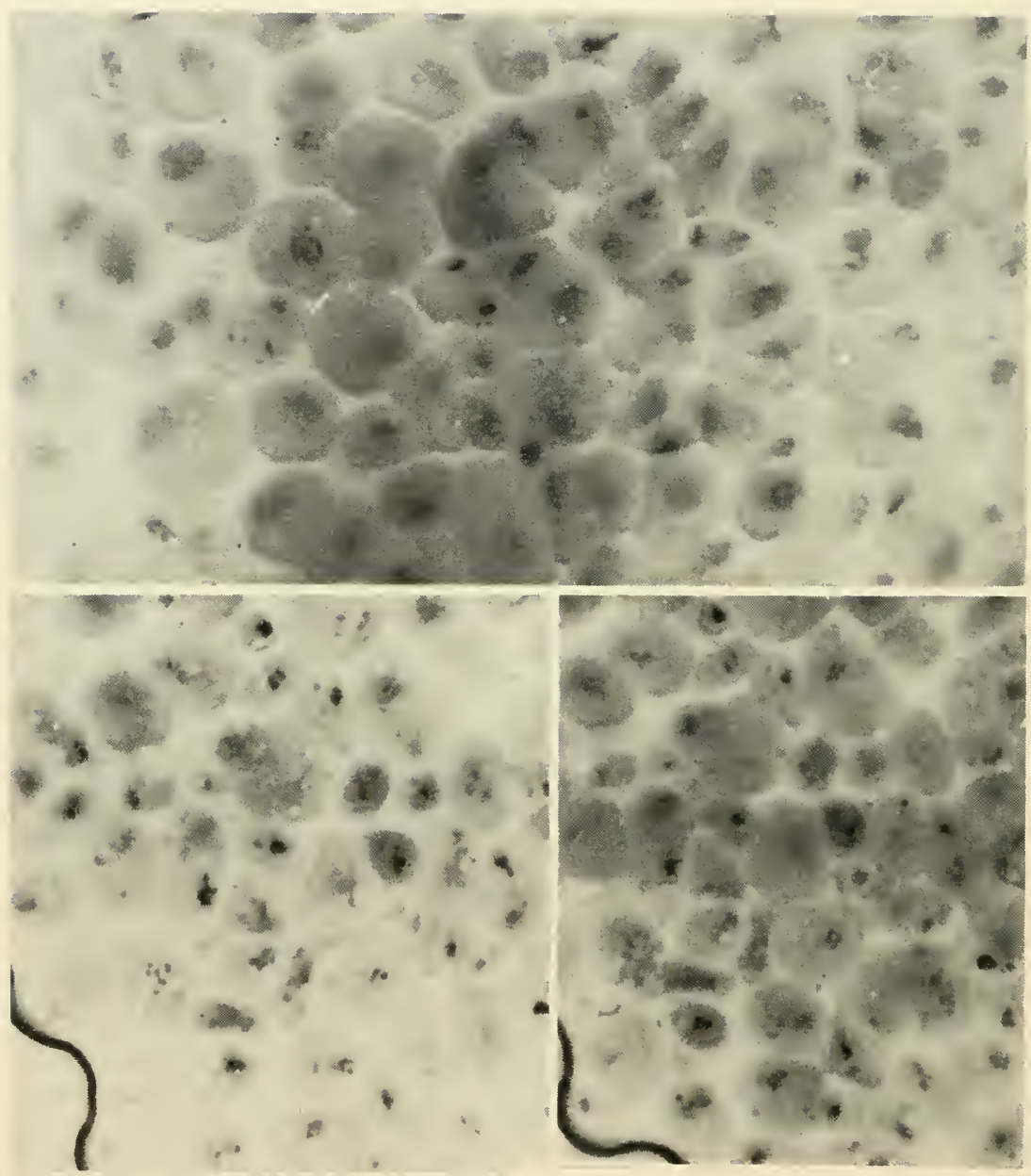

Fig. 6-4 Alkali-toluidine Blue Stain. In many cells the "net-knots" of heterochromatin can be seen. Some cells contain more than 20. In some cells these have condensed to 2. In other intermediate cells the coiling has progressed to a point where the number of knots is reduced but complete coiling into two bodies has not yet been achieved. 
centrosome. Occasionally the bud-opening is too small to permit the contents of the vacuole to enter the bud and the canal is distended at this point like the esophagus of an ostrich swallowing an orange. A bulb is produced at the end of this process to form the budvacuole. During this period the centrosome is a hemispherical solid unyielding structure that is not deformed by movements of bodies near it. After the bud-vacuole is formed the centrosome divides. After the division of the centrosome is completed and the establishment of contact of bud-vacuole and bud-centrosome has been attained, the interconnecting canal between the mother- and bud-vacuole disappears.
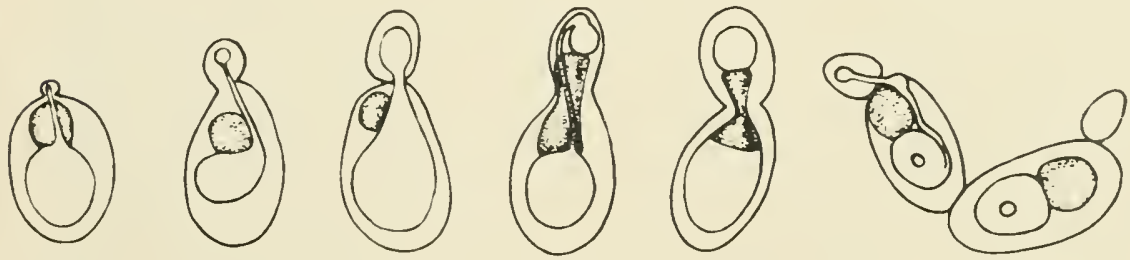

Fig. 6-5. Outline Drawings Showing the Vacuole and its Processes in Yeast Cells. The centrosome is stippled.

As soon as the bud approaches the size of the parent cell, the nuclear apparatus in bud and mother cell reorient themselves so that the centrosome in each cell is distal to the bud partition.

\section{THE MITOCHONDRIA}

In the cytoplasm of a resting (nonbudding) yeast cell one finds a variable number of mitochondria; some cells contain fifty or more (fig. 6-6). When the cell begins to divide they dissolve and the cytoplasm becomes optically homogeneous. These bodies in Saccharomyces were called basophilic granules by Guilliermond (1910) because they stained with basic dyes. In an ultramicroscope study of Saccharomycoeds (1932) he called the identical granules "lipoidal granules" because of their high refractivity. Fig. 6-7 shows a phase difference photograph of Saccharomycodes demonstrating the mitochondria. Wager and Peniston (1910) and Caspersson and Brandt (1941) called them volutin granules. I (1947) have pointed out that this is erroneous, since volutin does not occur in cytoplasm of living cells.

Caspersson and Brandt showed by precise ultraviolet microscopy that the cytoplasmic granules contain ribosenucleoprotein. 

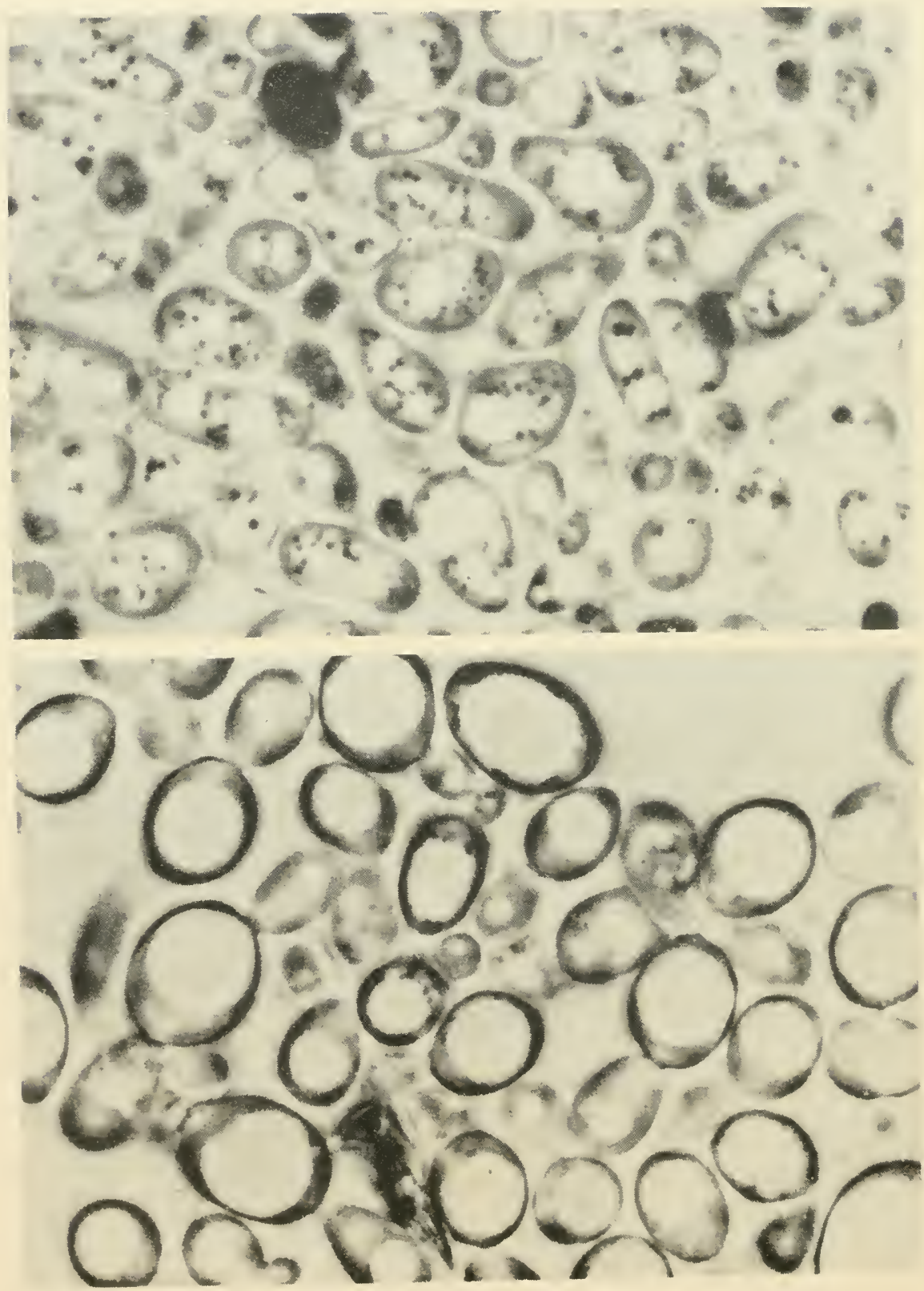

Fig. 6-6 Phase Difference Photograph of (a) Nondividing Cells Showing the Considerable Accumulation of Mitochondria and (b) Rapidly Dividing Cells Showing only a Few Mitochondria. 


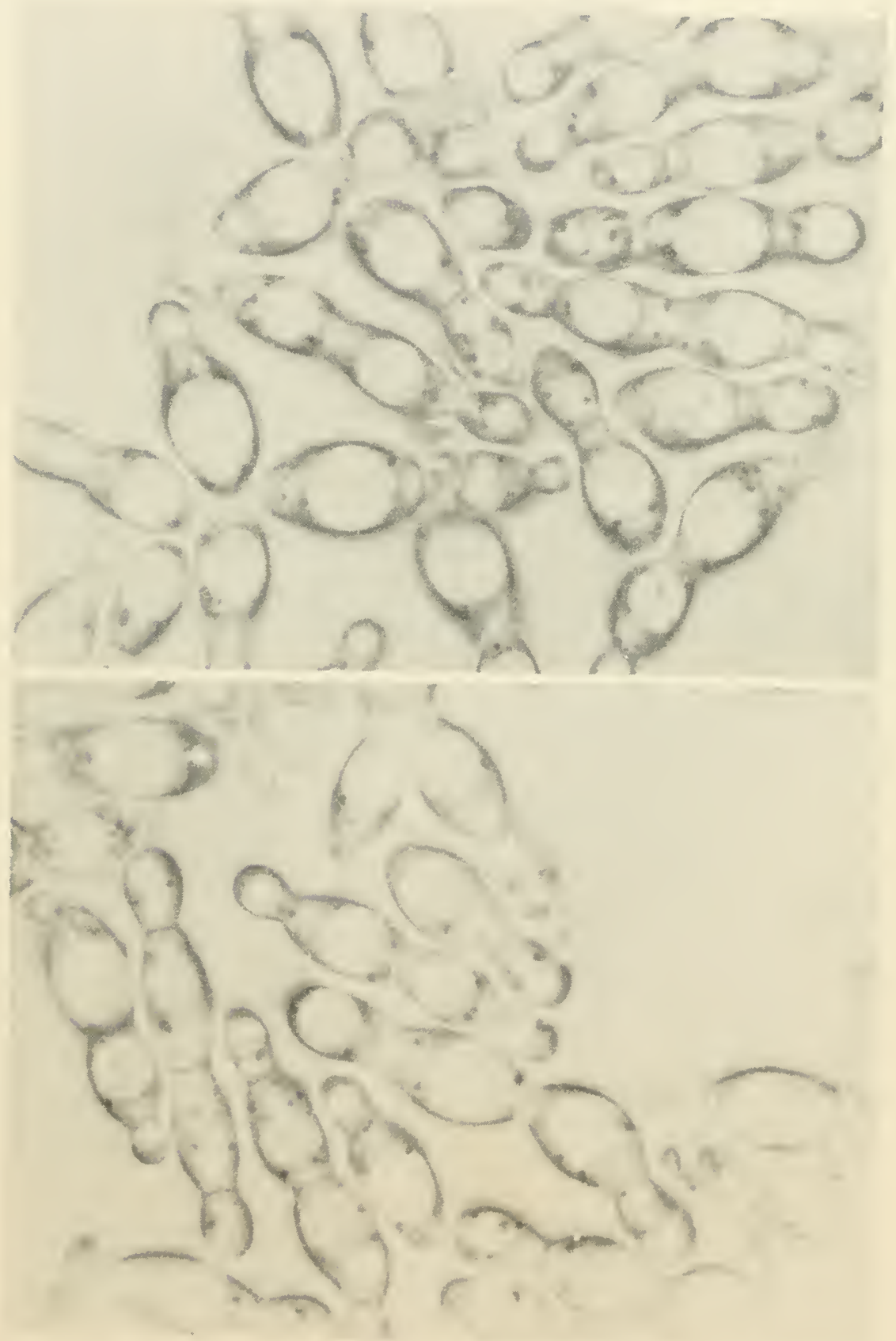

Fig. 6-7 Phase Difference Photograph of Saccharomycodes Ludwigii Showing Typical Budding and Mitochondria. 
Henneberg (1926) and I (1945) have called them fat granules because they are highly refractive; they coalesce when the cell is heated, or dead, or treated with alcohol, and are conspicuous in cells whose fat content is high. They are probably lipoidal coacervates with ribosenucleoproteins. Caspersson suggested that the cytoplasmic granules are the equivalents of both heterochromatin and the nucleolus, since heterochromatin is associated in higher forms with the production of ribose nucleoproteins. However, a true nucleolus is present in the vacuole and I consider the Feulgen-positive bodies in in the centrosome the equivalents of heterochromatin.

I (1948) have identified the cytoplasmic granules with mitochondria. They disappear when the cell is treated with acetic acid, which would not be expected if they contained only ribose nucleoprotein, but is characteristic of mitochondria. They coalesce when the cell is heated, or treated with alcohol, or subjected to pressure, which is also consistent with the view that they contain lipoids or fats. They increase while fat storage occurs and are abundant in cells containing large amounts of fat. Precise information on their reaction to fat stains is difficult to obtain, because most fat stains enter the living cell only slowly or not at all. The surrounding cytoplasm contains considerable amounts of fat, so that fat stains quickly overstain the entire cell. In dead cells the structure is so changed that localization of fat may easily lead to false conclusions concerning its actual disposition in the living cell. Caspersson and Brandt pointed out that the granules (mitochondria) show an irregular boundary with the ultraviolet microscope. With the Spencer phase-difference lens that I have been using, the boundary between the cytoplasm and the mitochondrium is very distinct (fig. 5-1). These facts suggest that the mitochondria are lipoidal or fatty bodies containing ribose nucleoprotein. Caspersson and Brandt found optically empty fat vacuoles in the cytoplasm in certain old cells. These can easily be produced by heating or merely drying and fixing yeast containing a large number of mitochondria on a slide in the usual manner. They state that the optically empty bodies are probably the fat granules described by Henneberg, kut Henneberg's figures show clearly that he meant the smaller granules in the cytoplasm when he spoke of "fat granules." Figs. 6-8 to 6-11 show mitochondria in various types of cells.

There seems to be a relation between the mitochondria and the centrosome. The last mitochondria to disappear when budding begins are those at the base of the centrosome (fig. 6-8). Mitochondria often form a mantle surrounding the centrosome. These facts suggest that the mitochondrial ribosenucleoproteins may be synthesized from the centrosomal Feulgen-positive heterochromatin. Caspersson and Schultz (1940) and Schultz, Caspersson and Aquilonius 


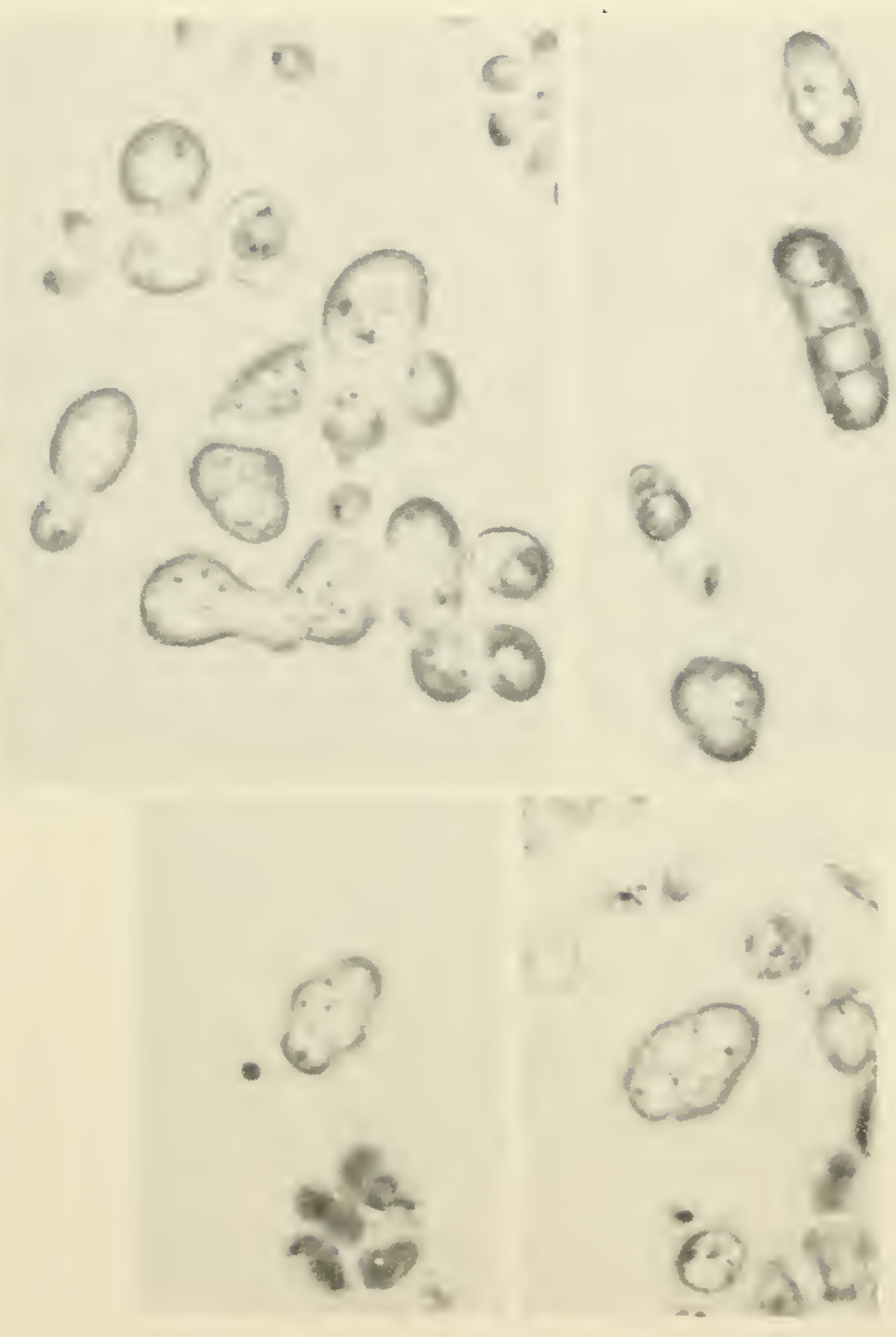

Fig. 6-8 Phase Difference Photograph Showing the Abundant Mitochondria in Copulating Cells and the Presence of one or more Mitochondria in each Spore. Note the centrosomes in bud and mother cell at left of copulating cells and mitochondria at junction of centrosome and nuclear vacuole. 


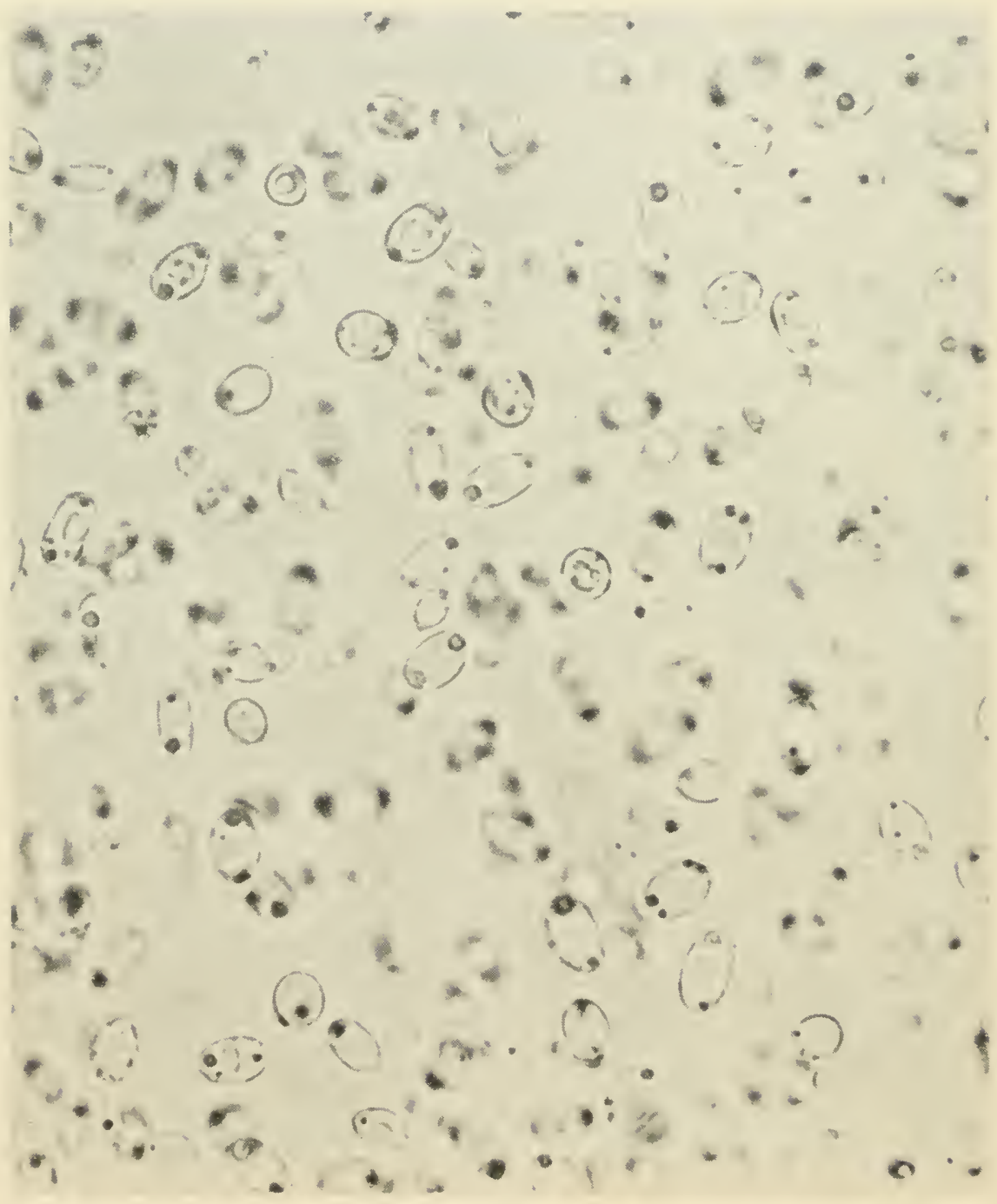

Fig. 6-9 Torulopsis Utilıs, Unstained, Photographed with Closed Diaphragm to Show Conspicuous Polar Mitochondria. Note nucleoli in some cells. 


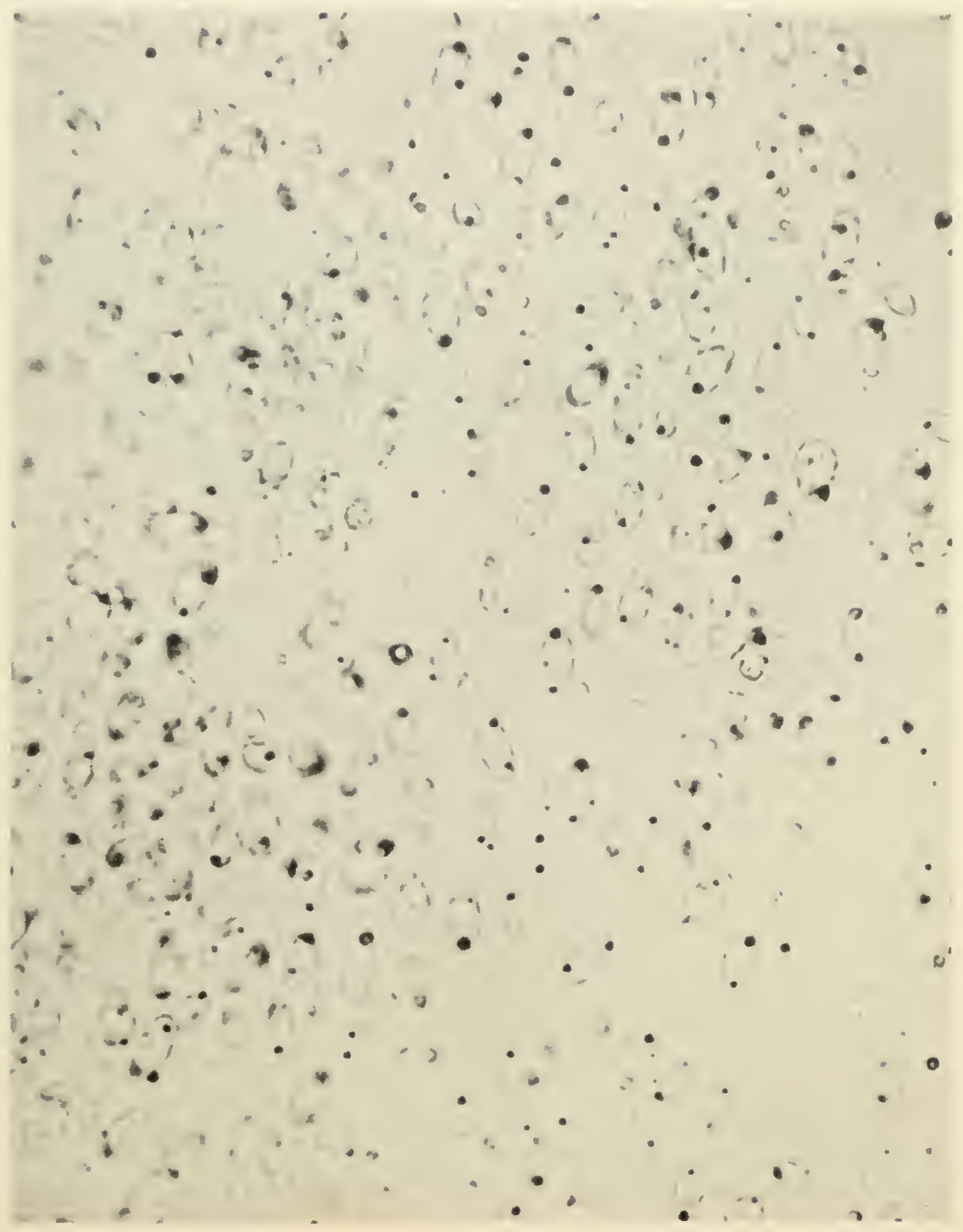

Fig. 6-10 Torulopsis Utilis, Unstained, Photographed with Closed Diaphragm to Show Conspicuous Polar Mitochrodria. Note nucleoli in some cells. 


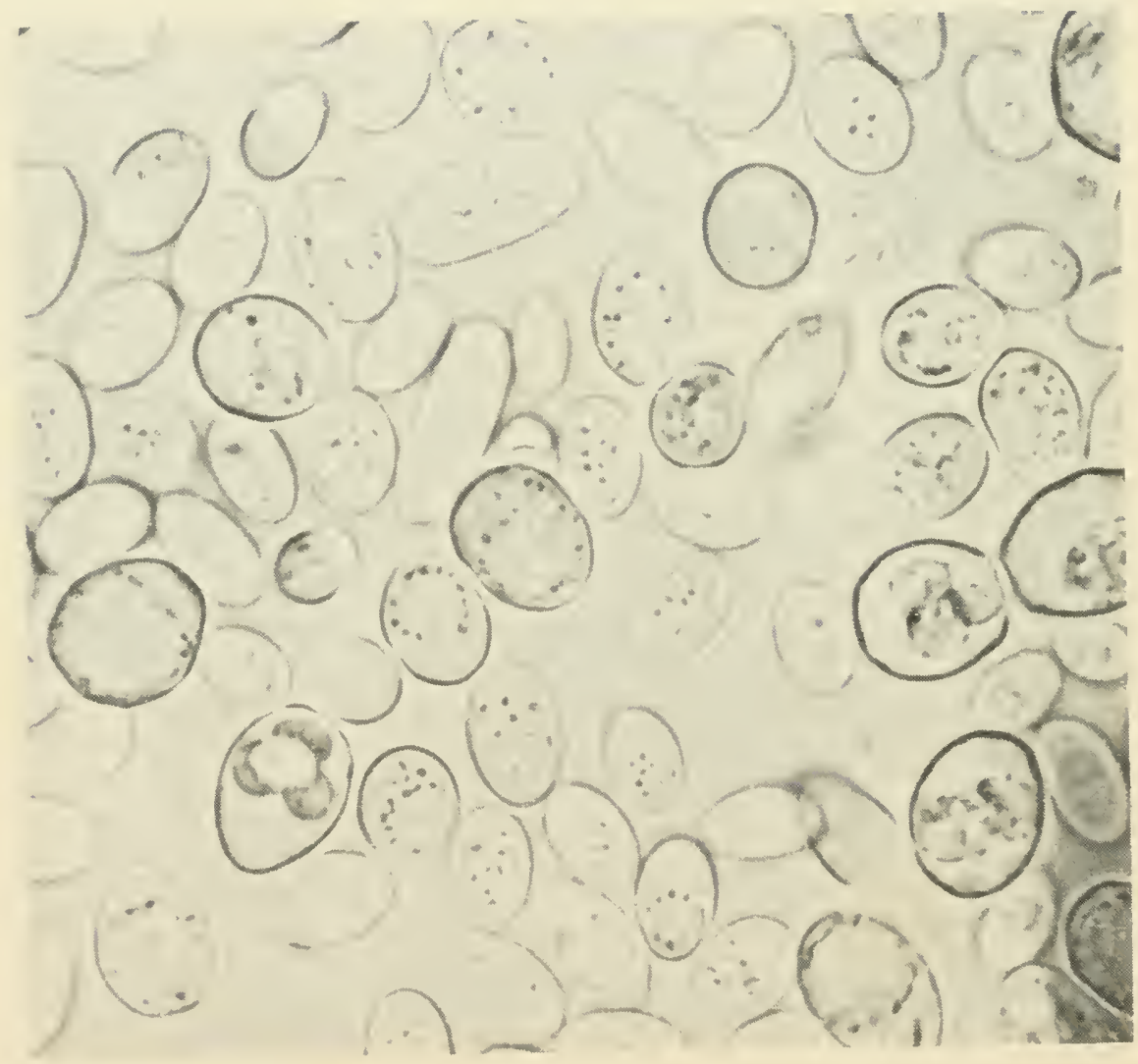

Fig. 6-11 Unstained cells of Saccharomyces Cerevisiae just Previous to Sporulation Contain Large Numbers of Mitochondria. Ordinary light microscope, diaphragm closed. 
(1940) suggested that the ribose nucleoproteins originated from the heterochromatic regions of the chromosomes.

\section{MITOSIS}

Pretreatment of cells by fluoride followed by treatment with diute toluidine blue has revealed structures in the vacuole which I identified as chromosomes. Fig. 6-12 shows 5 or 6 pairs of chromosomes in the vacuole. Fig. 6-13 shows two foci of a cell in which six pairs of chromosomes were counted. Fig. 6-15 shows six haploid chromosomes in the nuclear vacuole of a spore. Fig. 6-16 shows the fusion of nuclear vacuoles in copulation. Fig. 6-17 shows paired chromosomes in the nuclear vacuole of newly formed zygotes. Fig. 6-18 is a Feulgen stained preparation (by Rafalko) showing that the vacuole contains Feulgen positive chromosomes. Fig. 6-19 shows a series of photographs of yeast cells stained with toluidine blue. On the basis of these and similar observations I have proposed that yeast mitosis occurs according to the steps shown in fig. 6-20.

Fig. 6-12 Paired Chromosomes Stained with Toluidine Blue.

Observations of living cells have shown that the chromosomes in the non-dividing cell are each attached by one end to the inner wall of the vacuole and wave around in the nuclear sap. They seem to be relatively rigid and are apparently rather evenly spaced about 

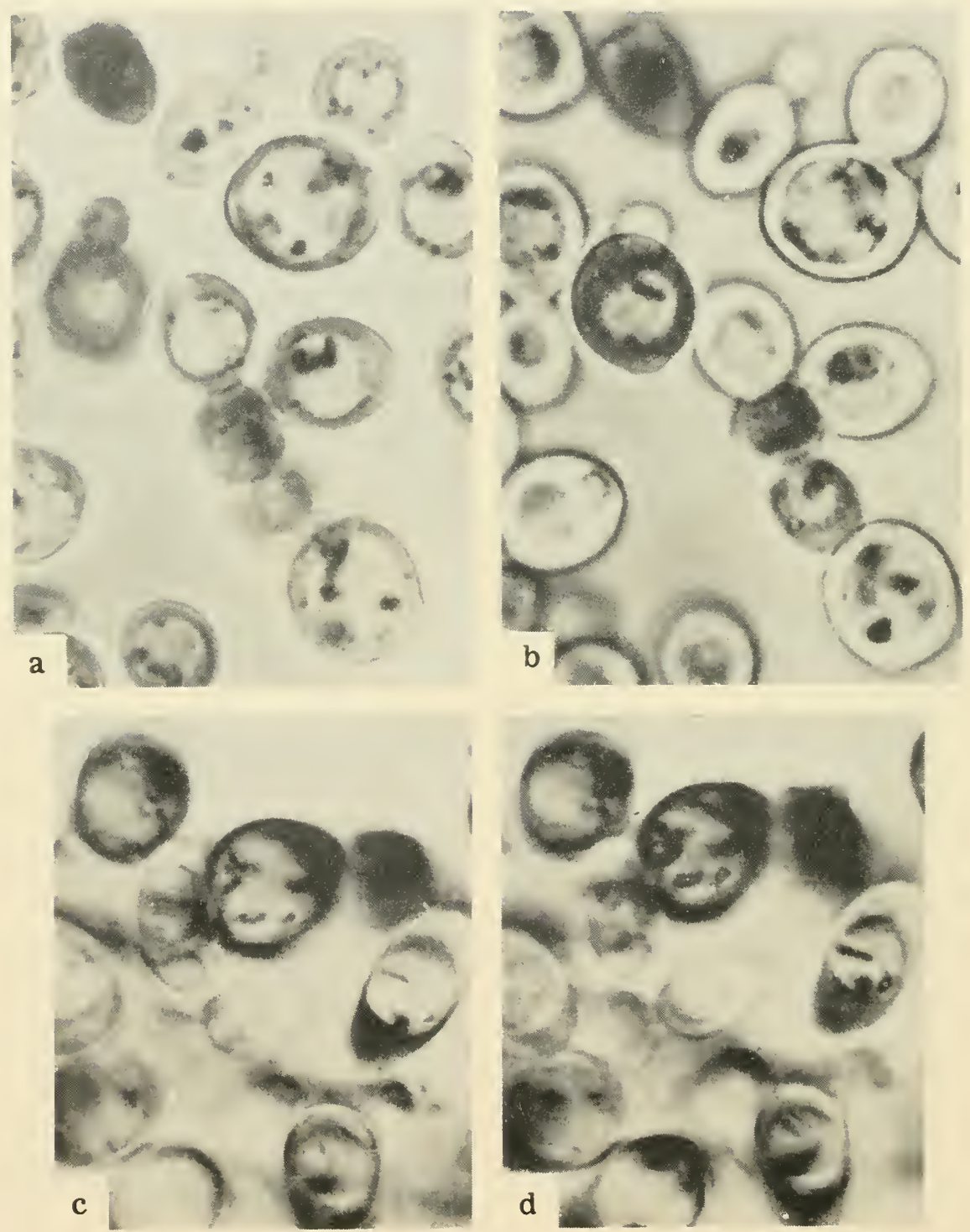

Fig. 6-13 Various Foci of Preparations Showing Chromosomes in the Nuclear Vacuole Stained with Toluidine Blue. 
the inner wall of the nuclear membrane. They are extraordinarily long in the living state, reaching across the entire vacuole and bending to conform to the inner aspect of the opposite side of the membrane. When the twelve long chromosomes are waving about in the nuclear sap, the vacuole is completely filled with vibrating chromosome threads. In most killed cells the chromosomes are rather short rods, which still retain their equidistant disposition around the inner aspect of the nuclear membrane, but remain pointing inwards. Occasionally several of the chromosomes are polarized towards the centrosome, but usually they are equidistantly distributed around the entire surface. This disposition of the chromosomes means that their ends are in close proximity either to the mitochondria or the ribose nucleoprotein dispersed from them.

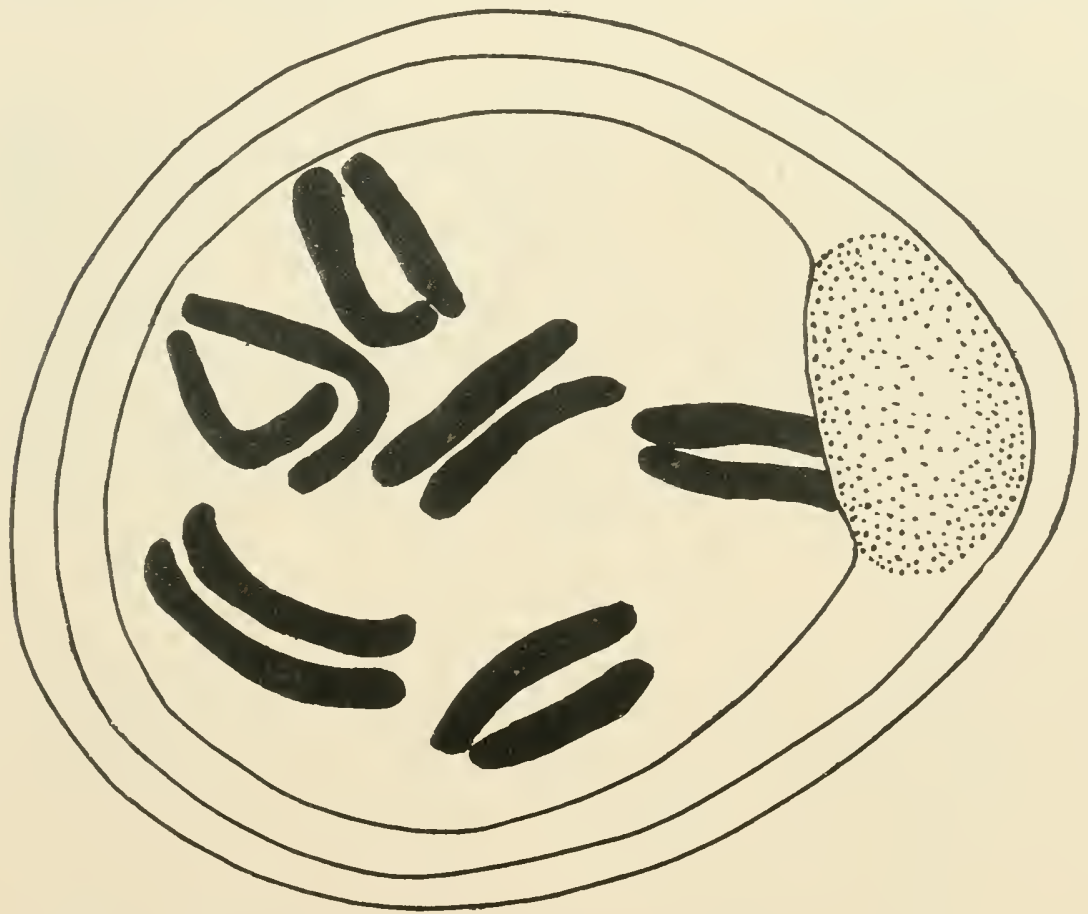

Fig. 6-14 Reconstruction of the Cell Shown in Fig. 6-13, c and d.

As mitosis begins, the homologous chromosomes approach each other but retain their attachment to the inner wall of the nuclear membrane (fig. 6-20), so that they are found in pairs with the distal ends waving around in the nuclear sap. Preliminary to mitosis the paired chromosomes shorten down to small cylindrical rods less than one-tenth their original length, presumabiy by the normal 
mechanism of coiling, producing six pairs. As soon as they are completely coiled the six small pairs detach themselves from the nuclear membrane. They vibrate in the vacuole in rapid Brownian movement, and finally begin to orient themselves in a single complex. This process can be observed in a series of steps in which each one of the six pairs is at first distinctly identifiable but finally becomes merged into a single structure, which appears to consist only of two long paired bands stretching across the entire diameter of the nuclear vacuole. Occasionally these bands are twisted about each other. The chromosome complex is flat and ribbonshaped, much wider than it is thick. It has a pronounced longitudinal split and the appearance of two flat ribbons attached to each other by their edges. This flat characteristic becomes apparent when the six pairs are being arranged in the complex. The next-

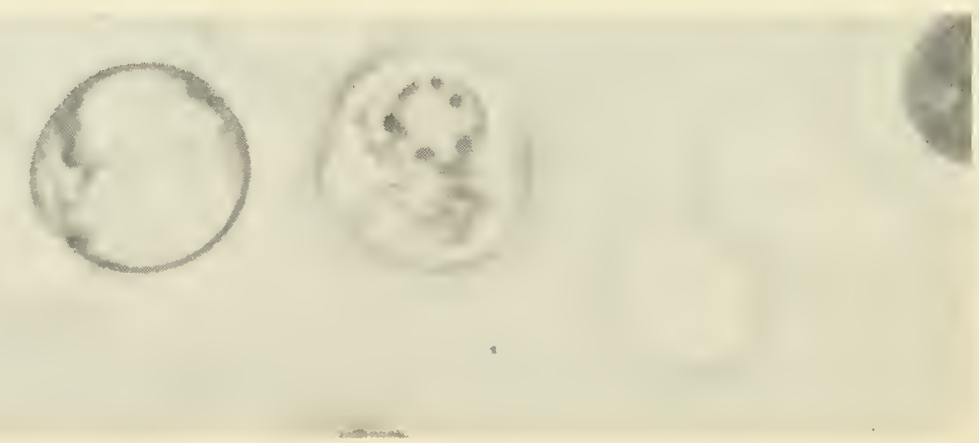

Fig. 6-15 The Central Structure is a Germinating Four-spored Ascus. One ascospore is in sharp focus and the wall of the spore can be seen enclosing the cell which consists of cytoplasm, crowded at one end, and the growing nuclear vacuole. Six haplnid chromosomes can be seen in the vacuole. Parts of two other spores, one below and one to the lower left are visible; the fourth is not visible in the optical section. The stain is toluidine blue.

step is the longitudinal splitting of the entire complex along the flat plane. Partially split complexes, resembling $\mathbf{Y}$-shaped figures, are often visible. It is also possible to find a pair of complexes in the cell, one lying across the other, each one extending the full distance of the vacuole, often bending when they meet the inner surface. One of these complexes passes into the bud vacuole by slipping through the bud canal; and immediately afterwards, or during this passage, begins to break down into twelve individual chromosomes. 

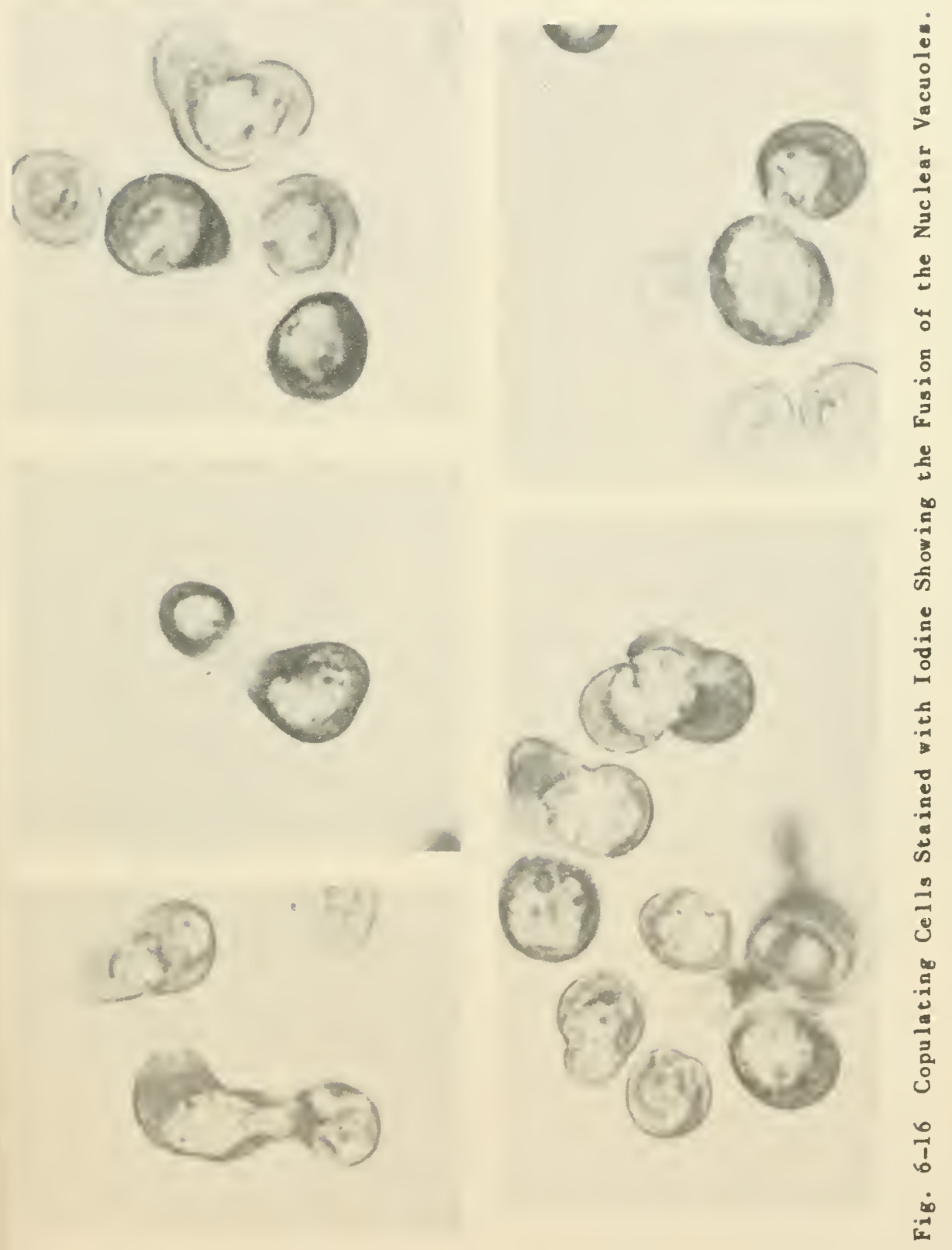


\section{DEMONSTRATION OF NUCLEOLUS, CHROMOSOMES, AND CENTROSOME BY STANDARD CYTOLOGICAL TECHNIQUES}

Mrs. Rafalko and I (unpublished) made cytological examinations of yeast cells by a variety of standard techniques. This involves dehydration of cells and considerable shrinkage, but is compensated for by better resolution and the possibility of comparison with conventional preparations. Most of our data are on Saccharomyces bayanus. The nucleolus lies in the nuclear vacuole. When stained with haemotoxylin, (Rafalko, unpublished) it is found to contain from one to three internal plastin bodies, characteristic of the conventional endosomes found in other forms. Haemotoxylin is effective in showing internal structures in the nucleolus probably by revealing differences in density. A halo of unstained material surrounds the nucleolus, similar to the halo surrounding the endosome of protozoa. Occasionally radiating threads can be seen extending from the nucleolus through the surrounding halo into the nucleoplasm. Fig. 6-21 is a photograph of one of Dr. Rafalko's Feulgen-stained preparations showing four pairs of Feulgen-positive chromosomes in the nucleus with a Feulgen-negative nucleolus attached to one pair.
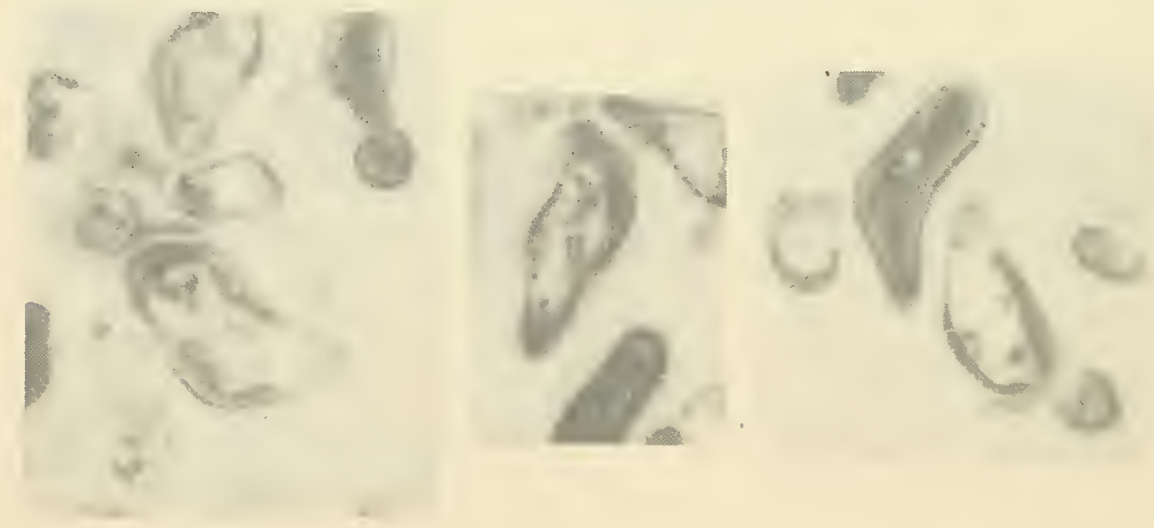

Fig. 6-17 Hecently Copulated Cells Stained with Toluidine Blue Showing Paired Chromosomes in the Zygote Nucleus.

In $\mathrm{S}$. cerevisiae, two round nucleoli are present in the early stages of growth. Eventually both nucleoli fuse.

In $\mathrm{S}$. bayanus, a single nucleolus is observed in the chromeacetic-formaldehyde Feulgen preparations and in chrome-acetic formaldehyde preparations stained with either methyl green and pyronin or methyl green and acid fuchsin; iwin nucleoli have not yet been found in $S$. bayanus. The nucleolus in S. bayanus is very large, and centrally located, and much larger than that of S. cerevisiae and more elongate, although in a young cell it may be rounder 


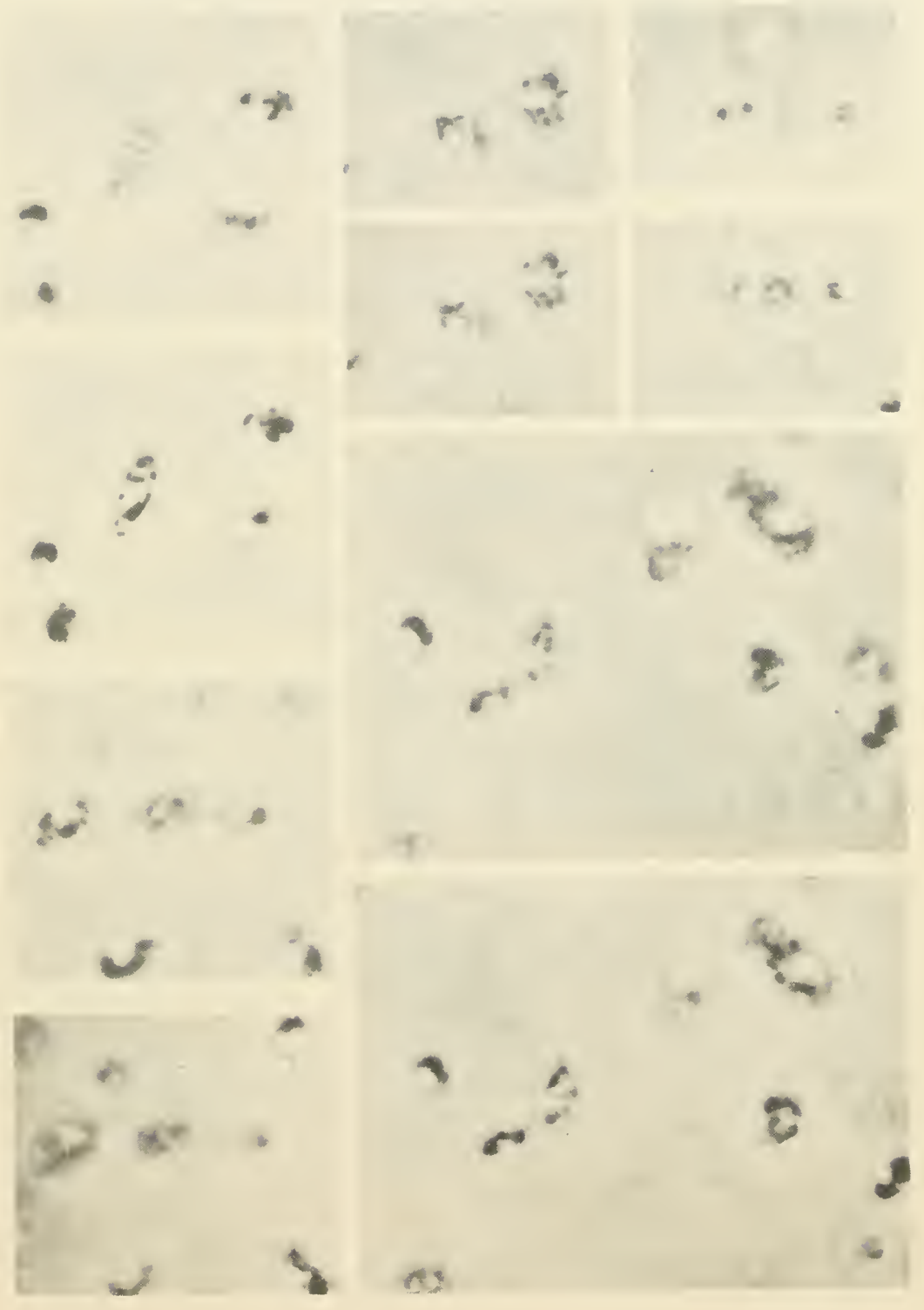

Fig. 6-18 Photographs of cella stained by Rafalko with his modified Feulgen. The deeply stained chromosomes in the vacuole are clearly visible. I am indebted to Dr. Rafalko for permitting me to photograph his preparations. The heterochromatin outside the vacuole can be seen clearly in two of the photographs. These photographs are made with the phase-difference microscope which intensifies the light color of the Feulgen stain. 

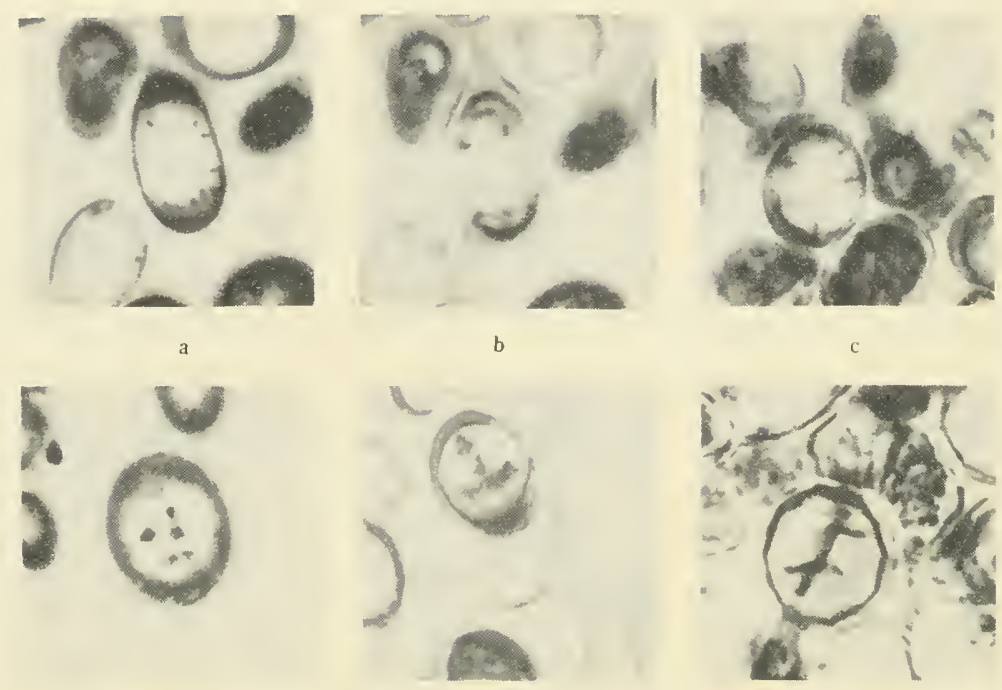

d
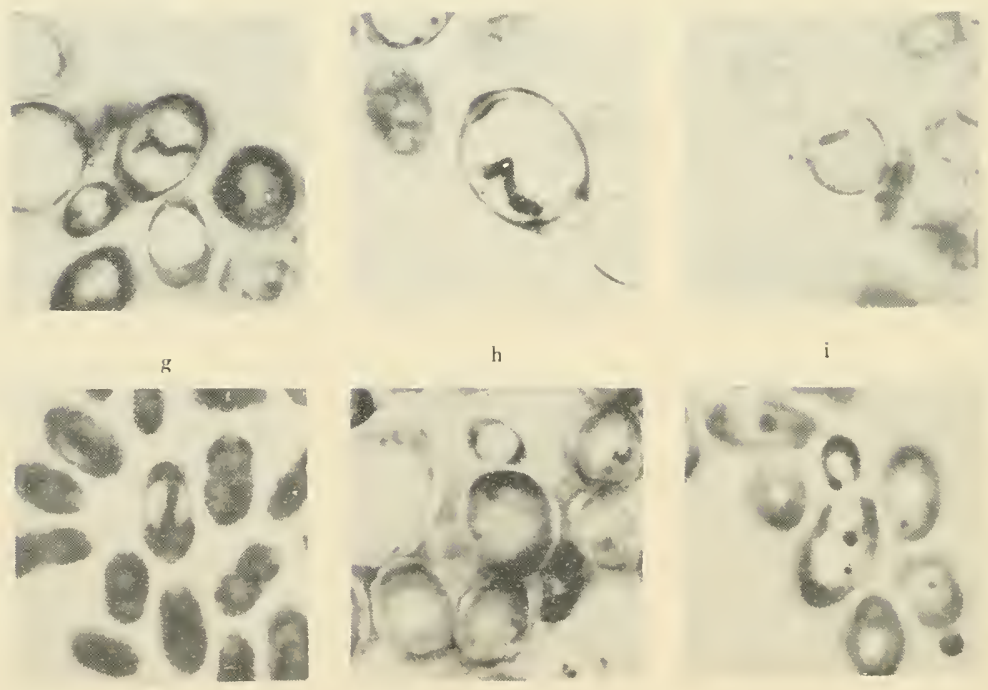

Fig. 6-19 (a) and (b) Twelve chromosomea attached to the wa!l of the nuclear vacuole. (a) Is high focus and (b) is low focus of the same cell. (c) Five pairs of chromosomes attached to the wall of the vacuole. (d) One focus of a cell containing six pairs of chromosomes; only five pairs are visible in this optical section. (e), ( $f),(g)$, stages in formation of complex. (b), (i), (j), newly formed complex with longitudinal split. (k) Cell with a chromosome with attached nucleolus (?), vibrating in the vacuole. (1), First division of a reating cell, showing two mitochondria, one in the mother cell and one in the bud. All but (f) are photographed with a Spencer phase-difference lens. 


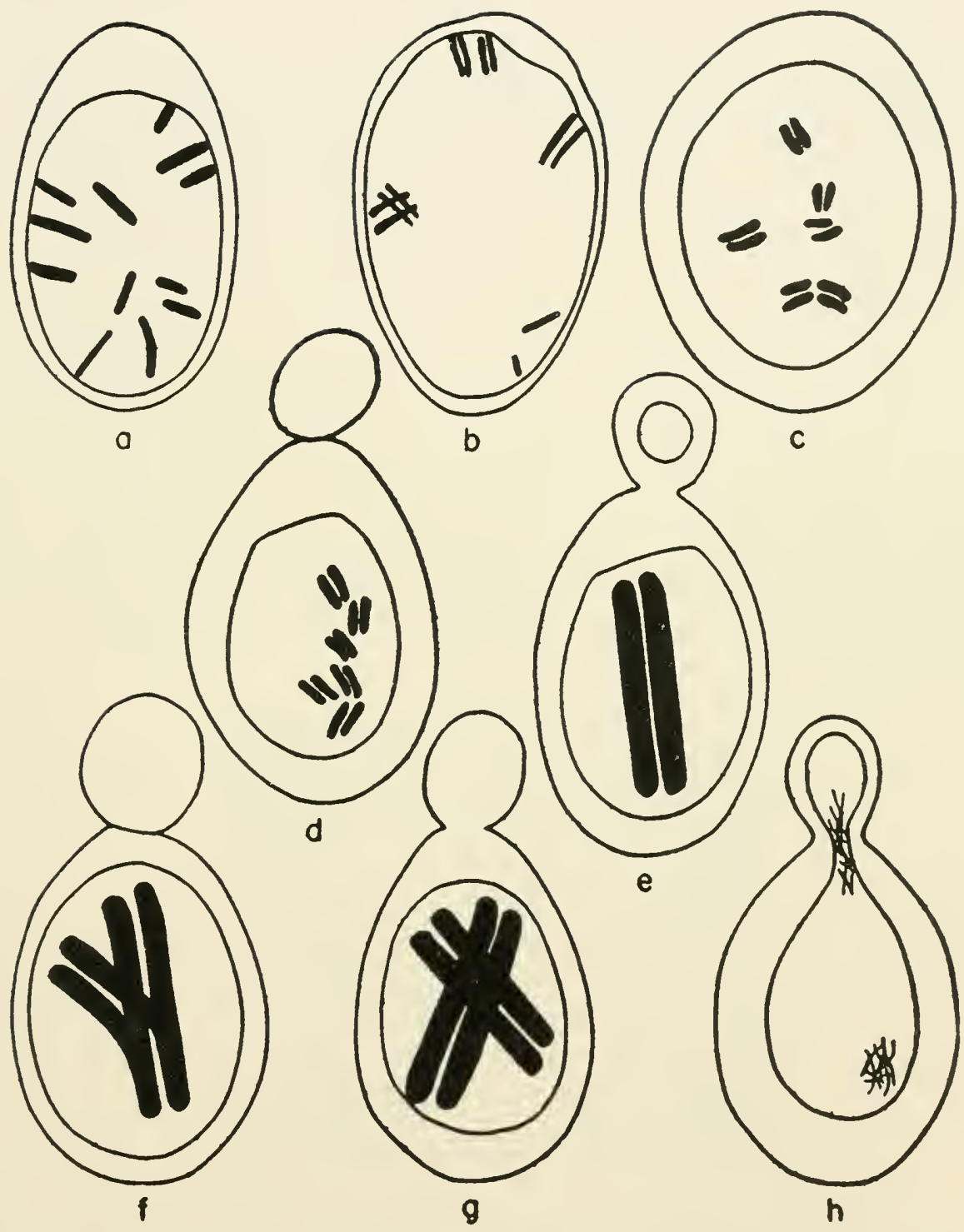

Fig. 6-20 Mitosis in the yeast cell. (a) Twelve chromoames attached to the wall of the nuclear vacuole. (b) The chromosomes pairing up, still attached to the wall of the vacuole. (c) Six pairs of chromosomes free in the nuclear sap. (d) Aggregation of the six pairs into a complex. (e) A fully formed nuclear complex. (f) Longitudinal split producing two complexes. (g) Two separated complexes following the longitudinal split. (h) Chromosomes from one complex passing into the bud vacuole. 
than in an older one. The chromosomes in the nuclear vacuole are practically always paired after the cells have been growing for six hours or more. There appear to be four or five pairs in S. bayanus and five or six in S. cerevisiae, Mrak 77. They appear to have the shape of paired cylindrical rods or crescent-shaped coffee-bean like structures. They stain a dark green with methyl green and a deep red with Feulgen following chrome-acetic-formaldehyde fixation. Methyl green is an acceptable chromatin stain and has long been recognized for its affinity for chromosomes. When chromeacetic-formaldehyde is followed by acid fuchsin-methyl green the centrosome always appears as a dense red body surrounded by an area which is pale pink. In these preparations the nuclear membrane can be traced to include all the centrosome. The nucleolus is is pale green and is always visible after $1 \frac{1}{2}$ hours incubation. Both nucleolus and chromosomes are not generally visible simultaneously. The pronounced and constant distinction between the centrosome and the chromosomes suggests that these structures which are both Feulgen-positive are different in some other respect than in content of desoxyribose-nucleoprotein.

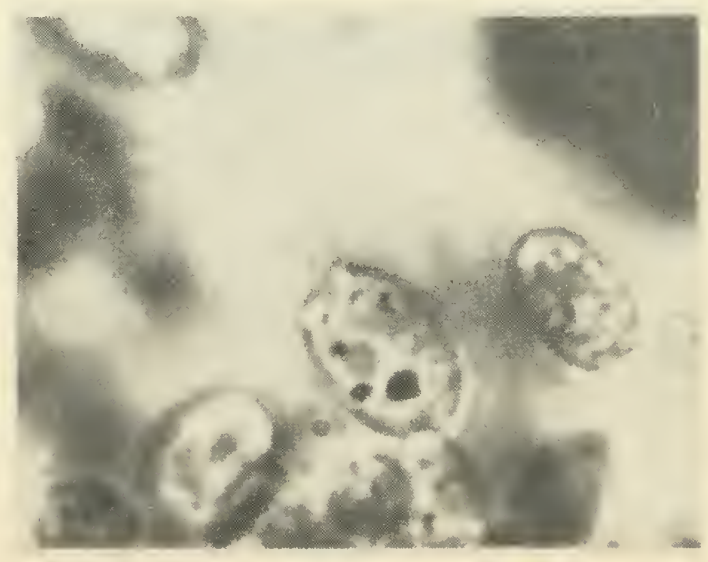

Fig. 6-2l A Feulgen-stained (using Rafalko's modification) yeast cell in a preparation made by Dr. Rafalko showing four pairs of Feulgen-positive chromosomes in the nuclear vacuole, one pair of which is attached to the Feulgen-negative nucleolus.

The methyl green-pyronin combination al ways shows the centrosome as a pale green structure. The chromosomes are dark green when visible and the nucleolus is pale green when visible. Either chromosomes or nucleolus are usually visible along with the constantly appearing centrosome. Sometimes all three are 
visible simultaneously. The cytoplasm is pink, indicating the presence of ribosenucleoprotein in the cytoplasm. The green coloration of the centrosome and chromosomes is consistent with the view that both contain chromatin. The green color of the nucleolus suggests that it may also contain chromatin on occasion. This view has been supported by the Feulgen stain which has on rare occasions seemed to reveal pink colored nucleoli.

The Feulgen method often stains the centrosome without staining the chromosomes; or the chromosomes may be stained while there is little or no stain in the centrosomes. We have rarely observed a pink coloration with Feulgen in the nucleolus, but have never found all three structures Feulgen-positive simultaneously. Chrome-acetic-formaldehyde followed by Feulgen and fast green reveal a typical Feulgen purple-red in the centrosome. The chromosomes were somewhat refractile and red-red, rather than the characteristic purple-red. The chromosomes were also a much fainter color than the purplish Feulgen-positive structures in the centrosome. Carnoy material stained with Feulgen shows the centrosome with excellent detail and reveals the crescent-shaped bodies so often described by earlier workers in the centrosome. Carnoy, however, is a poor fixative for vacuole and chromosomes; in fact, they are rarely visible after Carnoy. Chrome-acetic-formaldehyde is an excellent fixative for chromosomes. When the Feulgen stain was used on S. cerevisiae, Mrak 77 following mercuric chloride fixation, only the centrosome appears to take the Feulgen stain (these stains were made after 1 to $1 \frac{1}{2}$ hours). When, however, mercuric chloride and chromic acid are used in the killer, the centrosome is visible, with chromosomes occasionally visible in the same cells. Carnoy fixation followed by Feulgen and fast green has shown the nucleolus occasionally to be refractile and faintly pink. The following tabulation shows the results with different varieties of yeasts.

The demonstration of a structure in the nuclear vacuole identified as the nucleolus associated with Feulgen-positive threads confirms the view that the Feulgen-positive threads are chromosomes and eliminates the possibility that the Feulgen-positive structures in the centrosome are chromosomal. This suggests that the centrosomal chromatin may be the equivalent of the heterochromatin in higher organisms. The apparent shifts of chromatin from chromosomes to centrosome and vice versa suggest a cyclic relation between the chromosomal and the centrosomal chromatin, and the fact that the nucleolus sometimes, though rarely, contains chromatin suggests that it may be involved in the cycle. 


\begin{tabular}{|c|c|c|c|c|c|}
\hline Fixation & $\begin{array}{l}\text { Chrome- } \\
\text { acetic } \\
\text { formalde- } \\
\text { hyde }\end{array}$ & $\begin{array}{l}\text { Chrome } \\
\text { acetic } \\
\text { formalde- } \\
\text { hyde }\end{array}$ & $\begin{array}{l}\text { Chrome- } \\
\text { acetic } \\
\text { formalde- } \\
\text { hyde }\end{array}$ & $\begin{array}{l}\text { Chrome- } \\
\text { acetic } \\
\text { formalde- } \\
\text { hyde }\end{array}$ & Carnoy \\
\hline Stain & $\begin{array}{l}\text { Acld } \\
\text { fuchsin- } \\
\text { methyl } \\
\text { green }\end{array}$ & $\begin{array}{l}\text { Pyronin- } \\
\text { methyl } \\
\text { green }\end{array}$ & $\begin{array}{l}\text { Feulgen- } \\
\text { fast } \\
\text { green }\end{array}$ & $\begin{array}{l}\text { Safranin- } \\
\text { methyl } \\
\text { blue }\end{array}$ & $\begin{array}{l}\text { Feulgen- } \\
\text { fast } \\
\text { green }\end{array}$ \\
\hline Organism & $\begin{array}{l}\mathrm{S} \text {. } \\
\text { bayanus }\end{array}$ & $\begin{array}{l}\text { S. } \\
\text { bayanus }\end{array}$ & $\begin{array}{l}\text { Mrak } 77 \\
\text { S. cerev- } \\
\text { isiae, } \\
\text { S. } \\
\text { bayanus }\end{array}$ & $\begin{array}{l}\mathrm{S} \text {. } \\
\text { bayanus }\end{array}$ & $\begin{array}{l}\text { S. cerev- } \\
\text { isiae } \\
\text { Mrak } 77 \\
\text { S. } \\
\text { bayanus }\end{array}$ \\
\hline Nucleolus & $\begin{array}{l}\text { pale } \\
\text { green }\end{array}$ & $\begin{array}{l}\text { pale } \\
\text { green }\end{array}$ & $\begin{array}{l}\text { pale } \\
\text { green }\end{array}$ & $\begin{array}{l}\text { pale } \\
\text { blue }\end{array}$ & $\begin{array}{l}\text { pale } \\
\text { green }\end{array}$ \\
\hline Chromosome & $\begin{array}{l}\text { dark } \\
\text { green }\end{array}$ & $\begin{array}{l}\text { dark } \\
\text { green }\end{array}$ & $\begin{array}{l}\text { red-red } \\
\text { refractile }\end{array}$ & red & $\begin{array}{l}\text { red-red } \\
\text { and } \\
\text { somewhat } \\
\text { refractile }\end{array}$ \\
\hline Centrosome & $\begin{array}{l}\text { red } \\
\text { surrounded } \\
\text { by pale } \\
\text { area or } \\
\text { halo }\end{array}$ & $\begin{array}{l}\text { pale } \\
\text { green }\end{array}$ & $\begin{array}{l}\text { purple- } \\
\text { red }\end{array}$ & pink & $\begin{array}{l}\text { internal } \\
\text { structure; } \\
\text { purple- } \\
\text { red }\end{array}$ \\
\hline
\end{tabular}

\section{VOLUTIN (METAPHOSPHATE)}

Volutin is a cytological entity which is usually fixed with formaldehyde, and retains basic dyes after an acid rinse. Babes described it as metachromatin in bacteria in 1889. Meyer named it volutin in 1904 and described its staining qualities specifically. Guilliermond (1902) proposed changing the name volutin back to metachromatin. He called the intravacuolar bodies in yeast metachromatic granules. I have identified them as chromosomes or nucleoli coated with metaphosphate. He called the mitochondria (cytoplasmic granules which lose their stains when treated with dilute acid) basophilic granules.

Both Meyer and Guilliermond made extensive studies of the distribution of volutin in cells. Guilliermond (1910) concluded that volutin is a reserve material like glycogen and fat which is widely distributed, especially in the vacuoles of fungi and bacteria. 
He considered the yeast vacuole to be homologous to the vacuoles of higher organisms and did not consider it part of the nuclear apparatus. In spite of the fact that he states that his tests for volutin were the same as those specified by Meyer, the legends of his drawings often indicate that the stain used was haemalum, which would stain the mitochondria equally well. He states specifically that metachromatin (volutin) passes from the vacuole of the yeast cell into the spores.

Wager and Peniston engaged in a controversy with Guilliermond concerning the appearance of volutin in the cytoplasm. I have concluded that metaphosphate does not appear in the cytoplasm of living cells although it can be found in the cytoplasm of dead cells. Caspersson and Brandt (1941) have shown that ribose nucleoprotein is always present in the cytoplasm, either as granules, which I have identified with mitochondria, or dispersed, but they did not detect nucleic acid in the vacuole with the ultraviolet microscope, suggesting that it contained little nucleic acid. Since volutin is abundant in the vacuole, volutin in the sense of Meyer (metachromatin-Babes, Guilliermond) is different from the cytoplasmic ribose nucleoprotein granules which Caspersson and Brandt have called "volutin" and which I have identified with mitochondria.

Volutin is distinguished by its ability to retain basic stains when the cells are rinsed in dilute acid. When volutin is stained with methylene blue or toluidine blue, it retains the stain after treatment with 1 percent sulphuric acid. In addition to being "acid fast," volutin has the characteristic of staining red or purple with methylene blue or toluidine blue, in contrast to other basophilic components of the cell which stain blue with these dyes. Both metaphosphates and estersulphates retain dyes after destaining and stain red or purple with toluidine blue. Volutin is especially abundant in copulating yeast and in the newly formed zygote nuclei (fig. 6-22). Volutin is widely distributed in fungi and bacteria, while the estersulphates (Sylvén) are apparently widely distributed in higher animals. Wiame's work strongly suggests that the volutin in yeast cells is metaphosphate, because the addition of the phosphate to the cell increases the amount of volutin demonstrable in the cell, and extracts from these heavily stained volutin-containing cells yield reasonably large quantities of metaphosphate. There is, however, no cytological means of distinguishing between metaphosphates and estersulphates.

\section{THE TRANSFER OF VOLUTIN FROM CHROMOSOMES TO NUCLEOLUS}

The nucleolus and chromosomes can also be demonstrated with the volutin stain. [The nucleolus is a conspicuous structure in the nuclear vacuole. When present it is easily demonstrated with Lugol's 


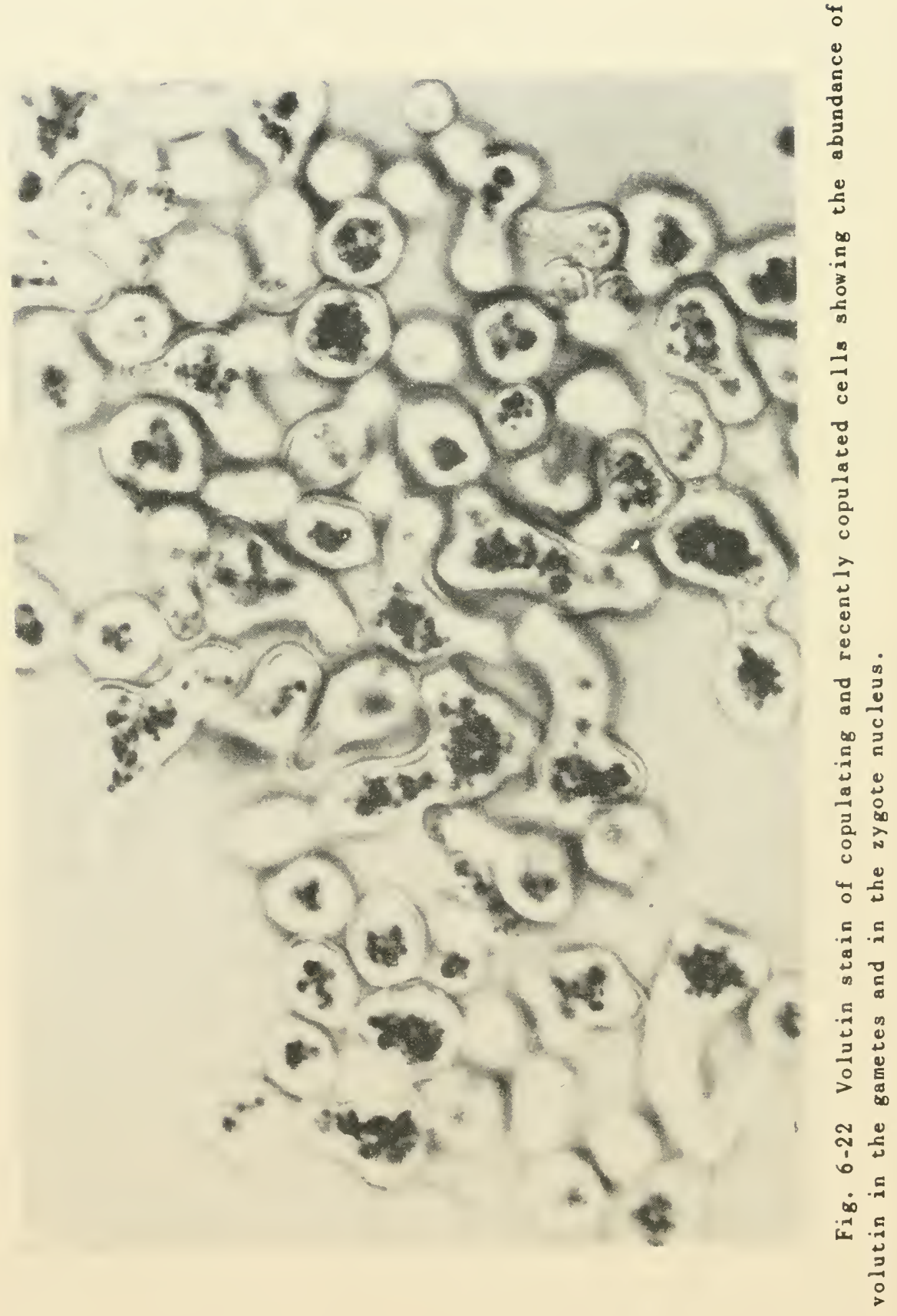


solution. Figs, 6-23 and 24 are preparations of yeast cells stained with Lugol's and reveal nucleoli in many of the vacuoles. The centrosomes are also visible in many cells.] If the chromosomes are coated with volutin, they are stained purple (fig. 6-25). If the nucleolus is coated with volutin, it is stained purple by the volutin stain (fig. 6-26). As soon as either the chromosomes or the nucleolus lose their volutin, they are no longer specifically stainable. The density of the stain also gives some idea of the quantity of volutin present. The best results are obtained when the volutin stain is adjusted to a $\mathrm{pH}$ of 2.5 , which prevents the proteins in the cytoplasm from also becoming stained.

The growth of S. cerevisiae was followed closely from resting to actively-growing, vigorously-fermenting cells (fig. 6-28). In resting cells, the chromosomes are unstained and unstainable by either the volutin stain or iodine, and the nucleolus is invisible or absent. After the cells have been in nutrient medium for a short time, (before volutin appears on the chromosomes) the chromosomes stain brown with iodine suggesting that they may contain carbohydrate. Fig. 6-27 is a photograph of cells fixed in dilute formalin and stained with iodine. Before budding begins, the chromosomes become coated with volutin, and two unstained nucleoli become visible in each cell. As budding begins, the nucleoli increase in size, volutin disappears from the chromosomes and appears on the nucleolus. Transfer of volutin occurs by a direct attachment of chromosome to nucleolus, for two or three small, dark purple dots can be seen attached to an unstained nucleolus. Generally, one nucleolus becomes coated with volutin before the other, and subsequently both fuse. These fused nucleoli are the commonly described refractile "dancing bodies" in the yeast vacuole. The nucleolus is a large, round structure, possibly 2 or 3 microns in diameter. At first the interior of the nucleolus is not deeply stained with the purple dye; the color is on the outer edge or surface of the structure. The nucleolus often becomes quite large before volutin appears on it.

The following typical results were obtained when 7 grams of compressed yeast were inoculated into $500 \mathrm{cc}$. of medium of the following composition:

$\begin{array}{lrl}\text { Liquid yeast extract } & 6 \mathrm{cc} \text {. } \\ \text { Peptone } & 5 \mathrm{gm} . \\ \mathrm{KH}_{2} \mathrm{PO}_{4} & 2 \mathrm{gm} . \\ \text { Corn steep water solids } & 2 \mathrm{gm} . \\ \text { cerelose } & 40 \mathrm{gm} . \\ \text { per liter of tap water. } & \end{array}$

The culture was grown in a 6-liter Erlenmeyer flask on a shaker.

Yeast cells in a cake of baker's yeast are small and compact and ordinarily show little or no volutin in the vacuole. During the 


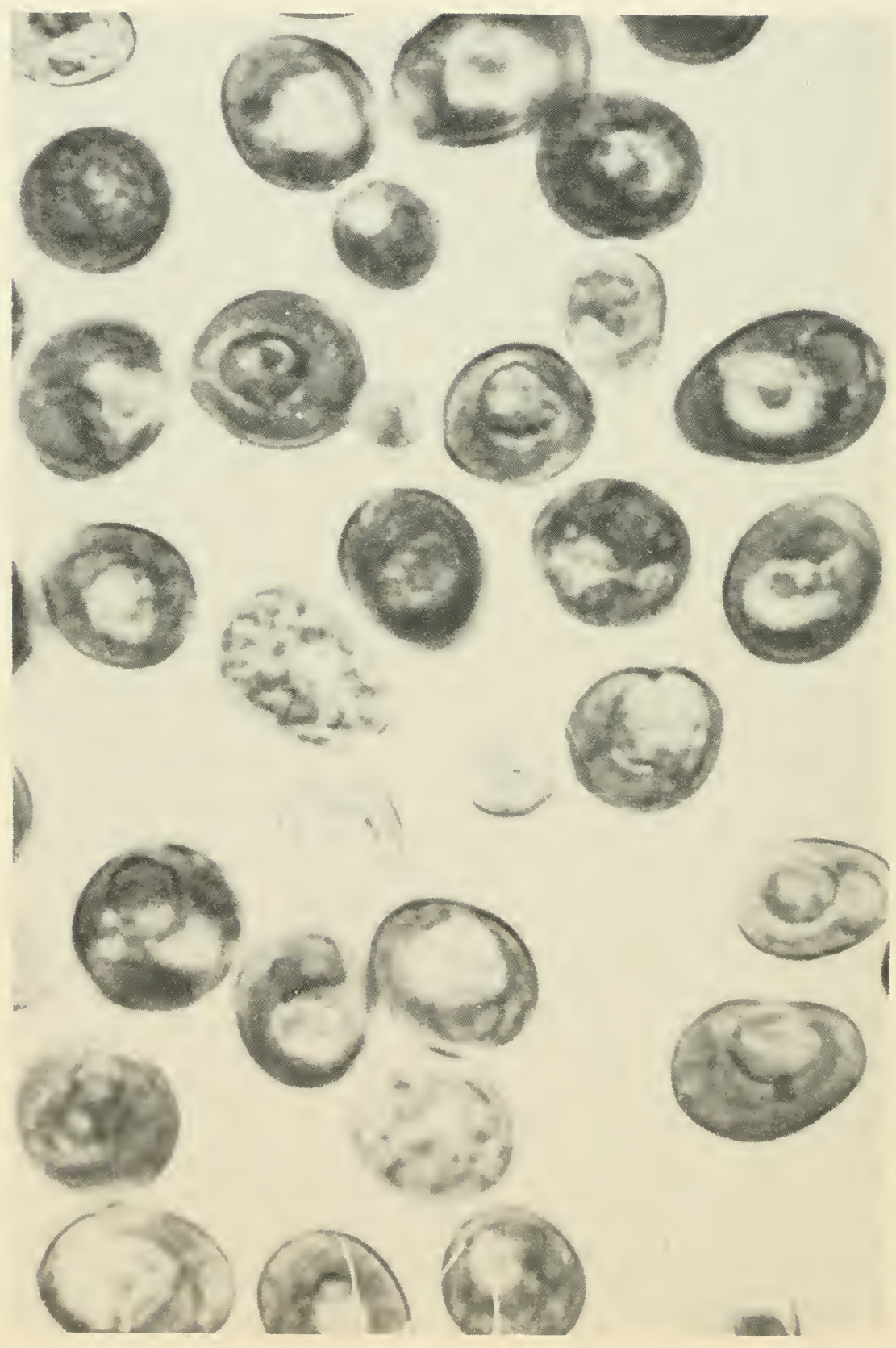

Fig. 6-23 Lugol's stained yeast cells containing large nucleoli and showing the centrosome in many cells. 


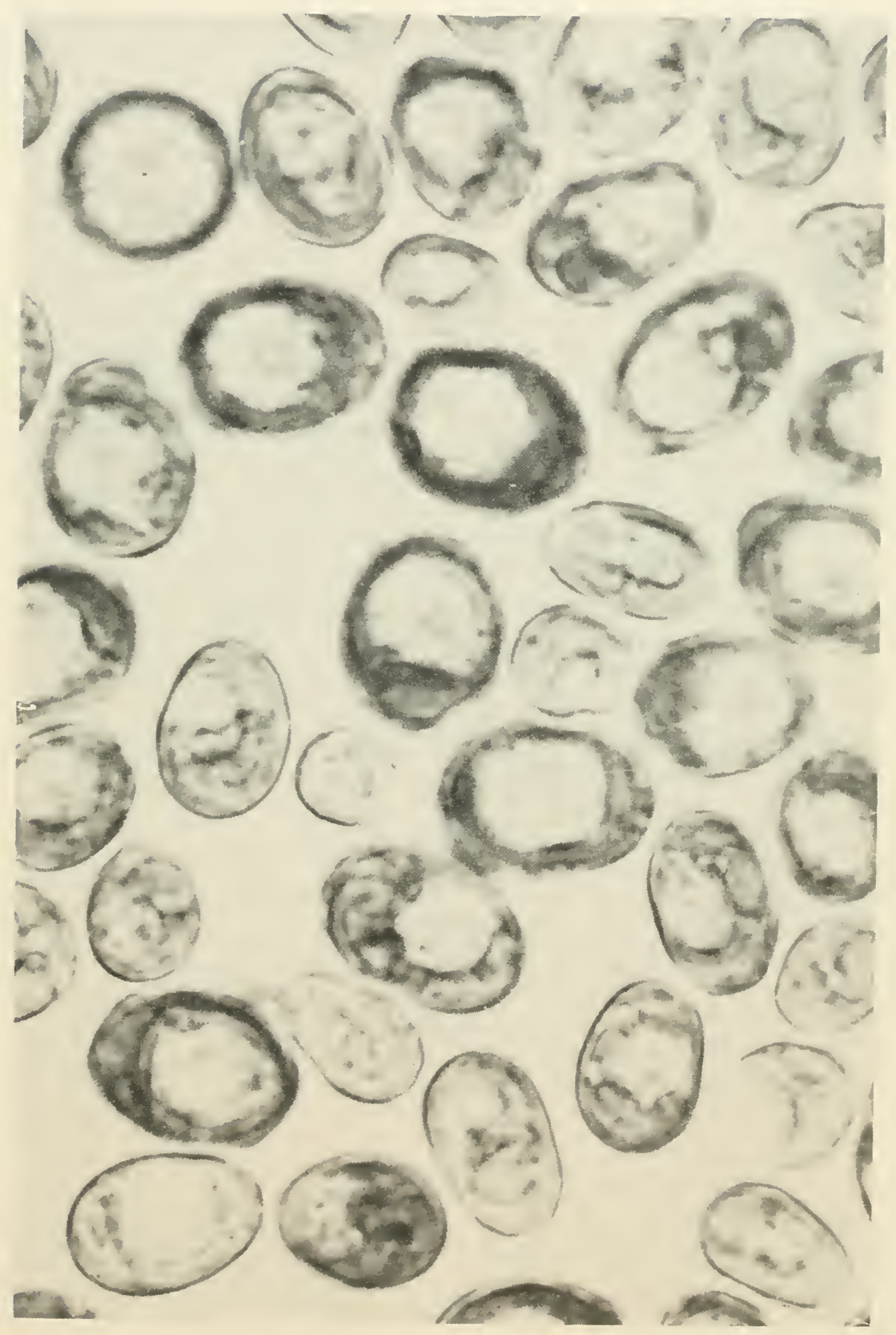

Fig. 6-24 Lugol's stained yesst cells containing lsrge nucleoli and showing the centrosome in many cells. 
first hour in the nutrient broth, the cells enlarge and show a faintly staining pink volutin deposit covering the chromosomal threads. As the cells continue to swell preparatory to division, the nuclear vacuole becomes much enlarged and the chromosomes begin to take the stain more deeply. In this stage, the shape and form of the chromo-

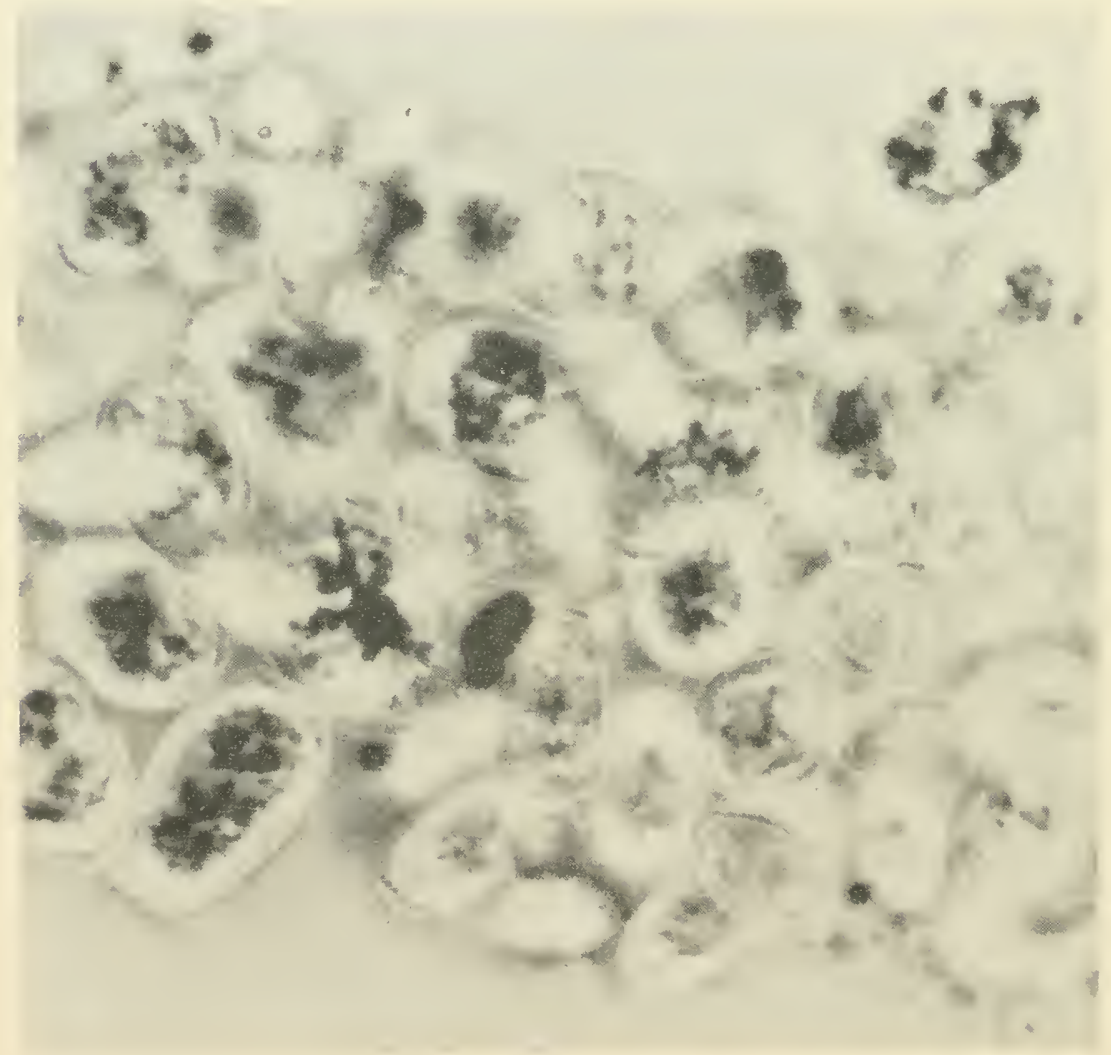

Fig. 6-25 Volutin coated chromosomes are visible within the vacuole.

somes can be clearly distinguished, and they can be made out as single strands or pairs of small, rather compact rods. As the vacuole enlarges still more, two nucleoli also appear, but during this period, they do not stain. About the time the first buds come up, one or two nucleoli are visible as large, light green, refractile bodies in the nuclear vacuole. The chromosomes now appear as a large number of individual bodies on the inner periphery of the vacuole. 
After a while, one nucleolus takes on the volutin stain, followed shortly by the other, or both fuse. At the same time, the color disappears from the chromosomes or becomes very much diminished. When the bud is growing at its most rapid rate, the nucleolus is swollen and densely stained, showing that it contains a large quantity of volutin. The chromosomes are visible as small, faintly stained peripheral bodies on the inner wall of the nuclear vacuole.

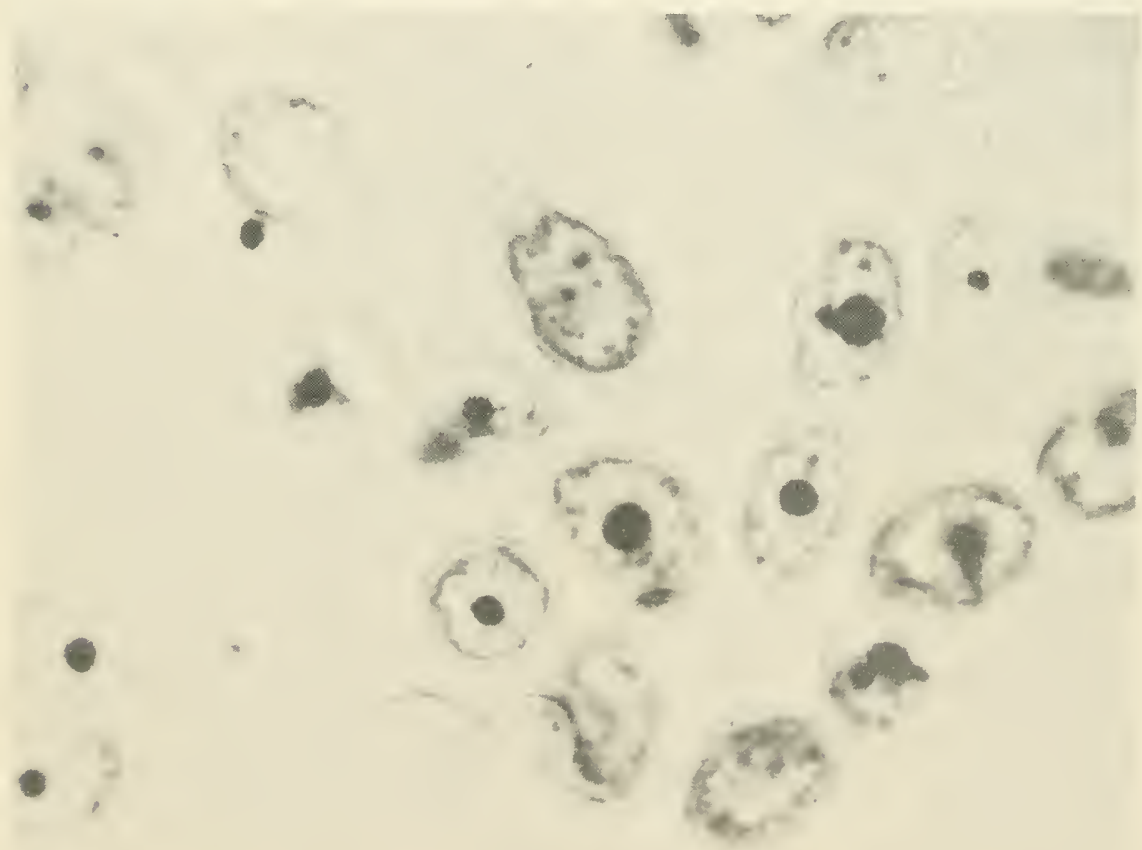

Fig. 6-26 The large spherical nucleolus in the center of the vacuole has taken the volutin stain and chromosomes are visible at the inner periphery of the vacuole or attached to the nucleolus.

The nucleolus is usually visible as a stained structure only in the vacuole of the mother cell, but in some cases the vacuole of the daughter cell also contains a stained nucleolus. After division has ceased, the nucleolus retains a dense coating of volutin for some time, while the chromosomes are lightly stained. On aeration the volutin disappears from the nucleolus but the chromosomes becorne coated as in fig. 6-25. 

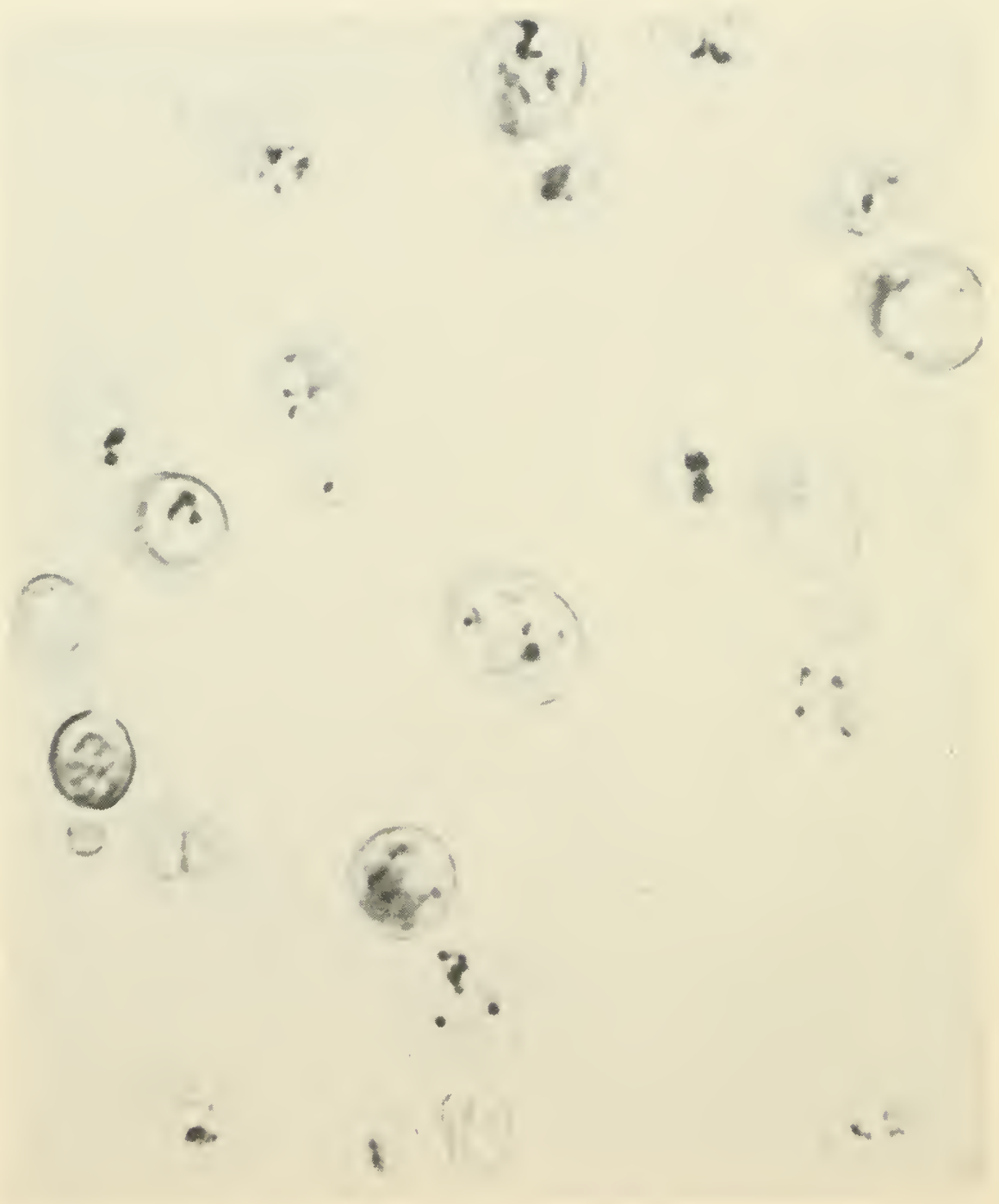

Fig. 6-27 Photograph of cells fixed in 1 part formalin plus 4 parts water and stained with Lugol's solution. The chromosomes stain dark brown suggesting that they are covered with carbohydrate. This precedes the accumulation of volutin on the chromow somes and suggests that the energy for the transformation of orthophosphate to metaphosphate is obtained from the localized carbohydrate. 


\section{“ABNORMAL" DISTRIBUTION OF VOLUTIN IN THE YEAST CELL}

Wiame has shown that yeast cells can be starved for phosphate by growing them in a medium deficient in phosphate, such as beet molasses. When growth finally ceases, chemical analysis reveals no metaphosphate in the cells, and the cells do not contain volutin. Furthermore, the cytoplasm is colorless showing a marked diminution of protein in the cytoplasm. He centrifuges these cells, washes them in water, and resuspends them in a 2 per cent sugar solution, adding $\mathrm{M} / 30 \mathrm{KH}_{2} \mathrm{PO}_{4}$. Wiame kindly performed this experiment with me in my laboratory and allowed me to follow the process cy-

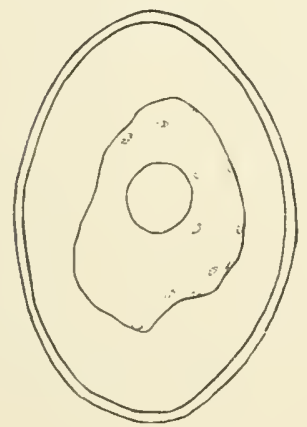

(a)

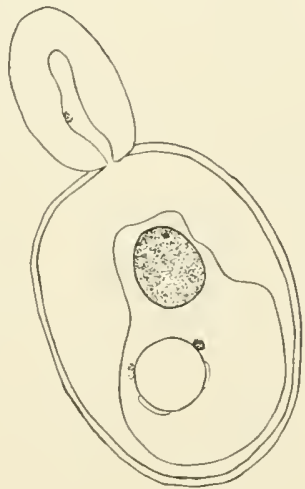

(b)

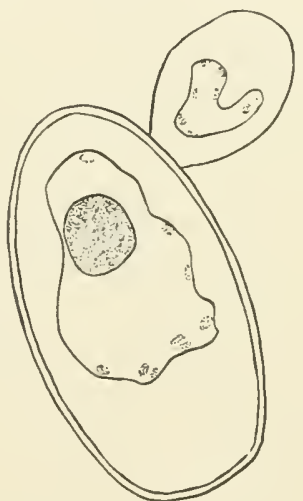

(c)

Fig. 6-28 Yeast cells stained for volutin; the vacuole is shrunken by fixation. (a) A single large unstained nucleolus with many small, light pink peripheral chromosomes. (b) A budding cell with one stained and one unstained nucleolus. Two stained and two faintly stained chromosomes are attached to the unstained nucleolus. (c) A budding cell with a deeply stained nucleolus and very faintly stained peripheral chromosomes. No nucleolus visible in bud.

tologically. The chromosomes rapidly became coated with volutin, staining red by the volutin stain. Volutin sometimes appeared on the chromosomes within three minutes after the addition of the phosphate. After twenty minutes, volutin begins to appear in the cytoplasm and the volutin in the vacuole decreases rapidly as the entire cytoplasm becomes faintly pink. Wiame's chemical tests show that there is a steady increase in the total metaphosphate in the cells and that the overall concentration of metaphosphate in the cells con- 
tinually increases in spite of the fact that volutin first appears in the chromosomes and then disappears from them and continues to accumulate later exclusively in the cytoplasm. Concurrent stains with the methylene blue dead cell stain reveal that the cells containing volutin in the cytoplasm are dead. This is consistent with the fact that metaphosphate is capable of precipitating proteins. The appearance of volutin in detectable amounts in the cytoplasm is a definite criterion of death. The rate of death can be reduced by adding the phosphate to the stained cells very slowly, but in a medium containing only 2 per cent sugar the phosphate eventually results in the death of the cells. When normal cells (in an adequate medium) take up phosphate, the volutin stain reveals that as growth progresses the cytoplasm becomes blue and the chromosomes red, indicating that there is protein but no stainable volutin in the cytoplasm of the healthy yeast cell. Healthy growth only occurs if a properly balanced nutrient is added slowly; phosphate alone produces a condition of imbalance which eventually results in death.

\section{VOLUTIN AND RIBOSENUCLEOPROTEIN SYNTHESIS}

The loss of stain from the chromosomes and its accumulation in the nucleolus indicates that the volutin formed on the chromosomes has been transferred to the nucleolus. Apparently, the synthesis of volutin occurs on the surface of the chromosomes, and as soon as the chromosomes becomes densely loaded, the transfer to the nucleolus occurs. Caspersson and Schultz have described the nucleolus of higher plants and animals as the center for the synthesis of ribosenucleoprotein. They showed that after ribosenucleoprotein is synthesized in the nucleolus in the cell of a higher plant or animal, it travels through the membrane into the cytoplasm. Caspersson and Brandt did not observe the nucleolus in the yeast cell with ultra violet light, possibly because the cytoplasm contains so much nucleoprotein that the smaller amount in the nucleolus is overshadowed. They did show (with ultra violet light) that ribosenucleoprotein accumulates on the outside of the nuclear vacuole. They thought it was synthesized at this point, but the demonstration of a conventional nucleolus in the nuclear vacuole suggests that the accumulation of ribosenucleoprotein, demonstrated on the surface of the nuclear membrane, may be the synthesis of this substance, due to the diffusion of ribose nucleic acid from the nucleolus through the nuclear membrane and into the cytoplasm. This view is in general agreement with Caspersson and Schultz's ideas concerning the role of the nucleolus in the synthesis of ribosenucleoprotein in higher plants and animals, but Caspersson and Brandt's findings concerning the yeast cell were based on the incomplete cytological information on the yeast cell available at that time. 


\section{THE POSSIBLE ROLE OF VOLUTIN (METAPHOSPHATE) AND NUCLEIC ACIDS IN CONFERRING SPECIFICITY TO CELLULAR ENZYMES}

Wiame has presented convincing evidence supporting the view that volutin is metaphosphate. Assuming that his theory is correct, one may develop the following hypothesis:

The chromosomes in resting cells do not stain either with iodine or with the volutin stain. However, shortly after resting cells are placed in a nutrient medium the chromosomes stain dark brown with iodine (fig. 6-27). After this has occurred they stain pink with the volutin stain. This suggests that the energy for transferring orthophosphate to metaphosphate is obtained from the localized carbohydrate. The metaphosphate which is synthesized from orthophosphate on the chromosome is transferred to the nucleolus. The synthesis of nucleic acids occurring in the nucleolus depends upon a supply of metaphosphate originating in the chromosomes, and the specificity of cellular enzymes may be associated with this phenomenon, since genes are known to control the specificity of various cellular enzymes. Von Euler and Janssens showed that the apoenzyme (protein) components of cellular enzymes are responsible for their specificity, and this important fact was recently confirmed by Spiegelman, Reiner, and Morgan; Caspersson and Schultz showed that nucleic acids are synthesized in the nucleolus and transferred from the nucleolus to the cytoplasm. They have suggested that these nucleic acids transfer the genetic specificities from the nucleus to the cytoplasm. I would like to propose that the transfer of the polymerized metaphosphate from the chromosome to the nucleolus determines the specificity of enzymes whose synthesis is under genetic control. The energy-rich phosphate bond in metaphosphate furnishes the energy which is required for the synthesis of the nucleic acids in the nucleolus, as well as of the materials which make up the chromosome itself. The syntheses occurring in the nucleolus initiate the production of specific enzymes, suggesting that the metaphosphates carry specificity-conferring groups with them from the chromosome to the nucleolus, which give specificity to the nucleic acids synthesized in the nucleolus.

Bauer and Caspersson have shown that a transfer of Feulgenpositive, and ultraviolet-absorbing substance take place from the chromosomes to the nucleolus in Chironymus. Rafalko and I have shown that the nucleolus in the yeast cell is on rare occasions Feulgenpositive. These facts suggest that metaphosphate and desoxyribose nucleic acid are transferred from the chromosomes to the nucleolus to confer specificity to nucleolar material. Callan has shown that in frog eggs the nuclear membrane is too fine to permit the passage of proteins. If this be true in yeasts the specificity conferring compound which passes into the cytoplasm may not be very large. 


\section{Chapter 7}

\section{STORAGE OF RESERVES IN THE CELL}

When a yeast cell is suspended in a medium containing an excess of available carbohydrate, glycogen is deposited in the cytoplasm. The ability to store reserves is a complicating factor in all attempts to analyze the growth and fermentative ability of yeast cells. The following more or less general rule seems to hold: Most available nutrients and vitamins when present in excess of maintenance requirements are stored in the cell as a reserve and the accumulated reserves generally interfere with growth, fermentation and respiration. When the proper reserves accumulate in sufficient variety, the cell becomes dormant.

One would expect that a small excess of each metabolite must be available in the cell, otherwise the mechanism would stop frequently because of the absence of an essential metabolite. This complicates the problem of studying growth, or indeed any metabolic process, tor the tendency to store metabolites that are not immediately used may be a general phenonenon.

Many free-living, single-celled organisms can suspend animation when one nutrient is deficient; under similar conditions a metazoan organism or even a more complex fungus would succumb. The ability of the free-living yeast cell to store nutrients against a future need may be a characteristic difference between free-living cells and many-celled organisms. In higher organisms the function of storage is usually assigned to specialized cells and the functionally active organs may be composed of cells containing minimal amounts of reserve material. Cells whose cytoplasm is not "cluttered up" with stored reserves can operate at a maximal rate without the interference of reserve materials with cytoplasmic reactions. Therefore differentiation in metazoans involves keeping those cells, which must maintain maximal activity, without stored reserves, but nutrients must be kept available for them in some easily assimilable form as in the blood stream. By contrast each yeast cell must perform both metabolism and storage and since these functions are mutually exclusive, periods of dormancy or of low metabolic activity occur frequently depending on the environmental conditions.

We are now familiar with at least three types of reserve substances which are stored in the cytoplasm of the yeast cell. They are glycogen, fat and ribose nucleoprotein. Metaphosphate accumulates on the chromosomes and this may also be considered a reserve substance, although its presence in the cytoplasm is a sign 
of death of the cell. Similarly, the presence of fat or excessive glycogen in the nucleus is a sign of death. There is one substance which seems to be present in relatively unvarying amounts (insofar as can be judged by the microscope) in all yeast cells irrespective of the amounts of stored substances in the cell. This is the basophilic nucleoprotein (?) in the centrosome. The heterochromatin stain seems to reveal about the same amount in every cell under all different conditions.

\section{GLYCOGEN}

Meissner (1900) studied the appearance and disappearance of glycogen in the yeast cell and showed that cells filled with glycogen produce more than the theoretical a mount of $\mathrm{CO}_{2}$ in fermenting a sugar substrate, because they produce $\mathrm{CO}_{2}$ from their own reserves in addition to that arising from the fermented carbohydrate. He also found that glycogen accumulates in the cell and attains a maximum at the end of the principal fermentation when it begins to disappear from the cell, even before all the sugar is consumed. He designated glycogen as a temporary reserve used by the cell through an endogenous diastatic enzyme. He pointed out that the deposition of an insoluble carbohydrate inside a semipermeable membrane enables the cell to take in soluble carbohydrate continuously by os mosis.

Wager and Peniston (1910) studied the same question with cytological techniques. They found that glycogen was deposited in the cell in the form of small granules which coalesced to form a solid mass of glycogen almost completely filling the cell.

McAnally and Smedley-Maclean (1935) and Smedley-Maclean and Hoffert $(1923,1924)$ showed that both carbohydrates and fats accumulated in the cell as a result of continued feeding with sugars. They found that phosphates increased the deposition of both reserves and that maltose seemed to increase the carbohydrate reserve. In addition, they pointed out that an excess of oxygen favored storage of fat.

Henneberg (1926) also pointed out that the presence of phosphates favored the deposition of glycogen, while chlorides seemed to inhibit its deposition. He found that the maximal protein content of the cell was 67 per cent but that yeasts containing stored reserves might contain only 22 per cent protein. He stated that the amount of protein in a cell stands in inverse relation to the amount of glycogen (and presumably fat). He pointed out that the yeast cells collected directly from fruits are generally rich in glycogen and furthermore that yeasts kept in moist condition on filter paper live longer if they contain large amounts of glycogen. Some apiculate and lactose-fermenting yeasts were unable to store glycogen. 


\section{STAINABLE GLYCOGEN}

The granular carbohydrate reserve in yeasts stains dark reddish brown with Lugol's iodine-potassium iodide solution. In many cells one can observe twenty or more small isolated granules (fig. 7-1). Occasionally, these granules are linked by connecting bands. Hundreds of small glycogen granules can be seen occasionally in some cells in addition to fifteen or twenty larger ones. These observations suggest that, in most of the cells containing a solid mass of dark-staining glycogen, the distribution is similarly nonhomogeneous with the basic granular structure obscured by overstaining. Under some circumstances the glycogen is deposited in granules right under the plasma membrane; under other circumstances it forms a flocculent deposit throughout the cytoplasm.

Unstained cells containing glycogen can be recognized by the high refractive index of the cytoplasm. The nuclear vacuole in a glycogen-containing cell is often concealed by the glycogen. The visible vacuoles of ten appear to be multiple, but critical observation shows that the small vacuoles are all interconnected with each other by fine canals and are merely separate compartments of one major vacuole. This is consistent with the view that the vacuole is the nucleus and that each yeast cell contains only a single vacuole; if the vacuole is divided, all the separate compartments connect with the centrosome. In many unstained glycogen-containing cells no vacuole is visible, but staining with Lugol's solution always reveals the vacuole either compressed into the middle of the cell by a surrounding sheath of glycogen, or at one pole of the cell. The vacuole in a glycogen-containing cell is usually much reduced in size.

Budding is retarded or inhibited in cells containing much glycogen, and occurs only after the glycogen has begun to disappear from the cell. Growing cells during the lag phase contain enough unidentified reserve to obscure or deform the vacuole. After the cell has completed one or two divisions, the refractive index drops and the vacuole reappears or loses its deformity.

Deposition of granular glycogen is irregular at high (12 per cent) concentration of sugar and rarely fills the whole cell. The stained granules are darker than those observed at lower concentrations of sugar. Many granules may coalesce, often forming two large polar deposits. Small granules of glycogen may be linked by arcs of glycogen. At lower sugar concentrations (4 per cent), the deposition of glycogen continues until it fills the entire cell with the exception of a small region at the one end into which the vacuole is crowded, or the vacuole may be concealed in the center of the cell inside the spherical envelope of glycogen. After the deposit has reached a maximum, the glycogen disappears on aeration, by peripheral dis- 


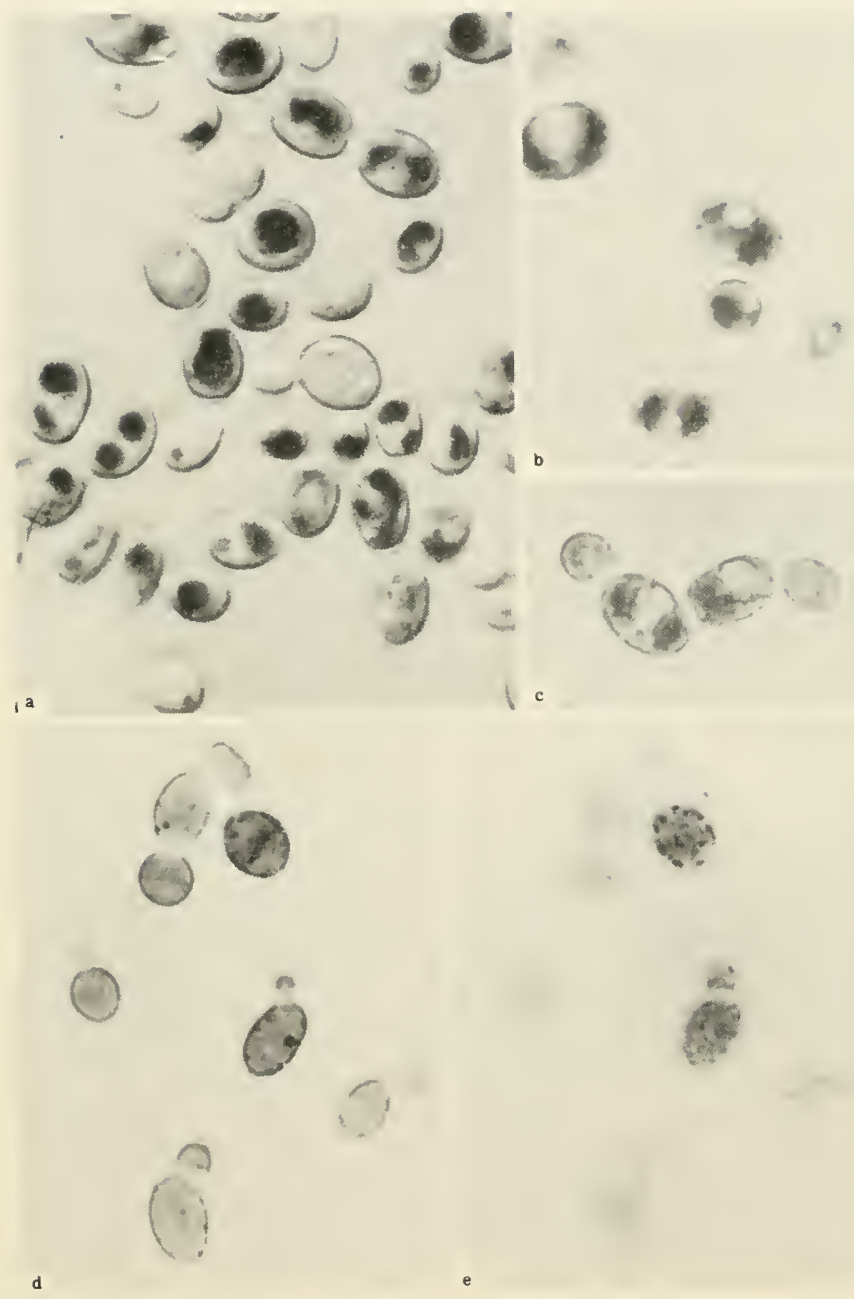

Fig. 7-1 Yeast Cells Containing Stored Glycogen Stained with Lugol's Solution.

a. Densely aggregated compact masses of glycogen characteristic of cells held in high concentrations of carbohydrate.

b. and c. Loosely aggregated glycogen accumulated while shaken in a Warburg apparatus containing 4 per cent of glucose for 2 hours.

d. and e. Two optical levels through a field showing an unusual type of accumulation of glycogen characterized by the deposition of numerous small compact deposits immediately in aide the plasma membrane. 
integration or solution, with a decrease in the density of the glycogen mass. Finally a single large diffuse granule is found in the cell, and the vacuole regains its original size. Under favorable conditions, involving low oxygen tension and continued addition of sugar, all the cells may become filled with glycogen.

Stainable glycogen does not accumulate in well-aerated yeast cultures grown in 1 per cent glucose broth. The nuclear vacuole attains its maximal size under these conditions.

Some dead cells contain glycogen in their vacuoles. Most of the dead cells remaining in a culture, which has grown until all the glycogen has disappeared from the living cells, contain glycogen in the cytoplasm. Either glycogen tends to deposit in dead cells or dead cells are unable to metabolize their accumulations of glycogen.

When a cell is fixed with formalin and stained with iodine the chromosomes often turn dark brown suggesting that they may contain carbohydrate on occasion. (See chapter 6.)

\section{MITOCHONDRIA}

The cytoplasm of the yeast cell contains many mitochondria if the culture is well aerated and well supplied with phosphate and sugar. The mitochondria contain fat and ribose nucleoprotein. When the cell is fermenting rapidly mitochondria are not visible. Staining with Nile-blue sulphate and osmic acid generally indicates that the fat is also evenly distributed throughout the cytoplasm. The mitochondria are often deposited at either pole of the vacuole, or comprise a network of streptococcal like threads of granules which sometimes branch and are closely appressed to the outer surface of the vacuole. In most types of $\mathrm{S}$. cerevisiae the mitochondria tend to increase in numbers and to enlarge individually as the culture becomes older and storage increases. The addition of alcohol or the application of heat causes many of the globules to coalesce. Dead cells usually contain coalesced globules, just as they often contain glycogen. Mitochondria invariably accumulate in yeast cells grown in shallow well-aerated Erlenmeyer flasks. Mitochondria apparently contain fat together with ribose nucleoprotein and may provide a mechanism for storing the reserve ribose nucleoprotein so that it is not in contact with the cytoplasm directly and therefore inactive in metabolism.

Wiame (1947) has defined the total basophily of a yeast suspension in terms of the total amount of toluidine blue taken up by a given amount of cells from a standard concentration of the dye. $\mathrm{He}$ dries a suspension containing a known number of cells on an etched glass slide. Then he stains the dried smear with toluidine blue. The suspension is next washed with normal hydrochloric acid and becomes completely destained. The amount of dye taken up is collected and measured arbitrarily in a photoelectric colorimeter. 
This gives a measure of the total basophily, presumably the total amount of nucleoprotein (which is principally ribose nucleoprotein for the desoxyribose nucleoprotein makes up only a small fraction of the total nucleoprotein) plus the metaphosphate. Since metaphosphate retains the dye more effectively than the nucleoprotein (it is not removed by a short exposure to 1 per cent sulphuric acid), a rough estimate of the metaphosphate fraction is achieved by deter mining the total basophily and subtracting the fraction of dye removed by washing with 1 per cent sulphuric acid. The nucleoprotein stains blue and the metaphosphate red, so it is possible to get some idea of the relative amounts by direct observation of the stained cells. Cells grown in media deficient in nitrogen but supplied with a maximum of all the other necessary ingredients show a marked diminution in basophily. Ordinarily, the yeast cell contains so much reserve ribose nucleoprotein in the cytoplasm and this substance has such an affinity for basic dyes that attempts to use a stain like aceto-orcein or methylene blue result in over-staining the entire cell. However, in cells starved for nitrogen, aceto-orcein may differentiate the heterochromatin in the centrosome very well. In the actively growing cell, the ribose nucleoprotein is generally dispersed throughout the cytoplasm; in the nondividing cell, it accumulates in the mitochondria forming the cytoplasmic granules. The total amount of ribose nucleic acid in the yeast cell varies, probably from 1 to 5 per cent of the dry weight, so the ribose nucleoprotein may vary roughly between about 5 to 25 per cent of the dried weight. Ribose nucleoprotein is readily dissolved by dilute sodium hydroxide while desoxyribose nucleoprotein is not. This is the basis of heterochromatin stain.

\section{THE FEULGEN-POSITIVE HETEROCHROMATIN} IN THE CENTROSOME

Under various different conditions of the cell with regard to its stored reserves about the same amount of basophilic (desoxyribose?) nucleoprotein, as far as can be judged by microscopic observation, appears in the centrosome, when one uses the heterochromatin stain (extract in dilute alkali and stain with dilute toluidine blue). This suggests that the centrosome is one of the structures which is relatively independent of variations in the storage of other reserves. The chromosomes are too delicate to stain, so we have no information on the constancy of their basic protein. However, we assume that they are present at all times and that the heterochromatin along with the chromosomes forms part of the basic or "skeletal" structure of the cell relatively unaffected by the storage of reserve. Von Euler and Hahn (1947) have shown that bakers' yeast contains about 7 per cent ribose nucleic acid and 0.3 per cent desoxyribose nucleic acid. 


\section{Chapter 8}

\section{THE INDUCTION OF DORMANCY AND THE CONDITIONS FOR REACTIVATION}

\section{DORMANCY}

Diploid cells which have been grown on presporulation agar for a week or more (but have not yet sporulated) become filled with mitochondria and glycogen (fig. 8-1), and as a result of these reserve accumulations are dormant. When tested in a Warburg apparatus they are unable to give off $\mathrm{CO}_{2}$ or consume $\mathrm{O}_{2}$. Accessory substances are probably also involved in the induction of dormancy since the presporulation medium is a very rich source of vitamins. Dormancy can only be broken by presenting the dormant cells with a nutrient containing vitamins and a nitrogen source in addition to glucose sufficient to insure continued growth. Vegetative cells which have grown on rich natural substrates, such as ripened fruits, fill with reserves and may be similarly dormant. Dormant cells either germinate from dormancy by a simple vegetative procedure in an adequate medium, or the diploid dormant cells may sporulate when placed on a gypsum slant along with a mass of other cells in an acid ( $\mathrm{pH} 4$ ) milieu. In the latter case, conditions are unfavorable for continued vegetative growth just as they would be in a sandy soil. These observations suggest that the vegetative cells grown on ripe fruit become loaded with reserves and become dormant. If they fall on another fruit or into a rich, sugary nutrient, they germinate and grow vegetatively. At the end of the season when the fruits finally fall on the soil, sporulation occurs and the spores germinate the following spring.

It has long been known that yeasts store both fats and carbohydrates, and the principal conditions controlling the storage of these reserve materials have been fairly well worked out. It was not known, however, that cells containing abundant accumulations of reserve materials are in a state of dormancy; they are unable to take up $\mathrm{O}_{2}$, to give off $\mathrm{CO}_{2}$, or to bud, either in a phosphate buffer solution or in a buffer-glucose solution in the Warburg apparatus; but they begin to grow when they are brought into a complete nutrient medium. The mechanism has a high survival value; it prevents cells from "wasting" their reserves, since growth can only begin under conditions in which continued or considerable growth is possible. 


\section{STORAGE OF GLYCOGEN UNDER DIFFERENT CONDITIONS}

The effects of low and moderate aeration and different concentrations of sugar, on glycogen deposition, were studied by comparing growth in $50 \mathrm{cc}$. of nutrient broth placed in an Erlenmeyer of $500 \mathrm{cc}$. capacity with growth in $30 \mathrm{cc}$. broth placed in an $8 \times 1$ inch test tube. The following percentage concentrations of sugar were used: 12 , $8,4,2$, and 1. Each culture was observed daily to determine the percentage of dead cells and the percentage containing glycogen. The final dry weight of yeast in mgm./cc. was measured. Table 8-1 shows that (1) in low concentrations of sugar glycogen disappears much more rapidly than in high, (2) the cells die much more rapidly in high than in low concentrations of sugar, and (3) the efficiency of converting sugar to yeast is much higher in low sugar concentrations than in high.

After the granular glycogen had disappeared from the cells grown in 12 or 8 per cent sugar solutions, the cytoplasm still contains the nongranular carbohydrate which is not found in yeasts grown in 1 or 2 per cent sugar.

\section{DORMANT VEGETATIVE CELLS}

After most yeasts have grown for several weeks on slants of our pre-sporulation agar, the cells contain an abundance of mitochondria and glycogen, and although all the cells are alive, they are dormant (fig. 8-1). Subsequent experiments were undertaken to reproduce dormancy by loading the cells with reserve materials in broth cultures, but we were unable to obtain cells with precisely the appearance of those taken from pre-sporulation agar, which appear to be much more heavily packed with larger mitochondria. The vacuoles in a dormant cell from pre-sporulation agar are usually spherical, and centrally located, indicating that deposition of reserves on this medium occurs without deformation of the vacuole. After an hour in nutrient medium, most of the mitochondria break down and after the second hour half of the cells show buds and some contain only a few mitochondria. The vacuoles appear to be multiple or obscured in the growing cells. In three hours there are very few granules left in the cells, nearly all of which contain lobed or obscured vacuoles. Practically all the cells bud, proving that the culture is viable.

Manometric studies were made with the cells taken directly from the pre-sporulation slant (fig. 8-1) and shaken with phosphate buffer containing 4 per cent glucose, but with no other nutrients. The respirometer values showed that the cells were incapable of taking up oxygen and incapable of producing $\mathrm{CO}_{2}$ either aerobically or anaerobically over a 150 -minute period. During the same period, all the cells in the nutrient broth budded. Ninety-eight per 

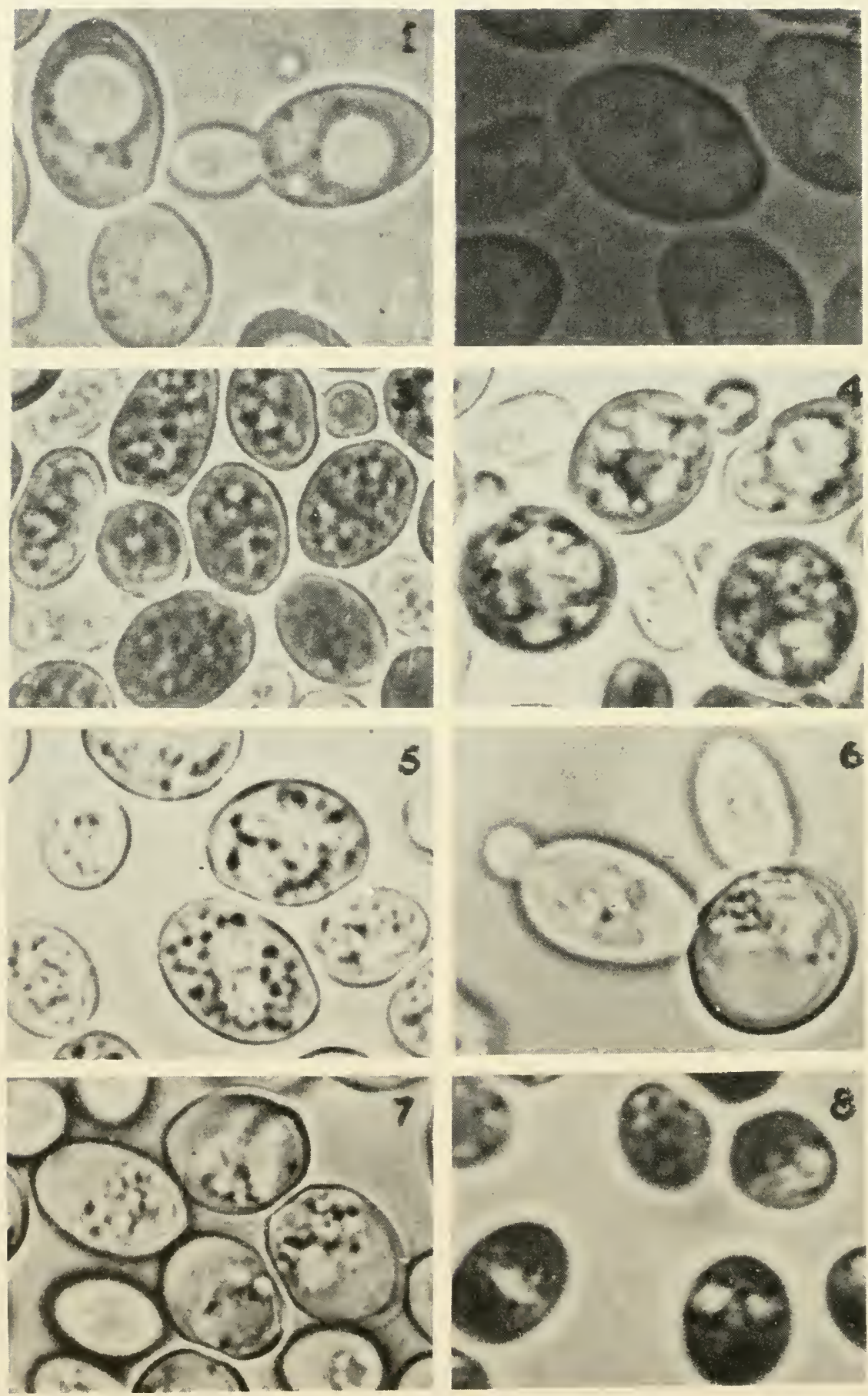
cent of the cells from the Warburg vessel showed little or no change.

Cells of a standard baking yeast, strain $\mathrm{A}$, which had been grown on pre-sporulation agar, were collected from the agar surface and washed with $\mathrm{M} / 15 \mathrm{KH}_{2} \mathrm{PO}_{4}$. Three Warburg vessels were inoculated with equal amounts of dormant cells. Each vessel contained 4 per cent of glucose in solution. The first received phosphate buffer, the second received 1 per cent of corn-steep-water solids, and the third received 0.3 per cent ammonium sulfate, biotin ( $2 \gamma$ per liter) and pantothenic acid (200 y per liter) (fig. 8-2).

Since our culture of S. cerevisiae is a "non-synthesizer" of biotin and pantothenic acid, these substances were added, together with ammonia, to see if they would break the dormancy of the fat-filled yeast cells. The cells suspended in buffer were dormant and gave off no $\mathrm{CO}_{2}$ after over five hours in the Warburg apparatus; but the cultures in the other vessels fermented the sugar, the action being much more rapid in the richer nutrients. This experiment cannot always be duplicated because cells from the pre-sporulation agar slants are not always in precisely the same condition for some cultures sporulate directly on the slant and in some cultures all the cells do not fill uniformly with reserves.

\section{FORMATION OF FAT AND MITOCHONDRIA}

Formation of fat and mitochondria occurs most rapidly in a medium in which fermentation and growth have nearly ceased. A 1 per cent glucose broth was prepared with half the standard amount of nutrient broth to insure the early cessation of growth. Fifty $\mathrm{ml}$. of broth in $500 \mathrm{ml}$. Erlenmeyer flasks were inoculated

Fig. 8-1 1. Budding vegetative yeast cells at the logarithmic stage of growth showing a single vacuole characteristic of this condition.

2. Budding yeast cell in the lag phase showing the apparently multiple vacuole resulting from deformations of the single vacuole by interference of reserve material.

3. Dormant vegetative yeast cells loaded with mitochondria and glycogen, grown on presporulation agar; the dark color is glycogen stained with iodine.

4. Germinating dormant cells trom presporulation agar, showing the vacuole spreading through the enclosing net-work of mitochondria and glycogen.

5. Cells loaded with mitochondria by growth in aerated sugar solution.

6. Cell of the type shown in 5 germinating.

7. Cell grown in sugar under conditions of reduced oxygen tension loaded with glycogen, a few mitochondria surrounding vacuole.

8. Cell of the type shown in 7 stained with iodine, revealing the deformation of the vacuole by the reserve materials. 
and shaken for 48 hours. The suspension of cells from some of the Erlenmeyer flasks was placed in $8 \times 1$-inch tubes. Tests with Fehling's solution were made to determine when the sugar disappeared, and sugar was added as soon as a deficiency was indicated. Phosphate was also added, since this is known to increase the deposition of both fat and glycogen. The addition of sugar was continued for four days.

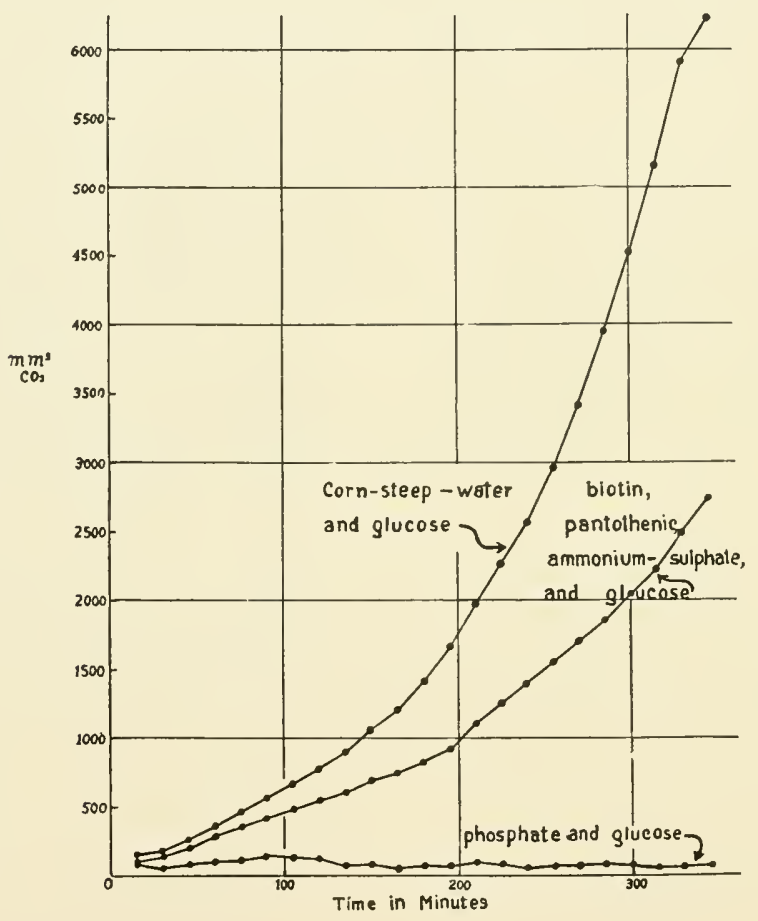

Fig. 8-2 Curves Showing the Evolution of $\mathrm{CO}_{2}$ by Three Comparable Suspensions of Dormant Cells in Different Nutrients over a 345-minute Period.

Three cultures were used, two standard baking yeasts, strains $\mathrm{U}$ and $\mathrm{R}$ (S. cerevisiae) and a hybrid (S. cerevisiae $\mathrm{x}$ S. globosus). Strain $U$ stored both mitochondria and carbohydrate relatively uniformly as indicated by microscopic examination. Strain $\mathbf{R}$ stored carbohydrate well, but the accumulations of mitochondria were irregular and the granule-containing culture was discarded. The hybrid stored mitochondria in large clusters of extremely tiny particles approaching the limits of visibility. The cells appeared to be 


\section{Table $8-1$}

The Percentages of Dead Cells and of Cells Containing Glycogen over a 5 day Period in Shallow Broth in Brlenmeyers and in Deep Broth in Test Tubes in Nutrients Containing Different Concentrations of Sugar.

\begin{tabular}{|c|c|c|c|c|c|c|c|}
\hline \multirow{2}{*}{$\begin{array}{l}\text { Per Cent } \\
\text { sugar }\end{array}$} & \multirow{2}{*}{ Day } & \multicolumn{2}{|c|}{ Erlenmeyer } & \multirow{2}{*}{$\begin{array}{c}\text { Final dry } \\
\text { wt. }\end{array}$} & \multicolumn{2}{|c|}{ Deep tube } & \multirow{2}{*}{$\begin{array}{c}\text { Final dry } \\
\text { wt. }\end{array}$} \\
\hline & & Dead & Glycogen & & Dead & Glycogen & \\
\hline 12 & $\begin{array}{l}1 \\
2 \\
3 \\
4 \\
5\end{array}$ & $\begin{array}{l}1 \\
30 \\
60 \\
70 \\
80\end{array}$ & $\begin{array}{r}50 \\
100 \\
100 \\
40 \\
20\end{array}$ & gm.per $c c$. & $\begin{array}{l}40 \\
40 \\
60\end{array}$ & $\begin{array}{l}25 \\
70 \\
30 \\
50 \\
20\end{array}$ & manper cc \\
\hline 8 & $\begin{array}{l}1 \\
2 \\
3 \\
4 \\
5\end{array}$ & $\begin{array}{l}70 \\
80 \\
80\end{array}$ & $\begin{array}{r}95 \\
100 \\
100 \\
\text { none } \\
\text { none }\end{array}$ & 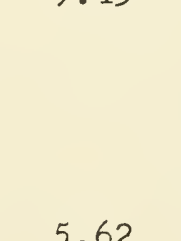 & $\begin{array}{l}25 \\
15 \\
40\end{array}$ & $\begin{array}{l}10 \\
50 \\
10 \\
60 \\
80\end{array}$ & 2.0 \\
\hline 4 & $\begin{array}{l}1 \\
2 \\
3 \\
4 \\
5\end{array}$ & $\begin{array}{l}20 \\
50 \\
70\end{array}$ & $\begin{array}{r}90 \\
100 . \\
80 \\
\text { none } \\
\text { none }\end{array}$ & 3.27 & $\begin{array}{r}5 \\
5 \\
25\end{array}$ & $\begin{array}{l}50 \\
70 \\
95 \\
40 \\
40\end{array}$ & 2.47 \\
\hline 2 & $\begin{array}{l}1 \\
2 \\
3 \\
4 \\
5\end{array}$ & $\begin{array}{l}0 \\
0 \\
0 \\
0\end{array}$ & $\begin{array}{r}90 \\
10 \\
\text { none } \\
\text { none } \\
\text { none }\end{array}$ & 2.51 & $\begin{array}{r}0 \\
0 \\
20\end{array}$ & $\begin{array}{l}80 \\
50 \\
20 \\
50 \\
20\end{array}$ & 1.44 \\
\hline 1 & $\begin{array}{l}1 \\
2 \\
3 \\
4 \\
5\end{array}$ & $\begin{array}{l}0 \\
0 \\
0 \\
0\end{array}$ & $\begin{array}{l}80 \\
\text { none } \\
\text { none } \\
\text { none } \\
\text { none }\end{array}$ & 1.36 & $\begin{array}{r}0 \\
0 \\
20\end{array}$ & $\begin{array}{r}100 \\
50 \\
5 \\
1 \\
5\end{array}$ & 0.95 \\
\hline
\end{tabular}


crowded with stored materials, but chemical analysis (ether extraction) revealed that only 6.16 per cent fat was present. The hybrid stored carbohydrate poorly. Nitrogen analyses were converted to protein by multiplying by the factor 6.25 (Table 8-2). The sample was ashed, and the difference was estimated to be carbohydrate. Table 8-3 reveals that the reserves of glycogen hindered the respiratory and fermentative activity of the cells considerably.

Before understanding the harmful effects of high sugar concentrations, we grew yeasts in a medium containing 8 per cent sugar, peptone, and yeast extract. In this medium 50 to 90 per cent of the cells died, especially if the cultures were well-aerated. The dead cells gave normal $Q_{\mathrm{CO}}$ and $\mathrm{Q}_{\mathrm{CO}}^{\mathrm{N}}$ values ranging from 300 to 450 ; but with $\mathrm{QO}_{2}$ values of 0 . The dead cells were unable to consume $\mathrm{O}_{2}$, although they were able to ferment. Some of our dormant cultures contained nearly 100 per cent viable dormant cells. These viable dormant cells, loaded with reserve materials, were unable to consume $\mathrm{O}_{2}$ or to evolve $\mathrm{CO}_{2}$, but their inability to consume $\mathrm{O}_{2}$ is obviously different from that of cultures containing many dead cells. Death of the cell apparently destroys its ability to take up $\mathrm{O}_{2}$ without affecting seriously its ability to ferment.

The reproducibility of results obtained by the Warburg respirometer depends upon the absence of accumulations of reserve materials in the cells. Cells should be aerated and transferred to the Warburg apparatus when a.minimum number of dead cells is pressent and a considerable proportion is actually budding. In order to make reproducible analyses, growth must be stopped in an active phase and the cells washed with $\mathrm{M} / 15$ phosphate. If the cells are allowed to stand in a nutrient medium, they will accumulate some reserve fat or carbohydrate, or both, depending on the aeration and the concentration of sugar.

Table 8-2

Chemical Analyses of Cells Containing Visible Deposits of Cells

\begin{tabular}{c|l|r|r|r|c}
\hline \hline Culture & $\begin{array}{l}\text { Visible } \\
\text { Deposit }\end{array}$ & Protein & Fat & Ash & $\begin{array}{c}\text { Carbo- } \\
\text { hydrate }\end{array}$ \\
\hline \multirow{3}{*}{ U } & None & 61.3 & 4.83 & 8.48 & 25.4 \\
& $\begin{array}{l}\text { Mitochondria } \\
\text { Glycogen }\end{array}$ & 25.0 & 31.25 & 5.22 & 38.5 \\
& None & 31.9 & 16.96 & 5.33 & 45.7 \\
M XII & Mitochondria & 39.4 & .17 & 9.29 & 36.1 \\
& None & 53.8 & 7.47 & 9.15 & 29.5 \\
Glycogen & 31.9 & 5.24 & 5.22 & 57.7 \\
\hline
\end{tabular}


Table $8-3$

Respiratory and Fermentative Activity of Yeast Containing Visible Depostis of $\nu$ itochondria and Glycogen*

\begin{tabular}{|c|c|c|c|c|c|c|c|c|c|}
\hline $\begin{array}{l}\text { Visible } \\
\text { Deposit }\end{array}$ & $\begin{array}{l}\text { Sam- } \\
\text { ple }\end{array}$ & $\begin{array}{l}\text { Cul- } \\
\text { ture }\end{array}$ & $\begin{array}{l}\text { Pro- } \\
\text { tein }\end{array}$ & $\mathrm{QO}_{2}$ & $Q_{\mathrm{CO}}^{\mathrm{O}}$ & $Q^{\mathrm{N}}$ & $\mathrm{Q}_{2}$ & ${ }^{2} \mathrm{CO}_{2}(\mathrm{~N})$ & ${ }^{2} \mathrm{CO}_{2}(\mathrm{~N})$ \\
\hline $\begin{array}{l}\text { Glyco } \\
\text { Glyco } \\
\text { Glyco }\end{array}$ & $\begin{array}{l}1 \\
2 \\
3\end{array}$ & $\begin{array}{l}\mathrm{R} \\
\mathrm{C} \times \mathrm{GII} \\
\mathrm{U}\end{array}$ & $\begin{array}{l}33.2 \\
38.7 \\
33.5\end{array}$ & $\begin{array}{r}0 \\
52 \\
47\end{array}$ & $\begin{array}{r}63 \\
156 \\
82\end{array}$ & $\begin{array}{r}116 \\
117 \\
83\end{array}$ & $\begin{array}{r}0 \\
840 \\
880\end{array}$ & $\begin{array}{l}1178 \\
2520 \\
1523\end{array}$ & $\begin{array}{l}2187 \\
1885 \\
1550\end{array}$ \\
\hline $\begin{array}{l}\text { Mitochon- } \\
\text { dria } \\
\text { Mitochon- } \\
\text { dria } \\
\text { Mitochon- } \\
\text { dria }\end{array}$ & $\begin{array}{l}5 \\
6\end{array}$ & $\begin{array}{l}\mathrm{R} \\
\mathrm{C} \times \mathrm{GII} \\
\mathrm{U}\end{array}$ & $\begin{array}{l}56.3 \\
57.5 \\
50.0\end{array}$ & $\begin{array}{r}76 \\
127 \\
125\end{array}$ & $\begin{array}{l}500 \\
151\end{array}$ & $\begin{array}{l}322 \\
377\end{array}$ & $\begin{array}{r}850 \\
1353 \\
1565\end{array}$ & $\begin{array}{l}2760 \\
5340\end{array}$ & $\begin{array}{l}3580 \\
4030 \\
3261\end{array}$ \\
\hline
\end{tabular}

* The $Q$ values of these yeasts without visible deposits (in glucose) were: $\mathrm{Q}_{2} 10-137, \mathrm{Q}_{\mathrm{CO}_{2}}^{\mathrm{O}} 160-432, \mathrm{Q}_{\mathrm{CO}}^{\mathrm{N}} 241-308$. The one low $\mathrm{QO}_{2}$ value probably indicates that this culture contained dead cells.

Accumulated carbohydrates are responsible for the lag in growth observed on the inoculation of the fresh medium. The lag can be minimized or eliminated if the cells are transferred before storage has occurred.

Hansen developed a technique of preserving yeast cultures by suspending them in 10 per cent sucrose solution and sealing the flask to prevent evaporation. Observation of such suspensions shows many autolyzed cells and others richly loaded with mitochondria. Presumably, the strong sucrose solution causes the autolysis of some cells and the formation of fat occurs in the presence of of the autolysate and the high sugar concentration. This results in the production of dormant cells. Hansen's procedure could not be used to supply dormant cells in our experiments because a very large percentage of the cells die in high concentrations of sugar and only a few attain full dormancy. Winge and Hjort (1935) recovered living cells from 47-year-old sucrose suspensions of yeast cells prepared by Hansen.

The fact that dormant cells do not break dormancy except in medium containing a relatively full complement of nutrients prevents the dormant cells from growing except under conditions that assure continued growth. The fact that they require specific vitamins which they are unable to synthesize may have some importance in solving the problem of inducing other fungal spores to germinate.

These facts are consistent with the view that the mitochondria contain ribose nucleoprotein insulated from the cytoplasm by lipoid. 
When all the ribose nucleoprotein is stored in the mitochondria, the cell is dormant. Breaking of dormancy would involve the dissolution of the mitochondrium and the dispersal of ribosenucleoprotein in the cytoplasm. This is consistent with the current view that mitochondria are coacervates of lipoidal and protein materials. 


\section{Chapter 9}

\section{TECHNIQUES FOR MICRODISSECTION AND PRESERVATION OF CULTURES}

\section{THE DISSECTING NEEDLE}

We use a solid glass needle with a flat tip about 20 microns in diameter for the isolation of single ascospores. The needle can be made by hand as follows: A pyrex rod is drawn out in a flame to make a small rod about a millimeter in diameter. This is broken up into six-inch lengths. Two burners are used to make the tip: one is the pilot light of an ordinary Bunsen burner and the other is a 26gauge hypodermic needle. The hypodermic needle and the Bunsen burner are connected to the same gas jet by a $\mathrm{T}$-tube; the flame of the hypodermic needle is controlled by a screw clamp on the tubing. The hypodermic needle is mounted by clamping it into the small jaw of a burette holder. The room must be without drafts, otherwise the small flame will blow out. A small guard to shield the flame from drafts may be necessary. The six-inch pyrex rod, one millimeter thick, is thinned out to about one tenth its thickness by melting and drawing out in the pilot flame. The next step involves thinning out the small end again down to a 20-micron tip and bending at the same time. This is done by bringing the small end of the pyrex rod over the flame from the hypodermic needle while maintaining a slight tension so that as soon as the glass softens it pulls out of the flame. This produces a thin rod and a right angle bend at the same time. The needle can be cut off using small manicure scissors. Several trials may be required before a needle the right size with an even end is produced.

The technique of using flat-tipped needles for microdissection was originally developed by Dickinson. With such a needle it is possible to move cells around over an agar or a glass surface, or to remove them from it by the capillary water that adheres to the flat needle surface.

The needles can also be made by the de Fonbrune microforge (fig. $9-1$ ). In this apparatus an incandescent platinum wire, in which the heat can be controlled, is mounted before a microscope. The operator can move the glass needle and the incandescent platinum wire by a number of knurled knobs and perfect control of the melted glass with continuous observation through the microscope is possible. (fig. 9-2). 


\section{THE DE FONBRUNE FNEUMATIC MICROMANIPULATOR}

Several types of micromanipulators operating on simple mechanical principles are available and may be used with advantage in the dissection of asci. Most of these instruments involve the use of three levers to control movement of the dissecting needle in each dimension, and the necessity of constantly changing from one lever

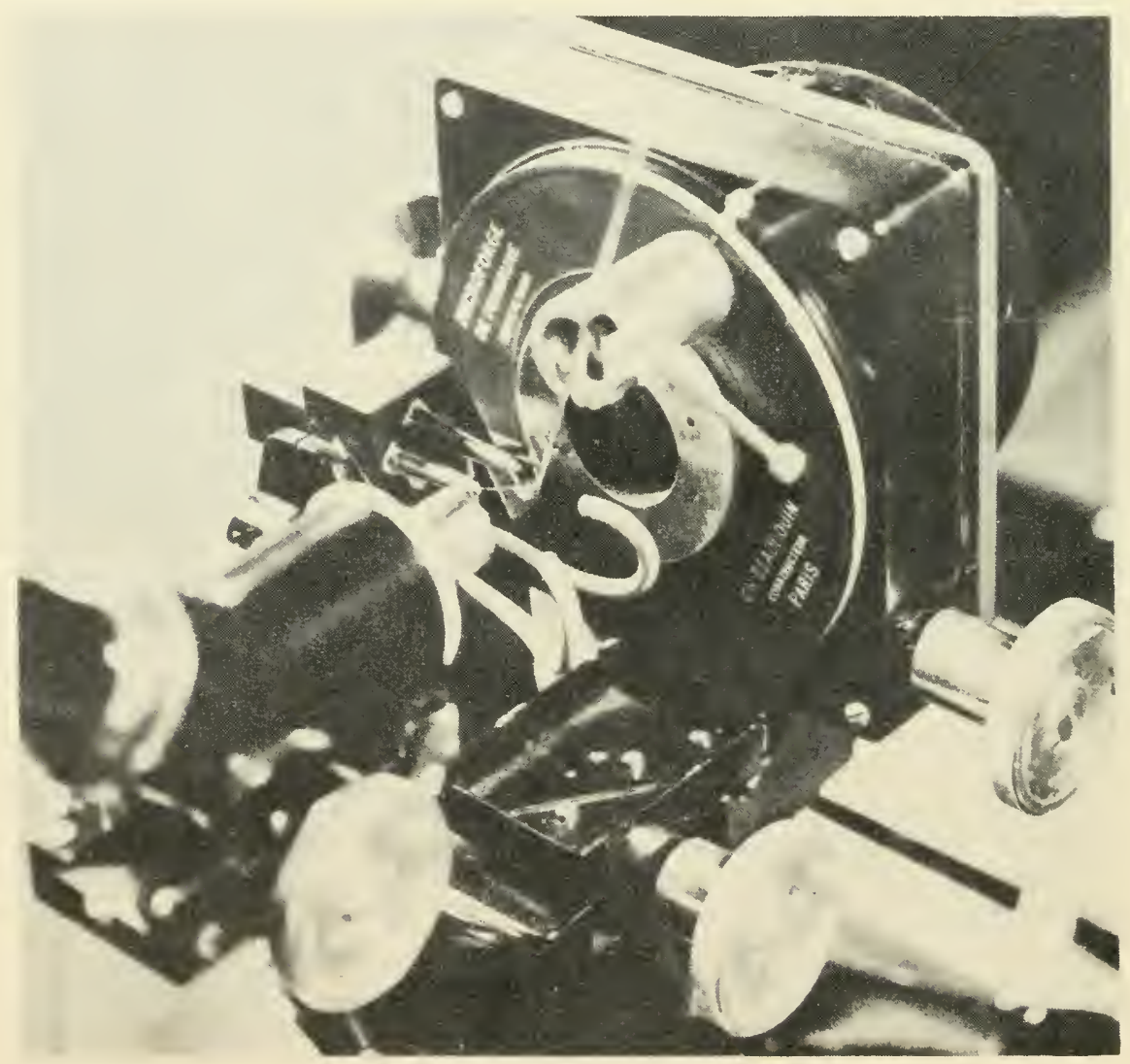

Fig. 9-1 The de Fonbrune Microforge Showing the Platinum Wire and the Glass Rod in Position to be Melted.

to the other during manipulation makes it difficult to learn to operate. The de Fonbrune is an "upright" instrument, for it can be so arranged that each movement is precisely the same as one would make if he held the needle in his hand and viewed it without lenses. This makes learning easy. In addition all movements are on one 
lever. The micromanipulator (fig. 9-3) is pneumatically controlled. It consists of two parts: (a) a set of three interconnected cylinders bearing pistons and (b) a chamber housing three anaeroid foils. The three pistons are so oriented with relation to each other that the movement of each piston alone, or of the three simultaneously, can be regulated by the use of the same lever. Each of the piston cylinders encloses a volume of air. Air compression within the cylinders is effected by piston movements and transmitted by means

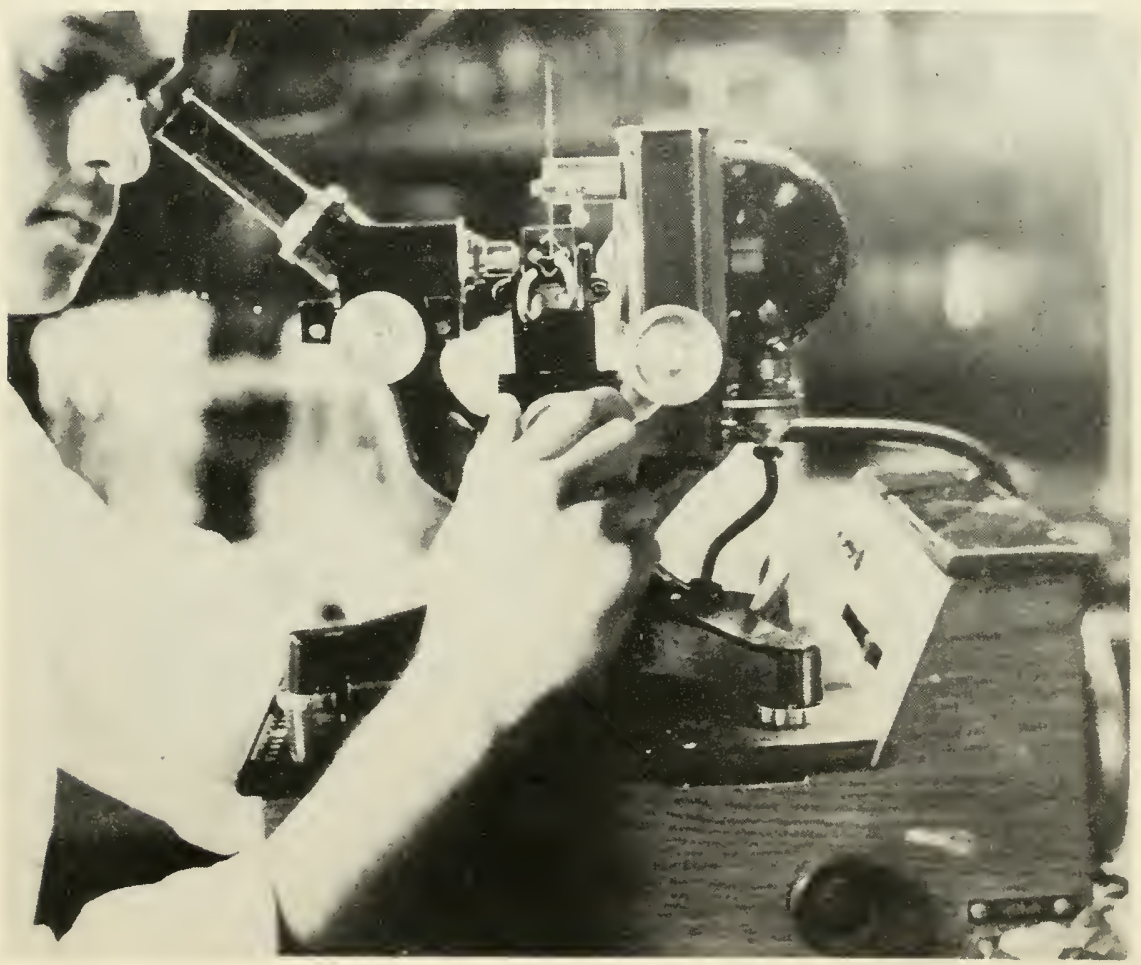

Fig. 9-2 The Microforge in Operation. A needle is being drawn out under observation through the microscope.

of three independent pneumatic tubes of inextensible rubber to the anaeroid foils. The degree of bulge of the anaeroids can be regulated to the desired extent by alterations of the air pressures within the foils by piston movements. Each anaeroid foil in turn is in connection with a lever, and the three levers from the three foils end in a common nose-piece that bears the dissecting needle. A 


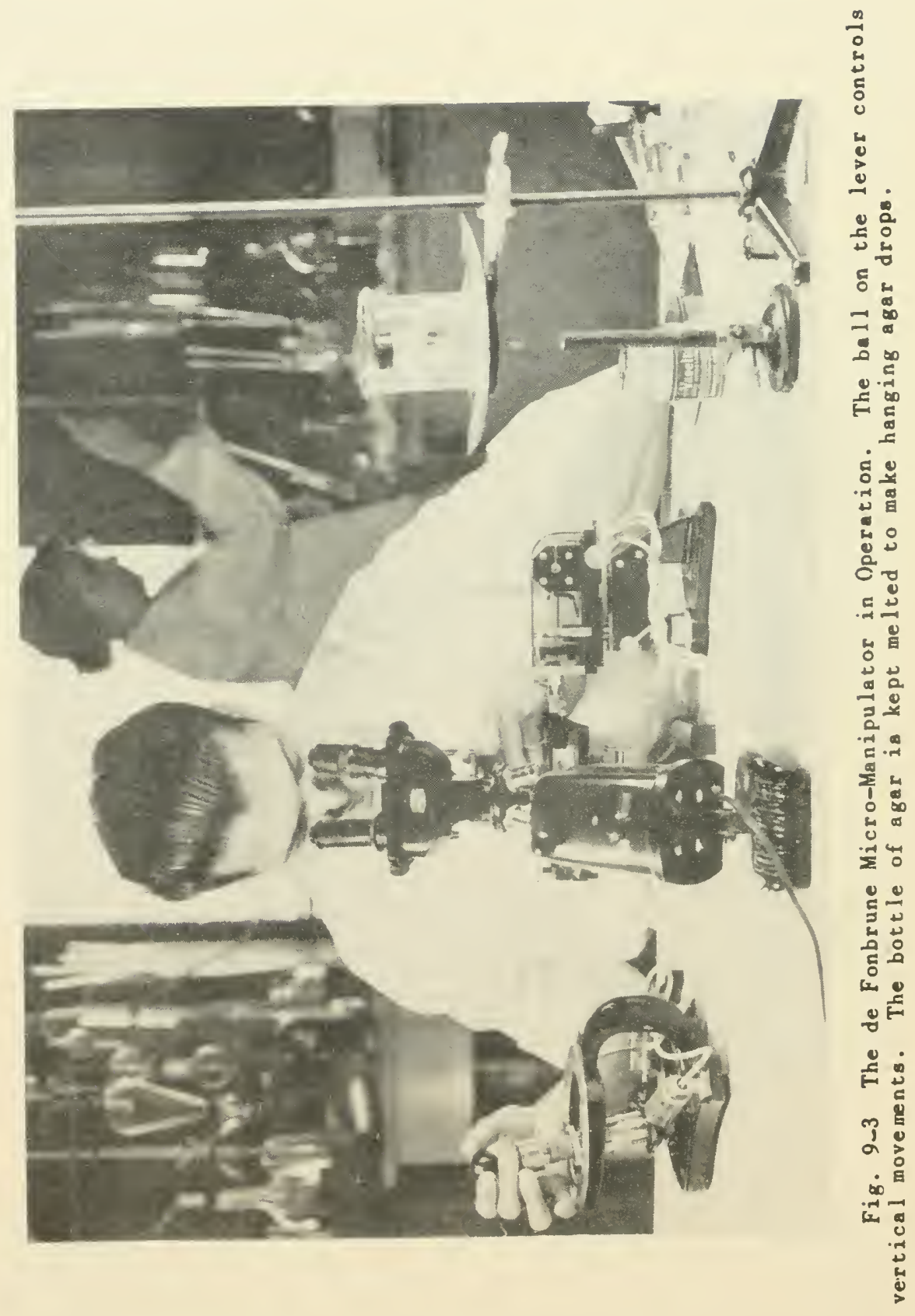


course adjustment screw in the nose-piece permits an initial upward-downward needle movement prior to operating the instrument.

A particular advantage in the de Fonbrune instrument is the provision of a collar around the vertical piston cylinder. An adjustment of this collar regulates the working distance of the pistons and, consequently, the latitude of movement of the dissecting needle in the field of the microscope. Such an adjustment is especially desirable since, lifting asci from a mass of cells in a water droplet one requires a rapid movement of the dissecting needle (effected by increasing the latitude of needle movement), while the actual dissection of the ascus and lifting of the ascospores needs to be done within a narrower range of needle movements in order to avoid injury to the spores during manipulation.

\section{THE MOIST CHAMBER}

An essential device used in micro-dissection is a rectangular open-top moist chamber made of plastic cemented onto a 3 by 2 inch glass slide and placed on the mechanical stage of the microscope. The outside measurements of the chamber are 2 by 1 inch and the walls are about $3 \mathrm{~mm}$. thick and $12 \mathrm{~mm}$. high. It supports two one inch No. 2 coverslips placed close to each other. One end of the chamber has a short wall ( $8 \mathrm{~mm}$. high) so that the dissecting needle can be inserted to work on the bottom of the coverslip. This is sufficient to reduce air currents within the chamber and lessens the chances of contaminations while the latter is in actual use.

When in use, a humid atmosphere is maintained in the chamber to prevent drying up of the droplet bearing the sporulated cells, and the dessication of the agar droplets. The top lens of the condenser is removed to increase the working distance of the condenser and accurate focusing of the light source is obtained by laying a piece of lens paper on the cover glass covering the moist chamber and focusing the condenser until the maximum intensity of light appears on the lens paper on top of the moist chamber. Control of the light intensity by a rheostat makes it possible to use different magnifications without too much eye-strain. A small bottle of agar is kept melted, using a $50 \mathrm{cc}$. beaker, as a water bath, over the pilot light of a Bunsen burner (fig. 9-3). The sides of the moist chamber are lined with filter paper kept moist by adding water from an eyedropper.

A very small quantity of sporulated yeast cells is collected from a gypsum block by means of a sterile inoculation needle and quickly transferred to a drop of clean water on a sterile cover glass. The cover glass is lifted with a pair of sterile forceps and inverted, sporulated cell suspension downwards, over the rim of the dissection chamber. Next, another cover glass is flamed while held with forceps, and four droplets of melted sterile agar are quickly de- 
posited on this cover glass (fig. 9-4) and the latter placed, droplets downward, over the rim of the moist chamber close to the coverslip bearing the droplet of sporulated yeast (fig. 9-5).

\section{DISSECTION}

When the coverslips have been placed in position, the micromanipulator is ready for use. The tip of the dissecting needle is centered in the field of the microscope with the aid of the low power lens. Spores and needle point are now centered under the 16-millimeter lens. Once this has been achieved, needle and cells are centered under a 4-millimeter, 0.65 N.A., objective. An 0.85 objective has too short a working distance for this purpose. Using the course adjustment screw on the manipulator nosepiece, the needle is brought to within approximately the same plane of focus as the cells in the water droplet. A large four-spored ascus from the fringe of the water droplet is next pulled out deftly and brought to the dry part of the cover glass. This is done with a rapid movement of the needle. Increased latitude (and therefore rapidity) of the needle movement can be secured by adjusting the collar attached to the vertical piston cylinder. It is particularly essential that one employs a quick movement to separate asci from the water droplet since forces of surface tension tend to prevent a single cell from being pulled out from the fringe of the water drop onto the dry part of the coverslip. The tip of the dissecting needle is then brought directly beneath the isolated ascus and with a careful movement the ascus is lifted from the coverslip and the needle lowered clear of the lower surface of the coverslip. The ascus is now on the needle and ready to be deposited on the second coverslip which bears the agar droplets. To do this, the moist chamber is moved with the mechanical stage until the agar droplets are within the field of the lowest power objective. The edge of one of the agar drops is then brought within the focus of the high power objective and the ascus is deposited very close to it. With sufficient practice it is always possible to locate the exact site of deposition of the ascus even after it has moved out of the field of the microscope during the succeeding operations. It is a good plan always to deposit an ascus at a fixed border of any one of the four agar droplets, as this makes it easier to return to the same site after separation and deposition of each spore onto an agar drop.

Dissection of an ascus must be performed very delicately; else injury to the ascus will result and cause the collapse of one or more spores. In order to rupture the ascus wall and liberate the contained ascospores, direct pressure is never exerted on the ascus. Rather, the latter is gently rolled about until the ascus wall collapses. Freshly sporulated cells are more amenable to this operation for young spores withstand manipulation to a greater degree than do those 





of older asci. When the ascus has ruptured, a spore is gently lifted away from its companions and the dissecting needle lowered clear of of the lower surface of the coverslip. The low power objective is now placed in position and an agar droplet brought within the field. The agar is centered, brought into focus, and the higher power objective replaced. The needle carrying the single spore is gently raised toward the surface of the drop to deposit the spore onto the agar. After deposition, the needle is again lowered and the low power lens is brought into position to locate the approximate site of deposition of the ascus. Using the high dry objective, one returns to the remaining three spores and each of them is deposited on the agar droplets in the same manner. When all four spores have been isolated on agar, the coverslip is removed from the chamber with a pair of sterile forceps and placed (agar downward) over the greased rim of a Van Tieghem cell and incubated at $29^{\circ} \mathrm{C}$. until microcolonisc of haploid yeast visible to the naked eye appear (fig. 9-6).

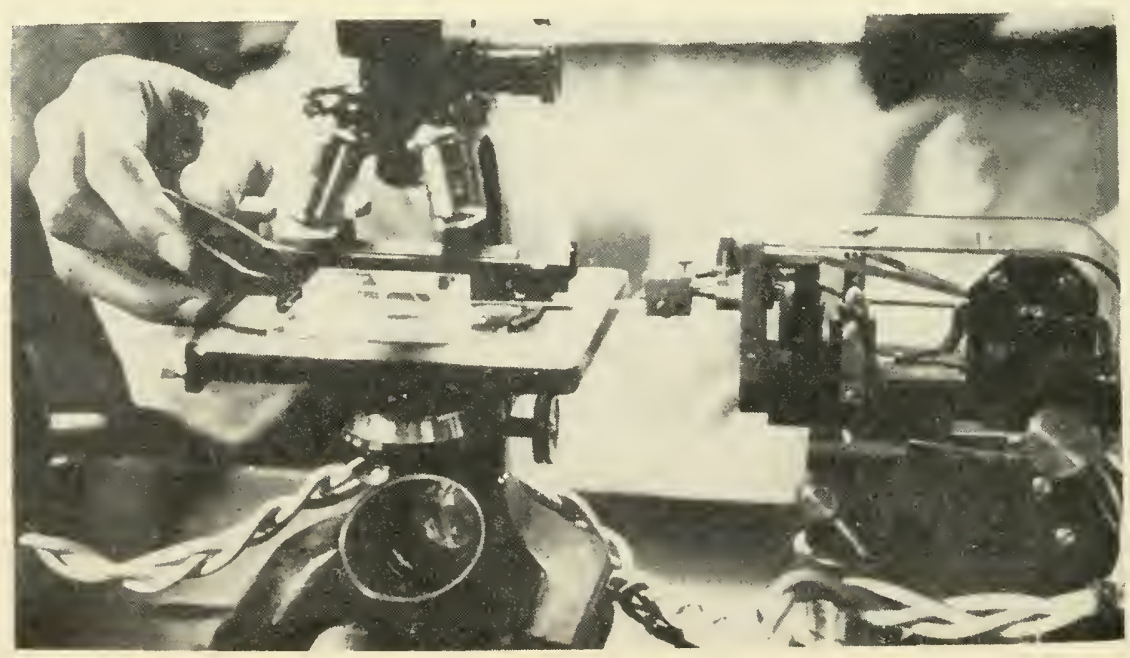

Fig. 9-5 Placing the Coverslip with Hanging Drops on the Moist Chamber.

Each microcolony, or the agar drop bearing it, is removed from the coverslip under a dissecting microscope with a spatulate inoculation needle, and the inoculum streaked over the surface of an agar slant. Each haplophase receives a consecutive number as it is streaked on the slant and the four isolates from a single ascus are held together with an elastic band. 


\section{DRYING CULTURES}

Yeast cultures can be preserved indefinitely by the drying method described by Brown (1925). This method is in use in Dr. Bronfenbrenner's laboratory at Washington University Medical School

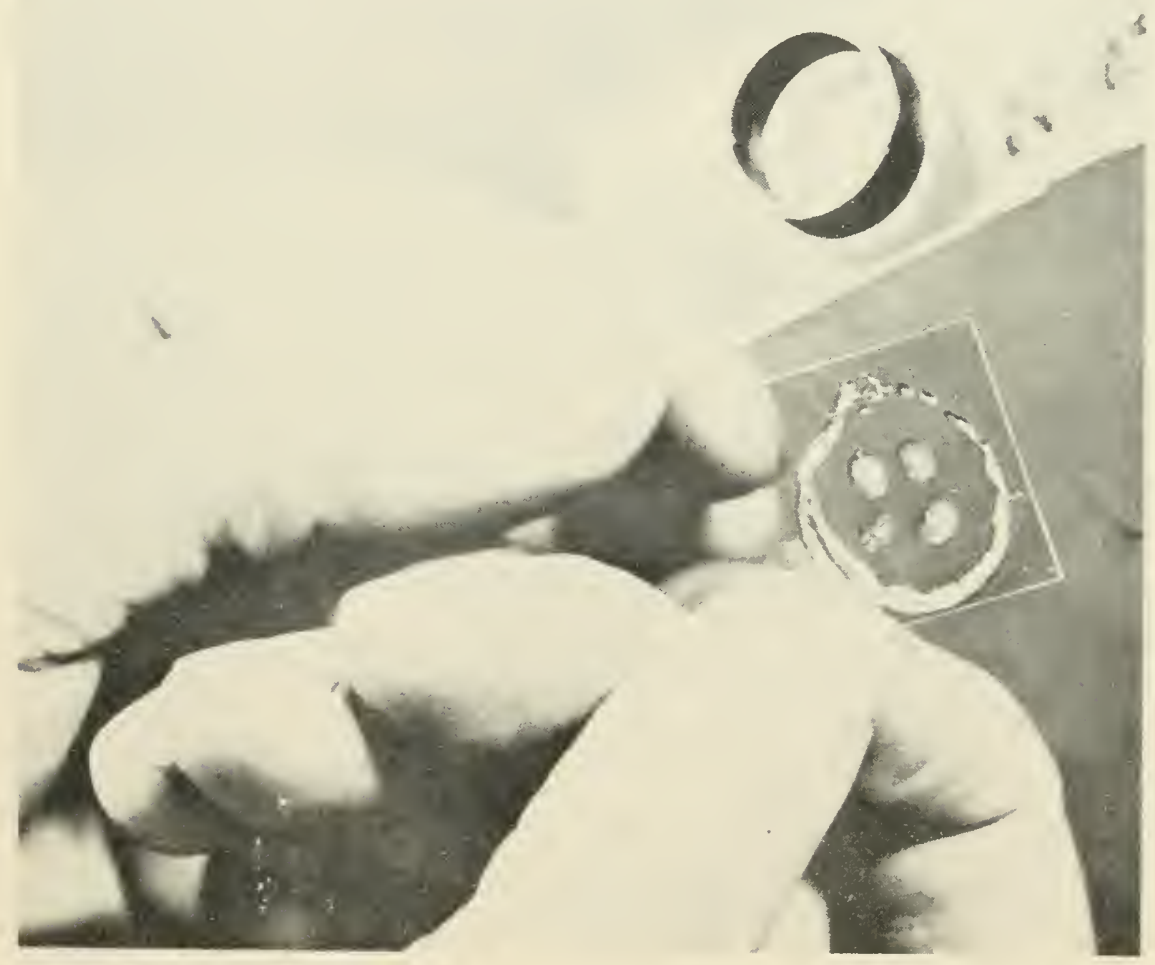

Fig. 9-6 Removing the Cover Glass from the van Tiegham cell. Each agar drop contains a small single yeast colony grown from a single ascospore.

and has been used with considerable success in our laboratory. Blood serum which has been sterilized by filtration is poured on the slant of a young culture (fig. 9-7) with a pipette drawn from a $7 \mathrm{~mm}$. glass tube. (The tubes are kept in the cotton-stoppered acid bottle shown in fig. 9-10). The blood serum is stirred around on the surface of the slant and the suspension of yeast cells in serum 
is drawn up into the tube again. About 3 drops of this suspension of yeast cells in blood serum is introduced into a sterilized cottonplugged, $7 \mathrm{~mm}$. pyrex tube (fig. 9-8). The tube is made by sealing one end of a $7 \mathrm{~mm}$. glass tube, six inches long, in an oxygen flame. It contains two single-hole glass beads. After adding the yeast suspension, the tube is plugged and the cotton is burnt off at the end of

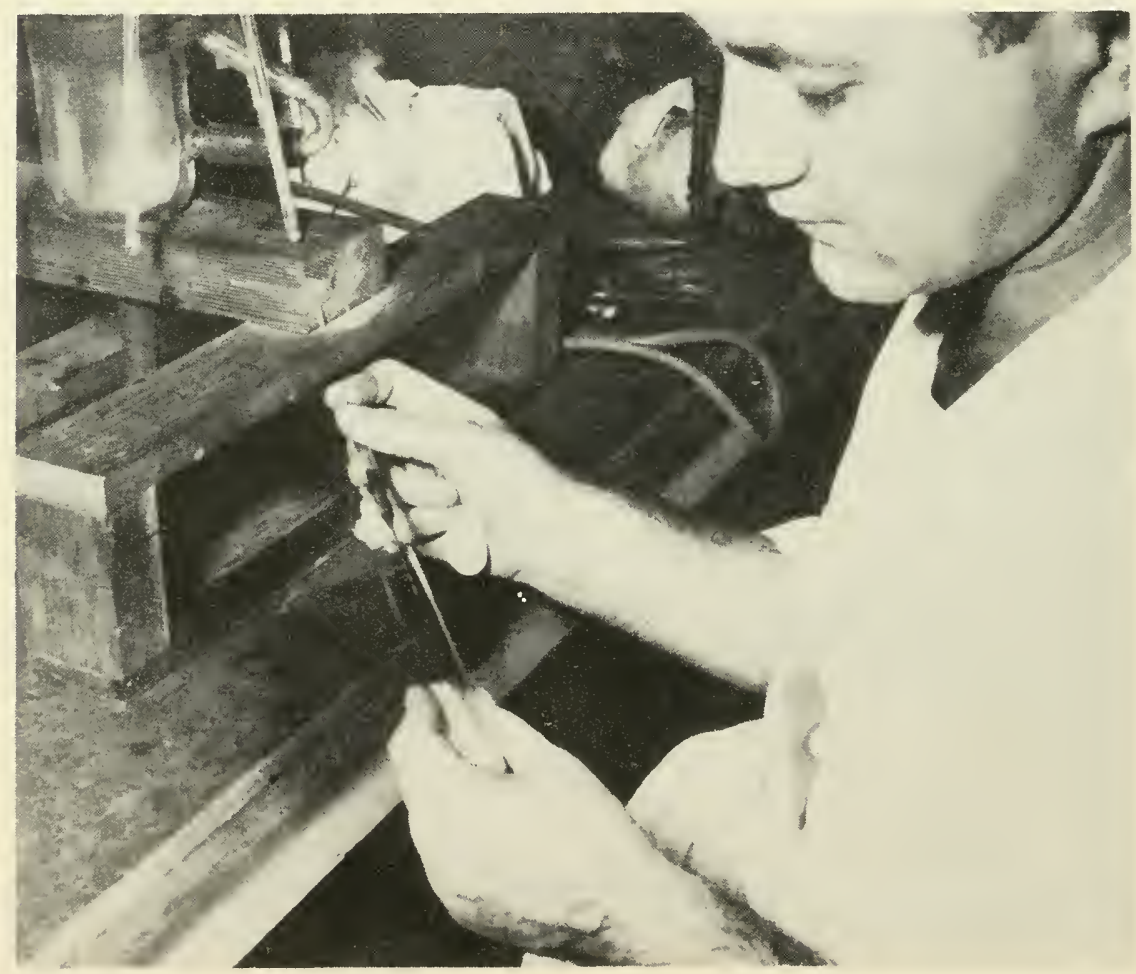

Fig. 9-7 Dropping the blood serum from a pipette on the surface of an agar slant covered with a growth of yeast cells.

the tube and shoved down into the tube to about one inch above the yeast cell suspension. The tube is now filled with a drying agent above the cotton plug (fig. 9-9). We use a patented drying agent called Hydralo, but dehydrated $\mathrm{CaCl}_{2}$ will probably serve as well. A second cotton plug is now added above the drying agent and shoved down into the tube. The tubes are connected with rubber tubing to a vacuum dessicator with a hollow center (fig. 9-10) .in which $\mathrm{CaCl}_{2}$ and ice are placed to condense the water. Vacuum is applied with 


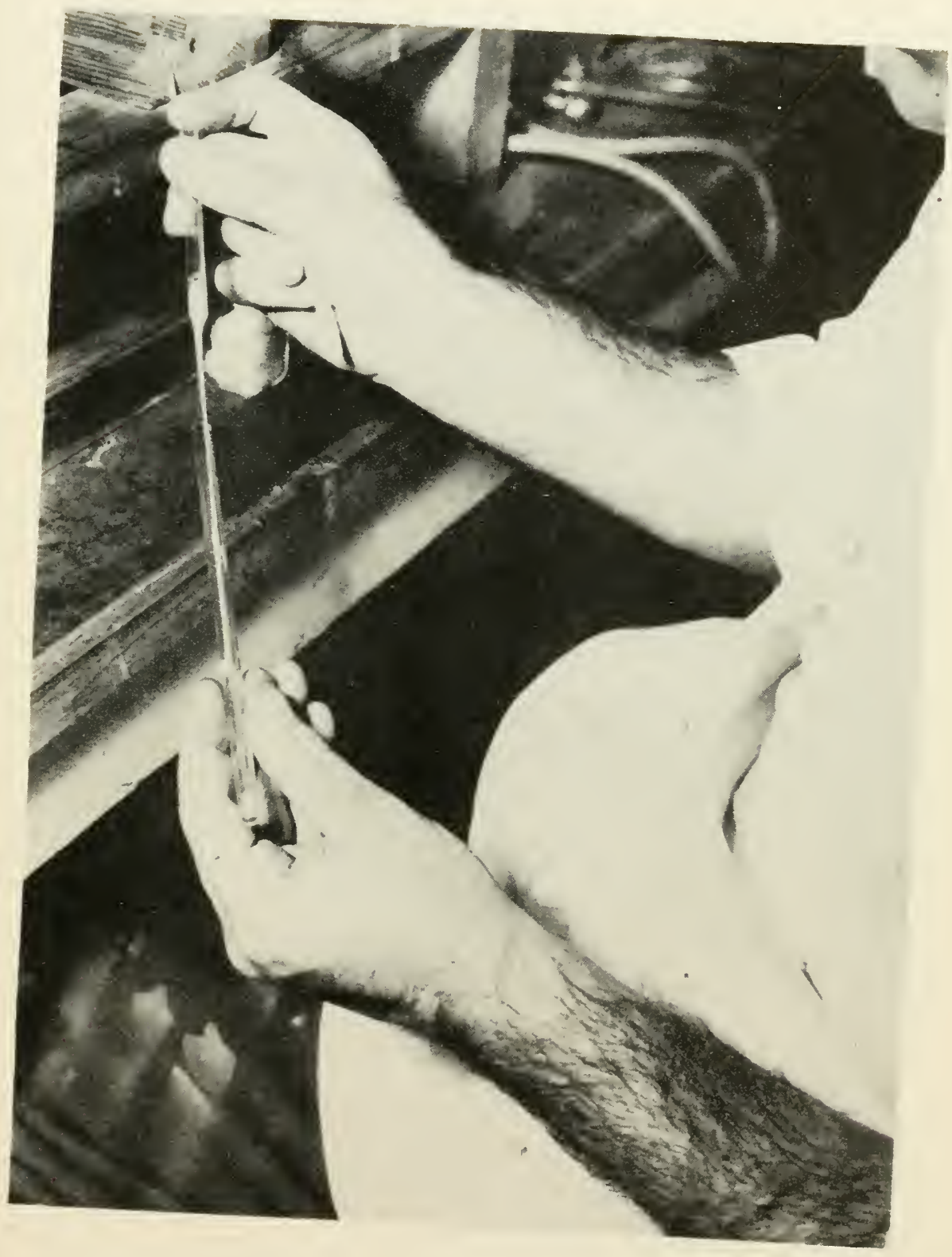
Fig. 9-8 Placing three drops of suspension of yeast cells
in blood serum in a slender glass tube containing two the operator holds the plug between the littining two glass beads. beads, the hand After the suspension has been finger and palm of the , the plug is inserted into the tube placed over the beads. about an inch above the 


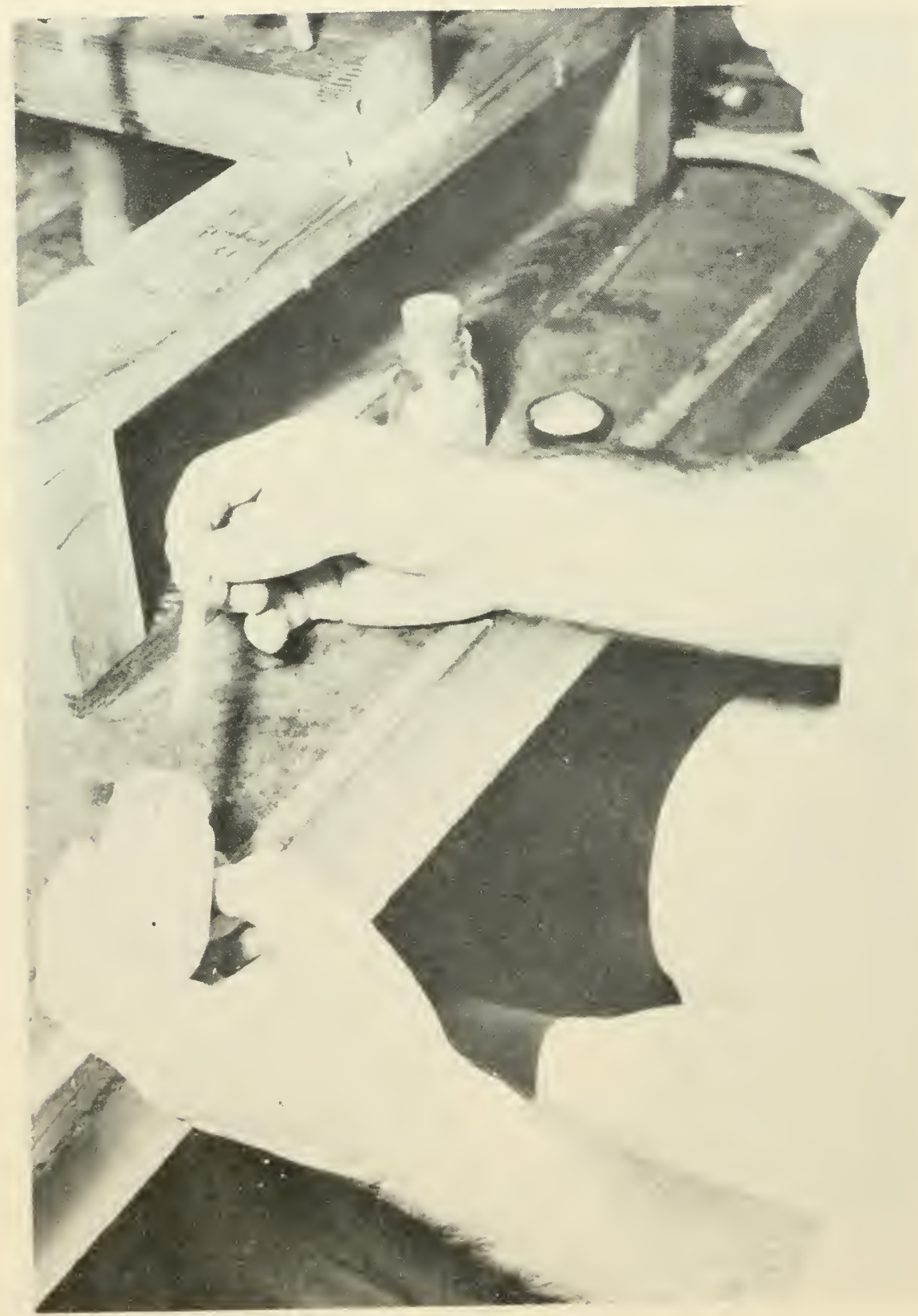

Fig. 9-9 Placing Drying Agent Above the Cotton Plug. The operator holds a bottle of drying agent in the left hand and shoves the open end of the tube into the drying agent and shakes the material down into the tube. 


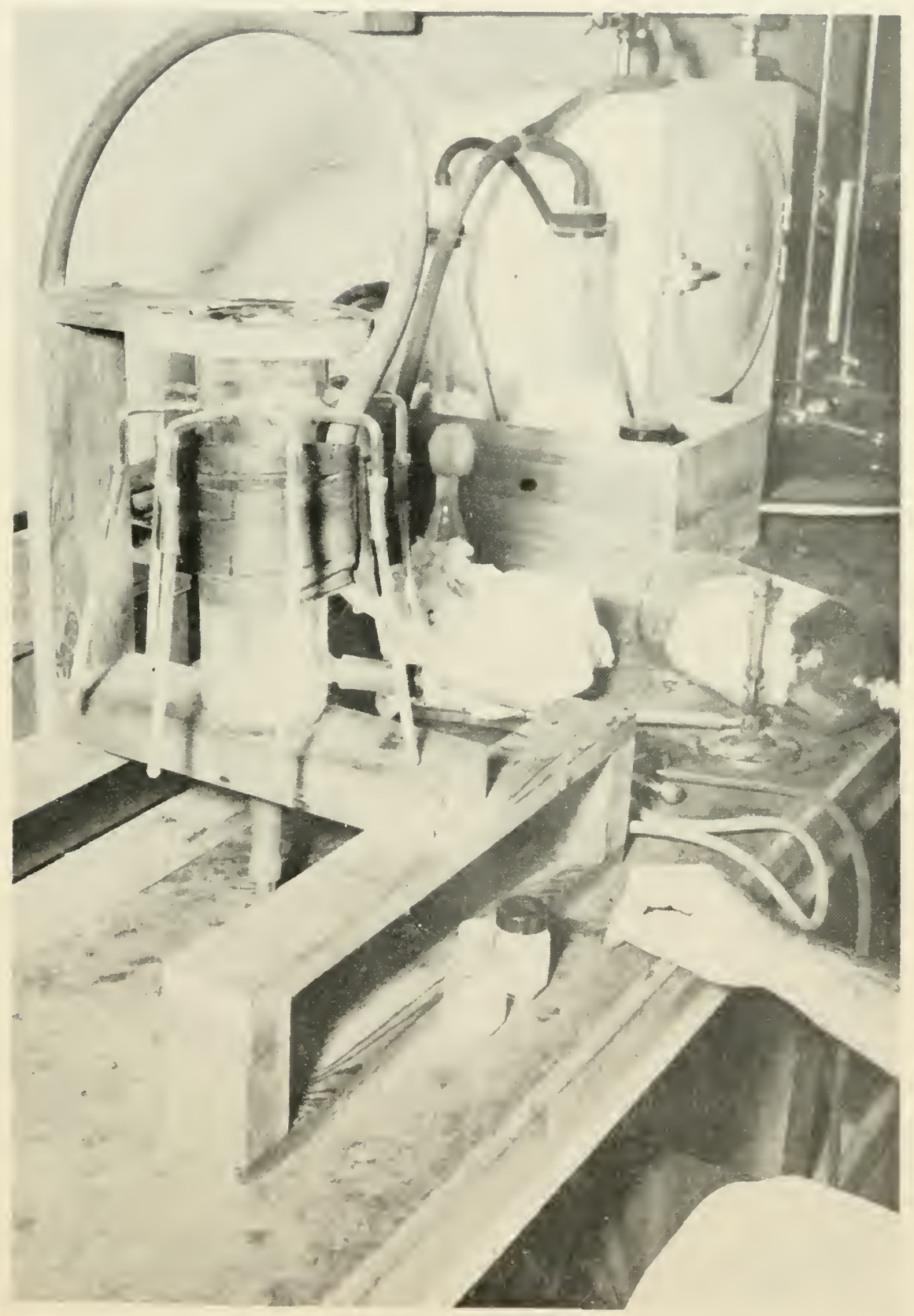

Fig. 9-10 Tube Attached to the Vacuum Dessicator. As soon as the last tube has been attached to the vacuum apparatus, the first one has boiled sufficiently to seal of $f$. 
a Cenco Megavac pump and as soon as the serum boils vigorously enough to flush out the oxygen, the tubes are sealed off with an oxygen torch (fig. 9-11). The drying agent removes the remaining water from the yeast cells and the tubes are stored in envelopes. The complete apparatus is shown in fig. 9-12.

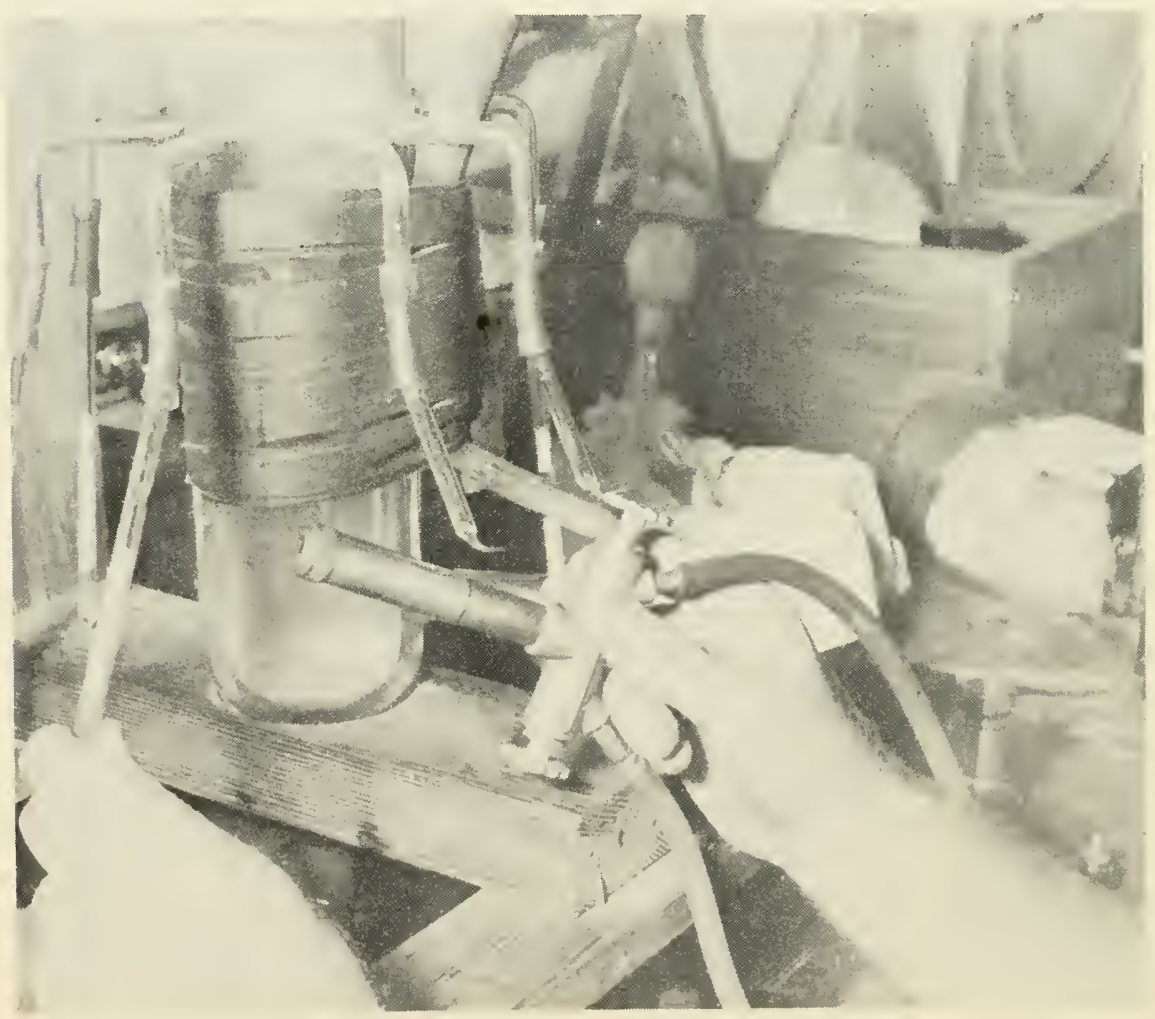

Fig. 9-11 Sealing off Glass Tube While Under a Vacuum with an Oxygen Torch.

These cultures are revived by scratching the tube with a file in the center of the cotton plug. The tube is broken, the cotton plug is removed and dropped in one tube of broth medium; the glass beads are fished out with a wire and dropped into a second tube of broth culture medium. After a few days the broth in the tubes becomes cloudy with yeast cells. If only the tube containing the cotton produces growth, a pure culture can be obtained by plating the culture in a petri dish. 


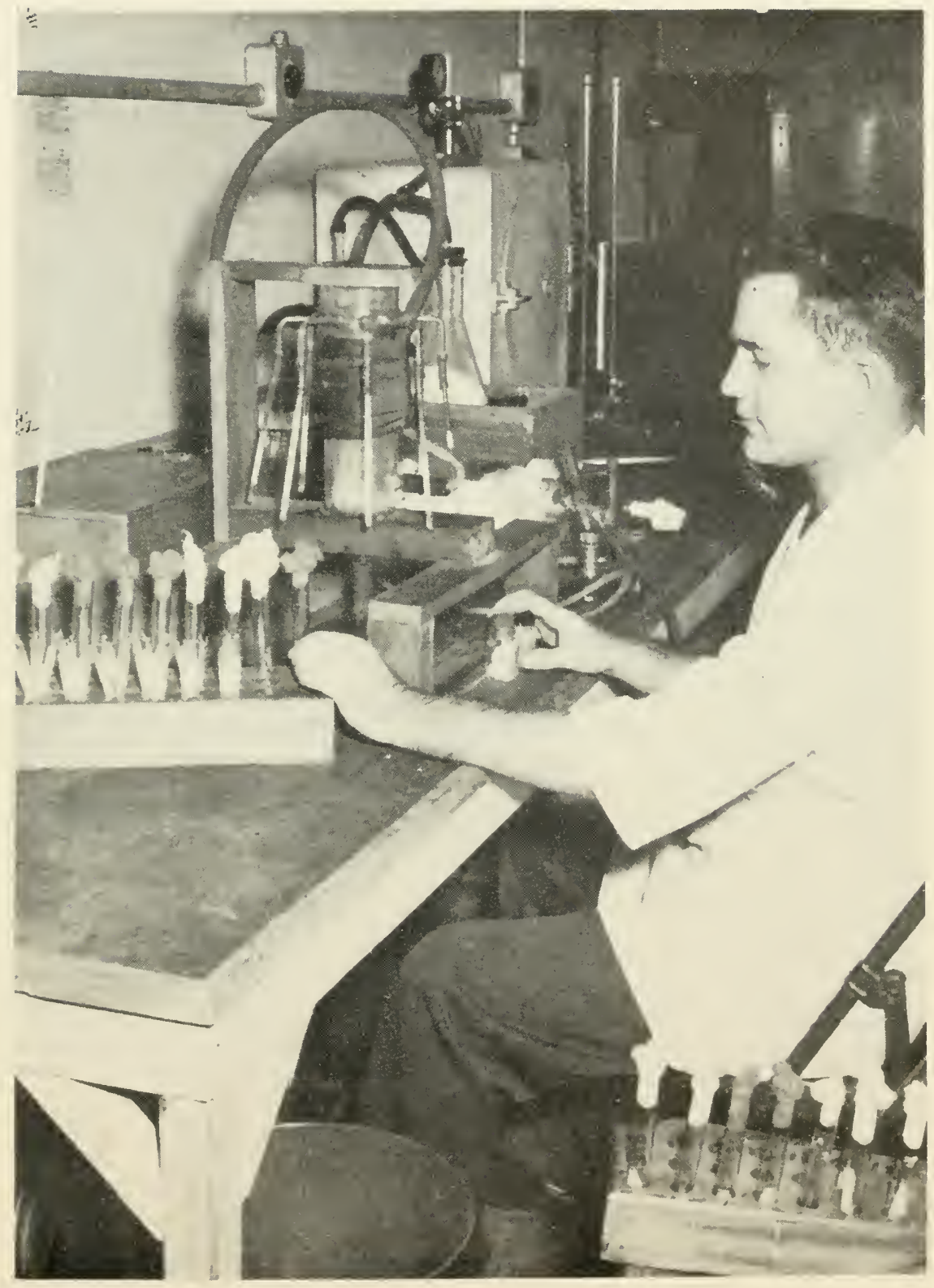

Fig. 9-12 Complete Assembly for Drying Cultures. The vacuum pump is below the table and the large filter flasks in the rear contain calcium chloride to prevent moisture from accumulating in the oil in the pump. In the foreground are cultures which have been dried and the tubes from which the cells were obtained. 


\section{Chapter 10}

\section{HYBRIDIZATION}

Winge and Laustsen (1939) hybridized yeasts by placing a haploid ascospore from one strain in close proximity to an ascospore of a second strain by use of the micromanipulator. When all conditions are favorable, the two spores fuse to produce a diploid hybrid cell. We (1943) have used this method, but obtained relatively few hybrids by ascospore to ascospore matings. The method has the peculiar disadvantage that the characteristics of the parents cannot be determined since the single haploid spore used as a parent is consumed in the mating process. This is particularly important in genetical analysis.

We developed a new procedure based on the fact that single as cospores from Saccharomyces cerevisiae often produce persistently haploid cultures. It is possible to hybridize these with other similarly derived haploid cultures simply by mixing the cells together in an appropriate medium. These mixtures result in copulation if each culture is paired with a complementary type. One parent culture can be mated to a large number of other clones. The parents can be classified as to biochemical and other characteristics previous to the matings and the progeny can be classified subsequently.

We make hybrids between clones of different mating types by mixing the haploid cells from the different cultures together en masse and after the diploid cells have been produced and have sporulated, we select 4-spored asci from the mixture, dissect them and grow each haploid ascospore separately in pure culture. This technique has been condemned by Winge and Roberts because they hold that illegitimate hybridizations occur too frequently to make the method reliable. We have done two things which validate our technique.

(1) Our stocks have been selected for types which practically never produce asci from single ascospore cultures.

(2) Our hybrids are currently heterozygous for from 9 to 12 alleles and the detection of illegitimacy in such well marked matings is direct and unambiguous. Among more than a thousand tetrads so marked, we have not found any illegitimate ones.

The special advantage of this method over the technique of sporeto-spore matings is that both parents can be fully characterized before the matings are made and parents can be selected which will insure that the hybrid be heterozygous for all the known loci. This 
is almost impossible if spore-to-spore mating is practiced, for unless the parents are classified, heterozygosis is merely a matter of chance. Furthermore, the same parent can be preserved indefinitely in lyophil tubes.

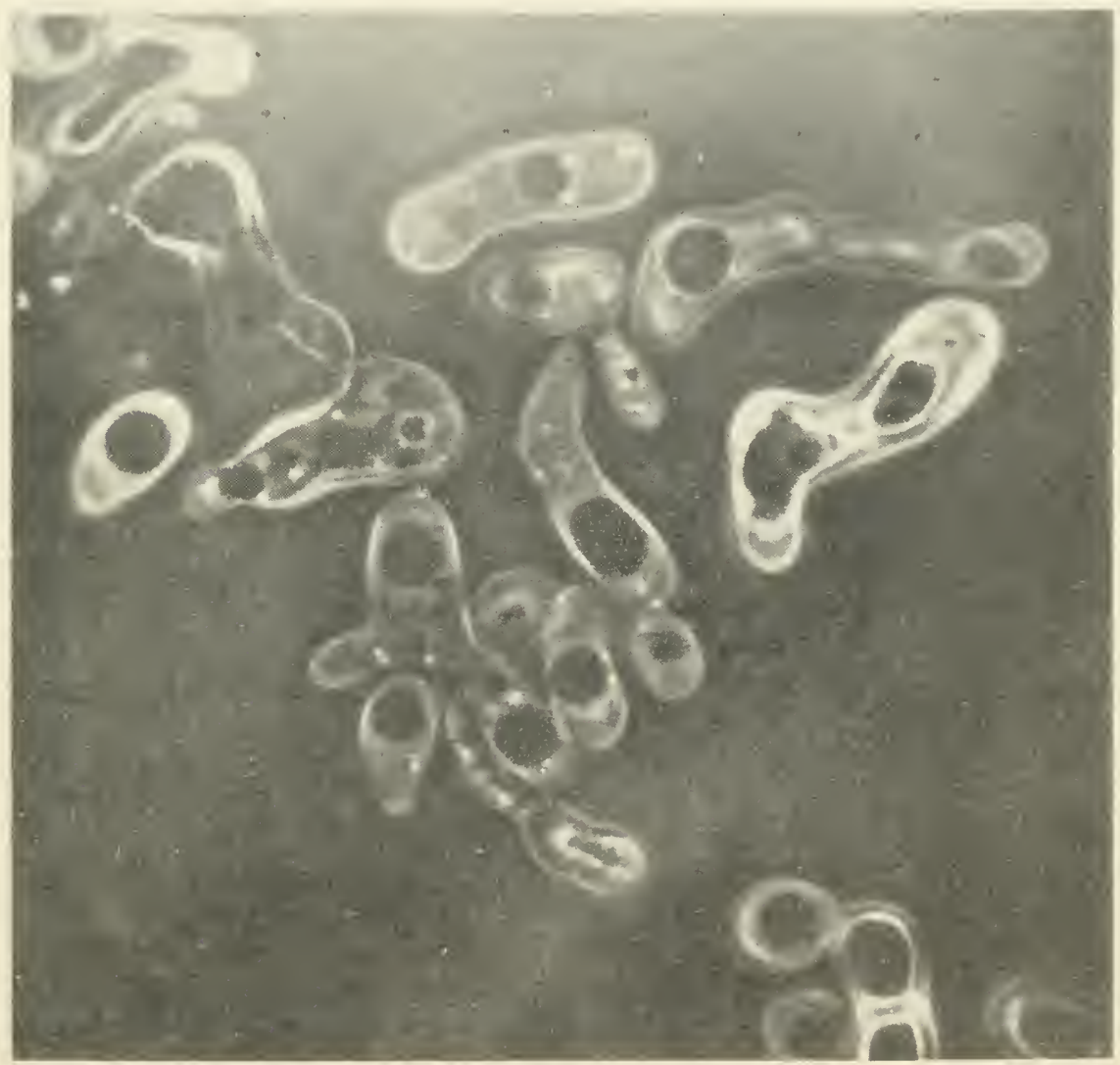

Fig. 10-1 Copulations Between a and a Haploid Cells of S. cerevisiae, by thase-Difference Iicroscopy.

\section{MATING TYPES}

Haplophase cultures of S. cerevisiae fall into two main groups which we have designated a and $\alpha$. Each haploid culture is made up of potential gametes which can be mated with other cells of appropriate genetic composition from another culture. As the pure haplophase cultures age, illegitimate copulations occur between two cells of the same reaction in the same culture, but these matings rarely result in the production of characteristically large diploid cells. Diploid cells produced by illegitimate copulations are gen- 
erally distinguished by smaller size and diminished ability to produce 4-spored asci containing viable spores. Legitimate matings between a and $\alpha$ haplophase cultures derived from a variety of strains of $\mathrm{S}$. cerevisiae usually produce large diploid cells which sporulate to form 4-spored asci containing viable spores. The procedure is as follows: One cc. of broth ${ }^{1}$ is placed in a $150 \times 18 \mathrm{~mm}$. tube. The broth is first inoculated with a large loop of cells from an agar slant of the tester culture and shortly thereafter with a large loop from the haploid culture to be tested. Copulations sometimes appear within six hours. The tube is kept overnight in an incubator at $16^{\circ} \mathrm{C}$. and then inspected under the microscope for the presence or absence of copulations.

A large-scale experiment has demonstrated that two principal alleles control mating-type specificity in S. cerevisiae. Fig. 10-2 shows the results of mating 58 different single ascospore cultures derived from a variety of industrial bakers' yeasts. Ly, HD, M, FLD, D and B represent standard legitimate diploid strains of commercial baking yeasts; 800 is one of the baking strains of yeast obtained from Dr. Wickerham of the Northern Regional Research Laboratory, Peoria, Illinois. Two other cultures are hybrids, one of $800 \times \mathrm{LK}$ and the other $(800 \times \mathrm{LK}) \times \mathrm{Ly}$. Haplophases isolated from the " $(800 \times \mathrm{L}) \times \mathrm{L}$ " hybrid were generally quite fertile. Three belong to mating type a and seven belong to mating type $\alpha$. Two were sterile. Copulations invariably occurred when an $\alpha$ and an a culture were mated, and haplophase $1 \mathrm{~A}$ produced illegitimate matings with three other a type cultures. The Ly strain was also generally quite fertile. Three a type haplophases and seven a type haplophases were found in this culture. Only once did an $\alpha \mathrm{x}$ a mating fail to produce fusions. When the " $(800 \times \mathrm{L}) \times \mathrm{L}$ " haplophases were mated with the Ly haplophases, a high degree of fertility was demonstrated,

1 The standard broth used in this laboratory at the present has the following corposition:

$\begin{array}{lr}\text { Peptone } & 3.5 \mathrm{~g} . \\ \mathrm{hH}_{2} \mathrm{PO}_{4} & 2.0 \mathrm{~g} . \\ \mathrm{MgSO}_{4} & 1.0 \mathrm{~g} . \\ \mathrm{Liquid} \text { yeast extract (Anheuser-Busch) } & \\ \quad 50 \% \text { solids } & 2.0 \mathrm{cc} . \\ \text { Thiamin } & 200 \mathrm{\gamma} \\ \text { Pyridoxine } & 200 \mathrm{\gamma} \\ \text { Niacin } & 200 \mathrm{\gamma} \\ \text { Pantothenate } & 200 \mathrm{\gamma} \\ \text { Biotin } & 2 \mathrm{y} . \\ \text { Cerelose (glucose) } & 40 \mathrm{~g} \cdot \\ \quad \text { Make up to l liter } & \\ \text { Solid medium is made by adding } 30 \mathrm{~g} . \text { of agar per liter. }\end{array}$


$\infty$

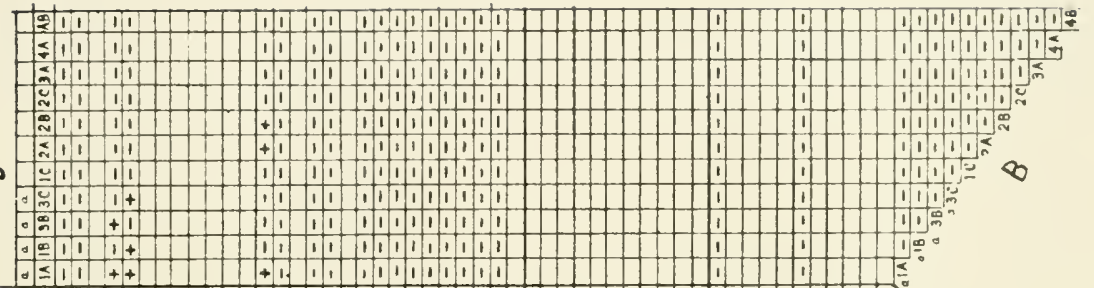

¿.

00

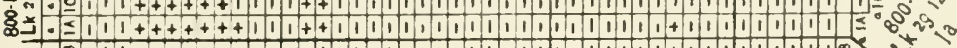

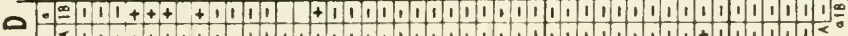

$-11,++++1,1+++1,1,1,1,1,1,1,1+1,1+1,111 \leq \leq^{\circ} 0$

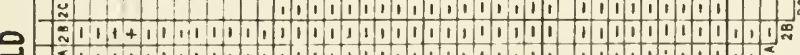

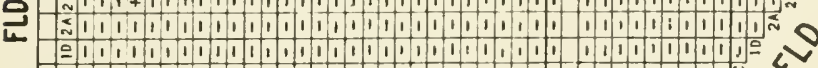

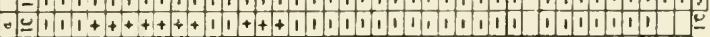

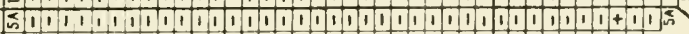

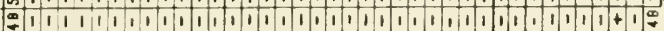

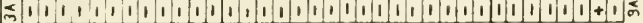

2

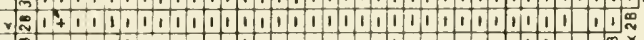

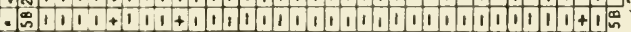

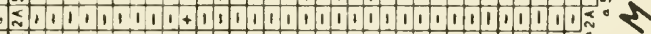

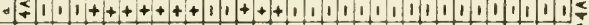

- $\leq 1,1++++1,1+1,1++1,1,1,1,1,1,1,1,1,1,1,1 \leq$

0

I

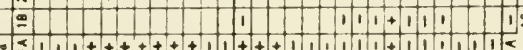

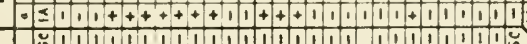

is

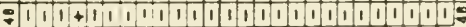

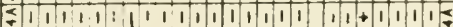

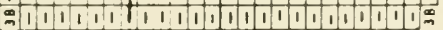

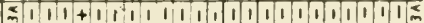

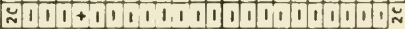

$-\approx|1| 1|+++1++11+++1||||| 1 \mid 1^{\infty}$

- $\approx 111++1++11++\left.111111\right|^{2}$

3

$=|1|++1++++11+++1|1| 1=$

- $2111+++1+1+11++111 \pm 0$

- : $1111++|1|+|1|++10_{0}^{\circ}$

- $10111+++++++11+++1 \mathbb{D}_{0}^{\circ}$

- K $1111+++++++11+++2<0$

$00+1+1+1|1| 1|1| 1 \mid 1110$

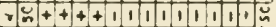

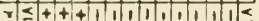

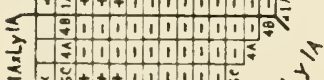

$\leq \frac{0}{6++1,1}|1| 115$

$=0+++1,11111$

$\sqrt{x+t+11}+1115$

$\therefore \sqrt{m_{1}+++1}=$

का $=2++++12$

$\forall=++++\leq$

$r \leq+++\leq$

$-51 !$

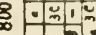

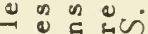

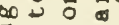

ह $\pi \cdot \pi$

$\overrightarrow{0}+$ in

$0 \rightarrow-1$

()

क.

0 -

$\frac{1}{0}=0 \pi$

$\circ$ \& 000 出

$\vec{a} \cdot \vec{n}=\sum_{0}^{\infty}$ क

a $\infty$

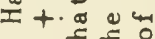

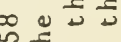

$\infty$

F $=0$

\& का बै

(1) $=1$

का क क

ए 51

- 00

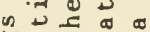

कo का एु

E 5 .

ป ... 둗

त $\mathrm{E}$ कान

$\geq 050$

- 10 을

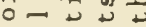

$= \pm$

$=3$

$=500$

בै.

(1) थ 0 a

थै 40.

- 0 ह

F

N

$0=5$

-1 0 के

- 1 E

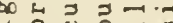

4 원

n 0

0 (1)

U U L

क ट 
with only seven failures out of forty-eight tests. With this strain also, culture $1 \mathrm{~A}$ produced illegitimate diploids. This highly fertile culture was also able to mate with $2 \mathrm{C}, 3 \mathrm{~A}$, and $4 \mathrm{~B}$, which were incapable of producing fusions with any other culture with which they were tested. When the a haplophases of the Ly and the " $(800 \times \mathrm{L})$ $x L^{\prime}$ hybrid were outcrossed to the other strains of yeast, only three hybrids were produced in several hundred matings, but outcrossings with the a strains weremuch more successful and resulted in a large number of hybrids. This occurred in spite of the fact that the HD, M, FLD, D and B cultures were apparently quite infertile among themselves. It appears, therefore, that the a strains could be successfully outcrossed to produce hybrids with foreign strains. The control of mating type by the a/ $\alpha$ alleles has been corroborated by the dissection of more than two thousand asci to date. A standard strain is used to test all the haplophase cultures dissected. A considerable number of sterile cultures were formerly found but now that our cultures have been selected and inbred, sterility and spontaneous illegitimacy occur only rarely.

The fact that the haplophase culture from a single spore can be used for an indefinitely large number of matings is particularly important when one wishes to introduce new genes into a stock. When an ascospore is isolated from a natural species of Saccharomyces, its haplophase cells are often found incapable of copulation when tested against our inbred stocks. However, persistence is sometimes rewarded and occasionally one rare individual copulated to produce a hybrid. These rare clones are used to bring new characters into the pedigree. The desirable haplophase cultures are preserved by drying. Dried haplophase cultures retain their full copulative strength indefinitely, although vegetative propagation of a haplophase culture may be accompanied by mutations which reduce mating strength and transform the original type into a sexually impotent haploid.

Some mutants obtained by continued selection involving numerous platings and transfers over a prolonged period (usually more than a year) become sterile. Prolonged competition and selection probably resulted in loss of fertility because the genes which insure fertility do not have a high survival value in competition with other rapidly growing mutants.

These facts suggest that a moderately proionged haplophase is a distinct advantage in Saccharomyces because it permits competition among the new mutants that arise in the haplophase, leading to selection of the most vigorous before copulations occur. This mechanism may account for the fact that Saccharomyces is the most cosmopolitan specles of yeast. If the mating type strength is great, as in Saccharomycodes ludwigii, fusions occur between the gametes in the ascus without selection or competition and the plasticity of 
the species is limited; for in the diplophase (which constitutes almost the entire life cycle in Saccharomycodes) mutations do not appear so frequently. It is relatively weak mating strength evidenced in Saccharomyces which makes an extension of the haplophase possible. The greater vigor of the diplophase enables it to outgrow the haplophases after the diplophase has been produced. The variety of genes affecting mating strength results in the production of a large variety of sterile haploids (which are essentially "blind alleys" in terms of the continuity of the species), and many of these haploids become stabilized in the form of the genus Torulopsis. Some freshly isolated haplophase cultures are incapable of mating and these would normally be placed in one of the asporogenous genera.

\section{SELECTION VERSUS HYBRIDIZATION}

Previous to the development of a system of hybridizing yeast all improvements resulted from selection. One can carry on a continuous selection with the unstable haplophase culture and get continued improvement for a long time. Such continued selection generally involves much mutation and the resulting culture may become sterile and incapable of hybridizing. It is, therefore, difficult to carry out a program of yeast improvement involving intensive selection followed by hybridization. Matings are most easily made between haplophase cultures that have been recently derived from single ascospore cultures. Since haploid yeast cultures may become sterile when subcultured vigorously, the test for mating type is best performed with single ascospore cultures obtained from freshly isolated spores. Each haplophase culture is either sterile or belongs to either the a or a mating type; a culture which copulates with both a and $\alpha$ haplophases is very unusual. In spite of occasional illegitimate copulations, the mating types are differentiated primarily by a single pair of alleles, which may lose their potency presumably by mutation and which are only rarely transformed into each other, i.e., a into a. We have evidence, however, that this does occur for some illegitimate diploids which we tested produced both a and $\alpha$ cultures, indicating that even in illegitimate copulations, mutation probably precedes the act of copulation.

\section{SPAN OF LIFE CYCLE}

A short generation time is an advantage in genetical studies. The following schedule reveals the minimum time intervals involved in yeast breeding:

Days

0 Isolation of ascospores.

1 Transfer of haplophase colony to agar. 
2 Mating of haplophase with tested strains.

3 Observation of positive matings and transfer to presporulation medium.

5 Transfer to gypsum.

6 Isolation of ascospores.

\section{HYBRIDIZATION PROCEDURES FOR THE PRACTICAL YEAST WORKER}

In the genetical analysis of yeast, we rely exclusively on single ascospore cultures isolated from the asci with the micromanipulator. However, for the applied scientist in the industrial laboratory, this refinement is not necessary. One can obtain an enormous variety of different segregants from a diploid culture by growing it on presporulation medium and transferring it to gypsum until an abundance of spores are obtained. The spores are more heat resistant than the vegetative cells. If they are suspended in water and held at $58^{\circ} \mathrm{C}$. for from 2 to 4 minutes, most of the vegetative cells will be killed and only the spores will survive. The suspension is cooled quickly and plated on solid medium. The large variety of small, rough colonies which appear are isolated and tested for their characteristics. This should be an effective method for selecting new varieties of beer or wine yeasts with different flavors or bouquets. One could either start with a proven yeast of known commercial value or could begin with any sporulating yeast.

Interest is usually centered on the new hybrids, but the haploid cells which are produced by breaking down a diploid also have distinct possibilities, and many of them may be found to be valuable in industry. Generally speaking, wine and baking yeasts are diploid, and beer yeasts are haploid.

\section{HYBRIDIZATION IN UNFA VORABLE MEDIA}

Hybrids capable of growing in an unfavorable medium such as paper mill sulphite liquor could probably be obtained by mixing masses of spores directly in the sulphite liquor itself and allowing the copulation of those capable of survival to occur directly in this unfavorable medium. Millions of copulations could occur, but only the hybrids which are able to grow in sulphite liquor conditions should survive.

The possibility exists of improving the yield of alcohol from beet molasses by hybridization. Much industrial alcohol is produced from beet molasses which contains considerable amounts of raffinose. S. cerevisiae is incapable of completely fermenting raffinose, so all the pctential alcohol is not realized. S. carlsbergensis, however, ferments melibiose and is distinguished from S. cerevisiae by this characteristic. One could produce a raffinose-fermenter 
with some of the desirable characteristics of S. cerevisiae by mixing the ascospores of both $\mathrm{S}$. cerevisiae and $\mathrm{S}$. carlsbergensis in a broth tube containing only melibiose as a source of sugar. Plating out the cell suspension from such a mixture should give some hybrids with the required desirable characteristics of both species. 


\section{Chapter 11}

\section{GENETICAL CHARACTERS}

The following tabulation divides genetical characters into five arbitrary categories:

Terminal characters

Quantitative characters

Lethal genes

Biochemical and physiological

Modifiers
1. Morphology and color (Higher plants and animals)

2. Colonial morphology (Microorganisms)

1. Weight

2. Size

3. Number of parts

The affected haploid segregant or homozygote dies.

1. Fermentative ability

2. Vitamin deficiencies

3. Amino acid deficiencies

4. Nucleic acid component deficiencies

5. Antigenic composition

6. Male sterility

7. Self-sterility

8. Mating types

1. Suppressors

Recessive genes which transform recessive phenotypes into wild type.

2. Plus and minus modifiers

Enhance or diminish the expression of a given character

\section{TERMINAL CHARACTERS}

Terminal characters affect structures produced late in development without reducing the viability of the organism under ordinary culture conditions; eye color in flies, flower color in plants, and colonial morpholozy in bacteria and yeasts, are standard examples of this type of mutant. There is considerable variation of colonial 
morphology among the progenies of hybrid yeasts; each haplophase usually produces a relatively distinct colonial type; but in rather extensive pedigrees we have not succeeded in making a genetical analysis of genes controlling colonial characters, possibly because interaction between these alleles occurs with high frequency.

In Neurospora multiple factors interfere seriously with genetical analysis of morphological characters. The colonial morphology of a clone carrying two different genes cannot be predicted on the basis of the morphology of each haploid parent previous to the mating. Each gene affects the entire thallus; genes controlling the shape of a colony or thallus do not express their effects in an isolated locality. In Drosophila a character which affects the structure of the wing may have little perceptible effect on eye color; when eye color and wing character are expressed independently classification is easy. Much of the success achieved in Drosophila genetics is due to cross-breeding stocks characterized by characters which make their appearance after differentiation is almost completed on widely separated regions of the animal. The only general principle concerning the effect of combining morphological genes in Neurospora is that multiple mutants are generally weaker than the single mutants. When more than two morphological characters are involved the analysis of ten fails. Neurospora hybrids heterozygous for three morphological characters, gap, crisp, and pale, could be analyzed by inspecting the progeny, and an additional physiological character, mating type, which could only be detected by test matings, could be superimposed on the morphological characters without complicating the analysis, but attempts to add cne additional character to this hybrid complicated the results beyond the nossibility of analysis.

We have only used a few morphological characters in yeasts; one of these is mucoid colonial growth. Variations in the substrate affect some colonial characters considerably. We have photographed hundreds of colonies of the haplophase cultures isolated in yeast pedigrees. Some clear-cut distinctive, morphological patterns reappeared frequently among the progeny (fig. 11-1) but except for mucoid, no simple ratios were obtained. There are two techniques of studying colonial morphology; one is to plate out a culture and examine the small, individual colonies, the other is to grow giant colonies, preferably in gelatin at low temperatures. We have used the former technique principally, for this also detects the other variations that occur with high frequency in haplophase yeast cultures. Winge and Laustsen (1937) have had some success using giant colonies grown on gelatin, but for the analysis of extensive pedigrees this might prove rather cumbersome. 

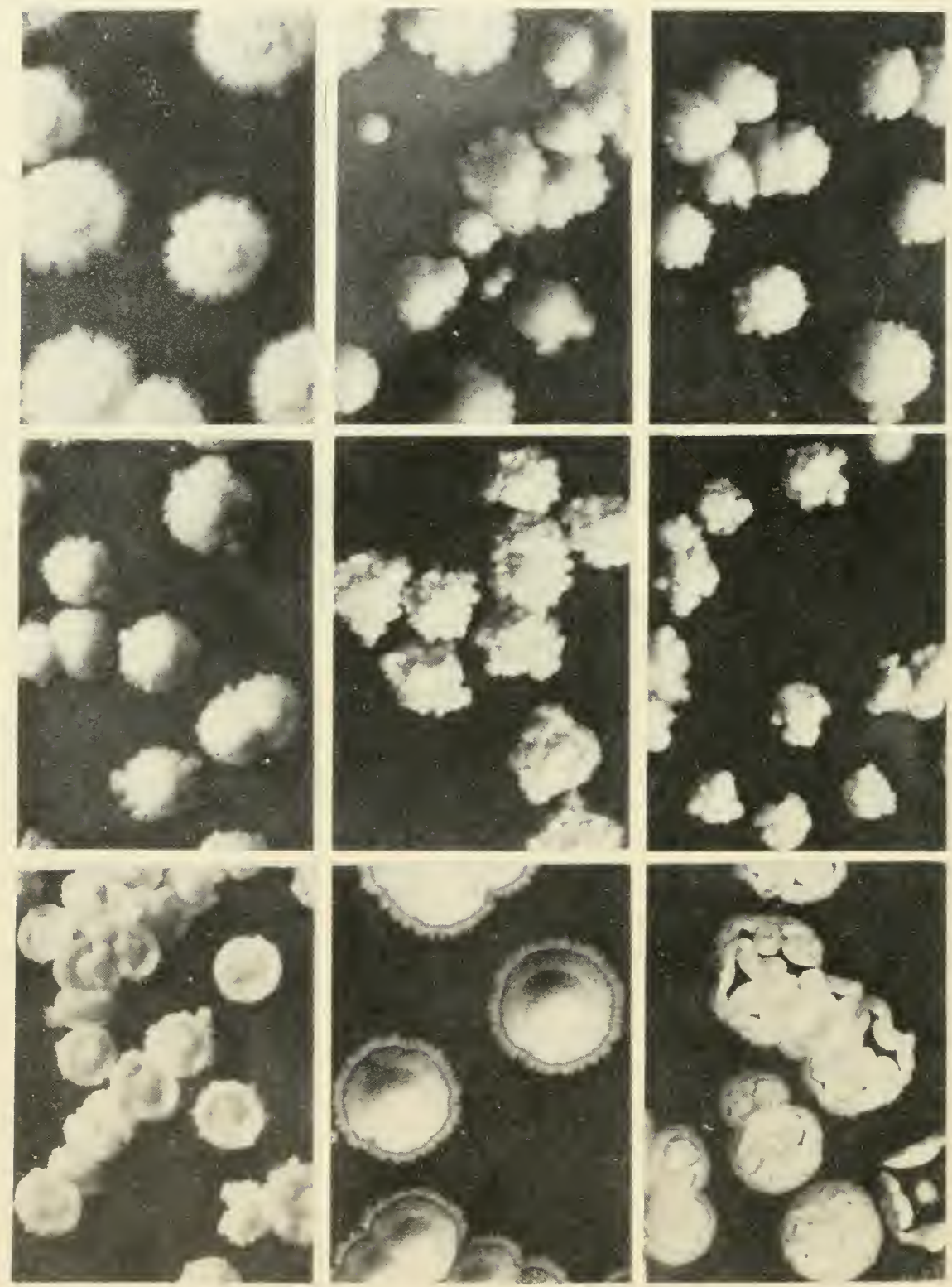

Fig. 11-1 Characteristically different colonial types displayed by genetically different haplophase cultures of Saccharomyces cerevisiae. 


\section{ROUGH COLONIES}

The "roughness" of colonies grown of solid medium is a diagnostic character differentiating various haplophase cultures. Smoothness is characteristic of the diplophase when grown on agar. Winge and Laustsen have shown that diplophase colonies grown on gelatin at low temperature produce rough colonies with characteristic morphological topography, but our cultures were grown on agar at room temperature and under these conditions, diplophase cultures are smooth. The smooth characteristic of the large-celled diplophase depends on the fact that the daughter cells separate quickly from mother cells and each new unit falls evenly into place, making the colony smooth. The contrast between the large-celled diplophase smooth cultures and the small-celled haplophase rough cultures probably depends primarily on cell size, for it appears as if the smaller cells tend to cohere in clusters much more readily than the larger ones. The difference in the types of aggregates characteristic of diplophase and haplophase cells makes it difficult to relate the colonial characteristics of the diplophase culture to its haplophase progeny, and since cell aggregation is so closely related to cell size, haploid colonies must be compared to other haploid colonies and diploids to other diploids. Roughness of the haplophase culture is due to the fact that the smaller cells remain together in clusters in such a way that a definite pattern results when the colony reaches its full growth. The basic pattern of aggregation is observable in microscopic examination of the cells from broth cultures, for rough types generally bud in various "rosette" forms.

Winge (1935) has described the "figure 8" arrangement of haploid cells prior to homozygous copulation. After a bud has reached full size, two new buds appear (one from the mother and one from the daughter cell) near the point of union of daughter and mother cell, producing a "4-leaf clover" effect. Most "rosettes" originate in a variation of the "figure 8 " formation without the subsequent copulations. Many of these cultures make what appears to be homoseneous suspensions because the "rosettes" are too small to affect the turbid appearance produced when the culture is suspended in fluid medium or grown on broth, but the extremely rough colonies cannot be brought into a homogeneous suspension, and when extreme roughtype yeasts are grown in liquid medium. the supernatant liquid is completely clear. This is a character much desired for beer and wine yeasts, especially for champagne yeasts. Such yeasts are called "bottom" yeasts. There is a basic pattern of cohesion even in extremely smooth cultures, for nearly all colonies show sorie distinctive topographical. structure if grown on solid medium long enough to form a giant colony. Conversely, when moderately rough colonies are sown heavily enough on agar to prevent the formation of large colonies, only smooth ones appear. On an unevenly spread 
plate one finds an outer fringe of extremely rough, large colonies surrounding a central group of small, smooth ones. All belong to the same genotype but the rough character cannot come into expression until the colony attains considerable size.

We have observed several hundred different types of roughcolonied yeasts, and although each one is distinctive and recognizable and can be duplicated and recognized when transplanted, we seldom encounter duplicates except in closely inbred cultures. The range of variation is extremely great. In addition to the fundamental "rosette" or budding pattern, differences in shapes and sizes of the cells affect the colonial form. In all colonies the variation in cell size increases with age, generally in the direction of producing larger, longer cells. As a rule, extremely rough colonies contain more elongate cells than smoother colonies, and part of the basis for extreme roughness is probably the maintenance of end-to-end connections after cell division which has been described so frequently in the genus Bacillus.

All four cultures from the single ascospores isolated from a 4-spored ascus originating from stable, smooth-colonied, wild-type diploid cultures of Saccharomyces cerevisiae are usually roughcolonied. Although we have dissected many asci from the same diploid culture, duplicate cultures are seldom found among the colonies grown from the single ascospores, indicating that the diploid cell is heterozygous for many loci which affect "roughness." There are some pedigrees, however, in which one allele apparently controls such an extremely coherent growth that the haplophases carrying it often settle out rapidly on growth in broth, making it possible to differentiate this culture from its allelic type by the speed with which flocculation (autoagglutination) occurs. However, since most haplophase cultures are slowly autoagglutinable, it is difficult to get good Mendelian segregation. In the pedigrees described in Chapters 19 and 26, the symbols $F$ and $f$ (flaky) are used to designate rapidly autoagglutinable and less rapidly autoagglutinable cultures. "F" cultures are always very rough. Since many factors apparently affect roughness or autoagglutination, we have not attempted to work out linkage relations.

\section{QUANTITATIVE CHARACTERS}

Quantitative characters such as weight and height (in man, for examole) are under genetic control but are also influenced considerably by the environment. Similarly, quantitative characters such as the dry weight of yeast produced in a specified medium are under genetic control but are dependent on environmental conditions while a character like mating type is relatively independent. Rate of growth is a character of great economic importance but it is also very difficult to measure accurately because of the influence of the environ- 
ment on the character. The rate of growth is probably considerably influenced by the ability to synthesize amino acids, vitamins, and other essential substances. As will be pointed out subsequently, it is very difficult to find yeasts completely unable to synthesize any any one of these substances, suggesting that the different cultures vary primarily in the quantitative rate of synthesis rather than in absolute deficiencies. Quantitative differences of this type are generally those most important to the breeder attempting to achieve improvements in animals or plants for industrial or agricultural uses, but they are used only rarely by the classical geneticist because they are too difficult to analyze.

\section{LETHAL GENES}

Winge and Laustsen (1939) described a lethal gene in Saccharomycodes ludwigii. Lethal genes cause the death of the haploid segregant or the diploid homozygote. For this reason they cannot pass through the haplophase in S. cerevisiae, although diplophase cultures might accumulate a large number of lethal genes by mutation and survive because the normal allele of the lethal would suffice to maintain the organism. Lethal genes are very common in Drosophila and can be transmitted through either the egg or the sperm because neither of these carries on an independent existence. As soon as fusion occurs the normal allele of the lethal gene supplies the essential substance, the deficiency of which causes the homozygous lethal to die. Dodge and Lindegren showed that haploid nuclei containing lethal genes are easily maintained in heterokaryons, and Beadle and Coonradt exploited this method in maintaining balanced Neurospora stocks on minimal medium. A thallus containing two kinds of nuclei one unable to synthesize thiamine and a second unable to synthesize pantothenate could survive in a medium lacking both of these essential vitamins by mutually supplying each other.

Spore lethals are relatively common in Saccharomyces. They apparently are variations of the haplophase which do not affect the viability of the haplophase, but which render the spore incapable of germination.

\section{BIOCHEMICAL AND PHYSIOLOGICAL CHARACTERS}

In a sense all characters are either biochemical or physiological but the term is applied to specific fermentative and synthetic abilities. Such characters have an important advantage, namely, they can be superimposed in one individual without complicating genetical analysis. For this reason we have concentrated on genes differentiating stocks with regard to the fermentation of carbohydrates and the synthesis of vitamins, amino acids, and nucleic acid components. These single gene differences, however, have not distin- 
guished fermenters and synthesizers from cultures comple te Iy incapable of fermentation or synthesis, as will appear in the subsequent discussion.

\section{FERMENTATIVE ABILITY}

The ability of Saccharomyces to ferment different sugars is gene-controlled and our breeding stocks are differentiated by their abilities to ferment galactose, melibiose, maltose, sucrose, raffinose and methyl alpha glucoside. We have used these fermentative differences as genetical markers and have concluded that a single gene-pair differentiates two stocks when in a large number of tetrads isolated from a hybrid, two cultures from each tetrad are "fermenters" and two are "nonfermenters."

\section{THE FERMENTATION TEST}

The fermentation test is performed in a $12 \times 100 \mathrm{~mm}$. Durham fermentation tube containing $3 \mathrm{cc}$. of a 4 per cent concentration of the carbohydrate and an inverted insert, $7 \times 50 \mathrm{~mm}$. The Durham tubes are prepared as follows: Each receives $2 \mathrm{cc}$. of the following medium without added sugar, plugged with cotton and sterilized with the inverted insert in place:

Components

Peptone
$\mathrm{MgSO}_{4}$
$\mathrm{KH}_{2} \mathrm{PO}_{4}$
Liquid Yeast Extract
Inositol
Thiamin
Pyridoxine
Pantothenate
Nicotinic
Paraminobenzoic
Biotin
Adenine
Methionine

Amount per liter

$3.5 \mathrm{~g}$.

$1 \mathrm{~g}$.

$2 \mathrm{~g}$.

2 cc.

10,000 micrograms 200

200

200

200

50

2

2,000

2,000

One cc. of a Seitz-filtered 12 per cent solution of Pfanstiehl carbohydrate is added to make a final concentration of 4 per cent. The Durham tube is inoculated with a large loop of organisms from an agar slant (fig. 11-2). Most yeasts are able to ferment dextrose, fructose, and mannose, and any hydrolysis whi $i$, $h$ splits off one of these hexoses, results in a positive fermentation test. 


\section{GALACTOSE-MELIBIOSE COMBINATIONS}

The fermentation of galactose and the hydrolysis of melibiose are controlled by two independently segregating genes. This means that four kinds of progeny are produced when a gamete capable of fermenting both galactose and melibiose (G ME) is mated to one incapable of fermenting either sugar ( $\mathrm{g} \mathrm{me}$ ).

\section{PRODUCTION OF $\mathrm{CO}_{2}$ WHEN DIFFERENT SUGARS ARE USED IN THE FERMENTATION TEST}

Genotype
G ME
G me
g ME
g me

Galactose

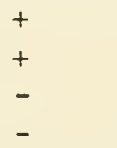

Nielibiose

$+$

$-$

$+$

$-$

The fermentation test can also be made in Smith tubes (fig. 11-3) by inoculating a nutrient broth containing a single specific carbohydrate. After incubation the following results may occur:

1. Rapid fermentation. Growth occurs within 24 to 48 hours and the tubes fill with gas within that time.

2. Slow fermentation. Growth occurs slowly and the tubes are filled with gas within from 5 to 20 days.

3. Growth occurs slowly but no gas appears in the tube. (This may occur frequently if the concentration of sugar is less than 1 per cent.)

4. No growth occurs and no gas appears.

In the genetical analysis of the haploid members of our pedigrees we have encountered phenomena 1 and 4 most frequently. The other types occur rarely and the distinction between types 1 and 4 is controlled by a single pair of genes in many pedigrees. We have called type 1 "fermenters" and type 4 "nonfermenters." Until recently we thought that type 4 was incapable of producing the specific enzyme. However, when the "nonfermenters" are placed in Smith tubes on mercury they produce gas albeit slowly. This means that the single gene difference between "fermenter" and "nonfermenter" does not involve presence or absence of the ability to produce the enzyme but that a gene pair exists whose dominant allele controls a high rate of enzyme production while its opposite number recessive allele is incapable of producing sufficient enzyme to register as a positive test in the Durham tube (fig. 11-2). However, cells carrying the recessive gene are able to produce enough enzyme to register a positive test in a Smith tube on mercury (fig. 11-4).

The "slow" fermenters (type 2 present still another problem. Winge and Roberts have described "slow" iermenting cultures of 
Saccharomyces which require from 3 to 12 days to ferment galactose in a Durham tube with galactose yeast extract agar. We have encountered similar slow fermenters of galactose and Mr. B. D. Mundkur has undertaken a study of them. These cultures when inoculated into a Smith tube (fig. 11-3) containing 4 per cent galactose and the medium described on page 11-7, produce gas in the inverted insert.in from 5 to 20 days. This is undoubtedly the same phenomenon described by Winge and Roberts. They found in addition that after fermentation had occurred, the fermenters could be "deadapted" by transfer to maltose broth and were "readapted" by transfer back
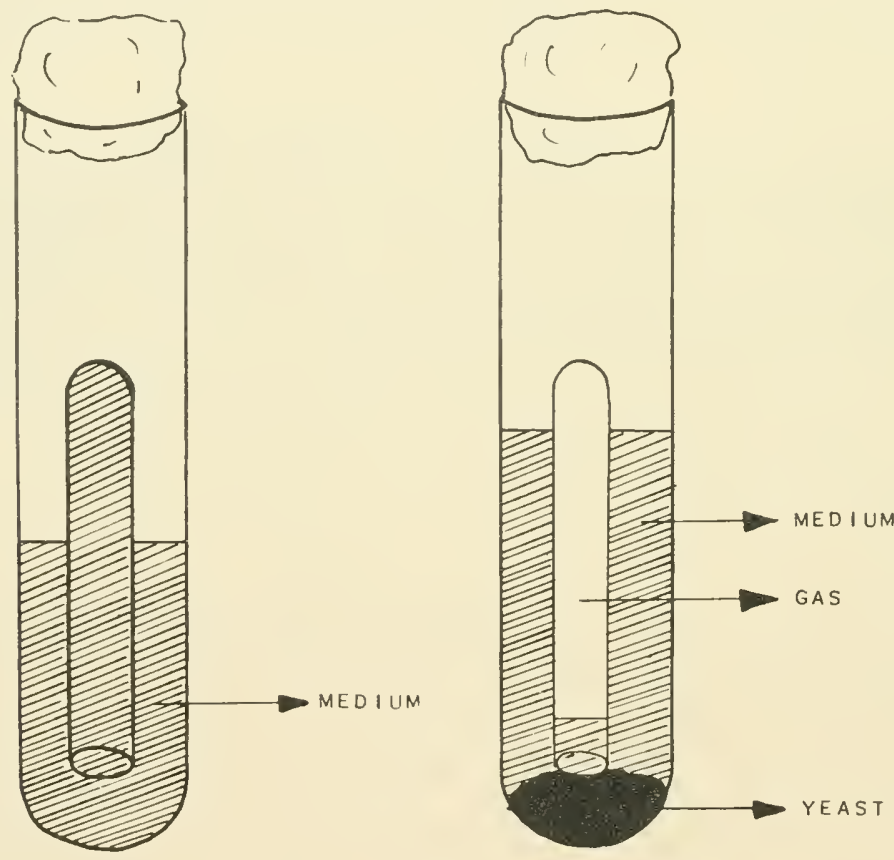

Fig. 11-2 Fermentation in Durham Fermentation Tubes. Growth of yeast occurs in nutrient broth and results in accumulation of carbon dioxide in the small inverted tube. A complete nutrient with only a single carbohydrate source is used. This is the test used by Lindegren and Lindegren. It depends on the browth of yeast to a sufficient amount to produce gas more rapidly than ditfusion trom the medium can occur. A small inoculum increases during the process; the growth of the yeast depends on the presence of air so the test is not anaerobic.

to galactose broth. They interpreted this result as indicating that each cell produced a small amount of enzyme and that the population as a whole slowly achieved or lost fermentative ability. 
Whatever the merits of their interpretation may be in the case which they have considered, it is quite clear it does not apply to what we have described as "slow" or "weak" fermenters. This is
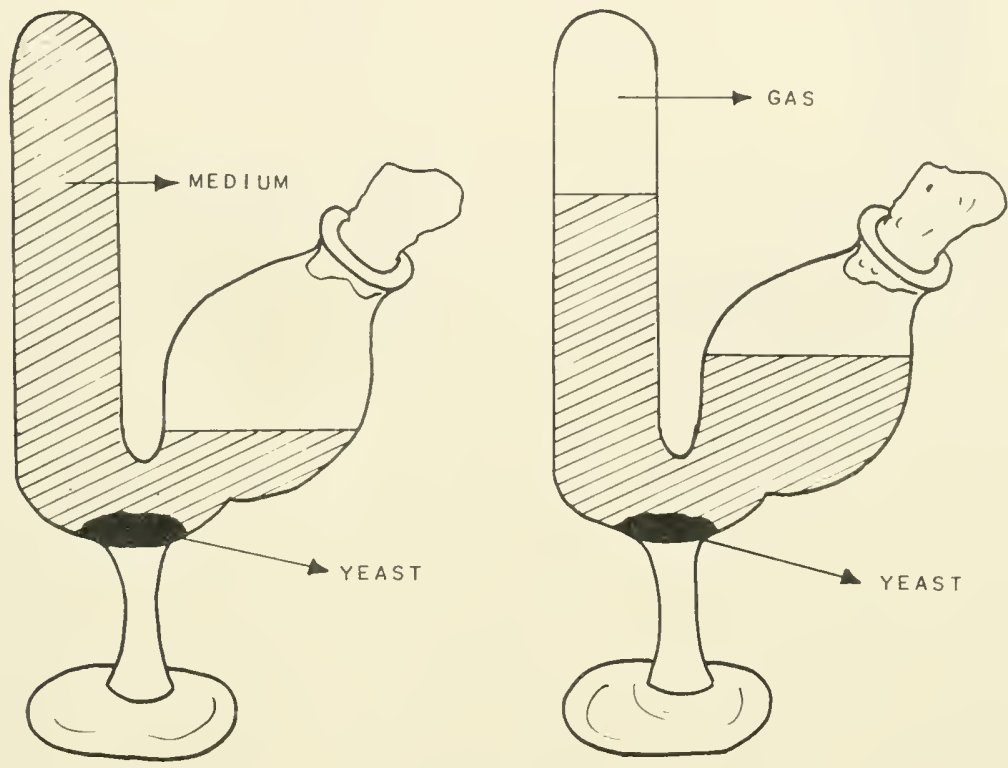

Fig. 11-3 Smith Fermentation Tube (Also Called Einhorn Tule). This is the test used by $K$ inge and Roberts. They used two media, (1) galactose yeast extract broth, in which the principal carbohydrate source is pure galactose, (2) Leer wort, in which the principal carbohydrate source is naltose but presumably considerable amounts of glucose are also present. This test also depends on the increase of yeast from a small amount to a large amount and the production of gas more rapidly than it can be lost by diffusion. In these tuies also the growth of sufficient cells to give a positive test depends on the presence of sutficient oxygen. This test and the one illustrated in Fig. 11-2 are essentially the same and will give the same results. They are both limited by the requirement that the concentration of sugar must be sufficiently high so that the evclution of $\mathrm{CO}_{2}$ will be rapid enough to permit accumulation of gas in the tuives? A rubber stopper may prevent diftusion and convert a test that might be negative (it only a sinall amount of sugar were present) into a positive.

revealed by the following experiment. Mr. Mundkur inoculated many of our type 2 "slow" fermenters of galactose into tubes containing $0.5 \mathrm{cc}$. of glucose broth. The inoculum was about 200 cells and the cultures were allowed to grow to completion at which time about $3,000,000$ cells were present. Pour plates of galactose agar were then made with the result shown in fig. 11-5. Similar pour 


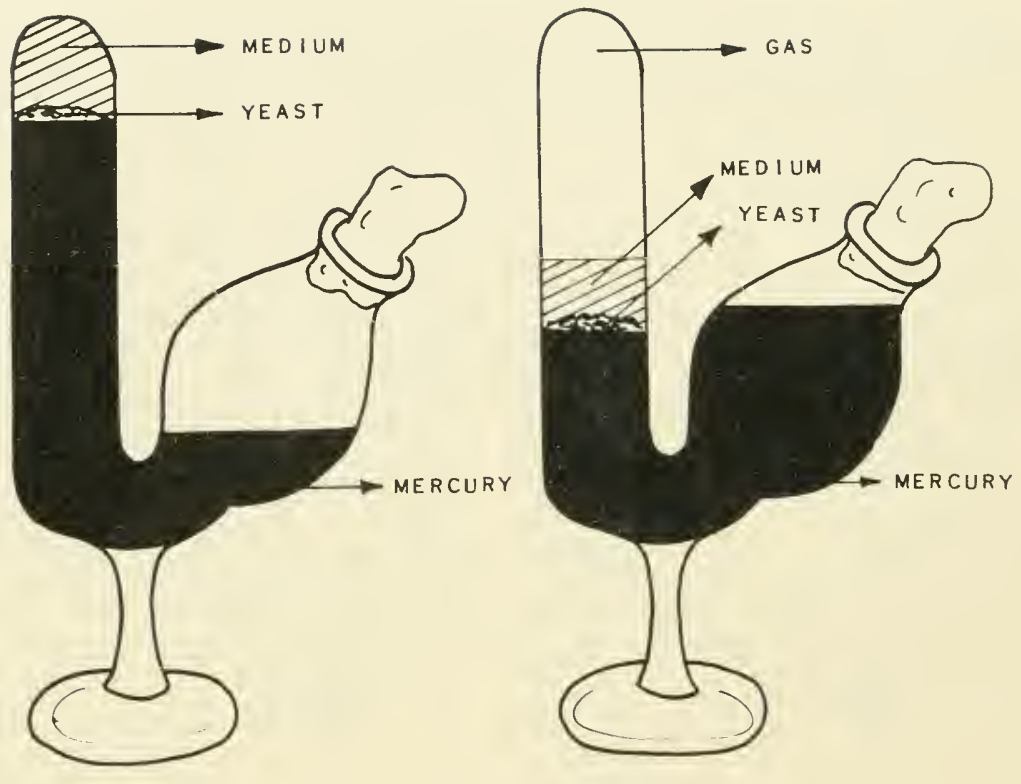

Fig. 11-4 Smith Tube Test for the Presence of (or the Prodiction of) Enzymes in Yeast Cells Under Anaerobic Conditions. The absence of nutrients precludes any considerable amount of growth. A very large inoculum consisting of the growth from an entire agar slant may be used in a single tube. The presence of constitutive or adaptive enzymes can be determined by observing the rate of gas production in the tube. Since there is no significant increase in cell numbers selection is excluded. If gas production begins inmediately and continues without a change of rate, a constitutive enzyme is present; if gas production begins after a lag period and follows a sigmoid curve an adaptive enzyme is produced.

In the tests shown in Fig. 11-2 and Fig. 11-3 it is impostsidle to determine what causes the lag since growth must occur before gas is produced. An opportunity for the selection of a mutant from a predominantly nonfermenter population may occur in tests involving growth, but is excluded in the Smith tube when mercury is used. In this test the anaerobic evolution of $\mathrm{CO}_{2}$ can be calculated for a stationary population just as it can in the Wartburg apparatus.

The van Iterson-Kluyver apparatus is another device which accomplishes the same end as the Smith tube with mercury. It is, however, more complicated since it contains several glasscocks and in our experience seems less desirable because of the loss of mircry on table tops and floor. These authors use a sugar solution containing nutrient, but we have found that the evolution of gas occurs quite readily in the Smith tube over mercury with only phosphate and sugar, if $50 \mathrm{mgm}$ (dry weight) of yeast is used.

The more elaborate Wartburg apparatus presents (1) a measure of the rate of evolution of $\mathrm{CO}_{2}$ in the presence of $\mathrm{N}_{2}$ or (2) in air and (3) the sate of consumption of oxygen. When the dryweight of yeast used is obtained the rate can be expressed in terms of $\mathrm{mm}^{3}$ of gas per hour per mgm of dryweight.

$11-11$ 
plates yielded from 0 to 19 large colonies per plate with a background of millions of minute colonies. Fig. 11-6 shows the region around one of the large colonies revealing the enormous number of tiny colonies embedded in the agar. Plating the large colony on galactose agar produced large and small colonies and the large colonies inoculated into Durham tubes filled the inverted insert

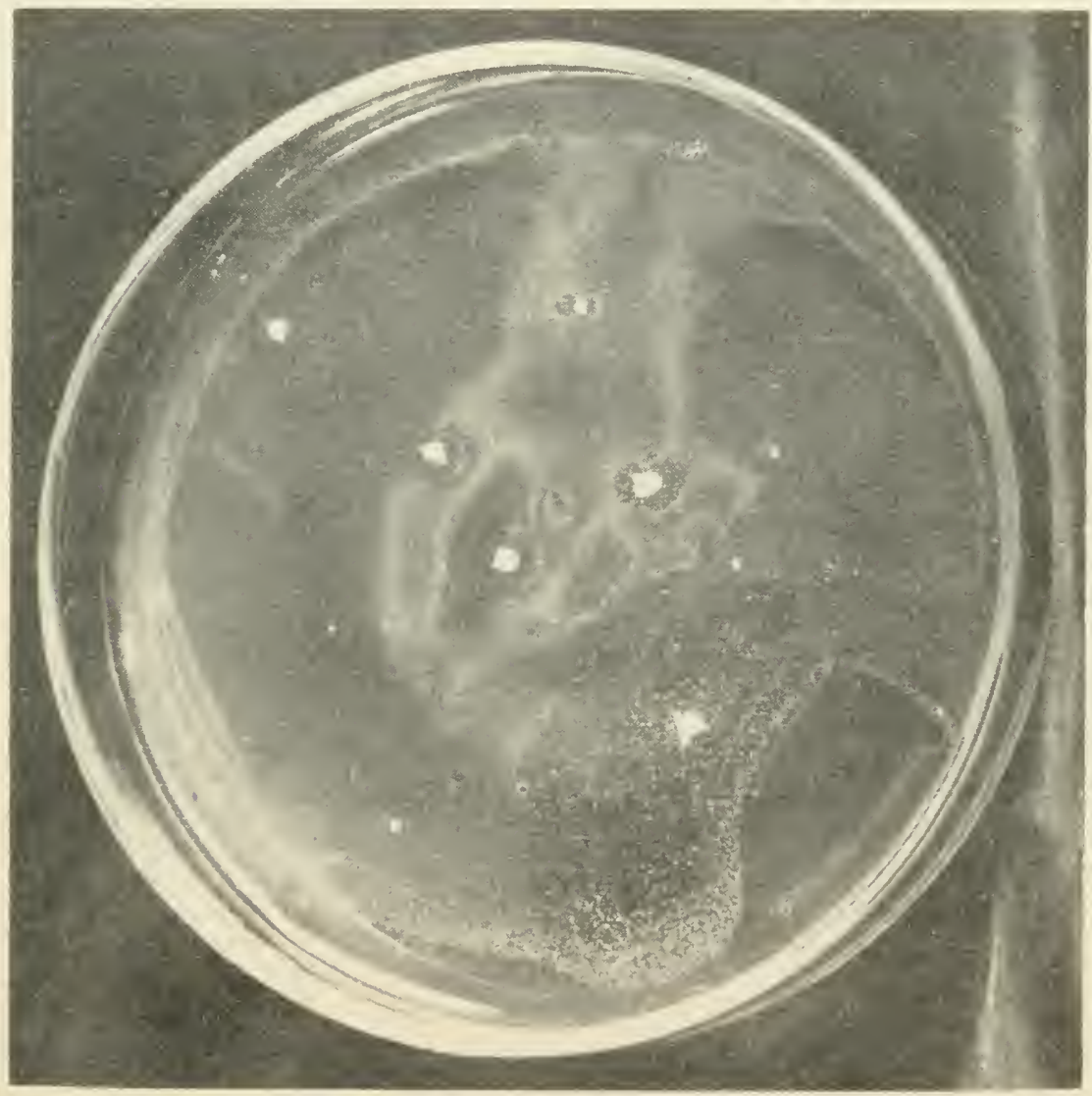

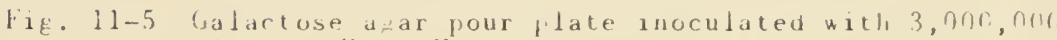
blucose yrown rells ot a "slow" termenter of falactose. Tixteen large colonies visiule.

with gas in about 24 hours obviously belonging to what we had called type 1 , or rapid fermenters. This is interpreted to mean that the "slow" fermentation in the Durham tube containing nutrient medium 
and galactose is "slow" not because each cell slowly achieves fermentative ability in the presence of galactose but that mutation (at the rate of about one per million cells) produces a small population which is selected and finally becomes large enough to produce gas in the tube. We may assume that the population from which these mutants are produced is not incapable of fermentation, but is not capable of producing sufficient gas to register a positive test in the Durham tube (fig. 11-2). We can say this because a large

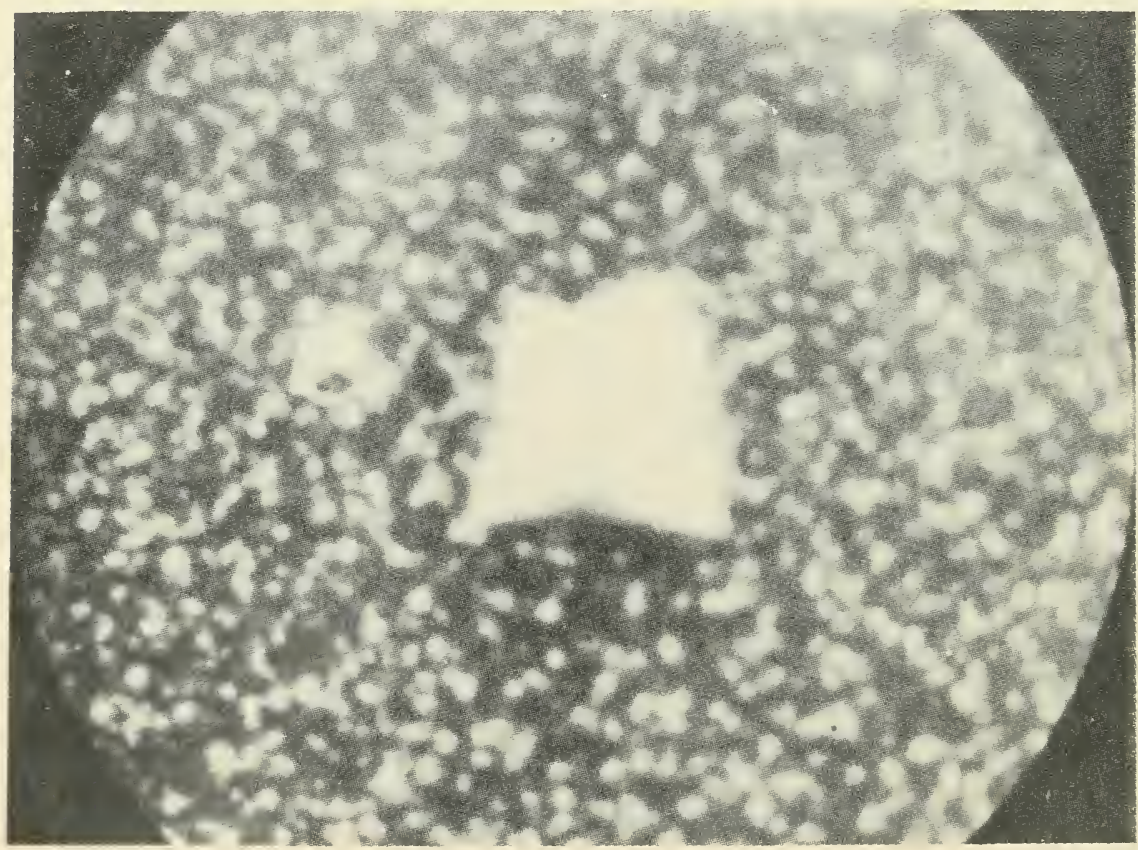

Fig. 11-6 Magnified area about one of the large colonies shown in Fig. 11-5 revealing the bachground of tiny colonies surrounding the large colony.

number of cells is capable of producing gas from galactose when allowed to stand in a Smith tube above mercury (fig. 11-4). in the absence of growth. This statement does not imply that a small amount of enzyme is always present for on this point we do not have conclusive evidence. The ambiguity here lies in the fact that when the so-called "nonfermenter" cells lie in galactose and buffer solution above the mercury in a Smith tube, they produce gas slowly with a long lag, i.e. they adapt to the presence of the sugars. Whether this means that they slowly produce the enzyme or it takes some time for a small amount of enzyme to take action, we do not know. This is a very critical question on which we do not as yet have a definite answer. 
This introduction will serve to polnt out the many complications which have arisen in the genetical analysis of fermentative ability of yeasts. Our analyses have concentrated on the discovery of single gene differences and we have been successful in developing stocks differentiated by single gene differences with regard to galactose, melibiose, maltose, alpha methyl glucoside and sucrose. These stocks are referred to as "fermenters" and "nonfermenters" with the reservations noted above.

\section{GALACTOSE - MELIBIOSE FERMENTATION}

Over 1,000 asci have been analyzed in which these two alleles segregate independently of each other; the gene controlling ability to ferment galactose does not interaçt with the gene controlling the ability to hydrolyze melibiose. The existence of a yeast capable of fermenting melibiose, but incapable of fermenting galactose raised an interesting point since melibiose is a disaccharide containing dextrose and galactose. Fermentation tubes were inoculated with the four different genotypes and after ten days, each tube was tested for the presence of residual galactose by the mucic acid test. The following table shows the results:

\section{TESTS FOR THE PRESENCE OF GALACTOSE IN FERMENTATION TUBES AFTER TEN DAYS}

$\begin{array}{lcc}\text { Genotype } & \text { Galactose } & \text { Melibiose } \\ \text { G ME } & - & - \\ \text { G me } & - & + \\ \text { g ME } & + & + \\ \text { g me } & + & +\end{array}$

Residual melibiose is hydrolyzed by the nitric acid and also produces mucic acid. These results suggest that the ME gene controls the production of a hydrolytic enzyme which breaks melibiose down to dextrose and galactose. In the absence of the gene, the dextrose is fermented and the galactose is left behind.

\section{RAFFINOSE}

Raffinose is a trisaccharide in which fructose, dextrose, and galactose are linked together, writh dextrose as the central molecule. The fructose and dextrose molecules in raffinose are united by a sucrose linkage and the ability to ferment one-third raffinose depends on the presence of the sucrose gene, which controls the production of a hydrolytic enzyme that hydrolyzes raffinose into fructose and melibiose. The fructose molecule is fermented and the residual melibiose is left behind. 
We have extensive yeast pedigrees in which the SU/su genes segregate regularly, producing offspring half of which are fermenters of sucrose and raffinose and half of which are nonfermenters. We also have stocks distinguished by their ability and inability to ferment melibiose; ME su yeasts are incapable of fermenting raffinose.

\section{PRODUCTION OF $\mathrm{CO}_{2}$ WHEN DIFFERENT SUGARS ARE USED IN THE FERMENTATION TUBES}

$\begin{array}{cccc}\text { Genotypes } & \text { Melibiose } & \text { Sucrose } & \text { Raffinose } \\ \text { ME SU } & + & + & + \\ \text { ME su } & + & - & - \\ \text { me SU } & - & + & + \\ \text { me su } & - & - & -\end{array}$

Unless some evidence is forthcoming on differential permeabilities, these data indicate that (1) the hydrolysis of raffinose depends on the presence of sucrase, which is capable of hydrolyzing fructose from the raffinose molecule, leaving residual melibiose (2) melibiase is not capabie of hydrolyzing melibiose in the raffinose molecule, (3) the complete hydrolysis of raffinose depends on the presence of both melibiase and sucrase, the latter acting first.

\section{ALPHA GLUCOSIDES}

In methyl alpha glucoside, and maltose, glucose is linked to the molecule by an alpha glucoside bond. Methyl alpha glucoside is used as an indicator of maltase because of the widely accepted view that they are both hydrolyzed by the same enzyme. We have dis covered, however, that two different genes control the ability to hydrolyze aipha methyl glucoside, and maltose. The gene controlling the hydrolysis of methyl alpha glucoside is segregated independently of that controlling the hydrolysis of maltose. From heterozygous hybrids, we obtain four kinds of progeny with regard to the fermentation of methyl alpha glucoside, and maltose: MG MA, MG ma, mg MA, and mg ma. The MG ma and mg MA genotypes are unexpected since methyl alpha glucosidase is supposedly capable of hydrolyzing both maltose and methyl alpha glucoside. It has frequently been shown that enzyme extracts from yeast are capable of hydrolyzing both substances in vitro. The existence of this variety of genotypes suggests either (1) that different or specifically modified enzymes control the hydrolysis of each of these alpha glucosides or (2) that the genes involved control specifically different permeabilities for the different alpha glucosides. It has also been suggested that some disaccharides may be fermented directly without hydrolysis. 
Dr. Caroline Raut (unpublished) has found a difference in the rate of hydrolysis of maltose and of alpha methyl glucoside by enzyme preparations from yeasts of the four different genotypes, with high activity always associated with the dominant gene. Therefore, it appears that there are two enzymes, one specific for maltose and the other for alpha methyl glucoside.

\section{VITAMIN, AMINO ACID, AND NUCLEIC ACID COMPONENT DEFICIENCIES}

Yeasts differ in their ability to grow in synthetic medium in the absence of different $\mathrm{B}$ vitamins, amino acids, and nucleic acid components. We have found that in most instances prolonged incubation of an apparently deficient culture has finally resulted in full growth. This indicated that relative differences in capacity for synthesis are encountered more frequently than absolute differences. However, we have found single gene differences which distinquish those which grow quickly and those which grow after delay in deficient medium. The method for classifying yeasts is to grow them in $18 \times 100 \mathrm{~mm}$. Kimble tubes which have been acid cleaned and sterilized and filled with $15 \mathrm{cc}$. of Burkholder's vitamin-free medium to which the various essential nutrilites have been added (chapter 21). Deficient medium is prepared by eliminating one of these substances. The amount of growth is determined by inspecting the cultures in a Klett photoelectric colorimeter using a green filter. The Klett instrument was rebuilt with a hole to accomodate the large type test tube. By this method, it is possible to keep tubes under observation for more than a month. Inoculation is made by preparing a dilute suspension of cells in distilled water and making inoculation with a loop containing a uniform number of cells (300 to 1500) as indicated by control plates and by direct counts.

The deficiencies are usually relative for the control test (without the nutrilite) eventually achieves full growth. Therefore, it is necessary to make the photometric reading at an optimal time. The time varies for different deficiencies. Cultures respectively dependent and independent of paraminobenzoic acid show a maximum difference on the second or third day after inoculation, but the dependent cultures begin to grow rather well on the fourth day so readings must be made before that time. Methionine and adenine deficiencies can be diagnosed on the second or third day but not on the fifth. Pyridoxine dependent and independent cultures can be distinguished on the third or fourth day and the differences persist for about two weeks. Pantothenate dependent cultures are distinguishable from independent cultures on about the fifth or sixth day and the dependent cultures do not begin to grow until the third week. 


\section{MATING TYPE SPECIFICITY}

Mating type specificity is determined by observing the appearance of copulations in $0.5 \mathrm{cc}$. of standard broth in a $6 \times 3 / 4$-inch test tube. Cells from each of the four spores from a single ascus are transferred into four separate tubes each containing $0.5 \mathrm{cc}$. of broth, by inoculating heavily with a needle from an agar slant of the haplophase culture. Each of the four tubes receives a similar amount of the cells of our standard mating-type tester. The mixed cultures are placed in an incubator at $16^{\circ} \mathrm{C}$. overnight and are observed in the morning. When a critical test is required eight tubes are used, one set of four is mated to a standard a type culture, and the other set to an a type culture. We routinely use only an a type culture. In some asci all four spores fail to produce copulations when mated to the standard a strain. This is probably due to infertility. There are always a few copulations even in the haplophase cultures obtained from a single ascospore and a clone mated to a standard a strain is only classified as a if from 25 to 50 per cent of the cells have copulated in the test tube. This character has proved extraordinarily stable in inbred pedigrees. Exceptions, although encountered, are not frequent enough to be troublesome; if fact, they sometimes prove very useful as in the analysis of ascus $167,168,169$, and 170 ; in which 169 , originally classified as a, was subsequently capable of mating with another a type culture from the same ascus; and in which culture 170 , originally classified as $\alpha$, finally produced some illegitimate progeny.

\section{SUPPRESSORS}

This designation was given by Bridges (1932) to recessive genes which transform a recessive character at another locus into its normal wild-type form. For example, the wild-type color of Drosophila eyes is red; a recessive gene in homozygous form is capable of transforming this red color to purple, which may be looked upon as a reduced capacity for color production. The suppressor of purple (when homozygous) changes the purple (deficient) color to red (normal) color. The purple gene interferes with the complete normal color production. When a purple fly is made homozygous for the suppressor of purple, the wild-type color appears; the fly achieves complete ability to produce the normal wild-type pigment in spite of the presence of the purple genes which normally reduce its ability. The suppressor gene makes a relatively weak organism capable of producing the full quantity of pigmentation. Suppressors do not actually suppress a character, but enable a recessive gene incapable of completing a synthesis to bring the synthesis to completion, or to bring into function an alternative route of synthesis. 


\section{PLUS AND MINUS MODIFIERS}

These genes (especially minus modifiers) are closely related to suppressors and are so named because they increase or diminish the phenotypic expression of a given gene without themselves having any effect on the wild-type character. Their presence is only detected when the gene which they modify is present in homozygous condition. They are abundant in Neurospora as modifiers of fluffy and other nonconidial mutants. When a cross heterozygous for fluffy is analyzed, the four fluffy cultures from one ascus nearly always fall into two categories, revealing the presence of genes which affect fluffy without affecting normal. If we look at the recessive gene as usually involving the loss of some synthetic ability, then plus modifiers decrease the synthetic ability still farther. A suppressor transforms the recessive into normal and is therefore a minus modifier which diminishes the character to zero expression by increasing the synthetic capacity of the organism to normal. Minus modifiers result in different degrees of diminution of the character without producing a complete transformation to normal. In this respect they resemble the Anaphragmic mutation of Lwoff (1946).

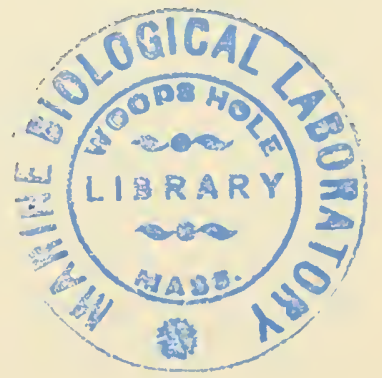




\section{Chapter 12}

\section{A COMPARISON OF SEXUAL REPRODUCTION IN YEASTS AND OTHER MICROORGANISMS}

The object of this chapter is to consider the respective advantages of different microorganisms for genetical research. Although a thorough understanding of the life-cycle of any organism always yields unexpectedly valuable and interesting facts, each investigator chooses the object of his research with a view to its peculiar advantages. Whatever he may find out about the biology of a plant or animal which produces no viable sexual offspring and can only be propagated asexually, he will be unable to make chromosome maps or to exploit tetrad analysis. He may succeed in accomplishing a very valuable research in vegetative life-cycles or physiology or biochemistry, but it cannot demonstrate the relation between a chromosomal gene and an hereditary characteristic except by employing analogy - a device which is universally frowned upon in scientific circles. An organism with a number of other peculiar advantages may be handicapped by an extremely large number of chromosomes so that the amount of labor involved in making chromosome maps may be prohibitive. It was a fortunate accident that Drosophila unexpectedly had a simple life-cycle and essentially only 3 chromosomes: Sciara which might equally well have been chosen has proved quite difficult to exploit successfully. Many workers are limited in the choice of their material by other considerations. One important factor which cannot be ignored is the amount of capital invested in a given project although we are never permitted to forget the old adage that it does not pay to throw good money after bad. Corn and Drosophila are peculiarly valuable because such a great variety of stocks is available and the techniques are so simple and so widely known that a person who had spent half his working life time on one or the other cannot afford to change.

It has been argued that one reason for studying the genetics of maize is the great importance of this type of work from an economic point of view. However, I do not believe it is possible to prove that theoretical genetics has contributed in a large way to the hybrid corn program. This program has fallen to a large extent within the peculiar province of the plant breeder between whom and the theoretical geneticist there has always been a major schism. Hybrid vigor is a problem for which classical genetics has pro- 
posed no acceptable solution. Both maize and Drosophila suffer from the handicap that tetrad analysis cannot be made and this fact has left undiscovered a fundamental genetical mechanism which has been brought to light by our work on yeast.

Tetrad analysis is so great an advantage in genetics that $I$ believe we must accept it as essential to research into the fundamental mechanism of the hereditary apparatus. The reasons for this statement will be apparent when we consider the arguments in Chapters 23 to 26.

\section{MUCORS}

Genetics of the fungi takes its origin from the discovery by Blakeslee that copulation between two thalli of opposite mating types precedes the formation of zygospores in Rhizopus. Burgeff collected over 20 variants of Phycomyces Blakesleeanus and analyzed the results of hybridizing these mutants. The Phycomycetes are called the siphonaceous fungi because of the absence of crosswalls in the mycelium. The gametangia are multinucleate and when the zygospore is cut off, large numbers of nuclei are enclosed in the young zygospore. Copulations are multiple, and many pairs of nuclei fuse in the zygospore, while many haploid nuclei are left over; the latter apparently disintegrate.

Tetrad analysis is not feasible because several diploid nuclei are produced within a single zygospore, and no cells comparable to ascospores, which isolate individual haploid nuclei, are found in Phycomycetes. After hybrid zygospores were formed Burgeff allowed them to germinate, and the individual haploid spores which were produced in the aerial sporangium were collected and diagnosed. Burgeff made and analyzed numerous crosses. From a doubly heterozygous hybrid one expects either two or four types of haploids from each zygote. However, many zygospores produced only one, two, or three of the expected types. He showed that segregation in mucors occurred in both the first and the second reduction division, but he was unable to detect or calculate linkage. Burgeff also discovered the interesting fact that zygospores can be put away in absolute alcohol and preserved in a viable state for a considerable length of time. His 1927 paper is the most ambitious attempt to analyze the genetics of the Phycomycetes, and it revealed that tetrad analysis, which is the principal advantage of fungal genetics, was not feasible in these organisms.

\section{SCHIZOMYCETES}

Lederberg, working with Tatum, discovered that it is possible to mate genetically different mutants of the colon bacillus, Escherichia coli, by mixing the cultures together in nutrient medium and 
plating out on specially designed agars. He discovered 15 genes in one linkage group, apparently in a linear order, suggesting that all the loci lie on one chromosome. This discovery gives genetical analysis in the bacteria almost the same validity that it has in higher organisms. Prior to this, most of the genetical work in the bacteria was by plating and selecting variants which resemble mutants known to occur in other organisms. However, there is no way of distinguishing most bacterial variants from the depletion mutations, but Lederberg's work suggests that regular segregations of some bacterial mutations can occur.

The enormous importance of bacteria as pathogenic agents makes any new technique for understanding their life cycles especially valuable. However, the approach to basic theoretical genetical problems is technically simpler using yeasts, and with yeasts one has the advantage of unambiguous tetrad analyses.

\section{HYMENOMYCETES}

The Hymenomycetes include the mushrooms and toadstools. They grow from an underground mycelium to produce a mushroom fruiting body on whose gills (hymenium) the basidia are borne. Generally, up to the time of production of the basidium, the cells are dicaryons with two separate complementary haploid nuclei in each cell. In the basidium, the nuclei fuse, undergo reduction, and produce four basidiospores on each basidium. The four spores from a single basidium can be collected by making a "spore print" and tetrad analysis of the fungi had its origin in the development of this technique by Kniep. Kniep discovered that many toadstools have tetrapolar "sex" and showed that the mating type alleles are Mendelian characters.

In the classical case, the zygote is heterozygous for two pairs of genes and has the constitution $\mathrm{Aa} \mathrm{Bb}$. There are three types of basidia: (1) $\mathrm{AB}, \mathrm{ab}, \mathrm{Ab}, \mathrm{aB}$; or (2) $\mathrm{AB}, \mathrm{AB}, \mathrm{ab}, \mathrm{ab}$; or (3) $\mathrm{Ab}, \mathrm{Ab}$, $a B, a B$. Each basidiospore produces a mycelium which can be mated with a complementary mycelium to produce a dicaryotic (often incorrectly called a diploid) mycelium according to the scheme below.

\begin{tabular}{c|c|c|c|c}
\hline & $\mathrm{AB}$ & $\mathrm{ab}$ & $\mathrm{Ab}$ & $\mathrm{aB}$ \\
\hline $\mathrm{AB}$ & & Clamps & & Barrage \\
\hline $\mathrm{ab}$ & Clamps & & Barrage & \\
\hline $\mathrm{Ab}$ & & Barrage & & Clamps \\
\hline $\mathrm{aB}$ & Barrage & & Clamps & \\
\hline
\end{tabular}



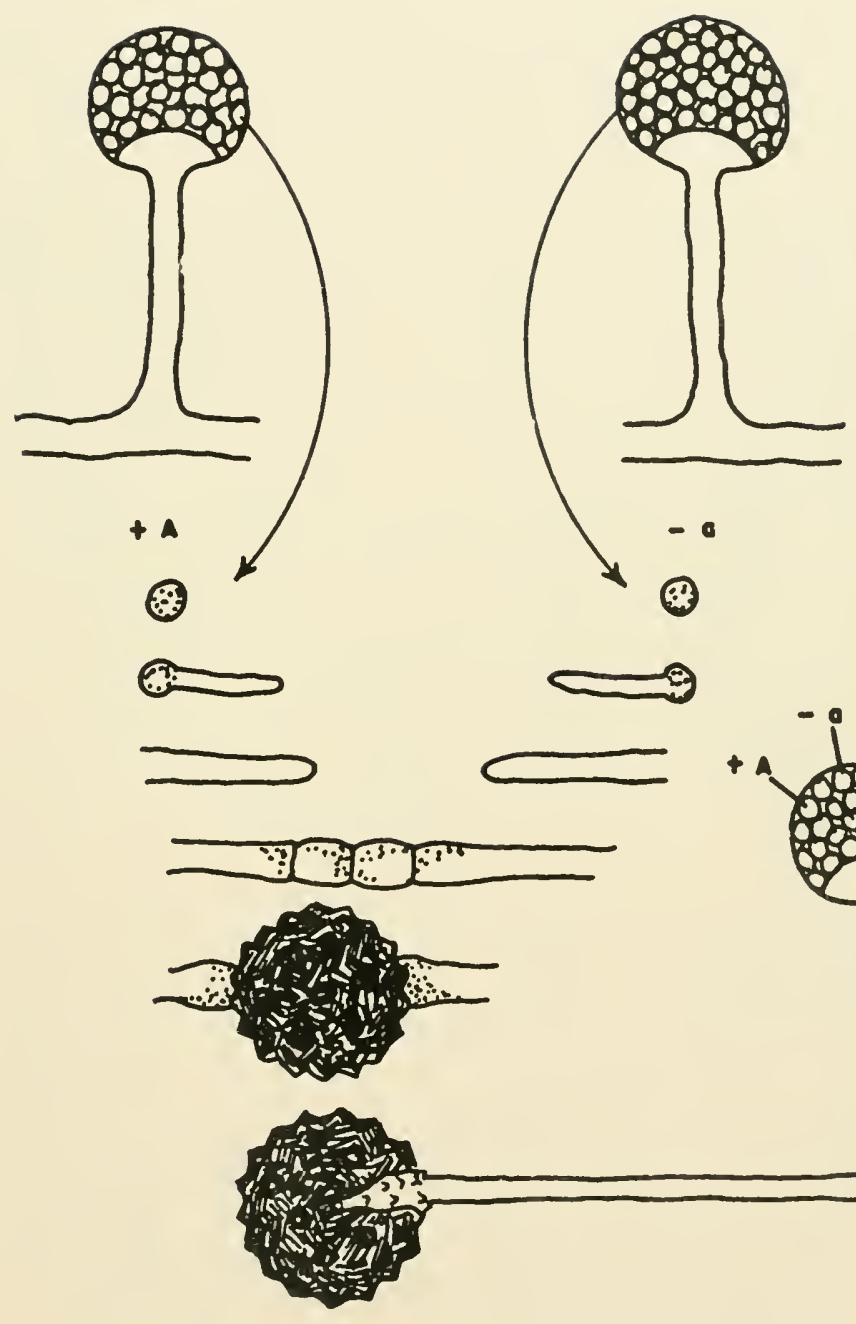

Fig. 12-1 Copulation and Segregation in a Mucor 
Bensuade discovered that the clamps (which indicate a dicaryotic mycelium) insure the production of cells, each of which contains two complementary nuclei.

Vandendries discovered that when two mycelia with common B factors are in opposition, they grow to within a few millimeters of each other and then stop without mingling due to some inhibiting substance. He named this phenomenon "barrage."

Brunswik made an elaborate study of mating type specificity in about 20 species of Coprinus. He found great differences from the classical type of clamp connections, some species being without clamps until the formation of the basidium, while others have precise and regular clamp connections throughout the life cycle. $\mathrm{He}$ discovered many irregularities in the behavior of mating types, and this work was much extended later by Vandendries and Hanna. Two mycelia of the same mating type often copulate and produce clamps, due to an "illegitimate" mating ("Durchbrechingskopulation"). Cultures grown from spore prints from a single fruiting body may interact with great regularity according to the tetrapolar scheme, but when a culture from a toadstool collected a few yards away (whose spores likewise behave regularly inter se) are tested, the two groups from the neighboring dungheaps are often found to be completely inter-fertile. This has been interpreted as due to multiple allelism of the genes A and B. Collections of mycelia originating from different continents when cross mated often reveal much sterility.

Buller discovered that a fully grown haploid AB mycelium in contact with a haploid ab mycelium is invaded by ab nuclei and becomes a diçaryotic mycelium. Migration of ab nuclei through the $A B$ mycelium apparently accounts for this effect. The AB mycelium can also be dicaryotized by an $A b / a B$ dicaryon, although neither an $A b$ nor an aB mycelium can achieve this effect. Since only an ab haplophase can dicaryotize the $A B$ mycelium, this phenomenon is puzzling. Many proposals have been made concerning the mechanism by which the reaction occurs, but none is completely satisfactory. My own preference is for an explanation which I have not seen. published, but which Quintanhila proposed in the course of a conversation: either the $\mathrm{aB}$ or the $\mathrm{Ab}$ nucleus in the dicaryon is converted into an ab nucleus by a directed mutation similar to that which occurs in the transformation of pneumococcal types.

Harder produced a dicaryotic mycelium by mating two variants of Schizophyllum commune, and operated on the dicaryon to produce a haploid mycelium which contained the nucleus of one variant in the mixed cytoplasm of the two variants. This is easily achieved by cutting off the clamps of the penultimate cell before it fuses with the ultimate cell and isolating the mononucleate ultimate cell by cutting through the penultimate cell. He discovered 


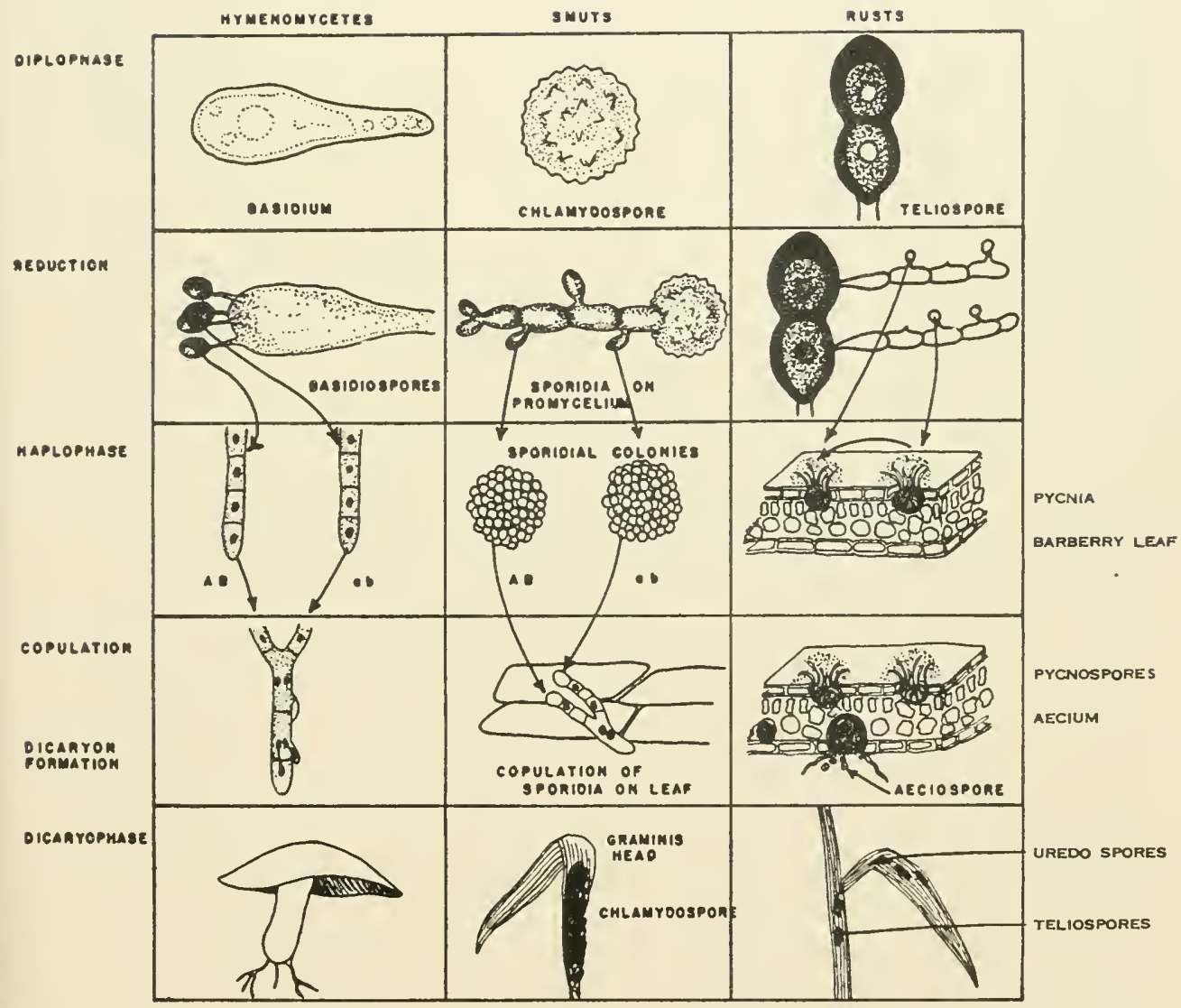

Fig. 12-2 Life Cycles in The Basidiomycetes 
that the addition of cytoplasm produced a stable change in the culture. The operation produces an $X$ nucleus in $X+Y$ cytoplasm, or a $\mathrm{Y}$ nucleus in $\mathrm{X}+\mathrm{Y}$ cytoplasm. These cultures can be compared with the original haploids: $X$ nuclei in $X$ cytoplasm and $Y$ nuclei in $Y$ cytoplasm. The additional cytoplasm produced stable mutants. Harder interpreted this as a cytoplasmic effect. In view of the fact that McCarty, Taylor, and Avery; and Boivin, Delaunay, Vendrely, and Lehoult have shown that simple chemical substances can produce stable transformations, Harder's conclusions may require qualification. The experimental procedure which Harder used to produce his "operated" variants is quite simple, and it would seem that this is one of the important discoveries in the Hymenomycetes that could be profitably repeated.

In spite of the abundance of genetical work done on the hymenomycetes, no chromosome maps are available; this is a strong indication that many technical difficulties must be overcome before they can compete with yeasts as objects of choice for genetical analysis.

\section{PARAMECIUM}

Paramecium has the peculiar advantage that cytoplasmic exchange occurs to a variable and roughly controllable amount during each copulation. Tetrad analysis in Paramecium is impossible for immediately after meiosis three of the haploid nuclei disintegrate. At this time two paramecia are copulated and the remaining haploid nucleus in each undergoes a mitotic division. Then an exchange of nuclei occurs between each conjugant, simultaneously with a cytoplasmic exchange, and in the two conjugants the nuclei fuse producing two diploid paramecia with presumably the identical diploid nuclei. Chen has shown, in a remarkably competent cytological investigation, that Paramecium has about 80 chromosomes and, therefore, no possibility exists of unravelling chromosomal aberration if such may occur. In nearly 20 years of study Sonneborn and his numerous coworkers have demonstrated apparent Mendelian inheritance for only one pair of alleles so the prospect of chromosome maps seems out of the question. The exchange of cytoplasm between the conjugants has led to a number of very interesting discoveries and a series of different explanations have been proposed involving relations between the gene and its components or supposedly autonomous cytoplasmic entities. However, since some workers believe that all cytoplasmic activity, except that of parasites and what one might classify as symbiotic plastids, like the chloroplasts, is directly under genic control the possibility of demonstrating the independent inheritance of the cytoplasmic entities and the chromosomal genes is remote if not impossible 
in an organism in which linkage is unknown and neither chromosome maps nor tetrad analysis is feasible.

However. cytoplasmic exchange can be readily achieved in the Hymenocycetes as Harder has so brilliantly demonstrated, and if adequate chromosome maps were available in them, many of the major questions which have been posed by Sonneborn's work might find a less equivocal answer.

\section{SMUTS}

The smuts are parasites on grassy plants and are economically important because they attack corn and small grains. The seeds of an infected plant are replaced by a sooty dust called smut; the soot is composed of chlamydospores, each of which contains a diploid fusion nucleus. In the spring a reduction occurs and the chlamydospore germinates to produce a promycelium containing four nuclei. The promycelium is divided into four cells and sporidia bud from each of the cells. Sporidial cultures resemble yeasts and can be grown in artificial media. There are two mating types in most smuts and sporidial cultures copulate to produce the dicaryons which infect the growing plant, which is almost always parasitized by a dicaryon. Ustilago levis attacks only the oat plant while U. hordei attacks only barley. The dicaryon produced by the copulation of $U$. levis $x \mathrm{U}$. hordei attacks both. Among the tetrapolar smuts, Bauch discovered that an $\mathrm{AB} / \mathrm{ab}$ dicaryon on an agar plate produces Suchfaden which are recognized by their rapid filamentous extension across the plate; they correspond to the dicaryotic hyphae which infect the host. When two sporidial cultures with common A factors but different B factors are mated, Wirrfaden grow out of the mixed culture on the agar plate. Wirrfaden are twisted copulation tubes resembling weak, distorted Suchfaden. Bauch discovered that both the $\mathrm{A}$ and $\mathrm{B}$ mating type factors exist in a multiple allelic series producing a great variety of pathogens, and that the same alleles were present in cultures isolated from England, Germany, and Siberia.

Bauch found that the chlamydospores from each naturally infected plant were all of one genotype, but that neighboring plants contained genetically different fungi. Christensen made an extended study of the problem of solo-pathogens. In yeast they would be called illegitimate diploids; solo-pathogens are monosporidial cultures which are capable of infecting the plant by producing a diploid or dicaryotic mycelium which grow throughout the plant and finally produces chlamydospores. The chlamydospores do not, however always produce solo-pathogenic sporidia.

Chlamydospores are rather easy to collect and can be germinated on agar. Huttig has classified the types of promycelia produced by the different types of smuts. There are several varia- 
tions from the classical type. In one, the promycelial cells send out germ tubes instead of producing sporidia. These germ tubes copulate with each other and by this means, it is possible to distinguish first and second-division segregation by direct inspection. These varieties have bipolar mating type. If we number the cells of the promycelium $1,2,3$, and 4 from the tip downward, there are two types of copulations: (1) the germ tube originating from 1 may copulate with 2 , and the one originating from 3 may copulate with 4 , or (2) the germ tube originating from 2 may copulate with 3 and that originating from 1 may copulate with 4 . These are the only types of copulations which are found. On the basis of calculations derived from observations of this phenonenon, Huttig found that the normal ratio of first and second division of segregation was 23:77.

In Neurospora Lindegren showed that the maximum expected frequency of second-division segregation is $67 \%$, and this figure is not exceeded in the analysis of the segregation of a large number of factors. This depends on the assumption that the chromosomes cross over freely along their entire lengths. The high frequency of post reduction in Ustilago found by Huttig is of considererable theoretical interest. His calculations are based on the assumption that copulation is between germ tubes originating from cells of opposite mating type and although confirmation of this as sumption is not presented, it is difficult to criticize the point of view. Huttig plated the chlamydospores on medium containing a large variety of chenicals and found in every case that seconddivision segregation occurred in excess of expectation. A possible explanation of the apparent discrepancy with Neurospora was suggested in a conversation with Dr. McClintock: the chromosomes in Ustilago may form a pair of chiasmata spanning the centromere, with only a single cross over occurring in each arm of each chromosome. This would make possible a 100 per cent post reduction depending upon the position of the locus.

Several attempts have been made to study the segregation of colonial characters such as color and colony shape in the promycelia of smuts. To study the segregation in Ustilago, it is necessary to inoculate the living host with paired cultures and collect the chlamydospores resulting from the infection. None of the workers has attempted to interpret the results in conventional genetical terms, although much of the data is obviously susceptible to this approach. The best data are those of Dickinson (1931), who studied the segregation of five characters. He only analyzed a limited number of promycelia, but my calculations of his data indicate that all five factors are in a single linkage group, although the data are not statistically significant. This suggests that the smuts may be excellent genetical material, in spite of the fact that 
they have not been exploited to this end, presumably because the workers in this field are more directly concerned with problems of economic importance. It may be, however, that the problems could be more quickly solved if a genetical analysis of the organism were available. The photographs presented by Stakman, Kernkamp, King, and Martin show that this analysis would not be difficult.

An idea which has persisted in smut literature is the view that segregation may occur in the third division. This view is based primarily on the extreme variability of many of the sporidial cultures. I believe that if one analyzed the data on the assumption that this variability was due to mutation and that third division reduction does not occur, a linkage map could readily be developed, in spite of the fact that progress to date has been disappointingly slow.

The cytological evidence presented in support of the third division segregation is quite irrelevant. Four chromosomes have been reported on the metaphase plate and an occasional third division has been observed in which two chromosomes were reported going to the poles. Since the haploid number of chromosomes always appears at the metaphase plate of Meioses I and II (due to synapsis), there are at least four chromosomes in the haplophase and the supposed observation of two chromosomes at a later division can only be due to inability to resolve small closely approximated bodies. That this must occur frequently is shown by the failure to find four chromosomes always going to the poles in Meioses I and $I$.

The fact that parasitism on the host only occurs when two sporidia of opposite or complementary mating type copulate and produce a dicaryon suggests that complementary genes linked to the mating type genes are essential for production of infective hyphae. The complementary genes must exchange infrequently, a condition which could be achieved by the presence of an inverted section (in which crossing over was inhibited) adjacent to the mating type alleles. Complementary pathogenicity genes would be expected to accumulate in such an inverted section. The fact that the dicaryon is an obligate parasite and, therefore, at least one growth cycle of a small grain is required to complete the smut life cycle is an obvious and serious disadvantage of these organisms as compared to yeasts for genetical study.

\section{RUSTS}

The rust fungi comprise 3500 known species attacking a great variety of plants. At least 200 distinct strains of wheat rust are now known, and it seems probable that an equal variety may exist for each of the 3500 species. Because of the great economic im- 
portance of wheat rust, there has been abundant support for research on the genetics of rusts. Wheat rust is a characteristic heteroecious rust spending part of its life cycle as a parasite on the barberry and part as a parasite on wheat. The sexual cycle takes place on the barberry while the economically important wheat rust represents the vegetative phase of the hybrids produced on the barberry. The barberry is the reservoir of variability. Control of the disease by the development of resistant wheat varieties has not been successful to date because the new hybrids arising from the barberry have always been effective against any previously resistant wheat. The two principal difficulties in the analysis of heredity in rusts are: (1) these fungi are obligate parasites and have not as yet been grown on artificial media, and (2) the only forms which can be characterized are the dicaryotic hybrids growing on wheat, i.e., haplophase cultures cannot be studied, thus one of the principal advantages of fungal genetics is not available in the rusts.

There were four important steps in the history of rust genetics:

1. DeBary showed that the pustules on barberry and the rust on wheat are both caused by the same organism. He even recognized the true significance of the pycnospores as sperm and although his ideas were disregarded for 50 years, they were finally abundantly confirmed by Craigie. DeBary's genius is one of the highlights of the 19th century.

2. Ericksson discovered that there were different physiological races of the vegetative stage of wheat rust. Stakman and Piemeisel extended this work and developed techniques for characterizing the different strains parasitic on wheat by infecting different varieties of wheat with a given strain of rust and distinguishing the different strains of rust on the basis of their effect on different wheat varieties. A rust strain may be extremely virulent with regard to one wheat variety and relatively ineffective on the second. There are no rusts that are virulent against all strains of wheat, and there are no wheat varieties that are resistant to all types of rusts. Stakman and his co-workers have cataloged numerous biological races by testing different wheat varieties and are principally responsible for the present census of 200 biological races of wheat rust.

3. Craigie discovered that rusts are heterothallic. He found that the small pustules produced on the upper surface of the barberry leaf by the infection of the sporidia actually function as sperm, and that the so-called pycnospores can be used to fertilize strains of opposite mating type. The pycnospores are homologous to the spermatia of Neurospora; each sporidial culture on the barberry is itself self-sterile but can be cross-fertilized by transfer of pycnospores from a pycnium produced by a sporidium 
of complementary mating type. In Nature, cross-fertilization is achieved by insects.

4. Newton and Johnson perfected techniques for the growth of the rust on barberry and artificial hybridization under controlled conditions. They demonstrated that pathogenic characters are segregated in a Mendelian manner, and'Johnson and Newton discovered evidence of maternal inheritance of some characters. Occasional reciprocal crosses produced different kinds of hybrids, depending upon which parent contributed the sperm, indicating that some characteristics cannot be transmitted through sperm.

At present, the rusts are not promising material for the solution of fundamental problems in genetics, because the only characters available are the teliospore color and pathogenicity of the dicaryotic hybrids and tetrad analyses cannot be made. Therefore, they are inferior to smuts which produce similar promycelia, but whose sporidial cultures can be grown on artificial medium. The most important problem in rust genetics is obviously the development of an artificial medium for the growth of sporidial cultures, and considering the present state of our knowledge of culture media, this should not be a difficult task. Its solution would immediately open up the field for tetrad analysis.

\section{NEUROSPORA}

Shear and Dodge named the genus, Neurospora, which contains both 4-spored and 8-spored species. Dodge discovered that the 8spored species were heterothallic, while the 4-spored species produced homothallic spores, due to the inclusion of one nucleus of each mating type in the spore.

The perithecium of Neurospora crassa produces a large number of asci, each containing 8 spores. The spores fall into two categories with regard to mating type. Each spore gives rise to a thallus which produces a mycelium, on which male and female sex organs, as well as asexual conidia, are developed. The female sex organ is called a bulbil and contains an oogonium and trichogyne. The male sex organ produces sperm called spermatia. The spermatia from an A type thallus are incapable of fertilizing the oogonium developed on the thallus of the same type, but will cross-fertilize an oogonium of a complementary mating type. The superimposition of sex organs and mating type shows that mating type genes are self-sterility alleles and bear no relation to either sex organs or the evolution of sex.

Single ascospore cultures are characterized either on morphological or biochemical characters and hybrids are made either by transferring spermatia from a tube of one mating type to a tube of the complementary mating type, or by inoculating a single tube with conidia from each culture and allowing them to grow together. Lin- 
degren and Lindegren analyzed crossing-over in 4 regions marked by 5 loci bridging the centrosome of N. crassa. They concluded that crossing-over was a non-random process; that local regions of the chromosome had specific patterns controlling the types of exchanges which occurred. This was not in agreement with the findings of Beadle and Emerson in Drosophila. It was suggested that they may have studied regions too long to permit the detection of all possible exchanges. The stocks of Neurospora now available should make a reinvestigation of this problem a simple matter.

The treatment of Neurospora with ultra violet and $\mathrm{X}$-rays has been used for the production of mutations. Lindegren and Lindegren treated individual spermatia and found that ultra violet produced very few chromosomal abberations, while X-rays produced a large proportion. Many of the variants reverted on subculture, but nearly one third of the ultra violet variants were stable on subculture, but produced only normal progeny when outcrossed. Therefore, the function which had been destroyed by treatment was restored in the heterozygous condition. At the time this was interpreted as a manifestation of cytoplasmic inheritance, but now the simpler concept of depletion mutation seems preferable. An extensive study of the effects of monochromatic radiation of Neurospora spermatia by Sansome, Hollaender, and Demerec has confirmed these results.

Beadle and Tatum irradiated Neurospora conidia and fertilized complementary thalli with irradiated conidia and isolated the asci subsequently developed. When an individual ascus produced $4 \mathrm{mu}-$ tant and 4 normal cultures, the mutant cultures were further analyzed for their ability to synthesize vitamins. This work in biochemical genetics has been summarized by Beadle and has led to a great interest in the use of Neurospora for the analysis of biochemical mechanisms. It does not involve any discrepancy with Lindegren and Lindegren's finding of depletion mutations following radiation, since Beadle's mutants were only isolated after hybridization, at which time all depletions would have been restored.

Beadle and his co-workers have used Neurospora for the study of biochemical syntheses by selecting stocks differing in ability to produce enzymes which control steps in biochemical syntheses. Beadle and his group have proposed the one-gene-one-enzyme hypothesis which states that each gene synthesizes a specific and different enzyme. Delbruck has pointed out that since no test has been devised by which this hypothesis would be disproved, additional evidence supporting it is not so much to be desired as the development of a critical test of its validity.

Lindegren showed that one of the natural devices for preserving variation in Neurospora and presumably in other fungi is the formation of heterocaryons. Most of the fungi are multinucleate coeno- 


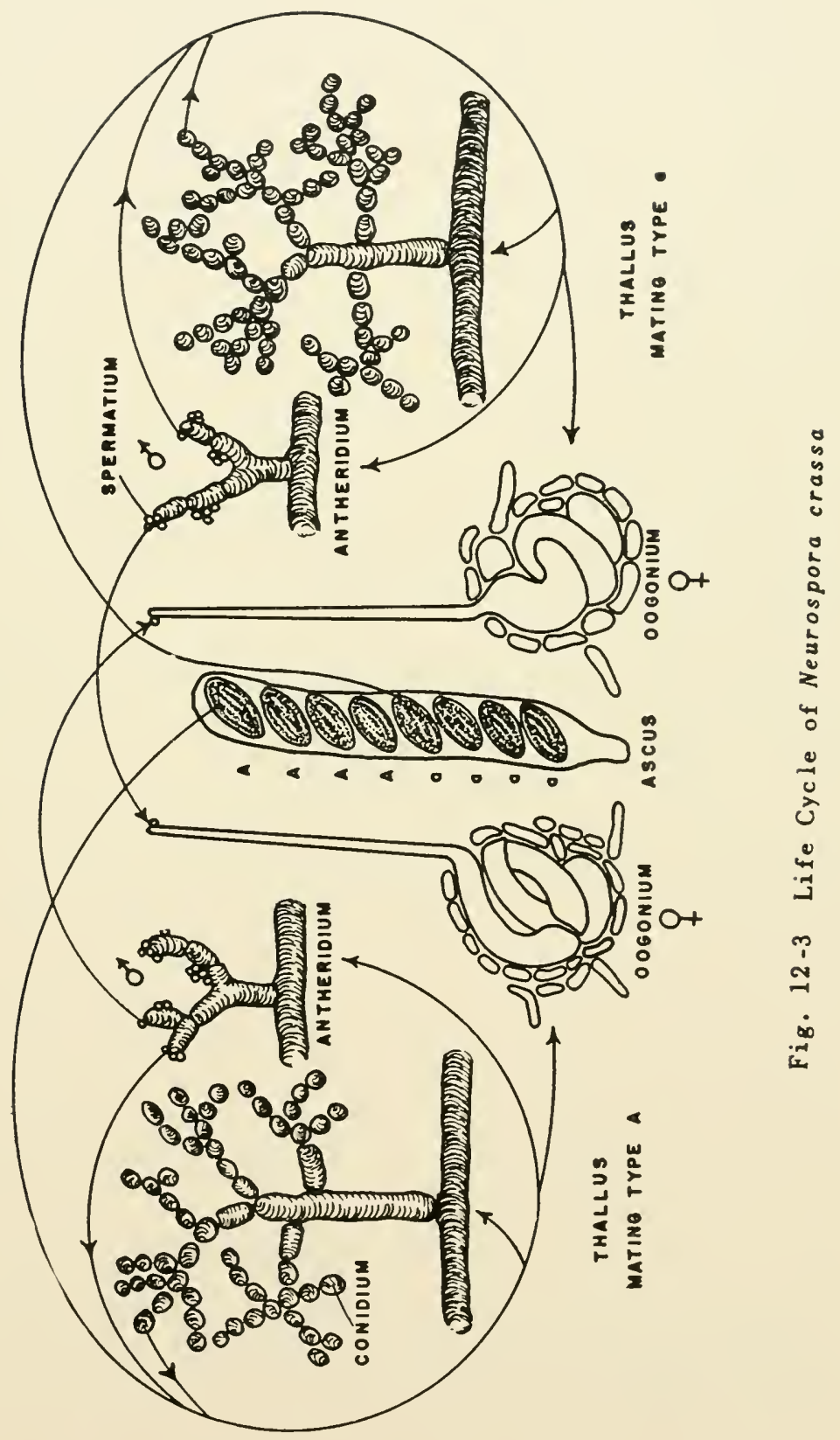


cytes without cross walls in the hyphae, so that all the nuclei are commingled in a common cytoplasm, enabling the fungus to maintain great genetic variability. In nature, the heterocaryons are usually composed of nuclei of complementary mating type, with superimposed sterility genes which minimize or prevent the formation of perithecia until fertilization by another stock occurs. Beadle and Coonradt pointed out the advantage of testing different mutants for allelism by making heterocaryons and observing whether or not the wild type were reconstituted. Ryan exploited the differences in rate of growth between mutants and heterocaryons as a means of testing for allelism. It should be pointed out, however, that neither the heterocaryon test for allelism nor the standard genetical test constitute proof of allelism. This problem is a fundamental problem of genetics. The same objections which Delbruck makes to the one-gene-one-enzyme hypothesis apply to assuming the existence of multiple allelic series. Thus far, no test has been devised which has established with certainty whether two mutants can be produced by changes at a single locus, and there are reasons for suspecting that multiple allelism does not exist. The principal advantages of yeasts over Neurospora are:

1. Yeasts are easily adaptable to respirometer analyses with the Warburg apparatus, while the filamentous Neurospora thallus is disintegrated by the shaking. This is one advantage which all freeliving cells have over the filamentous fungi.

2 . Yeasts are fermenters of carbohydrates and much modern biochemistry is based on the analysis of fermentation, and this knowledge may be applied to genetically different yeast strains. Neurospora is an oxidizer of carbohydrates and does not distinguish between different carbon sources such as sucrose, dextrose, galactose, lactose, etc.

Beadle's and Tatum's brilliant research on Neurospora have done much to enlarge our understanding of biology and have added considerably to our knowledge of biochemistry, but they have been pursued on the basic assumption that the classical concept of the gene is fundamentally correct. There is much evidence, however, that these classical views are not adequate. The facts on which the much debated one-gene-one-enzyme hypothesis are based were clearly established by Scott-Moncrieff and Haldane in their classical work on the relation between flowers and genes and plant pigments. They showed that a single gene may differentiate between a plant containing a pigment in which an $\mathrm{OH}$ group is replaced by an $\mathrm{OCH}_{3}$ group, and that numerous other equally specific chemical syntheses were under direct control of the gene.

\section{VENTURIA}

The brightest spot in the attempts to exploit the genetics of the fungi in solving the problem of pathogenesis is Venturia. The dif- 
ficulties in solving problems of this type with the rusts have been pointed out. The smuts are better material than the rusts, but the fact that the parasite is a dicaryon and an obligate parasite is a limiting factor. Venturia is the most favorable organism in which the relation of a parasite to its host can be studied. It is an 8spored ascomycete which produces haploid spores, the haplophase mycelium constituting the parasitic phase on the apple. The haplophase grows well on ordinary media and there is no complicating dicaryophase in the life cycle. The ability to act as a parasite must depend on some nutrient which the host produces that is essential to the parasite, or some relation between metabolic rates of host and parasite, and the extensive work on the nutrition of the ascomycetes suggest that relations of this type may be rather easily worked out. Venturia genetics has been attacked by Keitt and his co-workers. There are many varieties of apples on which this parasite is pathogenic, and the pathogenicity of the different genotypes of Venturia is highly specialized and clearly under genetical control. It is the ideal situation for an analysis of the interplay of host and parasite and the genetical and nutritional interaction of two organisms bound together by this relationship. Venturia is a typical ascomycete, whose life cycle is practically identical with that of Neurospora, except that the haplophase is pathogenic to various apples. However, the genes controlling pathogenicity segregate regularly in the ascus and precise ratios are obtained.

In the analysis of a selected stock Keitt has shown that one gene controls parasitism on Haralson and Wealthy apples, while cultures carrying its allele are not pathogenic against these varieties, but are pathogenic for McIntosh and Yellow Transparent, suggesting that gene $\mathrm{A}$ controls parasitism on the first two varieties, and its allele, gene a, controls parasitism on the other two. This situation only applies to the specific inbred stock of Venturia reported on, for earlier papers showed that the same gene did not control parasitism against these two varieties in all Venturia cultures. These indications suggest that study of the genetics of this fungus, especially the nutritional and vitamin deficiencies involved in the pathogenic stocks, give great promise of solving many problems involved in the host-parasite relationship.

\section{GLOMERELLA}

Glomerella is an ascomycete similar to Neurospora, except that it produces homothallic haploid spores. The fungus has been studied quite thoroughly by Edgerton, Chilton, and Lucas, by Huttig, and by Andes. They have all encountered the homothallic light cultures which revert to the self-sterile dark stocks incapable of producing large quantities of fertile perithecia from a single ascospore culture. The homothallic light stock ordinarily produces 
asci containing 4 light and 4 dark progeny, or 8 dark progeny. All the asci in a given peritheclum contain either four light or four dark or 8 dark cultures. Lindegren has shown that in Neurospora all the asci in a single perithecium (when matings are made as they are in the Glomerella work) arise from a single pair of nuclei associated at the initiation of the perithecium. On this fact, the work in Glomerella can be interpreted in terms of mutation from homothallic light to dark, and the association either or two mutant nuclei or one light and one dark nucleus at the initiation of the perithecium. The homothallic light mutates to dark and two kinds of perithecia are produced, the light/dark, or dark/dark. Mutation occurs so frequently that the light/light combination is only rarely found.

\section{PENICILLIUM AND ASPERGILLUS}

The discovery of penicillin has resulted in considerable interest in variations among species of Penicillium in penicillin productior Derx proved by mating single ascospore cultures isolated from Penicillium luteum that this species is heterothallic, but it is also homothallic. Penicillium notatum, from which penicillin is currently produced, is a form species rather than a true species. It probable originated from Penicillium ascospores and is perpetuated in nature asexually. The existence of heterothallic species in Penicillium suggest that the sexual mechanism may be exploited to obtain penicillin-producing strains.

Pontecorvo has devised a technique for the analysis of homothallic fungi which was applied to Aspergillus nidulans but which could easily be applied to Penicillium luteum.

"The technique as developed for A. nidulans is as follows: 1) A "balanced heterokaryon" is formed between two strains differing by two pairs of "biochemical"alleles and one or more pairs of other alleles ("biochemical" or "morphological"). Indicating with $L / 1$ and $L / / I^{\prime}$ the former, and $A / a$ and $B / b$ the latter, a balanced heterokaryon will carry two kinds of nuclei (I) and (II), for instance $\left(L, 1^{\prime}, A, B\right)$ and $\left(1, L^{\prime}, a, b\right)$. The balanced heterokaryon will be able to grow on a medium which does not supply either nutrilite required by genotypes 1 and $1^{\prime}$.

2) The heterokaryon forms zygotes by fusion of any two nuclei. Selfed zygotes (I,I) and (II, II), and crossed zygotes (I, II) will be produced with frequencies $(p+q)^{2}$ (where $p$ is the proportion of $n u-$ clei (I) and $q$ of nuclei (II) in the heterokaryon) provided fusion of nuclei is at random. $p$ and $q$ can be determined independently; e.g. in A. nidulans by sampling the uninucleate conidia.

3) The zygotes produce haploid ascospores following meiosis. As regards the "biochemical" alleles these spores will be of four kinds: $L, L^{\prime} ; L, 1^{\prime} ; 1, L ' ;$ and $1,1^{\prime}$. On a medium supplying neither of the nutrilites required by the 1 or l' genotypes, only $L, L$ ' recombinants 
will grow; on a medium supplying one nutrilite, one of the parental combination and the $L, L^{\prime}$ recombinants will grow; on a medium supplying both nutrilites, all four types will grow.

4) Segregation of the $A / a$ and $B / b$ alleles is studied only among the "biochemical" recombinants, and these must necessarily originate from cross mating."

\section{CROSS-FERTILIZATION IN SACCHAROMYCES AND OTHER ORGANISMS}

There is a variety of mechanisms in different plants and animals for insuring cross-fertilization, but all of these differ somewhat from that found in yeasts; the symbols a and $\alpha$ were given to the yeast alleles to indicate their uniqueness.

\section{Self-Sterility Alleles}

Most hermaphroditic, flowering plants are self-sterile due to a genetic mechanism which prevents pollen shed by the flower from growing down the styles of the parent plant. A mechanism that may be fundamentally similar prevents the sperms of an individual hermaphroditic sea squirt, Ciona, from fertilizing eggs produced by the ovaries of the same individual.

\section{Sexual Dimorphism}

In higher animals and some plants sexual dimorphism insures cross-fertilization. The genetic mechanism simply operates to reduce the probability of functional intersexes or hermaphrodites occurring.

\section{Plus-Minus Factors}

This mechanism in Rhizopus is not a sexual mechanism because no unmistakable sex organs are involved and therefore it cannot be called a self-sterility mechanism. It is more precise to consider this a special case in which a single pair of alleles controls copulation.

\section{Neurospora}

Fig. 12-3 describes the iife cycle of Neurospora and shows that the alleles controlling cross-fertilization are a specific type of self-sterility allele. Both plus and minus thalli contain both male and female sex organs but self-fertilization does not occur. The Neurospora mechanism differs from the self-sterility mechanism found in flowering plants in that the zygote is invariably heterozygous for the same pair of plus-minus alleles, and only one pair exists. In flowering plants a series of multiple alleles exists and a great variety of heterozygotes abounds. 


\section{Hymenomycetes}

The hymenomycete mechanism resembles the plus-minus Rhizopus mechanism rather closely since no obvious sex organs exist in these forms. It differs in involving two loci in many species, thus producing a minimum of four mating types. Furthermore, mutation is common and a multiple series of alleles at these loci further complicates the picture.

\section{Paramecium}

The mechanism which assures cross-fertilization in the singlecelled diploid Paramecium resembles the plus-minus mechanism found in fungi since no sex organs are present, but the heredity seems to be more complex. The fact that the copulating cells are diploid is a still further difference from the most closely comparable fungal mechanism.

Cross-fertilization has considerable survival value since it insures the incorporation of a variety of genes in each zygote and makes possible the production of a number of new recombinations. However, there are many organisms, such as the close-pollinated flowers, in which cross-fertilization does not occur or occurs only rarely. Inbreeding has made these genera somewhat less variable and therefore less plastic or adaptable than cross-pollinated forms. I have described (1942) copulation in a micrococcus in which crossfertilization did not occur. In this case the zygote was invariably homozygous since it was formed by the fusion of two genetically identical gametes, which originated from the division of a single haploid nucleus. The fusion occurred between two adjacent cells after cell division and solution of the intervening cross wall. This type of copulation has survival value in providing an opportunity (1) for unequal crossing over (Chapter 17) which can increase the number of loci or (2) for chromosomal rearrangements to occur through aberrations of the meiotic mechanism.

I prefer to define sex as Allen does, only in terms of true male and female sex organs. When we use this definition, mating type, self-sterility alleles, and plus-minus factors take on their true significance. They are not essential to the sexual mechanism but are simply means of assuring cross-fertilization. The fact that they may occur either in the absence of sex organs, as in the $\mathrm{Hy}-$ menomycetes, or may be superimposed on true male and female sex organs as in Neurospora (fig. 12-3), prove that they are devices of different origin than the sexual mechanism.

\section{THE ADAPTABILITY OF YEASTS FOR UNITING BIOCHEMISTRY AND EXPERIMENTAL CYTOLOGY}

Genetics has been found useful in biochemical analysis, especially in elucidating the steps which occur in the synthesis or break- 
down of biological chemicals in metabolism. This has tended to divert the activity of Neurospora workers to biochemistry rather than on other phases of biology, and has brought their work into the orbit of the biochemist. Yeast affords the best opportunity to unite biochemistry and biology because the morphological components of the cell have been rather completely described, and it is possible to study very precisely the effect of various nutrients on the cell organelles.

This is a marked advantage of yeast over Neurospora for biological study; the work which we have been doing in relating the morphology of the cell structure to the metabolic activity is not possible in Neurospora. We have clearly defined the chromosomes, the mitochondria, the nucleolus, and the heterochromatin of the yeast cell, which differs from the conventional cell only in the fact that the heterochromatin is located in a separate compartment.

The handicap of small size is more than overcome by the fact that the cells can be brought from rest to full division in less than two hours, thus affording an opportunity to observe directly the change in the organelles involved in the initiation of cell division. Furthermore, because they are free cells and can be grown in a variety of media under different environmental conditions, it is possible to alter their fat or protein content or their stored reserves, thus making possible observation under a variety of cell conditions controlled by the operator; cells organized into tissues or even in a thallus are much more sensitive to alterations of the environment.

\section{THE NECESSITY FOR DIRECT TETRAD ANA LYSIS}

One advantage of yeasts shared by other fungi is the opportunity which they afford for tetrad analysis. After the reduction division occurs in the ascus, each of the four haploid nuclei arising after reduction produces a haploid culture which can be grown separately and observed directly for characteristic hereditary differences. This is not possible either with spermatophytes or metazoa. Mendelism is based on the assumption that each sperm mother cell of a heterozygous (Aa) male produces $2 \mathrm{~A}$ and $2 \mathrm{a}$ sperm and that each Aa egg mother cell undergoes reduction to produce four egg nuclei (2A and $2 a$ ), three of which pass at random into the polar body and one of which functions as the egg nucleus fusing with the sperm nucleus to produce the next diploid generation. The only direct evidence supporting this assumption is that obtained by tetrad analysis of heterozygous fungal hybrids. Actually, however, the four haploid cultures originating from a single individual diploid cell following reduction do not always produce regular Mendelian ratios. If AAAa tetrads were produced occasionally by a fly, they would be difficult or impossible to detect, or to dif - 
ferentiate from cases in which the recessive gametes or the homozygous recessive progeny were slightly less viable. Deficiencies in the number of expected recessive progeny are assumed to result from low viability of the recessive allele rather than non-Mendelian segregations producing an excess of dominant gametes.

The assumption that each diploid sperm mother cell of a heterozygous $\mathrm{Aa}$ individual always produce $2 \mathrm{~A}$ and 2 a sperms is an inference based on statistical evidence. Although the direct evidence derived from the tetrad analysis of hybrid yeasts proves that regular Mendelian tetrads occur often, non-Mendelian segregations are not infrequent occurrences; heterozygous $\mathrm{A} / \mathrm{a}$ hybrids may produce other than AAaa tetrads, in opposition to the induction derived from statistical analysis of indirect evidence.

In a discussion with $\mathrm{Dr}$. Auerbach, she pointed out that the absence of detectable crossing-over in male Drosophila shows that conversion does not occur in male Drosophila. If one recessive gene, for example " $b$ ", in an $\mathrm{abc}$ d heterozygote were converted into the dominant $\mathrm{B}$, it would appear as a double cross-over. She has surveyed the literature and found no evidence of crossing-over in male Drosophila and, therefore, no conversion of alleles. However, this does not negate the positive evidence derived from tetrad analysis of yeasts. 
Chapter 13

\section{SEGREGATION, VARIATION AND RECOMBINATION}

\section{SEGREGATION OF MATING TYPE ALLELES}

Fig. 13-1A, B, C, and D show the cells grown from the four separate spores from a single ascus. Each is a haplophase culture. The four haplophase cultures were paired in all combinations as indicated. A and D were arbitrarily designated as belonging to mating type $a$, while $B$ and $C$ belong to $\alpha$. Copulation tubes and zygotes were produced when $\mathrm{A}$ and $\mathrm{B}, \mathrm{A}$ and $\mathrm{C}, \mathrm{B}$ and $\mathrm{D}$, and $\mathrm{C}$ and $D$ were paired. When transferred to gypsum the diploid cells produced 4-spored asci, while the A and D, B and C combinations failed to produce either diploid cells or ascospores. No spores were obtained from any of the unmated single ascospore cultures.

\section{SEGREGATION OF COLONIAL TYPES}

The colonies originating from the four single ascospore cultures are shown in fig. 13-2. In addition, the colonies of two diplophase cultures are shown on the same scale for comparison with colonies of the four haplophase cultures. In fig. 13-3 outline drawings of the cells of these six cultures appear. The diploid cells are long and ellipsoidal in contrast to the small round cells from the haploid cultures.

Colonies of diploid cultures are uniformly large and smooth provided they are plated on agar before spores form spontaneously. If they are left a few weeks on an agar plate and then plated again on agar, numerous small rough colonies also appear due to the germination of ascospores which have formed in the colony. The large colony size is evidence of the greater vigor of the diplophase.

The variation in the colonies of the four haplophase cultures shows the degree of heterozygosis of the diplophase from which they were derived. The different colony types are genetically different and result from the segregation of genes before spore formation.

The photographs of the haploid cells (fig. 13-1) reveal that the cells of the roughest colony (D) produce "rosettes" characteristic of most rough colony types. Culture A grows slowest on agar and the first plating produced only small colonies all of which were apparently uniform. In small colonies morphological characteristics are difficult to determine, since diagnostic characters are clearly shown only by relatively large colonies. 

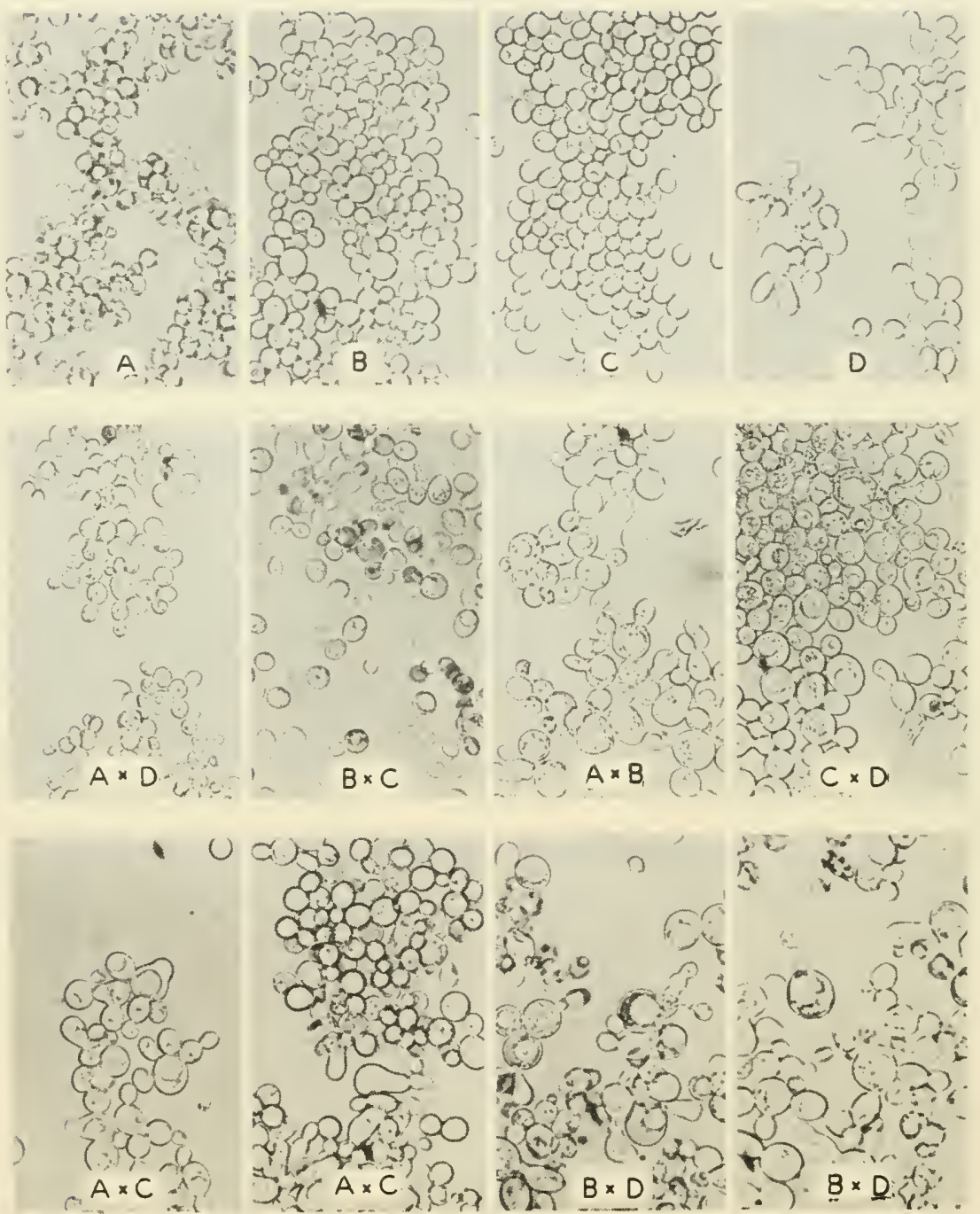

Fig. 13-1 The four haplophase culturea derived from the four apores of a single ascus of Saccharomyces cerevisiae (A, B, C and D) and the results of pairing these cultures in all possible combinationa. A $x$ D and $B \times C$ pairings do not result in copulations. The $A \times B$ pairing results in copulation, and the zygotes are much larger than the cells found in either $A$ or $B$ alone, suggesting that the hybrid is more vigorous than either parent. The same increase in 8 ize is observed in the $C \times D, A \times C$, and $B \times D$ pairing. 

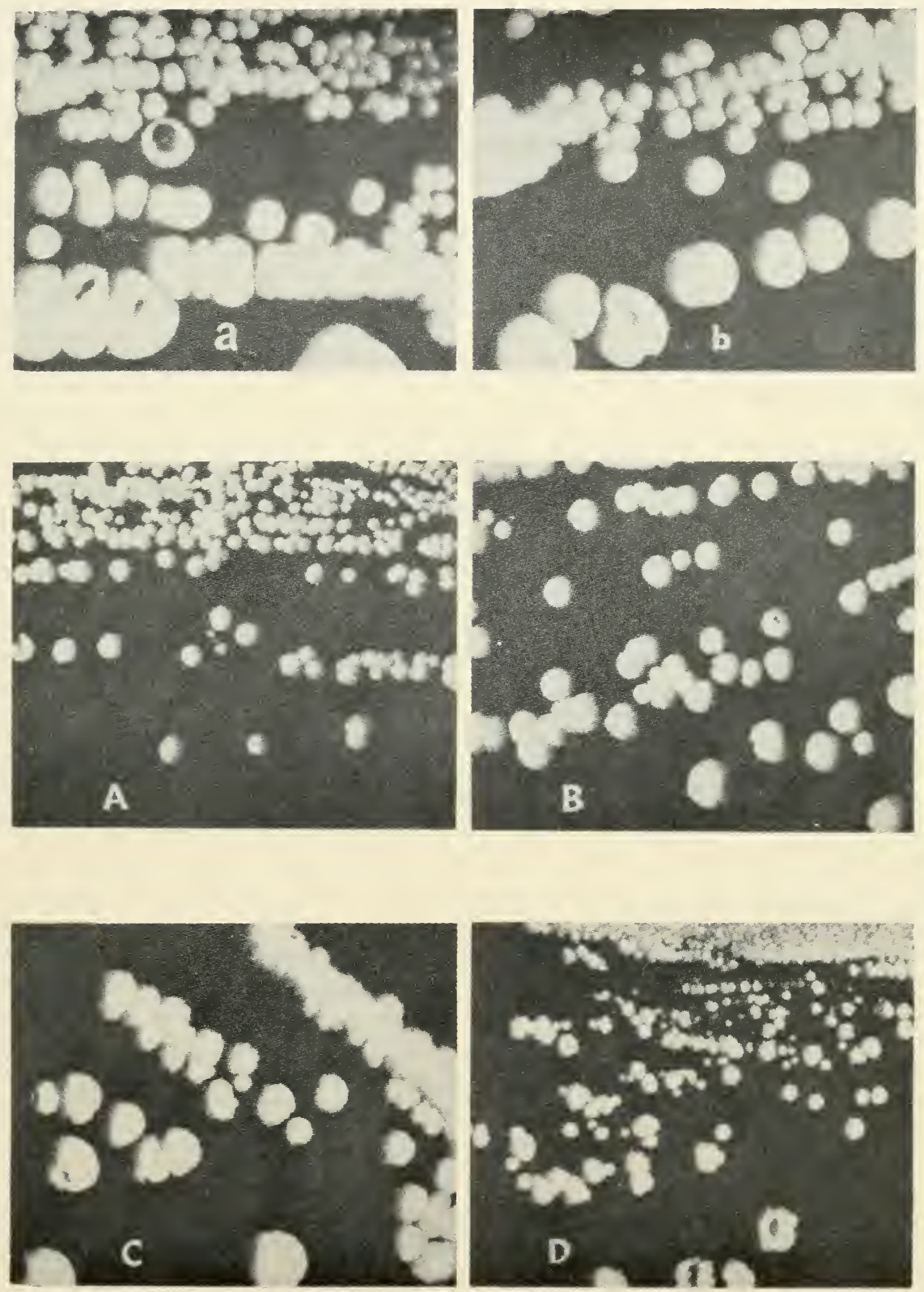

Fig. 13-2 Colonies of two diplophase and four haplophase cultures grown on agar showing differences in size and morphology of colonies. The four haplophase cultures arose from the four spores of a single ascus and correspond to the cultures A, B, C, and D shown in Fig. 13-1. 


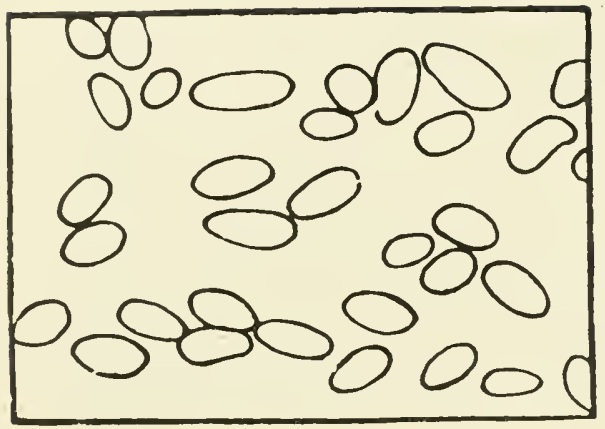

a

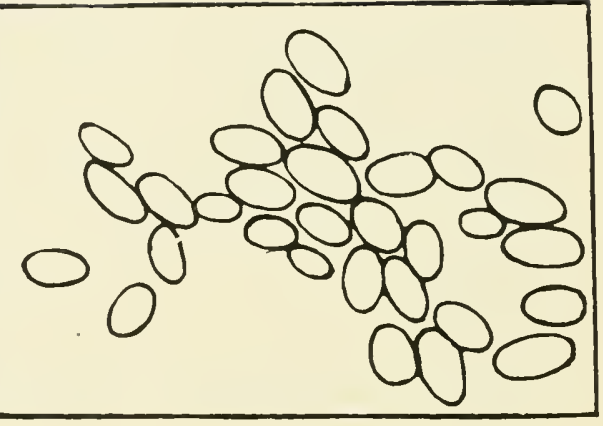

b

DIPLOPHASE

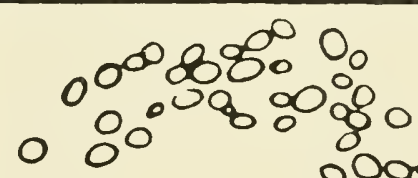

- 0 o098

$\sum_{000}^{\circ} 8_{00}^{0000 .}$

A

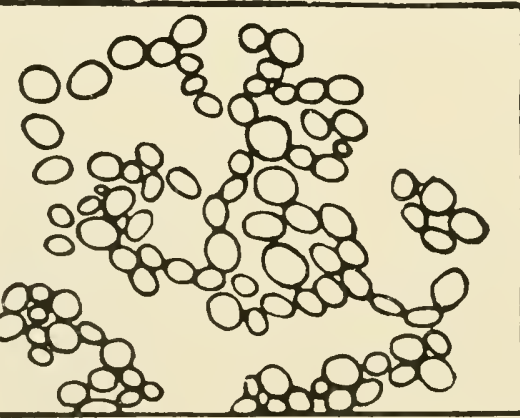

C

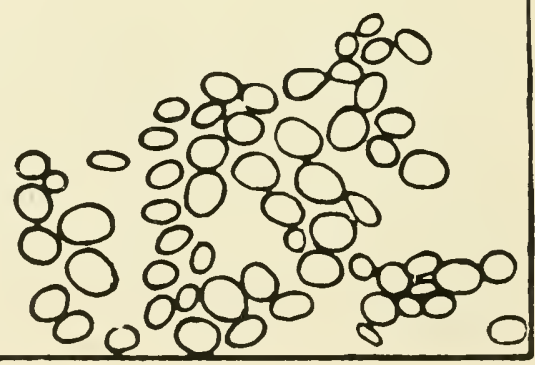

$\mathbf{B}$

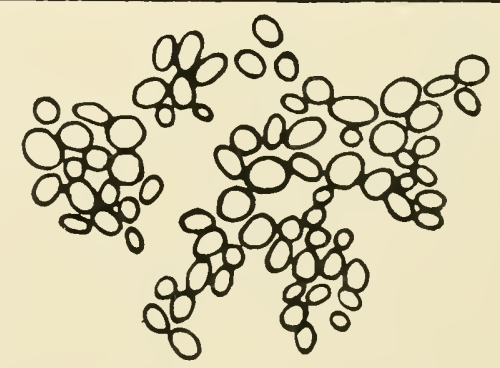

D

HAPLOPHASE

Fig. 13-3 Outline Drawings of Haploid and Diploid Cells from the Colonies Shown in Fig. 13-2. 
VARIATION AND COLONIAL TYPES

On serial plating a large variety of colonial types appeared from each of the four haploid cultures. Plating and selection of the colonies were continued for several weeks and several stable forms were obtained. The cells in these colonies were all round-celled like their progenitors and therefore haploid. The slow-growing, small-colonied culture (A) produced more different types of colonies than the other three This observation is in line with those on other strains, which indicate that weak cultures usually produce many more variations than vigorous cultures, probably because the new mutations meet with less competition from the original type.

Cultures B, C and D show a characteristic feature of the more vigorous haplophase strains. When plated on agar the characteristic large colony appears accompanied by a smaller smooth form. (The cavities in the colonies reveal the places from which transfers have been made.) Transfer of the large rough colony produced the original rough type again accompanied by the small smooth form. The original rough type is the primary segregant while the small smooth form is a variant of the original segregant. The new variant forms a small colony because it grows slowly; its smoothness is due to the small size rather than being related to any specific morphological colonial type. Characteristic colonial morphology does not appear in young or small colonies. Subculture of the smooth produces the smooth form along with a few colonies which appear to be directly derived from this variant now adapted to grow on agar and capable of producing a colony large enough to display its specific morphological characteristics. The new variants from the original segregant pass through the small round phase before becoming adapted. These new types may be either true mutations or what we have called depletion mutations. Depletion mutations are variants which can be propagated indefinitely in the vegetation haplophase but which revert to normal in the heterozygous condition. They cannot pass through the sexual cycle. Much of the variation found when haplophase colonies are plated on agar is due to depletion mutation rather than true mutation. This is proved by the fact that the characteristic variation does not reappear among the offspring of hybrids produced from matings of the variants.

The four segregants shown in fig. 13-2 are characteristically different in colony shape and topography. The D culture is the roughest. The $C$ culture produces the largest colonies and subsequent testing on a variety of sugars indicated that it grew most rapidly and produced most gas. The A culture, even after long subculturing, was much less vigorous. 
The colonies of the four primary types obtained by subculture six months after the original isolation are shown in fig. 13-4. The three photographs labelled "A" all show variants of the A culture. Two of these are relatively stable while considerable variation is visible in the third. The large colony size shows that some of these new variants have become fairly well adapted to the medium. The primary segregants from ascospores B, C and D are still clearly recognizable in spite of several serial transfers. They are generally much more stable than the A culture although many new variants were obtained from each of them. However, the slow growing A culture produced so many variants during the serial transfers that neither the original segregant nor the lines of descent can be traced.

\section{VARIATIONS IN RELATION TO LIFE CYCLE}

Haploid yeast cells are much smaller and more variable than diploid cells, varying more both from culture to culture and within a single culture than diploid cells. These differences are also reflected in the colonies.

Segregation of genes occurs when the chromosomes of the diploid cells are segregated at the reduction division just prior to sporulation. The haplophase originates by the reduction of the diplophase at spore formation, and the segregation of a heterozygote produces segregants of different genotypes. Yeasts are extraordinarily heterozygous, and a great variation of colonial forms is obtained by the isolation of single ascospore cultures. Each of the four spores formed in a single ascus is usually genetically different. The haploid segregants are usually rough-colonied. The segregant cultures also vary in fermentative ability, in ability to synthesize amino acids, vitamins, and nucleic acid components, in color, and in the size and shape of the haploid cells. The type of cell aggregation is also characteristically different. Haplophase clones generally tend to produce aggregated or agglutinated cells much more frequently than diploid clones.

Haplophase yeasts are nearly always inferior in synthetic abiliity when compared quantitatively, or qualitatively, to the diploid parent from which they originated; many of them have lost certain specific characteristics. For example, a single ascospore culture originating from S. cerevisiae may be unable to ferment sucrose, or maltose, or galactose, or even glucose, although the original cultures fermented these sugars successfully.

Variation in the haplophase enormously increases the number of colonial forms, but the original segregant can generally be distinguished from the secondary variant when the culture is plated. At first, the variants are usually slow-growing and produce small, round colonies, but on transfer they become adapted and stabilized 
and their specific colonial character becomes apparent, distinguishing them from the original segregant. Whether this is a process involving several genetical steps has not been determined. Variations occurring in the haplophase can be selected and propagated. In spite of the wide variety of types that occur, the existing genotype of any cell limits its potentialities and the range of its possible variations. This fact has been especially brought out in experiments aimed at adapting haplophases by selection. S. cerevisiae is unable to ferment melibiose, and prolonged exposure of haplophase cultures of $\mathrm{S}$. cerevisiae to melibiose failed to produce any mutants capable of fermenting this sugar. A haplophase variant of S. cerevisiae incapable of fermenting galactose, could not be induced through a four-month period to produce mutants capable of fermenting galactose, although this strain produced an abundance of colonial variants during the same period. Although "losses" occur easily, "gains" as in the case of specific fermentative or synthetic abilities, apparently do not occur at all under certain conditions.

\section{RECOMBINATION}

Segregation and mutation produce a great variety of haploid 8 gametes. Copulations between these gametes selected at random produce recombinations different in a number of characters from the diploid from which they originated. Genetical variation in yeasts is produced by (1) segregation, (2) variation, and (3) recombination. The large ellipsiodal cells which produce a smooth colony on solid medium are usually heterozygous for a number of characters which are segregated at the reduction division and transmitted to four different ascospores. Each of these four spores generally produces a characteristically different colony; the original segregant. The haploid cells are usually rough and much smaller than the original diploid cell. When colonies are grown from the haploid cells, a variety of colonial types appears, indicating that stable gene mutations or depletion mutations have occurred. Occasionally diploid cells appear in some of the single ascospore cultures, revealing that copulations had produced an illegitimate diplophase. The illegitimate diploid cells are usually somewhat smaller than the legitimate diploids.

Legitimate diploidis generally produce an abundance of viable ascospores (fig. 1-7), while illegitimate diploids generally sporulate less vigorously and produce fewer and less viable ascospores. The asci of illegitimates often fail to produce 4-spored asci so necessary for genetical analysis and if they sporulate at all may produce mostly 2 -spored asci. 


\section{TRANSFORMATION OF SACCHAROMYCES INTO TORULOPSIS (CRYPTOCOCCUS, TORULA)}

The four cultures in fig. 13-1, two of which were originally a and two $a$, arising from the four ascospores of a single ascus, were carried in culture in the laboratory for over a year and were observed to produce a great variety of morphological mutants during that period. That they were still haploid was shown by the shape and size of the cells (fig. 13-4). After this extended subculturing the cultures were mated to each other in all possible pairs, following exactly the same procedure shown in fig. 13-1. These matings all failed to produce either copulations or diploid cells. No asci were obtained by transferring the mated cultures to our presporulation medium and gypsum slants. These cultures have become completely neutralized as to sex and have been transformed into typical members of the genus Torulopsis. They are incapable of producing diploid sporulating cultures either by legitimate or illegitimate copulation. This undoubtedly is due to mutation either of the principal a/ $\alpha$ alleles or of modifying genes which inhibit copulation.

The origin of Torulopsis from Saccharomyces was suggested by Satava (1934), and Winge and Laustsen (1939) suggested that the genus Zygosaccharomyces also originated from Saccharomyces. Winge and Laustsen (1937) paired all the Torulopsideae available to them in all combinations and failed to obtain any copulations.

\section{THE INSTABILITY OF THE MATING TYPES ON SUBCULTURE}

The fact that many of the cultures from single ascospores which we carried in culture for a year or more were unable to copulate with freshly isolated tester strains capable of copulation suggests the view that standard Saccharomyces capable of copulation may be transformed into neutral Torulopsidae (Cryptococci). Apparently, the genes controlling copulation become altered by mutation under the selection pressure exerted on haploid cultures. When cultures are isolated in the haploid state, the survival value of the genes controlling copulation disappears and mutations which might be deleterious to copulative ability may be favored. The new mutation might give an advantage in growth rate which would be detrimental to copulative strength or specificity.

Another type of transformation with respect to capability for copulation is found among single ascospore cultures. These variants produce an abundance of copulation tubes as soon as the culture reaches full growth. The cells from cultures of this type also copulate with the cells of any other cultures of either mating type and produce diploid zygotes, irrespective of whether or not they are mated with either a or a strains. The variants have lost the characteristic of discriminating between the a and a types. 

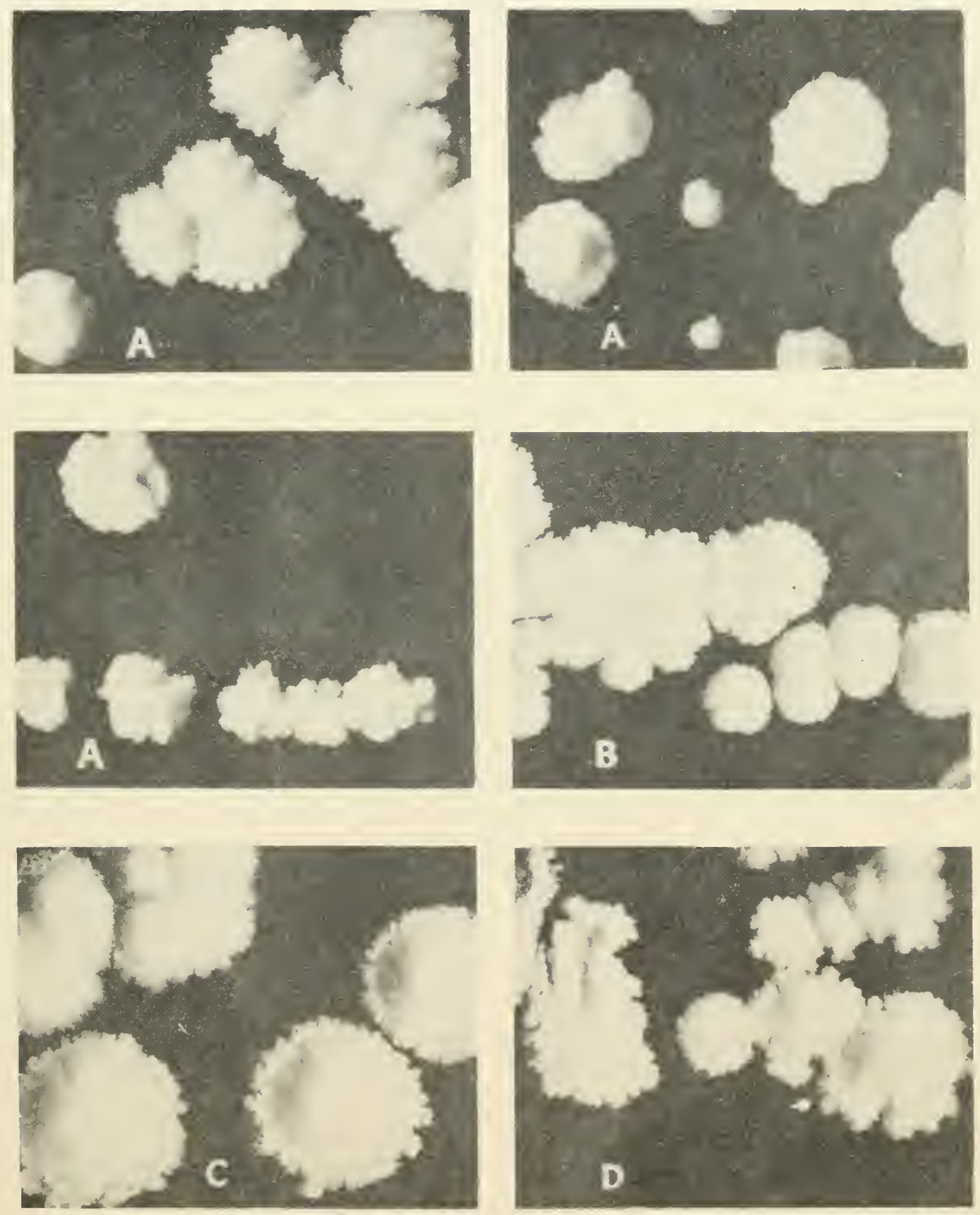

Fig. 13-4 Colony types of the haplophase cultures shown in Fig. 13-2 after serial transfer over a six months' period. The original A type has been lost and none of its descendents resemble it morphologically. Cultures $B, C$, and D still resemble the original parents quite closely. 
In our genetical studies we have selected vigorously against this character and in our breeding stocks a high degree of stable mating type specificity has been achieved.

The genes controlling copulation in yeasts are much more stable than the alleles at the AB loci in the Hymenomycetes. Two cultures of Coprinus lagopus collected from different dung heaps are completely interfertile (Hanna, 1925) due to mutations at the AB loci. Vandendries (1937) extended this study to show that sterility factors can be demonstrated when Hymenomycetes from different continents are mated. The self-sterility alleles in Neurospora are extraordinarily stable (Lindegren, 1932, 1934), we found no mutations of + to - or vice versa in nearly 100,000 single ascospore cultures. However, single ascospore cultures of Neurospora often lose their fertility when carried in culture in precisely the same way that yeasts do, probably because of genes modifying the +/factors. The fact that multiple mutants of Neurospora are less fertile than the wild type suggests that most mutant genes modify the $+/$ - alleles to reduce fertility (Lindegren, Beanfield, and Barber, 1939).

Haploid yeasts are astonishingly variable. Subculturing a haplophase yeast produces a large variety of morphological mutants. If the $\mathrm{a} / \alpha$ alleles are modified by these variations, changes in fertility might occur. This would be especially true in a yeast culture, since the selection pressure is great and constantly varying, due to the effect of the growth of the organisms on the substrate. Diploid cultures are protected against variation possibly because the presence of paired chromosomes minimizes the probability of depletion mutations occurring (see Chapter 15). Illegitimate diploids are stable by another device. They generally fail to produce viable haploid ascospores but produce diploid ascospores which germinate to form clones indistinguishable from the parent type. 


\section{Chapter 14}

\section{VARIATION IN GROWTH AND FERMENTATIVE CAPACITY}

\section{THE “D” FAMILY}

Lindegren and Lindegren (1944) developed a large family of haploid and diploid cultures originating from a single diploid cell of $\mathrm{S}$. cerevisiae. Cultures originating from (a) vegetative cells, (b) single ascospores dissected from 4-spored asci, (c) intact 4 spored asci, and (d) 1-spored asci were critically examined in an analysis of four inbred generations of the "D" family (p. 14-2). The individual cultures in the pedigree are designated as follows: Arabic numerals indicate asci; capital letters designate the ascospores isolated from asci. For example, D1A is the culture from ascospore A isolated from ascus D1. Four spores were dissected from each 4-spored ascus; in ascus D1 only two spores (D1A and D1B) produced viable colonies. D1C and D1D died and are not listed in the pedigree. Only one spore, D2B, from ascus D2 produced a viable colony. Single ascospore cultures may be either haploid or illegitimately diploid or mixed, but data concerning the genetic composition of the single ascospore cultures in this pedigree are not available.

Small Roman letters designate subcultures arising from individual colonies obtained by sowing a suspension of cells on an agar plate. For example, the original D culture was plated on agar and fourteen colonies, Da - Dn, selected, The yeast was grown in $8 \times 1$ inch tubes in $20 \mathrm{cc}$. of broth containing 1.6 per cent sucrose and 1.4 per cent corn steep water solids. After 48 hours the contents were shaken up and $10 \mathrm{cc}$. poured into a Hopkins vaccine tube and centrifuged. The volume of yeast was read off directly in hundredths of a cc. per $10 \mathrm{cc}$. of medium. All fourteen subcultures yielded more than 6.0 hundredths of a cc.

The original D culture was placed on gypsum, and two 4-spored and three 1-spored asci were isolated. The two 4-spored asci were dissected and the spores planted separately in culture tubes. Only three of the ascospores from the 4-spored asci produced cultures, while all three 1 -spored asci germinated and each produced a visible culture. The yield, that is, the crop of cells collected by centrifuging $10 \mathrm{cc}$. of the broth, of the cultures originating from the 1spored asci approximated that of the single colonies selected by plating diploid vegetative cells. This indicates that 1 -spored asci are genetically similar to the vegetative cells from which they arise. 


\section{FIRST GENERATION}

$$
\text { Dissected }
$$$$
\text { 4-spored asci }
$$

$\begin{array}{ll}\text { D 1A } & \text { Yield } \\ \text { D 1 B } & 1.0 \\ \text { D 2B } & 5.5\end{array}$

$\begin{array}{lc} & \text { 1-spored sei } \\ & \frac{\text { Yield }}{6.7} \\ \text { D3A } & 6.7 \\ \text { D4A } & 6.0 \\ \text { D5A } & 6.0\end{array}$

\section{SECOND GENERATION}

4-Spored asci dissected

1-Spored asci

$\begin{array}{ll}\text { Db } 33 A & \begin{array}{l}\text { Yield } \\ 5.5\end{array} \\ \text { Db } 34 A & 6.2 \\ \text { Db35A } & 6.9 \\ \text { Db36A } & \mathrm{n} . \mathrm{g} \\ \text { Db38A } & 6.7 \\ \text { Db39A } & 6.8 \\ \text { Db4DA } & 6.3 \\ \text { Db41A } & 6.2\end{array}$

Yield Spores

$\begin{array}{llc}\text { DbiA } & 6.5 & 15 \% \\ \text { Db1B } & 6.0 & 15 \% \\ \text { Doic } & 4.5 & 0 \\ \text { Do } & & \end{array}$

$\begin{array}{ll}O b 2 A & 4.1 \\ D b 28 & 6.0\end{array}$

DOBA 4.0

Obi7A

Dbi7c

Dbi70
Do18A

Db19A

Dol98

Dbl9c
Db19D

Db20A

Db208

Db2iA

Db21B

Ob21C

Db210

Db22C

OD23A

Do 238

Db23C

DD230

DD258

Db25C

Db250

Db26C

Db37A
$15 \%$

$5 x$
$5 x$

5.5

5.0
5.8
5.5

5.5

5.5

6.0

5.8

5.9

5.8

5.1
5.8

4.8

4.8

5.8

5.1

5.2

\section{0}

5.3

5.8

5.9

6.4
5.3

6.0

6.0

5.4
5.6

5.6

rare
rare
Single colonies

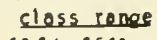

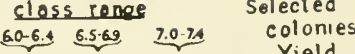

Da to on 310,1, Db 6.8

Dm 6.8

Db24 No spores getminted

4-spored asci selfed class range

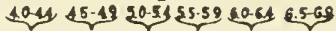
Ascus Colonies

\begin{tabular}{|c|c|c|c|c|c|c|c|}
\hline $\mathrm{DbS}$ & $a-i$ & & & 1 & 1 & & 3 \\
\hline Db G & $a-j$ & & & 5 & 5 & & \\
\hline D67 & a-1 & & & 1 & 7 & 1 & \\
\hline Db8 & $a-h$ & & & 2 & 4 & 2 & \\
\hline Db9 & $a-h$ & & & 4 & 2 & 2 & \\
\hline Dblo & $a-g$ & & & & $t$ & 5 & \\
\hline Dbil & $8-h$ & & 1 & & & 7 & \\
\hline $\begin{array}{l}\mathrm{Db} 12 \\
\mathrm{Ob} 13\end{array}$ & $a-\hat{r}$ & & i & 2 & $\begin{array}{l}3 \\
4\end{array}$ & $\begin{array}{l}2 \\
3\end{array}$ & \\
\hline 0614 & $a-h$ & & & 2 & $i$ & 4 & 1 \\
\hline Dbl5 & $a-h$ & & & $i$ & 2 & 5 & \\
\hline Db 16 & $a-h$ & 1 & & & 2 & 3 & \\
\hline $\mathrm{Db} 28$ & $8-p$ & & & 1 & 5 & 4 & \\
\hline Db29 & $3-5$ & & & 3 & 6 & 2 & 3 \\
\hline Db30 & $a-n$ & & 2 & 1 & 7 & 4 & \\
\hline Db31 & $a-s$ & & 1 & 2 & 3 & 7 & \\
\hline
\end{tabular}

Selected Colonies

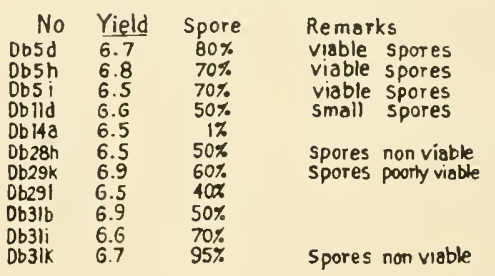

Pedigree of the "D" Family. 


\section{THIRD GENERATION}

Dosd

4-Spored Asci Dissected

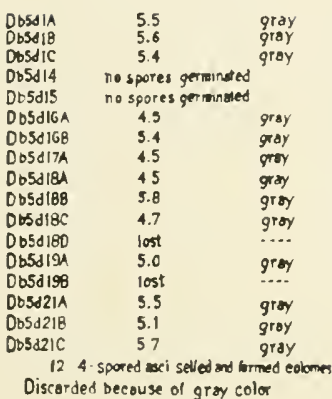

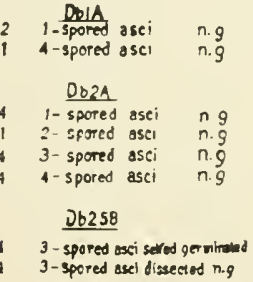

Db5h

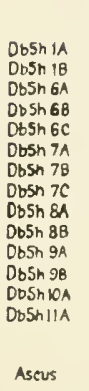

4 spored Asci Dissecled

5.7

5.6

5.6

3.2

5.6

5.0

3.5
5.4

5.0

5.4

5.0

5.6

63

4-spored Asci Selfed

Colonies class Range

Doshn
Dbsh 3
Doshy
Obsh 5

$\begin{array}{lll}a-n & 1 \\ 0-n & 5 \\ 0-p & \\ 0 \cdot 0 & \end{array}$

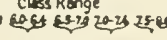

10

Colonies selected Yield spores idectiol

Dosh3e 2.0 20\% 1,2

$\begin{array}{llll}\text { Dosh } 3 \text { e } & 6.0 & 205 & 1,2\end{array}$

$\begin{array}{llll}\text { Dbsh3j } & 60 & 80 \% & 1,2\end{array}$

Dusin4c $68 \quad 3 \mathrm{z} \quad 1,2 \mathrm{sed}$

DbSh4d $6.3 \quad 107 \quad 1.2,3 \mathrm{~s}$

Dbsint] $6.6 \quad 305 \quad$ i2,3 3 ma

Dbsh40 $6.5 \quad 20 \%$

Db5h5b $6.8 \quad 50 \%$ and's

$\begin{array}{llll}\text { Dosh5s } & 7.0 & 70 \times & 1.2\end{array}$

$\begin{array}{llll}\text { Dbsh5i } & 6.8 & 50 \times & 1.2 .3\end{array}$

Dosh 501

DbSh 30

$\begin{array}{ll}50 x & 1,2,3 \\ 70 x & 2,3\end{array}$

Dbsi

4 spored osci dissected

Dbill germinated

Random Ascospores from ruptured asci

\begin{tabular}{|c|c|}
\hline & Yines \\
\hline & 5.5 \\
\hline $\begin{array}{l}\text { Dosilic } \\
\text { DoSi6f }\end{array}$ & 5.5 \\
\hline Dosiac & 5.0 \\
\hline Db51GH & 5.0 \\
\hline Do5i6! & 6.2 \\
\hline 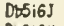 & 5.6 \\
\hline DbSiGK & 4.0 \\
\hline DBSIGL & n. 9 \\
\hline DbSi6M & n. 9 \\
\hline $\mathrm{O} 65, \mathrm{GN}$ & $n_{-g}$ \\
\hline Db5i60 & n.g \\
\hline DbSi6P & n.g \\
\hline
\end{tabular}

4-spored Asci Satiod

Ascus Colonies class Range

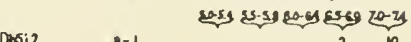

Dosi2

D65.14

Dosis

\section{FOURTH GENERATION} OS5h5b
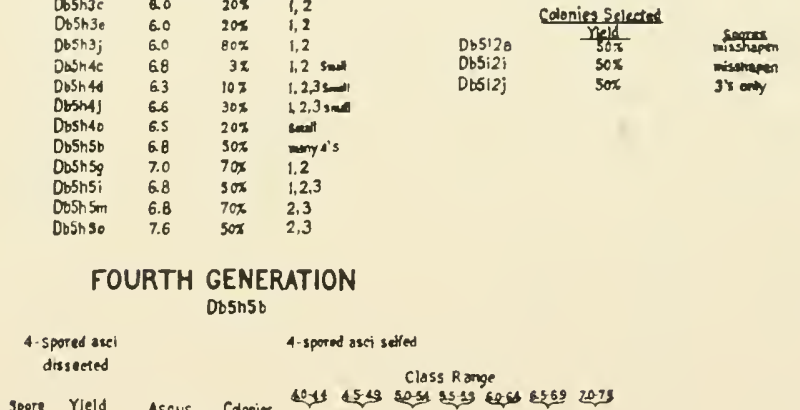

Do5h5biA 5

DoSinsb28 56

DDSnSb2C 5.9

Disonsb2A 5.1

D65hSb3A 5.3

Desh5b48 5.5

Dosistatc 55

DoShSbS -

Doshsba $-j$

D.5h5W1

OLSTSW2

Do5h 506 ,

Dorh5b7 all gerainated No selactions mode

Db5n5tog all germinated No selactions mode

$005 h 5660)$

4-3poned asci disseeted

Dosi2A

Yield

4-spored osci selfed

$\begin{array}{ll}\text { Db512a1A } & 5.0 \\ \text { Do512a18 } & 5.5 \\ \text { Db512alc } & 5.3 \\ \text { Db512a2A } & 5.0 \\ \text { Do5i2a2B } & 5.0 \\ \text { Do5i2a2C } & 50 \\ \text { Db512d2D } & 52\end{array}$

Do5: $2 \mathrm{as}$

Dosizat all germinstod No selactions mat

$0 \times 12$ as

D.512a6)

Dosi2d2D

Pedigree of the "D" Family. 
The yield of the single ascospore cultures from 4-spored asci is variable, indicating that the original culture is heterozygous for the factors affecting the ability of the cells to grow in the prescribed medium.

The second generation originated from culture $\mathrm{Db}$, which gave a high yield and produced viable 4 -spored asci on gypsum. Seven of eight 1-spored asci germinated, and the yield of the cultures from 1-spored asci was approximately the same as that of the cultures from which they originated. Fourteen 4 -spored asci were dissected and the fifty-six single spores isolated; only thirty-three spores produced viable cultures. Both yield and ascospore production were rather variable among these cultures. Only two of the thirty-three single ascospore cultures produced 4-spored asci; the others generally produced 1 - or 2 -spored asci, or failed to sporulate.

Sixteen intact 4-spored asci were isolated and planted in hanging drops. All germinated to produce cultures. The resulting colonies were suspended in water and plated on agar. Individual colonies were selected and tested for yield. Although only 19 per cent of single ascospore cultures gave a yield of 6.0 or more, 45 per cent of the cultures from self-fertilized asci fell in this category. The following shows the data summarized from fig. 14-0:

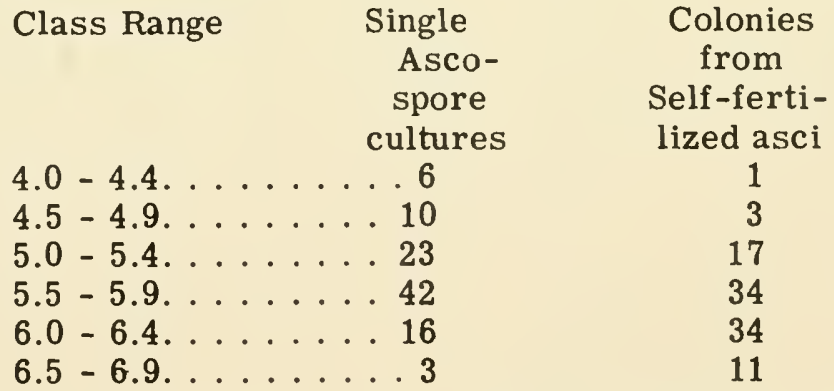

The highest-yielding colonies from the self-fertilized asci were placed on gypsum, and all but one of them produced an abundance of spores. Three of them ( $\mathrm{Db} 5 \mathrm{~d}, \mathrm{Db} 5 \mathrm{~h}$, and $\mathrm{Db} 5 \mathrm{i}$ ) produced viable 4 -spored asci and were used to progagate the third generation.

Only two of the 34 single ascospore cultures in the second generation (DblA and Db2A) produced 4 -spored asci. Five 4-spored asci originating from these single ascospore cultures were isolated but no viable cultures were obtained. Sixteen 1-spored, eleven 2spored, and four 3 -spored asci from these single ascospore cultures also failed to produce cultures. A few of the asci irom single ascospore culture $\mathrm{Db} 25 \mathrm{~b}$ were viable but the low viability of the ascospores from single ascospore cultures arising from normally heterozygous diploid cultures seems clearly established by these 
data. Only twelve viable cultures were obtained from ninety-four spores originating from single ascospore cultures. In contrast, thirty-five cultures were obtained from seventy-one ascospores isolated from cultures arising from self-fertilized asci.

One of the self-fertilized asci from culture Db5h (Db5h5b) produced viable 4-spored asci and was carried into the fourth generation. Culture Db512a also produced viable 4-spored asci and was propagated into the fourth generation.

In the pedigree of the " $\mathrm{D}$ " family, viable 4-spored asci were usually obtained from self-fertilized intact asci, and such cultures were generally more vigorous in both yield and ascospore viability than cultures from single ascospores. On this basis, it is possible to distinguish yeast cultures arising from single ascospores and those arising from intact asci.

A survey of forty cultures of apparently diploid baking yeasts revealed that 24 of them sporulated poorly and produced no 4spored asci, suggesting that they were illegitimate diploids. Sixteen of them sporulated abundantly and produced 4-spored asci, suggesting that they were legitimate diploids.

\section{VARIATION IN GROWTH AND GAS PRODUCTION}

Cultures from various pedigreed sources totalling over 1450 genetically different strains were studied by Lindegren, Spiegelman, and Lindegren (1945) with regard to the volume (crop or yield) of cells produced in $10 \mathrm{cc}$. of peptone, yeast-extract, sugar medium and the ability to produce $\mathrm{CO}_{2}$ from various sugars. The progenies from 12 strains of bakers' yeasts were examined. The progenies of only six of the strains will be considered here in detail. They are representative of the range of biochemical heterogeneity found in the fermentative characteristics in all twelve. As will be indicated at the proper point, some of the studies reported here were made on random sample strains obtained from all 12 pedigrees. The 12 diploid sporulating yeasts, symbolized by letters (G, M, FD, F, D, L, $\mathrm{BE}, \mathrm{HD})$, stem from commercial baking yeasts collected on the market, whereas strains $800,801,806,812$ were obtained from the yeast collection of the Northern Regional Research Laboratory at Peoria, Illinois, through the courtesy of Dr. L. J. Wickerham.

The medium in which the cells were grown consisted of 0.5 per cent peptone, $\mathrm{M} / 15$ phosphate buffer adjusted to $\mathrm{pH} 6.4$, and $2 \mathrm{cc}$. of liquid yeast extract per liter. Carbohydrate was added to the above to make a 2 per cent solution. In the medium labelled " 3 -sugar broth," dextrose, sucrose, and maltose were used in a ratio of $2: 1: 1$, Results obtained on single sugars are labelled in the figures with the name of the sugar used. Yield and gas evolution characteristics were examined on sucrose, glucose, and galactose, levulose and maltose. 
The yield of cells was determined in about the same manner as in the preceding section but the peptone yeast-extract medium was used. Cultures were inoculated into $20 \mathrm{cc}$. of medium contained in $25 \times 200 \mathrm{~mm}$. test tubes. Each tube contained an inverted gas tube $11 \mathrm{~cm}$. long with a capacity of $4 \mathrm{cc}$. The openings of the gas tubes were cut off at a slant to prevent a seal forming at the base. The gas evolved was read at the end of 24 hours by measuring the length of the gas column to the nearest $0.5 \mathrm{~cm}$. The results on gas volume were recorded and plotted in terms of these arbitrary units, each of which is equivalent to $0.36 \mathrm{cc}$. After 48 hours the amount of growth was determined. The contents of each test tube were agitated to attain uniform distribution of yeast cells and a $10 \mathrm{cc}$. sample removed and placed in a graduated Hopkins vaccine tube. This was then centrifuged for 20 minutes at 2,000 r.p.m. and the yield read off in terms of arbitrary units of yeast volume. Each such unit represents $0.012 \mathrm{cc}$. of yeast volume per $10 \mathrm{cc}$. of culture medium. These arbitrary units were employed in the various plots used in comparing the different strains.

The results are summarized by spot diagrams which give a pictorial survey of the distribution of the various physiological types. The position of each point characterized a particular strain.

\section{GAS EVOLVED VERSUS GROWTH}

Figs. 14-1 to 14-6 inclusive, give the gas-yield data obtained when the strains were inoculated into the " 3 -sugar" broth medium containing glucose, sucrose, and maltose. The data are plotted in terms of the arbitrary units of gas and yeast volume described above. By far the vast majority of the strains from all sources can apparently utilize at least one of the sugars to support growth. With a few exceptions yields of 4.5 and over were obtained. While the yields under these conditions are restricted in the main to a relatively narrow region lying between 4.5 and 7.0 the gas measurements are spread over the entire range of measurements. In all the pedigrees examined, there exist some members with relatively high yields and poor (between 0 and 0.5 ) gas production. These strains are obviously different from those which, though giving the same yield, produce 10-20 times as much $\mathrm{CO}_{2}$. Insofar as the general distribution is concerned, there is no apparent difference in the physiological types derivable from the original twelve diploids. Figs. 14-7 to 14-12 inclusive show similar data for growth and gas evolution on single sugars. In these and all subsequent diagrams, the strains represented in each figure do not all belong to the same pedigree. Sample strains were selected at random from the twelve pedigrees and these were used in the single sugar experiments. Studies similar to those depicted in figs. 14-7 to 14-9 


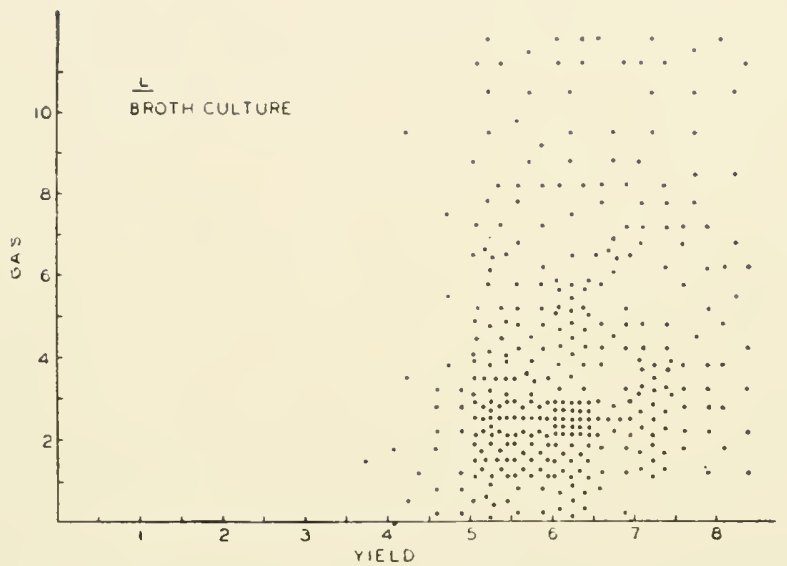

Fig. 14-1 Biochemical Types in Family L Growing on "3Sugar Broth;" each Spot Represents a Different Strain.

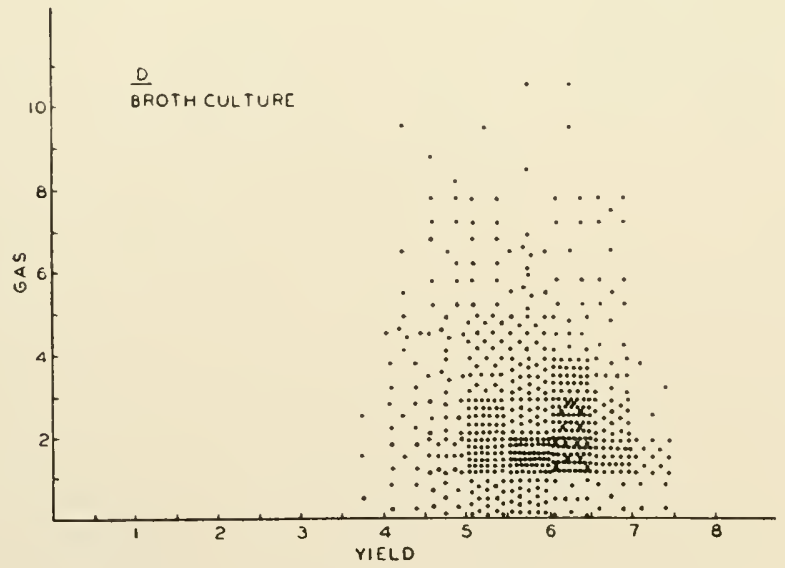

Fig. 14-2 Biochemical Types in Family D Growing on "3Sugar Broth;" each Spot Represents a Different Strain. 


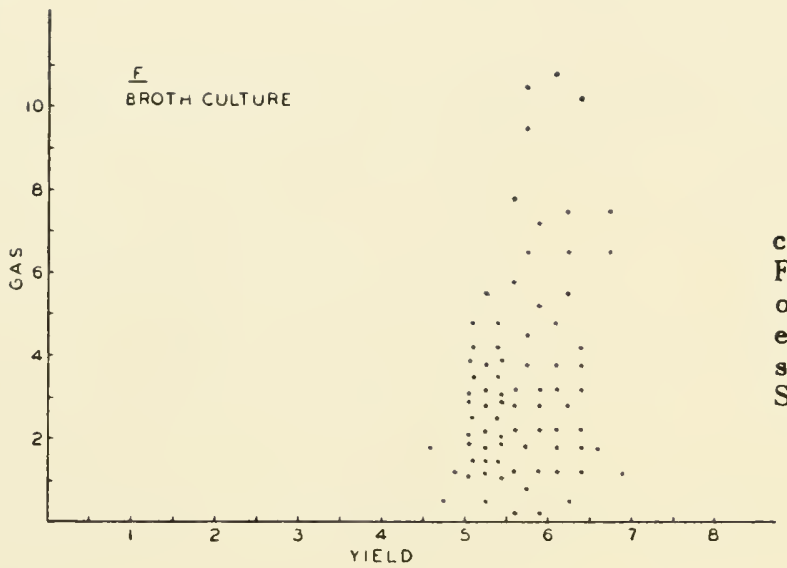

Fig. 14-3 Biochemical types in Family $F$ Growing on "3-Sugar Broth;" each Spot Represents a Different Strain.

Fig. 14-4 Biochemical Types in Family FD Growing on "3-Sugar Broth;" each Spot Repre sents a Different Strain.
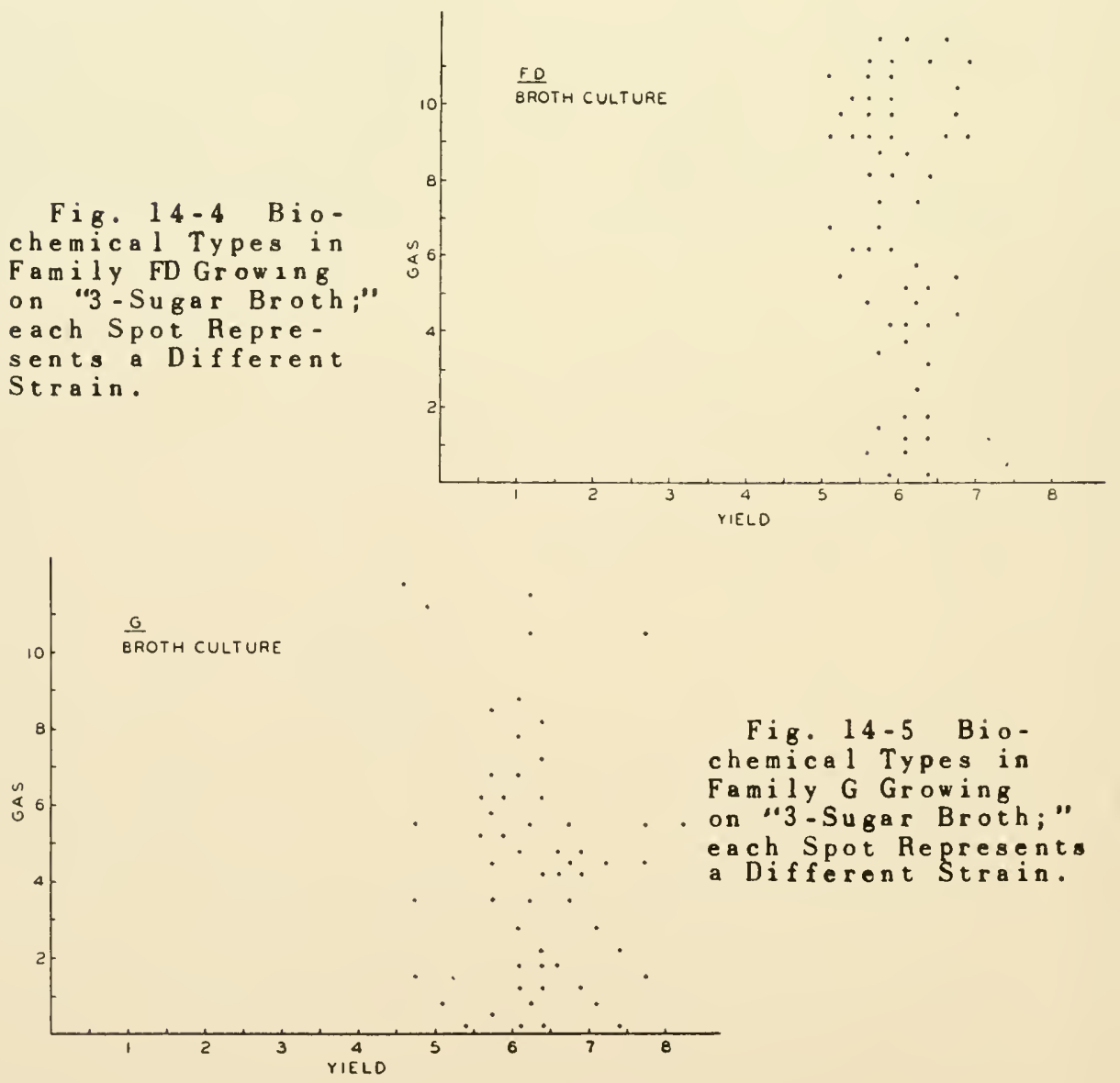

Fig. 14-5 Biochemical Types in Family G Growing on "3-Sugar Broth;" each Spot Represents a Different Strain. 


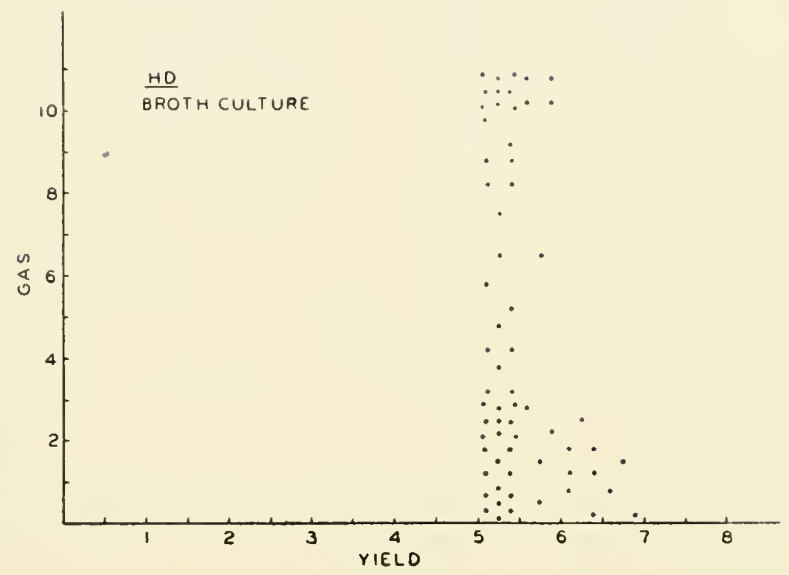

Fig. 14-6 Biochemical Types in Family HD Growing on "3Sugar Broth" each Spot Represents a Different Strain.

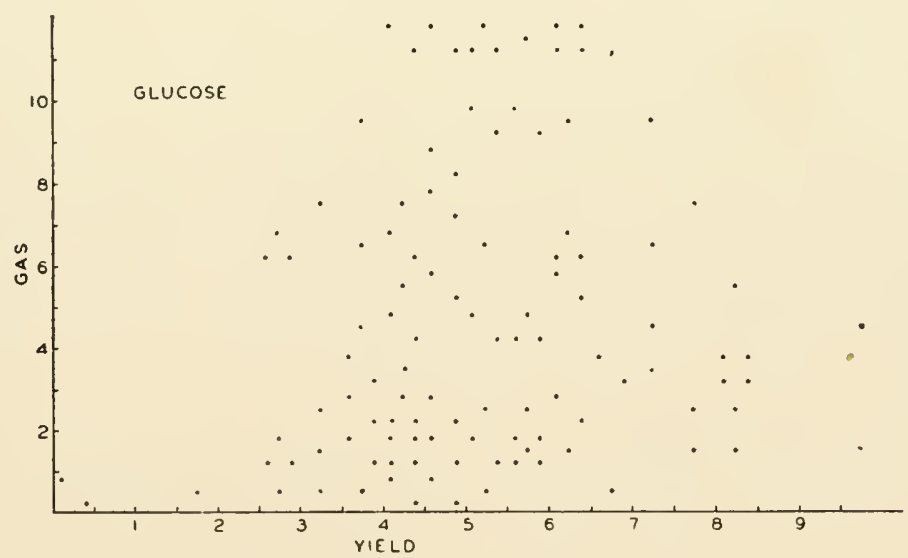

Fig. 14-7 Representative Strains from all 12 Progenies Growing on Glucose Broth. 


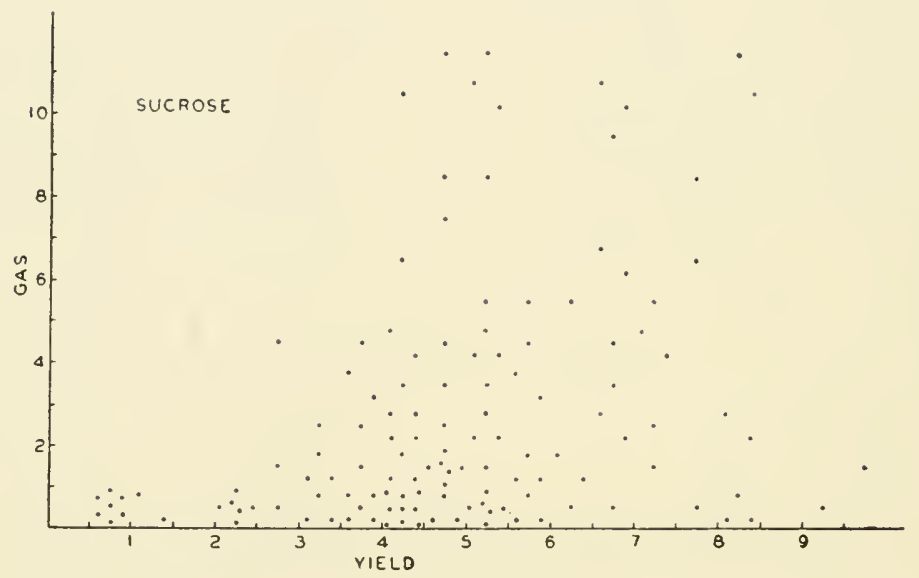

Fig. 14-8 Representative Strains from all 12 Progenies Growing on Sucrose Broth.

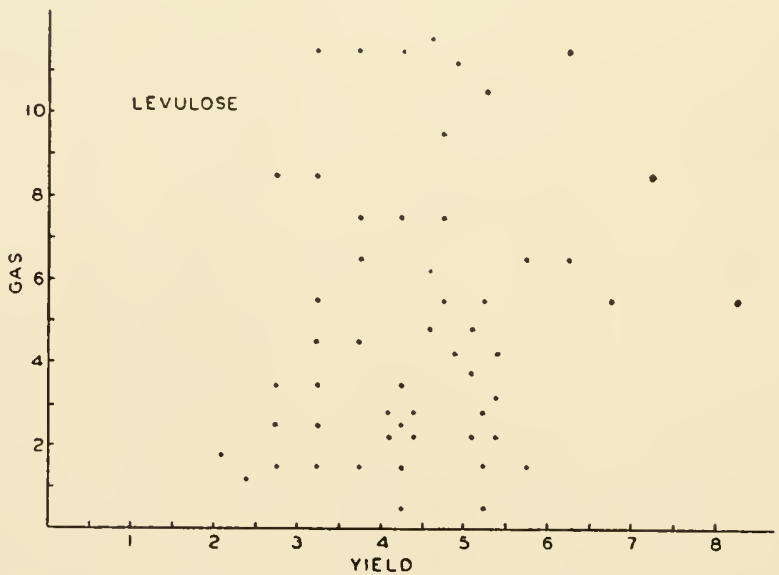

Fig. 14-9 Representative Strains from all 12 Progenies Growing on Levulose Broth. 


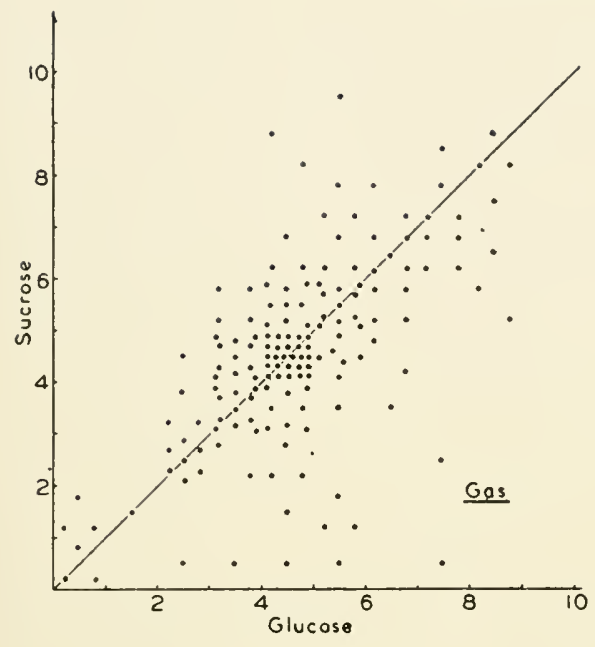

Fig. 14-10 Comparison of Gas Production on Sucrose and Glucose.

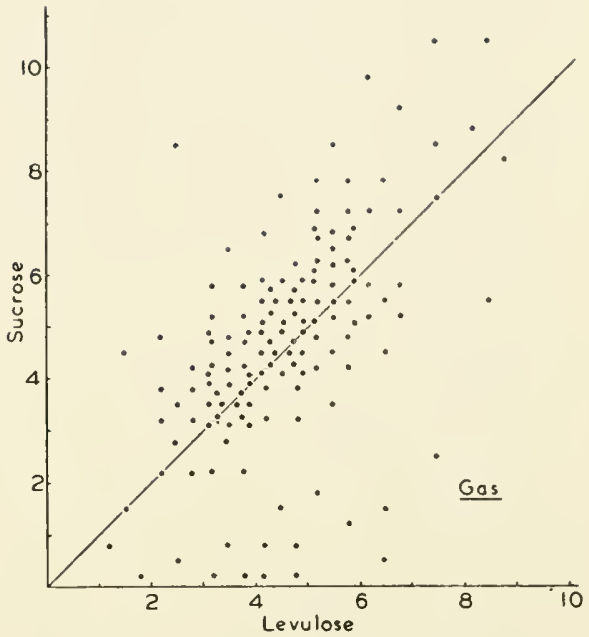

Fig. 14-11 Comparison of Gas Production on Sucrose and Levulose.

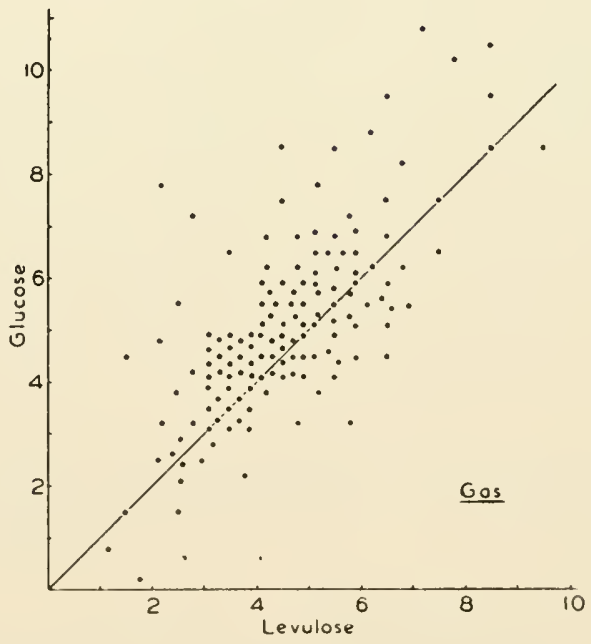

Fig. 14-12 Comparison of Gas Production on Glucose and Levulose. 
were also made with galactose and maltose and showed the same general distribution of points.

It is evident from the figures that the same independence of yield and gas production exists for single sugars as was found in the three-sugar broth medium. One striking difference appears, however, in the wide spread along the yield axis when single sugars are used, as compared with that observed when the " 3 -sugar broth" was used. This is due to the fact that there are relatively few yeast strains which cannot metabolize any of the three sugars used in the "3-sugar broth," whereas a fair number can be found which find it difficult to grow well on glucose, sucrose, or levulose alone. For all sugars examined both vigorous and poor gas producers were found among the comparatively high yielding cultures. Here again it is difficult to escape the conclusion that a strikingly different physiology exists amongst closely related types.

The yield and gas production of the same strains were compared on different sugars. Figs. 14-10 to 14-12 inclusive give such comparisons for gas production. In these diagrams both coordinate axes represent units of gas produced, the abscissae corresponding to behavior on one sugar, the ordinate being used for the other. The relative distances of any given point from the two axes denote then the comparative behavior of the corresponding strain on the two carbohydrates being compared. Points lying on or close to the 45 degree line drawn in each diagram correspond to strains which can handle the two sugars equally well.

As is illustrated by the even distribution about the 45 degree line, fig. 14-10 shows an expected general equivalence between sucrose and glucose. There are, however, a small number of strains unable to ferment sucrose successfully although they have no difficulty with glucose. This is probably due to a comparatively low content of the sucrose-splitting enzyme in the cells of these strains. Several strains demonstrate the interesting biochemical character of fermenting sucrose significantly better than glucose. One in particular producing 8.3 units on sucrose and only 4.1 on glucose.

The plot of sucrose against levulose given in fig. 14-11 yields the same general picture as was obtained in the comparison of the sucrose against glucose. Here also strains are found that can ferment sucrose significantly better; one strain produces 8.2 units on sucrose and 2.2 units on levulose.

A comparison of the two hexoses, glucose and levulose, given in fig. 14-12 reveals a small but definite shift toward the glucose side. Certain strains demonstrate this preference for glucose in an exaggerated manner. One strain produced 7.6 units of gas on glucose and only 2.1 on levulose.

Yield spot diagrams were also made for the same strains growing on the same sugars. Except for the minor movement of certain 
strains, the pair-wise comparison of the growths on the different sugars exhibited exactly the same patterns as the corresponding gas diagrams. For the purpose of this presentation a detailed discussion of the yield distribution would add little that is new to the information already obtained from the analysis of the gas diagrams.

Comparisons similar to those on glucose, levulose, and sucrose given in fig. 14-10 to 14-12 were also carried out with both galactose and maltose. The spot diagrams will not be given, but since certain interesting biochemical types were noted, the results may be briefly summarized.

When behavior on glucose was compared with galactose, it was found, as was to be expected, that the vast majority of strains fell on the glucose side of the 45 degree line. Out of 150 strains examined only one showed marked preference for galactose. This particular strain produced 5.7 units of gas on galactose and only 1.4 on glucose. The comparison of galactose against levulose showed a decided preference for levulose in the vast majority of strains. Four strains were found, however, that showed a significantly better fermentative capacity when galactose was the substrate as compared to that measured on the levulose medium.

When gas production on maltose was compared with that on glucose, it was found that the majority of strains could ferment glucose with greater ease. Five of the 150 strains tested were unable either to split maltose or to use it directly in some other fashion. As in the case of the glucose-sucrose comparisons (fig. 14-10), several biochemically anomalous strains were found. For example, one strain produced only 1.2 units of gas on glucose and 5.3 units on maltose. This would be difficult to understand if this strain metabolized maltose by first splitting it into two glucose molecules.

In the comparison of maltose versus levulose, the tendency was toward better fermentation of levulose in 82 per cent of the strains tested. This differs from the sucrose-levulose comparison (fig. 14-11) in which there exists a slight preponderance on the sucrose side of the 45 degree line. When the two disaccharides, maltose and sucrose, were compared, it was found that 87 per cent of the strains fermented sucrose with greater ease. However, here as elsewhere, a wide divergence of biochemical types was found. Seven strains could not ferment sucrose at all but had little difficulty with maltose.

\section{RANGE OF VARIABILITY}

For a proper evaluation of the data presented, it is important to realize that many of the strains examined in the present study are haploid. The range of physiological behavior exhibited by any family is thus conditioned not only by the types obtainable by segregation and recombination of the segregant from the original par- 
ent diploid, but is also dependent on the mutational potentialities of the haploid strains studied. Despite the additional flexibility attained through haploid variation, it is perhaps a little surprising that such a wide variety of physiological types are found within a given family.

Meyerhof (1925) compared the physiology of various yeasts and. found that they differed markedly in their metabolic characteristics. However in the case of his investigation, the comparison was not between members of the same family. Highly selected brewing and wine yeasts were compared with standard Torulopsideae (Cryptococci). In the present study, the twelve original strains were commercial bakers' yeasts. They were thus the result of intensive selection toward a particular physiological property, namely, aerobic $\mathrm{CO}_{2}$ production on sucrose. Despite intense selection they still possess the potentiality for giving rise to strains possessing almost diametrically opposed behavior with respect to the very physiological character for which they were selected.

These findings have implications for studies of the biochemistry of yeast strains. Any experimental procedure which permits sporulation to occur with the consequent introduction of the haplophase may give rise to widely divergent results on what may have been considered the same strain. In this connection, it may be of interest to note that abundant sporulation may occur on the surface of package yeasts sold commercially. Reproducibility of physiological measurements on a sporulating strain can be attained only if it is transferred sufficiently frequently to avoid sporulation. Although the variability observed, if not controlled, prevents an adequate description of strain physiology, it can serve as a useful tool for the isolation of biochemically interesting types. 


\section{Chapter 15}

\section{DEPLETION MUTATIONS}

The abundant variation which occurs when one plates a haplophase culture has been described in Chapter 13. This phenomenon seems to be in direct opposition to the reliability which we have demonstrated in our mass mating technique. The answer to this apparent contradiction is that many of these variants, although stable on vegetative reproduction, cannot be transmitted through the sexual cycle. In other words, when such variants are mated to a normal individual they produce not half normal and half variant progeny, as one would expect, but only normal progeny. We have called this type of variation a depletion mutation, implying that something in the genome is lost which can be restored in the zygote. The present chapter describes the analysis of the first depletion mutation which we discovered.

\section{RELATION OF PINK COLOR TO ADENINE AND METHIONINE SYNTHESIS}

Culture No. 4, an a mating type galactose-, maltose-fermenting haplophase segregant of Saccharomyces cerevisiae (Mrak 93) was subjected to mustard gas treatment by Tatum and Reaume (in ms.) and produced an adenine-dependent mutant with pink colonies. The symbol ad $(P)$ indicates the adenine-dependent variant producing pink colonies; ad indicates the same allele carried by a white phenotype. The symbol AD indicates the dominant allele; no secondary symbol is necessary for these are always white. The adenine-dependent pink (ad (P)) culture was shown to be a gene mutation by hybridizing it with an adenine-independent white (AD) haplophase of $\mathrm{S}$. cerevisiae.

MATING HYBRID SEGREGANTS haploid $x$ haploid diploid haploid haploid haploid haploid gene $\quad \operatorname{ad}(P) \times \quad A D \quad a d(P) / A D \quad a d(P) ~ a d(P) \quad A D \quad A D$ color pink $\mathrm{X}$ white white pink pink white white

Forty-six asci were dissected from the white hybrid and in 12 asci, two white and two pink cultures arose from each ascus, proving that gene mutation was involved (Table 15-1). In these 42 asci the pink cultures were adenine-dependent and the white cultures were adenine-independent. 
Three asci produced fewer than the expected number of pink segregants. One contained three white and one pinkculture; the pink segregant and one white culture were adenine-dependent. Two asci produced 4 white cultures; two were adenine-dependent and two were adenine-independent. These exceptional adenine-dependent white cultures will be discussed in detail below.

From one ascus more than the expected number of pink cultures was obtained (three pink and one white culture) and the three pink segregants were adenine-dependent. Most of the adenine-dependent cultures adapt after four days and grow in the adenine-deficient medium. The differences in growth in adenine-deficient and adenine-containing medium are generally diagnostic on the second and third days, but growth of the so-called adenine-dependent cultures in the adenine-deficient medium becomes fairly dense on the sixth and seventh day.

The hybrid was also heterozygous for mating type, galactose-, maltose-, and melibiose-fermentation and there were no exceptions of these characters to Mendelian segregation.

The hybrid from which the 46 asci were analyzed was homozygous for genes controlling the synthesis of methionine, so that relatively adequate amounts of methionine were available to all four segregants. Tatum and Reaume also discovered a methioninedependent mutant produced by mustard gas treatment and this gene was introduced into the stock by a series of matings. A hybrid heterozygous for adenine-dependence and methionine-dependence was produced. Analysis of 74 asci from the reciprocal crosses ad (P) MT $\times$ AD mt (pink $\times$ white) and ad $m$ t $\times$ AD MT (white $x$ white) revealed that the development of pink color required methionine. The adenine-dependent $w h t t e$ cultures were also methionine-dependent and all the adenine-dependent $p i n k$ cultures were methionine-independent proving that methionine was required for the development of the pink color.

A doubly heterozygous hybrid produces only three kinds of asci (Chapter 24) and the frequency of these three types can be used to detect linkage of either of the genes with each other or with their respective centromeres. The data in Table 15-1 show that the two reciprocal matings, one producing 35 and the other producing 29 asci, both follow the same pattern and the three kinds of asci are present with a total frequency of $14: 10: 51$, which is statistically equivalent to a 1:1:4 ratio, proving that the two genes are not linked. Asci containing 2 pink : 2 white; 0 pink : 4 white; 1 pink: 3 white, correspond to the same three categories and reveal that pink is produced as a result of a two-factor interaction.

Six asci from a pink by pink hybrid produced four pink cultures from each ascus. Three asci dissected from a homozygous adeninedependent, methionine-dependent hybrid produced only white cultures. 
Table 15-1

Genetical Analysis of Asci from Various Crosses involuing Pink and hite Color, and Adenine and lethionine Synthesis

\begin{tabular}{|c|c|c|c|c|c|c|}
\hline \multirow{2}{*}{ MATINGS } & \multicolumn{5}{|c|}{ SEGREGAiTS } & \multirow{2}{*}{ COLOR } \\
\hline & $\begin{array}{l}\text { No. } \\
\text { asci }\end{array}$ & A & B & $C$ & D & \\
\hline$\underset{\text { pink }}{\operatorname{ad}(\mathrm{P}) \mathrm{MT}} \times \underset{\mathrm{AD}}{\mathrm{wT}}$ & $\begin{array}{r}42 \\
1 \\
2 \\
1\end{array}$ & $\begin{array}{l}\operatorname{ad}(P) \\
\operatorname{ad}(P) \\
\operatorname{ad} \\
\operatorname{ad}(P)\end{array}$ & $\begin{array}{l}\operatorname{ad}(P) \\
\operatorname{ad} \\
\operatorname{ad} \\
\operatorname{ad}(P)\end{array}$ & $\begin{array}{l}A D \\
A D \\
A D \\
\operatorname{ad}(P)\end{array}$ & $\begin{array}{l}A D \\
A D \\
A D \\
A D\end{array}$ & $\begin{array}{l}2 \text { pink : } 2 \text { white } \\
1 \text { pink : } 3 \text { white } \\
0 \text { pink : } 4 \text { white } \\
3 \text { pins : } 1 \text { white }\end{array}$ \\
\hline $\begin{array}{c}\operatorname{ad}(\mathrm{P}) \mathrm{MT} \\
\text { pink }\end{array}$ & $\begin{array}{r}7 \\
5 \\
23\end{array}$ & $\begin{array}{l}\text { ad(P)MT } \\
\text { ad } m t \\
\operatorname{ad}(\mathrm{P}) M \mathrm{~T}\end{array}$ & $\begin{array}{l}\operatorname{ad}(P) M T \\
\text { ad } m t \\
\text { ad } m t\end{array}$ & $\begin{array}{ll}A D & m t \\
A D & M T \\
A D & m t\end{array}$ & $\begin{array}{ll}\mathrm{AD} & \mathrm{mt} \\
\mathrm{AD} & \mathrm{MT} \\
\mathrm{AD} & \mathrm{MT}\end{array}$ & $\begin{array}{l}2 \text { pink : } 2 \text { white } \\
0 \text { pink : } 4 \text { white } \\
1 \text { pink : } 3 \text { white }\end{array}$ \\
\hline $\begin{array}{l}\text { ad } m \text { t } \times A D \quad M T \\
\text { white white }\end{array}$ & $\begin{array}{r}7 \\
5 \\
27\end{array}$ & $\begin{array}{l}\operatorname{ad}(\mathrm{P}) \mathrm{MT} \\
\mathrm{ad} \\
\mathrm{ad}(\mathrm{P})\end{array}$ & $\begin{array}{l}\text { ad }(P) M T \\
\text { ad } m t \\
\text { ad } m t\end{array}$ & $\begin{array}{ll}\mathrm{AD} & \mathrm{mt} \\
\mathrm{AD} & \mathrm{MT} \\
\mathrm{AD} & \mathrm{rt}\end{array}$ & $\begin{array}{ll}\mathrm{AD} & \mathrm{mt} \\
\mathrm{AD} & \mathrm{MT} \\
\mathrm{AD} & \mathrm{MT}\end{array}$ & $\begin{array}{l}2 \text { pink : } 2 \text { white } \\
0 \text { pink : } 4 \text { white } \\
1 \text { pink : } 3 \text { white }\end{array}$ \\
\hline 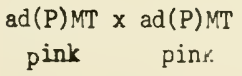 & 6 & $\operatorname{ad}(\mathrm{P}) \mathrm{MT}$ & $\operatorname{ad}(\mathrm{P}) \mathrm{MT}$ & $\operatorname{ad}(\mathrm{P}) \mathrm{MT}$ & $\approx d(P) M T$ & 4 pink : 0 white \\
\hline $\begin{array}{l}\text { ad } m t \times \text { ad } m t \\
\text { white white }\end{array}$ & 3 & ad $m t$ & ad $m t$ & ad $m t$ & ad int & 0 pink : 4 wnite \\
\hline 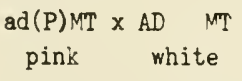 & 7 & $\operatorname{ad}(\mathrm{P}) \mathrm{MT}$ & $\operatorname{ad}(\mathrm{P}) \mathrm{MT}$ & $\mathrm{AD} \quad \mathrm{MT}$ & $A D \quad M T$ & 2 pink : 2 white \\
\hline $\begin{array}{l}\text { ad } M T \times \text { ad }(P) M T \\
\text { white } \quad \text { pink }\end{array}$ & 11 & $\operatorname{ad}(\mathrm{P}) \mathrm{MT}$ & $\approx d(P) M T$ & $\operatorname{ad}(\mathrm{P}) \mathrm{MT}$ & $\operatorname{ad}(P) M T$ & 4 pink : 0 white \\
\hline $\begin{array}{l}\text { ad } M T \times \text { ad(P)MT } \\
\text { white pini }\end{array}$ & 10 & $\operatorname{ad}(P) M T$ & $\operatorname{ad}(\mathrm{P}) \mathrm{MT}$ & $d(P) N T$ & $\operatorname{ad}(\mathrm{P}) \mathrm{MT}$ & 4 pirk : 0 white \\
\hline $\begin{array}{l}\text { ad } M T \times \text { ad } m t \\
\text { white wite }\end{array}$ & 8 & $\operatorname{ad}(P) M T$ & $\operatorname{ad}(\mathrm{P}) \mathrm{MT}$ & $\mathrm{mt}$ & ad $m t$ & 2 pink $: 2$ vihite \\
\hline $\begin{array}{l}\text { ad } M \mathrm{~T} \times \mathrm{AD} \mathrm{mt} \\
\text { white wite }\end{array}$ & $\begin{array}{l}1 \\
5\end{array}$ & $\begin{array}{l}\operatorname{ad}(P) M T \\
\operatorname{ad}(P) M T\end{array}$ & $\begin{array}{l}\operatorname{ad}(P) M T \\
\text { i.d } m t\end{array}$ & $\begin{array}{ll}A D & m t \\
A D & m t\end{array}$ & $\begin{array}{ll}A D & m t \\
A D & M T\end{array}$ & $\begin{array}{l}2 \text { pink : } 2 \text { wnite } \\
1 \text { pirk : } 3 \text { white }\end{array}$ \\
\hline
\end{tabular}


Seven asci from an ad(P)MT x AD MT back cross hybrid produced 2 pink and 2 white per ascus. This further confirms the regular segregation of the two genes.

Adenine-dependence is the effect of the action of a single gene; pink pigment is a correlated effect which depends on the synergistic effect of other genes as well. Pink pigment is apparently produced following the interaction of a precursor of adenine and an excess of methionine plus other substances. Pigment is usually produced in organisms incapable of completing the synthesis of adenine and capable of producing a considerable amount of methionine. The variation in intensity of color in different pink organisms indicates that many other factors affect color intensity.

\section{FALSE MUTATIONS}

Some of the adenine-dependent, methionine-dependent white cultures were transferred to peptone agar to which an excess of methionine had been added. Pink cultures appeared thus confirming the dependence of the pink character on the presence of methionnine. Added methionine did not induce the development of a pink color in any of the adenine-independent white organisms. When the cultures arising from homozygous ad $\mathrm{mt}$ stocks were grown on agar, numerous small secondary pink papillae often appear suggesting local accumulation of sufficient methionine to produce the pink color.

Variations in bacteria following environmental changes have often been called "mutations" but the present experiments show that variations may also be due to a deficiency either of external or internal origin which prevents the development of the characteristic phenotype on a deficient medium. False "mutations" from pink to white may appear when growth occurs in the absence of sufficient methionine to insure the production of the pink color; many pink cultures have white borders which may arise when the supply of methionine in the medium becomes exhausted. Transfer of these false "mutants" to a medium containing sufficient methionine may result in a false "reverse mutation" from white to pink without any change occurring in the gene itself.

\section{DEPLETION MUTATIONS}

Tatum and Reaume discovered that white (ad MT) variant colonies which retain their methionine synthesizing ability often arise from pink cultures on vegetative propagation, - a fact which we have confirmed, and which they will discuss in greater detail elsewhere. Fig. 15-1 shows a typical plate of one of our pink segregants. Numerous white variants of the original pink have appeared. These white variants are ad MT like the white variants of Tatum and 


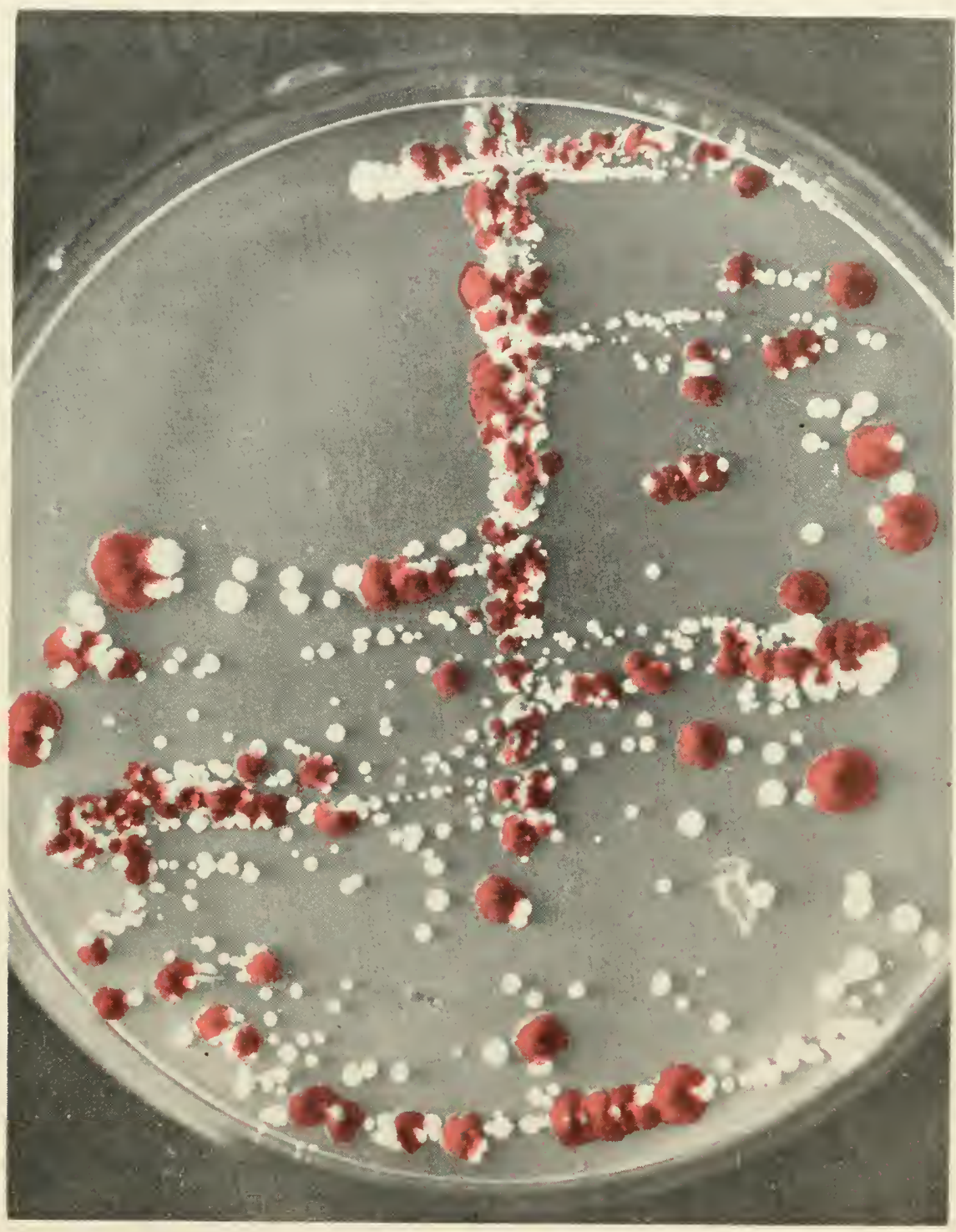

Fig. 15-1 A Petri Plate on Which a Variable ad(P)MT Colony has been Streaked. The white colonies are also ad MT. They are smaller in size indicating a somewat diminished vigor. This is a depletion mutation ince outcrosing the whites with any normal will restore the pink color. The pink color "runs out" either in inbreeding or in asexual reproduction and can be restored by outcrossing. 
Reaume. Similar white segregants arising in the pink pedigree were subjected to genetical analysis.

The hybrids of one of the exceptional adenine-deficient methionine sufficient ( $\mathrm{ad} \mathrm{MT)}$ ) whi te cultures (obtained among the 46 asci from the first hybrid in Table 15-1 by standard pinks, ad(P)MT, produced 21 asci containing 4 pink cultures each. Eight asci from a hybrid of the exceptional white (ad MT) by a standard adeninedependent, methionine-dependent white produced two pink and two white cultures per ascus; the white cultures were methionine-dependent. Six asci of an exceptional white (ad MT) by an adenineindependent, methionine-dependent white produced the progeny that would be expected if the exceptional white were a normal pink. This analysis indicates that the inability of these exceptional adeninedependent, methionine-independent cultures to produce the pink pigment was due to a loss which is restored following hybridization.

The hypothesis which I have invoked to explain the occurrence of the "mutation" from pink to white and its restoration on outcrossing depends upon the general theory of heredity presented in chapter 28. This theory proposes that the gene produces its effect by initiating the synthesis of a gene-product which passes into the cytoplasm; maintenance of a specific character depends upon a specific gene-product-to-gene equilibrium. Pink color depends upon the presence of the two genes ad and MT and gene-product x. The ad gene indicates adenine-dependence and, therefore, the inability of the organism to complete the synthesis of adenine, resulting presumably in the accumulation of a precursor of adenine in the cell. In the presence of methionine the precursor unites with the gene-product $(x)$ of some non-allelic gene (see fig. 28-1) producing the pink color; this drastically diminishes the concentration of gene-product $x$. The metabolism of the cell, which is now white because gene-product $\mathrm{x}$ is no longer present in the required amount, becomes stabilized at this new low gene-product-to-gene equilibrium (for gene $\mathrm{x}$ ) and the clone continues to multiply at the new low level. "Mutation" of pink to white is frequent for continuous production of the adenine precursor frequently diminishes gene-product $\mathrm{x}$ and results in "running out" of the pink character. If the normal geneproduct-to-gene equilibrium (for gene $\mathrm{x}$ ) is present in the stock to which the outcross is made, the pink color is automatically restored. 


\section{Chapter 16}

\section{ADAPTATION IN A YEAST UNABLE TO UTILIZE GLUCOSE}

Nonod (1942) analyzed the mechanism of adaptation of microorganisms to media containing mixtures of carbohydrates and described the phenomenon of "Diauxie."

Diauxic growth occurs when an organism adapts to two different carbohydrates in sequence using first only one and after that has been consumed using only the other.

The substances for which the cell contains "constitutive" enzymes are used first and those for which it must produce "adaptive" enzymes are used secondly. Diauxie is not demonstrable for every mixture of carbohydrates of the above combination but was frequently demonstrated. He considers the difference between "constitutive" and "adaptive" enzymes quantitative rather than qualitative.

Spiegelman and Dunn (1947) confirmed the interaction of enzymes attacking carbohydrates in yeasts and showed that adaptation to galactose reduced the amount of the constitutive enzyme, glucozymase.

Glucozymase is one of the "constitutive" enzymes of the yeast cell. However, if its production is under genetical control, it should be possible to obtain mutants lacking one or more of the essential enzymes. Skoog and Lindegren (1947) derived a culture incapable of fermenting glucose from a hybrid between $\mathrm{S}$. microellipsoideus and $\mathrm{S}$. cerevisiae. This culture was isolated in synthetic medium containing lactate as a carbon source. The culture was stable for nonfermentation of glucose as long as it was maintained with frequent transfer on lactate medium but reverted to glucose fermentation if left in glucose for about 10 days or even if left in lactate for a long time. It reverted immediately in peptone. This is apparently a typical depletion mutation which can undergo repair in vegetative reproduction.

The culture grew in lactate alone without any evidence of a lag period. That is, there was no adaptive period; the enzyme controlling the use of lactate was "constitutive." In glucose alone a long lag period of from 8 - 10 days was required. In mixtures of lactate and glucose an irregular diauxic growth curve was produced. But the presence of glucose inhibited the cell in its ability to utilize lactate, for in mixtures of lactate and glucose a lag period always intervened before growth began. 
The non-glucose culture was isolated by suspending spores of the hybrid in distilled water, heated at 58 degrees C. for four minutes to kill vegetative cells, and then plating on agar media containing organic acids in concentrations of 1 per cent substituted for glucose in Burkholder's synthetic medium including vitamins and with alkali added to make the initial $\mathrm{pH}$ of the media approximately 6. After four days' incubation at 30 degrees C. individual colonies were transferred to tubes containing liquid media of the same composition. After a few days sufficient growth occurred in these tubes to make possible loop transfers to tubes containing the standard synthetic medium with 2 per cent glucose. These tubes were incubated under $\mathrm{CO}_{2}$ at 30 degrees $\mathrm{C}$. From over four hundred such transfers from individual colonies, twelve were found which grew on the organic acid substrates, i.e., on lactate, succinate, or malate, or in combinations of salts of these acids, but which failed to grow when subsequently transferred to glucose. Subcultures were maintained on lactate medium and were tested repeatedly for their abili ty to grow on glucose either in the presence or absence of atmospheric oxygen. It was found that all but two of the twelve organisms quickly reverted to glucose utilization, and these were not further investigated. Nearly all the subcultures of one of the two remaining isolates reverted to sugar utilization within a week and generally within a few days after exposure to glucose in liquid medium. With rare exceptions, reisolations from the reverted cultures yielded only glucose utilizing organisms.

The remaining culture, isolate No. 97, was carried for three months on a lactate medium and was kept stable with respect to nonutilization of glucose by frequent transfers to new medium. It was unable under the conditions of these experiments either to ferment or respire glucose (at least at a significant rate). The same holds for fructose and sucrose. As will be seen below, however, this stability would not be maintained in standard culture practice.

\section{Adaptation to Glucose Utilization}

In tests of the stability of non-glucose utilization, loop transfers were made from the lactate medium to synthetic medium with glucose. Many such transfers of strain No. 97 after standing for some weeks would show a sudden rapid growth and fermentation; i.e., a reversion to glucose utilization occurred in individual tubes after different long periods of incubation in contact with the substrate.

On the other hand, it was observed that the addition of glucose to the organic acid substrates tended to repress the growth of the organism. Thus glucose not only is not assimilated by the unadapted organism but it actually inhibits the utilization of the organic acids. 
To study these phenomena in more detail strain No. 97 was cultured on a series of media containing lactate or glucose in different concentrations or the two substances combined in different proportions added to the synthetic medium. The growth in $10 \mathrm{cc}$. volumes in $6 \times 3 / 4$-inch test tubes was measured photometrically at suitable intervals. Some typical results with an adapted strain, isolate No. 322 , are included as parts B of the figures.

Considering first the growth on media with lactate alone added it may be seen (fig. 16-1) that the growth of strain No. 97 is greater the lower the concentration of lactate between 4 and 0.5 per cent, whereas with the glucose-adapted strain No. 322 , the reverse is true. The results with No. 97 might appear to mean that lactate is not utilized but rather interferes with the utilization of some other component of the substrate. However, the fact that only traces of growth are obtained in control tubes without lactate excludes this interpretation and indicates rather that the concentration of lactate tends to decrease both the growth rate and final yields. In the case of strain No. 322 on the other hand even 4 per cent, the highest concentration used, may not be sufficient for optimum growth.

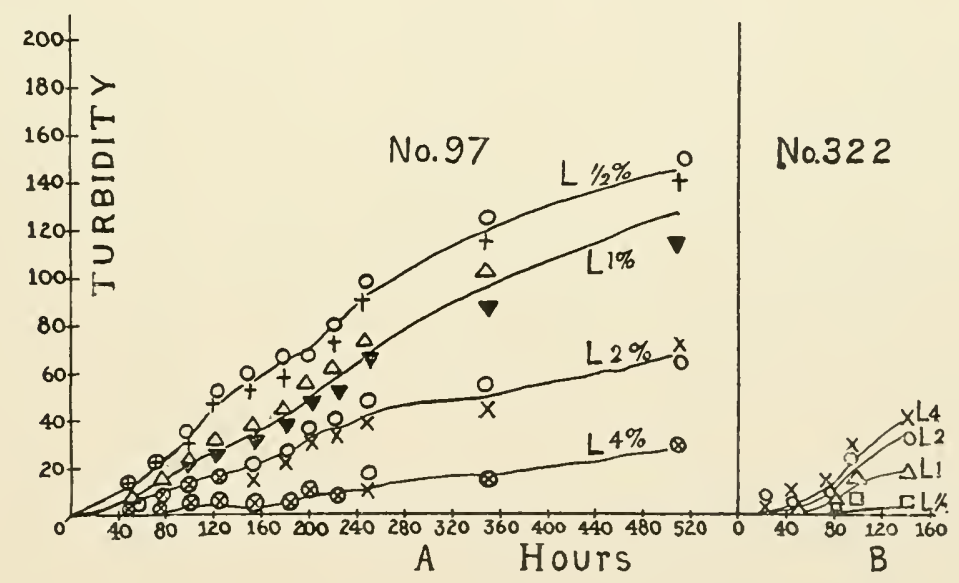

Fig. 16-1 A. Growth on eight subcultures of No. 07 (previously maintained on lactate) on different concentrations of lactate in synthetic medium. Ordinates, density of yeast suspension; abscissae, hours.

B. Growth of four subcultures of No. 322 under similar conditions.

Comparing next the growth on lactate with that on glucose (figs. 16-2A, 16-3A, 16-4A, and 16-5A) the following striking differences are to be noted: 


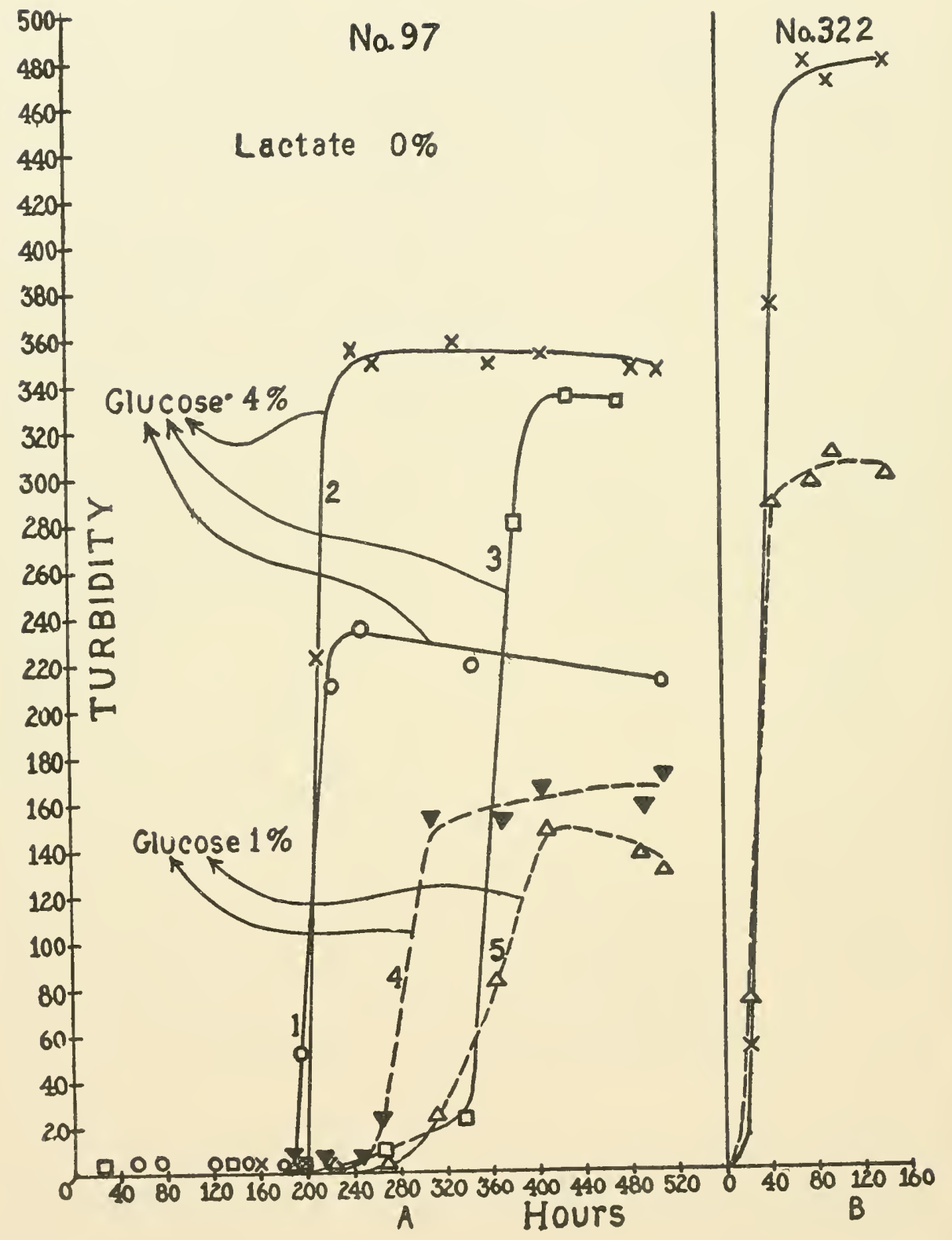

Fig. 16-2 A. Growth of subcultures of glucose unadapted - train No. 97 in synthetic medium containing no lactate. Solid lines, 4 per cent glucose; broken lines, 1 per cent glùcose.

B. Growth of abcultures of the adapted strain No. 322 on glucose. 
When glucose is added to a medium containing lactate (which without glucose would support immediate growth) there is a very long period between inoculation and the appearance of measurable growth followed suddenly by a period of very rapid growth. On the average 150 to 170 hours were required on mixtures of lactate and glucose; one culture adapted after only 40 hours but several failed to grow appreciably until after 10 or more days.

In the absence of lactate No. 97 requires an incubation period of nearly 200 hours before it begins to grow on 4 per cent glucose. However, after that incubation period the growth is extremely rapid, indicating that adaptation is complete. On 1 per cent glucose a longer adaptation period is required and the adapted cultures do not grow nearly so rapidly. By comparison No. 322 requires no adaptation and growth progresses at a maximum rate.

Fig. 16-3 shows that when half per cent lactate is added to 4 per cent glucose No.97 begins to grow much sooner, but the growth rate is not so great as in the absence of lactate, and after an initial rapid increase there is a period of lag and a second period of increasing growth rate. One per cent glucose with half per cent lactate shows a greater variation in the secondary phase of growth, and a corres pondingly longer delay before growth begins. A comparison of figs. 16-2 and 16-3 reveals that adaptation to glucose is complete in the absence of lactate after a relatively long lag period, and that higher concentrations of glucose produce more effective adaptation than low concentrations. When lactate is added to glucose the nonutilizer of glucose begins to grow a little sooner but the growth rate is less rapid, indicating that adaptation to glucose is not complete and that the presence of lactate inhibits this adaptation. A secondary phase in growth indicates that subsequent steps occur in adaptation to glucose. It is also clear that the presence of glucose interferes with utilization of lactate because on lactate culture No. 97 begins to grow immediately without any lag, and 120 hours are required before growth begins on the mixture.

Figs. 16-4 and 16-5 in which increasing concentrations of lactate are used, confirm the above conclusions and show in addition that the growth of the glucose utilizer is inhibited on high concentrations of lactate, although 0.5 per cent has no harmful effect, suggesting that harmful effects of lactate do not account for the irregularities of growth on No. 97 shown in fig. 16-3. The sta rting time of rapid growth varies greatly from one culture to the next. Some data for individual cultures are presented in Table 16-1. The adaptation times, the points of intersection of the abscissa with the slopes of the growth curves in the regions of rapid linear growth, representapproximately the periods required for adaptation to glucose utilization. In most cases they correspond to the times of appearance of sufficient yields to be measurable in the Klett photometer. 


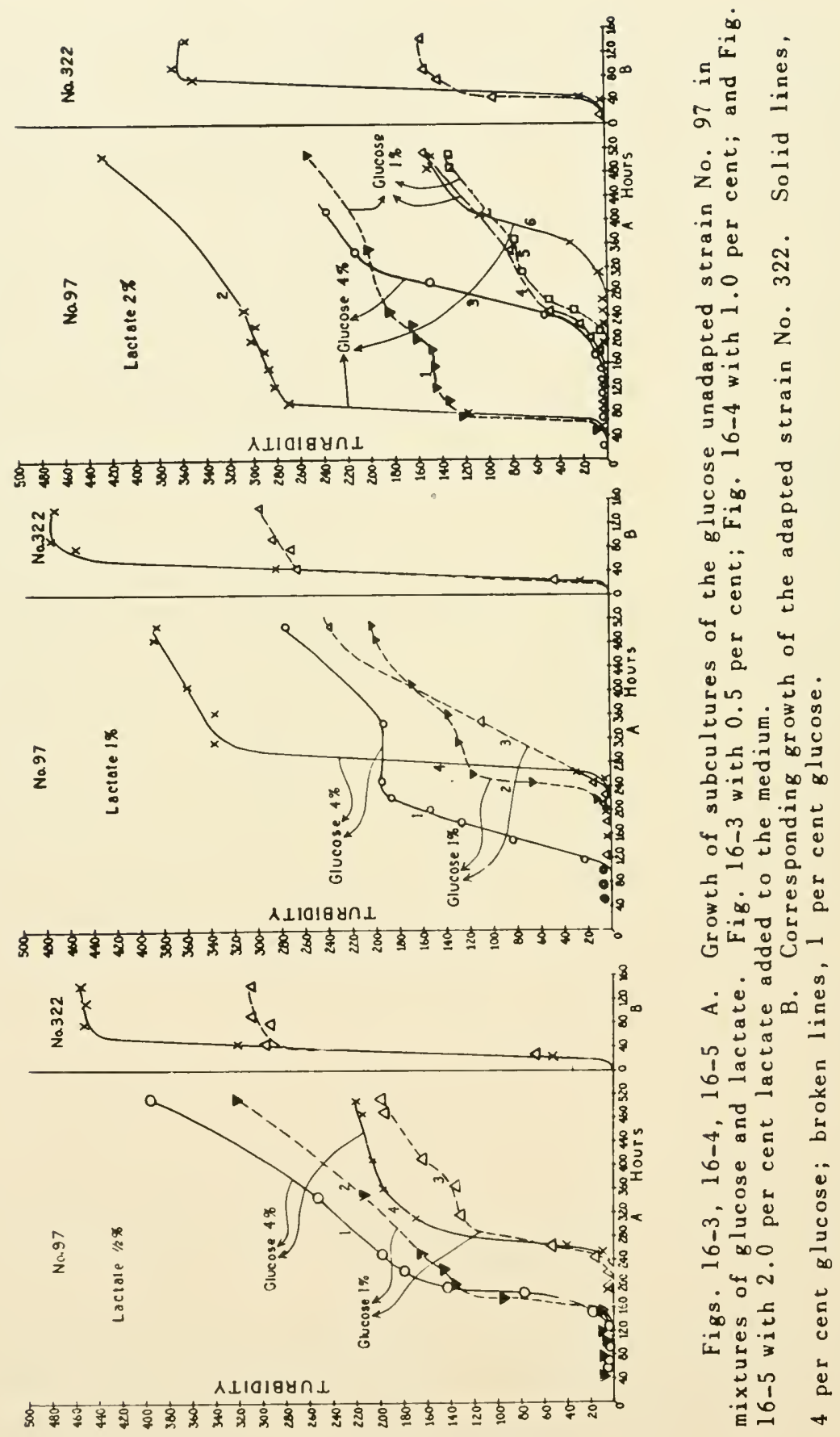


They do not, however, correspond to the times of initiation of growth. Traces of yeasts, much less than measurable with the instrument, are generally observable earlier, usually within three to four days after inoculation on the mixtures of lactate and glucose. Occasionally, as is illustrated by some curves in figs. 16-2A and 16-5A and checked by loop transfers to glucose medium, conside rable yields are obtained before adaptation to glucose occurs.

The time required from the beginning of rapid growth to the attainment of maximum rate (flex points of curves) is difficult to estimate exactly but is certainly also very variable from one culture to the next. The average values are shown under the heading "Periods of increasing growth rates." Perhaps the length of time required increases with the concentration of lactate in the medium, but this tendency is certainly less marked than the extreme differences exhibited between individual cultures of a given set, which will be discussed below.

The later portions of the curves show that with the addition of glucose alone (fig. 16-2) to the medium the growth approaches the final yields very quickly once it has started, whereas with mixtures of lactate and glucose (figs. 16-3, 16-4, 16-5) the growth decreases very markedly as a rule long before the final yields are attained. This decrease in growth rate is evidently due to a number of different causes which operated to different extents in individual cultures. In the first place where adaptation to glucose has occurred before the lactate has been depleted subsequent growth may result from further utilization of remaining lactate. See curves 1 and 3 in Fig. 16-3A; curves 1 and 2 in fig. 16-4A; and curves $1,2,4$, and 6 in fig. 16-5. Secondly, it is clear from a comparison of the figures with increasing lactate concentrations that there is a correspondingly slower rate of utilization of the glucose and that the growth rates frequently begin to decrease long before the glucose has been depleted, i.e., inhibition occurs when the amount of growth is only a fraction of the final yield, so that glucose as well as the lactate present must be required for the subsequent yield. This decrease in growth rate before the final yield is attained with increasing concentration of lactate, also holds for strain 322. It appears, therefore, that high lactate concentrations interfere to some extent with the utilization of glucose. Hence, the phase of subsequent slow growth and the secondary rise in growth rate may be due to an adaptation to glucose utilization in the presence of lactate, i.e., to the development of a tolerance to lactate as well as to the utilization of lactate. Numerous other changes must occur in the medium which may impede the rate and also in speclfic instances may give preference to the rapid increase of mutant types in the cultures. 
The final total yield of a culture is primarily determined by the glucose content of the medium. Thus the largest yields on the average were obtained on 4 per cent glucose, and approximately the same yields were eventually obtained on mixtures with lactate. In the case of 1 per cent glucose the addition of lactate may give significant increases. Mean values for the total yields of four sets of strain No. 97 measured after between 600 and 800 hours and the results obtained with a set of strain No. 322 after 142 hours are shown in Table 16-1. Many factors affect the final yields. Most important of these is perhaps the different extents of fermentation versus assimilation in growth on the available substrate occurring in the individual cultures. Furthermore, the long growth periods and different growth rates introduce variable changes in the medium leading to modifications in yields, partial autolysis, presence of ghost cells and perhaps even reutilization of substrate by new types. These difficulties do not arise with the glucose adapted strain, where the relation of final yield to glucose content is very definite except in so far as the presence of 2 per cent lactate inhibits the growth on glucose. The cultures were not kept long enough to determine whether this lag in yields on 2 per cent lactate was ultimately recovered.

To summarize: Inoculation in glucose or glucose-lactate mixtures does not result in immediate growth as inoculation from lactate to lactate does. The delay is the incubation period required to adapt to the utilization of glucose. The incubation period may be shortened by additions of lactate, but when lactate is present the total growth and the growth rate is generally less and soon suffers a temporary stoppage. After this stoppage a secondary increase in growth occurs with a correspondingly slower rate of utilization of the remaining glucose and lactate.

Adaptation to glucose on lactate medium. The above results show that adaptation to glucose utilization occurs on synthetic medium with added glucose. It may also occur after very long periods on lactate media without added glucose. Thus, of some 50 cultures which have been tested for glucose adaptation after 600 or more hours of incubation on 1 and $\frac{1}{2}$ per cent lactate media, 3 and possibly several others were adapted at least to slow growth on glucose. It is not certain, however, that this adaptation has occurred in the absence of the substrate. More likely, some glucose has been produced in the cells as a result of hydrolysis of trehalose and perhaps of other polysaccharides so that a condition comparable with that of added glucose obtains in old cultures of the unadapted strain.

Adaptation on standard peptone glucose broth. It was found that transfers of culture No. 97 from lactate to standard glucose broth containing yeast extract and peptone (in contrast to the behavior on synthetic medium) grew rapidly and as a rule exhibited 
Table 16-1

Growth on lxture of Lactate and Glucose

\begin{tabular}{|c|c|c|c|c|c|c|c|c|c|}
\hline & $\begin{array}{l}\text { Culture } \\
\text { No. }\end{array}$ & $\begin{array}{l}\mathrm{G}_{4} \\
\mathrm{~L}_{2}\end{array}$ & $\begin{array}{l}\mathrm{G}_{4} \\
\mathrm{~L}_{1}\end{array}$ & $\begin{array}{l}\mathrm{G}_{4} \\
\mathrm{~L}_{\frac{1}{2}}\end{array}$ & $\begin{array}{l}\mathrm{G}_{1} \\
\mathrm{~L}_{2}\end{array}$ & $\begin{array}{l}\mathrm{G}_{1} \\
\mathrm{~L}_{1}\end{array}$ & $\begin{array}{l}\mathrm{G}_{1} \\
\mathrm{~L}_{\frac{1}{2}}\end{array}$ & $\mathrm{G}_{4}$ & $\mathrm{G}_{1}$ \\
\hline \multirow{2}{*}{$\begin{array}{l}\text { Adaptation } \\
\text { Times* }\end{array}$} & 97 & 157 & 162 & $163 \mid$ & 129 & 176 & 169 & 299 & 214 \\
\hline & 322 & 25 & 10 & 10 & 25 & 10 & 20 & 10 & 10 \\
\hline \multirow{2}{*}{$\begin{array}{l}\text { Periods of In- } \\
\text { creasing Growth } \\
\text { Rates** }\end{array}$} & 97 & 63 & 59 & $(38)$ & 66 & 48 & 37 & 57 & 69 \\
\hline & 322 & 25 & 20 & 20 & 15 & 25 & 30 & 20 & 20 \\
\hline \multirow{2}{*}{$\begin{array}{l}\text { Relative Total } \\
\text { Yields*** }\end{array}$} & 97 & 390 & 333 & 327 & 339 & 236 & 302 & 325 & 222 \\
\hline & 322 & 355 & 470 & 455 & 480 & 160 & 310 & 320 & 310 \\
\hline
\end{tabular}

Each mean value was determined from 8 tubes of No. 97 and 2 tubes of No. 322 .

* Mean time in hours from inoculation to initiation of rapid growth on mixtures of lactic acid and glucose and on glucose.

** Mean time in hours from initiation of rapid growth to attainment of maximum rate (flex point of curves) on mixtures of lactic acid and glucose.

***Final photometer readir.gs: Mean values after between 600 and 800 hours incubation of No. 97 and 142 hours incubation of No. 322 . 
vigorous fermentation after 24 to 48 hours incubation at 30 degrees C. This rapid adaptation could be due to either a specific factor in the natural medium or simply to the inclusion of different substrates which permit multiplication of the unadapted cells thus providing a large population for the selection of mutants.

\section{CROSSES AND BACKCROSSES WITH STRAIN NO. 97}

Several crosses of the unadapted strain No. 97 with glucoseutilizing yeasts were made by Mrs. Lindegren. Only a few ascospores were obtained in each successful case. The ascospores were isolated on a lactate medium like that described above but matings were made in standard peptone glucose broth and the natural presporulation medium was used to induce sporulation. No clear segregation of the progeny has been found. In cases where all four spores from an ascus were viable, two often grew rapidly while two grew slowly, but when tested later for glucose utilization all would be positive. Viable progeny from back-crosses of these cultures with straln No. 97 were similarly positive. It is clear, however, from the evidence obtained later that rapid reversion to glucose utilization occurs on standard glucose broth and that such reversions would take place on the complex medium required for sexual fusion (Chapter 3). The progeny not only may have been quickly adapted but may have been derived in the first place only from glucose-adapted parent cells.

\section{THE MECHANISM OF ADAPTATION}

These results fit the view that adaptation occurs by the recovery of a recently lost ability; it seems likely that not only the multiplication of the mutants but the mother cells themselves are influenced by the presence of the substrate, and that the mutant does not arise merely by chance. The reversion to glucose utilization is probably not a single step change occurring uniformly in individual cultures to restore the mutant to the original wild type. This suggests that the strain incapable of utilizing glucose did not arise by specific single step change in the metabolic apparatus of the wild type. 


\section{Chapter 17}

\section{BACK-MUTATION AND PROGRESSIVE MUTATION}

The standard Mendelian mutant ordinarily used in genetical analysis usually involves the loss of some synthetic ability; it is deficient as compared to normal. Back-mutation to normal, therefore, involves the reacquisition of a capacity which has been temporarily lost. A progressive mutation involves the acquisition of an entirely new ability. The present chapter describes back-mutation of a non-fermenter of galactose to a fermenter of this sugar. It is proposed that back-mutation probably involves the repair of a gene which is only partially deficient or the reactivation of a non-allelic dormant gene which can be brought into functional activity in the presence of certain essential components (Chapter 22). Although it seems clear that progressive mutations must be the basis of evolutionary advances, the frequency of which they occur may be very low. Apparent progressive mutation so frequently encountered among microorganisms in the laboratory are probably back-mutations or reactivations rather than progressive mutations.

\section{ADAPTIVE FERMENTATION OF GALACTOSE WITHOUT FORMATION OF NEW CELLS}

S. cerevisiae is an active fermenter of galactose. One of our diploid cultures of $\mathrm{S}$. cerevisiae ( $\mathrm{Lk}$, a derivative of the $\mathrm{L}$ family, fig. 14-1, ferments galactose vigorously as measured by the evolution of gas in nitrogen. In preparing for Warburg analyses the cells grown on glucose were washed in $\mathrm{M} / 15 \mathrm{KH}_{2} \mathrm{PO}_{4}$ and resuspended in this buffer containing 4 per cent purified galactose. Under such conditions, cell counts remain constant over long periods of time. Spiegelman, Lindegren, and Hedgecock (1944) showed that adaptation to galactose fermentation occurs quickly and almost explosively after 180 minutes in the Warburg vessel (fig. 17-1). Total counts of the population showed no change in the number of individuals present and are plotted on the same graph. This is adaptation without the formation of new cells. The three hour lag period is reproducible under standard contitions and is characteristic for this strain.

\section{ADAPTATION BY "BACK-MUTATION" \\ (FROM NON-FERMENTATION TO FERMENTA TION OF GALACTOSE) FOLLOWED BY SELECTION}

Practically all haplophase stocks of S. cerevisiae ferment galactose. However, one haplophase culture of S. cerevisiae from the 
D family (fig. 14-2), most of whose members ferment galactose, was a nonfermenter of galactose; when cells of this culture (Db23B, Chap. 14) grown on glucose were suspended in 4 per cent galactose in a Warburg respirometer under anaerobic conditions no carbon dioxide was produced. However, when it was planted in galactose broth and allowed to stand for several weeks fermentation nearly always occurred; it is a "slow" fermenter of galactose. This was due to the fact that a small fraction of its vegetative cells became capable of fermenting galactose and in the presence of galactose they have a selective advantage. This was shown by the following experiments.

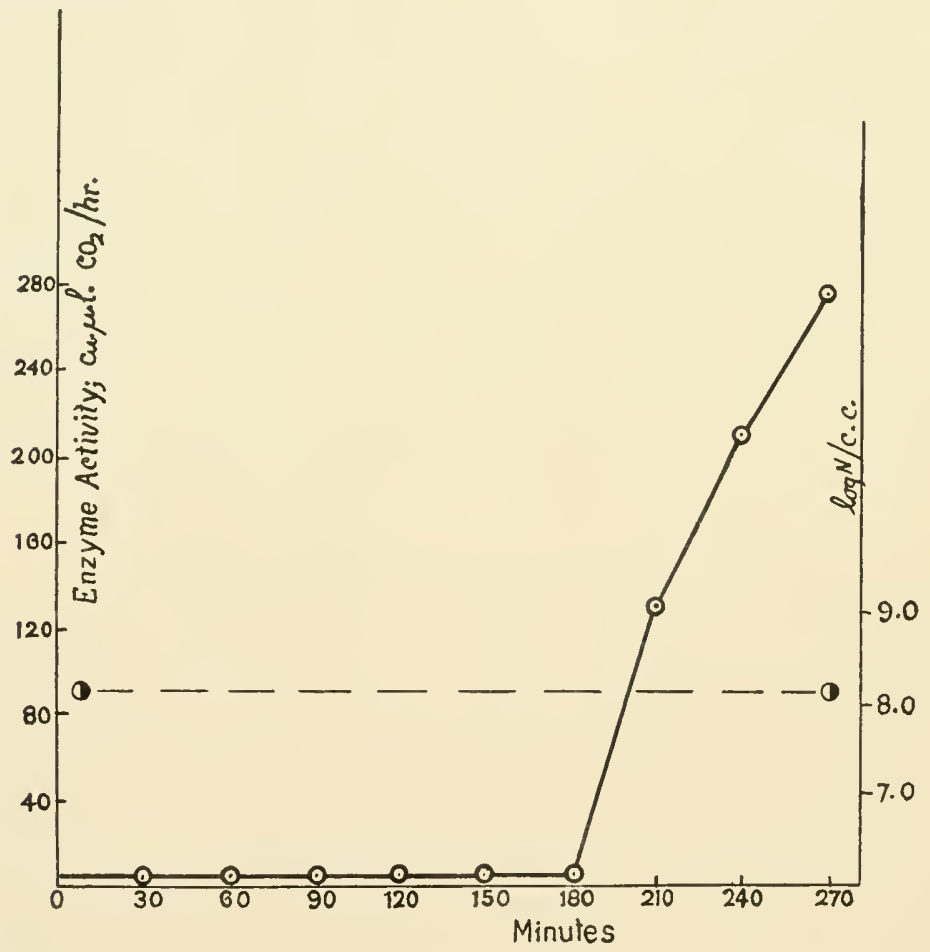

Fig. 17-1 Three-Hour Adaptation Period of Culture Lk when Stationary Population is Exposed to Galactose. $\odot=$ Enzyme activity, $\mathbb{C}=\log$ of number of cells per cubic centimeter.

Cells from a colony growing on an ordinary glucose plate were suspended in chilled galactose broth and diluted to contain about 5000 cells per cc. as determined by direct count with a haemocytometer. Of this suspension, $0.2 \mathrm{cc}$. was placed on the surface of an agar plate from which all excess fluid had been allowed to drain. The solid medium contained 4 per cent agar and 4 per cent galactose. A sterile bent rod was then rotated over the surface to produce an 
even distribution of cells. A 5 per cent agar medium containing 4 per cent galactose cooled to $39^{\circ}$ was poured over the inoculated surface. These test plates were incubated at $28^{\circ} \mathrm{C}$. for at least 48 hours.

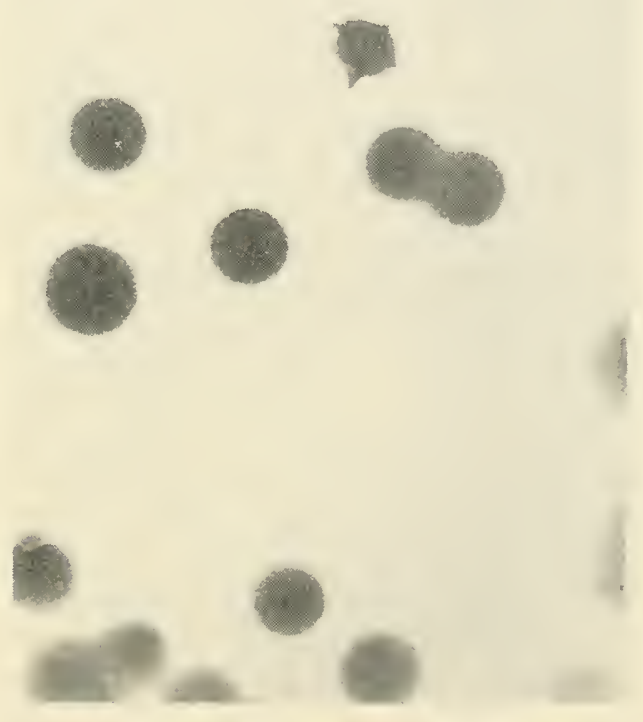

Fig. 17-2 Field of a Test Plate in which Colonies Grow Embedded Between Two Layers of Agar. Positives (gas-producers) are easily distinguished by the typical star-shaped crack in the agar surrounding them.

Colonies growing on a plate of this type either produced a sufficient amount of gas to make a star-shaped crack in the agar or grew to make a flat colony lying horizontally between the two layers of agar (fig. 17-2). Counts of ten test plates, each containing about 600 colonies, revealed that an average of from 2 to 15 per cent of the colonies produced cracks. This warrants the conclusion that from 2 to 15 per cent of the so-called nonfermenter haplophase cultures are actually capable of fermenting galactose. When cells from a fermenter colony of $\mathrm{Db} 23 \mathrm{~B}$ which had produced cracks on galactose were similarly tested, 75 to 99 per cent of the cells produced cracks.

When the diploid culture (Lk), grown on glucose, was similarly tested on galactose plates 100 per cent of the colonies produced the star-shaped cracks in the agar. 
The nonfermenter Db23B was a haploid member of the galactose fermenting " $D$ " family of S. cerevisiae and practically all other members of this family (Chap. 14 and fig. 14-2) were fermenters. Individual cells of $\mathrm{Db} 23 \mathrm{~B}$ which became termenters in galactose probably did not acquire an ability of which they were basically incapable. The gene controlling fermentation of galactose may have been temporarily degraded and when it was repaired in the presence of galactose the fermenters took over; the de novo acquisition of new capacity is probably an extraordinarily unusual occurrence. We have made thousands of tests of different genetical strains which are incapable of producing mutants capable of fermenting galactose even when they are held in galactose broth until it dries up (about four weeks).

\section{PROGRESSIVE MUTATION}

Mutations discovered in the laboratory examination of flies and fungi were at first considered comparable to the changes occurring in progressive evolution. Stadler (1932) suggested that X-ray mutations may not be comparable to those involved in progressive evolution, but may in fact amount to destruction of the gene. The acquisition of a fermentative character may depend upon an elaborate series of changes in the organism. The haploid $S$. cerevisiae culture Db23B which produced an increased number of fermenter progeny from a weakly fermenting clone may never have been completely deficient in this capacity; from 2 to 15 per cent of the population derived from a simple "non-fermenter" cell were capable of fermentation, suggesting that most of the remainder ot the population may have been capable of slow and irregular adaptation by a mechanism similar to that which gave the diploid strain the ability to adapt with a high degree of regularity. The inability of the haploid strain to ferment soon and regularly after exposure to substrate may be due to a relative deficiency of some essential substance required for the formation of measurable amounts of the enzyme rather than total inactivation of the gene controlling the fermentative process. Back-mutation or reactivation may appear to occur in a random manner, but the process itself need not be fundamentally random. This view will be developed further in Chapter 28. Real progressive changes in yeasts may be very rare phenomena. Yeasts have probably been adapted to grow as saprophytes for about half a billion years. During this period they have developed forms capable of fermenting most of the common sugars. The ability to ferment any one of these, even including glucose, may be lost; once it has been completely lost it appears impossible under ordinary laboratory oonditions to recover the ability. This is indicated by the fact that our unadaptable galactose and melibiose strains never produce a suffucient number of mutants to interfere with genetical 
analysis in pedigrees in which regular Mendelian segregation occurs. They are apparently non-reverting cultures, but they may simply have a more complex and therefore a slower adaptation mechanism than their allelic sibs which ferment more readily.

\section{THE ORIGIN OF LACTOSE FERMENTERS}

There are many Saccharomyces capable of fermenting lactose. Kluyver pointed out that no lactose fermenter is capable of fermenting maltose, and vice versa. The fact that these two characters are mutually exclusive is strong evidence that they are controlled by the same gene. Many lactose fermenters are Zygosaccharomyces and the Zygosaccharomyces are now accepted as originating from Saccharomyces. The original Saccharomyces was a maltose fermenter incapable of fermenting lactose; lactose fermentation probably originated by the transformation of the maltose-fermenting gene in Saccharomyces into a lactose-fermenting gene, rather than by the de novo production of a lactose-fermenting locus. It is possible to make a rough estimate of the time involved in the perfection of this mutation. Yeasts are not abundant in the intestinal tract of mammalian infants and this suggests that lactose-fermenting yeasts did not develop until humans began to collect milk in containers. Chance contamination of milk with wild yeasts may have resulted in the germination of haplophases whose maltose-fermenting genes mutated to lactose fermenters and became established in the new environment. The collection of milk by humans probably began between twenty and fifty thousand years ago and sometime during that long period in which lactose was available as a substrate, yeasts have been able to alter the gene controlling maltose fermentation into one which was capable of fermenting lactose. Up to the present time, however, no Saccharomyces has evolved capable of fermenting both lactose and maltose. This step in evolution would require the duplication of the lactose and the maltose alleles by unequal crossing over.

\section{UNEQUAL CROSSING OVER AND EVOLUTION}

Alexander and Bridges (1928) supposed that chromosomes become longer by duplicating small sections repeatedly through unequal crossing over. Fig. 17-3 shows how unequal crossing over (originally discovered by Sturtevant, 1925) would result in producing a slightly longer chromosome with two genes in place of the original one and a corresponding chromosome deficient for the gene is question. Bridges (1935) finally found in the salivary glands of Drosophlia dramatic evidence supporting his original view concerning the mechanism by which chromosomes increase the number of their genes. Fig. 17-4 is a reproduction of a part of the 
salivary X-chromosome of Drosophila copied from Bridges' original article. The extraordinarily high frequency of the double bands ("doublets") is most striking; each of these presumably originated by unequal crossing over. In addition, relatively large regions are repeated. In division 3 , subdivision $C$, bands 1,2 , and 3 are obviously duplications of bands 5,6 , and 7 . In subdivision $D$, bands 1 and 2 are duplicates of bands 3 and 4 . In the enlarged region in division 2, subdivision B, two pairs of conspicuous "repeats" can be observed. Since Drosophila have undergone relatively few changes over geological periods as evidenced by the discovery of typical forms in amber, it is apparent that this replication of chromosomes was well advanced hundreds of thousands of years ago. The frequently occurring recessive mutations characteristic of wild type Drosophila are practically all losses rather than progressive improvements. The losses occasionally have an advantage in fitting the organism into an ecological niche and thus have
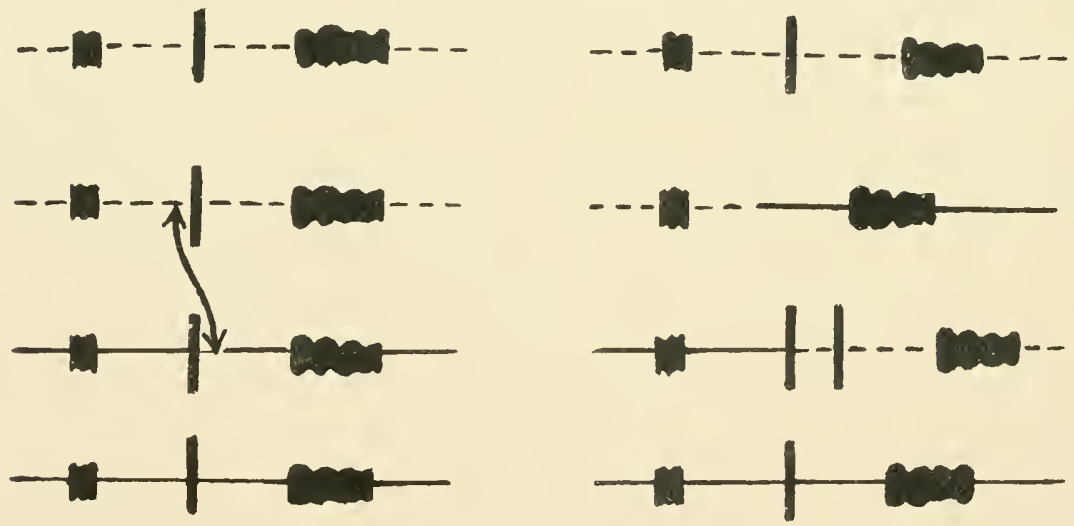

Fig. 17-3 A Diagram Showing how Unequal Crossing-Over Could Produce a Duplication of one Locus and a Slightly Longer Chromosome.

a selective advantage. Dobzahnsky (1946) has produced evidence supporting this view by showing that seasonal fluctuations occur in Drosophila genotypes and that populational shifts occur with a predominance of one genotype at one season and the alternative type at another season. Loss mutations offer a certain advantage to the lactobacilli which are unable to synthesize the vitamins and amino acids found in milk; this may reduce the synthesizing "load" on metabolism of the organism (Lwoff, 1943). Such deficiencies also serve to "lock" the organism into its particular niche, making it completely dependent on milk and thus forcing it exclusively to developing mutant forms increasingly better adapted to this substrate (if the teleology may be excused). 


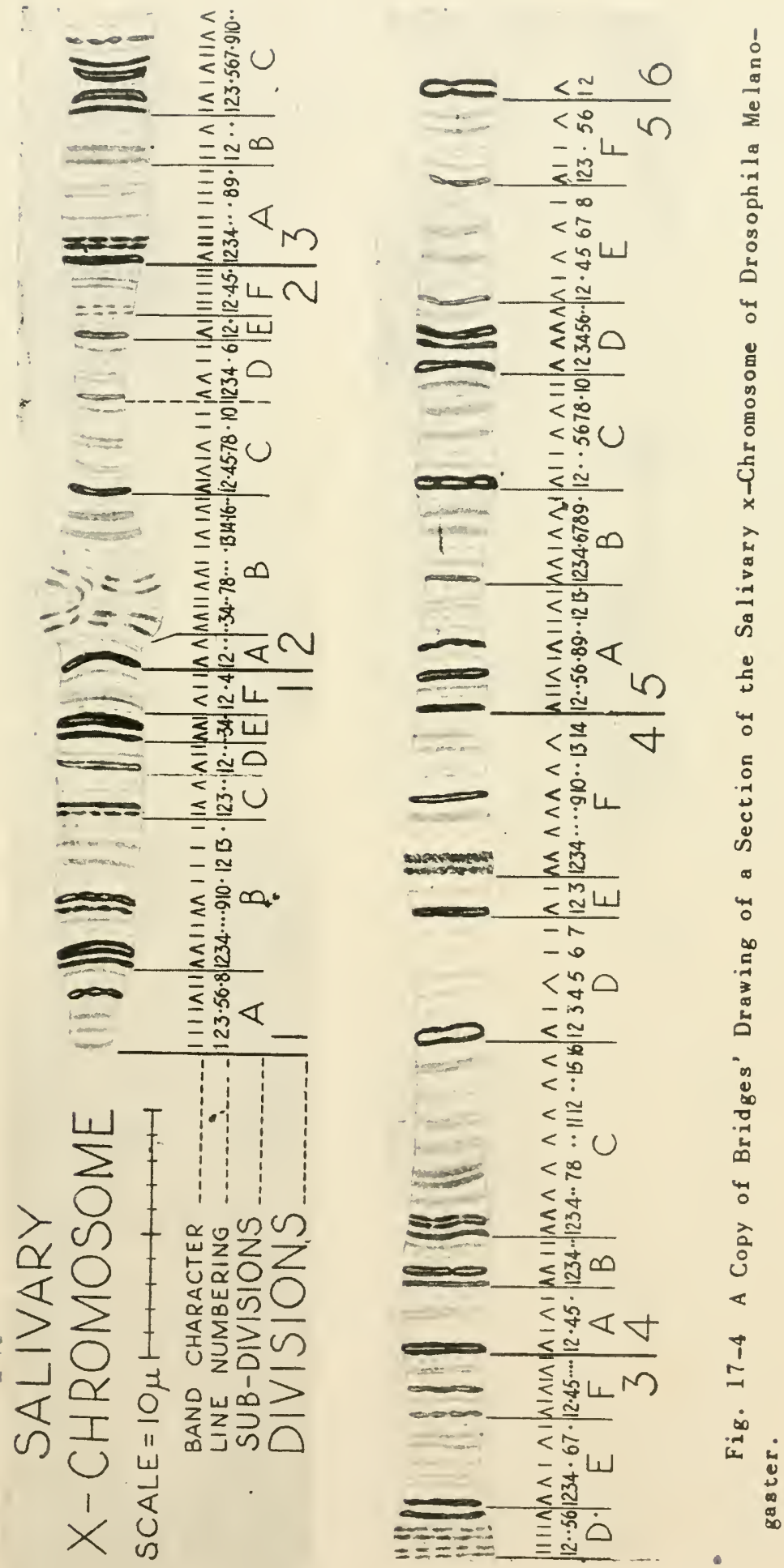


The development of a new progressive characteristic (like the ability to ferment lactose) is in a different category from these minor "loss" mutations. The Drosophila evidence indicates that the evolution of a new character probably begins with the production of a "repeat." The "repeat" produces an extra gene which is available for mutation. After the "repeat" has occurred the organism may continue for an indefinitely long time with this extra gene unchanged. When a maltose-fermenting yeast drops into milk, the maltose gene is quite useless since milk contains no maltose. The previous discussion suggests that some time within the last twenty thousand years a maltose-fermenting gene achieved the ability to ferment lactose. This information, however, is not encouraging from the standpoint of developing new progressive mutations in the laboratory. Apparently no "repeat" has occurred in Saccharomyces producing a strain able to ferment both maltose and lactose (Brettanomyces ferments both sugars slowly).

It seems probable that duplication and mutation have led to the development of an organism capable of fermenting galactose, from one originally able to ferment only glucose, but hundreds of millenia may have been required for this progressive step. The high degree of stability of the ability to ferment glucose suggests that this gene was the original mutation and the galactose gene developed from it by a repeat and a mutation. The hydrolytic mechanism capable of breaking down disaccharides may have developed independently. The sexual mechanism is capable of throwing off a variety of haplophases, such as Zygosaccharomyces lactis, which can become adapted to milk and then could be reincorporated into the wild-type organism. Judging from the failure of the available apparatus to incorporate the lactose fermenting ability into the standard organism over a period of approximately fifty thousand years, other similar progressive changes may have required extraordinarily long periods of time.

These considerations have led to the conclusion that mutations . involving an ability to ferment galactose or other sugars do not occur in the laboratory. When an experiment seems to indicate that a mutation from nonfermenter to fermenter has occurred, I prefer to interpret the result as indicating that the fermentative ability had never been lost, but that the gene controlling it had been partially degraded and some component of the medium had supplied the essential element necessary for bringing the gene into full functional activity.

\section{A HYBRID BETWEEN S. CEREVISIAE AND S. FRAGILIS}

We have attempted to hybridize lactose and maltose fermenters numerous times, but generally found the spores of lactose fermenters to be nonviable. One hybrid between the lactose fermenter, 
Saccharomyces fragilis, with kidney-shaped spores, and the maltose fermenter Saccharomyces cerevisiae, with round spores, was

Fig. 17-5 An Ascus from a Hybrid between S. Cerevisiae (Maltose-Fermenter, Round Spores) and S. Fragilis (LactoseFermenter, Kidney-Shaped Spores) Containing Two Round and Two Kidney-Shaped Spoles. They were nonviable.

successful and yielded a diploid which sporulated to produce two round and two kidney-shaped spores per ascus, fig. 17-5. The spores were nonviable, however, and the culture has been lost. 


\section{Chapter 18}

\section{DEVELOPMENT OF FERTILE BREEDING STOCKS}

The genus Saccharomyces contains species which differ in their ability to ferment various simple sugars, but there are none which decompose complex carbohydrates and generally none whose intact cells secrete proteolytic enzymes. Different species are differentiated by their requirements for the B-vitamins and mutants requiring amino acids, and nucleic acid components have been found. There is, however, considerable interspecific sterility and the project of developing fertile breeding stocks has involved an enormous amount of labor.

\section{FERMENTATIVE CHARACTERS}

Many cultures of $\mathbf{S}$. cerevisiae are incapable of producing an abundance of viable ascospores. We tested a great variety of $\mathbf{S}$. cerevisiae cultures, but a strain which we obtained from Dr. E. M. Mrak (93) and one which we isolated (FLD) are among the most vigorous which we have discovered. Since many species of Saccharomyces are differentiated from $\mathbf{S}$. cerevisiae by differences in ability to ferment different carbohydrates, we sought to bring the genes controlling these differences into our stocks. Many species are described which are incapable of fermenting galactose, but in our first atte mpts we were unable to obtain viable ascospores from any of the deficient species. Our first success was in the pedigree involving S. microellipsoideus (Chapter 19). It is a weak fermenter of galactose. We obtained a culture from Dr. L. J. Wickerham and one ascospore dissected from it produced a culture capable of fermenting galactose weakly. A hybrid between it and a haplophase from S. cerevisiae (FLD), a strong fermenter of galactose, produced only one viable ascospore. This haplophase culture was not a weak fermenter, but was apparently incapable of fermenting galactose even after standing 30 days in the fermentation tube. An outcross of the non-fermenter to a haplophase fermenter from S. cerevisiae (Mrak 93) produced a pedigree in which regular 2:2 segregations of both the $\mathrm{a} / \alpha$ and the $\mathrm{G} / \mathrm{g}$ alleles occurred in each ascus. The non-fermenters did not produce gas from galactose although they were observed continuously until they finally dried up, a period of from 40 to 50 days. Fig. 18-1 shows the steps by which the breeding stocks have been developed.

Both S. cerevisiae and S. microellipsoideus are non-fermenters of melibiose. S. carlsbergensis (Mrak 126) fermented this sugar 
and several viable ascospores were dissected from it. One of these (C1A) produced a haplophase culture which fermented both galactose and melibiose and copulated abundantly with our a haplophase No. 35 ( $\mathrm{g}$ me) and produced a pedigree in which the a/ $\alpha$ alleles segregated regularly proving the a allele in $\mathrm{S}$. carlsbergensis is identical to that in our breeding stock. However, the fermentative ability of the cultures dertved from the asci was segregated in 4:0 and $3: 1$ ratios in addition to the $2: 2$ ratios expected if a monohybrid mating had been made. Our first interpretation of these data was that more than a single pair of genes controlled the fermentation of both galactose and melibiose. Since that time many additional data have been accumulated from hybrids analyzed in the pedigree shown in fig. 18-1; they will be considered in detail in the subsequent discussion.

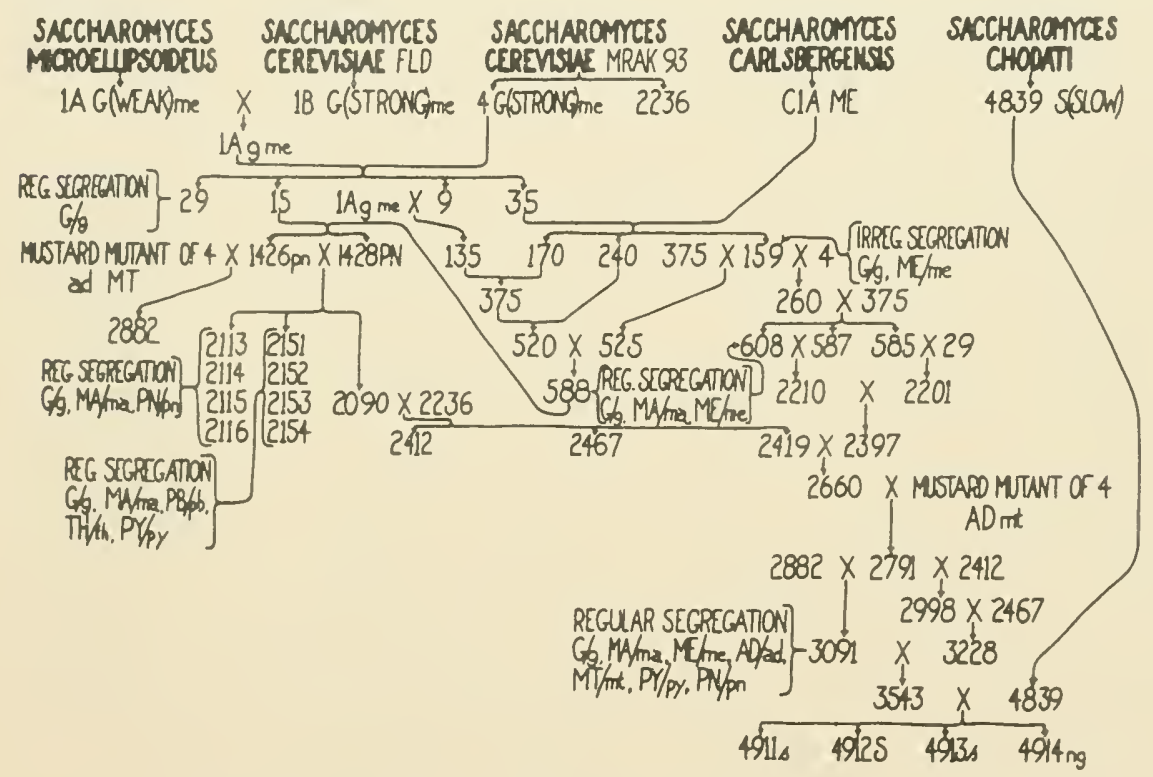

Fig. 18-1 Pedigree Showing how our Breeding Stocks have been Developed. Four different "species" were crossbred to incorporate their various differences in the stock. At several stages in the process irregular segregations occurred, especially when a heterozygote was first made, later selection produced 2:2 segregations.

S. microellipsoideus is a non-fermenter of maltose, but most of the haplophase cultures which were tested from No. 9 to No. 500 were capable of fermenting maltose. Occasional non-fermenters were found and in some asci the ratio was 2:2. A backcross to the original non-fermenter of galactose ( $1 \mathrm{~A} \mathrm{~g} \mathrm{me)} \mathrm{eventually} \mathrm{pro-}$ duced No. 520 which was mated to No. 525 , and in this pedigree regular 2:2 segregations of mating type alleles and genes con- 
trolling the fermentation of galactose, maltose, and melibiose occurred.

\section{VITAMIN DEFICIENCIES}

In addition to the ability to ferment different sugars, yeasts are differentiated by their ability or inability to grow in defined media lacking specific vitamins, amino acids, or other growth substances. Most of these nutritional characters are relative. A haplophase culture which we designate as capable of growth in the absence of pantothenate will produce full growth in the deficient medium in about 36 hours, while the so-called non-synthesizer may require 5 or 6 days to attain full growth.

The mating of No. 588 by No. 15 produced a pedigree in which some asci showed regular segregation of genes affecting the ability to grow in media lacking specific vitamins. S. cerevisiae is capable of rapid growth in a defined medium lacking pyridoxine, while S. carlsbergensis is not. S. carlsbergensis can grow in the absence of biotin, although S. cerevisiae cannot. S. microellipsoideus also requires biotin. S. microellipsoideus cannot grow in the absence of inositol, which is not required by either S. carlsbergensis or S. cerevisiae. The pedigree including the segregants from the C1A by No. 35 hybrid did not regularly show 2:2 segregations of factors controlling vitamin synthesis. However, the backcross of No. $588 \times$ No. 15 produced some asci in which $2: 2$ segregations of the ability and inability to grow in pantothenate deficient medium occurred. The mating No. $1426 \times$ No. 1428 produced a hybrid in which the genes controlling mating type, fermentation of galactose and maltose, and the ability to grow in defined medium lacking of the following nutrilites: pantothenate, paraminobenzoic acid, thiamin, and pyridoxine were all segregated 2:2 in nearly every ascus. Two to two segregation of genes affecting the synthesis of biotin did not occur in the particular pedigree although the stock was descended from clones differentiated in regard to biotin.

Haplophase No. 2236 was derived from S. cerevisiae (Mrak 93). A hybrid of No. $2236 \times$ No. 2090 eventuaily produced No. 2660. Haplophase No. 4 was treated by Dr. E. L. Tatum and Mr. S. E. Reaume with nitrogen mustard gas and a mutant incapable of growing in the absence of methionine was obtained by them. This was mated to No. 2660 and introduced methionine dependence into the stock. A second mustard mutant of No. 4, adenine dependence was produced by them and we introduced it into the stock by mating it 
with No. 1426 to produce No. 2882. A mating of No. 2882 and No. 2791 introduced adenine dependence into the methionine dependent stock. The mating of No. $3091 \times$ No. 3228 produced a hybrid heterozygous for at least 9 alleles, all of which showed regular 2:2 segregation in nearly every ascus.

Saccharomyces chodati (fig. 18-2) is ordinarily described as a slow fermenter of sucrose. The culture which we obtained from Dr. L. J. Wickerham was a slow fermenter and the only copulating haplophase (No. 4839) which we derived from it was also a slow fermenter. The original culture produced only two spores per as-

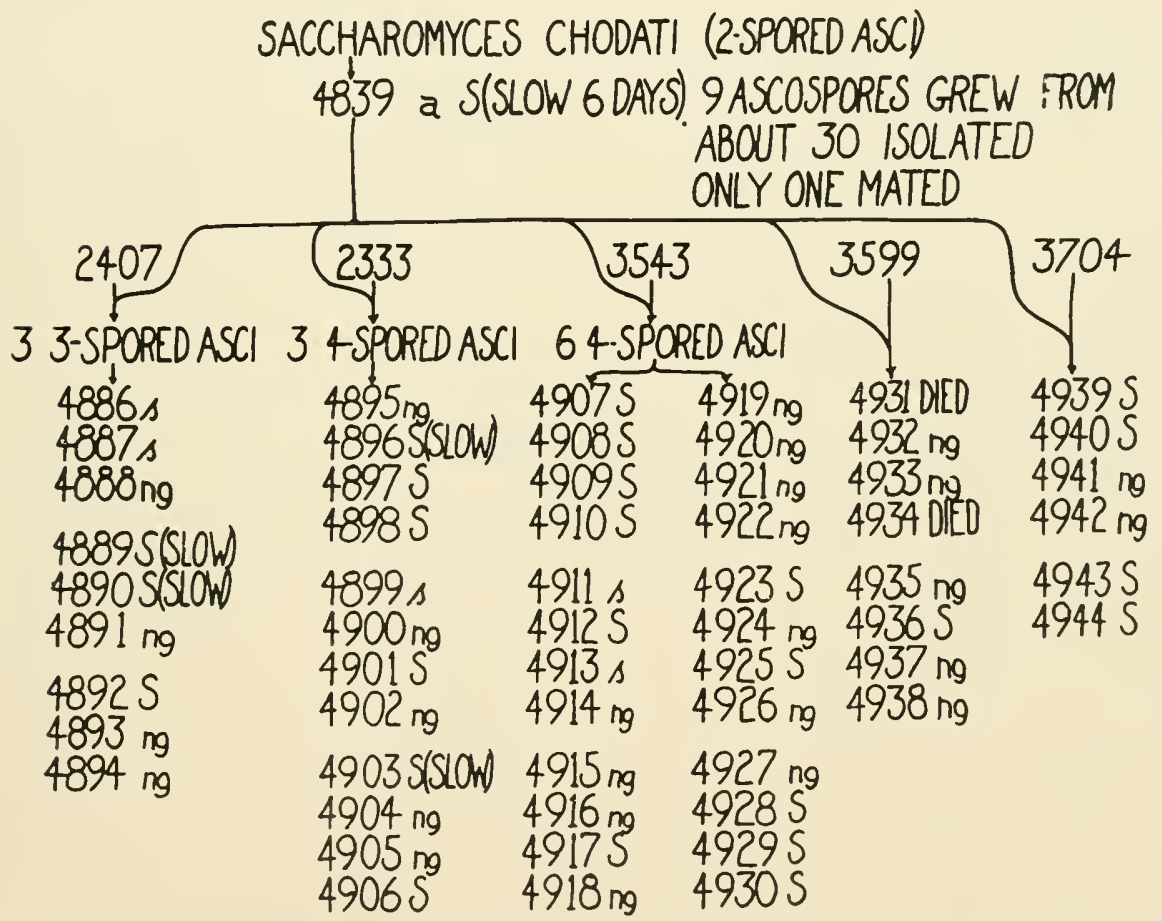

Fig. 18-2 Pedigree Showing the Origin of a Non-Fermenter of Sucrose by Mating a Haplophase of S. Chodati with our Fertile Haplophase.

cus but its descendents of it eventually produced hybrids from which asci containing 4 viable spores were obtained. A hybrid (No. 4839 $\mathrm{x}$ No. 3543 ) produced a 4 -spored ascus from which 3 viable spores were isolated. Two were non-fermenters of sucrose. Five other asci isolated from the hybrid are shown in fig. 18-2. One contained 4 sucrose fermenters but many irregularities in viability occurred. In the figure "n.g." means that the spore did not germ- 
inate after isolation, "died" means that the spore produced a small haploid culture which could not be transferred. Haplophase No. 4911 was a fertile non-fermenter of sucrose and it was used to introduce the character into our stocks in the series of steps shown in fig. 18-3. Many irregular segregations occurred but eventually

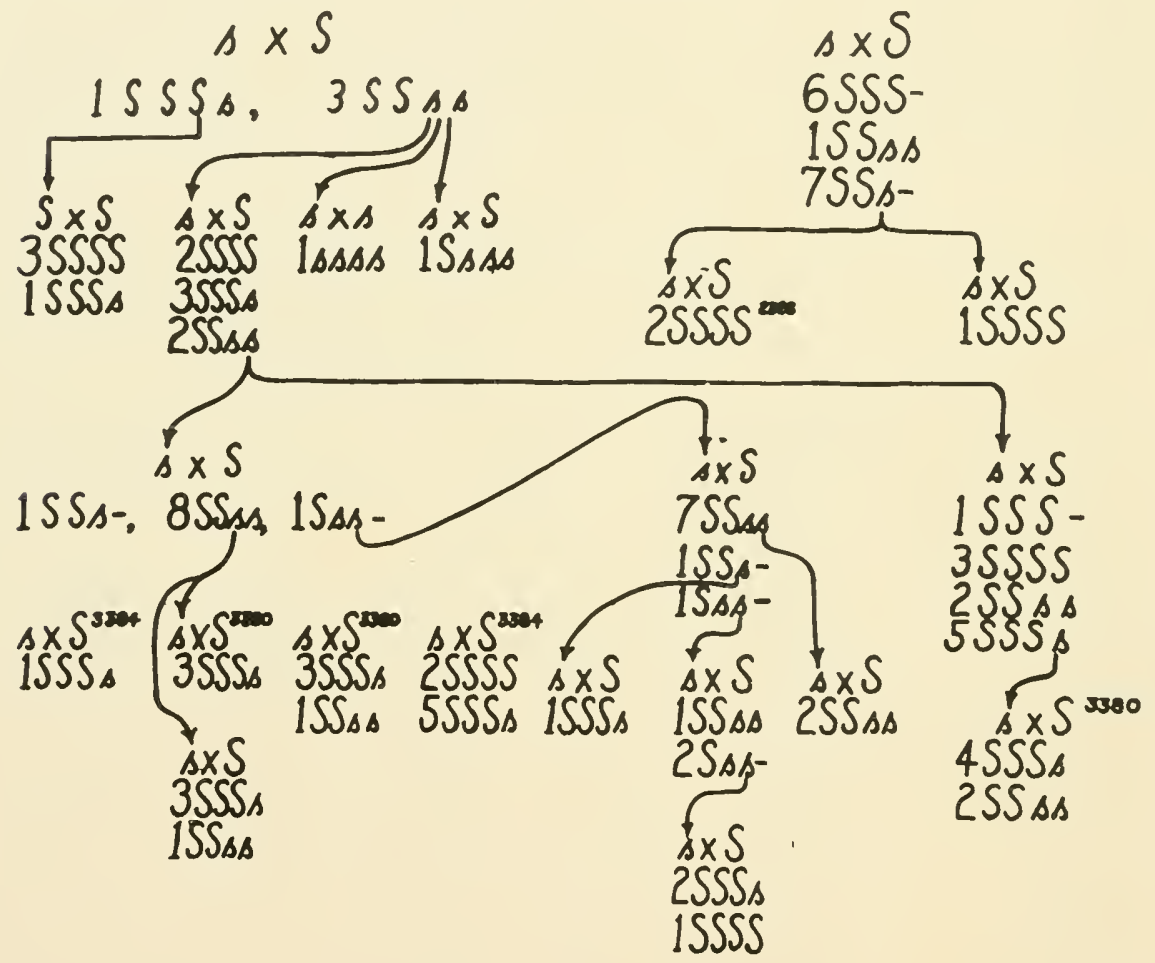

Fig. 18-3 Irregular Segregations in the Sucrose Pedigree.

a hybrid produced 2:2 segregations and the sucrose non-fermenting allele was introduced into the complex stock. Some of our present stocks contain 14 alleles, all of which segregate 2:2 in nearly every ascus.

HAPLOID NON-FERMENTERS FROM A WEAK FERMENTER BY A STRONG FERMENTER HYBRID

It is important to note that both the original nonfermenter of galactose and the original nonfermenter of sucrose were derived 
from mating of weak or slow fermenters by strong fermenters. The simplest explanation according to classical Mendelian theory is that two different alleles were involved, both controlling the fermentation and the nonfermenter was the segregant carrying both negative alleles. This explanation will be tested (Chapter 26) and shown to be inadequate. It is proposed that only a single pair of alleles is involved and that the phenomenon results from the transfer of fermentative ability from the weaker to the stronger allele in the heterozygote. 


\section{Chapter 19}

\section{REGULAR MENDELIAN INHERITANCE}

\section{A GENE-CONTROLLED ADAPTIVE ENZYME}

The fermentation of galactose by $\mathrm{S}$. cerevisiae is an adaptive reaction (fig. 17-1). Galactozymase is not present in measurable amounts in cells grown in glucose, and only appears in cells placed in galactose after an incubation period of from 3 to 8 hours. Cells which have been adapted to ferment galactose lose this ability when removed from the substrate and have to be readapted to use it fermentatively. The following genetical analysis shows that the difference between an adaptable culture and an unadaptable culture is controlled by a single Mendelian gene. The ability to adapt is not transferred through the cytoplasm as the adaptive process might suggest; cells carrying the recessive gene are incapable of fermenting galactose.

\section{THE ORIGIN OF A NONFERMENTER OF GALACTOSE}

The demonstration that a given property is under genetic control requires the production of a hybrid between an individual possessing the property and one without it and the demonstration of regular segregation of individuals with and without the property among the progeny of the hybrid. However, the ability to ferment galactose is widely distributed in the genus Saccharomyces, and we had great difficulty in finding a suitable nonfermenting haplophase capable of producing hybrids in which we could study the inheritance of this character. We obtained from Dr. L. J. Wickerham a culture of S. microellipsoideus (NRRL, No. 210) which was a relatively weak fermenter of both galactose and melibiose. Four asci dissected from $\mathrm{S}$. microellipsoideus produced 9 cultures which fermented galactose slowly. A hybrid (i x z, Table 19-1), Lindegren and Lindegren, 1947) was made between one of these and S. cerevisiae (F.L.D.), which ferments galactose vigorously. The hybrid was brought to sporulation and the single ascospore (No. 1A) grown from it was found to be incapable of fermenting galactose.

\section{TETRAD ANALYSIS OF AN $\propto \mathrm{G} / \mathrm{ag}$ HYBRID}

The nonfermenter was mated with a haplophase galactose fermenter originating from S. cerevisiae (Mrak, No. 93). An a-mating 


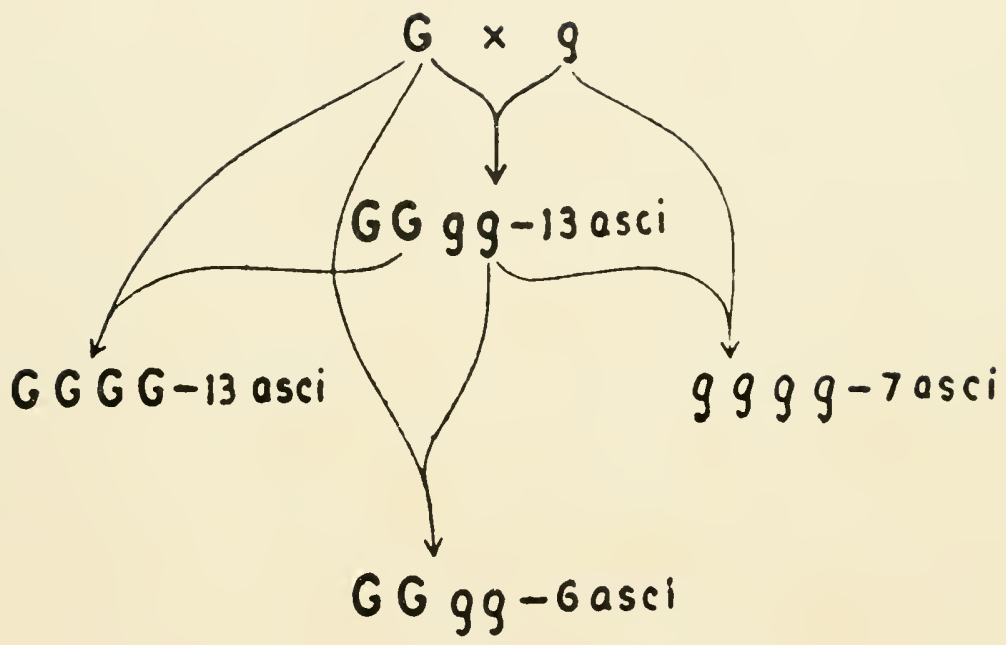

Fig. 19-1 A Diagram Sumarizing the Data Presen ted on the Inheritance of Galactose-Fermenting Abiity in Table 19-2. No exception to standard Mendelian inheritance was found. Thirteen asci were analyzed from a heterozygous hybrid made by mating a galactose fermenter (G) by a nonfermenter ( $g$ ); two spores in each of these asci carried the dominant gene controlling fermentation of galactose, and two carried the recesaive a llels. A backcross of fermenter to the fermenter parent produced thirteen asci; all four spores in each of these asci carried the fermenting gene. A backcross of the nonfermenter to the nonfermenting parent produced seven asci, each of which contained four nonfermenting spores. A heterozygous zygote was produced by backcrossing a nonfermenter to the fermenting parent; six asci were analyzed and each contained two fermenting and two nonfermenting spores. This analysis shows quite convincingly that in some pedigrees the genes controlling fermentation of galactose may behave in a regular Mendelian manner. 
type, galactose-fermenting $(\alpha G)$ culture, was mated with the amating-type nonfermenter (ag). Thirteen asci were analyzed from the a Gxag hybrid which was demonstrated to be heterozygous for mating type $(a / a)$ and galactose fermentation $(\mathrm{G} / \mathrm{g})$.

Table 19-1

The Origin of a Nonfermenter of Galactose

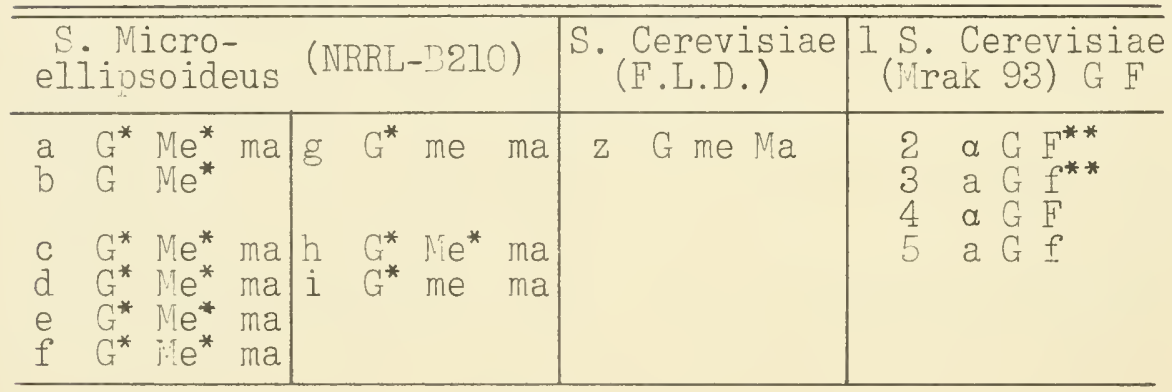

$$
\begin{aligned}
& G^{*} \text { me ma } x \text { G me Ma } \\
& \text { IA a g me Ma }
\end{aligned}
$$

* Slow fermentation.

* "F" and "f" symbolize the "flaking" or clumping characteristic of haplophase cultures. See discussion in Chapter 9 to Chapter 11.

The haplophase cultures derived from the asci were designated a or a by mating them with standard mating-type strains derived from Mrak's culture No. 93 of S. cerevisiae. Galactose fermentation was tested by inoculating small tubes of nutrient galactose broth containing smaller inverted tubes. In all these and subsequent tests the sugar solution was sterilized by filtration through a Seitz filter and added to the autoclaved medium to make a 4 per cent solution of the carbohydrate in the finished medium. Fermentation in the positive cultures was recognizable within 24 or 48 hours, but the negative cultures were not discarded until they had been under observation for more than three weeks.

Thirteen asci were obtained by mating an a galactose-fermenter by an a galactose-fermenter (fig. 19-1). Mating type segregated in all asci but the zygote was homozygous for galactose fermentation. Another hybrid was made by backcrossing a non-fermenter to the original fermenter parent. Both mating type and galactose fermentation segregated regularly in 6 asci. Finally a hybrid homozygous for the nonfermenter alleles was produced by backcrossing a nonfermenter to the original parent. Mating type segregated regu- 
larly and no fermenters appeared among the progeny. These data proved that the ability to ferment galactose is controlled by a conventional Mendelian gene.

\section{MENDELIAN INHERITANCE OF GENES AFFECTING FERMENTA- TIVE AND VITAMIN-SYNTHESIZING ABILITY}

Burkholder's medium (Chapter 21) is an excellent diagnostic medium for distinguishing pantothenate "synthesizers" from "nonsynthesizers," because a so-called nonsynthesizer grows rapidly in this medium containing pantothenate, but requires weeks or months to produce growth in its absence. However, genes affecting vitamin synthesis are apparently transmitted in some pedigrees in a non-Mendelian way. The first pedigree on the inheritance of "Vitamin-synthesizing" ability in Saccharomyces (Lindegren, 1945) failed not only to reveal regular Mendelian inheritance of this ability but also of genes controlling the fermentation of carbohydrates. In our selected inbred strains, the ability to ferment galactose and maltose is transmitted in a regular Mendelian manner, and the genes affecting the synthesis of paraminobenzoic acid, pantothenate, pyridoxine, and thiamin are transmitted with corresponding regularity. These genes are described as "affecting" rather than "controlling" the synthesis of vitamins, because we have not discovered any absolute deficiencies in yeasts. A socalled nonsynthesizer of pantothenate eventually will grow in a medium without the addition of pantothenate, although some cultures do not begin growth until they have stood in the tubes for nearly a month.

In genetical analysis, it is relatively unimportant whether absolute or partial deficiencies are dealt with; all that is required is a clear-cut differentiation of the haploid offspring of a hybrid into two different categories. This is easily effected in our present yeast pedigrees by using Burkholder's medium with and without added pantothenate. Genes affecting pantothenate and pyridoxine synthesis are easily diagnosed; the "nonsynthesizers" do not begin to grow until a week after planting while the "synthesizers" attain nearly full growth after 48 hours. The former may produce a turbidity reading of between 200 and 300 , while the latter still show a reading of between 0 and 5 . After the tubes have been held for two months it is difficult to distinguish them, but at 4 or 5 days the difference is pronounced. Cultures differing in genes which affect the synthesis of thiamin and paraminobenzoic acid show distinct differences at the end of 48 hours, but by the fourth day it is difficult to tell them apart. However, any clear-cut segregation of the progeny into two classes supplies the geneticist with an adequate gene "marker." 
Table 19-2

Pedigree Showing the Inheritance of Hating Type $(a / a)$, Galactose Fermentation $G / g$, and $A$ utoagglutination $(f / F)$

\begin{tabular}{|c|c|c|c|c|c|c|c|c|c|c|}
\hline & & $a G \mathrm{me}$ & F (Tabl & le $19-1)^{4}$ & $\begin{array}{l}\mathrm{x} \\
\mathrm{x}\end{array}$ & $1 \mathrm{~A}$ & a g me & (Table 19-1) & & \\
\hline $\begin{array}{r}9 \\
10 \\
11\end{array}$ & $\begin{array}{lll}a & \dot{b} \\
a & g \\
a & G & F\end{array}$ & $\begin{array}{l}12 \\
13 \\
14 \\
15\end{array}$ & $\begin{array}{lll}a & g & F \\
a & G & f \\
a & G & f \\
a & g & F\end{array}$ & $\begin{array}{l}16 \\
17 \\
18 \\
19\end{array}$ & $\begin{array}{l}\mathrm{a} g \\
\mathrm{a} \\
\mathrm{a} \\
\mathrm{a}\end{array}$ & $\begin{array}{l}g \\
G \\
g \\
F\end{array}$ & $\begin{array}{l}20 \\
21 \\
22\end{array}$ & $\begin{array}{lll}a & g & f \\
a & G & f \\
a & G & F\end{array}$ & $\begin{array}{l}23 \\
24 \\
25 \\
26\end{array}$ & $\begin{array}{lll}a & & \\
a & G & f \\
a & g & F \\
a & G & F\end{array}$ \\
\hline $\begin{array}{l}27 \\
28 \\
29 \\
30\end{array}$ & 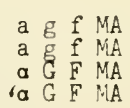 & $\begin{array}{l}31 \\
32 \\
33 \\
34\end{array}$ & $\begin{array}{lll}a & G & f \\
\alpha & G & f \\
\alpha & g & F \\
a & g & F\end{array}$ & $\begin{array}{l}35 \\
36 \\
37 \\
38\end{array}$ & $\begin{array}{l}a g \\
\alpha \\
a \\
a\end{array}$ & $\begin{array}{l}g \\
G \frac{F}{F} \\
g \\
G \\
f\end{array}$ & $\begin{array}{l}124 \\
125 \\
126 \\
127\end{array}$ & $\begin{array}{lll}a & G & f \\
\alpha & G & f \\
a & g & F \\
\alpha & g & F\end{array}$ & $\begin{array}{l}128 \\
129 \\
130\end{array}$ & $\begin{array}{lll}a & g & F \\
a & G & f \\
\alpha & g & f\end{array}$ \\
\hline $\begin{array}{l}146 \\
147 \\
148 \\
149\end{array}$ & 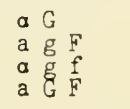 & $\begin{array}{l}150 \\
151 \\
152 \\
153\end{array}$ & $\begin{array}{lll}a & G & f \\
a & g & F \\
a & G & F \\
a & g & f\end{array}$ & $\begin{array}{l}154 \\
155 \\
156 \\
157\end{array}$ & $\begin{array}{ll}\alpha & g \\
\mathrm{a} & \mathrm{g} \\
\mathrm{a} & \mathrm{G} \\
\mathrm{a} & \mathrm{g}\end{array}$ & $\begin{array}{ll}g \\
G \\
G\end{array}$ & & & & \\
\hline & & . & $a$ & G me $\quad \stackrel{4}{F}$ & $\begin{array}{l}\mathrm{x} \\
\mathrm{x}\end{array}$ & 13 & a $G$ me $f$ & & & \\
\hline $\begin{array}{l}40 \\
41 \\
42 \\
43\end{array}$ & $\begin{array}{lll}a & G & f \\
a & G & F \\
a & G & F \\
a & G & f\end{array}$ & $\begin{array}{l}44 \\
45 \\
46\end{array}$ & $\begin{array}{lll}a & G & f \\
a & G & f \\
a & G & F\end{array}$ & $\begin{array}{l}47 \\
48 \\
49\end{array}$ & $\begin{array}{l}a G G \\
\alpha G G \\
a G\end{array}$ & $\begin{array}{l}G \\
G \underset{F}{F}\end{array}$ & $\begin{array}{r}98 \\
99 \\
100\end{array}$ & $\begin{array}{lll}a & G & f \\
a & G & F \\
a & G & f\end{array}$ & $\begin{array}{l}101 \\
102 \\
103\end{array}$ & $\begin{array}{lll}a & G & f \\
a & G & f \\
a & G & F\end{array}$ \\
\hline $\begin{array}{l}104 \\
105 \\
106 \\
107\end{array}$ & $\begin{array}{lll}a & G & f \\
a & G & F \\
a & G & F \\
a & G & f\end{array}$ & $\begin{array}{l}108 \\
109 \\
110 \\
111\end{array}$ & $\begin{array}{lll}a & G & F \\
a & G & f \\
a & G & F \\
a & G & f\end{array}$ & & & & & & & \\
\hline & & & & G me $\stackrel{4}{F}$ & $\begin{array}{l}\mathrm{x} \\
\mathrm{x} \\
\end{array}$ & 11 & a G me F & & & \\
\hline $\begin{array}{l}51 \\
52 \\
53 \\
54\end{array}$ & $\begin{array}{lll}\text { a } & G & F \\
a & G & f \\
a & G & F \\
\alpha & G & F\end{array}$ & $\begin{array}{l}55 \\
56 \\
57\end{array}$ & $\begin{array}{lll}a & G & f \\
a & G G \\
a & G & f\end{array}$ & $\begin{array}{l}59 \\
60 \\
61 \\
62\end{array}$ & 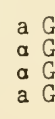 & $\begin{array}{ll}G & f \\
G ~ \\
G ~ \\
G F\end{array}$ & $\begin{array}{l}71 \\
72 \\
73 \\
74\end{array}$ & $\begin{array}{llll}a & G & F & M a \\
a & G & F & M a \\
a & G & F & M a \\
a & G & f & M a\end{array}$ & $\begin{array}{l}75 \\
76 \\
77 \\
78\end{array}$ & $\begin{array}{lll}a & G & F \\
a & G & F \\
a & G & F \\
a & G & f\end{array}$ \\
\hline $\begin{array}{l}112 \\
113 \\
114 \\
115\end{array}$ & $\begin{array}{lll}a & G & F \\
\alpha & G & f \\
a & G & F \\
a & G & F\end{array}$ & & & & & & & & & \\
\hline & & & & G me $\stackrel{4}{F}$ & $\begin{array}{l}\mathrm{x} \\
\mathrm{x} \\
\end{array}$ & 15 & a g me F & & & \\
\hline $\begin{array}{l}64 \\
65 \\
66 \\
67\end{array}$ & $\begin{array}{lll}\alpha & G & F \\
a & g & F \\
a & G & F \\
\alpha & g & F\end{array}$ & $\begin{array}{l}68 \\
69 \\
70\end{array}$ & $\begin{array}{lll}\alpha & g & F \\
a & G & F \\
\alpha & g & F\end{array}$ & $\begin{array}{l}86 \\
87 \\
88 \\
89\end{array}$ & 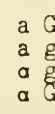 & $\begin{array}{ll}G & F \\
g & F \\
g & F \\
G & F\end{array}$ & $\begin{array}{l}90 \\
91 \\
92 \\
93\end{array}$ & $\begin{array}{lll}a & G & F \\
\alpha & g & F \\
a & g & F \\
a & G & F\end{array}$ & $\begin{array}{l}94 \\
95 \\
96 \\
97\end{array}$ & $\begin{array}{lll}a & G & f \\
a & g & F \\
a & G & F \\
a & g & f\end{array}$ \\
\hline $\begin{array}{l}142 \\
143 \\
144 \\
145\end{array}$ & $\begin{array}{lll}a & g & F \\
\alpha & G & F \\
\text { a } & G & F \\
\alpha & g & F\end{array}$ & & & & & & & & & \\
\hline & & & & a g me $M$ & $\begin{array}{ll}\mathrm{A} & \mathrm{x} \\
\mathrm{CA} & \mathrm{x} \\
\end{array}$ & $\begin{array}{ll}x & 9 \\
x & \\
\end{array}$ & $a g f$ & & & \\
\hline $\begin{array}{l}79 \\
80 \\
81 \\
82\end{array}$ & $\begin{array}{llll}a & g & F & M A \\
a & g & f & M A \\
a & g & F & M A \\
a & g & f & A\end{array}$ & $\begin{array}{l}83 \\
84 \\
85\end{array}$ & $\begin{array}{lll}a & g & f \\
a & g & f \\
a & g & F\end{array}$ & $\begin{array}{l}116 \\
117 \\
118 \\
119\end{array}$ & $\begin{array}{l}a \\
a \\
a \\
a \\
a \\
a \\
a\end{array}$ & $\begin{array}{ll}g & f \\
g & f \\
g & f \\
g & f\end{array}$ & $\begin{array}{l}120 \\
121 \\
122 \\
1<3\end{array}$ & $\begin{array}{lll}a & g & f \\
a & g & f \\
a & g & f \\
a & g & f\end{array}$ & $\begin{array}{l}131 \\
132 \\
133\end{array}$ & $\begin{array}{lll}a & g & f \\
a & g & f \\
a & g & f\end{array}$ \\
\hline $\begin{array}{l}134 \\
135 \\
136 \\
137\end{array}$ & $\begin{array}{lll}a & g & f \\
a & g & f \\
\alpha & g & f \\
a & g & f\end{array}$ & $\begin{array}{l}138 \\
139 \\
140 \\
141\end{array}$ & $\begin{array}{lll}a & g & f \\
a & g & f \\
a & g & F \\
a & g & f\end{array}$ & & & & & & & \\
\hline
\end{tabular}


Table 19-3 describes 8 asci dissected from a hybrid heterozygous for mating type, galactose fermentation, maltose fermentation, and a pair of genes affecting the ability of the organism to grow in Burkholder's medium wi thout added pantothenate. The - and + signs under the columns G and MA indicate whether or not the organism produced gas in a medium containing galactose of maltose respectively. The figures under the column PAN show the turbidity reading registered in a Klett Photoelectric Colorimeter, after four days in a culture tube of Burkholder's medium without added pantothenate.

\section{Table 19-3}

Analysis of Asci from a Hybrid Heterozygous for Hating Type, Fermentation of Galactose and Maltose, and Genes Affecting the Synthesis of Pantothenate (Ascospores from Hybrid $1426(\mathrm{a} \mathrm{g} M A P A N) \times 1428(\mathrm{a} G \mathrm{ma}$ pan))

\begin{tabular}{|c|c|c|c|c|c|c|c|c|c|}
\hline \multirow{2}{*}{$\begin{array}{l}\text { Culture } \\
\text { No. }\end{array}$} & Type & G & MA & PAN & \multirow{2}{*}{$\begin{array}{l}\text { Culture } \\
\text { No. }\end{array}$} & Type & G & $\mathrm{MA}$ & PAN \\
\hline & \multicolumn{4}{|c|}{1} & & \multicolumn{4}{|c|}{2} \\
\hline 2101 & a & - & - & 274 & 2105 & $a$ & + & - & 5 \\
\hline 2102 & a & $\overline{-}$ & $=$ & 160 & 2106 & 2 & $\underline{I}$ & + & 190 \\
\hline 2103 & a & + & + & 3 & 2107 & $a$ & - & $t$ & 2 \\
\hline 2104 & $a$ & & + & 4 & 2108 & a & + & - & 254 \\
\hline \multicolumn{5}{|c|}{3} & \multicolumn{5}{|c|}{4} \\
\hline 2109 & a & - & + & 6 & 2113 & $a$ & - & - & 3 \\
\hline 2110 & $\alpha$ & + & - & 2 & 2114 & $a$ & - & + & 210 \\
\hline 2111 & $a$ & \pm & $\overline{-}$ & $145+$ & 2115 & 2 & + & - & 220 \\
\hline 2112 & 2 & + & + & 274 & 2116 & 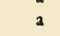 & + & + & 0 \\
\hline \multicolumn{5}{|c|}{$s$} & \multicolumn{5}{|c|}{6} \\
\hline 2121 & a & - & + & 0 & 2125 & a & & & 2 \\
\hline 2122 & $a$ & + & - & 200 & 2126 & $a$ & + & + & 270 \\
\hline 2123 & $a$ & $\mp$ & $\overline{-}$ & 5 & 2127 & a & - & - & 4 \\
\hline 2124 & 2 & - & + & 345 & $2 \mathrm{i} 28$ & $a$ & - & - & 200 \\
\hline \multicolumn{5}{|c|}{7} & \multicolumn{5}{|c|}{8} \\
\hline 2147 & $a$ & + & + & 290 & 2151 & $a$ & & + & 4 \\
\hline 2148 & a & - & + & $137+$ & 2152 & a & & + & 250 \\
\hline 2149 & a & & - & 3 & 2153 & a & & - & $140+$ \\
\hline 2150 & a & & - & 8 & 2154 & a & & - & 4 \\
\hline
\end{tabular}


Table 19-4

Analysis of Asci from a Hybrid Heterozygous for Wating Type, Permentation of Galactose and Maltose, and Genes Affecting the Synthesis of Paraminobenzoic Acid. Thiamin and Pyridoxine (Ascospores from Hybrid. $2236 \times 2090$ (a $G M A$ pab th py $x$ a $g$ ma $P A B$ TH $P Y)$ )

\begin{tabular}{|c|c|c|c|c|c|c|c|c|c|c|c|c|c|}
\hline $\begin{array}{c}\text { Culture } \\
\text { No. }\end{array}$ & Type & G & MA & PAB & TH & PY & $\begin{array}{c}\text { Culture } \\
\text { No. }\end{array}$ & Type & G & MA & PAB & TH & PY \\
\hline \multicolumn{7}{|c|}{1} & \multicolumn{7}{|c|}{4} \\
\hline 2409 & $a$ & + & - & - & - & + & 2419 & $a$ & - & - & + & + & - \\
\hline 2410 & $a$ & - & - & + & + & - & 2420 & a & - & + & + & - & - \\
\hline 2411 & $a$ & + & + & - & - & + & 2421 & a & + & + & - & + & + \\
\hline 2412 & 2 & - & + & + & + & - & 2422 & $\alpha$ & + & - & - & - & + \\
\hline \multicolumn{7}{|c|}{5} & \multicolumn{7}{|c|}{6} \\
\hline 2423 & $a$ & - & - & - & - & - & 2427 & a & + & - & + & + & - \\
\hline 2424 & $a$ & + & + & + & + & + & 2428 & $a$ & - & + & - & - & + \\
\hline 2425 & a & + & - & - & - & + & 2429 & $a$ & - & - & - & + & - \\
\hline 2426 & a & + & + & + & + & - & 2430 & a & + & + & + & - & + \\
\hline \multicolumn{7}{|c|}{7} & \multicolumn{7}{|c|}{8} \\
\hline 2431 & 3 & + & + & - & + & - & 2435 & a & + & - & - & - & + \\
\hline 2432 & a & + & - & + & + & + & 2436 & 3 & - & + & + & + & - \\
\hline 2433 & $a$ & - & + & + & - & + & 2437 & $a$ & - & - & + & - & + \\
\hline 2434 & $\alpha$ & - & - & - & - & - & 2438 & $\alpha$ & + & + & - & + & - \\
\hline \multicolumn{7}{|c|}{9} & \multicolumn{7}{|c|}{10} \\
\hline 2439 & $a$ & + & - & - & - & - & 2443 & a & + & + & + & - & - \\
\hline 2440 & $a$ & - & + & + & + & + & 2444 & $a$ & - & + & - & - & + \\
\hline 2441 & 3 & - & + & - & + & + & 2445 & $a$ & + & - & + & + & - \\
\hline 2442 & died & & & & & & 2446 & died & & & & & \\
\hline \multicolumn{7}{|c|}{11} & \multicolumn{7}{|c|}{12} \\
\hline 2447 & 2 & - & - & + & - & - & 2451 & $\mathbf{a}$ & - & - & + & - & + \\
\hline 2448 & $a$ & + & - & - & + & + & 2452 & $a$ & + & + & + & + & + \\
\hline 2449 & a & - & + & + & + & - & 2453 & a & + & + & - & + & - \\
\hline 2450 & $a$ & + & + & - & - & + & 2454 & a & - & - & - & - & - \\
\hline
\end{tabular}


Table 19-4 (Continued)

\begin{tabular}{|c|c|c|c|c|c|c|c|c|c|c|c|c|c|}
\hline $\begin{array}{c}\text { Culture } \\
\text { No. }\end{array}$ & Type & G & MA & PAB & TH & PY & $\begin{array}{c}\text { Culture } \\
\text { No. }\end{array}$ & Type & G & MA & $\mathrm{PAB}$ & TH & PY \\
\hline \multicolumn{7}{|c|}{13} & \multicolumn{7}{|c|}{14} \\
\hline 2455 & $a$ & + & - & - & - & + & 2459 & $a$ & + & + & - & - & - \\
\hline 2456 & 2 & - & + & + & - & $\dot{t}$ & 2460 & a & + & - & - & - & + \\
\hline 2457 & $a$ & - & + & + & + & - & 2461 & $a$ & - & $\bar{I}$ & + & + & \pm \\
\hline 2458 & & & & & & & 2462 & & & & & & - \\
\hline \multicolumn{7}{|c|}{15} & \multicolumn{7}{|c|}{16} \\
\hline 2463 & a & + & + & - & - & + & 2467 & a & + & - & - & - & - \\
\hline 2464 & $a$ & - & + & + & + & - & 2468 & $a$ & - & - & + & + & + \\
\hline 2465 & 3 & - & - & + & + & - & 2469 & 3 & + & + & - & - & + \\
\hline 2466 & $a$ & + & - & - & - & + & 2470 & $a$ & - & + & + & + & - \\
\hline \multicolumn{7}{|c|}{17} & \multicolumn{7}{|c|}{18} \\
\hline 2471 & $a$ & + & - & - & + & - & 2474 & $a$ & - & + & - & - & - \\
\hline 2472 & a & - & + & + & + & - & 475 & 2 & + & - & + & + & + \\
\hline 2473 & $a$ & + & - & + & - & + & 2476 & 2 & + & + & - & - & + \\
\hline \multicolumn{7}{|c|}{19} & \multicolumn{7}{|c|}{20} \\
\hline 2477 & 2 & + & + & + & + & - & 2481 & 2 & + & - & - & + & - \\
\hline 2478 & $\alpha$ & - & - & - & + & - & 2482 & a & - & - & + & - & + \\
\hline 2479 & 3 & + & + & + & - & + & 2483 & a & - & + & - & + & - \\
\hline 2480 & a & - & - & - & - & + & 2484 & 2 & + & + & + & - & + \\
\hline \multicolumn{7}{|c|}{21} & \multicolumn{7}{|c|}{22} \\
\hline 2485 & $a$ & + & + & - & + & + & 2489 & $a$ & + & + & + & + & - \\
\hline 2486 & 2 & - & + & + & - & + & 2490 & a & - & - & - & + & + \\
\hline 2487 & 3 & + & - & + & + & - & 2491 & a & + & + & - & - & - \\
\hline 2488 & $\alpha$ & - & - & - & - & - & 2492 & 3 & + & - & + & - & + \\
\hline \multicolumn{7}{|c|}{23} & \multicolumn{7}{|c|}{24} \\
\hline 2493 & $a$ & + & - & - & + & - & 2497 & $a$ & + & + & - & + & - \\
\hline 2494 & $a$ & - & - & - & - & + & 2498 & $a$ & - & - & + & $t$ & + \\
\hline 2495 & a & + & + & + & + & - & & 2 & - & - & - & - & - \\
\hline 2496 & 2 & - & + & + & - & + & 2500 & 2 & + & + & + & - & + \\
\hline \multicolumn{7}{|c|}{25} & \multicolumn{7}{|c|}{26} \\
\hline 2501 & 2 & - & - & + & - & + & 2505 & a & + & & + & & + \\
\hline 2502 & 2 & - & - & + & + & - & 2506 & $a$ & - & - & - & + & 1 \\
\hline 2503 & $a$ & + & + & - & + & - & 2507 & $a$ & + & + & + & - & + \\
\hline 2504 & $a$ & + & + & - & - & + & 2508 & a & - & + & - & + & - \\
\hline
\end{tabular}


Cultures 2111,2148 , and 2153 produced the recorded turbidity in the pantothenate-free medium after 48 hours and were discarded. They would doubtless have grown more, this being indicated by the + sign after the turbidity reading. Each ascus produced two cultures with a turbidity reading of less than 8 and two with more than 160 four days after inoculation. The genes controlling mating type, galactose fermentation, and maltose fermentation also segregated regularly in each of the eight asci.

Table 19-4 is a pedigree describing the cultures grown from the ascospores dissected from 24 asci. These asci are derived from a hybrid heterozygous for mating type, galactose fermentation, maltose fermentation, and genes affecting the synthesis of paraminobenzoic acid, thiamin, and pyridoxine. The + and - signs indicate whether or not the cultures ferment galactose or maltose, and whether they grow in Burkholder's vitamin-free medium. The readings on the paraminobenzoic - and thiamin-free media were made after 48 hours, while those in the pyridoxine-free media were made after four days. Two of the cultures from each ascus produced practically no growth at the time diagnosis was made. The readings were all recorded numerically just as were the pantothenate readings shown in Table 19-3, but for the purposes of clarity were converted into + and - signs in the table.

In these selected pedigrees, regular segregation is the rule. In every ascus except Nos. 5 and 22, all characters segregated in a $2: 2$ ratio. This is adequate proof that the characters are under genic control. In asci 5 and 22 , only galactose was affected; the regular segregation of the other characters proves that these asci were truly hybrid. The significance of the irregular segregations will be discussed in detail in Chapters 25 and 26. 


\section{Chapter 20}

\section{IRREGULAR RATIOS}

\section{NON-MENDELIAN INHERITANCE OF ABILITY TO FERMENT GALACTOSE}

In contrast to the regularity with which fermenter and nonfermenter alleles were segregated in the hybrids described in Chapter 19, it is interesting to note the irregular segregations when the nonfermenter allele is introduced from Saccharomyces bayanus. $\mathrm{S}$. bayanus is incapable of fermenting galactose, hybrids between it and S. cerevisiae make it possible to study the inheritance of galactose fermentation. S. bayanus produces large cylindrical cells both in the haplophase and diplophase, providing an additional genetical marker. The hybrid between the large cylindrical gametes of $\mathrm{S}$. bayanus and the round gametes of $\mathbf{S}$. cerevisiae produced a large cylindrical diplophase, proving that the bayanus-type cell is dominant (fig. 20-1). One difficulty is that our culture of S. bayanus sporulated only rarely and only one ascospore of a very large number that was isolated grew. The fact that many of the single ascospore cultures of the hybrid produced viable 4-spored asci considerably complicated the genetical analysis. It is notable as an evidence of hybrid vigor that the original hybrid and the progeny all sporulated very abundantly in spite of the poor sporulation of the original $\mathrm{S}$. bayanus.

Table $20-1$ is a pedigree showing the progenies of a hybrid between S. bayanus and S. cerevisiae. All of the haplophase cultures from the hybrid fermented galactose. Half of the single ascospore cultures had large cylindrical cells like $S$. bayanus and half resembled haplophases of S. cerevisiae. It is apparent that the gene controlling cell shape segregated in a regular Mendelian way and af fords the necessary proof that a hybrid between the two species had been produced. However, none of the expected segregations of $G$ from $g$ occurred. All the asci were GGGG when all were expected to be GGgg. I first interpreted this phenomenon as a result of cytoplasmic transfer. Since that time accumulating evidence has revealed a number of similar circumstances and a more satisfactory interpretation will be brought forth in later chapters. It will be sufficient at this time to establish the fact that irregular ratios occur in yeasts. As an example of a loss, the haplophase culture No. 9 (which fermented galactose) produced an illegitimate diploid which on dissection yielded four spores all nonfermenters of galactose. Three produced the typical long cylindrical cell shape confirming their descent from their haploid parent. In the fourth the cell shape 

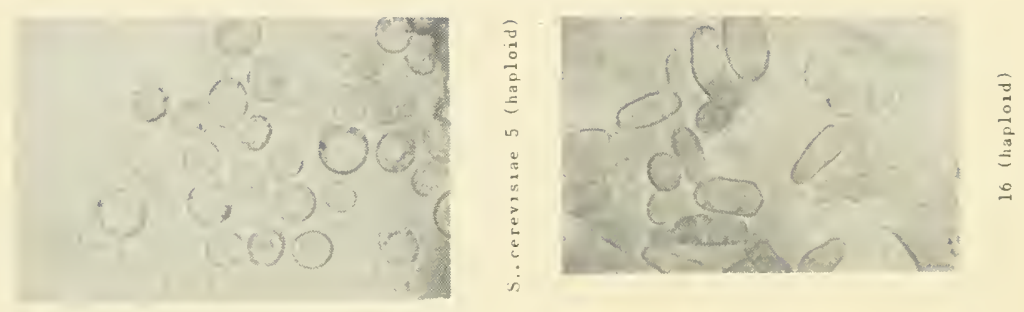

ํำ
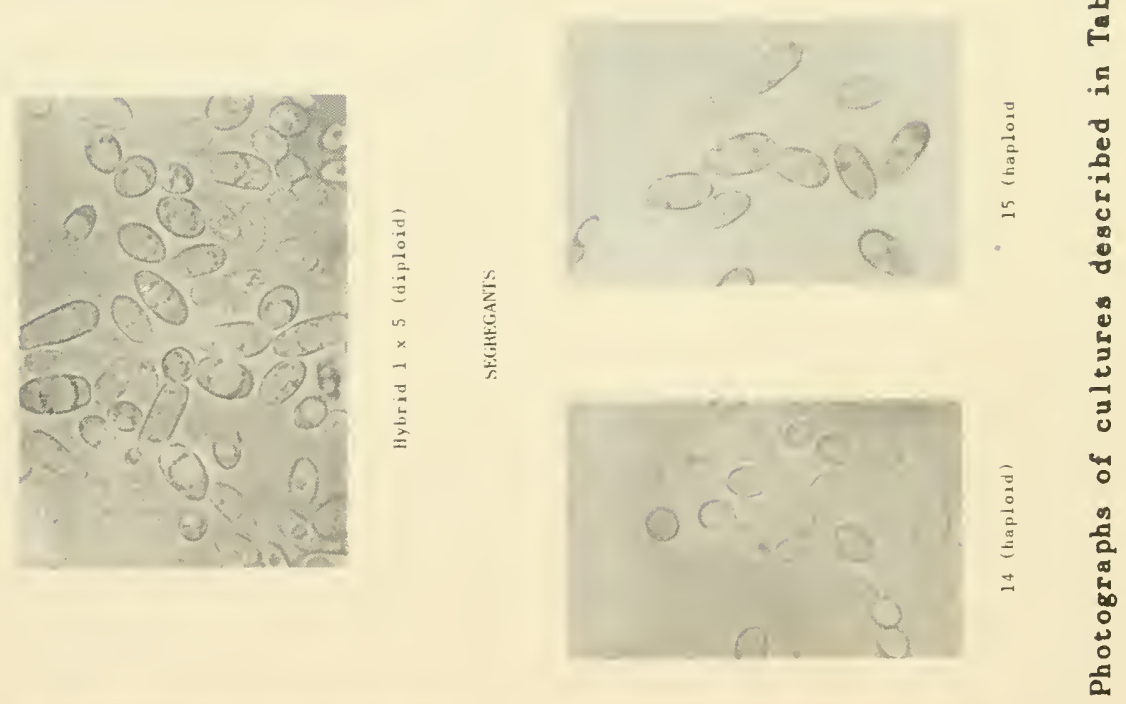

$\stackrel{0}{\Leftrightarrow}$
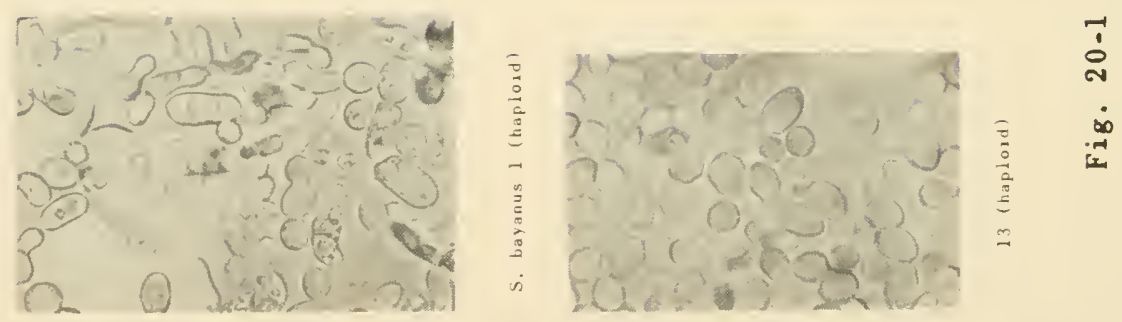
Table 20-1

Pedigree describing the progenies obtained from a hybrid of $S$. bayanus (cylindrical-cells, $L$; nonfermenter of galactose, g) by S. cerevisiae (round celled, 1; galactose-fermenter, G). Each column of 4 consecutive numbers represents a single ascus.

S. bayanus

$$
\begin{array}{ll}
1 & \text { Ig } \\
2 & \text { died } \\
3 & \text { died } \\
4 & \text { died }
\end{array}
$$

S. cerevisiae

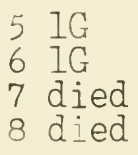

$1 \times 6$

$\begin{array}{rl}9 & \mathrm{LG} \\ 10 & \mathrm{LG} \\ 11 \mathrm{IG} \\ 12 \mathrm{IG}\end{array}$

9

illegitimate

diploid

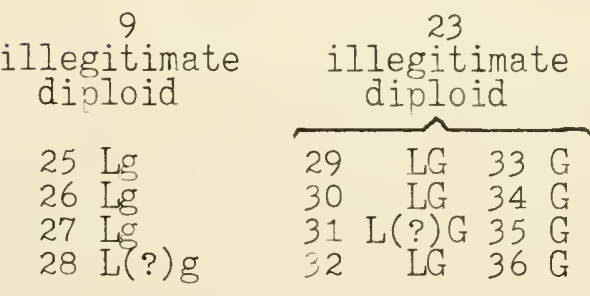

$\begin{array}{ll}13 & 1 G \\ 14 & I G \\ 15 & \mathrm{LG} \\ 16 \mathrm{LG}\end{array}$
$171 \mathrm{G}$

$181 G$

19 LG

$20 \mathrm{LG}$
$21 I G$

$22 \mathrm{LG}$

$23 \mathrm{LG}$

$24 I G$

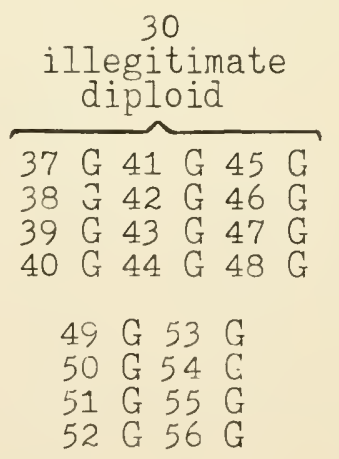

$\begin{array}{ccc}1 & x & 23 \\ L G & x & L G\end{array}$

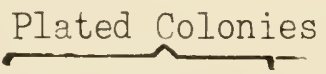

57 LG

$58 \mathrm{LG}$

$6 \mathrm{G}$, and $0 \mathrm{~g} 61 \mathrm{G} 65 \mathrm{G}$

$59 \mathrm{LG}$

$5 \mathrm{G}$, and $\mathrm{Og}$

$O \mathrm{G}$, and $17 \mathrm{~g}$

60 LG

$13 \mathrm{G}$, and $11 \mathrm{~g}$

62 G 66 G

$63 \mathrm{G} 67 \mathrm{G}$

64 d 68 G

The serial numbers of crltures in this table do not correspond to those in any other pedigrees in this book. 
was relatively irregular. The important point is that illegitimate diploidization of a fermenter produced only nonfermenter progeny. By contrast haploid culture 23 produced illegitimate diploids which threw only fermenter progeny (one with irregular cell shape L(?) and one of these in turn (haploid culture 30 ) produced illegitimate diploids from which twenty haplophase fermenter progeny were recovered.

Haplophase culture 23 from which only galactose fermenters were recovered when illegitimate diploids were produced was backcrossed to haplophase 1 which was a nonfermenter. Three asci were dissected, eleven fermenter haplophases were recovered, and one ascospore failed to germinate. Haplophase cultures derived from ascus (57-60) were plated out and individual colonies tested for their ability to ferment; 57 and 58 threw only fermenter descendants; 59 threw only nonfermenters, and 60 threw both fermenters and nonfermenters.

It is clear from these data that the control of galactose fermentation in S. cerevisiae which segregates in a regular Mendelian manner when hybridized with the nonfermenter allele originating from $\mathrm{S}$. microellipsoideus behaves in a very irregular manner when hybridized with the nonfermenter allele originating from $\mathrm{S}$. bayanus.

\section{NON-MENDELIAN INHERITANCE OF ABILITY TO FERMENT MELIBIOSE}

S. cerevisiae is incapable of fermenting melibiose, and its haploid segregants fail to ferment this sugar even after continued growth in broth containing melibiose. S. carlsbergensis is capable of fermenting melibiose by an adaptive enzyme (fig. 20-2), as are all its haploid segregants. This is the principal character upon which S. cerevisiae and S. carlsbergensis are differentiated. Subsequent genetic analysis established the fact that the haploid cultures of S. carlsbergensis carried only a single gene controlling the fermentation of melibiose. Hybrids between these two species would therefore be expected to produce two fermenter and 2 nonfermenter spores per ascus. None of the first seven asci dissected from the hybrid, S. carlsbergensis haplophase (No. 7) by S. cerevisiae haplophase (No. 4) (Table 20-2), were of the expected type. Three were Me Me Me Me, two were Me Me Me me. A backcross of one of these to the nonfermenter haploid No. 4 produced regularly segregating asci and the back-cross $5 \times 23$ did the same. However, when 20 and 23 were mated $(\mathrm{Me} / \mathrm{Me})$ several nonfermenter progeny were obtained. No fermenters were obtained, however, from the me/me crosses. The unconventional behavior of the members of this pedigree was first interpreted as the result of multiple genes controlling melibiose fermentation and is considered by 
Pedigree of a Hybrid between S. cerevisiae (Lk) (No. 1) S. carlsbergensis (Mrak, 126) (No.2). The numbers in parenthesis. (No. 1), etc. correspond to those used in Chapters 21, 22 and 26. The other numbers in this pedigree correspond 20 those in Table 21-2 but are not used elsewhere in this book.

\begin{tabular}{|c|c|c|c|}
\hline $\begin{array}{c}\text { S. cerevisiae } \\
\text { me diploid (Lk) } \\
\text { (No. 1) }\end{array}$ & $\begin{array}{c}\text { S. carlsberge } \\
M E \text { diploid (Mral } \\
\text { (No. 2) }\end{array}$ & $\begin{array}{l}\text { is } \\
126)\end{array}$ & \\
\hline 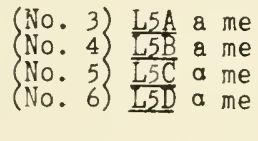 & $\begin{array}{rrr}\text { (No. 7) } \frac{\mathrm{C}_{1} \mathrm{~A}}{76} & \text { a } \\
& \mathrm{ME} \\
77 & \mathrm{ME} \\
78 & \mathrm{ME} \\
79 & \mathrm{ME}\end{array}$ & $\begin{array}{l}80 \\
81 \\
82 \\
83\end{array}$ & $\begin{array}{l}M E \\
M E \\
M E \\
M E\end{array}$ \\
\hline
\end{tabular}

(No. 4) $\underline{L 5 B} \times$ (No. 7) $\underline{C 1 A}$ (a me $x$ a ME)

\begin{tabular}{lllllllll} 
No. 20 & a $\mathrm{ME}$ & $12 \mathrm{ME}$ & $16 \mathrm{ME}$ & $35 \mathrm{ME}$ & $90 \mathrm{ME}$ & $94 \mathrm{ME}$ & $110 \mathrm{ME}$ \\
$\mathrm{No} .21$ & $\mathrm{ME}$ & $13 \mathrm{ME}$ & $17 \mathrm{ME}$ & $36 \mathrm{ME}$ & $91 \mathrm{me}$ & $95 \mathrm{ME}$ & $111 \mathrm{me}$ \\
No. 22 & a ME & $14 \mathrm{ME}$ & $18 \mathrm{ME}$ & $37 \mathrm{me}$ & $92 \mathrm{ME}$ & $96 \mathrm{ME}$ & $112 \mathrm{ME}$ \\
No. 23 & a ME & $15 \mathrm{ME}$ & $19 \mathrm{ME}$ & $38 \mathrm{ME}$ & $93 \mathrm{ME}$ & & \\
\hline
\end{tabular}

(No. 4) $\underline{L 5 B} \times($ No. 20) (a me $x$ a ME)

$\begin{array}{llll}25 \mathrm{ME} & 29 \mathrm{ME} & 33 \mathrm{ME} & 39 \mathrm{me} \\ 26 \mathrm{ME} & 30 \mathrm{me} & 34 \mathrm{me} & 40 \mathrm{n} \cdot \mathrm{g} . \\ 27 \mathrm{me} & 31 \mathrm{ME} & 35 \mathrm{ME} & 41 \mathrm{ME} \\ 28 \mathrm{me} & 32 \mathrm{n.g} . & 36 \mathrm{me} & 42 \mathrm{me}\end{array}$

(No. 5) L5C $\times$ (No. 23) (a me $\times$ \& ME)

\begin{tabular}{lllllllll}
$45 \mathrm{ME}$ & $49 \mathrm{ME}$ & $53 \mathrm{me}$ & $57 \mathrm{ME}$ & $61 \mathrm{n} \cdot \mathrm{g}$. & $65 \mathrm{n} \cdot \mathrm{g} \cdot$ & $69 \mathrm{me}$ & $86 \mathrm{ME}$ \\
$46 \mathrm{n} \cdot \mathrm{g}$. & $50 \mathrm{me}$ & $54 \mathrm{me}$ & $58 \mathrm{me}$ & $62 \mathrm{n} \cdot \mathrm{g} \cdot$ & $66 \mathrm{me}$ & $70 \mathrm{ME}$ & $87 \mathrm{ME}$ \\
$47 \mathrm{me}$ & $51 \mathrm{n.g}$. & $55 \mathrm{ME}$ & $59 \mathrm{ME}$ & $63 \mathrm{me}$ & $67 \mathrm{n} \cdot \mathrm{g}$. & $71 \mathrm{ME}$ & $88 \mathrm{me}$ \\
$48 \mathrm{me}$ & $52 \mathrm{n} \cdot \mathrm{g} \cdot$ & $56 \mathrm{n.g}$. & $60 \mathrm{me}$ & $64 \mathrm{ME}$ & $68 \mathrm{ME}$ & $72 \mathrm{me}$ & $89 \mathrm{me}$ \\
\hline
\end{tabular}

(No. 20) $\times$ (No. 23) (a ME x a ME)

$\begin{array}{lllllllll}124 \mathrm{ME} & 128 \mathrm{ME} & 136 \mathrm{ME} & 140 \mathrm{ME} & 144 \mathrm{ME} & 150 \mathrm{n} \cdot \mathrm{g} . \\ 125 \mathrm{ME} & 129 \mathrm{ME} & 137 \mathrm{ME} & 141 \mathrm{me} & 145 \mathrm{ME} & 151 \mathrm{ME} \\ 126 \mathrm{me} & 130 \mathrm{ME} & 138 \mathrm{ME} & 142 \mathrm{ME} & 146 \mathrm{ME} & 152 \mathrm{ME} \\ 127 \mathrm{n.g} . & 131 \mathrm{ME} & 139 \mathrm{ME} & 143 \mathrm{ME} & & 153 \mathrm{ME}\end{array}$

(No. 4) $\underline{\mathrm{L} 5 \mathrm{~B}} \times 49$ (a me $\times$ a ME) (No. 4) $\underline{\mathrm{L} 5 \mathrm{~B}} \times 55$ (a me $\times$ a ME)
104 ME
$105 \mathrm{me}$
$106 \mathrm{me}$
117 me
$118 \mathrm{ME}$
$118 \mathrm{n} . \mathrm{g}$.
$120 \mathrm{ME}$

(No. 5) $\underline{L C} \times 72$ (a me $\times$ a me) (No. 7) $\underline{C 1 A} \times 72$ (a ME $\times$ a me)

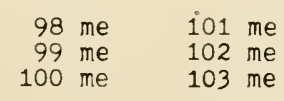

$\begin{array}{ll}M E & M e \\ M E & M E \\ M E & M E\end{array}$


Spiegelman (1946) to be due to cytoplasmic transfer, but the explanation in chapter 26 may fit the facts more adequately.

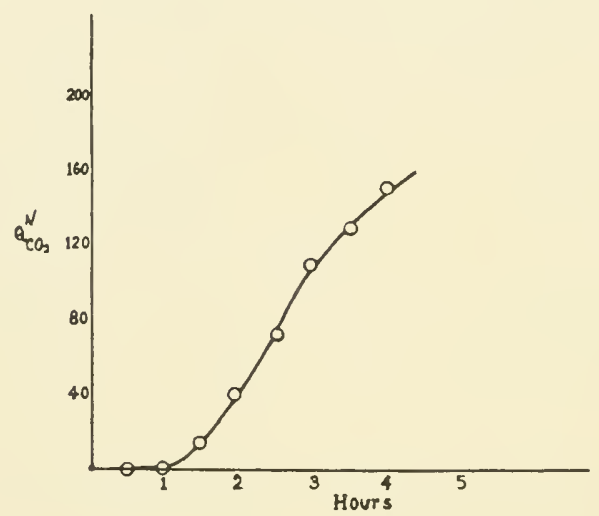

Fig. 20-2 Graph Showing the Adaptive Nature of Melibiose Fermentation by S. Carlsbergensis. Glucose-grown culture placed in a Warburg vessel containing 4 per cent melibiose in $M / 15$ $\mathrm{KH}_{2} \mathrm{PO}_{4}$. Cell division does not occur during this period so adaptation occurs in a stationary population.

\section{IRREGULAR RATIOS IN OTHER ORGANISMS}

Irregular ratios, which are frequently encountered by geneticists, are generally assumed to result from (1) multiple factor inheritance (Nillsson-Ehle), (2) suppressors (Bridges) or (3) modifying factors. That these genetical mechanisms can produce irregular ratios has been amply demonstrated, but the present analy. sis suggests that irregular ratios may arise from other causes as well. The genetical data on which the modern conception of the gene is based are intensively selected data. When genetical experiments do not give the expected ratios, the worker explores a number of possibilities known to disturb regular segregation of the character, and may eventually resolve his difficulties by discovering that multiple factors, or suppressors, or modifying factors, are involved; in the event that he fails to ao so he often discards the material in search for some that is more easily amenable to Mendelian analysis.

When the progeny of a mating cannot be classified, one cannot determine what effects are responsible for the irregular ratios unless specific tests are made. It is possible, however, that the tests may not have validity because the act of preparing for them (purifying the stocks) may alter the situation to the extent of in- 
validating them. The search for precisely segregating genes compels the selection of genetical material. In our own work on Neurospora we were unable to classify the progeny of over two-thirds of our matings.

The economy that is achieved in describing living organisms by genetical terms is enormous, for with morphological characters that can only be designated descriptively, one cannot present a pedigree involving many hundreds of individuals unless some shorthand "all-or-none" designation can be discovered. If each culture differs individually from every other one it is not feasible to develop categories to fit them all. When we found crosses in Neurospora whose progeny we could not analyze, we assumed that too many factors were involved. Thus the only step that could be taken was to inbreed for a few more generations until the stocks were "cleaned up" and the characters were recognizable in their different combinations. The question is whether this process of "cleaning up" stocks does not introduce some new factor or remove some normal mechanism.

Even in Drosophila when directly classifiable terminal characters such as the number and position of bristles are studied, an abundance of unclassifiable data accumulate which are presumed to arise from multiple factors or other causes. In the genetical analysis of the Smuts, Dickinson studied colonial characters and color and found that segregation occurred in an irregular fashion and he assumed irregularities of chromosomal behavior such as a third division segregation to explain the effects. This is another example of the pervading assurance that all genetical characters segregate regularly. In our own collection of yeast pedigrees we have hundreds of photographs of colonies but we have thus far been unable to develop a publishable analysis. Descriptive characters are simply too difficult to handle.

However, biochemical characters such as ability to ferment carbohydrates are easier to handle. In some fermentation pedigrees in yeast the inheritance was so precisely and regularly Mendelian that it was clearly gene-controlled. In the pedigrees in Chapter 19 , the $a / \alpha$, and $G / g$ alleles segregated precisely according to Mendelian expectations. This proved that the characters were referable to two different loci which segregated regularly in the reduction division. The irregular ratios involving the fermentation of galactose and melibiose which were discovered later (Chapters.20 and 26) raised the question of whether or not some of the irregular ratios which occur in other organisms arise in a similar manner.

\section{THE "GOOD GENE" CONCEPT}

Geneticists frequently speak of genes as if they fall into two natural categories: "good" and "bad." A "good" gene is easy to 
diagnose (especially in combinations with others), is relatively unaffected by environment, does not diminish vigor to an unusual extent, and usually gives regular ratios. "Bad" genes have one or more of the opposite characteristics. Classical Mendelian genetics is based on the analysis of data involving hybrids heterozygous for combinations of "good" genes. Genetical analysis of material in which only "bad" genes can be found is difficult if not impossible, along classical lines; geneticists often say that such organisms are "poor" genetical material and therefore cannot be studied properly. At least part of the difficulties involved in transferring classical genetics to useful purposes has arisen from the fact that what plant and animal breeders consider their "best" organisms are what a classical geneticist would call "poor" genetical material.

\section{GRADED IMPAIRMENT OF GENE FUNCTION}

Our experiments with yeast and Neurospora suggest that the difference between "good" and "bad" genes may be in the degree of impairment of gene function that has occurred. When haploid Neurospora microconidia are treated individually on agar plates with ultraviolet and X-rays and then transferred to culture tubes, a large number fail to grow but the remainder fall into four categories: (1) Some are normal and remain so. (2) Many are variants which revert to normal on the first of second transfer, suggesting an injury which persisted for some time after the environment had become normal; a typical Dauermodifikation. (3) Many variants are stable on vegetative reproduction, but most of these apparently stable haploid mutants when mated with a haploid wild type produce only wild type progeny, suggesting that repair on the injury occurred in the heterozygote. (When we first discovered this phenomenon we concluded that the injury was to the cytoplasm: at that time, the absence of any segregation character was considered proof for the view that no change in a gene had been involved. Analysis of yeast genetics, however, now suggests that an impairment of gene function had occurred which was repaired in the heterozygote.) (4) A few variants were good Mendelian genes--easy to diagnose and regularly segregating. On the present theory it is supposed that "good" genes are produced by an irreparable injury to the gene. 


\section{THE EFFECT OF THE MEDIUM ON APPARENT VITAMIN-SYNTHESIZING DEFICIENCIES}

The importance of vitamin deficiencies in the diagnosis of genetically different yeasts led us (Lindegren \& Raut) to study a variety of synthetic media in an attempt to find the one most suitable for our purposes. Beadle and Tatum's work on biochemical mutants of Neurospora has resulted in wide acceptance of the view that it is relatively easy to distinguish strains genetically capable from those genetically incapable of vitamin synthesis. They discovered many mutants apparently incapable of performing specific syntheses. Skoog and Lindegren (Chapter 16) showed that the ability or inability of one culture to ferment glucose depended on the substrate. Lindegren and Raut (1947) showed that different synthetic media so affect the growth of organisms that conclusive demonstration of specific deficiency requires a much more critical study of the environment than has hitherto been achieved. The fact that one culture may grow and another may fail to grow in a medium deficient of a specific vitamin in a synthetic medium has proved very useful for genetical diagnosis but it does not necessarily give reliable information concerning the synthetic ability of the organism under other conditions. Without critical testing it is difficult to say whether a given deficiency is absolute. Lindegren and and Raut found that the standard test of ability to grow in the presence and inability to grow in the absence of a given vitamin in synthetic medium is inadequate and that the criterion of equal increments of growth for equal additions of the vitamin may give an ambiguous answer.

\section{A COMPARISON OF TWO SYNTHETIC MEDIA}

Table 21-1 shows the formulas of the synthetic media used by Burkholder (1943), Hutner (unpublished), Wickerham (1946), and Beadle and Tatum (1945). Many experiments have shown that Hutner's medium is a very different substrate for yeast growth than Burkholder's. This was clearly revealed by growing cultures No. 3 and No. 10 (Table 20-2) on two batches of each medium, one to which no pantothenate was added and the other containing $50 \gamma$ of pantothenate per liter (fig. 21-1). Culture No. 3 does not begin to grow in Burkholder's medium without added pantothenate until after 200 hours, but growth is completed in Burkholder's medium con- 
Table 21-1

Formulas of Different Synthetic Vedia

\begin{tabular}{|c|c|c|c|c|}
\hline & $\begin{array}{l}\text { Burk- } \\
\text { Holder's }\end{array}$ & Hutner's & $\begin{array}{l}\text { Beadle \& } \\
\text { Tatum's }\end{array}$ & $\begin{array}{c}\text { Wicker- } \\
\text { ham's }\end{array}$ \\
\hline & \multicolumn{4}{|c|}{ Grams Per Liter } \\
\hline $\begin{array}{l}\text { Dextrose } \\
\text { Sucrose } \\
\text { Recrystallized } \\
\mathrm{K}_{2}^{2} \mathrm{HPO}_{4} \\
\mathrm{KH}_{2} \mathrm{PO}_{4} \\
\mathrm{MgSO}_{4} \cdot 7 \mathrm{H}_{2} \mathrm{O} \\
\mathrm{CaCl}_{2} \cdot 2 \mathrm{H}_{2} \mathrm{O} \\
\left(\mathrm{NH}_{4}\right)_{2} \mathrm{SO}_{4} \\
\mathrm{KI} \\
\mathrm{NaCl} \\
\mathrm{Sodium} \text { citrate } \\
\mathrm{Citric} \text { acid } \\
\left(\mathrm{NH}_{4}\right)_{2} \mathrm{HPO}_{4} \\
\mathrm{Ammon}_{4} \mathrm{Hum}_{\text {tartrate }} \\
\left(\mathrm{NH}_{4}\right)_{\mathrm{NO}_{3}}\end{array}$ & $\begin{array}{l}20.0 \\
2.0 \\
\\
1.5 \\
0.5 \\
0.33 \\
2.0 \\
0.0001\end{array}$ & $\begin{array}{l}1.0 \\
0.2 \\
0.037\end{array}$ & $\begin{array}{l}1.0 \\
0.5 \\
0.1 \\
0.1\end{array}$ & $\begin{array}{l}10.0 \\
1.0^{*} \\
0.125 \\
0.875 \\
0.5 \\
0.1 \\
1.0 * \\
0.0001 \\
0.1\end{array}$ \\
\hline
\end{tabular}

Parts Per Million

\begin{tabular}{l|l|l|l|l}
\hline Boron & 0.01 & & 0.01 & 0.01 \\
Manganese & 0.01 & 0.5 & 0.02 & \\
Zinc & 0.07 & 4.0 & 2.00 & 0.07 \\
Copper & 0.01 & 0.1 & 0.10 & 0.01 \\
Molybdenum & 0.01 & & 0.02 & \\
Iron & 0.05 & 4.0 & 0.20 & 0.05 \\
\hline
\end{tabular}

Micrograms Per Liter

\begin{tabular}{|c|c|c|c|c|}
\hline $\begin{array}{l}\text { Thiamin } \\
\text { Pyridoxine } \\
\text { Nicotinic acid } \\
\text { Pantothenate } \\
\text { Biotin } \\
\text { Inositol } \\
\text { Riboflavin } \\
\text { p-amino- } \\
\text { benzoic acid }\end{array}$ & $\begin{array}{r}200 \\
200 \\
200 \\
200 \\
2 \\
10,000 \\
\\
\\
\\
\text { pH } 5.0 \\
\text { with } \\
\text { NaOH }\end{array}$ & $\begin{array}{r}200 \\
200 \\
200 \\
200 \\
2 \\
10,000 \\
\\
\\
\text { pH } 6.0 \\
\text { with } \\
\text { citric } \\
\text { acid }\end{array}$ & 5.0 & $\begin{array}{r}400 \\
400 \\
400 \\
400 \\
2 \\
2,000 \\
200 \\
200 \\
\text { pH } 5.3\end{array}$ \\
\hline
\end{tabular}

* Either asparagine or $\left(\mathrm{NH}_{4}\right)_{2} \mathrm{SO}_{4}$ was used. 
taining $50 \gamma$ of pantothenate per liter after 50 hours. Readings taken at 72 hours would be interpreted to mean that it was a "nonsynthesizer." Culture No. 10 is capable of more rapid growth than culture No. 3 in Burkholder's without added pantothenate. Culture No. 10 grows much more slowly in the absence of pantothenate than in its presence. Culture No. 10 synthesizes pantothenate after a considerable lag in Burkholder's medium; in Hutner's medium the lag is much shorter. Burkholder's medium is a much better diagnostic medium than Hutner's, since growth occurs more rapidly on it than on Hutner's when pantothenate is supplied, but when pantothenate is not added growth occurs less rapidly on Burkholder's than on Hutner's. That is, Burkholder's is a better medium when pantothenate is added and a poorer medium without added pantothenate.

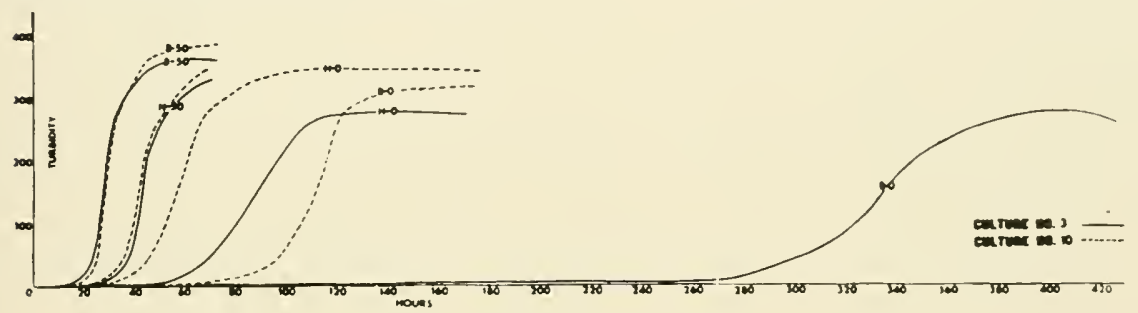

Fig. 21-1 Comparison of the Amounts of Growth on Burkholder's and Hutner's media. The turbidity indicated by a Klett Photoelectric Colorimeter is plotted against the time in hours after inoculation. Culture No:- 3 was grown on two batches of Hutner's medium, one containing $50 \gamma$ of pantothenate per liter and the other containing no added pantothenate. It was also grown on two different batches of Burkholder's medium, one containing $50 \gamma$ per liter of pantothenate and one no added pantothenate. Culture No. 10 was also grown on these four different kinds of media.

\section{THE "VITAMIN" PEDIGREE}

Table 20-2 (Chapter 20) describes the melibiose-fermenting capacity of the various cultures which were subsequently investigated in some detail for their "vitamin-synthesizing" activity on Burkholder's medium according to his technique. The original diploid culture of S. cerevisiae (No. 1) was incapable of fermenting melibiose and its offspring, cultures Nos. 3, 4, 5 and 6 were similarly incapable. In this pedigree the numbers are used to indicate the different cultures produced by the four spores of a single ascus. Thus cultures $3,4,5$ and 6 were originally from one diploid ascus of S. cerevisiae. Culture No. 2 (S. carlsbergensis) fermented melibiose as did all its haploid progeny. Culture No. 7 was the only survivor of a single ascus and a mating between it and No. 4 produced a hybrid supposedly heterozygous for the ability to ferment melibiose. 


\section{HYBRIDS BETWEEN YEASTS SUPPOSEDLY DIFFERENT IN VITAMIN-SYNTHESIZING ABILITY}

The members of this pedigree were characterized (Lindegren and Lindegren, 1945) as "vitamin-synthesizers" and "vitamin-nonsynthesizers." The distinction between ability and inability to synthesize was made by Burkholder's method with his medium. This procedure defines readings taken at 72 hours as diagnostic. We have since discovered that this method does not give conclusive results, for if growth were allowed to continue, the supposedly nonsynthesizing yeasts will eventually begin to grow and will finally, in most cases, attain a level equal to that of the so-called synthesizers. However, at the end of 72 hours a 10 or 20 -fold difference in growth often exists as indicated by reading the turbidity with a Klett Photoelectric Colorimeter. Haploids of S. carlsbergensis were characterized as biotin + , pantothenate + , and pyridoxine -; haploids of S. cerevisiae were characterized as biotin -, pantothenate -, and pyridoxine + , according to Burkholder's technique.

Table 21-2 shows the Klett reading 3 obtained after 72 hours by Burkholder's technique of these different cultures. It appears that culture No. 4 is a non-synthesizer of pantothenate, while No. 7 is a synthesizer. A hybrid between No. 4 and No. 7 (culture No. 10) appears capable of synthesizing pantothenat;, but the four haploid progeny, Nos. 20,21, 22, 23 do not reveal regular segregation for this character. It was subsequently discovered that none of these cultures was a nonsynthesizer and that the data appearing in Table 21-2 do not give an adequate picture of the synthetic ability of the organisms. The inadequacy of this characterization will be dealt with in detail below.

\section{VITAMIN-SYNTHESIZING ABILITY OF SUPPOSEDLY DEFICIENT YEASTS IN A NATURAL MEDIUM}

Dr. F. W. Tanner, Jr. (unpublished) grew various members of the pedigree shown in Table 20-2 (Chapter 20) in a natural medium containing molasses and corn-steep liquor for 72 hours. When the yeast and the medium were assayed it was found that under these conditions all the cultures synthesized similar amounts of the different vitamins. Apparently the cultures were not clearly differentiated by ability and inability to synthesize the vitamins, but were all capable of synthesis under certain conditions.

\section{CONTINUOUS OBSERVATION OF GROWTH}

Our present technique, an example of which has been presented in fig. 21-1, requires a much longer observation period. Many of the same cultures were reinvestigated and were sometimes observed for as long as a month, readings being made over the entire 
Table 21-2

The Amount of Growth after 72 Hours of Cultures from Table 20-2 on Burkholder's Medium Deficient in the Indicated Vitamins

\begin{tabular}{|c|c|c|c|c|c|c|c|}
\hline \multirow{2}{*}{ Culture No. } & \multicolumn{3}{|c|}{$\begin{array}{l}\text { Photometer } \\
\text { Reading }\end{array}$} & \multirow{2}{*}{ Culture No. } & \multicolumn{3}{|c|}{$\begin{array}{l}\text { Photometer } \\
\text { Reading }\end{array}$} \\
\hline & -Py $•$ & $-\mathrm{Pa}$. & $-B i$ & & -Py • & -Pa. & $-B i$ \\
\hline $\begin{array}{l}1 \\
2\end{array}$ (diploid diploid & $\begin{array}{r}350 \\
24\end{array}$ & $\begin{array}{l}220 \\
280\end{array}$ & $\begin{array}{r}11 \\
280\end{array}$ & $\begin{array}{l}4 \\
5 \\
7\end{array}$ & $\begin{array}{r}350 \\
310 \\
22\end{array}$ & $\begin{array}{r}15 \\
20 \\
200\end{array}$ & $\begin{array}{r}12 \\
8 \\
125\end{array}$ \\
\hline
\end{tabular}

\begin{tabular}{|c|c|c|c|c|c|c|c|}
\hline 10 (diploid) & 315 & 240 & 135 & \multirow{3}{*}{$\begin{array}{l}12 \\
13 \\
14 \\
15\end{array}$} & \multirow{3}{*}{$\begin{array}{r}355 \\
80 \\
350 \\
25\end{array}$} & \multirow{3}{*}{$\begin{array}{r}293 \\
65 \\
220 \\
15\end{array}$} & \\
\hline $\begin{array}{l}20 \\
21 \\
22 \\
23\end{array}$ & $\begin{array}{l}274 \\
312 \\
290 \\
300\end{array}$ & $\begin{array}{r}45 \\
202 \\
200 \\
210\end{array}$ & $\begin{array}{r}50 \\
55 \\
59 \\
110\end{array}$ & & & & $\begin{array}{r}170 \\
160 \\
75 \\
7\end{array}$ \\
\hline $\begin{array}{l}90 \\
91 \\
92 \\
93\end{array}$ & $\begin{array}{l}318 \\
100 \\
300 \\
345\end{array}$ & $\begin{array}{l}212 \\
210 \\
235 \\
235\end{array}$ & $\begin{array}{l}140 \\
140 \\
134 \\
110\end{array}$ & & & & \\
\hline
\end{tabular}

$4 \times 20$

\begin{tabular}{|c|c|c|c|c|c|c|c|}
\hline $\begin{array}{l}25 \\
26 \\
27 \\
28\end{array}$ & $\begin{array}{l}310 \\
325\end{array}$ & $\begin{array}{l}25 \\
28\end{array}$ & $\begin{array}{l}145 \\
145\end{array}$ & $\begin{array}{l}33 \\
34 \\
35 \\
36\end{array}$ & $\begin{array}{l}340 \\
410 \\
355\end{array}$ & $\begin{array}{r}12 \\
140 \\
9\end{array}$ & $\begin{array}{r}10 \\
100 \\
9\end{array}$ \\
\hline \multicolumn{8}{|c|}{$5 \times 23$} \\
\hline $\begin{array}{l}57 \\
58 \\
59 \\
60\end{array}$ & $\begin{array}{r}305 \\
37 \\
38 \\
347\end{array}$ & $\begin{array}{r}16 \\
94 \\
100 \\
16\end{array}$ & $\begin{array}{l}33 \\
14 \\
10 \\
20\end{array}$ & $\begin{array}{l}69 \\
70 \\
71 \\
72\end{array}$ & $\begin{array}{r}340 \\
93 \\
358\end{array}$ & $\begin{array}{r}237 \\
15 \\
16\end{array}$ & $\begin{array}{l}275 \\
230\end{array}$ \\
\hline $\begin{array}{l}86 \\
87 \\
88 \\
89\end{array}$ & $\begin{array}{l}400 \\
380 \\
400 \\
460\end{array}$ & $\begin{array}{r}315 \\
150 \\
300 \\
50\end{array}$ & $\begin{array}{r}15 \\
150 \\
10 \\
200\end{array}$ & $\begin{array}{l}154 \\
155 \\
156 \\
157\end{array}$ & $\begin{array}{r}355 \\
12 \\
425 \\
3\end{array}$ & $\begin{array}{r}200 \\
165 \\
140 \\
20\end{array}$ & $\begin{array}{r}120 \\
30 \\
300 \\
365\end{array}$ \\
\hline \multicolumn{8}{|c|}{$20 \times 23$} \\
\hline $\begin{array}{l}136 \\
137 \\
138 \\
139\end{array}$ & $\begin{array}{l}318 \\
360 \\
340 \\
320\end{array}$ & $\begin{array}{l}256 \\
222 \\
240\end{array}$ & $\begin{array}{l}160 \\
193 \\
200 \\
170\end{array}$ & & & & \\
\hline
\end{tabular}


period. This is quite different from the standard practice of discontinuing the experiment after 72 hours. When the period of observation was extended for a longer time, cultures of S. cerevisiae (as well as C. carlsbergensis) were found to be able to grow on Burkholder's medium in the absence of pantothenate. This was equally true of all the progeny of the hybrid, many of which had previously been described as "nonsynthesizers." In some cases there was a delay of more than 600 hours before growth began. We grew the cultures in $6 \times 3 / 4$-inch Kimble tubes and determined the amount of growth by measuring turbidity on the Klett Photoelectric Colorimeter adapted to take the larger tubes. This made it possible to make readings over the entire period without discarding the cultures.

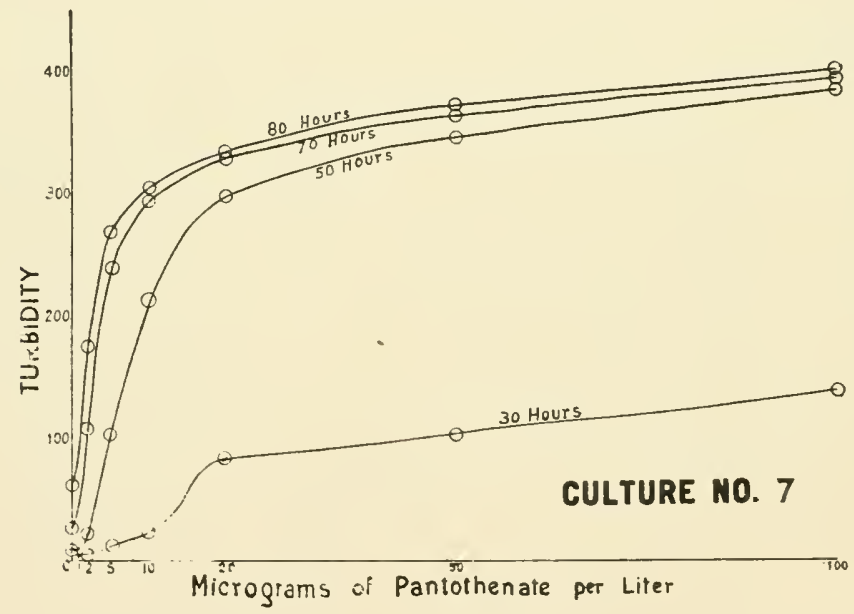

Fig. 21-2 The Relationship Between Increments of Pantothenate in Burkholder's liedium and the Amount of Growth of Culture No. 7 at different time intervals. In some phases of the curves a straight-line relationship exists between the amount of growth and the amount of added pantothenate but in all the culture tubes the growth eventually rose to approximately the same level.

THE INADEQUACY OF THE CRITERION, EQUAL INCREMENTS OF GROWTH FOR EQUAL INCREMENTS OF VITAMIN

Culture No. 7 was planted in a series of media containing different concentrations of pantothenate and the data produced a family of parallel curves (fig. 21-2). If the experiment were terminated at an arbitrary time (standard practice in nearly all assay experiments), curves could be obtained in which equal increments of vitamin appear to produce equal increments of growth. The curves in 
fig. 21-2 were obtained by taking readings at various times. At 50 hours there appears to be a straight line relation between the amount of added vitam in and the amount of growth over a considerable portion of the curve.

\section{THE EFFECT OF MULTIPLE DEFICIENCIES OF THE B VITAMINS}

A haploid culture of S. cerevisiae which grew well on agar containing Hutner's synthetic medium with the vitamins biotin, inositol, thiamin, and pantothenate, but which was unable to grow on the same agar medium lacking all these vitamins, was tested on agar containing various combinations of vitamins. Pour plates following Lederberg and Tatum's (1946) technique were made. In this technique a layer of sterile agar is poured in the bottom of the petri dish. Then a layer of agar seeded with 500 cells is poured on top, and a third layer of sterile agar on top of the second. This technique prevents colonies at the top and bottom of the agar from growing diffusely over and under the agar. A penicillin assay cup placed in the center of each plate was filled with a solution containing the combination of vitamins.

Colonies were counted and a number of methods of scoring were tested, but simple + and - scoring is probably the most informative. The following tabulation gives the results:

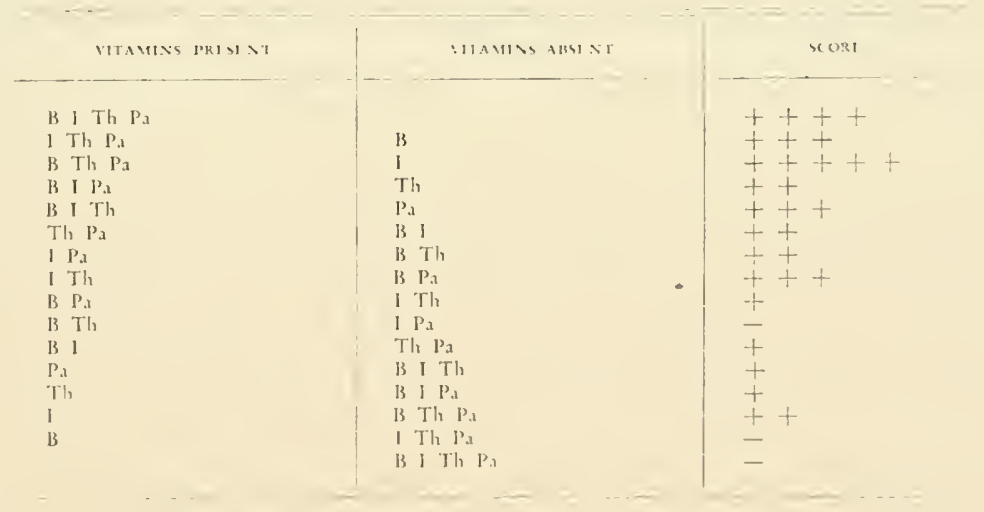

The addition of inositol is apparently not very helpful in this concentration $(10,000 \gamma$ per liter) when biotin, thiamin and pantothenate were also present for there was more growth when it was absent than when it was present. Actually there were fewer colonies when it was absent, but those which grew did much better. However, the culture supplied pantothenate alone grew less well than that sup- 
plied with inositol and pantothenate. There were probably conside rable amounts of biotin in the agar, for removal of biotin did not usually reduce growth greatly. Removal of biotin and inositol simultaneously was serious. When either inositol or pantothenate were removed singly no serious effect occurred, but when both were removed together there was considerably less growth. The cells could synthesize both inositol and pantothenate easily when only one was absent but in the absence of both they synthesized poorly. These facts indicate that ability to grow in the absence of vitamins may involve simply lack of capacity to grow, or begin to grow, under the prescribed conditions rather than inability to synthesize the absent vitamins under all conditions (Williams, 1941).

\section{THE RELATION BETWEEN VIABILITY OF CELLS AND INABILITY TO GROW ON DEFICIENT MEDIUM}

The ability or inability to grow in a deficient medium may merely involve inviability in the new medium rather than absolute inability to synthesize the vitamin in question. If the cells are inviable in the deficient medium they will be unable to begin synthesis of the required vitamin. The fact that most yeasts begin to synthesize when they are permitted to stand a sufficiently long time suggests that continued examination of cultures is necessary. This points up an important difference between Neurospora and yeasts. A conidium of Neurospora can put out a germ tube and begin growth in distilled water but if it is to continue growth it must be immediately supplied with the substances which it requires. It is relatively difficult to establish a dormant mycelium capable of awaiting future developments. Probably one of the most important advantages of a single-celled form over a filamentous fungus is that the single-celled form can go through one or two divisions and settle down to dormancy while a filamentous organism with specialized spores must continue to grow once the filament is produced or it will perish. This may give the appearance of absolute deficiencies to many mutants which die shortly after producing a filament in a medium lacking a specific vitamin. This suggests that many "vitaminless" mutants which are incapable of producing the enzyme completing one step in the synthesis of the specific vitamin, might be able to synthesize the vitamin if the medium were adequate.

These results have been supported by further work on three other mutants differentiated by ability to synthesize p-aminobenzoic acid, pyridoxine and uracil. So-called nonsynthesizers of pyridoxine eventually grew in a medium containing no added pyridoxine. Diploids heterozygous for a gene controlling synthesis of $\mathrm{p}-\mathrm{a}$ minobenzoic acid produced two classes of offspring, one which grew rapidly and one which grew slowly in the absence of p-aminobenzoic acid. Although these two classes were clearly differentiated, 
the weak synthesizers were always able to grow eventually. An even more important bit of confirmatory evidence was obtained from the stock incapable of synthesizing uracil. The uracil-deficient cultures responded quickly to large amounts of uracil added to Bukholder's medium, but in the medium without uracil they died. Transfer from uracil deficient medium to a complete medium a few days after inoculation, proved that the cells had died.

Lindegren and Raut's results indicate that all so-called "nonsynthesizers" probably require qualification because synthesis might have been discovered if observation were made over a longer period. Many reversions of Beadle and Tatum's "vitaminless" mutants in the deficient medium have been reported by Bonner, Tatum, and Beadle (1943) and other workers in this field, but they are usually regarded as exceptional cases rather than the standard expected behavior while the reverse is apparently the case with yeasts. The above results suggest the possibility that some other improvements of the medium than the addition of the vitamin for which the stock appears to be deficient might often lead to a higher frequency of reversions.

There are two important differences in the vitamin deficiencies described by us and those described by Beadle and Tatum: (1) Our variants were derived from naturally occurring forms while theirs were induced by radiation. (It is possible that deficiencies induced by radiation may be more complete than natural ones and have less capacity for reversion.)

(2) Our stocks were selected after a series of mating in which irregular segregations had occurred. Beadle and Tatum did not begin work with a treated culture unless it segregated regularly at the first trial. 


\section{Chapter 22}

\section{THE SEPARATE FUNCTIONS OF RECESSIVE AND DOMINANT}

\section{ALLELES IN ADAPTATION TO PANTOTHENATE DEFICIENCY}

The classification of genetically different yeasts into dominant "synthesizers" and recessive "non-synthesizers" of the different B vitamins is merely a relative distinction, for the so-called "nonsynthesizers" eventually grow in a test medium deficient for the vitamin in question. In an investigation of the effect of environment and competition on the adaption of cultures carrying the recessive allele it was found that the adaptation occurred in two steps: (a) A primary adaptation during which the recessive ("non-synthesizer") grew at a low rate presumably by synthesizing the absent vitamin, until the population attained a relatively high density. (b) A secondary adaptation initiated by the mutation of one or more recessive cells to the dominant so-called "synthesizer". Small amounts of added vitamin were effective in reducing the time required to complete these steps.

In high concentrations of pantothenate the dominant ("synthesizer") cells were unable to compete with cells carrying the recessive allele and, therefore, adaptations were only apparent in low concentrations of pantothenate. This latter observation is consistent with the observations of Lwoff, who pointed out that the "inability" to synthesize a specific nutrilite may be a selective advantage in high concentrations of the specific substance.

\section{THE EFFECT OF THE ENVIRONMENT \\ ON ADAPTATION TO A DEFICIENT MEDIUM}

Microorganisms in an "unfavorable" environment frequently become adapted and survive and grow in that environment. Bacteria exposed to antibacterial agents such as penicillin (Demerec 1945a, b) or sulfonamide (Oakberg and Luria 1947) acquire a resistance which is transmitted. In many cases, the reason for the increased resistance is unknown. In some cases it is due to increased synthesis, as for example, of p-aminobenzoic acid in Staphylococcus aureus resistant to sulfonamide (Landy et al 1943) and increased synthesis of pantothenate in pantoyl taurine resistant Corynebacterium diphtheriae (McIlwain 1943).

Mutant strains of Neurospora crassa unable to grow in the absence of a specific nutrilite will sometimes adapt while being grown 
in a deficient medium, and acquire the ability to grow normally without the added nutrilite. This appears to be due to an increase in synthetic ability. For instance, Ryan (1946) has demonstrated chemically the presence of $1(+)$ leucine in the mycelium of leucineless Neurospora which adapted and became able to grow in the absence of leucine.

The effect of the environment poses a problem, for the environment will select any spontaneously occurring variant better suited to survive under the specific environmental conditions. Several problems are involved in attempting to determine definitively whether the environment does or does not act to induce change in the organism.

Critical evidence on this point was contributed by Luria and Delbruck (1943) in their study of the occurrence of phage resistance in Escherichia coli. A series of separate cultures of bacteria were grown from an inoculum of 50 to 500 cells in the absence of phage and then each entire culture was plated separately on a plate containing phage and a study made of the number of colonies which grew on each plate.

The experiment was designed to determine (1) whether exposure to phage induced the bacteria to mutate or (2) whether exposure to phage merely selected mutants which had occurred spontaneously prior to the exposure. If the phage induced a heritable resistance in the cells, one would expect this change to be induced in approximately the same proportion of bacteria in a group of similar cultures, except for variation due to sampling error. On the other hand, if the resistant colonies were due to chance mutations, then one would expect a wide variation in the number of resistant cells found in each culture depending on how many mutations had occurred and on the time at which each mutation occurred. If a mutation occurred early in the growth of the culture, by the time growth was completed there would be many mutant cells in the clone derived from the early mutation. On the other hand, if the mutation occurred near the end of growth, there would be few mutant cells derived from it. This assumes that the mutants and the original grow at the same rate.

They found wide variation in the numbers of resistant colonies and concluded from this that the resistance was caused by random mutations prior to exposure to the phage. This variance test has since been applied to other kinds of adaptations, including resistance of Staphylococcus aureus to penicillin (Demerec 1945a, b) and sulfonamides (Oakberg and Luria 1947), mutation from histidine dependence to independence in Escherichia coli (Ryan et al 1946) and from uracil dependence to independence in Clostridium septicum (Ryan et al 1946).

However, it would be difficult to detect if phage were capable of affecting or inducing mutations by this method. While the occurrence of spontaneous mutations prior to exposure to the phage can account 
for the adaptation in the above instance, it is important to note that in this particular case, because of the lethal effect of the phage, it is not possible to determine the mutation rate in the continued presence of the phage, but only prior to its application. Lysis takes place very quickly. Only bacteria which are in the process of division at the time of plating may sometimes complete the division, and the division time is 19 minutes (in broth). Therefore, if the phage were able to induce resistance in bacteria, it would have to be by an almost instantaneous induction.

A particularly interesting case of adaptation is that studied by Ryan (1946) of the adaptation of a leucineless Neurospora mutant obtained after treatment with ultraviolet light. Genetic tests supplied evidence that the adaptation was a mutation of the recessive leucineless allele back to the normal dominant wild type allele. The number of adaptations which occurred increased as the concentration of leucine was lowered. This is opposite to what would be expected if the mutation were spontaneous and random. One would then expect a larger number of adaptations in higher concentrations of leucine, since the leucineless strain should be able to multiply more rapidly producing a larger number of nuclei and hence a greater chance of mutation.

When the non-synthesizing and the synthesizing nuclei were combined in a heterocaryon and grown on nutrient agar t thout leucine, the fungus grew at the wild-type rate, showing that although both types of nuclei were present, the synthesizing nuclei enabled the mycelium to synthesize leucine and thus grow at the normal rate. However, when the same heterocaryon was grown on nutrient agar containing a limiting concentration of leucine, the growth rate was that of the non-synthesizing organism. It was shown further that on this limiting medium the non-synthesizing nuclei actually displaced the synthesizing nuclei in the hyphal tips during growth, so that when hyphal tips are cut off, they contain only leucineless nuclei. This replacement accounts for the growth occurring at the leucineless rate.

Two groups of leucineless cultures were grown, one on a high and the other on a low concentration of leucine, so that when each group had completed growth, the first consisted of a larger amount of mycelium than the second. Then small clots of wild-type mycelium were inserted into the middle of the masses of leucineless mycelium and allowed to grow to completion. The small clot of wildtype mycelium inserted into the small mass of leucineless mycelium grew to a greater size than that inserted into the large mass. Apparently the large mass of leucineless mycelium is more effective than the small mass in suppressing the growth of the wild-type mycelium.

From the foregoing data the conclusion was drawn that, in the heterocaryon, there is direct competition between the synthesizing and the non-synthesizing nuclei, and that the greater the number of 
non-synthesizing nuclei, the more effective they are in inhibiting the synthesizing nuclei. Hence, in a high concentration of leucine there is a very large number of leucineless nuclei, so that if a mutation to synthesizing ability did occur, it would be effectively suppressed, while if one occurred in a smaller number of leucineless nuclei (at the lower leucine concentration) the mutation would not be effectively suppressed and adaptation would occur.

This selection phenomenon is sufficient to explain the increase in number of adaptations on lower concentrations of leucine. It is impossible, however, to determine whether the concentration of leucine affects the mutation rate because of the competition between the nuclei.

The above studies show that random mutation and selection by the environment can account for some adaptations but the evidence does not exclude other possibilities, particularly the possibility of non-random induction of mutation by specific agents. In the experiments of Luria and Delbruck (1943) it was not possible to determine the mutation rate in the presence of the phage because of the lethal action of the phage; in Ryan's (1946) work, competition between the nuclei prevented study of the rate of occurrence of mutations in the presence of various concentrations of leucine.

Specific induction of mutations by external agents is a possibility which must be considered, for various workers, particularly Avery and Biovin, have demonstrated what may be transformation of certain types of pneumococci and Escherichia coli into other types by the specific action of desoxyribose nucleic acid extracts.

\section{SLOW ADAPTATION TO THE ABSENCE OF PANTOTHENATE BY A PANTOTHENATELESS MENDELIAN RECESSIVE}

Lindegren and Raut (1947a, b) studied a haploid pantothenatedependent strain of Saccharomyces cerevisiae which adapted to pantothenate independence in about ten days when inoculated into medium to which no pantothenate had been added. The time required for adaptation was shortened by the addition of minute amounts of pantothenate, but was prevented entirely by the addition of larger amounts of pantothenate. The regularity of occurrence of adaptation and its correlation with increases in small amounts of pantothenate were interpreted to indicate induction of adaptation by pantothenate. However, it was pointed out that the data did not exclude the possibility of selection of infrequently occurring mutants.

They compared the growth curves of genetically different yeasts in media containing different concentrations of pantothenate. Various members of the pedigree are shown in Table 20-2 with the following amounts of pantothenate added per liter: $100,50,20,10,5,2,1,0.5$, 
and $0 y$. Each tube was inoculated in a uniform manner with a loop. Three hundred colonies grew from each loopful of inoculum used, but since the haploid cells were typically aggregated, the total number of cells in each inoculation was probably about 1500 .

Fig. 22-1 shows the results with a diploid culture of S. cerevisiae (No. 1); the turbidity is plotted against time in hours. The graphs are made by plotting the average of the turbidity produced in the duplicate tubes, except in a few cases in which the tubes were so widely different that averaging did not seem to be a permissible practice. Usually the readings differed by only a few units and averaging was obviously acceptable. After $\mathbf{4 5}$ hours, growth is practically completed in the media containing 50 and $100 \gamma$ of pantothenate, but it is fully 75 hours before appreciable growth is recorded in the tube without added pantothenate. This culture had previously been characterized as a synthesizer of pantothenate. These data show that diagnosis depends largely on the time at which readings are taken. Comparison of the $100 \gamma$ and $0 \gamma$ tubes at the end of 45 hours would have resulted in characterizing this particular organism as a "non-synthesizer" of pantothenate. The relationship between the amount of added pantothenate and the time at which growth begins is quite clear since the curves are all closely parallel during early and logarithmic growth and overlapping of the curves only occurs after the logarithmic phase of growth has been completed. There is a sharp difference between the time at which growth begins in the tubes containing 0.5 and $1 \gamma$ of pantothenate per liter as well as between growth in tubes containing 1 and $2 \gamma$ of pantothenate per liter.

The culture of S. cerevisiae, whose reactions are recorded in Fig. 22-1, was induced to sporulate, and similar tests with the four haplophase cultures are shown in Figs. 22-2 and 22-3. Cultures No. 3 and No. 4 are remarkably similar in behavior. According to previous techniques these would have been classified as "non-synthesizers" because growth in the absence of pantothenate did not begin until after 250 hours. The particularly interesting feature of the behavior of these cultures is the close relation between the length of the delay before growth begins and concentration of pantothenate in the medium.

In culture No. 5 (Fig. 22-2) the different concentrations of pantothenate also bear a direct relation to the delay before growth begins. A similar picture exists for culture No. 6, except that in the concentrations of $1 \gamma$ and $0 \gamma$ per liter the duplicate tubes differed so markedly from each other that it was not permissible to average the results. This is one of the few cases in which growth in $1 \gamma$ per liter in one of the duplicate tubes occurred later than growth in the tube containing $0.5 \gamma$ per liter.

The behavior in culture No. 5 (Fig. 22-2) is an almost ideal example of the phenomenon. Growth in media containing $100 \gamma$ and $50 \gamma$ per liter takes place at a very rapid rate. In the medium containing 
$20 \gamma$ the rate is somewhat decreased and in $10 \gamma$ a considerable decrease has occurred. A further decrease occurs in $5 \gamma$ per liter, so that a continual decrease in iate of growth occurs in the $50 \%$, $20 \gamma, 10 \gamma$ and $5 \gamma$ media, where beginning of growth is delayed more and more as the concentration decreases, but after growth begins in the $2 \gamma$ to $0 \gamma$ tubes the rate is uniform and more rapid than in the $5 \gamma$ medium. It is clear that the amount of growth when more than $5 \gamma$ of pantothenate is present bears a direct relation to the concentration. The rates of growth in the $2 \gamma, 1 \gamma, 0.5 \gamma$ and $0 \gamma$ tubes are very similar, but the delay before growth begins bears a close relation to the concentration of pantothenate.
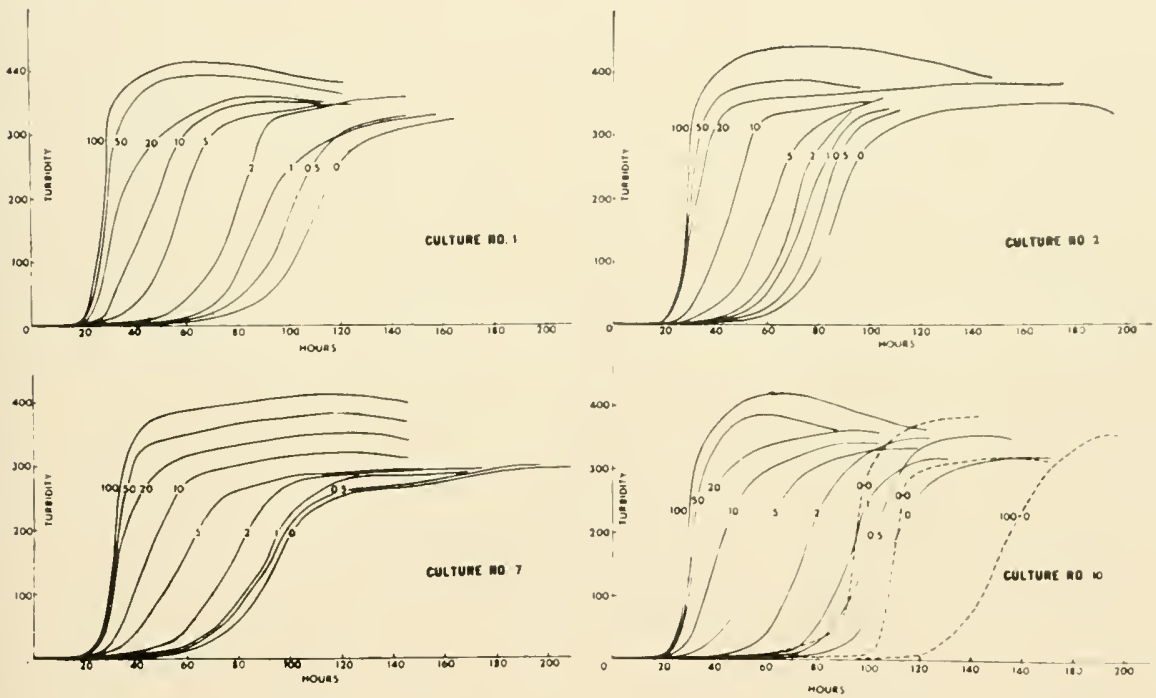

Fig. 22-1 The Growth of Cultures Nos. 1 (S. Cerevisiae, Diploid), 2 (S. Carlstuergensis, Diploid), 7 (S. Carlsbergensis, Haploid, ClA) and 10 (S. Carlsbergensis x S. Cerevisiae, Diploid Hybrid) in Burkholder's Medium Containing Different Concentrations of pantothenate. The solid lines represent the original inoculations; the dotted lines represent transfers from these inoculations as indicated, $100-0$ being a transfer from $100 \gamma$ to $0 \gamma$.

When transfers from the culture tubes without added pantothenate were made to similar tubes likewise without added pantothenate $(0-0)$ growth occurred about 150 hours sooner in the second than in the original test. These curves of growth are shown as dotted lines on the graph. As the yeast was transferred serially in 0 medium, the delay before growth started was further shortened. The fifth transfer $(0-0-0-0-0)$ began to grow sooner in the medium without added 

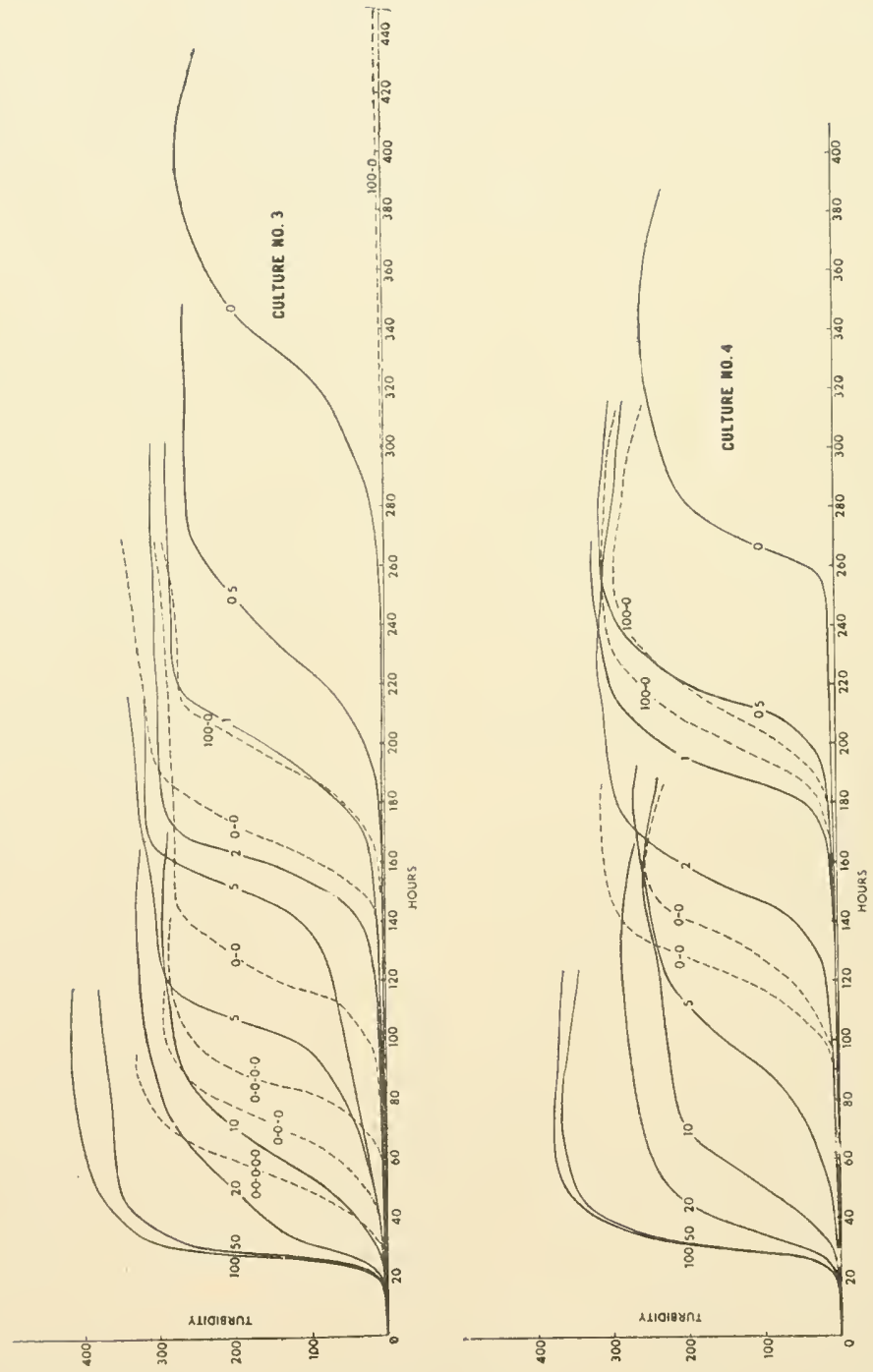

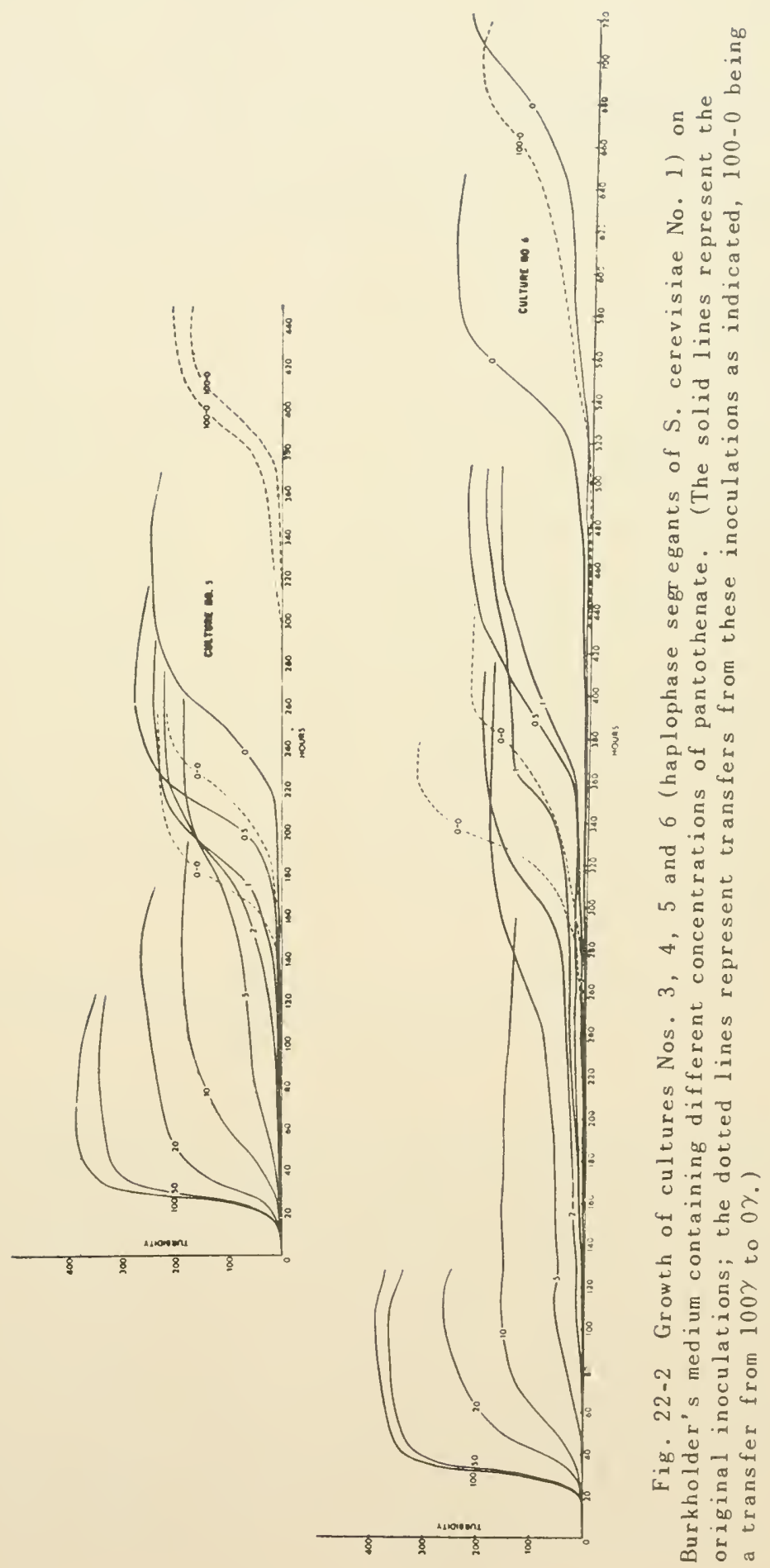
pantothenate than the cultures which had been originally classified as "synthesizers."

Inoculum came from a slant of yeast extract agar. Cells were suspended in $10 \mathrm{cc}$. of sterile distilled water and transfers to Burkholder's medium were made with a loop of this diluted suspension to insure against transfer of vitamins. A small but uniform number of cells was transferred in each loop. Ir respective of the concentration of pantothenate in Burkholder's synthetic media none of these original transfers failed to grow, but each grew after the delay indicated on the graphs. Many other transfers were subsequently made from one tube of Burkholder's synthetic medium to another with the same concentration of pantothenate; all these resulted in growth.

S. carlsbergensis (culture No. 2, fig. 22-1) completed growth in all concentrations of pantothenate before 100 hours. The single haploid offspring of S. carlsbergensis (No. 7, fig. C1A) is capable of utilizing any available pantothenate as is shown by the beautifully parallel curves on the different concentrations. A hybrid between culture No. 7 and No. 5 produced the hybrid, culture No. 10, which grew rapidly in deficient medium (fig. 22-1). When four haploid progeny from hybrid No. 10 , cultures No. 20 , No. 21 , No. 22 and No. 23 (fig. 22-3) were tested, all showed the ability to use whatever pantothenate was available as evidenced by the parallel nature of the curves for different concentrations. However, these four progeny were all "synthesizers" of the vitamin; no clear-cut Mendelian segregation occurred. This does not mean that the difference is not under gene control, for this pedigree is one in which gene transformation occurs frequently.

\section{ADAPTATION TO THE ABSENCE OF PANTOTHENATE BY A REGULARLY SEGREGATING, SLOWLY ADAPTING CULTURE}

Since the strains described in the preceding section did not segregate in a regular Mendelian manner, the experiments were continued. by Raut (1949) with pantothenate-dependent haploid strain 2154, from a regularly segregating pedigree, in order to determine the genetic nature of the adaptation. In addition to segregating regularly, strain 2154 also requires a longer time for adaptation to take place (over a month). This strain was derived from the pedigree shown in Table 19-3. Critical selective breeding for several generations had produced regular segregations and considerably lengthened the time required for adaptation. Haploid clone 2154 came from an ascus containing two dependent and two independent spores, as follows: 2151 -, $2152+, 2153$ + and 2154 -, where "+" indicates ability to grow without pantothenate added to the medium and "-" indicates inability to grow under these conditions. In this pedigree whenever a pantothenate 
independent strain is crossed by a dependent strain, the segregation obtained is always 2 dependent and 2 independent spores in each four spored ascus. Ability to grow rapidly without pantothenate is determined, in the pedigree, by the segregation of a single gene. Clone 2154 is further characterized by being a non-fermenter of both maltose and melibiose, and of mating type "a".
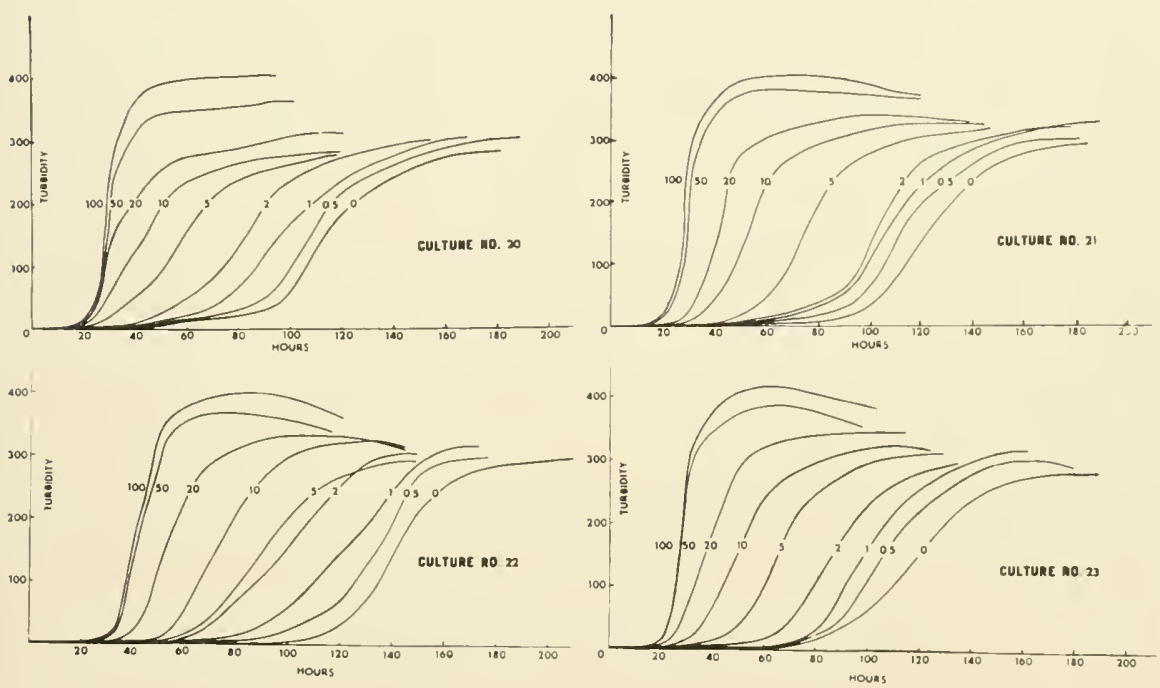

Fig. 22-3 Growth of cultures Nos. 20, 21, 22, and 23 (haplophase segregants from S. carlsbergensis by $S$. cerevisiae hybrid) on Burkholder's medium containing different concentrations of pantothenate.

The experiment to determine the effect of concentration of pantothenate on adaptation was repeated with culture 2154. Figure 22-4 is the series of curves obtained by plotting amount of growth (in colorimeter readings) against time in hours. From $200 \gamma$ per liter down to $2 \gamma$ per liter, both the rate of growth and the total amount of growth decreases as the amount of pantothenate decreases. This is what would be expected if the organism requires pantothenate for growth but is unable to synthesize it, at least in sufficient amount. However, as the concentration reaches this critical point $(2 \gamma$ per liter) or drops below it, there is a long lag after which the culture. adapts and attains a rate and total amount of growth greater than those at the intermediate concentrations.

The adaptation is a permanent heritable change for the increased rate of growth which occurs in the lowest concentrations is main- 
tained on inoculating a small number of cells into a new tube of medium without added pantothenate. Serial transfer on minus pantothenate nedium increases the rate of growth even further. The increase in adapted rate of growth is not lost after transfer from medium containing pantothenate.

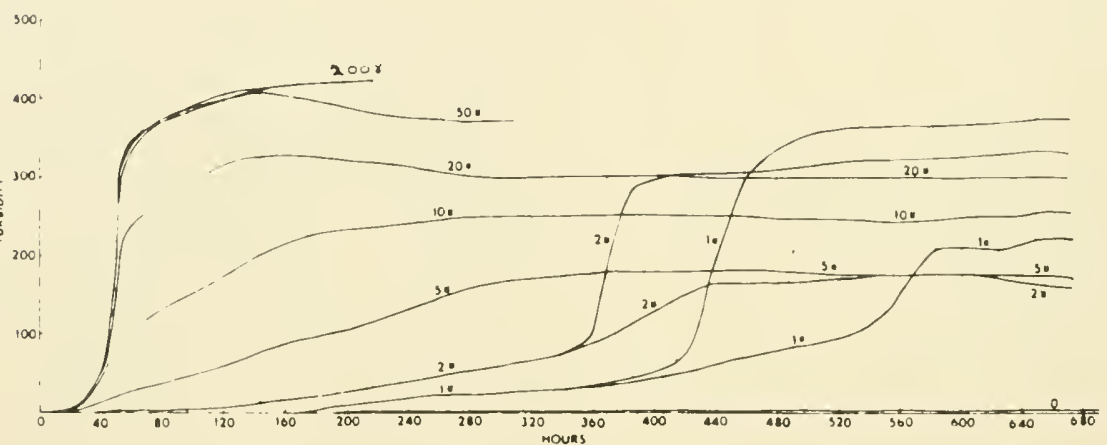

Fig. 22-4 Growth of pantothenate-dependent culture 2154 in the presence of various concentrations of pantothenate.

The zero concentration curves are incomplete in the time covered by the graph. They are shown in fig. 22-4A and are discussed in the section entitled, "Growth of the Recessive Culture in the Absence of Pantothenate".

A second important aspect of the adaptation curves (fig. 22-4) is that below the critical concentration of pantothenate, although there is considerable irregularity, adaptation takes place sooner in the higher concentration ( $2 \gamma$ and $1 \gamma$ per liter) than in the $0 y$ concentration.

These points bring up the following questions:

1. Is the heritable change a gene mutation?

2. Why does adaptation take place at $2 \gamma$ per liter or below, but not above this point?

3. Why is adaptation speeded up by increases of pantothenate at concentrations below the critical level?

4. If this proves to be a gene mutation, is it specifically induced by the minute amount of pantothenate present or does it occur spontaneously and at random? 


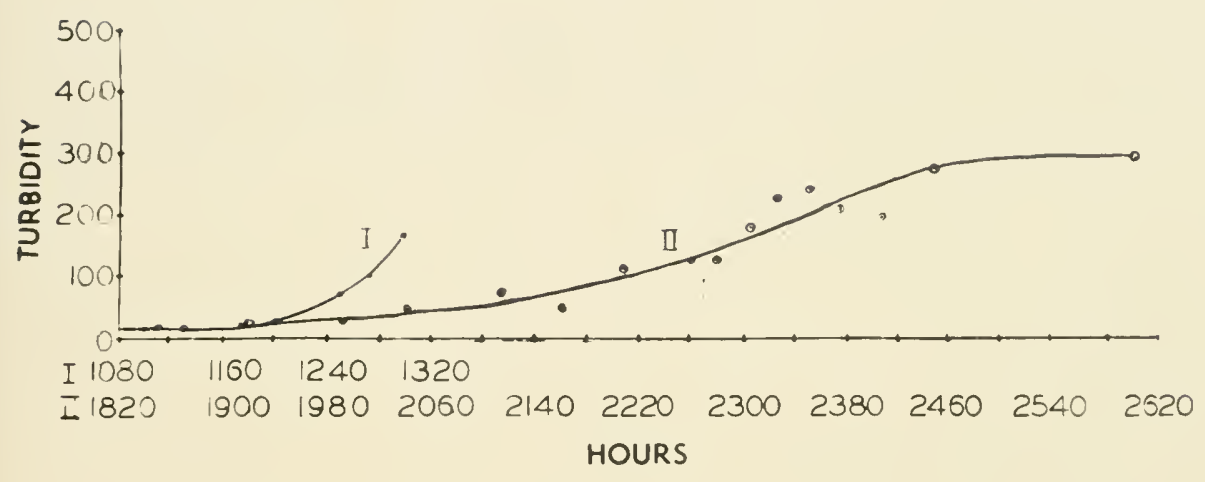

Fig. 22-4A Growth of 2154 cells in pantothenate-free medium $(0 y)$. In duplicate tubes adaptation occurred at different times. The number of cells are shown in Table $22-3$. These numbers are converted into Klett readings to make this curve comparable to fig. $22-4$ of which fig. 22-4A is an extension. Two different time scales are shown; one for Culture I and one for Culture II. Sampling was discontinued at the points indicated. It is rlear that I was already adapted at about 1200 hours. Adapted cells were found in Il at around 2400 hours, but it is clear from fig. $22-7$ that considerable growth occurred previous to the "secondary" adaptation.

\section{DEMONSTRATIONS OF THE GENIC NATURE OF THE ADAPTATION}

To determine if a gene mutation were involved, one of the adapted (2154) cultures was crossed with different synthesizing and non-synthesizing haploid strains. The following tabulation lists the eight cultures from two asci described in table 19-3 showing which grew in Burkholder's medium with added pantothenate and which did not. The superscript over the plus indicates the number of days before full growth appeared. The figure in parenthesis after the minus indicates the time required for the cultures to adapt in the absence of added pantothenate. Culture 2154 becomes capable of growth in a medium without pantothenate after 5 days once it has become adapted to the deficient substrate.

$\begin{array}{lllll}2113- & (>40) & 2151- & (>30) \\ 2114+4 & & 2152+3 & \\ 2115+4 & & 2153+3 & \\ 2116- & (>40) & 2154- & (>30) \rightarrow 2154\left(+^{5}, \text { adapted }\right)\end{array}$




\section{Table 22-1}

Genetical analysis of hybrids between non-synthesizers and synthesizers of pantothenate of indicated origins.

\begin{tabular}{|c|c|c|c|c|c|}
\hline & 215 & $\begin{array}{l}54 \text { ad } \\
+5\end{array}$ & $\begin{array}{c}2154 \\
(>30)\end{array}$ & $\begin{array}{c}2153 \\
+3\end{array}$ & $\begin{array}{c}2115 \\
+4\end{array}$ \\
\hline $\begin{array}{l}2113 \\
(>40)\end{array}$ & $\begin{array}{l}+5 \\
+4 \\
+5 \\
+5 \\
+4 \\
+7\end{array}$ & $\begin{array}{l}+5-- \\
+8-- \\
+7-- \\
+8-- \\
+8- \\
-*--\end{array}$ & $\begin{array}{l}-- \\
-- \\
-\end{array}$ & & \\
\hline $\begin{array}{l}2114 \\
+4\end{array}$ & $\begin{array}{l}+3 \\
+5 \\
+3\end{array}$ & $\begin{array}{l}+4+6- \\
+5+11- \\
+3+8\end{array}$ & $\begin{array}{l}+3+4- \\
+3+3-\end{array}$ & & $\begin{array}{l}+3+3+4+4 \\
+3+3+3+4 \\
+3+3+3\end{array}$ \\
\hline $\begin{array}{l}2151 \\
(>30)\end{array}$ & $\begin{array}{l}+7 \\
+8 \\
+6 \\
+6 \\
+5 \\
+9\end{array}$ & $\begin{array}{l}+9- \\
+11- \\
+11- \\
+11- \\
-\quad- \\
-\quad-\end{array}$ & 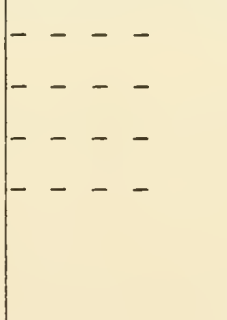 & & \\
\hline $\begin{array}{l}2152 \\
+3\end{array}$ & $\begin{array}{l}+4 \\
+3 \\
+4 \\
+4 \\
+3 \\
+3 \\
+4\end{array}$ & $\begin{array}{r}-+7+4^{* *} \\
+6+6- \\
+4+8- \\
+4+6- \\
+4-+5^{* *} \\
+5+5- \\
+4-\quad-\end{array}$ & $\begin{array}{l}+3+4-- \\
+3+4-- \\
+3+4-\end{array}$ & $\begin{array}{l}+5+5+6+10 \\
+4+4+4 \\
+5+5\end{array}$ & \\
\hline
\end{tabular}

Control tube containing pantothenate did not grow; apparently loss mutation involving some unspecified substance.

** 4063, 4064, 4065, 4066

*** $4175, \quad 4176, \quad 4177,4178$ 
Culture 2154 (-) required more than 30 days to adapt to pantothenate synthesis in Burkholder's medium with no added pantothenate. A culture of 2154 grown for 7 weeks in this medium produced full growth on a second transfer to Burkholder's medium without added pantothenate six days after adaptation. This culture was designated 2154 (+ adapted). After several transfers on the deficient medium, this adapted culture and 2113 (-) produced fertile asci, six of which were dissected (table 22-1). Pantothenate independence vs. dependence segregated 2:2 in each ascus and full growth of the dominants was achieved in between four and eight days revealing that adaptation resulted from mutation of a single gene.

A control test is always made by inoculating a tube containing pantothenate. This makes it possible to detect cases in which failure to grow is not due only to pantothenate dependence, but to some additional deficiency arising by mutation. Such cultures may be pantothenate independent but unable to grow due to the effect of the new mutation. The culture marked with a single asterisk failed to grow in the control tube containing pantothenate presumably due to its inability to synthesize some essential substance not present in Burkholder's medium.

Matings of unadapted 2154 (-) by 2113 (-), and by 2151 (-), on the other hand, produced asci which contained only dependent ascospores.

In order to determine whether the mutation involved back mutation of the recessive allele to the dominant allele of the gene for pantothenate independence, or was a mutation at a new locus, crosses were made between 2154 (t, adapted) and both $2114(+)$ and $2152(+)$. If two different loci are involved, three kinds of asci may be obtained. If we designate one pair of alleles for independence, as $A$ and $a$, and the other as $B$ and $t$, the three types are $A B A B a b a b, A b A b a B a B$, and $A B A b a B a b$. Then the first type of ascus listed ( $A B A B a b a b$ ) will contain two independent and two dependent spores, the next all independent and the last three independent to one dependent. If the genes are freely assorting, these asci will be obtained in a ratio of 1:1:4 (Lindegren 1948).

A hybrid of dominant $2114\left(+^{4}\right)$ by $2154(+$, adapted) produced three asci each containing three independent ascospores. This is the most frequent type of culture obtained when two freely mating non-allelic genes are hybridized. If $2114(+)$ is $\mathrm{Ab}$ and $2154(+, \mathrm{a}-$ dapted) is $\mathrm{aB}$, the double dominant can be indicated by $\mathrm{AB}$, the double recessive by $a b$, then the asci are $A B A b a B$ ab. A mating of $2114\left(+{ }^{4}\right)$ by 2154 ( - , unadapted) reveals that the original dominant (Ab) stock contains a single independent gene. The mating of 2114 $(+)$ by $2115(+)$ shows that each carries a single dominant gene, the cross of $\mathrm{Ab}$ by $\mathrm{Ab}$ producing four $\mathrm{Ab}$ dominants in each ascus. $\mathrm{A}$ mating of 2154 (+, adapted) by its unadapted sister 2151 (-) showed 
that these two cultures differed from each other by a single allele; the mating was a B by ab. A mating of unadapted 2154 (-) by unadapted 2151 (-) gave 4 asci each of which produced only recessives; this is a homozygous ab by ab cross. A mating of 2154 (+, adapted) by its independent sister $2152(+)$ produced six asci containing three independents and one ascus containing two independents. The asci are $A B A b$ a $B a b$, and $A B A B$ ab ab respectively. The third type $A b$ $\mathrm{Ab}$ a $\mathrm{B}$ a B did not appear. A mating of $2152(+)$ by the unadapted 2154 (-) revealed that 2152 contained only a single dominant allele. A mating of 2152 by 2153 revealed that 2152 and 2153 each carried a single dominant gene and that these genes were alleles since no dependent spores were obtained.

This analysis makes it possible to assign the genotype $\mathrm{a} B$ to 2154 (+, adapted) and ab to 2154 unadapted; 2153, 2115, 2114 and 2152 are $\mathrm{Ab}$, and 2151 and 2113 are also $\mathrm{ab}$.

Two of the asci obtained from the mating of 2152 by 2154 adapted were subjected to further analysis shown in the following tabulation. Cultures $4175,4176,4177$, and 4178 , from a single ascus, contained three independent and one dependent clone. $4176(\mathrm{AB})$ mated to 2151 (ab) dependent produced one ascus containing four independent spores, two asci containing three, and one ascus containing two. This is obviously a mating of a double mutant independent clone by a double mutant dependent, AB by ab. Therefore, 4175 is the double mutant independent. Mating of the two other independent clones, 4175 and 4178 , by dependents, produced asci indicating that 4175 and 4178 each contained a single independent allele.

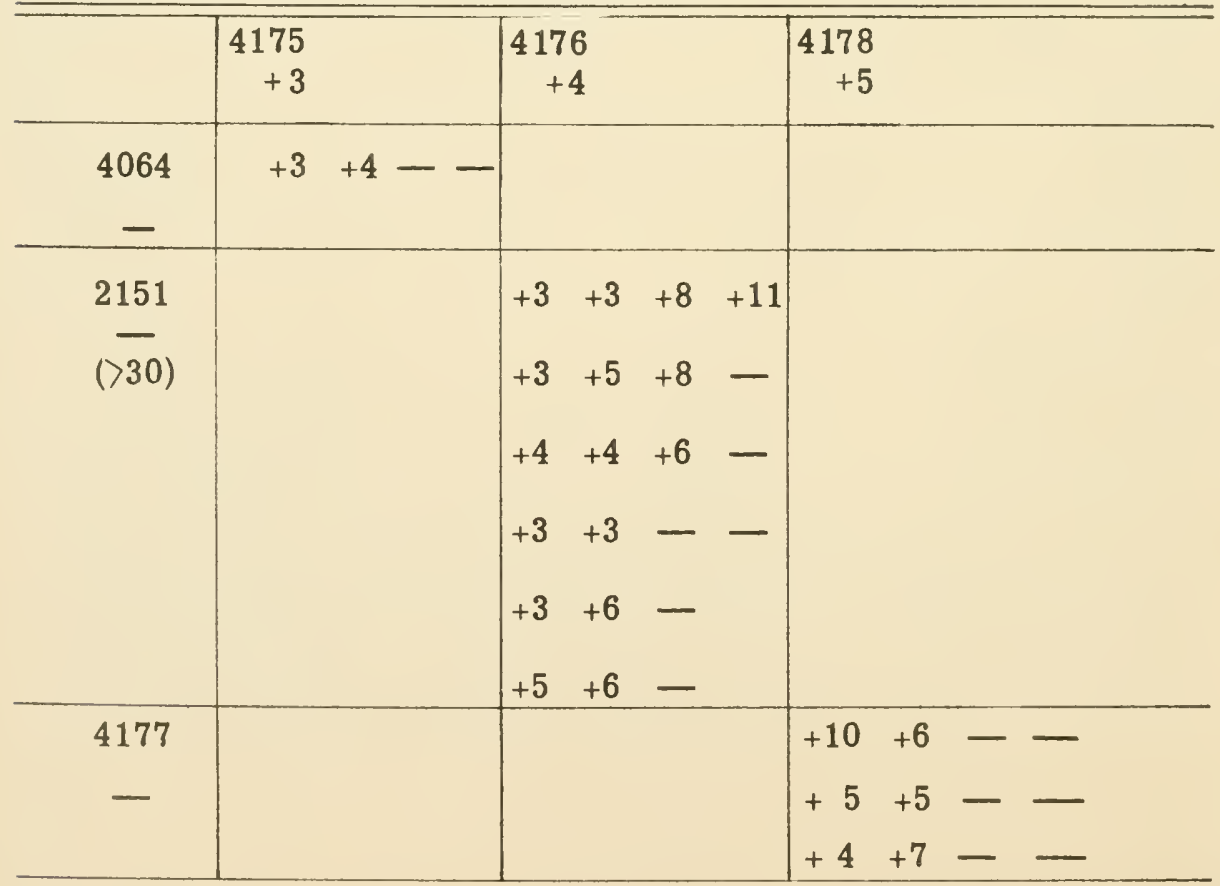


This analysis indicates that adaptation to pantothenate independence in 2154 resulted from a mutation of dependent locus b to independent locus B. If the original unadapted 2154 carried the genes $\mathrm{ab}$, and its synthesizing sisters 2151 and $2153 \mathrm{Ab}$, then the mutation during adaptation changed $a b$ to $a B$. A mating of $a B(2154+$, adapted) by $\mathrm{Ab} 2152(+)$ can produce three types of asci containing two, three and four independent spores respectively. Those containing three independent spores would be like the ascus $4175,4176,4177,4178$ which is of the constitution $\mathrm{AB}$ ab $\mathrm{Ab}$ aB. Cultures 4175 and 4178 are both single mutants, either $\mathrm{Ab}$ or $\mathrm{aB}$.

The two genes can be roughly distinguished by their growth rates. The original (Fig. 22-4) synthesizers (Ab) usually begin the rapid observable phase of growth in pantothenate free medium before the adapted 2154 genotype (aB). Among the asci in which 3:1 segrega tions occurred, two of the clones usually grew more rapidly than the third. Hence, apparently, the clone of the genotype $A B$ and that of the genotype $\mathrm{Ab}$ generally grow at the same rate while the genotype aB grows more slowly. Fig. 22-5 shows growth curves for the $B$ gene ( 2154 adapted) on a series of concentrations of pantothenate; as compared with the original unadapted 2154 (ab), the adapted strain (aB) grows more slowly when sufficient pantothenate is supplied than the original. The ab clones showed no evidence of growth for over a month, but all were subject to the adaptation phenomenon described in detail for culture 2154.

\section{SYNTHESIS OF PANTOTHENATE BY GENE B}

To determine if the $\mathrm{B}$ gene controls the synthesis of pantothenate, culture $2152(\mathrm{Ab})$, which synthesizes pantothenate, and adapted 2154 (aB), were assayed.

The cultures were prepared for assay by growing them on Burkholder's synthetic medium minus pantothenate, washing, centrifuging and digesting in $10 \mathrm{ml}$. distilled water at $\mathrm{pH} 5.5$ in the autoclave at 20 pounds pressure for 15 minutes. An aliquot was dried and weighed to determine the dry weight of the sample.

The extracts thus prepared were assayed with culture 2154. Extracts from both cultures produced growth. indicating the presence of free pantothenate in both of the cultures. The presence of pantothenate in the adapted strain indicates that the new gene B does not function by allowing the yeast to grow without pantothenate, but instead makes possible the synthesis of pantothenate.

\section{ALTERNATE ROUTE OF SYNTHESIS}

Since pantothenate is synthesized rapidly in the presence of either A or B, or both, the new gene must enable synthesis to take place over an alternate route, unless both $A$ and $B$ affect the same step. 
A

B

A

$\downarrow$

$\mathrm{V} \rightarrow \mathrm{W} \rightarrow \mathrm{X} \rightarrow \mathrm{Y} \rightarrow \mathrm{Z} \rightarrow$ pantothenate $\mathrm{W} \rightarrow \mathrm{X} \rightarrow \mathrm{Y} \rightarrow \mathrm{Z} \rightarrow$ pantothenate

$$
\mathrm{W}^{\prime} \rightarrow \mathrm{X}^{\prime} \stackrel{\substack{\downarrow \\ \mathrm{X}^{\prime}}}{\rightarrow} \mathrm{Z}^{\prime} \rightarrow \text { pantothenate }
$$

If the genes were involved in two different steps in a series of steps in the synthesis of pantothenate, as diagrammed in I, the lack of either gene would slow up the synthesis. As it is, synthesis occurs rapidly when eithe ${ }_{1}$ gene is present. Thereiore, the situation must be as diagrammed in II, where $A$ is involved in one possible mode of synthesis, and B in another alternate route.

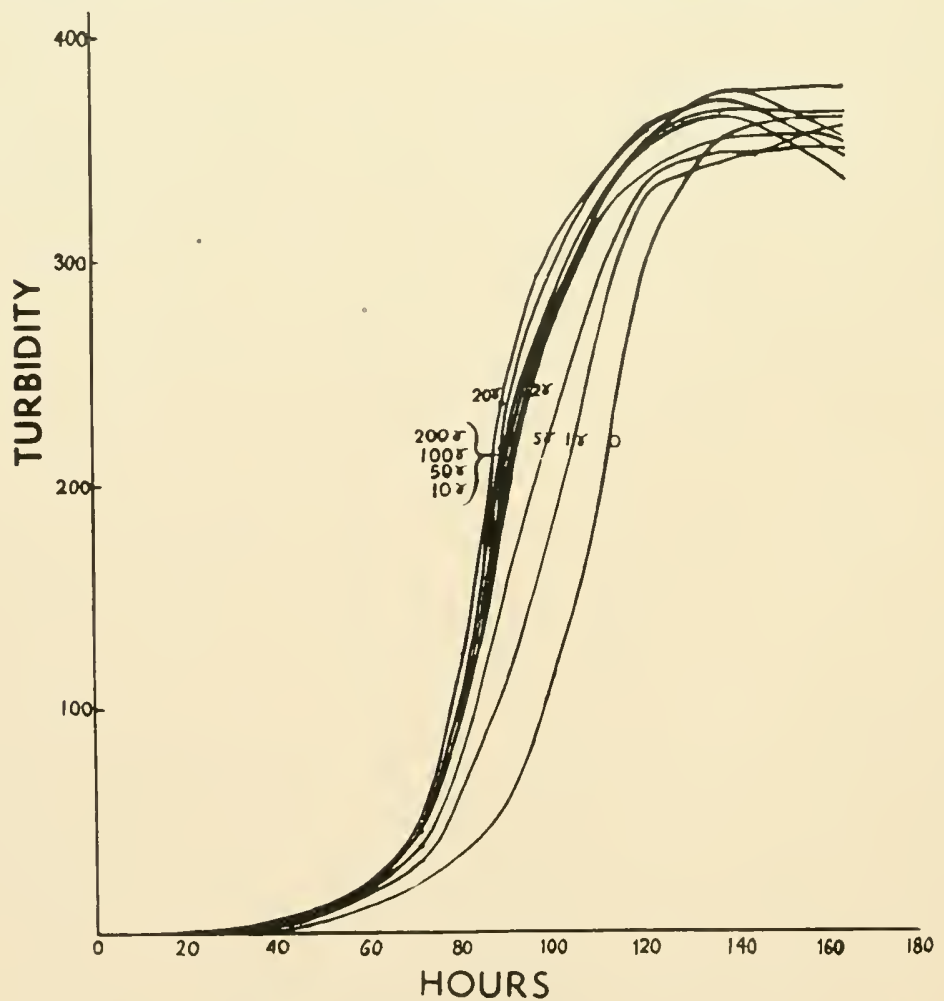

Fig. 22-5 Growth of Adapted Culture 2154 in a Series of Concentrations of Pantothenate.

The mutation $\mathrm{B}$ involves an addition to the synthetic abilities of the organism. One would assume that a number of steps were in- 
volved in the new route of synthesis. It is difficult to conceive of the process being established by a spontaneous chance one-step gene mutation, unless most of the route were previously established. It seems most likely that the complete mechanism for pantothenate synthesis was operative in some of the progenitors of culture 2154, but that a step was blocked in the non-synthesizer and this block was overcome by the adaptation.

\section{MUTATION IN THE PRESENCE OF PANTOTHENATE}

An attempt was made to discover why adaptation occurs at 2 ? per liter or lower, but not above this point. The first question is whether mutations occur at all in high concentrations of pantothenate. To determine this two groups of test tubes containing $4 \mathrm{ml}$. of complete medium were inoculated with around 500-1000 cells of 2154 and allowed to grow to completion. The yeast was then washed five times with sterile water and the entire cultures plated out on pantothenate-free agar. The plates were incubated at $30^{\circ} \mathrm{C}$ and observed for appearance of colonies. The results obtained are given in table 22-2.

Table 22-2.

\section{occurrence of Wutants in the Presence of Pantothenate.}

Experiment I

\begin{tabular}{c|c|c|c|c|c|c}
\hline \multirow{2}{*}{ Culture } & \multicolumn{6}{c}{ Days to appearance of colonies } \\
\cline { 2 - 6 } & 10 & 13 & 16 & 20 & 23 & 25 \\
\hline 1 & $2 \mathrm{~s} 2 \mathrm{vs}$ & $4 \mathrm{~s}$ & 4 & 4 & $4,8 \mathrm{vs}$ & $4,8 \mathrm{vs}$ \\
2 & 0 & 0 & 0 & $3 \mathrm{vs}$ & $3 \mathrm{~s}$ & 3 \\
3 & 0 & 0 & 2 & 2 & $2,4 \mathrm{vs}$ & $2,4 \mathrm{vs}$ \\
4 & 0 & 1 & $2,3 \mathrm{~s}$ & $1,6 \mathrm{~s}$ & $1,7 \mathrm{~s}$ & $7,3 \mathrm{~s}$ \\
5 & $1 \mathrm{~s}$ & 1 & $1,1 \mathrm{~s}$ & $1,3 \mathrm{vs}$ & $1,4 \mathrm{vs}$ & $2,4 \mathrm{~s}$ \\
6 & 0 & $1 \mathrm{~s}$ & 1 & 1 & 1 & $1,2 \mathrm{~s}$ \\
7 & 0 & $3 \mathrm{vs}$ & $4 \mathrm{vs}$ & $8 \mathrm{~s}$ & $1 \mathrm{~s}, 9 \mathrm{vs}$ & $1,10 \mathrm{~s}$ \\
8 & 0 & 1 & $1,4 \mathrm{vs}$ & $1,4 \mathrm{~s}$ & $1,4 \mathrm{~s}$ & $1,4 \mathrm{~s}$ \\
9 & 0 & 1 & $1,4 \mathrm{~s}$ & 6 & 7 & cont. \\
\hline
\end{tabular}

Average number of cells seeded on each plate-5.78 x $10^{7}$. 
Experiment II

\begin{tabular}{|c|c|c|c|c|c|c|}
\hline \multirow{2}{*}{ Culture } & \multicolumn{6}{|c|}{ Days to appearance of colonies } \\
\hline & 7 & 9 & 13 & 16 & 18 & 21 \\
\hline 1 & 0 & 0 & 0 & 1Vs & $1,1 \mathrm{~s}$ & $1,6 \mathrm{~s}$ \\
\hline 2 & $0^{\circ}$ & 0 & $>50 \mathrm{VS}$ & $>50 \mathrm{Vs}$ & $>50 \mathrm{Vs}$ & $108 \mathrm{~s}$ \\
\hline 3 & 0 & 0 & $3 \mathrm{vs}$ & $6 \mathrm{vs}$ & $6 s$ & $6 s$ \\
\hline 4 & 0 & 0 & 0 & $7 \mathrm{vs}$ & $8 \mathrm{vs}$ & $9 \mathrm{vs}$ \\
\hline 5 & 0 & 0 & $6 \mathrm{vs}$ & $10 \mathrm{vs}$ & $10 \mathrm{~s}$ & $13 \mathrm{~s}$ \\
\hline 6 & 0 & 0 & $2 \mathrm{VS}$ & $4 \mathrm{VS}$ & $4 \mathrm{VS}$ & $4 s$ \\
\hline 7 & 0 & 0 & $9 s$ & $24 \mathrm{~S}$ & $24 \mathrm{~s}$ & 24 \\
\hline 8 & 0 & 0 & 0 & $30 \mathrm{vs}$ & $40 \mathrm{Vs}$ & $180 \mathrm{~s}$ \\
\hline 9 & 0 & 0 & 0 & $1 \mathrm{VS}$ & $1 \mathrm{VS}$ & $1 \mathrm{~s}$ \\
\hline 10 & 0 & 0 & 0 & 0 & 0 & 0 \\
\hline 11 & 0 & 0 & $<1000 \mathrm{vs}$ & $<1000 \mathrm{vs}$ & $3 s<1000 v s$ & $4,970 \mathrm{~s}$ \\
\hline 12 & 0 & $1 \mathrm{vs}$ & 1 & 1 & $1,4 \mathrm{~s}$ & $4,5 \mathrm{VS}$ \\
\hline 13 & 0 & 0 & 0 & 0 & 0 & 0 \\
\hline 14 & 0 & 0 & $50 \mathrm{vs}$ & $50 \mathrm{vs}$ & $>100 \mathrm{vS}$ & $130 \mathrm{vs}$ \\
\hline 15 & 0 & 0 & 0 & $1 \mathrm{~s}$ & $1 \mathrm{~S}$ & 1s \\
\hline 16 & 0 & 0 & 0 & 0 & 0 & 0 \\
\hline 17 & 0 & 0 & 0 & 0 & 0 & 0 \\
\hline 18 & $2 \mathrm{vs}$ & $2 \mathrm{~s}$ & $3,5 \mathrm{vis}$ & $3,15 \mathrm{vs}$ & $3.23 \mathrm{vs}$ & $3,34 \mathrm{VS}$ \\
\hline 19 & 0 & 0 & $8 \mathrm{vs}$ & $<100 \mathrm{vs}$ & $>100 \mathrm{vs}$ & $340 \mathrm{~s}$ \\
\hline 20 & 0 & 0 & 0 & 0 & 0 & $3 \mathrm{Vs}$ \\
\hline 21 & 0 & 0 & 0 & 0 & $2 v s$ & $2,41 \mathrm{~s}$ \\
\hline 22 & 0 & 0 & 0 & $<100 v s$ & $>100 \mathrm{~s}, \mathrm{VS}$ & $11,717 \mathrm{vi}$ \\
\hline
\end{tabular}

Average number of cells seeded on each plate--3.7 $\times 10^{8}$. Mutation rate $=3.21 \times 10^{-9}$ per cell per generation . 
The numbers designate the numbers of colonies appearing on the plate. The letters " $S$ " and "vs" designate small and very small colonies. A number alone designates a large colony, i.e., 0.4 to $0.6 \mathrm{~mm}$. Three large and three small colonies is designated $3,3 \mathrm{~s}$.

This method does not determine whether the mutations take place in the tubes prior to plating or whether they take place on the plate, particularly since a time elapses before any colonies appear. A slight film develops from the approximately $5 \times 10^{7}$ to $5 \times 10^{8}$ non-synthesizing cells which are seeded on each plate. Microscopic examination showed that each cell had increased on the average to about 30 cells during several weeks on the plate. The film appeared within two or three days after plating, after which there was no further apparent macroscopic change until individual colonies began to appear (fig. 22-6). Microscopic examination of the minute colonies after several weeks on the plate showed that the cells were vacuolated, as is characteristic of old cells, and staining with dilute methylene blue showed that with few exceptions the cells were all dead.

The fact that the cell population seems to multiply very little after the first few days suggests that the mutations do not take place on the plates, but occur in the culture tubes prior to plating. It appears further that the crowding of the cells may actually slow down the growth rate of the mutant cells which are plated out, and thus account for the long delay before their appearance, since crowding generally slows down the growth of individual colonies. As may be observed from table 22-2 there were a number of instances in which 50 or 100 , or even more colonies appeared simultaneously on one plate. It seems unlikely that so large a number of mutants would occur simultaneously, as would have to be the case if they occurred on the agar plate.

In addition to the variation in numbers of colonies there is also variation in the size and the time of appearance of the colonies, which suggests that all the mutants are not identical. Further testing will be required to determine how many classes of mutants are involved.

A few hundred cells of some of the colonies appearing on the test plates were inoculated into liquid test ${ }^{\prime}$ medium. Some colonies which required about two weeks to develop into small colonies on the test agar plates, on transfer to liquid medium reached maximum growth within four to eight days. Colonies requiring a longer time to develop on the plates, also grew more rapidly in liquid test medium than they had on the agar plates, though still not as rapidly as the faster-growing colonies. The more rapid growth rates in liquid test medium may have been due to the fact that the crowding on the plates inhibited the growth of the mutant cells on the plates as pointed out above. On the other hand, it is possible that secondary mu- 
tations may have taken place during the development of the colony on the plate, so that by the time the mutant was tested by transferrir to liquid medium its growth rate was more rapid than when it was originally isolated.

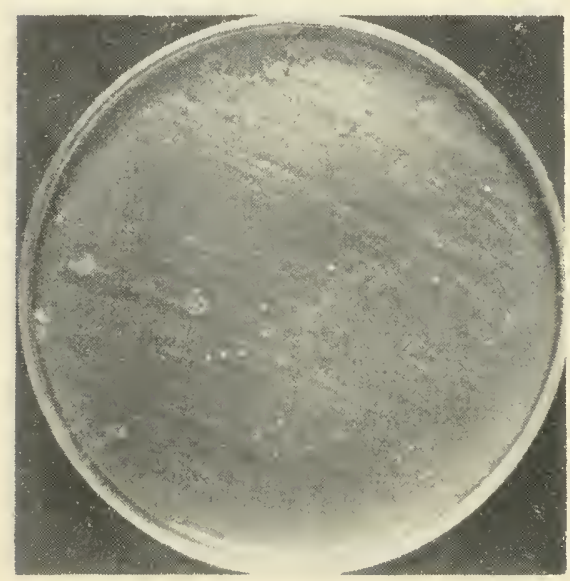

A

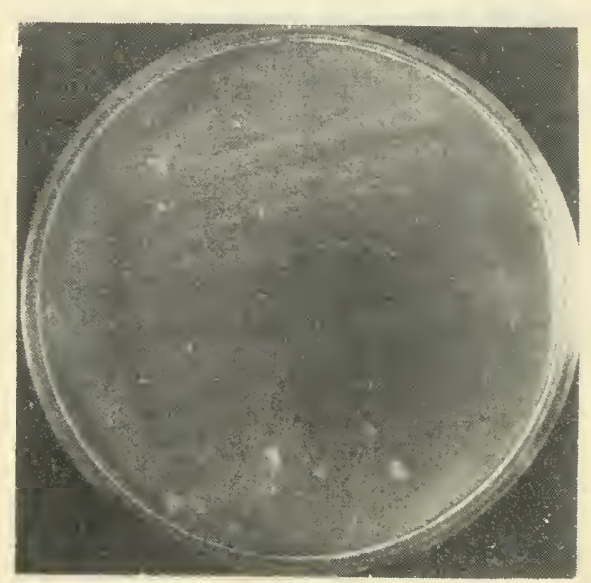

B

Fig. 22-6 Growth of Hutant Colonies on Test Agar Plates. A, Table 7, Exp: II, Culture 7; B, Table 7, Exp. II, Culture 18.

Since the mutants grow more slowly than the original in the presence of sufficient pantothenate there is selection against them during growth in complete medium prior to plating on the test agar, and because of this, it is not possible to calculate the variance from the data in table 22-2. It is apparent that mutations do occur in the presence of a high concentration of pantothenate and that there is considerable variation in the numbers of mutants. It appears that the mutations are random and because of the selection against them, are not ordinarily encountered. The mutation rate, calculated from the data from the experiment in which some of the cultures contained no mutants is $2.82 \times 10^{-9}$ per cell per generation. The mutation rate, calculated from the data from the experiment in which some of the cultures contained no mutants is $3.21 \times 10^{-9}$ per cell per generation. The mutation rate was calculated from the formula devised by Luria and Delbruck (1943) as given by Demerec and Fano (1945) in which the mutation rate $a,=\frac{-1.6 \log 10 \mathrm{P} 0}{\mathrm{~N}}$, where $\mathrm{P}_{0}$ is the proportion of cultures in which there are no mutants and $\mathrm{N}$ is the average number of cells per culture. Both large and small connnisc wars included in the calculation of the mutation rate. 
Table 22-3

The Number of Cells in Duplicate Cultures of \#2154 in Pantothenate-free Medium

\begin{tabular}{|c|c|c|c|}
\hline \multicolumn{2}{|c|}{ Culture I } & \multicolumn{2}{|c|}{ Culture II } \\
\hline Hours & Cells/ml & Hours & Cells/ml. \\
\hline 0 & $83^{*}$ & 0 & $83^{*}$ \\
\hline 1030 & $3.9 \times 10^{5}$ & 1584 & $4.0 \times 10^{4}$ \\
\hline 1104 & $4.6 \times 10^{5}$ & 1781 & $1.6 \times 10^{5}$ \\
\hline 1152 & $9.9 \times 10^{5}$ & 1853 & $5.6 \times 10^{5}$ \\
\hline 1200 & $1.9 \times 10^{6}$ & 1871 & $3.3 \times 10^{5}$ \\
\hline 1248 & $2.7 \times 10^{6}$ & 1919 & $7.6 \times 10^{5}$ \\
\hline 1271 & $9.5 \times 10^{6}$ & 1992 & $9.9 \times 10^{5}$ \\
\hline 1296 & $1.4 \times 10^{7}$ & 2040 & $2.3 \times 10^{6}$ \\
\hline \multirow{6}{*}{\multicolumn{2}{|c|}{ (Growth not complete at 1296 hours) }} & 2114 & $5.0 \times 10^{6}$ \\
\hline & & 2163 & $3.0 \times 10^{6}$ \\
\hline & & 2208 & $9.1 \times 10^{6}$ \\
\hline & & 2261 & $1.1 \times 10^{7}$ \\
\hline & & 2280 & $1.1 \times 10^{7}$ \\
\hline & & 2309 & $1.9 \times 10^{7}$ \\
\hline \multirow{6}{*}{\multicolumn{2}{|c|}{$\begin{array}{l}\text { * The inoculum was about } 1000 \\
\text { cells in } 12 \mathrm{ml} \text {. medium. }\end{array}$}} & 2327 & $2.8 \times 10^{7}$ \\
\hline & & 2352 & $3.1 \times 10^{7}$ \\
\hline & & 2376 & $2.5 \times 10^{7}$ \\
\hline & & 2407 & $2.3 \times 10^{7}$ \\
\hline & & 2448 & $4.0 \times 10^{7}$ \\
\hline & & & $4.2 \times 10^{7}$ \\
\hline
\end{tabular}

GROWTH OF THE RECESSIVE CULTURE IN THE ABSENCE OF PANTOTHENATE

In general, when culture \#2154 is inoculated into pantothenatefree medium, there is slow growth for a month or longer and then the culture "adapts" and grows fairly rapidly. When transfers are made from the culture after completion of growth into new tubes of test medium, a rapid rate of growth is maintained.

To study the nature of the adaptation in pantothenate-free medium, the following experiment was set up: Several tubes of panto- 
thenate-free medium were each inoculated with about 1000 cells of culture \#2154 and then followed closely to determine the number of cells present when the adaptation occurred and what proportion of the cells adapted. Samples were taken and the cells counted with a haemocytometer at frequent intervals during the growth period to determine if a change in growth rate occurred; at the same time samples were plated on plus and minus pantothenate agar plates to detect the presence of mutants. The data are shown in Table 22-3.

Cell counts from the beginning and the end of the logarithmic phase of growth were used in the calculation of generation time from the formula given by Buchanan and Fulmer (1928), in which generation time $=\frac{t \times \log _{10} 2}{\log _{10} b-\log _{10} B}$ where $t$ is the length of time over which the generation time is to be calculated, $b$ is the amount of growth at the end of the time, and $\mathrm{B}$, the amount of growth at the beginning. The generation time thus calculated is the average time required for cell division.

One of the duplicate tubes did not begin to show appreciable visible growth until about 1000 hours (46 days) and then exhibited a generation time of 42 hours. The other tube began to show appreciable growth at around 1900 hours (80 days), and had a generation time of 87 hours during the rapid phase of growth. The generation time of a fully adapted culture after several transfers on minus pantothenate medium is about 12 hours. Cell counts were used for the zero level of pantothenate as a measure of growth rather than turbidity readings. Evaporation of the water from the tube is a relatively unimportant factor during the first month of inoculation, but may become serious later, although more than one-half of the water is never lost and this only occurs in the tubes after 3 months or so. No effort was made to correct for this error.

Figure 22-7 shows the number of cells in one of these cultures grown about three months at $30^{\circ} \mathrm{C}$., plotted on semilogarithmic graph paper. The inoculum does not fall on the line, but there is fairly close fit of all the points plotted during the logarithmic phase of the growth of the culture. There is an obvious change in the rate of growth from that of the original inoculum, after which, the rate remains constant with a generation time of 82 hours.

The adaptation to the medium which establishes this generation time may be called the "primary" adaption. This adaptation is obviously different from the "secondary" adaptation which produces cells with a generation time of around 12 hours in pantothenate-free medium and which presumably originates by the mutation of a single cell subsequently demonstrated to be a segregating gene mutation.

Extrapolation of the curve shows that to achieve the primary adaptation at least one cell of the original inoculum must have adapted and does not exclude the possibility that the entire population may have adapted. This is an extraordinarily high mutation rate (if it be 
mutation) and suggests that the entire population adapted rather than a small fraction of it. The yeast containing recessive genes "ab", grows slowly in the pantothenate-free medium, and must be able either to grow at a very slow rate without pantothenate, or to synthesize pantothenate at a very slow rate. Experiments in this laboratory, with sugar fermentation have shown that in the presence of the recessive so-called "nonfermenting" alleies, fermentation actually does occur, but occurs much more slowly than when the dominant gene is present. This corresponds to the well-known example of the $c i$ gene in Drosophila, in which increased dosage of the recessive gene produces the same effect as the presence of a single wild-type allele (Stern). It seems probable, therefore, that the recessive allele does not involve complete absence of a reaction, but rather an extremely slow rate of reaction. On this basis, the slow growth on the pantothenate-minus medium during the primary adaptation may be due to very slow synthesis of pantothenate by the recessive alleles.

\section{MUTATION IN THE ABSENCE OF PANTOTHENATE}

Rapidly growing mutants were isolated from the culture tube at the points indicated (fig. 22-7). After 97 days, one colony which grew more rapidly than the rest was detected in a sample of approximately 44,000 cells plated on test agar plates. No colonies were detected in two intermediate platings with a total of 60,000 to 70,000 cells each, but in the next plating another mutant colony was found. This was 102 days after the original inoculation.

\section{Table 22-4}

Rapidly-growing Wutants Detected in Culture II

\begin{tabular}{r|c|c|c}
\hline Days & Cells/ml. & Cells plated & Mutants \\
\hline $1-96$ & & & 0 \\
97 & $2.8 \times 10^{7}$ & 44,000 & 1 \\
98 & $3.1 \times 10^{7}$ & 62,000 & 0 \\
99 & $2.5 \times 10^{7}$ & 76,000 & 0 \\
102 & $4.0 \times 10^{7}$ & - & 1 \\
\hline
\end{tabular}

The cell population was estimated from a haemocytometer count and the number of cells plated by a colony count of a diluted sample plated on plus-pantothenate nutrient agar. 


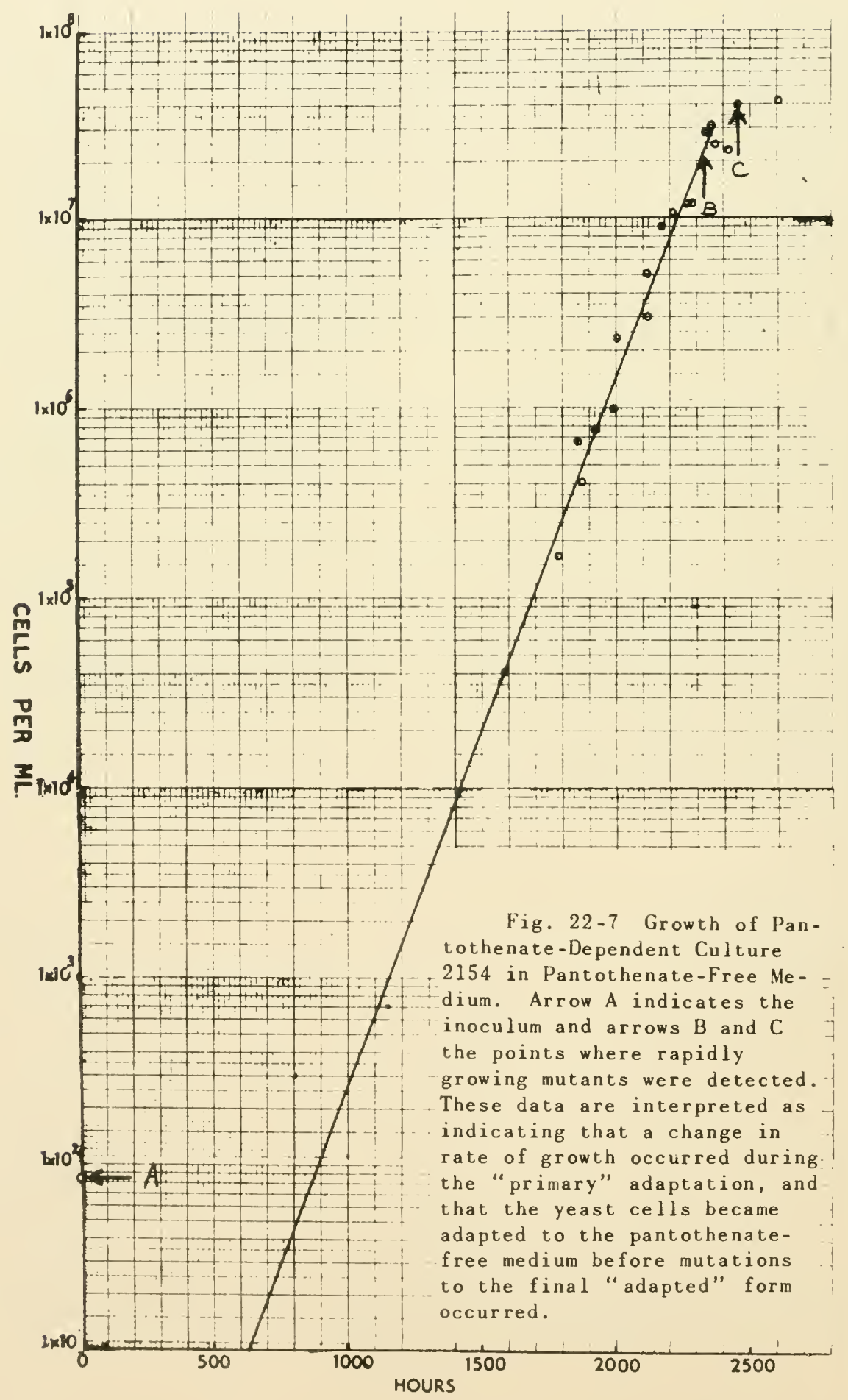


It is important to note that no rapidly-growing mutant cells were detected until the culture neared completion of growth and the cell population was very high. Of course, since only a small amount of the culture was sampled, it is unlikely that the adapted cells would be detected when they first occurred, but only after they had multiplied sufficiently to be fairly numerous in the population. The mutants maintained their rapid growth rate on transfer to pantothenatefree liquid medium and reached their maximum growth in about a week.

In addition to these two rapidly growing colonies some of the cells plated on pantothenate-free agar grew slowly and formed visible colonies (around $0.25 \mathrm{~mm}$.) in about two weeks. Thirty of these were tested by transfer into pantothenate-free liquid medium, where, except for five colonies which did not grow, they showed considerable growth (a turbidity reading of between 60 and 270) in about nine days. Three such colonies were detected in about 850 cells plated on the $83 \mathrm{rd}$ day after inoculation. The population at this time was about $6 \times 10^{5}$ cells per ml. Fifteen colonies were noted six days later (from about five times that number of cells); the cell population had increased to $1 \times 10^{6}$ per ml. Because of their slow growth, a good many of the colonies were probably overlooked. Apparently pantothenat $\epsilon$-independent "mutants" with intermediate growth rates may occur.

Another culture (III) similarly studied was incubated for 41 days at $30^{\circ} \mathrm{C}$. until the population was slightly less than $1 \times 106$ cells per $\mathrm{ml}$. and was then held at $7^{\circ} \mathrm{C}$. until completion of growth; under these conditions growth takes place more slowly and any change in the population can be more easily detected. The development was comparable to that obtained at $30^{\circ} \mathrm{C}$. The growth rate was constant (generation time 124 hours at $7^{\circ} \mathrm{C}$.) and no mutants were picked up in the samples plated until again the culture had reached its maximum growth. At this point, 76 days after inoculation, one small colony appeared after several days on a pantothenate-free agar plate in about 90,000 cells plated. No such colonies appeared in about 120,000 cells plated the next day, but 2 days later 3 colonies out of 90,000 plated grew more rapidly. On the next day 1 out of 100,000 , 4 days later, 3 out of $80,000,5$ days later, 3 out of 120,000, 6 days after this, 4 appeared, and in the last sample taken at 104 days, there were 5 colonies.

As before, the population was almost at its maximum point before the first rapidly growing colony was detected, so there was no increase in population size or in number of adapted cells.

These data show that the adaptation is not a simple one-step process. First, there is a primary adaptation which enables the culture to grow slowly in the pantothenate-free medium. When the cells are transferred to another tube of pantothenate-free me- 
Table 22-5

Rapidly-growing wutants Detected in Culture III

\begin{tabular}{c|c|c|c}
\hline \hline Days & Cells/ml. & Cells plated & Mutants \\
\hline 75 & $2.7 \times 10^{6}$ & 62,800 & 0 \\
76 & - & 74,000 & $1^{*}$ \\
77 & $3.2 \times 10^{6}$ & 120,000 & 0 \\
79 & $3.1 \times 10^{6}$ & 90,000 & $3^{*}$ \\
80 & $4.6 \times 10^{6}$ & 100,000 & 1 \\
83 & $3.9 \times 10^{6}$ & 80,000 & 3 \\
87 & - & 120,000 & 3 \\
89 & $4.3 \times 10^{6}$ & 50,000 & 0 \\
93 & - & - & 4 \\
98 & - & - & 0 \\
102 & - & - & 5 \\
\hline
\end{tabular}

* The 4 large colonies picked up on the 76th and 79th days showed only slight growth after 22 days when a few hundred cells were inoculated into liquid test medium. All of the colonies selected later showed appreciable growth in 3 to 4 days after transfer to pantothenatefree medium.

dium, the time to visible growth is reduced to three to four weeks, so this adaptation is transmitted vegetatively. The rate of growth following the "primary" adaptation has been shown to be constant. However, the rate is not the same in all cultures. The generation time for culture $\mathrm{II}$ at $30^{\circ} \mathrm{C}$. is 82 hours, for culture I it is 40 hours and for a third culture, not discussed further in this paper, it is 65 hours. The change occurs when there are relatively few cells present. The secondary adaptation (apparently a gene mutation), resulting in cells which grow much more rapidly in the absence of pantothenate, occurs when a large number of cells is present. There is variation in the rate of growth on pantothenate-free medium among the rapidly-growing colonies which make it appear that there are several levels of independence; both primary and secondary adaptations may consist of several steps. 


\section{RANDOMNESS OF MUTATION IN THE ABSENCE OF PANTOTHENATE}

A series of experiments were carried out to determine whether or not the "secondary-adaptation" mutations occur at random in the absence of pantothenate - that is, whether there is wide variation in the number of mutants in a group of similar cultures. However, in the absence of pantothenate, selection favors the synthesizing mutants so that the variation due to difference in time of occurrence of mutation is magnified. Therefore, it is not possible to calculate the variance, and it is possible to calculate the mutation rate only by means of the Luria and Delbruck (1943) method which depends on the number of cultures not containing mutations among a series of similar cultures.

A few hundred cells from separate colonies of culture III, isolated from agar containing pantothenate were inoculated into small tubes containing $5 \mathrm{ml}$. of pantothenate-free medium and were allowed to grow for about a month (table 22-6). At this time appreciable growth had occurred and the entire cultures were plated out on test agar plates to determine the number of mutants in each (experiments III, IV, V, and VI). (In experiments I and II, a loop of inoculum was taken directly from test tubes of culture III during the "primary" adaptation. The loop of cells was washed and diluted in $10 \mathrm{ml}$. of sterile distilled water and a loop of these washed cells was used to inoculate each of the $5 \mathrm{ml}$. of pantothenate-free broth in test tubes.) These cultures were allowed to grow to near completion of growth and then the entire cultures were plated on test agar plates. Since, in each case, the inoculum used consisted of cells which had undergone a considerable number of cell divisions in the selective environment of the test medium, a possibility of variation in the inoculum of the small tubes, which were plated out was introduced. This error does not seem to be very great, however, because the cultures grew quite uniformly and did not show very great differences in cell populations after growing in the pantothenate-free medium for 4 weeks (table 22-6). The number of mutants obtained from the various groups of cultures is given in the table.

From the great variation in the number of mutant colonies it appears that the mutations occur at random. From the variation in the sizes of the colonies it seems that growth rates of the mutants are not all the same. 


\section{Table 22-6}

\section{Occurrence of Wutants in Pantothenate-Free Medium}

Experiment I

(CuIture III)

Experiment II

(Culture III)

\begin{tabular}{l|c|l|c|c|c}
\hline No. cells & \multicolumn{2}{|c|}{$\begin{array}{c}\text { No. mutants } \\
17 \text { days }\end{array}$} & 28 days & No. cells & \multicolumn{2}{c}{$\begin{array}{c}\text { No. mutants } \\
17 \text { days }\end{array}$} & 28 days \\
\hline $1.98 \times 10^{7}$ & 0 & cont. & $1.77 \times 10^{7}$ & 0 & $7 \mathrm{~s}$ \\
$1.87 \times 10^{7}$ & 0 & $2 \mathrm{vs}$ & $1.60 \times 10^{7}$ & 39 & $70 \mathrm{~s}, \mathrm{vs}$ \\
$1.54 \times 10^{7}$ & 0 & $4 \mathrm{vs}$ & $4.17 \times 10^{7}$ & $>80,000$ & $>80,000 \mathrm{~s}$ \\
$2.13 \times 10^{7}$ & 0 & $2 \mathrm{~s}$, cont. & $1.35 \times 10^{7}$ & $>20,000$ & $>80,000 \mathrm{~s}$ \\
$1.77 \times 10^{7}$ & 3 & 5 & - & 0 & nu \\
$1.11 \times 10^{7}$ & 1 & $1,2 \mathrm{vs}$ & - & 0 & $25 \mathrm{vs}$ \\
$1.46 \times 10^{7}$ & 1 & 1 & - & 0 & cont. \\
\hline
\end{tabular}

Experiment III (Culture II)

\begin{tabular}{|c|c|c|c|c|c|c|c|c|}
\hline \multirow{2}{*}{\multicolumn{2}{|c|}{ Culture Total Cells }} & \multicolumn{7}{|c|}{ Lays to appearance ut colunies } \\
\hline & & 3 & 7 & 11 & 16 & 18 & 25 & 31 \\
\hline 1 & $1.4 \times 10^{6}$ & 1 & 2 & 2,13 & 3 & $3, j \cup s$ & $3,1 \cup \mathrm{s}$ & $3,15 \mathrm{~s}, \mathrm{nu}$ \\
\hline 2 & $17.3 \times 10^{6}$ & 0 & $3<5 \cup \mathrm{vs}$ & $4<5$ uvs & $4<, 0 \mathrm{~s}$ & $4<50 s$ & $4<50 s$ & $4,77 \mathrm{~s}, \mathrm{nu}$ \\
\hline 3 & $3.2 \times 10^{6}$ & 0 & 0 & $50 \mathrm{vs}$ & $50 \mathrm{vs}$ & $5 \mathrm{ivs}$ & $5<100 \mathrm{~s}$ & $y=100 s$ \\
\hline 4 & $5.6 \times 10^{6}$ & 0 & 0 & 0 & Is & $\therefore$, nu & ls & ls \\
\hline 5 & $9.2 \times 10^{6}$ & 0 & 0 & 0 & $1<1000 \mathrm{~V} 3$ & $4<1000 \mathrm{vs}$ & $7: 1000 \mathrm{vs}$ & $\begin{array}{l}4,225 \mathrm{~s} \\
4900 \mathrm{vs}\end{array}$ \\
\hline 6 & $16.7 \times 10^{6}$ & 0 & 0 & 0 & nu & nu & $\mathrm{nu}$ & nu \\
\hline 7 & $17.3 \times 10^{6}$ & 0 & 0 & 0 & nu & $7 \mathrm{~s}<1000 \mathrm{vs}$ & $y \cdot 1000 \mathrm{vs}$ & $y<320 \mathrm{vs}$ \\
\hline 8 & $25.9 \times 10^{6}$ & 0 & 0 & 0 & 0 & 0 & 0 & 0 \\
\hline 9 & $4.3 \times 10^{6}$ & 0 & 0 & 0 & 4 & 4 & $4, n u$ & $7, n u$ \\
\hline 10 & $1.8 \times 10^{6}$ & 0 & 0 & 0 & $1, n \cup$ & 1 100vs & $1<1$ JOVs & $7<1$ ᄂOvs \\
\hline Av. & $10.3 \times 10^{6}$ & & & & & & & \\
\hline
\end{tabular}

Mutation rate $=1.55 \times 10^{-7}$ per cell per generation. 
Experiment IV (Culture II)

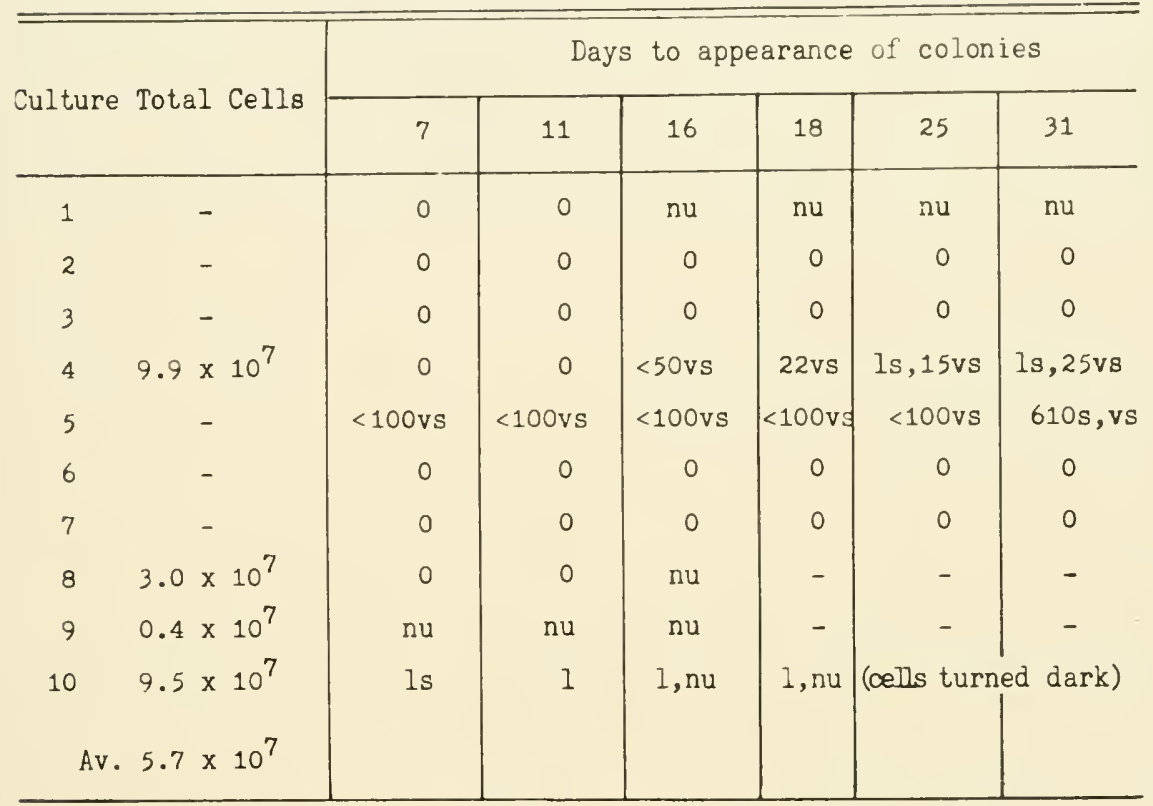

Mutation rate $=1.12 \times 10^{-8}$ per cell per generation

Counts were made of a number of identical cultures which were not plated, which are as follows: $2.1 \times 10^{7}, 7.9 \times 10^{7}, 8.5 \times 10^{7}$, $6.3 \times 10^{7}$, and $3.8 \times 10^{7}$, with an average of $5.5 \times 10^{7}$.

Experiment, V (Culture II)

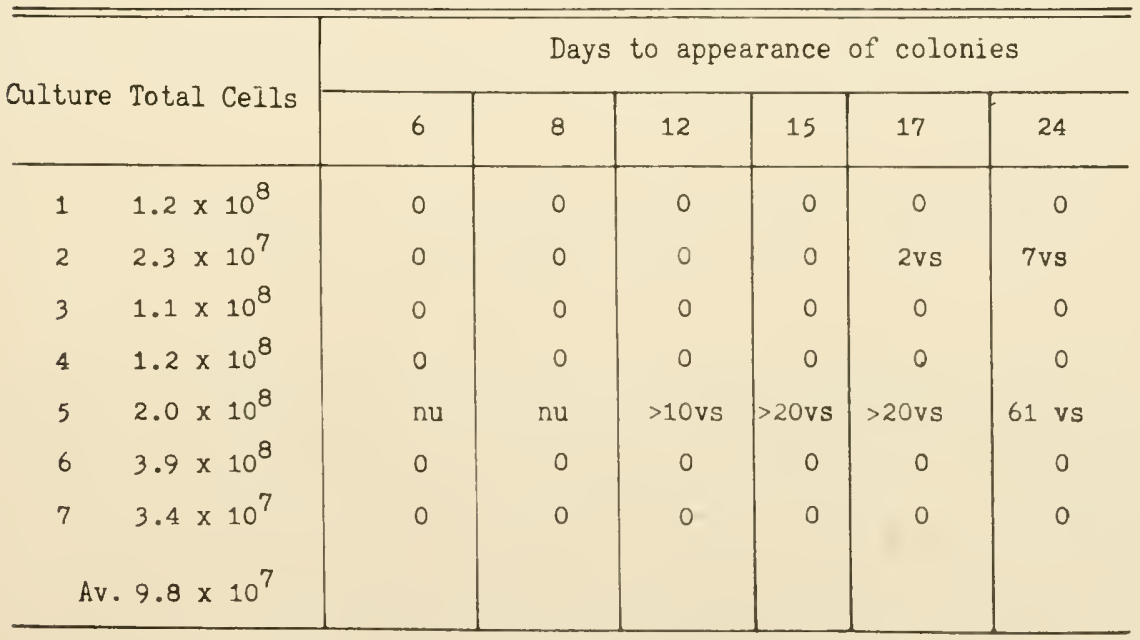

Mutation rate $=2.39 \times 10^{-9}$ per cell per generation. 
Experimerit VI (Culture II)

\begin{tabular}{cc|c|c|c|c|c}
\hline \hline \multirow{2}{*}{ Culture Total Cells } & \multicolumn{5}{|c}{ Days to appearance of colonies } \\
\cline { 3 - 7 } & 9 & 13 & 16 & 18 & 23 \\
\hline 1 & $7.3 \times 10^{7}$ & 1 s<1000vs & $2<1000 \mathrm{vs}$ & $3>1000 \mathrm{~s}$ & & $3,3600 \mathrm{~s}$ \\
2 & $12.0 \times 10^{7}$ & 0 & 0 & $1 \mathrm{~s}$ & $1 \mathrm{~s}$, vs & 2 \\
3 & $1.6 \times 10^{7}$ & 0 & 1 & 1 & 1 & $1,2 \mathrm{~s}, \mathrm{nu}$ \\
4 & $32.0 \times 10^{7}$ & 0 & $10 \mathrm{~s}$ & $17 \mathrm{~s}$ & 17 & 17 \\
5 & $15.0 \times 10^{7}$ & 0 & 0 & 0 & $($ contaminated) \\
6 & $4.4 \times 10^{7}$ & 1 & 1 & 1 & 1 & $1, \mathrm{nu}$ \\
7 & $34.0 \times 10^{7}$ & 0 & 12 & 17 & 17 & 17 \\
8 & $24.0 \times 10^{7}$ & 0 & 0 & 0 & 0 & 0 \\
9 & $9.2 \times 10^{7}$ & 0 & $>1000 \mathrm{vs}$ & $>1000 \mathrm{vs}$ & $>1000 \mathrm{vs}$ & $7200 \mathrm{vs}$ \\
10 & $3.3 \times 10^{7}$ & $1 \mathrm{vs}$ & $>1000 \mathrm{~s}$ & $>1000 \mathrm{~s}$ & $>1000 \mathrm{~s}$ & $2280 \mathrm{~s}$ \\
& & & & & & \\
\hline
\end{tabular}

Mutation rate $=8.25 \times 10^{-9}$ per cell per generation.

Numbers alone designate large colonies, ranging approximately from 0.4 to $0.6 \mathrm{~mm}$. in diameter. The letters $\mathrm{s}$ and vs designate from small and very small colonies. 3,3s means there are 3 large and 3 small colonies on the plate; "nu" means not uniform. The film of yeast cells on some of the plates appeared uneven although there were no colonies visible macroscopically. Microscopic examination revealed that a considerable number, around a fifth, of the colonies had reached a size of several hundred celis; these plates were classified "nu". The proportion of cultures showing o mutants at the end of the time given in the table was used in calculation of the mutation rates.

\section{COMPETITION BETWEEN ADAPTED AND UNADAPTED CELLS IN LIMITING CONCENTRATIONS OF PANTOTHENATE}

Gene mutations to pantothenate synthesis occur at random both in the presence and absence of pantothenate, but adaptations to growth in the absence of pantothenate only occur in media in which the concentration of pantothenate is less than two micrograms per liter and do not occur in concentrations above this point. An attempt was made to discover why adaptation occurs at the $2 \gamma$ of pantothenate per liter level or below, but not above this point. A series of duplicate tubes were set up containing decreasing amounts of pantothen- 
ate $(200 \gamma, 20 \gamma, 10 \gamma, 2 \gamma$ and $0 \gamma$ per liter) and inoculated with a mixed inoculum of cultures 2154 and adapted 2154 . The inoculum consisted of about 125 adapted cells and around 500 unadapted. Figure 22-8 shows the growth obtained as measured in colorimeter readings. The cultures were observed for another two weeks beyond the time shown on the graph, during which no appreciable change in the amount of growth occurred.

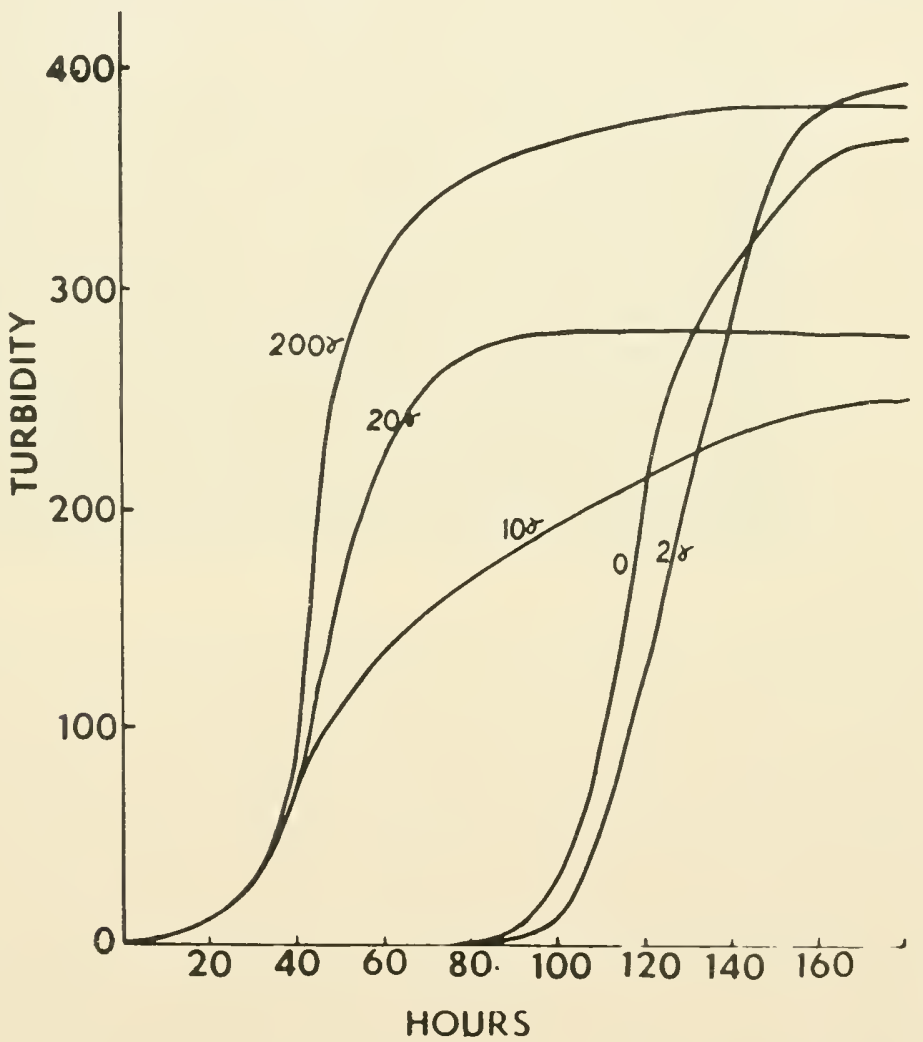

Fig. 22-8 Growth of Mixed Cultures of 2154 and Adapted 2154 in Various Concentrations of Pantothenic Acid.

Rate and total amount of growth of the mixed cultures decreases as the concentration of pantothenate decreases from $200 \gamma$ to $10 \gamma$, but as it is dropped to $2 \gamma$ and $0 \gamma$, there is a lag of 4 days after which rapid growth ensues. The total amount of growth is greater at $2 \gamma$ and $0 \gamma$ than at the intermediate amounts of $20 \gamma$ and $10 \%$. The shape of the curves in $200 \%, 20 \%$, and $10 \gamma$, are characteristic of growth curves for pantothenate-dependent culture 2154, and those at $2 \gamma$ and or comparable to those of adapted 2154 . When both types of cells are present, the non-synthesizing cells determine the growth in high 
concentrations of pantothenate while in very low concentrations the adapted cells make up the bulk of the population. It may be noted from table 22-7 that the total number of adapted cells in the mixed cultures at completion of growth increases as the concentration of pantothenate decreases. The ratio of the two types of cells in the final growth obtained was determined by plating samples on plus and minus pantothenate plates and the total number of cells estimated from baemocytometer counts.

Table 22-?

Analysis of $\boldsymbol{\nu}$ ixed Cultures

\begin{tabular}{c|c|c|c}
\hline $\begin{array}{c}\text { Pa. } \\
\text { Conc. }\end{array}$ & $\begin{array}{c}\text { Total } \\
\text { Cells }\end{array}$ & $\begin{array}{c}\text { Total } \\
\text { Adapted }\end{array}$ & \multicolumn{1}{|c}{$\begin{array}{c}\text { Ratio } \\
\text { ad:unad }\end{array}$} \\
\hline 200 & $2.1 \times 10^{8}$ & $1.9 \times 10^{4}$ & $1: 11,000$ \\
20 & $1.6 \times 10^{8}$ & $6.2 \times 10^{4}$ & $1: 2600$ \\
10 & $7.5 \times 10^{7}$ & $9.9 \times 10^{4}$ & $1: 760$ \\
2 & $1.6 \times 10^{8}$ & $1.6 \times 10^{8}$ & $76: 1$ \\
0 & $1.4 \times 10^{8}$ & $1.4 \times 10^{8}$ & $700: 0$ \\
\hline
\end{tabular}

If we refer to the growth rates of these two cultures grown separately, it is apparent from comparison of figure 22-4 showing growth rates for pantothenate-dependent strain 2154 alone and figure 22-5 for adapted 2154 alone, that the rate of growth of the dependent strain varies extremely with concentration of pantothenate while the rate of the independent strain varies only slightly. The unadapted dependent strain begins to grow sooner and grows more rapidly than the adapted strain at high concentrations. (See also figure 22-10). In low concentrations of pantothenate the adapted strain grows more rapidly. In the intermediate concentrations the growth rates of the two strains are approximately similar. Hence, in mixed cultures, while there is a possibility of competition between the cells of the two strains in all concentrations, it is effective only at intermediate concentrations, because at high concentrations the unadapted cells almost completely overgrow the adapted, while at low concentrations the adapted predominate.

The fact that mixtures of strains at intermediate concentrations do not reach maximum growth may be accounted for in two ways. (1) On limiting concentrations of pantothenate the dependent cells may not grow as efficiently as they do with sufficient pantothenate so that they exhaust one or more of the essential nutrients more rapidly than at optimal pantothenate concentrations and thus are unable to attain as high total growth as in the presence of sufficient pantothenate. When dependent cells are grown on limiting concen- 


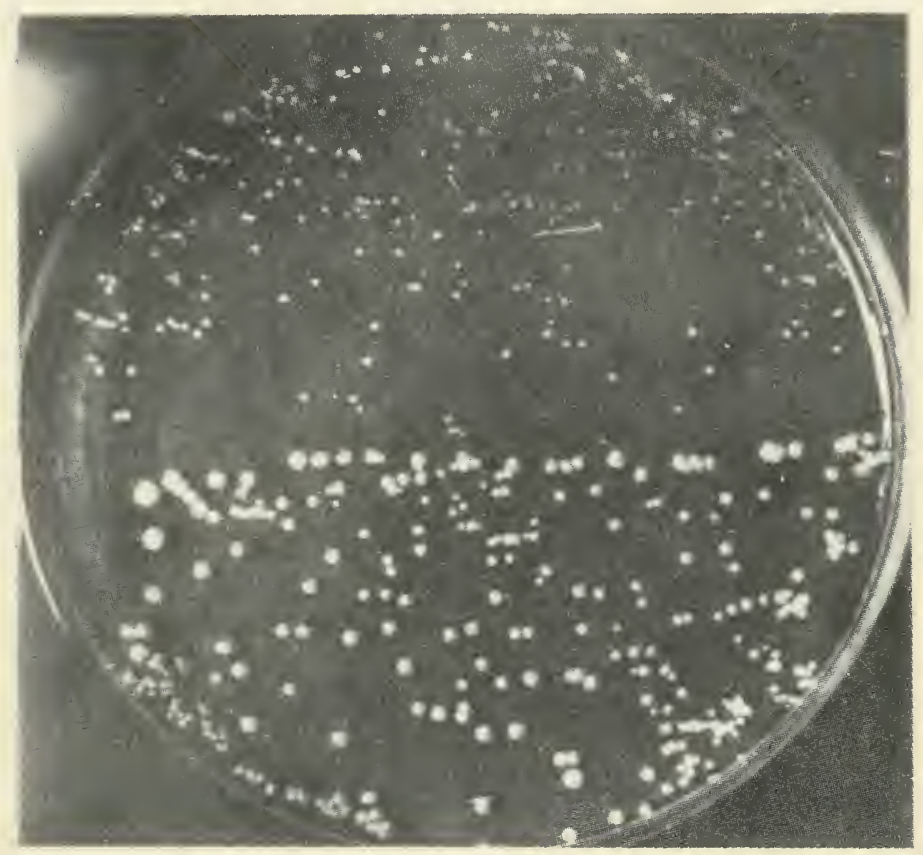

Fig. 22-9 A Week's Growth of Cultures 2154 and Adapted 2154 on a Complete Medium Agar Plate Showing the Difference in rates of Growth. The small colonies on the upper half of the plate are adapted 2154 and the larger ones on the lower half are 2154.

trations, in addition to the total cell number being reduced, the cells are much smaller, are irregularly shaped, and tend to clump. (2) The sick dependent cells may produce inhibitory metabolic products in unusually high amount. Either exhaustion of the medium or accumulation of by-products by the unadapted cells would inhibit growth of the adapted cells and thus account for the fact that maximum growth of mixed cultures is not obtained.

However, at $2 \gamma$ and $0 \gamma$ levels, very little growth of non-synthesizing cells can take place, so the adapted cells attain approximately the maximum amount of growth. There appears to be slight inhibition of adapted cells even at $2 \gamma$.

To test these possibilities, culture 2154 was allowed to grow up completion in duplicate tubes of medium containing $20 \gamma$ and $10 \gamma$ of pantothenate. A small inoculum of adapted 2154 cells was then introduced into the cultures grown at both concentrations and the same inoculum plus sugar into the duplicates of the two cultures. There was no further growth in the first case. When sugar as well as adapted cells was added, there was a small increase in growth. Since no growth at all was obtained when only adapted cells were 


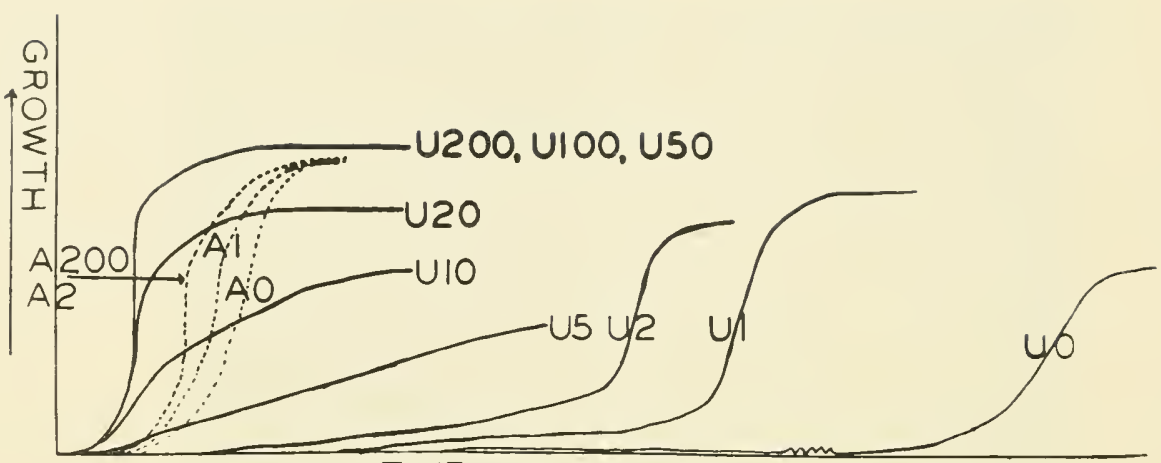

TIME

Fig. 22-10 Idealized Growth Curves Produces by Adapted and Unadapted Cells Inoculated into Media Containing Various Concentrations of Pantothenate. The numbers indicate the concentrations of pantothenate in micrograms per liter; $U$ and $A$, respectively, indicate unadapted and adapted inocula. At concentrations of 200 , 100 , and 50 microgr ams, the unadapted inocula give practically identical growth curves. When unadapted inocula are introduced into tubes containing 20, 10, and 5 micrograms per liter, the total final growth is roughly proportional to the amount of pantothenate present and no further growth occurs in the tubes. One can assume that adapted cells are almost certainly present in the U $20, U 10$, and $U 5$ tubes, but are unable to grow either because the inefficient growth of the unadapted cells has exhausted the nutrients or produced some inhibitory substance.

When adapted inocula are introduced into duplicate tubes containing from 200 to 2 micrograms of pantothenate per liter, the growth curves virtually superimpose, indicating that the growth rate of adapted cells is limited by factors other than pantothenate above 2 micrograms. The adapted organism exhibits a slightly slower growth rate in the absence of added pantothenate or in one microgram of pantothenate per liter, so that addition of pantothenate does have a slight effect in increasing growth rate. The growth curve of the adapted cells has both a longer lag and a slower rate than that of the unadapted cells given adequate pantothenate. Presumably, the long $\mathrm{lag}$ and the slow rate both depend on the synthesizing "load" carried by the adapted organism.

Curves U 2, U 1, and U 0 show the growth achieved by the un adapted cultures inoculated into media containing 2,1 , and no added micrograms of pantothenate, respectively. These curves are characterized at first by a long period of slow growth of unadapted cells and later by a sharp upward slope consisting of growth by the "adapted", mutant. The mutation to pantothenate synthesis occurs some time before the beginning of the upward swing. The cells dur ing the primary adaptation, although growing inefficiently and capable of producing inhibitory substances, or of exhausting the medium, 
added, it appears that all the sugar had been exhausted by the inefficiently growing unadapted cells. However, since addition of sugar made possible only a small incrcase in growth, it would appear that there were other factors in addition to exhaustion of sugar which prevented the adapted cells from attaining maximum growth. Further work will be required to determine whether this inhibition be due to exhaustion of other nutrients in addition to sugar or to accumulation of harmful by-products or both.

The reason the adapted culture does not reach the same high rate of growth as the unadapted in the presence of optimal concentrations of pantothenate may be due to a slight decrease in efficiency of growth caused by the extra "load" of synthesizing pantothenate. Other pantothenate synthesizing cultures bearing the A gene grow as rapidly as unadapted 2154 in the presence of a large amount of pantothenate so we cannot assume that ability to synthesize pantothenate in general depresses growth rate. In this particular case since we know the adapted strain synthesizes the vitamin by an alternate route it may be that this diverts an intermediate in the synthesis of some other growth factor so that the rate of growth is depressed.

This inhibition of growth of mixed cultures in intermediate amounts of the essential growth factor appears to be the same phenomenon as that studied by Ryan and Schneider (1948) of the interaction between histidineless and histidine independent cells of E. coli.

apparently have not reached a population large enough to limit seriously the secondary growth of the adapted mutant. The growth in the long primary phase may be due to synthesis of pantothenate by the recessive genes present in the unadapted cells. There is apparently little or no lag in the growth of unadapted cells in concentrations of pantothenate from: 200 to 5 micrograms per liter. However, when 2 micrograms of pantothenate are present, a short lag occurs during which the recessive (unadapted) organism is adapting to growth in the mediun and possibly to the slow synthesis of pantothenate. When 1 microgram is present a slightly longer lag occurs, indicating that the extra microgram of pantothenate is of considerable assistance in speeding up the growth of the unadapted cells. Correspondingly, in the absence of added pantothenate an even longer lag occurs than in the medium containing 1 microgram. The upward swing of these curves, after the primary adaptation, is due to the mutation from the unadapted to the adapted form. It depends on the production of a population suf ficiently large to assure the presence of one or more mutants. Small variations in the amounts of pantothenate below the critical level of 2 micrograms are directly reflected in the time at which the primary adaptations occur. 


\section{THE FUNCTIONS OF RECESSIVE AND DOMINANT}

\section{ALLELES IN ADAPTATION}

The analysis of the "slow" fermentation of galactose (Chapter 11) shows that the process of "slow" fermentation occurs in two phases. During the first phase of primary adaptation there is an abundant increase of cells with galactose the source of carbon, although none of the cells produced during this period is capable of rapid fermentation of galactose and no gas appears in the tube. However, when the cells are removed from the fermentation tube, placed in a Smith tube over mercury and provided with substrate, they produce gas. Therefore, they are actually capable of fermenting galactose, but at a much slower rate than the dominant genotype. The slow growth in galactose is the result of utilization of galactose by the cell through the agency of the recessive gene by the conventional fermentative apparatus; the recessive gene is not incapable of fermentation but merely ferments the sugar at a lower rate. The period during which the recessive cells are multiplying in the medium comprises the primary adaptation. The failure of the cells during the primary adaptation to produce visible gas in the fermentation tube results from the diffusion of the gas into the atmosphere more rapidly than it is evolved. That gas is actually produced is shown by the evolution of gas over mercury in the Smith tubes. Adaptation of the recessive by treatment with substrate has been shown to increase its ability to produce gas in the Smith tube. The frequency of mutation from recessive to dominant is in the order of $1 \times 10^{7}$ and when the population attains this magnitude a sufficient number of dominant cells is present to take over and the secondary adaptation begins. During secondary adaptation the dominant gene ferments galactose at such a rapid rate that the gas collects in the fermentation tube before it can diffuse into the atmosphere.

These facts appear to bear a direct relation to the phenomenon of adaptation to a pantothenate-free medium by the pantothenatedependent cultures. Fig. 22-1 shows that the growth rate in a medium containing a full supply of pantothenate follows a simple sigmoid curve, but that the growth in lower concentrations is a more complex phenomenon resembling the diaux $i e$ growth curves described by Monod. The phase of the primary adaptation involves long lag followed by a slow rate of growth; for example, in the first $1 \gamma$ curve the lag persists for about 170 hours and the slow growth following primary adaptation continues until about the 380th hour. From the 170th to the 380 th hour the recessive alleles are presumably synthesizing the pantothenate required for growth. About this time the mutation occurs initiating the rapid growth characteristic of the secondary adaptation. When a larger amount of pantothenate is present all these steps occur more rapidly because of the reduction of the synthesizing "load". 
The supposedly "random" gene mutations initiating the secondary adaptation are not necessarily fortunate accidents which occur without previous history in the phylogeny of the organism; they may be the result of a standard mechanism developed by selection.

\section{CONCLUSIONS}

Adaptation from pantothenate dependence to pantothenate independence of a haploid yeast was found to be due to mutation of a single gene. The new mutant gene is not a back mutation to the dominant gene originally present, but is a mutation at a different locus. The new mutant enables the yeast to synthesize pantothenate by an alternate route to that controlled by the original wild-type synthesizing gene.

There are two steps in the adaptation to pantothenate independence: (1) An adaptation to slow growth in minus pantothenate medium, which is transmitted vegetatively. (2) Gene mutation to pantothenate synthesis. The gene mutation was identified as a single gene by independent segregation of the wild-type and the mutant synthesizer. However, the growth rate of the mutant increased on serial transfer of the adapted culture prior to making the hybrid in which segregations occurred. The mutant locus for pantothenate synthesis may be capable of different levels of synthesizing capacity, and the environment may act to select the most active level, thereby increasing the rate of growth on serial transfer.

Mutation to synthesis, in this case, seems to occur at random both in the presence and in the absence of pantothenate. From the limited amount of data obtained so far, there appears to be no difference in mutation rates in the presence and in the absence of pantothenate. However, in the presence of intermediate or high concentrations of pantothenate the non-synthesizing cells overgrow the mutants and, therefore, adaptation occurs only in very low concentrations of pantothenate.

The primary adaptation to the medium, permitting very slow growth, has not been shown experimentally to occur at random. The fact that the generation times of cultures, during the primary adaptation, varied from approximately 40 hours to 80 hours is consistent with what would be expected with spontaneous mutations. However, there are two facts which make it seem most likely that the primary adaptation consists of a change in all or a large portion of the cells some time after inoculation into the deficient medium. (1) The change occurs with considerable regularity in a relatively small number of cells while gene mutations ordinarily occur extremely rarely and with great irregularity. (2) Samples of a few hundred cells taken at random from a culture growing slowly following the primary adaptation showed little variation in rate of growth. This slow growth may be 
accounted for by slow synthesis of pantothenate by the recessive presumably non-synthesizing alleles. As cell population increases, probability of a gene mutation occurring increases. When such a mutation occurs, there is a sharp increase in rate of growth, - the "secondary" adaptation. The decrease in time required for the secondary adaptation to occur on the addition of minute amounts of pantothenate is due to a slightly increased rate of growth and hence a greater number of cells and a greater probability of mutation. The time required for the primary adaptation to the medium to take place appears to be shortened by the addition of small amounts of pantothenate. 


\section{Chapter 23}

\section{THE BALANCED LETHAL - SACCHAROMYCODES}

Lindegren (1932) developed a technique for calculating linkage relations in Neurospora based on the cytological fact that centromeres always undergo reduction at the first meiotic division. In Neurospora crassa first-and second-division segregation are easily distinguishable by the arrangement of the spores in the ascus (Dodge, 1929); the spindles do not overlap and the first-division segregation produces an ascus in which four spores at one end differ from the four at the other end (fig. 23-1). Any gene-pair which always or nearly always undergoes first-division reduction is near the centromere. A single cross-over between the locus of the gene and the centromere results in second-division segregation (fig. 232). Each second-division ascus produces two cross-over and two non-cross-over chromatids and the percentage of crossing over (or the map distance from the centromere) is theoretically equal to one half the percentage of second-division segregation. This relationship may be affected by the non-random nature of exchanges between chromatids (Lindegren \& Lindegren, 1942).

In Neurospora tetrasperma (Dodge, 1927) the location of the gene controlling mating type near the centromere and the orientation of the spindles controls the production of homothallic ascospores. In this species, the self-sterility alleles segregate at the first-division and the spindles of the second division overlap as indicated in fig. 23-3. This produces an ascus containing four homothallic spores, since each contains nuclei of complementary mating types. Occasional mononucleate spores are produced which give rise to haploid clones; thus giving the species sufficient plasticity to make a high degree of variability available for adaptations to new conditions.

Winge and Laustsen showed that Saccharomycodes ludwigii is a heterozygous balanced lethal. S. ludwigii normally has no haplophase for the spores appear in the ascus in pairs and copulate before beginning growth (fig. 23-4). In S. ludwigii two genes, one of which has a lethal allele $(\mathrm{N} / \mathrm{n})$ and the other controlling length of cell $(\mathrm{L} / \mathrm{l})$, are both segregated at the first division of the zygote nucleus and the second spindles overlap. The zygote is always heterozygous for both pairs of alleles and the asci (which contain two spores at each pole) produce zygotes by the fusion of these which are similarly heterozygous. Only two kinds of asci are produced (fig. 23-5). 


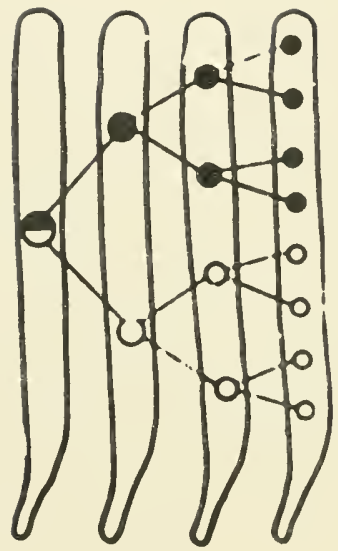

a

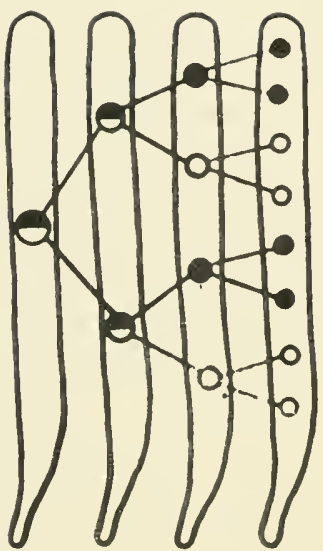

$b$

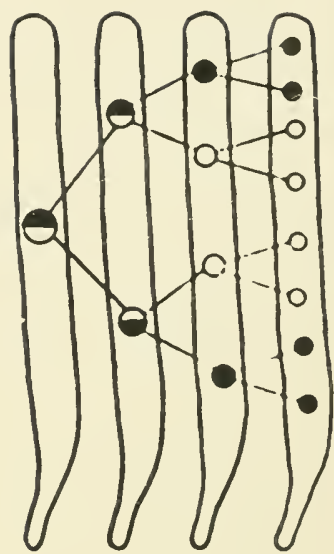

c

Fig. 23-1 First-and Second-Division Segregation in Neurospora.

a. When reduction occurs at the first division, the 4 spores at the upper end and the 4 spores at the lowe $r$ end of the ascus are different. Reversing the orientation of the first spindle would produce the opposite arrangement; namely, 4 white at the top and 4 black at the bottom.

b. This second-division segregation produces an alternate 2 by 2 arrangement of spores in the ascus and reversing the orientation of the second division spindles would reverse the arrangement from top to bottom.

c. This type of second division segregation produces 4 similar spores in the center of the ascus with two of the opposite types at either end. Reversing the second division spindles would exactly reverse the pattern. This diagram shows that 6 kind of asci can be produced in Neurospora, two character$j$ stic of first-division segregation and 4 characteristic of secoud-division segregation.

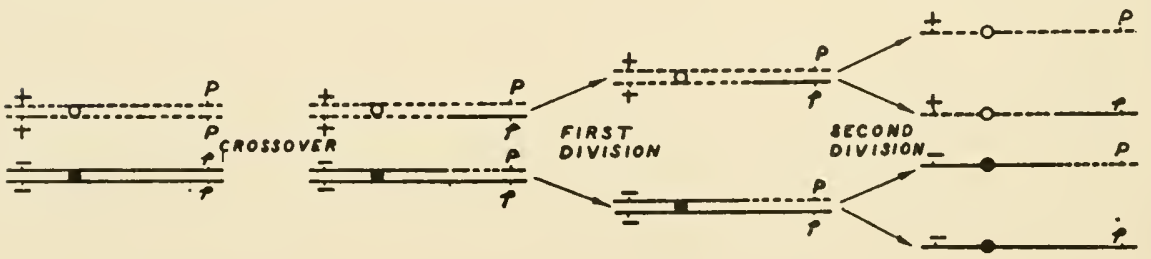

F1g. 23-2 Diagram of crossing-over in Neurospore at the four-strand stage showing how a single cross-over between the centromere and the $\mathrm{P} / \mathrm{p}$ locus resulta in second-division segregation of $P$ from $p$, while the absence of crossovers between the centromere and $+/$ - result in first-division segregation of + from -. 


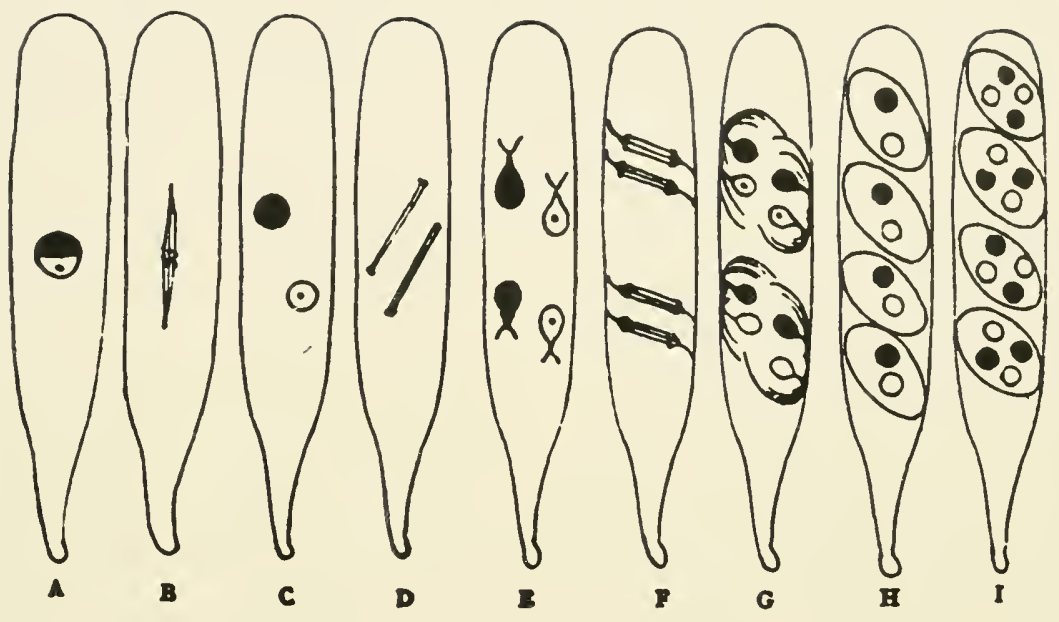

Fig. 23-3 Regular first-division segregation of mating type alleles and regular orientation of spindles in Neurospora tetrasperma combining to produce spores all of which contain four nuclei, two each of opposite mating type (copied from Dodge).

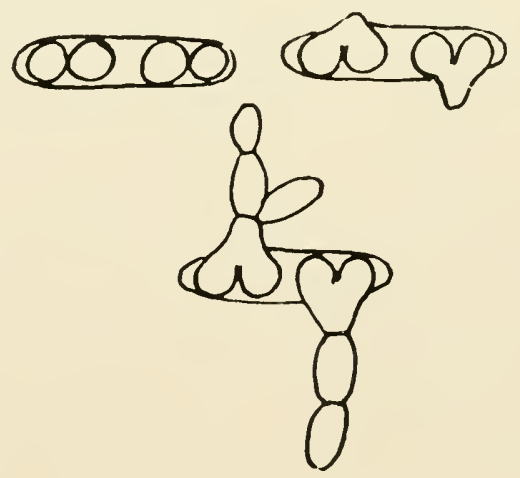

Fig. 23-4 Copulation in Saccharomycodes. (Gopied from Winge and Laustsen). 
The following analysis shows that this is due to the location of the genes $\mathrm{N} / \mathrm{n}$ on a different chromosome from the genes $\mathrm{L} / \mathrm{l}$, but both genes are probably sufficiently near the centromeres of their respective chromosomes so that crossing over between the locus and the centromere is very rare.

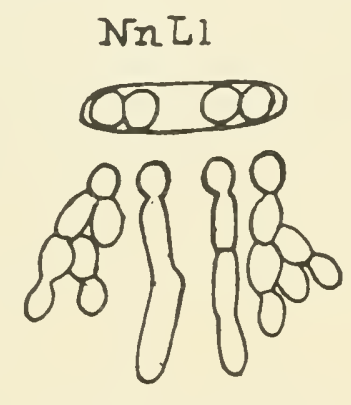

$\mathrm{N} 1 \mathrm{~nL} \mathrm{~nL} \mathrm{~N} I$
$N n \amalg I$

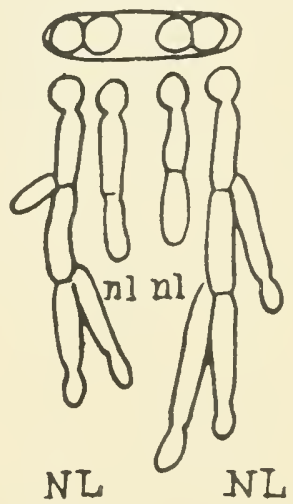

Fig. 23-5 Two Kinds of Asci Discovered by Winge and Laustsen in S. Ludwigii.

Since the centromeres always separate reductionally at the firsi meiotic division, a gene near the centromere usually undergoes reduction at the first division. The zygote is heterozygous for $\mathrm{N} / \mathrm{n}$ and $\mathrm{L} / \mathrm{l}$, both of which are near the centromeres of two different chromosomes (fig. 23-6). Just before the first reduction division, the entire chromosome (except the centromere) is duplicated, and the dyads carried to the poles of the first spindle produce two nuclei. It is obvious that at the two-nucleate stage only two kinds of asci are possible: (1) asci with an NNLL and an nnll nucleus, or (2) asci with an NNll and an nnLL nucleus (fig. 23-5). Both nuclei are homozygous for either $\mathrm{N}$ or $\mathrm{n}$ and either $\mathrm{L}$ or 1 . The first ascus can produce only NL and $\mathrm{nl}$ spores. The second ascus can produce only $\mathrm{Nl}$ and $\mathrm{nL}$ spores. No asci will be found in which all four types of spores are produced.

The spindles for the second division are oriented so that spores carrying complementary alleles are placed near each other and the fusion of adjacent spores produces heterozygous diploids.

In a subsequent paper, Winge (1947) extended his genetical analysis and indicated his agreement with the interpretation which I have outlined above. 


\section{THE ACCUMULATION OF LETHAL GENES}

Saccharomycodes represents an adaptation like that in Neurospora tetrasperma which insures the production of diploid or homothallic strains from each ascus. This device makes it possible for lethal genes to accumulate in the chromosomes further restricting the flexibility of the species in its ability to outcross. Winge has refuted Guilliermond's claim that the spindles in Saccharomycodes do not overlap and has insisted that the spindles in Saccharomycodes do overlap. It may be, however, that both situations obtain as in the genus Neurospora: the spindles overlap in Neurospora tetrasperma but do not overlap in either $N$. crassa or $N$. tetrasperma. Overlapping spindles are associated with first-division segregation of the mating type alleles (due to their location near the centromere) and favor the accumulation of the lethal genes. Overlapping spindles insure the orientation of spores in the ascus which makes it possible for $\mathrm{N}$. tetrasperma to produce homothallic ascospores (fig. 23-3). A lethal gene carried near one of the mating type alleles or

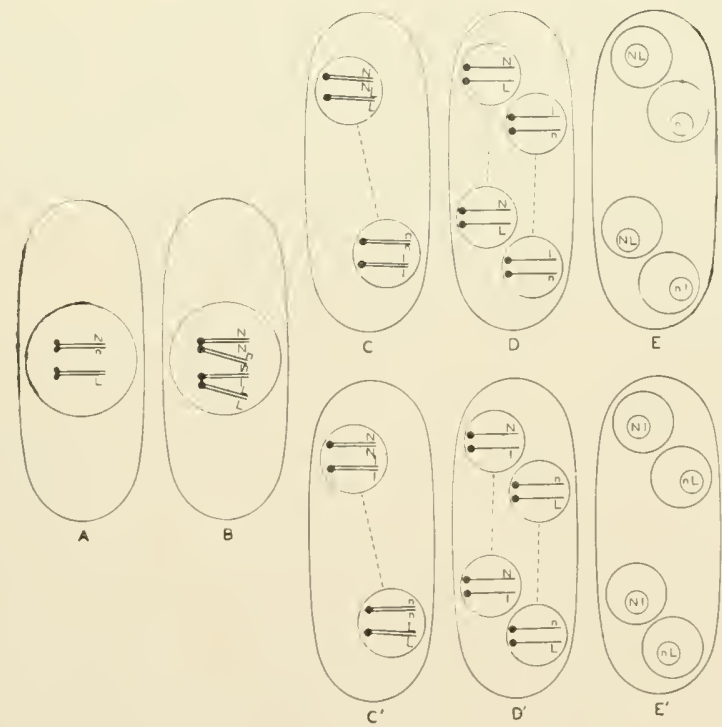

Fig. 23-6 Diagram of Meiosis in Saccharomycodes Ludwigii.

near the centromere of any other chromosome would always be introduced into a heterokaryon with another haploid nucleus carrying the normal allele and thus make it possible for the lethal to live a 
parasitic existence. Lethal genes have been found by Dodge to be carried in Neurospora tetrasperma by this device. The accumulation of degenerate alleles in Saccharomycodes is a good example of the value to a species of the interposition of a haplophase in the life cycle and helps to explain why haplophases are still carried in highly evolved plants such as the Spermatophytes.

\section{Reversion of $\mathrm{n}$ to $\mathrm{N}$}

Winge observed in his genetical analysis of S. ludwigii that the lethal locus $\mathrm{n}$ occasionally mutated to the dominant normal $\mathrm{N}$ allele. This is apparently another example of the repair of a degraded gene by its dominant normal functional allele in the heterozygous condition. The $\mathrm{n}$ allele, therefore, resembles the reverting and irregularly segregating genes we have found in Saccharomyces rather than the fully degraded alleles usually used in Neurospora genetics. 


\section{Chapter 24}

\section{CHROMOSOME MAPS}

\section{TETRAD ANALYSIS AND CENTROMERE LINKAGE}

By tetrad analysis it is possible to determine how closely two different freely assorting genes are linked to their respective centromeres. We have shown in Neurospora that the centromere undergoes reduction at the first division and, therefore, a gene close to the centromere usually undergoes reduction at the first division (Chapter 23). A mating of $\alpha \mathrm{G} \times$ ag produces three kinds of tetrads: (I) $\alpha$ G, $\alpha$ G, ag, ag, (II) aG, aG, $\alpha$ g, $\alpha$ g, (III) $\alpha \mathrm{G}, a g, \alpha g$, aG. Type I contains only parental combination spores, type II contains only recombination spores, and type III contains all four possible types. If types I and II are equal, the total number of original and recombination spores will be equal, proving that the genes are not linked. The "checkerboard" shown in the Table 24-1 proves that the random expectation of I:II:III equals 1:1:4. Fig. 23-1 shows that 6 kinds of tetrads are produced when the genes are so far from their respective centromeres that the random expectation of first-to second-division segregation occurs and when orientation in the ascus is determinable and the spindles do not overlap. Although order is not d terminable in Saccharomyces because the ascus is a small oval cell, the checkerboard serves to calculate the expected frequencies of the 36 different types of tetrads and from this, the 3 distinguishable types can be calculated. The first row at the top of Table 24-1 represents the 6 types of asci with the haploid nuclei in the 6 possible arrangements in the ascus (fig. 23-1). The column at the left side represents the arrangements possible for the second gene pair. Thus 12 arrangements combine to produce 36 total kinds of asci. Of these, 6 are type I, 6 are type II, and 24 are type III, so the random expectation of the 3 types is 1:1:4. Many tetrad analyses of two gene pairs yield the 1:1:4 ratio, but the $\alpha \mathrm{G} \times$ ag heterozygotes produced a total of I:II:III = 79:81:167. The equivalence of type $I$ and II prove that the $\mathrm{a} / \mathrm{a}$ and $\mathrm{G} / \mathrm{g}$ loci are not linked but the deviation of tetrad types from random expectation prove that some mechanism has prevented random distribution. We have interpreted this to mean that both loci are relatively close to their respective centromeres causing them to segregate reductionally at Meiosis I with higher than random frequency.

An examination of Table 24-1 reveals that the combination of first-division segregation of $\mathrm{A} / \mathrm{a}$ and $\mathrm{B} / \mathrm{b}$ produce only tetrads of 
types I and II. These are the 4 tetrads in the upper left hand corner of the table. Combinations of second-division segregations of both $\mathrm{A} / \mathrm{a}$ and $\mathrm{B} / \mathrm{b}$ produce types $\mathrm{I}: \mathrm{II}: \mathrm{III}$ in the ratio $1: 1: 2$. These are the 16 tetrads in the lower right hand corner of the table. Combinations of first- and second-division segregations produce only type III tetrads. These are shown in the two remaining blocks - the upper right hand and the lower left hand.

Since we know that second-division segregation represented as a percentage is equal to twice the distance of a locus from the centromere, it is possible to calculate the expected ratios of I:II:III for various loci at different distances from their centromeres.

$$
\text { Table 24-1 }
$$

Checkerboard showing the 36 different types of asct expected from the random segregation of 2 different pairs of alleles. The six types of asci for the $A / \alpha$ alleles are arranged in the first row: the six types for the $B / b$ alleles are in the first column. These arrangements are derived from the types of segregation found in tubular asci like Neurospora (fig. 23-1) when the genes are segregated at random because they are farther than 33 units from the centromere. The resulting arrangements.due to the combination of these types is shown in the squares. Since arrangenents are not detectable in a spherical ascus, the type (I, II, or III) is indicated also. Therefore, if both genes are far from the centromere, the calculated ratio of $I: I I: I I I=6: 6: 24$.

\begin{tabular}{|c|c|c|c|c|c|c|}
\hline & $\begin{array}{l}\text { A A a a } \\
\text { First }\end{array}$ & $\begin{array}{l}\text { a a A A } \\
\text { First }\end{array}$ & $\begin{array}{l}\text { A a }=A \\
\text { Second }\end{array}$ & $\begin{array}{l}\text { a A A a } \\
\text { Second }\end{array}$ & $\begin{array}{l}\text { a } A \text { a } A \\
\text { Second }\end{array}$ & $\begin{array}{l}\text { A a A a } \\
\text { Second }\end{array}$ \\
\hline $\begin{array}{l}\text { BBbb } \\
\text { First }\end{array}$ & $\begin{array}{r}A B \text { AB at at } \\
I\end{array}$ & $\begin{array}{r}a B \text { aB } A t \quad f . t \\
\text { II }\end{array}$ & $\begin{array}{r}A B \text { aB ab } A t \\
\text { III }\end{array}$ & $\begin{array}{r}a B \quad A \perp A b a b \\
\text { III }\end{array}$ & $\begin{array}{r}a B \text { AD a } A b \\
\text { III }\end{array}$ & $\begin{array}{r}\text { A5 } a B A b \text { ab } \\
\text { III }\end{array}$ \\
\hline $\begin{array}{l}\text { blBB } \\
\text { First }\end{array}$ & $\begin{array}{llll}A b & A b & a B & a B \\
& & & I I\end{array}$ & $\begin{array}{rrr}a b & a b & A B \\
& & A B\end{array}$ & 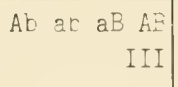 & $\begin{array}{r}a b \text { At } A E a B \\
\text { III }\end{array}$ & $\begin{array}{r}\text { at As aB AB } \\
\text { III }\end{array}$ & $\begin{array}{r}A b \text { ab } A B \text { aB } \\
\text { III }\end{array}$ \\
\hline \begin{tabular}{l|} 
Bbb? \\
Second
\end{tabular} & 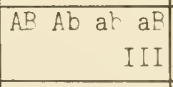 & $\begin{array}{rrr}a b \text { ab } & A b & A^{-} \\
& \text {III }\end{array}$ & 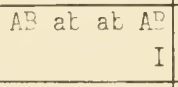 & $\begin{array}{rlll}a \bar{B} & A b & A b & a B \\
& & & I I \\
& & \end{array}$ & $\begin{array}{r}a B \quad A b \text { ab } A B \\
\text { III }\end{array}$ & $\begin{array}{r}\text { A3 ab At aB } \\
\text { III }\end{array}$ \\
\hline $\begin{array}{l}b B 2 z \\
\text { Second }\end{array}$ & $\begin{array}{r}A b \quad A^{D} \quad a B \text { at } \\
\\
\text { III }\end{array}$ & $\begin{array}{r}a b a^{D} A B \quad A b \\
I I I \\
\end{array}$ & $\begin{array}{llll}A b & a B & a B & A b \\
& & I I\end{array}$ & $\begin{array}{r}a b \\
\end{array}$ & $\begin{array}{r}a b \quad \mathrm{AB} a \mathrm{Ab} \\
\text { III }\end{array}$ & $\begin{array}{r}A b \text { aB } A B a b \\
\text { III }\end{array}$ \\
\hline $\begin{array}{l}\text { bBb3 } \\
\text { Second }\end{array}$ & $\begin{array}{r}\text { At } A^{\circ} \text { at } a B \\
\text { III } \\
\end{array}$ & $\begin{array}{r}a b \text { aB Ab } A^{R} \\
\text { III }\end{array}$ & $\begin{array}{r}A k a^{R} a b \quad A B \\
\text { III } \\
\end{array}$ & $\begin{array}{r}a b \quad A B \quad A b \quad a B \\
\text { III }\end{array}$ & 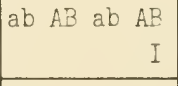 & $\begin{array}{r}A b \text { a } A b \text { aB } \\
\text { II } \\
\end{array}$ \\
\hline $\begin{array}{l}\text { EbEk } \\
\text { Second }\end{array}$ & $\begin{array}{r}A B \quad A b \quad a B \text { at } \\
\text { III }\end{array}$ & $\begin{array}{r}\mathrm{a}^{\mathrm{D} \text { at }} \mathrm{AP} \mathrm{Ab} \\
\text { III }\end{array}$ & $\begin{array}{r}A^{D} \quad a b \quad a B \quad a b \\
\text { III }\end{array}$ & $\begin{array}{r}a B \text { Ac } A B \text { at } \\
\text { III }\end{array}$ & $\begin{array}{r}\mathrm{aB} \text { At } \mathrm{aB} \text { At } \\
\\
\mathrm{II}^{\mathrm{T}}\end{array}$ & 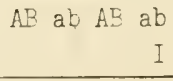 \\
\hline
\end{tabular}


The simplest way to represent this ratio as a single figure is $\frac{I+I I}{I+I+I I}$. Table 24-2 summarizes this ratio for various calculated combinations. These calculations are summarized graphically in fig. 24-1. This graph can be used to determine the distance of alleles from their centromeres if the different ratios I I + II + III are known for various combinations. Table 24-3 shows the ratios for all possible combinations of the $\mathrm{a} / x, \mathrm{G} / \mathrm{g}$, and $\mathrm{AD} / \mathrm{ad}$ loci are:

$$
\begin{aligned}
\times \mathrm{AD} \times \mathrm{a} \mathrm{ad} & =0.462 \\
\alpha \mathrm{G} \times \mathrm{a} \mathrm{g} & =0.490 \\
\mathrm{ADG} \times \mathrm{ad} g & =0.693
\end{aligned}
$$

An examination shows that these ratios agree with the following distances.

$\begin{array}{llll}\text { Ratio } & \mathrm{a} / \alpha & \mathrm{G} / \mathrm{g} & \mathrm{AD} / \mathrm{ad} \\ .693 & 23.6 & 6.25 & 11.25 \\ .490 & 23.6 & 6.25 & \\ .462 & 23.6 & & 11.25\end{array}$

We conclude, therefore, that the $\mathrm{a} / \alpha$ locus is 23.6 units from its centromere, the $\mathrm{G} / \mathrm{g}$ locus is 6.25 , and the $\mathrm{AD} / \mathrm{ad}$ locus is 11.25 . The $\mathrm{I}+\mathrm{II}+\mathrm{III}$ ratio for the G-PN combination is 0.466 . G and PN are not linked and since the distance of $\mathrm{G}$ from the centromere is known to be 6.25 and the graph indicates that PN is 25.6 units from its centromere. The distance of PB from its centromere was calculated from the graph in the same manner.

The AD chromosome contains 6 marked loci and the distances between them are indicated in fig. 24-2. The circled figures were calculated from the graph and the others by the standard recombination technique. 
Table 24-2

Calculated ratios $\frac{I+I I}{I+I I+I I I}$ of tetrad types for patrs of alleles on different chromosomes varylng distances frow their centromeres.

\begin{tabular}{c|c|c|c|c|c|c|c|c}
\hline & & \multicolumn{7}{|c}{ Distance A/a from Centromere } \\
\cline { 3 - 8 } & & 0 & 5 & 10 & 15 & 20 & 25 & 30 \\
\hline \multirow{4}{*}{$\begin{array}{c}\text { Distance } \\
\text { B/b }\end{array}$} & 5 & 1.00 & .90 & .80 & .70 & .60 & .50 & .40 \\
from & 10 & .90 & .815 & .73 & .64 & .56 & .475 & .39 \\
Centromere & 15 & .70 & .64 & .59 & .535 & .475 & .43 & .37 \\
& 20 & .60 & .56 & .515 & .475 & .44 & .40 & .36 \\
& 25 & .50 & .475 & .45 & .43 & .40 & .38 & .35 \\
& 30 & .40 & .39 & .38 & .37 & .36 & .35 & .34 \\
\hline
\end{tabular}




\section{Table 24-3}

The accumulated numbers of the three types of asci obtained by analyses of hybrlds heterozygous for at least 2 pairs of genes both of whtch segregated with a $2: 2$ ratio. The ratio $\frac{I+I I}{I+I I+I I I}$ is calculated for those

combinations in which the genes are not linked.

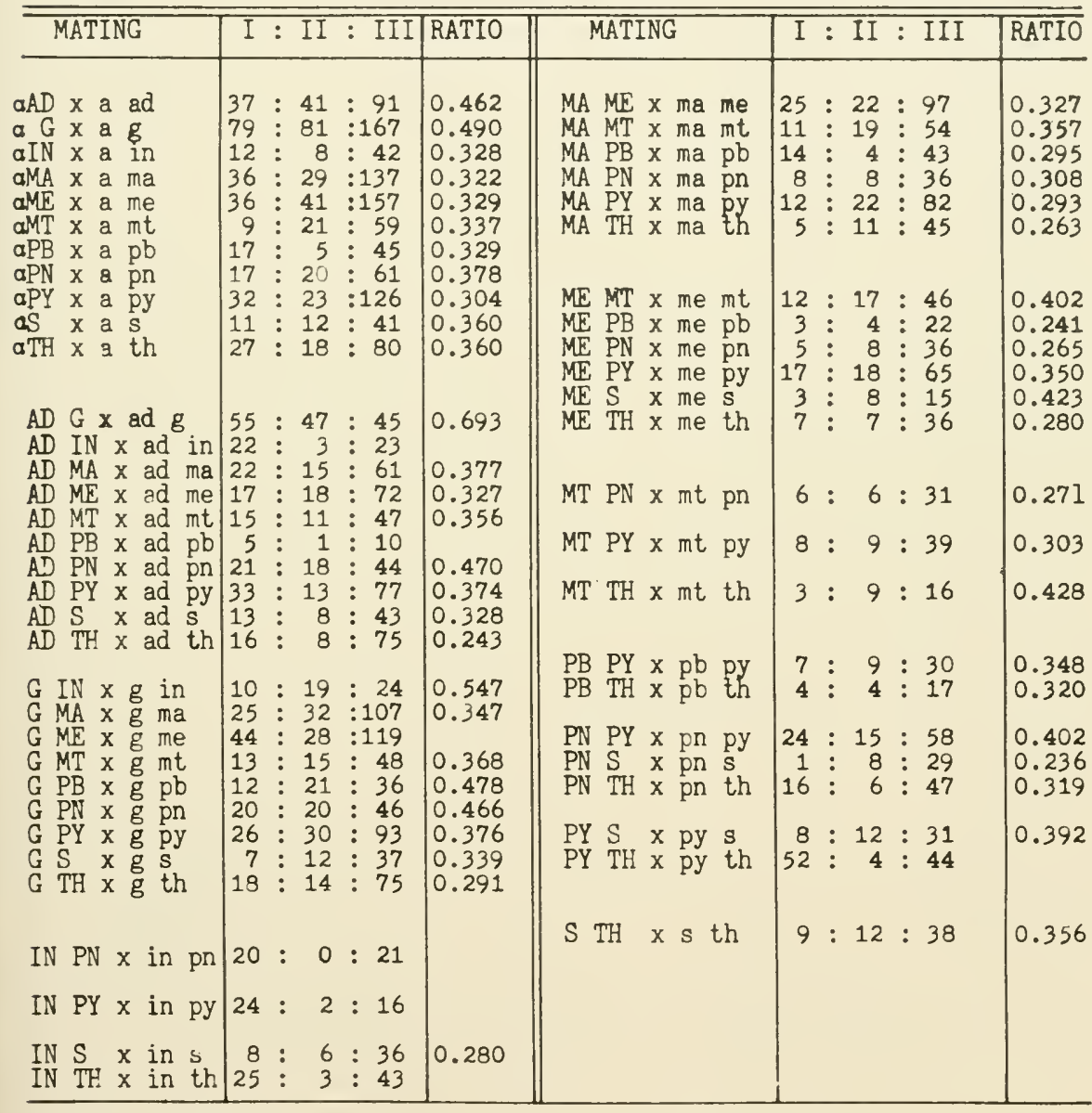

$\mathrm{AD}$ Adenine

G Galactose

IN Inositol

MA Maltose

ME Melibiose

MT Methionine
Paraminobenzoic Acid

Pantothenate

Pyridoxine

Sucrose

TH Thiamin 


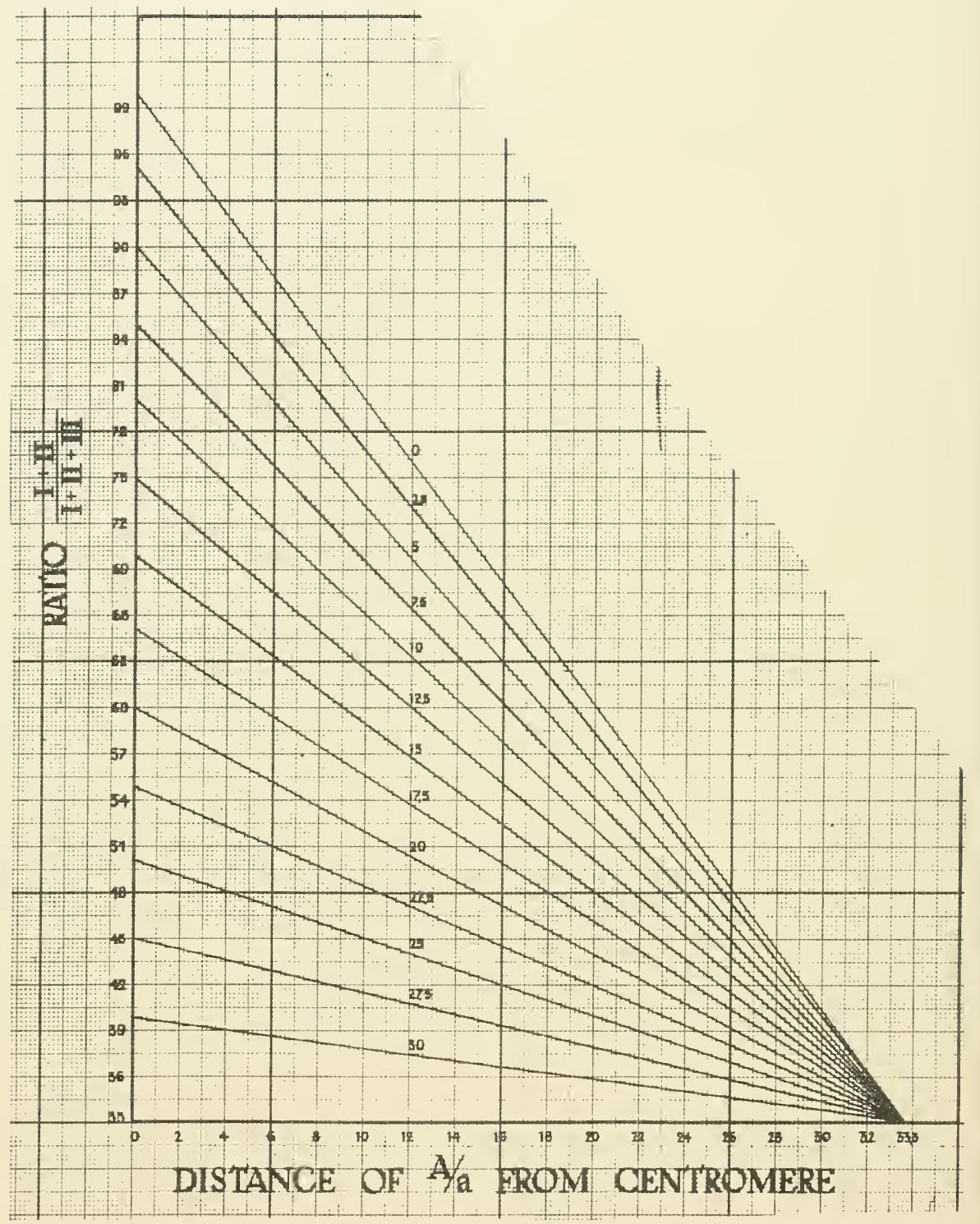

Fig. 24-1 Graphic Representation of the Calculations in Table 24-2. The abscissae represent varying distances of an allele A/a from its centromere, the ordinates represent the ratios I+ II

each curve plots the values of this ratio for the indicated distance of $\mathrm{B} / \mathrm{b}$ against various distances of the $\mathrm{A} / \mathrm{a}$ locus from its centromere. 

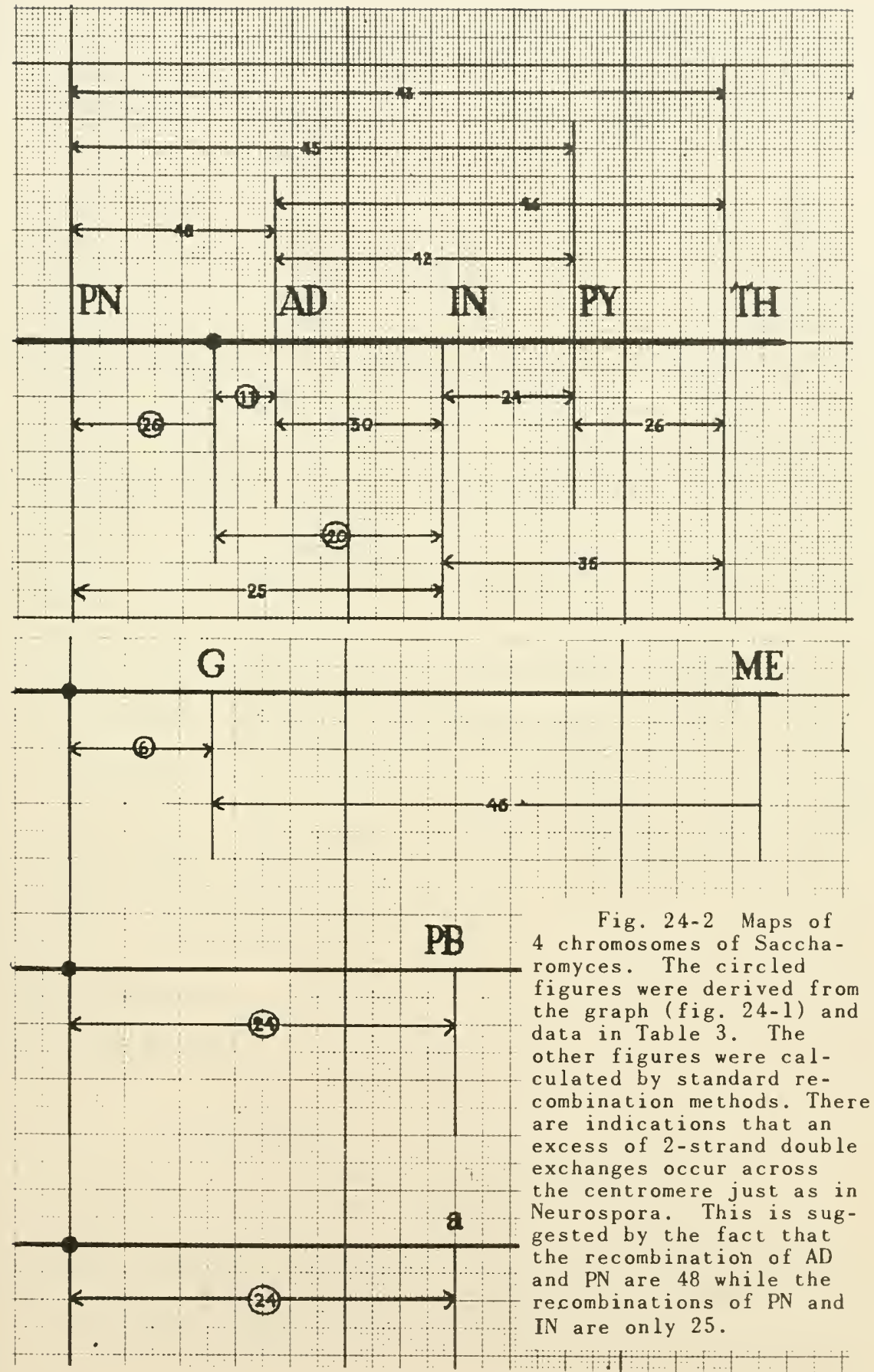


\section{Chapter 25}

\section{PROOF OF INTERCHROMOSOMAL TRANSFER OF HEREDITARY}

\section{MATERIAL BY TETRAD ANALYSIS}

Sixty asci from a hybrid heterozygous for a/ $\alpha, \mathrm{AD} / \mathrm{ad}, \mathrm{G} / \mathrm{g}$, $\mathrm{IN} /$ in, PN/pn, PY/py were analyzed and in 57 all eight alleles segregated regularly $2: 2$. The first 4 asci in fig. 25-1 are typical of the 57 and are characterized by $2: 2$ segregations at all eight loci. Each of these asci produced 2 pink and 2 white cultures. Pink cultures are adenine dependent. Pink mutations have not appeared spontaneously, our first pink culture was one obtained by treatment of haplophase No. 4 (fig. 18-1). Ascus Nos. 5918-22 and ascus Nos. 5979-82 both contained 3 pink cultures. Mutation of white to pink does not occur in this stock without treatment. The ad gene controls the production of pigment and it is in the well-marked chromosome shown in fig. 24-2. Four other loci in this chromosome segregated in a perfectly regular manner so the possibility of chromosomal irregularities causing the phenomena is eliminated. Adenine independent cultures ordinarily attain full growth in adenine deficient medium in two days, while adenine dependent (pink) cultures require 2 weeks. Two of the pink cultures in each ascus attained full growth in two weeks, while one in each grew up in 5 days. This pink culture is designated by ad circled. However, it is phenotypically pink in culture and this type is never encountered in our inbred white stocks.

This is an example of the type of irregular ratio which can only be detected by tetrad analysis (fig. 25-2). The simplest explanation is that in the $\mathrm{AD} / \mathrm{ad}$ heterozygote, one of the $\mathrm{AD}$ loci loses some of its essential substance to the two ad ioci thus degrading the AD locus and reducing its ability to contribute as effectively to the synthesis of adenine. The Konversion Hypothesis of Winkler suggested that exchanges of this type occurred and used this idea to explain crossing over. However, it is only in tetrad analysis that conversion and crossing over can be distinguished.

\section{CONVERSION OF RECESSIVES TO DOMINANTS IN THE HETEROZ YGOTE}

One of the 60 asci (No. 6040-43) produced 4 white cultures instead of the expected 2 white and 2 pink. When tested in defined medium lacking adenine, they all attained full growth in 2 days. Proof that an illegitimate hybridization has not occurred is the fact that 
$2: 2$ segregations occurred at the $\mathrm{G} / \mathrm{g}, \mathrm{IN} / \mathrm{in}$, and $\mathrm{PN} / \mathrm{pn}$ loci. In addition to transformations at the $\mathrm{AD} / \mathrm{ad}$ locus, three of the cultures were $S$ and one $S$ and all four cultures were thiamin and pyridoxine

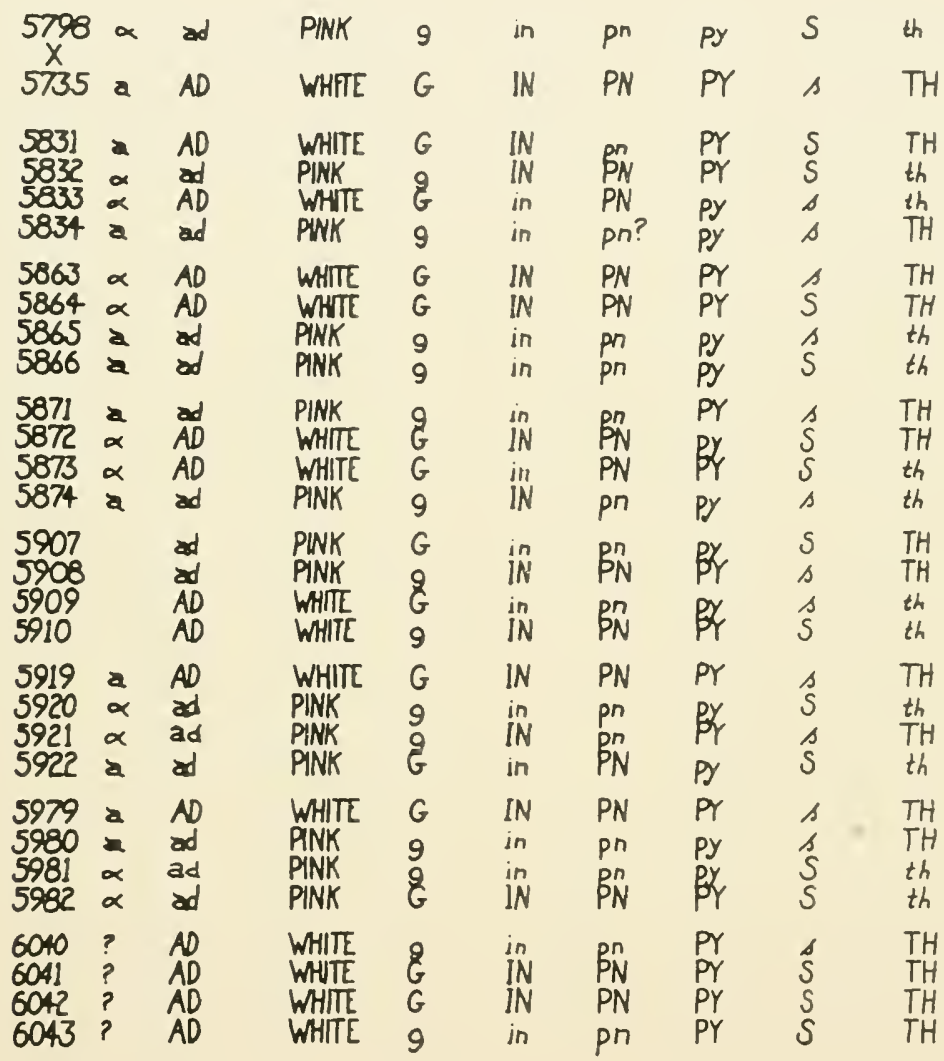

Fig. 25-1 Tetrad analysis of seven sample asci from a hybrid heterozygous for six alleles on one chromosome.

independent. Mating types were also disturbed. The test for mating type is ordinarily made by testing all 4 cultures with a single vigorously copulating haplophase culture and those which copulate are designated as opposite sex to the tester, while those which do not, are marked the same sex as the tester. If the tests are not clearcut, a second test using the opposite mating type usually clears up any ambiguity. However, No. 6040-43 was tested by mating to both $a$ and $\alpha$ mating types and only negative reactions were obtained in every case. These cultures, therefore, were practically sterile. An 
important fact confirming the view that No. $6040-43$ is not an illegitimate hybrid of No. 5735 is the presence of one non-fermenter of sucrose in the tetrad. I have pointed out the problems involved in introducing the sucrose gene into the stock and its appearance in this tetrad is proof of its truly hybrid origin. Whatever the cause for the high frequency of dominants (or synthesizers) it is clear (1) that they could not have been differentiated from other phenotypically similar cultures except on the basis of their origin in the same ascus and therefore are only detectable by tetrad analysis and are undetectable by any other means, and (2) that misdivisions are not

\begin{tabular}{|c|c|c|c|c|c|c|c|c|c|c|}
\hline WHITE & pn & $A D$ & IN & PY & TH & WHITE & $\mathrm{PN}$ & $A D$ & IN & PY \\
\hline WHITE & PN & ad & IN & PY & th & PINK & pn & ad & in & py \\
\hline PINK & PN & $A D$ & in & py & th & PINK & & & $1^{1 n}$ & py \\
\hline INK & pn & ad & in & py & $\mathrm{TH}$ & PINK & PN & ad & $\downarrow$ IN & PY \\
\hline
\end{tabular}

$\begin{array}{llllllllllll}\text { WHITE } & \text { PN } & \text { AD } & \text { IN } & \text { PY } & \text { TH } & \text { WHITE } & \text { PN AD IN } & \text { PY } & \text { TH } \\ \text { WHITE } & \text { PN } & \text { AD } & \text { IN } & \text { PY } & \text { TH } & \text { PINK } & \text { pn ad } & \text { in } & \text { py } & \text { th } \\ \text { PIN } & \text { pn ad } & \text { in } & \text { py } & \text { th } & \text { PINK } & \text { pn ad } & \text { IN } & \text { PY } & T H \\ \text { PINK } & \text { pn ad } & \text { in } & \text { py } & \text { th } & \text { PINK } & \text { PN ad in } & \text { py th }\end{array}$

Fig. 25-2 Four-strand Crossing Over Analysis of Four Sample Asci. Since the Mating was PN AD IN PY TH the first ascus is a pn ad in py th

non-crossover ascus in which no detected exchanges occurred. In the second ascus, three exchanges occurred at the indicated places. The first pair constitute a 3 -strand exchange and the second pair also constitute a 3-strand exchange. The details of analysis of this type have been fully described by Lindegren and Lindegren (1942). The third ascus is one of those containing 3 pink cultures. The circled ad attained full growth in 5 days and is apparently the degraded $A D$. Therefore, a 2-strand exchange occurred followed by a 3 -strand exchange at the points indicated. The fourth ascus is the other with 3 pink cultures and a simple exchange occurred in this ascus in the $\mathrm{PN}-\mathrm{AD}$ region.

likely to be involved since they would produce tetrads in which some members would be non-viable and in these tetrads every member was viable. Since it is clear that the ascus is truly hybrid one can only conclude that a transfer of chromosomal material has occurred changing the weakly synthetic (recessive) alleles into dominants. The fact that several alleles were affected in this ascus suggests 
that some non-specific local factor may be involved which either facilitates transfer or insures the adhesion of the transferred material to its new position.

Conversion is obviously controlled by heredity since it occurs with high frequency in some lines (e.g. C1A) and rarely in others. Conversion may result from the nature of the association of the heterozygous alleles at synapsis, either due to the duration of synapsis, the condition (stickiness) of the chromosome or some other general character. I say some general character, for several alleles are usually converted simultaneously. Since it is genetically controlled, a gene may influence the stickiness of chromosomes or duration of synapsis. Whether or not a converting mechanism is selected in higher organisms may depend upon the advantages accruing to the occasional appearance of recessives. The failure to demonstrate conversions in Drosophila may suggest that recessive alleles have been selected which serve specific functions, as Dobzhansky's evidence on seasonal selection of chromosomal aberrations indicates. The failure to discover recessive Mendelian genes in Sciara may mean that a converting mechanism is operating here which prevents the appearance of recessives. 


\section{Chapter 26}

\section{IRREGULAR SEGREGATIONS AS SUPPORTING EVIDENCE FOR GENE CONVERSION}

The tetrad analyses in Chapter 25 are conclusive evidence of the transformation of alleles because they involve well-marked chromosomes and exclude the possibility of illegitimacy or multiple factors producing the non-Mendelian ratios. Our first evidence supporting the idea of conversion was derived from the analysis of hybrids between galactose-melibiose-fermenters with "non-fermenting" haplophases. It has been pointed out in Chapter 11 that the socalled "non-fermenters" are capable of producing enzyme but do not produce detectable amounts of gas under the conditions of the test. They are called "non-fermenters" with this qualification. Many more data of this type are now available and are presented in this chapter. In the tetrad analysis of many yeast hybrids produced by matings of fermenter with "nonfermenter" stocks, each ascus contains 2 fermenter and 2 "nonfermenter" spores. In other pedigrees involving apparently similar matings $1,2,3$, or 4 fermenters are obtained from each ascus. The pedigree shown in Table 26-1 was developed by mating the haploid culture C1A (Table 18-1), a single ascospore culture derived from S. carlsbergensis (culture 126 from Dr. Mrak's collection), capable of fermenting both galactose and melibiose ( $\alpha$ GME), with "nonfermenter" cultures Nos. 35 and 85 (a g me) (Table 19-2). Both of the latter cultures did not produce gas from either galactose or melibiose; heterozygous diploids were produced. The a $\mathrm{G} \mathrm{Me} \mathrm{x} \mathrm{a} \mathrm{g}$ me matings revealed that these alleles segregated independently of each other. The a/ $\alpha$ alleles behaved regularly in nearly every ascus and the exceptions concerned principally the fermentative characters.

Nine complete 4-spored asci from the hybrid $35 \times \mathrm{C} 1 \mathrm{~A}$ (a $\mathrm{g}$ me $x$ \& $G M E$ ), were analyzed. One GGGG and one GGGg ascus and seven of the expected GGgg asci were obtained. This mating produced 10 complete 4 -spored asci in which $\mathrm{ME} / \mathrm{me}$ were diagnosed. Eight were the expected ME ME me me; one was ME ME ME ME and one was ME ME ME me. A second similar mating ( $85 \times \mathrm{C} 1 \mathrm{~A}$ ) produced three GGgg with 1 GGGG and two ME ME me me with two ME ME ME me.

Matings of an individual (184) from one of the irregular asci revealed non-Mendelian transmission of both G and ME (184 X 166 and $184 \times 187$ ). A mating of 16 by 164, and 4 by 159 each produced two regularly segregating asci. 


\title{
Table 26-1
}

\section{Irregular Ratios in a Fermenter by Nonfermenter Pedigree}

\author{
$35 \times \quad$ C1A \\ a g me $F$ a $\mathrm{X}$ E $f$
}

159 a g ME f MA 163 a G me $f$ MA 167 a G ME MA 171 a $G$ ME $f$ 160 a $G$ ME f ma 164 a G ME $f$ MA 168 a G ME MA 172 a $G$ me $f$ 161 a g me $f$ MA 165 a g ME F MA 169 a G ME $f$ MA 173 a $g$ ME $f$ 162 a G me $f$ MA 166 a g me F MA 170 a G ME F MA 174 a g me $f$

175 a $G$ me $f$ MA 179 a $G$ me $F$ 176 a $G$ me $f$ MA 180 a g ME $f$ 177 a $g$ ME $f$ MA 181 a $g$ me $f$ 178 a g ME F ma
230 a $G$ me $F$ 231 a G ME F 232 a $\mathrm{g} M E$
233 a $G$ me MA 234 a G ME MA 235 a $\mathrm{g} M E$ 236 a g me MA

237 a G ME ma 241 a g me MA 245 a g ME MA 249 a g me MA 238 a G ME F MA 242 a G ME MA 246 a g me ma 250 a G ME ma 239 a $G$ me ma 243 a G me MA 247 a G ME F ma 251 g ME MA 240 a g me MA 244 a g ME MA 248 a G me ma 252 me ma

\section{$85 \times \mathrm{C} 1 \mathrm{~A}$}

a g me $F$ a $x$ G $f$

\begin{tabular}{|c|c|}
\hline $\begin{array}{lllll}184 & \alpha & G & M E & f \\
185 & \alpha & G & M E & f \\
186 & a & G & m e & f \\
187 & a & G & M E & f\end{array}$ & 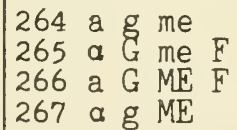 \\
\hline
\end{tabular}

268 a g me ma 272 a g me 269 a $G$ me MA 273 a $G$ me 270 \& G ME MA 274 a G ME

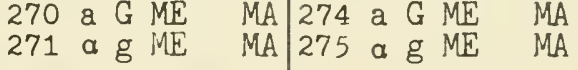

\section{$184 \times 166$ \\ a G ME $f$ a $g$ me $F$}

\section{$184 \times 187$ \\ a G ME $f$ x a G ME $f$}

295 a G ME F MA 299 G ME MA 289 a G ME

296 a G ME MA 300 G ME MA 290 a G ME

297 a G ME MA 301 G ME MA 291 a $G \mathrm{ME}$ 298 a G ME MA 302 a G ME MA 292 a G ME

293 a G ME 294 a g me a g me $F \quad \begin{array}{lll}16 & x & 164 \\ \text { a G ME } f\end{array}$

188 a g me $F$ 189 a G ME $f$ 190 a g me $f$ 191 a $G \mathrm{ME} f$
192 a g ME F 193 a $G$ me $f$ 194 a $G$ me $f$ 195 a g ME F

\section{$65 \times 161$}

a $g$ me $F \quad x$ a $g$ me $f$ 196 a g me F MA 200 a g me $f$ 197 a $g$ me $f$ MA 201 a $g$ me $f$ 198 a g me ma 202 a g me F 199 a $g$ me F MA 203 a $g$ me $f$

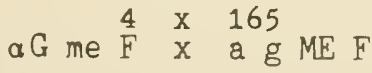

208 a G ME 209 a g me F 210 a $g$ ME F

\section{a $G$ me $\stackrel{4}{F} \quad \begin{array}{ll}x & 1.59 \\ x & \text { a } g \text { ME } f\end{array}$}

256 a $g$ me $M A 260$ a $G$ me MA 257 a g ME f MA 261 a $g$ ME F MA 258 a G ME F MA 262 a g ME F MA 259 a G me MA 263 a G me MA 
A mating homozygous for $\mathrm{g}$ and me produced no fermenter progeny. This is an example of the general rule that homozygous matings of nonfermenters do not produce fermenters.

One ascus from the doubly heterozygous hybrids (GME x gme) $35 \times$ C1A produced a GME GME GME GME ascus. The ascospores from the exceptional ascus $(167,168,169,170)$ were inbred and mated separately to both the recessive and the dominant stocks. When these data were first presented (Lindegren and Lindegren, 1946) backcrosses had only been made to recessive stocks and analysis of the hybrids indicated that each of the fermenter phenotypes in the ascus 167 to 170 behaved with certain conspicuous exceptions as dominants. The exceptions are apparent in a summary of the data, table 26-2. When the backcrosses were made to the fermenter parents only very rare nonfermenter offspring were encountered. Finally, when the intra-ascus matings were made a considerable number of recessive phenotypes was encountered. This latter fact was the clearest evidence that we were dealing with irregular inheritance, involving apparent dominants which could either lose their dominant character or imprint their dominant character on recessive individuals. The predominant ascus type produced by mating the original stocks to recessives are two fermenter and two nonfermenter spores per ascus. Those with four fermenters might be illegitimate offspring, but those in which 3:1 ratios occurred are difficult to interpret on any conventional basis. The higher frequency with which recessives appear in the backcrosses to the fermenter type are evidence of irregular behavior and this is reinforced by the frequency of recessives in the intra-ascus matings.

Illegitimates were obtained from culture 170 and showed an exceptionally high frequency of the recessive phenotypes; in this case, only two spores survived per ascus. Another unusual fact in this pedigree was that 167 also produced illegitimate spores which were not analyzed. Culture 169, at first diagnosed as an a mating type culture, behaved in some matings as an $\alpha$. In spite of the absence of other markers these data were considered convincing evidence of conversion and this view was fully corroborated by the recent work of Mundkur, 1949, in which the phenomenon of conversion is analyzed, in hybrids heterozygous for other genes than G and ME.

In the backcross of fermenter cultures 167, 168, 169, and 170 to the nonfermenter (Table 26-2), each of the matings yielded exceptions to the expected 2:2 ratio per ascus. For example, the 167 ( $\mathrm{G} \times \mathrm{g}$ ) mating yielded 1 GGGG ascus, the 168 ( $\mathrm{G} \mathrm{x} \mathrm{g}$ ) mating yielded 3 GGGG asci, the 169 ( $\mathrm{G} \mathrm{x}$ g) mating yielded 4 GGGg and 1 GGGG, and the 170 ( $\mathrm{G} \mathrm{x} \mathrm{g}$ ) mating yielded $1 \mathrm{gggg}$ and $1 \mathrm{Gggg}$ ascus. The G genes in 167,168 , and 169 are capable of transforming $g$ genes into phenotypic dominants, but the G gene in 170 is "weak" and often loses its own capacity to ferment. Many of the asci are of the ex- 
Table 26-2

Swmary of Backcrosses of Ascus 167-170

To Fermenters and Nonfermenters

\begin{tabular}{ll|lll|lll|ll}
\hline 167 & G ME & 168 & G ME & 169 & G ME & 170 & G ME \\
\hline
\end{tabular}

Backcross to $g$ me, nonfermenter.

\begin{tabular}{|c|c|c|c|}
\hline $15 \mathrm{G}, 9 \mathrm{~g}$ & $27 \mathrm{G}, 10 \mathrm{~g}$ & $36 \mathrm{G}, 22 \mathrm{~g}$ & $14 \mathrm{G}, 23 \mathrm{~g}$ \\
\hline 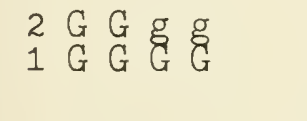 &  & $\begin{array}{lllll}4 & G & G & g & g \\
4 & G & G & G & g \\
1 & G & G & G & G\end{array}$ & $\begin{array}{lllll}5 & G & G & g & g \\
1 & G & g & g & g \\
1 & g & g & g & g\end{array}$ \\
\hline $\begin{array}{rl}13 & \mathrm{ME}, 12 \text { me } \\
2 & \mathrm{ME} \mathrm{ME} \text { me me } \\
1 & \mathrm{ME} \mathrm{ME} M \mathrm{ME} \\
\mathrm{ME}\end{array}$ & $\begin{array}{l}18 \mathrm{ME}, 19 \text { me } \\
5 \mathrm{ME} \mathrm{ME} \mathrm{me} \mathrm{me}\end{array}$ & $\begin{array}{l}23 \mathrm{ME}, 34 \text { me } \\
5 \mathrm{ME} \mathrm{ME} \text { me me } \\
3 \mathrm{ME} \text { me me me }\end{array}$ & $\begin{array}{l}19 \mathrm{ME}, 17 \mathrm{me} \\
8 \mathrm{ME} \mathrm{ME} \mathrm{me} \mathrm{me}\end{array}$ \\
\hline
\end{tabular}

Backcross to fermenter, G ME

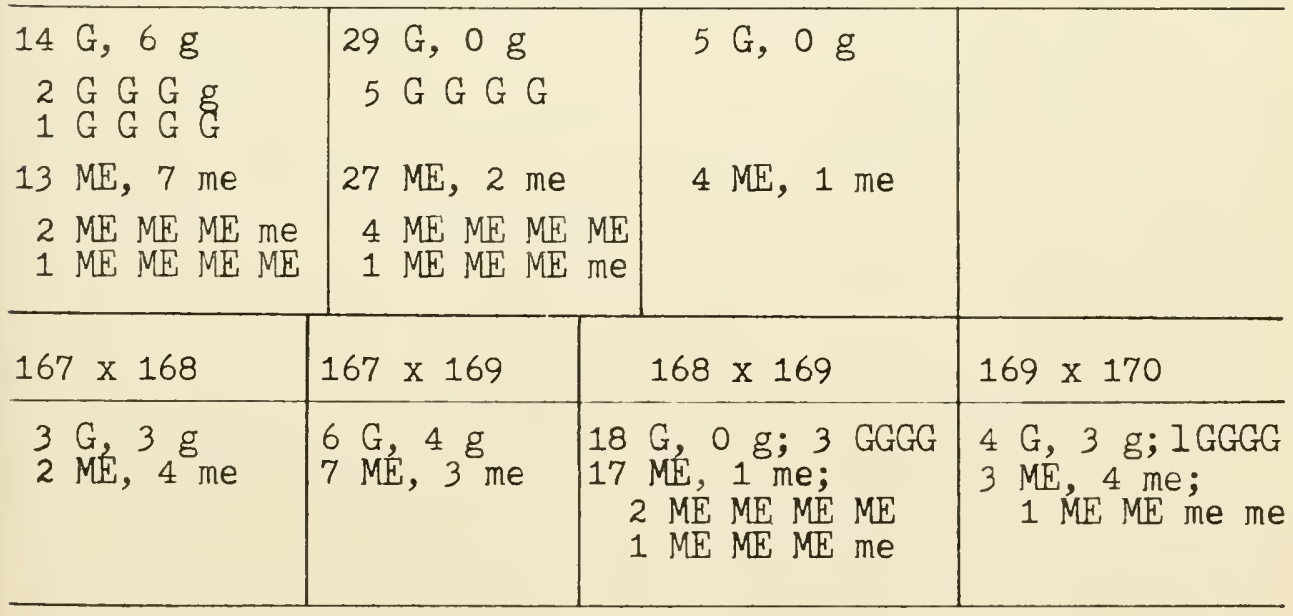

$170 \times 170$

$1 \mathrm{G}, 3 \mathrm{~g}$

$0 \mathrm{ME}, 4$ me 
pected (GGgg) type, but the numerous exceptions indicate that the Mendelian mechanism is not operating precisely. The ME alleles in 168 and 170 when backcrossed to me behave like standard dominants producing only ME ME me me asci. However, $167 \times \mathrm{me}$ produced one ME ME ME ME ascus and $169 \times$ me yielded $3 \mathrm{ME}$ me me me asci. In the backcross to the fermenter only cultures 167 , 168 , and 169 were studied. 168 and 169 behaved rather regularly as dominants with the $\mathrm{G}$ allele, but 167 produced several unexpected me progeny. The intra-ascus crosses which were matings of fermenters by fermenters also yielded a considerable number of nonfermenter progeny. An intra-ascus hybrid produced by mating $168 \times 169$ yielded only galactose fermenters and a single nonfermenter of melibiose among 18 progeny. (This mating was made possible by the fact that 169 acted as an a culture after many transfers.) The fact that the intra-ascus matings produced nonfermenter progeny indicates that the phenotypical fermenters in the ascus are not fully potent. The data suggest that spores 168 and 169 carry strong dominants for both $\mathrm{G}$ and $\mathrm{ME}$. The illegitimate mating of $170 \times 170$ threw more "nonfermenters" of galactose and melibiose than fermenters.

\section{THE ORIGIN OF NONFERMENTERS OF MALTOSE}

Numerous attempts were made to bring a maltose nonfermenter into the pedigree by selecting haploids from a number of natural nonfermenters of maltose. However, many of them failed to produce viable spores and none of those with viable spores was capable of producing hybrids. S. microellipsoideus from which the members of this pedigree are descended is ordinarily classified as a nonfermenter of both maltose and lactose, but no data are available now concerning the cultures which we used.

The progeny were diagnosed for ability to ferment maltose after the original cultures had been lost. Whatever the maltose fermenting ability of the parents, it is clear that the asci do not follow any regular behavior. With regard to maltose the first mating yielded 1 ma ma ma MA, 2 ma ma MA MA, 2 ma MA MA MA, and 3 MA MA MA MA segregations. Regular segregations occurred in some asci, notably 237-240, and 249-252. Other asci from the same hybrid were MA MA MA MA, or MA MA MA ma. However, this resembled the circumstances surrounding melibiose and galactose fermentation and did not negate the possibility that a reliable nonfermenting allele of maltose had been uncovered. Further analysis showed that ma $x$ ma matings generally produce only ma ma ma ma asci, indicating that the nonfermenter is not capable by itself of achieving fermentative ability. The precision with which the $\mathrm{MA} / \mathrm{ma}$ alleles segregate in the mating of $520 \times 525$, Table 26-3, proves that fermentation of maltose is under genic control. 
Table 26-3

Maltose Permenter by Nonfermenter

$246 \times 375$

a $g$ me ma $x$ a $g$ ME MA

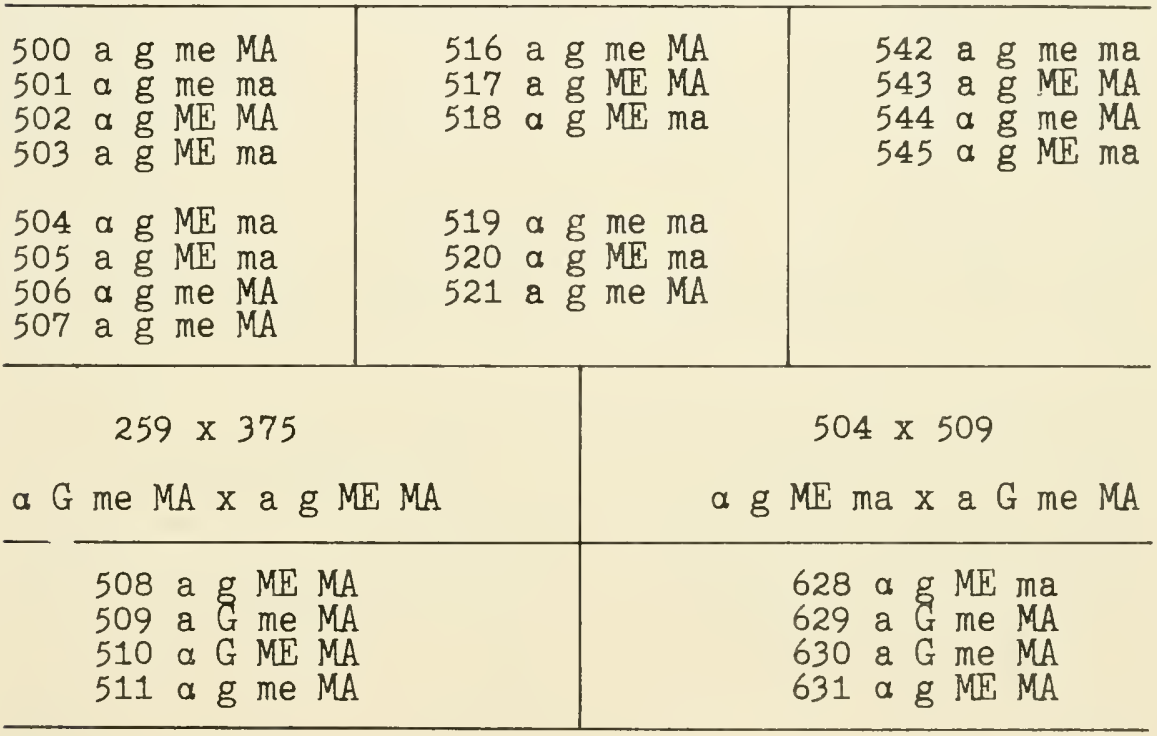

$373 \times 372$

a $G$ me $M A x$ a g me MA

512 a $G$ me MA

513 a $G$ me $M A$

514 a $G$ me MA

$515 \propto \mathrm{G}$ me $\mathrm{MA}$
546 a g me $M A$

547 a $G$ me $M A$

548 a $G$ me MA 
Table 26-3 (Continued)

$260 \times 375$

a $G$ me $M A x$ a $g$ ME $M A$

\begin{tabular}{l|llll}
\hline 522 a g me MA & 526 a $G$ me MA & 530 a G ME MA \\
523 a G ME MA & 527 a G ME MA & 531 a g me MA \\
524 a g ME MA & 528 a G ME MA & 532 a G me MA \\
525 a G me MA & 529 a g me MA & 533 a g ME MA \\
534 & 538 a G ME MA & & \\
535 a G me MA & 539 a g me MA & & \\
536 a g ME MA & 540 a G ME MA & & \\
537 a g me MA & 541 a G me MA & & \\
\hline
\end{tabular}

\section{$525 \times 520$}

a G me MA x a g ME ma (549 diploid)

\begin{tabular}{l|l|l}
\hline 550 a g ME MA & 554 a G me ma & 558 a G ME ma \\
551 a G ME ma & 555 a g ME MA & 559 a g MF ma \\
552 a g ME ma & 556 a G me ma & 560 a G me MA \\
553 a G ME MA & 557 a g ME MA & 561 a g me MA \\
562 a g me MA & 566 a g ME ma & 570 a G ME ma \\
563 a G ME MA & 567 a G ME ma & 571 a g me ma \\
564 a G me ma & 568 a g ME MA & 572 a g me MA \\
565 a g ME ma & 569 a G ME MA & 573 a G ME MA \\
574 a G me MA & 578 a G ME ma & 581 a G me MA \\
575 a g ME ma & 579 a G ME MA & 582 a g me ma \\
576 a G ME ma & 580 a g ME MA & 583 a G ME ma \\
577 a g me MA & & 584 a g ME MA \\
585 a G ME MA & 589 a g ME ma & 593 a g ME MA \\
586 a g ME MA & 590 a G me ma & 594 a G ME ma \\
587 a g me ma & 591 a g me MA & 595 a $\mathrm{ME} \mathrm{MA}$ \\
588 a G me ma & 592 a G ME MA & 596 a g ME ma \\
597 a G ME MA & 601 a G ME ma & 604 a g me MA \\
598 a g me ma & 602 a g me MA & 605 a G me ma \\
599 a G ME ma & 603 a g me MA & 606 a g ME ma \\
600 a g me ma & &
\end{tabular}




\section{GENETICAL ANALYSIS OF TRANSFORMED ME ALLELES}

Table 26-4 shows the analysis of ascus 550-551-552-553 which was obtained from a hybrid heterozygous for $\mathrm{G} / \mathrm{g} \mathrm{ME} / \mathrm{me}$ and $\mathrm{MA} / \mathrm{ma}$ alleles. Regular segrègations of the $\mathrm{G}$ and $\mathrm{MA}$ alleles occurred, but the ascús was ME ME ME ME. These data are summarized below.

550 gMEMA

$\mathrm{x}$ me

$6 \mathrm{ME}, 5 \mathrm{me}$

2 MEMEmeme
551 GMEma

$\mathrm{x}$ me

$6 \mathrm{ME}, 4 \mathrm{me}$

1 MEMEmeme
552 gMEma

$\mathrm{x}$ me

$4 \mathrm{ME}$

1 MEMEMEME
553 GMEMA

$\mathrm{x}$ me

$6 \mathrm{ME}$
$550 \times 553$

$5 \mathrm{ME}, 5 \mathrm{me}$

1 MEMEmeme
$552 \times 553$

$15 \mathrm{ME}, 1 \mathrm{me}$

3 MEMEMEME

1 MEMEMEMe

Backcross to the nonfermenter (me) revealed that 550 and 551 gave regular ratios; these two clones acted like regular dominants. However, 552 and 553 backcrossed to the nonfermenter threw only a dominant progeny, indicating that the $\mathrm{ME}$ alleles in 552 and $553 \mathrm{had}$ the ability to transform nonfermenters into fermenters. A mating of $552 \times 553$ ( $\mathrm{ME} \times \mathrm{ME}$ ) produced $15 \mathrm{ME}$ and 1 me clones, indicating that they were strong dominants. However, mating 550 to 553 (ME x ME) gave only ME ME me me asci, suggesting that 550 was a weak dominant which easily lost its fermentative ability. This might indicate that $\mathbf{5 5 0}$ had recently acquired its fermentative ability.

TESTS FOR CYTOPLASMIC EFFECTS (WITH THE COOPERATION OF DR. DOUDOROFF AND OUR STAFF)

The tetrad analyses presented in Chapter 25 have shown that irregular ratios of the type described in the preceding section can be interpreted as the result of the direct transfer of material from 
Table 26-4

Analysis of 550,551, 552, 553

$550 \times 515$

a $g$ ME MA $x$ a $g$ me MA

\begin{tabular}{l|l|l|l}
\hline 663 a g? me ma? & 667 a g ME MA & 671 a g me ma? \\
664 a g me MA & 668 a g MA & 672 a g ME MA \\
665 a g ME ma? & 669 a g ME ma? & 673 a g ME MA? \\
666 a g ME MA & 670 a g me ma & 674 a g me MA \\
\hline
\end{tabular}

$551 \times 521$

a G ME ma $x$ a $g$ me $M A$

\begin{tabular}{l|lll|ll|l}
\hline 658 a G ME ma & 661 a G ME MA & 685 G ME MA & 698 a G ME MA \\
& 662 a G ME ma & 686 G me MA & 699 a g me ma \\
& & 687 g me ma & 700 a g me MA? \\
& & & & 701 a G ME MA \\
\hline
\end{tabular}

$552 \times 609$

a g ME ma $x$ a $G$ me $M A$
$553 \times 509$

a G ME MA $x$ a $G$ me $M A$
651 a G ME MA

652 a G ME ma

653 a g ME ma

654 a g ME ma
655 a G ME MA

656 a G ME MA?

657 a G ME ma
688 a G ME ma 689 a G ME MA

$552 \times 553$

a g ME ma $x$ a $G M E M A$

\begin{tabular}{l|l|l|ll|ll}
\hline 635 a G ME & 639 a G ME ma & 643 a G ME MA? & 647 a & ME ma \\
636 a G ME & 640 a G ME & 644 a g ME ma & 648 a $M E$ ma \\
637 a g ME ma & 641 a g ME & 645 a G ME ma & 649 a me ma \\
638 a g ME ma & 642 a g ME ma & 646 a g ME MA? & 650 a ME ma \\
\hline
\end{tabular}

$553 \times 550$

a G ME MA x a g ME MA

\begin{tabular}{l|l|l}
\hline 675 a G ME ma? & 678 a g me ma & 681 a G me ma \\
676 a g me ma & 679 a g ME MA & 682 a g?me MA \\
677 a g me ma & 680 a G ME MA & 683 a g ME MA \\
& & 684 a g ME ma \\
\hline
\end{tabular}


one gene to its allele in the heterozygous condition. At one time I held the view that the fermenter character was maintained by a cytoplasmic mechanism, independent of the gene and could be perpetuated in the cytoplasm of the recessive nonfermenter by interaction between substrate and cytoplasm. If such were the case, fusion of cytoplasms during copulation could establish the factor in the hybrid cytoplasm and it could be transmitted to varying numbers of spores when these were cut out at sporulation from the mixed cytoplasm. If the number of cytoplasmic units were large, nearly all the progeny would be fermenters; if the number small, only a few would acquire the character. This view grew out of some joint work with Dr. Spiegelman in our laboratory.

We (Spiegelman, Lindegren, and Lindegren) published a paper stating that cytoplasmic transfer occurred when mating, meiosis and isolation of the spores of a heterozygous (fermenter/nonfermenter) diploid yeast occurred in the continuous presence of the specific substrate. A backcross mating which had previously produced only ME ME me me asci was repeated with the difference that the gametes, the copulating cells, the zygotes, and the sporulating cells were kept constantly in the presence of melibiose. This resulted in $6 \mathrm{ME}$ ME ME ME and one ME ME me me ascus; similar matings in the absence of melibiose had produced $10 \mathrm{ME} \mathrm{ME} \mathrm{me} \mathrm{me} \mathrm{asci.}$

The cultures from the asci containing 4 fermenters each were dissimilated in phosphate buffer in the absence of melibiose for from 7 to 20 days and then tested in the Warburg apparatus for their ability to ferment melibiose. At the same time the ability to ferment glucose was also examined. In 4 asci tested, 2 cultures each lost their ability to ferment melibiose while 2 retained it, although all 4 were capable of fermenting glucose. This was taken to indicate that these were cultures containing the recessive gene which had been carrying the enzyme in the cytoplasm. The fermenting cultures were not tested further, although as the experiments below indicate, they might likewise have lost their ability to ferment melibiose.

We (Spiegelman, Lindegren, and Lindegren) had concluded from the experiments cited above that the presence of melibiose had enabled a cytoplasmic enzyme to maintain itself and increase in the absence of the specific gene, normally controlling its synthesis, but this conclusion was shown to be untenable by the following experiments.

Our joint paper with Dr. Spiegelman aroused considerable interest and when Dr. Michael Doudoroff visited us, he expressed an intense interest to see the experiment reproduced and extended. When he arrived, we were analyzing the matings $557 \times 554$, and 573 $x$ 571. Since all these haplophases had arisen from the rather regular pedigrees shown in Table 26-3 (from matings $260 \times 375$ and $525 \times 520$ ) we predicted to him that only regular segregations would 
occur because we had finally "cleaned up" the cytoplasm. We were thoroughly surprised to find the results shown in Table 26-5. The mating $557 \times 554$ is of interest, since the independent segregation of the galactose gene indicates that ratios obtained for the melibiose and maltose genes were not due to illegitimate copulation or selfdiploidization. Dr. Doudoroff then proposed and supervised the following experiments:

Table 26-5

Irregular Ratios from "Clean" Stocks

\begin{tabular}{|c|c|c|c|c|}
\hline & $\begin{array}{r}557 \\
\alpha \text { g ME MA }\end{array}$ & $\begin{array}{l}554 \\
\text { a G me ma }\end{array}$ & & \\
\hline 1180 a G ME ma & $1183 \propto$ a G ME MA & $1186 \propto G \mathrm{ME} M A$ & 1190 & G \\
\hline 1181 a G ME ma & 1184 a g ME ma & $1187 \propto \mathrm{g} \quad \mathrm{MA}$ & 1191 & G ME MA \\
\hline 1182 a G me MA & 1185 a G MA & 1188 a G ME MA & 1192 & $\mathrm{~g} M E \mathrm{ma}$ \\
\hline & & 1189 a $\mathrm{g} \mathrm{ME} \mathrm{MA}$ & 1193 & G ME MA \\
\hline
\end{tabular}

1194 g ME MA 1198 G ME MA 1202 a G MA

1195 G ME MA 1199 G ME ma 1203 a G ME MA

1196 G me MA 1200 G ma 1204 a G ME ma

1197 G ME MA 1201 G ME MA

$\begin{array}{rll}573 & \times & 571 \\ \text { a G ME MA } & \text { x } & \text { a g me ma }\end{array}$

\begin{tabular}{|c|c|c|c|c|}
\hline 1205 \& G ME MA & 1208 a G ME MA & 1212 & G me $M A$ & $1216 \propto \mathrm{G} \mathrm{ME} \mathrm{MA}$ \\
\hline 1206 a G MA & 1209 a G ME MA & 1213 & G ME MA & 1217 a G ME MA \\
\hline 1207 G ME MA & $\begin{array}{l}1210 \text { a G ME MA } \\
1211 \text { a G ME } M A\end{array}$ & $\begin{array}{l}1214 \\
1215\end{array}$ & $\begin{array}{l}\text { G ME MA } \\
G M E M A\end{array}$ & 1218 a G me $M A$ \\
\hline
\end{tabular}

1219 G ME MA

1220 G ME MA

1221 G ME MA

$1222 \mathrm{G} M E \mathrm{ma}$ 
Experiment I. If the "extra" fermenters were the result of the transfer of a substrate-dependent cytoplasmic entity, they should lose their ability to ferment in the absence of substrate. Consequently, cultures $1194,1195,1196,1197,1198,1199,1200,1201$, $1208,1209,1210,1211$, and their parents 554,557, 571, and 573 were transferred daily in glucose broth for 11 successive transfers and then tested on glucose, galactose, melibiose, and maltose in the standard manner in small fermentation tubes. No previous nonfermenters fermented a new sugar. The only exception to the diagnosis made according to our regular custom immediately after isolation, was that culture 557 failed to ferment maltose until after 12 days, and a subculture remained negative. The interesting thing is that $1194,1195,1196,1197$, and 1198 which had recelved their ability to ferment maltose from 557 were apparently stable.

Experiment II. Cultures 1208, 1209, 1210, and 1211 were shaken in $\mathrm{M} / 30 \mathrm{KH}_{2} \mathrm{PO}_{4}$ without substrate for 8 days; then aliquots were taken from each flask and each aliquot was "fed" a few drops of either (1) glucose or (2) galactose or (3) melibiose or (4) maltose or (5) starved. This was done in the hope of "deadapting" the cells by forcing them to form adaptive enzymes to other substrates.

Starvation was then continued for 10 days. Then glucose was added to each for 1 day; the cultures were streaked, 2 colonies were selected at random from each plate; these were combined, suspended in buffer, and used to inoculate separate broth fermentation tubes containing the 4 different sugars: glucose, galactose, melibiose, and maltose. All cultures fermented rapidly (1-3 days) with the following exceptions: (1) 1210 from glucose to galactose, 6 days; (2) 1210 from maltose to galactose, 4 days; (3) 1208 from maltose to galactose, 4 days. However, 1210 initially required 5 days to ferment galactose and 1208 was also slow on galactose initially. Therefore, this extensive attempt to remove "cytoplasmic" components was completely negative. In fact, the only diminution was in a parent which must have carried a fermenter gene for many tetrads which originated from it subsequently segregated regularly. The precaution of setting up one glucose tube as a control for the detection of "dead" cultures was unnecessary.

Experiment III. Since mass transfers might have maintained mixtures (which could be separated by colony isolation) 5 individual colonies each from 573,1208, 1209, 1210 and 1211 were tested on the 4 sugars according to the procedure outlined above. There were only 2 exceptions: 2 isolates of 1208 failed to ferment maltose.

Experiment IV. Then five colonies were isolated from each of the "treated" cultures (starved or fed one of the four sugars) derived from culture 1208 from Experiment II (a total of 30 cultures). These 30 cultures were transferred in glucose broth for 11 successive days and each of the five transfers from (1) the original tube, 
(2) starved without sugar, (3) starved and fed glucose, (4) starved and fed galactose, (5) starved and fed melibiose, and (6) starved and fed maltose were then tested for their ability to ferment galactose. (Culture 1208 originally required 4 to 5 days to ferment galactose). Six of the 30 cultures fermented galactose in 4-5 days. All others were faster, usually from 1-2 days except one starved and fed melibiose, which failed to ferment, and a second also fed melibiose, which was contaminated.

Experiment V. Table 26-6 shows the results of an experiment in which cultures dissimilated in $\mathrm{M} / 30 \mathrm{KH}_{2} \mathrm{PO}_{4}$ on the shaker for 20 days (1) in phosphate buffer and (2) in phosphate buffer with glucose. In all but one case, 5 isolated colonies from each haplophase were tested. Each separate colonial isolate is indicated by a small letter, 1208-a, etc. The numbers indicate the days before fermentation occurred. One isolate of 1211 in phosphate buffer failed to ferment maltose and one of 1210 in phosphate buffer with glucose failed to ferment maltose; all others performed as well as the originals except three which were slow in maltose.

Experiment VI. The possibility that selecting colonies from a natural medium might have restored fermentation vigor was investigated by selecting colonies from plates of Hutner's synthetic medium and testing on the 4 sugars. Table 26-7 shows one failure on galactose and one on maltose, but most others have their usual speed of fermentation.

Experiment VII. Finally, the dissimilation and starvation technique was tested by isolating individual colonies from the original stock cultures on our standard peptone-yeast extract agar and Hutner's synthetic medium agar (Table 26-8). Three isolates from Hutner's medium were unable to ferment galactose and one was very slow (29 days). These results indicate that dissimilation and starvation give no result that cannot be obtained by direct isolation on a deficient medium.

Experiment VIII. Haplophase 557 (Table 26-5) is a parent which produced many progeny vigorously capable of fermenting both melibiose and maltose when mated to a nonfermenter of these sugars. It is genotypically designated $\mathrm{g}$ ME MA. In Experiment I, a culture of 557 was obtained which fermented maltose only after 12 days. Thirteen colonies were obtained from this culture. Cultures from each of these 13 colonies were transferred daily for 11 times through $5 \mathrm{cc}$ each of 4 per cent glucose broth. The cultures were plated on peptone-yeast extract agar. Single colonies were suspended in $\mathrm{M} / 15$ phosphate buffer and 2 drops of this suspension used to inoculate small fermentation tubes of galactose, maltose, melibiose, and sucrose. The cultures were observed from $11 / 21$ to $12 / 26$ for fer mentative ability, by which time the fermentation tubes had dried up. 
Table 26-6

Experiment $V$ by Dr. Doudoroff

9-16-46 suspenstons placed on shaker. Suspenslons taken from shaker 10-5-46 after 20 days dissinilation in $\mathrm{H} / 3 \mathrm{KH}_{2} \mathrm{PO}_{4}$, and plated. 2 colonies selected fro each plate (10-9-46) and placed together in phosphate buffer. 2 drops of suspenston put into each of 4 sugars. Numbers indicate days elapsing unt 1 fermentation uccurs.

\begin{tabular}{|c|c|c|c|c|c|c|c|c|c|}
\hline $\begin{array}{l}\text { In phosphate } \\
\text { buffer }\end{array}$ & Gal. & Glu. & Mal. & Mell. & $\begin{array}{l}\text { In phosphate } \\
\text { buffer with } \\
\text { glucose }\end{array}$ & Gal. & Glu. & Mal. & Mel. \\
\hline $1208-8$ & 3 & 2 & 3 & 3 & $1208-8$ & 3 & 2 & 2 & 2 \\
\hline$n-b$ & 3 & 2 & 3 & 2 & $n-b$ & 3 & 2 & 3 & 2 \\
\hline$n-c$ & 3 & 2 & 2 & 2 & $n-c$ & 3 & 2 & 2 & 2 \\
\hline$n-d$ & 3 & 2 & 2 & 2 & $n-d$ & 3 & 2 & 3 & 2 \\
\hline$n-e$ & 3 & 2 & 2 & 2 & $n-e$ & 2 & 2 & 3 & 2 \\
\hline $1209-a$ & 2 & 2 & 2 & 2 & $1209-8$ & 2 & 2 & 2 & 2 \\
\hline$\pi-b$ & 3 & 2 & 6 & 3 & $n-b$ & 2 & 2 & 3 & 2 \\
\hline$n-c$ & 2 & 2 & 3 & 2 & $n-c$ & 3 & 2 & 3 & 2 \\
\hline$n-d$ & 2 & 2 & 3 & 2 & $n-d$ & 2 & 2 & 3 & 2 \\
\hline$n-e$ & 2 & 2 & 3 & 2 & $n-e$ & 3 & 2 & 2 & 2 \\
\hline $1210-8$ & 4 & 2 & 2 & 2 & $1210-a$ & 5 & 2 & - & 3 \\
\hline$n-b$ & 4 & 2 & 3 & 3 & $n-b$ & 5 & 2 & 3 & 3 \\
\hline$\pi-c$ & 4 & 2 & 2 & 2 & $n-c$ & 4 & 2 & 3 & 2 \\
\hline$n-d$ & 4 & 2 & 2 & 2 & $n-d$ & 4 & 2 & 3 & 3 \\
\hline$n-e$ & 4 & 2 & 2 & 2 & $n-e$ & 4 & 2 & 3 & 2 \\
\hline $1211-8$ & 4 & 3 & 4 & 3 & $1211-b$ & 3 & 2 & 3 & 2 \\
\hline$n-b$ & 4 & 3 & - & 3 & $n-c$ & 3 & 2 & 3 & 2 \\
\hline$n-c$ & 3 & 3 & 3 & 3 & $n-d$ & 3 & 2 & 3 & 3 \\
\hline$n-d$ & 4 & 3 & 4 & 3 & & & & & \\
\hline$n-e$ & 4 & 3 & 3 & 3 & & & & & \\
\hline
\end{tabular}




\section{Table 26-7}

Isolates starved with buffer and glucose on shaker for 20 days and then streaked on Hutner's medium. Single colonies picked and put into buffer. Then .2 cc. put into each of 4 sugars using Hutner's medium. Second part of table shows results when starvation was with buffer alone. See Experiment VI.

\begin{tabular}{|c|c|c|c|c|c|c|c|c|c|}
\hline $\begin{array}{l}\text { With buffer } \\
\text { and glucose }\end{array}$ & Gal. & Glu. & Mal. & Mel. & $\begin{array}{l}\text { With buffer } \\
\text { alone }\end{array}$ & Gal. & Glu. & Mal. & Mel. \\
\hline $1208-a$ & 6 & 2 & 3 & 6 & $1208-a$ & 3 & 2 & 2 & 2 \\
\hline$n-b$ & 6 & 3 & 2 & 2 & $n-b$ & 6 & 3 & 6 & 3 \\
\hline$n-c$ & 6 & 2 & 3 & 2 & $n-c$ & 2 & 2 & 2 & 2 \\
\hline$-d$ & 6 & 3 & 3 & 3 & $"-d$ & 3 & 2 & 2 & 2 \\
\hline$n-\mathrm{e}$ & 6 & 2 & 3 & 6 & $n-\mathrm{e}$ & 6 & 2 & 3 & 3 \\
\hline$\pi-f$ & - & 3 & 3 & 3 & $n-f$ & 6 & 2 & 14 & 6 \\
\hline $1209-a$ & 2 & 2 & 2 & 2 & $1209-a$ & 2 & 2 & 2 & 2 \\
\hline$n-b$ & 3 & 2 & 2 & 2 & $n-b$ & 2 & 2 & 2 & 2 \\
\hline$n-c$ & 6 & 3 & 3 & 3 & $n-c$ & 2 & 2 & 2 & 2 \\
\hline$n-d$ & 3 & 2 & 2 & 2 & $n-d$ & 2 & 3 & 2 & 2 \\
\hline$n-e$ & 6 & 2 & 3 & 2 & $n-\mathrm{e}$ & 3 & 2 & 3 & 2 \\
\hline$\pi-f$ & 3 & 2 & 3 & 2 & $n-f$ & 3 & 2 & 3 & 2 \\
\hline $1210-a$ & 6 & 2 & 3 & 2 & $1210-a$ & 6 & 3 & 3 & 3 \\
\hline$\pi-b$ & 6 & 2 & 2 & 2 & $"-b$ & 6 & 2 & 2 & 2 \\
\hline$n-c$ & 6 & 2 & 3 & 2 & $\pi-c$ & 6 & 2 & 3 & 2 \\
\hline$n-d$ & 6 & 2 & 3 & 3 & $n-d$ & 6 & 2 & 3 & 2 \\
\hline & & & & & $n-e$ & 14 & 3 & 6 & 6 \\
\hline & & & & & $n-f$ & 14 & 3 & 6 & 6 \\
\hline $1211-\mathrm{a}$ & 3 & 2 & - & 2 & $1211-\mathrm{a}$ & 3 & 2 & 6 & 3 \\
\hline$\pi-b$ & 3 & 2 & 2 & 2 & $n-b$ & 3 & 3 & 3 & 3 \\
\hline & & & & & $\pi-c$ & 3 & 2 & ; & 3 \\
\hline & & & & & $n-d$ & 3 & 3 & 6 & 3 \\
\hline & & & & & $n-\mathrm{e}$ & 6 & 2 & 6 & 3 \\
\hline & & & & & $\pi-f$ & 6 & 3 & 6 & 6 \\
\hline
\end{tabular}




\section{Table 26-8}

\section{Experizent VII}

Plates of peptone-yeast agar streaked from orlginal 1208. 1209, 1210, and 1211 tubes. Single colontes plcked and put into buffer. Then .2 cc. of this suspension put into each of 4 sugars using Butner's med tum.
Plates of Butner's medium were streaked from orlginal tubes of 1208. 1209. 1210, and 1211. Single colonies were picked and put into buffer. Then.2 $c c$. of this suspension was put into each of 4 sugars using Hutner's medium.

\begin{tabular}{|c|c|c|c|c|c|c|c|c|c|}
\hline & Gal. & Glu. & Mal. & Mel. & & Gal. & Glu. & Mal. & Mel. \\
\hline 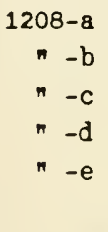 & $\begin{array}{l}4 \\
7 \\
7 \\
7 \\
4\end{array}$ & $\begin{array}{l}2 \\
9 \\
4 \\
2 \\
3\end{array}$ & $\begin{array}{l}2 \\
3 \\
3 \\
3 \\
3\end{array}$ & $\begin{array}{l}2 \\
2 \\
2 \\
3 \\
2\end{array}$ & $\begin{array}{c}1208-\mathrm{H}-\mathrm{a} \\
"-\mathrm{b} \\
"-\mathrm{c} \\
"-\mathrm{d} \\
"-\mathrm{e} \\
"-\mathrm{f}\end{array}$ & $\begin{array}{r}29 \\
- \\
- \\
7 \\
4 \\
-\end{array}$ & $\begin{array}{l}2 \\
2 \\
3 \\
2 \\
3 \\
2\end{array}$ & $\begin{array}{l}3 \\
3 \\
4 \\
3 \\
3 \\
2\end{array}$ & $\begin{array}{l}2 \\
3 \\
3 \\
3 \\
3 \\
2\end{array}$ \\
\hline $\begin{array}{r}1209-\mathrm{a} \\
n-\mathrm{b} \\
n-\mathrm{c} \\
n-\mathrm{d} \\
n-\mathrm{d}\end{array}$ & $\begin{array}{r}2 \\
4 \\
7 \\
7 \\
10\end{array}$ & $\begin{array}{l}2 \\
2 \\
2 \\
3 \\
3\end{array}$ & $\begin{array}{r}3 \\
3 \\
3 \\
10 \\
7\end{array}$ & $\begin{array}{l}3 \\
2 \\
3 \\
7 \\
7\end{array}$ & $\begin{array}{cc}1209-\mathrm{H}-\mathrm{a} \\
\pi & -\mathrm{b} \\
\pi & -\mathrm{c} \\
\pi & -\mathrm{d} \\
\pi & -\mathrm{e} \\
\pi & -\mathrm{f}\end{array}$ & $\begin{array}{l}3 \\
2 \\
2 \\
3 \\
3 \\
3\end{array}$ & $\begin{array}{l}2 \\
2 \\
2 \\
2 \\
2 \\
2\end{array}$ & $\begin{array}{l}2 \\
2 \\
3 \\
2 \\
2 \\
3\end{array}$ & $\begin{array}{l}2 \\
2 \\
2 \\
2 \\
2 \\
2\end{array}$ \\
\hline $\begin{array}{rr}1210-a \\
n & -b \\
n & -c \\
n & -d \\
n & -e\end{array}$ & $\begin{array}{l}7 \\
7 \\
7 \\
7 \\
7\end{array}$ & $\begin{array}{l}2 \\
2 \\
2 \\
2 \\
2\end{array}$ & $\begin{array}{l}3 \\
2 \\
2 \\
2 \\
3\end{array}$ & $\begin{array}{l}2 \\
2 \\
2 \\
3 \\
3\end{array}$ & $\begin{array}{cc}1210-\mathrm{H}-\mathrm{a} \\
n & -\mathrm{b} \\
n & -\mathrm{c} \\
n & -\mathrm{d} \\
n & -\mathrm{e} \\
n & -\mathrm{f}\end{array}$ & $\begin{array}{l}6 \\
6 \\
6 \\
6 \\
6 \\
6 \\
6 .\end{array}$ & $\begin{array}{l}2 \\
2 \\
2 \\
2 \\
2 \\
2\end{array}$ & $\begin{array}{l}3 \\
2 \\
2 \\
3 \\
3 \\
3\end{array}$ & $\begin{array}{l}2 \\
2 \\
2 \\
2 \\
2 \\
2\end{array}$ \\
\hline 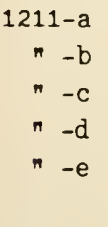 & $\begin{array}{l}4 \\
4 \\
4 \\
4 \\
4\end{array}$ & $\begin{array}{l}3 \\
3 \\
3 \\
3 \\
3\end{array}$ & $\begin{array}{l}3 \\
4 \\
3 \\
4 \\
4\end{array}$ & $\begin{array}{l}3 \\
3 \\
2 \\
3 \\
3\end{array}$ & $\begin{array}{cc}1211-\mathrm{H}-\mathrm{a} \\
\pi & -\mathrm{b} \\
\pi & -\mathrm{c} \\
\pi & -\mathrm{d} \\
\pi & -\mathrm{e} \\
\pi & -\mathrm{f}\end{array}$ & $\begin{array}{l}6 \\
6 \\
3 \\
3 \\
6 \\
3\end{array}$ & $\begin{array}{l}3 \\
2 \\
2 \\
2 \\
2 \\
2\end{array}$ & $\begin{array}{l}3 \\
2 \\
3 \\
3 \\
3 \\
3\end{array}$ & $\begin{array}{l}3 \\
2 \\
2 \\
2 \\
2 \\
2\end{array}$ \\
\hline
\end{tabular}


Table 26-9 shows the number of days before fermentation began. The minus sign indicates fermentation did not occur. Of 79 colonies tested 4 finally fermented galactose after 12, 27, 21, and 17 days respectively. Twenty-two nonfermenters of melibiose appeared while all cultures fermented sucrose on the second or third day. Therefore, 557 which carries an unmistakable $\mathrm{ME}$ gene can lose its ability to ferment in a fermentation tube in about one-fourth of the single colony isolates if carried long enough in the absence of melibiose. This is a very high rate of loss. However, conventional diagnosis is still possible because selection in the presence of the substrate favors the fermenters.

Experiment IX. The experiments (Splegelman, Lindegren, and Lindegren) which had been interpreted as indicating that the continued presence of a specific type of sugar could alter the phenotype of the progeny were repeated. Matings were made using haplophases grown with glucose maintaining glucose in the tubes in which matings occurred, keeping glucose on the gypsum slant and dissecting the hybrid asci in glucose in an attempt to determine if this would produce regular segregation of the glucose, melibiose or maltose alleles. The first mating of $4 \times 128$ produced six 2 : 2 galactose asci but gave 2 irregular maltose asci (Table 26-10). The mating L5C x 23 produced a large number of nonfermenters of melibiose while the mating C1A $\times 15$ produced 3 asci in which more than 2 galactose fermenters were found. When galactose was maintained throughout the cycle (except for the fact that the haplophase incapable of fermenting galactose was grown on glucose) 20 asci were obtained from $4 \times 128$ in which $2: 2$ ratios of fermenter to nonfermenter of galactose occurred. Three asci produced more than 2 galactose fermenters, while 1 produced 3 nonfermenters of galactose. Among 6 asci from a hybrid heterozygous for melibiose fermentation there were none with more than 2 spores capable of fermenting melibiose. The presence of maltose did not materially change the kind of phenotype produced. Except for the fact that fever than the expected number of fermenters of melibiose were obtained in glucose in the L5C x 23 mating, no significant evidence of the effect of substrate was obtained.

Conclusions:

In the above series of experiments, the earlier claim that the presence of a specific substrate could cause the maintenance of a character for which no gene was present could not be substantiated. Not only were inexplicable losses of fermentative ability occasionally observed, but also abnormal ratios of fermenters to nonfermenters in the segregants which were apparently independent of the substrate on which growth and sporulation was achieved. Such ratios were almost always in favor of fermenters, and at least in some experiments could not be explained by self-diploidization or illegitimate copulation. 
Table 26-9

Days required for 13 isolates from a "slow" (12 day) maltose fermenter derived by dissimilation (Expertment I) from $557\left(g\left\|_{A}\right\| E\right)$. originally a fast fermenter of altose, to ferment 4 different sugars in fermentation tubes containing peptone-yeast extract broth.

\begin{tabular}{|c|c|c|c|c|c|c|c|c|c|}
\hline & Gal. & Mal. & $\mathrm{Mel}$. & Suc. & & Gal. & Mal. & Mel. & Suc. \\
\hline $\begin{array}{rr}557-b-1 \\
" & -2 \\
\pi & -3 \\
n & -4 \\
n & -5 \\
\pi & -6\end{array}$ & $\begin{array}{l}- \\
- \\
- \\
- \\
-\end{array}$ & $\begin{array}{r}18 \\
13 \\
18 \\
22 \\
-\end{array}$ & $\begin{array}{l}2 \\
2 \\
2 \\
2 \\
2 \\
2\end{array}$ & $\begin{array}{l}2 \\
2 \\
2\end{array}$ & $\begin{array}{rr}557-G-1 \\
" 1 & -2 \\
\pi & -3 \\
\pi & -4 \\
n & -5 \\
" & -6\end{array}$ & $\begin{array}{l}- \\
- \\
- \\
- \\
-\end{array}$ & $\begin{array}{r}14 \\
13 \\
18 \\
- \\
14 \\
14\end{array}$ & $\begin{array}{l}2 \\
2 \\
- \\
4 \\
2 \\
4\end{array}$ & $\begin{array}{l}2 \\
2 \\
2\end{array}$ \\
\hline $\begin{array}{rr}557-J & -1 \\
n & -2 \\
n & -3 \\
n & -4 \\
n & -5\end{array}$ & $\begin{array}{l}- \\
- \\
- \\
-\end{array}$ & $\begin{array}{r}15 \\
- \\
22 \\
25 \\
13\end{array}$ & $\begin{array}{l}2 \\
2 \\
2 \\
2 \\
2\end{array}$ & $\begin{array}{l}2 \\
2 \\
2\end{array}$ & $\begin{array}{rr}557-d & -1 \\
" & -2 \\
" & -3 \\
" & -4 \\
" & -5 \\
" & -6\end{array}$ & $\begin{array}{l}- \\
- \\
- \\
- \\
-\end{array}$ & $\begin{array}{r}22 \\
- \\
- \\
- \\
- \\
-\end{array}$ & $\begin{array}{l}2 \\
2 \\
2 \\
2 \\
2 \\
2\end{array}$ & $\begin{array}{l}2 \\
2 \\
2\end{array}$ \\
\hline $\begin{array}{cc}557-e-1 \\
n & -2 \\
n & -3 \\
n & -4 \\
n & -5 \\
n & -6\end{array}$ & $\begin{array}{l}- \\
- \\
- \\
- \\
-\end{array}$ & $\begin{array}{r}- \\
- \\
\overline{8} \\
- \\
-\end{array}$ & $\begin{array}{l}2 \\
2 \\
2 \\
2 \\
2 \\
2\end{array}$ & $\begin{array}{l}2 \\
2 \\
2\end{array}$ & $\begin{array}{rr}557-\mathrm{H} & -1 \\
" & -2 \\
" & -3 \\
" & -4 \\
" & -5\end{array}$ & $\begin{array}{l}- \\
- \\
- \\
12\end{array}$ & $\begin{array}{l}19 \\
25 \\
25 \\
28 \\
35\end{array}$ & $\begin{array}{l}2 \\
2 \\
4 \\
2 \\
4\end{array}$ & $\begin{array}{l}2 \\
2 \\
2\end{array}$ \\
\hline $\begin{array}{rr}557-D-1 \\
n & -2 \\
n & -3 \\
n & -4 \\
n & -5\end{array}$ & $\begin{array}{l}- \\
- \\
- \\
- \\
-\end{array}$ & $\begin{array}{l}18 \\
35 \\
15 \\
11\end{array}$ & $\begin{array}{l}2 \\
2 \\
2 \\
2 \\
2\end{array}$ & $\begin{array}{l}2 \\
2 \\
2\end{array}$ & $\begin{array}{rr}557-g-1 \\
" & -2 \\
n & -3 \\
" & -4 \\
\pi & -5 \\
\pi & -6\end{array}$ & $\begin{array}{r}- \\
27 \\
- \\
- \\
- \\
-\end{array}$ & $\begin{array}{l}24 \\
17 \\
11 \\
14 \\
21 \\
21\end{array}$ & $\begin{array}{l}- \\
- \\
- \\
- \\
-\end{array}$ & $\begin{array}{l}3 \\
3 \\
3\end{array}$ \\
\hline $\begin{array}{cc}557-F & -1 \\
n & -2 \\
n & -3 \\
n & -4 \\
n & -5 \\
n & -6\end{array}$ & $\begin{array}{l}- \\
- \\
- \\
- \\
-\end{array}$ & $\begin{array}{r}15 \\
11 \\
11 \\
18 \\
6 \\
11\end{array}$ & $\begin{array}{l}2 \\
2 \\
4 \\
- \\
\overrightarrow{2}\end{array}$ & $\begin{array}{l}2 \\
2 \\
2\end{array}$ & $\begin{array}{rr}557-b-1 \\
" 1 & -2 \\
" & -3 \\
" & -4 \\
" & -5 \\
" & -6\end{array}$ & $\begin{array}{l}- \\
- \\
- \\
- \\
-\end{array}$ & $\begin{array}{r}1 \overline{1} \\
12 \\
- \\
21 \\
14\end{array}$ & $\begin{array}{l}- \\
- \\
- \\
-\end{array}$ & $\begin{array}{l}3 \\
3 \\
3\end{array}$ \\
\hline $\begin{array}{cc}557-c-1 \\
n & -2 \\
n & -3 \\
\pi & -4 \\
n & -5 \\
n & -6\end{array}$ & $\begin{array}{l}- \\
- \\
- \\
-\end{array}$ & $\begin{array}{l}11 \\
17 \\
21 \\
18 \\
11 \\
13\end{array}$ & $\begin{array}{l}3 \\
- \\
- \\
- \\
3\end{array}$ & $\begin{array}{l}3 \\
3 \\
3\end{array}$ & & & & & \\
\hline
\end{tabular}


Table 26-9 (Continued)

\begin{tabular}{rr|r|r|r|r}
\hline \hline & & Gal. & Mal. & Mel. & Suc. \\
\hline $557-I-1$ & - & - & 4 & 3 \\
$n$ & -2 & - & 13 & 4 & 3 \\
$n$ & -3 & - & - & 11 & 3 \\
$n$ & -4 & - & - & 4 & \\
$n$ & -5 & - & - & 4 & \\
$n$ & -6 & - & - & 4 & \\
\hline & & & & & \\
$557-c-1$ & - & 13 & - & 3 \\
$n$ & -2 & - & 7 & - & 3 \\
$n$ & -3 & - & 7 & - & \\
$n$ & -4 & 21 & 10 & 7 & \\
$n$ & -5 & - & 10 & - & \\
$n$ & -6 & - & 13 & & \\
$n$ & -7 & 17 & 11 & & \\
$n$ & -8 & - & 13 & & \\
$n$ & -9 & - & 34 & & \\
$n$ & -10 & - & 12 & & \\
\hline
\end{tabular}

Table 26-10

The effect on fermentative ability of the progeny produced by maintaining haplophase, carrying on matings and dissecting hybrid asci in the constant presence of different sugars.

a. Glucose maintained throughout cycle

(1) 4 (Fig. 18-1) $\times 128$ (Table 19-2)

( $\alpha G$ me $M A) \times$ (a g me MA)

G G g g 6

me me me me 2

MA MA MA MA 1

$G g g-1$

MA MA MA ma 1

(2) L5C (Table 20-2) $\times 2$; (Table 20-2)

$\alpha$ G me $x$ a G $M E$

ME ME me me 1

ME ME me - 2

ME me - 4

ME me me - 1

(3) $\mathrm{C} 1 \mathrm{~A} \times 15$ (Fig. 18-1)

$$
(\alpha G M E) \quad x \text { a } g \text { me }
$$

ME me me me 5

me me me me 2

me me me - 1

$\begin{array}{ll}G G g g & 2 \\ G G G G . & 2 \\ G G G- & 1\end{array}$

ME ME me me 2

ME ME ME ME 2

$M_{E} M_{E} M_{L}-1$ 


\section{Table 26-10 (Continued)}

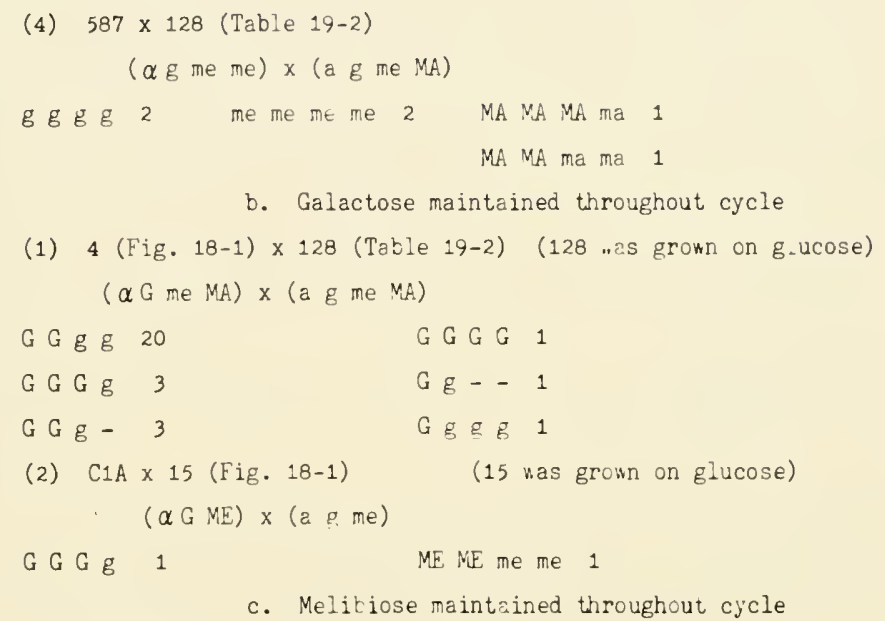
(L5B was grown
on glucose

(587 was grown on glucose

(1) L5B (Table 20-2) × 20 (Tatie 20-2)

$$
\begin{aligned}
& \text { (a } G \text { me MA) } \times(\alpha G M E) \\
& \text { ME ME me me } 4 \\
& \text { ME me me }-2 \\
& \text { d. Ma.tose maintained throughout cycle }
\end{aligned}
$$

(1) $587 \times 128($ Ta: le 19-2)

$$
\begin{array}{r}
(\alpha g \text { me ma) } x \text { (a g me MA) } \\
\text { MA MA MA MA } 1 \\
\text { MA MA ma ma } 3 \\
\text { ma ma ma }-1
\end{array}
$$

\section{GENETICAL ANALYSIS OF THE FOUR HAPLOPHASES FROM A TRANSFORMED TRIPLY HETEROZYGOUS ASCUS}

Table 26-5 shows an ascus (1208-1209-1210-1211) in which all four haplophases fermented galactose, melibiose and maltose although one parent was a nonfermenter of all three sugars. This ascus was produced by a mating between two haplophases from an ascus in which regular segregation for all three alleles occurred. It was subjected to genetical analysis and the following tabulation on page 26-21 shows the result of backcrossing the four clones from one ascus with both the nonfermenters and fermenters.

The backcross $\mathrm{G} \times \mathrm{g}$ produced a total of $64 \mathrm{G}$ 's and $19 \mathrm{~g}$ 's. Cultures 1208, 1209, 1210, and 1211 were not only fermenters of galactose but were able to transform the nonfermenters into fermenters, but the backcross to 1210 ( $\mathrm{G} \mathrm{x} \mathrm{g}$ ) behaved like simple 
Backcross to non fermenter $g$ me ma

$8 \mathrm{G}, 3 \mathrm{~g}$

1 G G G G

$10 \mathrm{ME}, 1$ me

1 ME ME ME ME

1 ME ME ME me

11 MA, 1 ma

2 MA MA MA MA
$12 \mathrm{G}, 0 \mathrm{~g}$

3 G G G G

$11 \mathrm{G}, 10 \mathrm{~g}$

4 G G g g

$12 \mathrm{ME}, \mathrm{O}$ me

3 ME ME ME ME

$11 \mathrm{ME}, 10 \mathrm{me}$

$1 \mathrm{ME}$ ME ME me

3 ME ME me me

$10 \mathrm{MA}, 2 \mathrm{ma}$

19 MA, $1 \mathrm{ma}$

1 MA MA MA MA

2 MA MA MA ma
3 MA MA MA MA

1 MA MA MA ma
$33 \mathrm{G}, 6 \mathrm{~g}$

6 G G G G

$2 G G G g$

$1 \mathrm{~g} g \mathrm{~g} g$

$21 \mathrm{ME}, 18 \mathrm{me}$

5 ME ME me me

$2 M E M E M E M E$

$1 \mathrm{ME}$ ME ME me

$25 \mathrm{MA}, 10 \mathrm{ma}$

4 MA MA MA MA

2 MA MA MA ma

1 MA MA. ma ma

1 ma ma ma ma

Backcrnss to fermenter G ME MA

$4 \mathrm{G}, \circ \mathrm{g}$

1 G G G G

$4 \mathrm{ME}$, 0 me

1 ME ME ME ME

$4 \mathrm{MA}, \mathrm{O} \mathrm{ma}$

1 MA MA MA MA
$12 \mathrm{G}, 0 \mathrm{~g}$

3 G G G G

$12 \mathrm{ME}$, $\mathrm{O}$ me

3 MF ME ME ME 4 ME ME ME ME

12 MA, 0 ma

$16 \mathrm{MA}, 0 \mathrm{ma}$

3 MA MA MA MA 4 MA MA MA MA
$20 \mathrm{G}, 0 \mathrm{~g}$

5 G G G G

$19 \mathrm{ME}, 1 \mathrm{me}$

4 ME ME ME ME

1 ME ME ME me

$20 \mathrm{MA}, \mathrm{O} \mathrm{ma}$

5 MA MA MA MA

heterozygous cross with regular segregations. The backcross to me produced a total of $54 \mathrm{ME}$ 's to $29 \mathrm{me}$ 's. The backcross to 1211 produced 5 of the expected ME ME me me asci; the mating with 1210 produced 3. Otherwise the asci all fell into unexpected categories. Both 1208 and 1209 transformed the nonfermenters, with which they were mated, to fermenters. The backcross to the triple recessive yielded 65 fermenters of maltose to 14 nonfermenters of maltose; only 1 ascus of the expected MA MA ma ma type was obtained. Transformation was the rule rather than the exception with the MA/ ma alleles.

Backcrossed to the galactose fermenter, only fermenters were obtained, indicating that the transformed cultures were complete dominants. The backcrosses to the dominant ME yielded 1 me to 
$50 \mathrm{ME}$ spores, indicating nearly complete transformation to the dominant form.

Two of the important matings in the complete pedigree (Table 26-11) are $608 \times 587$ and $571 \times 587$. In both these matings an a $\times$ a hybrid produces only aaaa asci, proving that recessive genes do not in themselves have the ability to transform nonfermenters into fermenters, but can only acquire fermentative capacity from a fermenter. This does not exclude the possibility that nonfermenter clones may carry a mechanism which endows the fermenter gene with transforming capacity.

\section{PROOF THAT THE MULTIPLE FACTOR HYPOTHESIS CANNOT EXPLAIN THE IRREGULAR RATIOS}

Table 26-12 shows data obtained in the course of tetrad analysis of heterozygous hybrids which have yielded irregular ratios. The asci were obtained from hybrids after mating two phenotypically different clones which yielded some 2:2 segregations and in addition other asci containing 4:0, 3:1, or other types of tetrads. Only asci in which all four spores are viable can be used in such an analysis. Table 26-13 shows the frequencies of phenotypes expected on tetrad analysis of hybrids when from one to four pairs of genes are involved and a single epistatic gene is sufficient to determine the phenotype. The data in Table 26-11 differ significantly from all these expected types; in fact, the Pppp and pppp tetrads do not fit into any scheme of conventional Mendelism and, therefore, make it impossible to apply any statistical tests.

These data prove that the multiple factor hypothesis does not provide an adequate explanation for the phenomenon. The large excess of PPpp asci strongly support the view that only a single pair of alleles are involved in determining the phenotype. Many matings of homozygous $\mathbf{P} \times \mathbf{P}$ and $\mathrm{p} \times \mathrm{p}$ hybrids support the view that these irregularities are confined almost exclusively to the heterozygotes. In the $\mathbf{P} \times \mathbf{P}$ hybrids there may be sufficient material present so that losses do not occur frequently, and in the $\mathrm{p} \mathrm{x} \mathrm{p}$ hybrids, the locus may be so degraded that it cannot be repaired in the absence of a P allele. The Pppp asci suggest that on occasion the $p$ locus may detach enough material from the $P$ locus to degrade it to the p phenotype, but that the amount removed either does not reach the p:locus or is insufficient to repair it.

\section{THE SIGNIFICANCE OF CONVERSION IN THE LONG-TERM ADAPTATION TO GALACTOSE BY SACCHAROMYCES}

It has long been known that Saccharomyces cerevisiae ferments galactose by an adaptive enzyme produced in the cell during the 
Table $26-11$

Genetical Analysis of Ascus 1208-1209-1210-1211

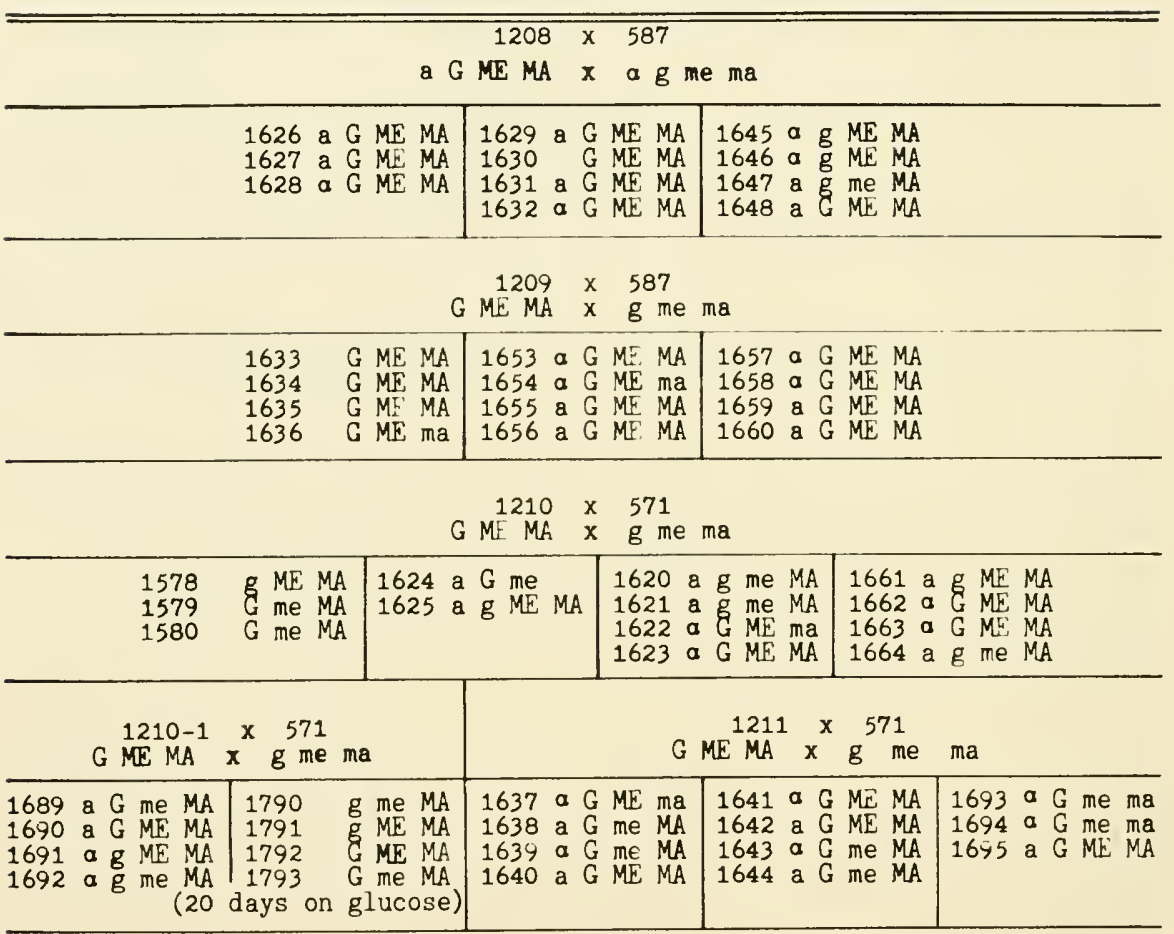

1211-1 X 571

$G$ ME MA $x$ g me ma

(20 days on glucose)

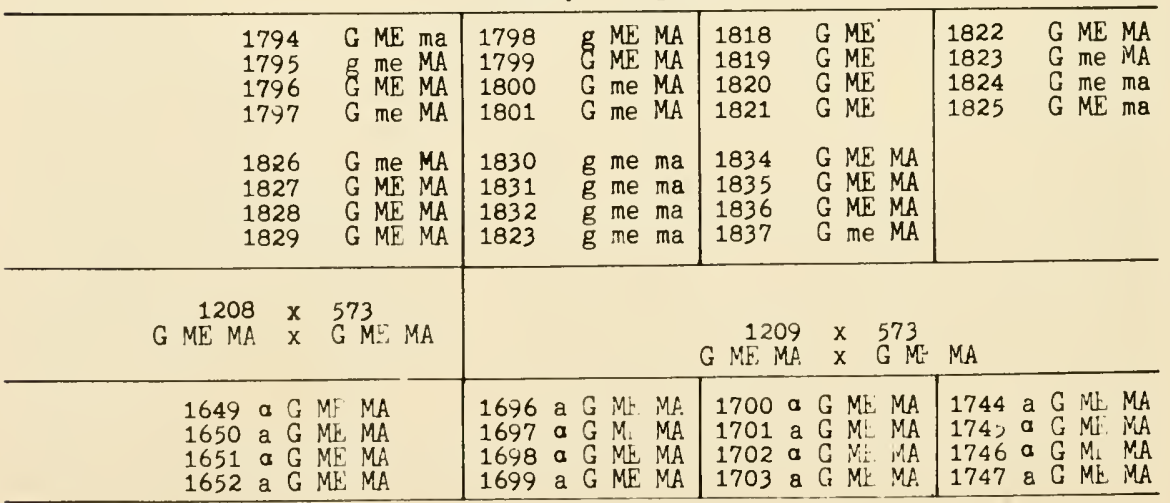


Table 26-11 (Continued)

$\begin{array}{rrr}1210 & x & 585 \\ \text { G ME MA } & x & G \\ \text { ME MA }\end{array}$

\begin{tabular}{|c|c|c|c|c|c|c|c|c|}
\hline \multirow[t]{2}{*}{$x^{2}$} & \multicolumn{2}{|c|}{ 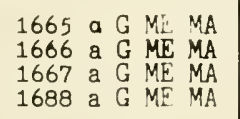 } & \multicolumn{2}{|c|}{ 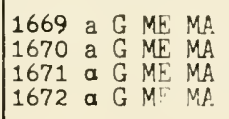 } & \multicolumn{4}{|c|}{ 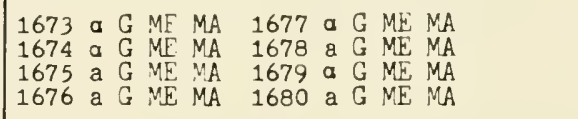 } \\
\hline & \multicolumn{4}{|c|}{ G $\begin{array}{r}1211 \\
M E\end{array}$} & \multicolumn{2}{|c|}{$\begin{array}{ll}x & 585 \\
x & G M E\end{array}$} & & \\
\hline $\begin{array}{ll}1681 & a \\
1682 & a \\
1683 & a \\
1684 & a\end{array}$ & 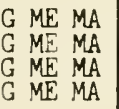 & $\begin{array}{l}1685 \\
1686 \text { a } \\
1687 \text { a } \\
1688 \text { a }\end{array}$ & $\begin{array}{l}G \\
G E \\
G E \\
G E \\
G E \\
G A \\
\text { GE } \\
M A\end{array}$ & $\begin{array}{l}1708 \text { a } \\
1709 \text { a } \\
1710 \text { a } \\
1711 \text { a }\end{array}$ & 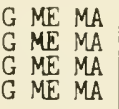 & $\begin{array}{l}1712 \text { a } \\
1713 \text { a } \\
1714 \text { a } \\
1715 \text { a }\end{array}$ & $\begin{array}{lll}G & M E & M A \\
G & m e & M A \\
G & M E & M A \\
G & M E & M A\end{array}$ & 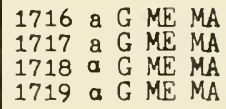 \\
\hline
\end{tabular}

$\begin{array}{cll}571 & x & 587 \\ \text { g me ma } & x & g \\ \text { me ma }\end{array}$

\begin{tabular}{|c|c|c|c|c|c|c|c|c|c|c|}
\hline & & & $\begin{array}{l}1704 \\
1705 \\
1706 \\
1707\end{array}$ & $\begin{array}{l}a \\
a \\
a \\
a\end{array}$ & $\begin{array}{l}g \text { me ma } \\
\text { g me ma } \\
\text { g me ma } \\
g \text { me ma }\end{array}$ & $\begin{array}{l}1736 \text { a } \\
1737 \text { a } \\
1738 \text { a } \\
1739 a\end{array}$ & $\begin{array}{l}\text { g me } \pi \\
\text { g me } n \\
\text { g me } \pi \\
g \text { me } \pi\end{array}$ & & & \\
\hline & & & & & $G M E^{607} \mathrm{ma}$ & $\begin{array}{rr}\mathrm{x} & 587 \\
\mathrm{x} & \mathrm{g}\end{array}$ & me ma & & & \\
\hline $\begin{array}{l}1223 \\
1224 \\
1225 \\
1226\end{array}$ & $\begin{array}{lll}\mathrm{G} & M E & \mathrm{ma} \\
\mathrm{G} & \mathrm{ME} & \mathrm{ma} \\
\mathrm{G} & \mathrm{ME} & \mathrm{ma} \\
\mathrm{G} & \mathrm{ME} & \mathrm{ma}\end{array}$ & $\begin{array}{l}1231 \\
1232 \\
1233 \\
1234\end{array}$ & $\begin{array}{l}G \quad M E m \\
G M E \\
G M E \\
G M \\
M E\end{array}$ & $\begin{array}{c}\text { ma } \\
\text { ma } \\
\text { ma } \\
\text { ma }\end{array}$ & $\begin{array}{l}1235 \\
1236 \\
1237 \\
1238\end{array}$ & $\begin{array}{l}G \mathrm{ME} \text { ma } \\
\mathrm{G} \text { ME ma } \\
\mathrm{G} \text { me ma } \\
\mathrm{G} \\
\mathrm{ME} \text { ma }\end{array}$ & $\begin{array}{l}1239 \\
1240 \\
1241\end{array}$ & 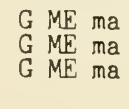 & $\begin{array}{l}1243 \\
1245 \\
1246 \\
1247\end{array}$ & $\begin{array}{l}\text { g } M E \text { ma } \\
G \text { me ma } \\
G \text { ME } \mathrm{ma} \\
\mathrm{G} M E \mathrm{ma}\end{array}$ \\
\hline
\end{tabular}

a g me MA $\begin{array}{rrr}608 & \times & 587 \\ \text { g me ma }\end{array}$

\begin{tabular}{|c|c|c|c|c|c|c|c|c|c|c|}
\hline $\begin{array}{l}1150 \\
1151 \\
1152\end{array}$ & $\begin{array}{l}\mathrm{g} \text { me MA } \\
\mathrm{g} \text { me MA } \\
\mathrm{g} \text { me ma }\end{array}$ & $\begin{array}{l}1153 \\
1154 \\
1155\end{array}$ & $\begin{array}{l}g \text { me } M A \\
g \text { me } M A \\
g \text { me } M A\end{array}$ & $\begin{array}{l}1157 \\
1158 \\
1159 \\
1160\end{array}$ & $\begin{array}{l}g \text { me } \\
g \text { me } \\
g \text { me }\end{array}$ & $\begin{array}{l}\text { MA } \\
\text { MA } \\
\text { MA }\end{array}$ & $\begin{array}{l}1161 \\
1162 \\
1163\end{array}$ & $\begin{array}{l}\mathrm{g} \text { me } \mathrm{MA} \\
\mathrm{g} \text { me } \mathrm{MA} \\
\mathrm{g} \text { me ma }\end{array}$ & $\begin{array}{l}1164 \\
1165 \\
1166 \\
1167\end{array}$ & $\begin{array}{l}g \text { me } M A \\
g \text { me } M A \\
g \text { me } M A \\
g \text { me } M A\end{array}$ \\
\hline & & & $\begin{array}{l}1168 \\
1169 \\
1170 \\
1171\end{array}$ & $\begin{array}{l}\text { me } \\
\text { me } \\
\text { me } \\
\text { me }\end{array}$ & $\begin{array}{l}1172 \\
1173 \\
1174 \\
1175\end{array}$ & $\begin{array}{l}\mathrm{g} \\
\mathrm{g} \\
\mathrm{g} \\
\mathrm{g}\end{array}$ & $\begin{array}{l}\text { me } \\
\text { me } \\
\text { me } \\
\text { me }\end{array}$ & & & \\
\hline
\end{tabular}


Table 26-12

Distribution of spores of contrasting phenotypes ( $P$ and p) among heterozygous asci produced by mating clones of the corresponding contrasting phenotypes.

\begin{tabular}{l|c|c|c|c}
\hline \hline & G/g & ME/me & AD/ad & $\mathrm{S} / \mathrm{s}$ \\
\hline PPPP & 9 & 4 & 1 & 8 \\
PPPp & 5 & 3 & 0 & 34 \\
PPpp & 28 & 33 & 57 & 12 \\
Pppp & 1 & 4 & 2 & 1 \\
Pppp & 1 & 0 & 0 & 0 \\
\hline
\end{tabular}

Table 26-13

Calculated frequencles of contrasting phenotypes expected on tetrad analysis of mono-, di-, tri-, and tetrahybrid heterozygotes when a single epistatic gene is sufficient to produce the phenotype.

\begin{tabular}{|c|c|c|c|c|}
\hline & $\begin{array}{l}\text { mono- } \\
\text { hybrid }\end{array}$ & $\begin{array}{l}\text { di- } \\
\text { hybrid }\end{array}$ & $\begin{array}{l}\text { tri- } \\
\text { hybrid }\end{array}$ & $\begin{array}{l}\text { tetra- } \\
\text { hybrid }\end{array}$ \\
\hline PPPP & 0.0 & 16.7 & 52.8 & 75.4 \\
\hline PPPP & 0.0 & 66.7 & 44.4 & 24.2 \\
\hline PPpp & 100.0 & 16.7 & 2.8 & 0.4 \\
\hline Pppp & 0.0 & 0.0 & 0.0 & 0.0 \\
\hline pppp & 0.0 & 0.0 & 0.0 & 0.0 \\
\hline
\end{tabular}


course of a few hours in the absence of cell division. Winge and Roberts (1948) have studied a culture of S. Chevalieri which ferments galactose by "long-term" adaptation which occurs after cells have grown from 3 to 12 days, in a medium containing galactose. After the adaptation the fermentation itself occurs at a rapid rate. They state that "long-term" adaptation of S. Chevalieri to galactose does not involve a shift in the population (mutation followed by selection) but is an adaptation of the entire original population. They consider the delay an indication of the time required by the cells to build up galactozymase to a sufficiently high concentration to make it demonstrable. They made hybrids between these two species and found a regular segregation of rapidly fermenting (1 to 2 days) to "long-term" adapting ( 3 to 12 days) spores from each ascus provided the ascospores were isolated in beer wort, a medium containing considerable amounts of maltose, but lacking galactose. If the ascospores were isolated in galactose broth, all four spores from each ascus fermented galactose immediately (1 - 2 days). However, they used only asci in which all 4 spores germinated and the possibility exists that some nonutilizers of galactose may have failed to grow. They described a series of experiments in which they deadapted and readapted galactose fermenting cultures and denied the significance of mutation as a factor in this process.

I have pointed out that our so-called "nonfermenter" (g) clones (Chapter 11) are capable of limited fermentation of galactose under conditions in which cell division does not occur, indicating that the recessive allele is capable of producing small amounts of enzyme. The fermentation by the $g$ cultures differs from the delayed fermentation by $\mathrm{S}$. Chevalieri since the $\mathrm{g}$ clones ferment at a very low rate after adaptation without cell division, while S. Chevalieri ferments as rapidly as normal $\mathrm{S}$. cerevisiae after a "long-term" adaptation involving growth. We have discovered occasional cultures in our pedigrees which resemble the cultures of S. Chevalieri described by Winge and Roberts, by fermenting galactose after a long exposure involving growth. Mr. Mundkur and I (Chapter 11) have shown that this involves mutation from the $\mathrm{g}$ to the $\mathrm{G}$ allele followed by selection of the mutant $G$ population in the presence of galactose. This process is made especially effective since the $\mathrm{g}$ cells are capable of using galactose slowly, thereby building up the population to a point at which a low mutation rate of $\mathrm{g}$ to $\mathrm{G}$ could produce a sufficient number of rapid fermenters to take over.

These facts have established the existence of three phenotypes (1) rapid fermenters (2) so-called "nonfermenters", and (3) "longterm" fermenters. These phenotypes will be designated $G, g$, and $\mathrm{g}_{\mathrm{S}^{*}}$. The designation of $\mathrm{g}_{\mathrm{S}}$ is used by Winge and Roberts for a gene which they consider an allele of G. We shall use the same designation, but we interpret this phenotype as a clone composed original- 
ly of g cells (previously called nonfermenters) capable of mutation to $G$ with sufficiently high frequency to insure long-term adaptation to galactose. The difference between $g_{S}$ and $g$ is therefore in the exceptionally high mutation rate of $g$ to $G$ in the $g_{S}$ clones.

\section{EVIDENCE FOR MUTATION OF g TO G}

The times required for long-term adaptation of $\mathrm{g}_{\mathrm{S}}$ clones from the same tetrad may vary from 4 to 25 days. These discrepancies are not consistent with the view that the lag represents the period during which the adaptive enzyme is accumulating in the entire population of cells, although discrepancies of this degree would be expected if mutation and selection occurred. In addition to the variations of time of gas formation for sibs, great variations occur in the time of fermentation when nearly uniform amounts of inocula are used and the same culture is used to inoculate a large number of different tubes. This further supports the view that selection of a mutation occurs in the positive tubes.

Cells of the $\mathrm{G}$ mutant derived from a $\mathrm{g}_{\mathrm{S}}$ culture evolved gas within 24 hours when inoculated into galactose broth. A mating was made using one of the $G$ mutants selected from a plate by a standard fast fermenter of galactose. Nine 4-spored asci of this hybrid were dissected and all the haploid progeny were fast fermenters of galactose. The mating was made by inducing mass copulations of haplophases heterozygous for mating type. The hybrid was heterozygous for mating type, the fermentation of melibiose, alpha methyl glucoside, maltose, and the synthesis of thiamin, inositol, and pyridoxine, and was homozygous for adenine dependence. The latter factor resulted in the production of only pink progeny. Proof that a hybrid had been obtained derived from the fact that regular segregations occurred in every ascus for the heterozygous alleles.

It could be argued, however, that the G mutant (selected from the plate) was a fast fermenter because it contained autonomous cytoplasmic enzymes (plasmagenes) and that a uniform distribution of these plasmagenes had occurred in the hybrid and among its progeny. To test this view the derived $G$ was mated to a standard g. Ten asci yielded two $G$ spores in each ascus. This hybrid was heterozygous for six genes; each ascus was characterized for four pairs of alleles: $\mathrm{a} / \mathrm{a}, \mathrm{G} / \mathrm{g}, \mathrm{AD} / \mathrm{ad}$, and $\mathrm{MG} / \mathrm{mg}$. AD indicates adenine independence and $M G$ indicates ability to ferment alpha methyl glucoside. The data appear in Table 26-14. The fact that every ascus except one yielded 2 pink and 2 white cultures proves that a hybrid had been produced. Each pink culture is adenine dependent; each white is adenine independent. That the exceptional ascus is also a hybrid is shown by the segregation of other characters. In this ascus conversion of both pink and $\mathrm{mg}$ genes has occurred. 


\section{Table 26-14}

Ten hybrid asci from a mating of a G mutant originating frow a $g_{s}$ culture by a stable $g$ parent. The hybrid was heterozygous for the four indicated alleles and all the asci are obviously true hybrids. The clones from ascus 452-455 (which produced 4 white cultures) were also sterile. In this ascus there was an extra fermenter of alpha methyl glucoside. The superscripts for the $g_{s}$ phenotype indicate the number of days intervening before fermentation occurred.

M-72 - aG (derived) MGPink (ad) x M-148 - a gmg White (AD)

420 a $g^{7}$ MG W

421 a $\mathrm{g}_{\mathrm{S}}^{14} \mathrm{mg} \quad \mathrm{P}$

$\begin{array}{lllll}422 & \alpha & G & \mathrm{mg} & P\end{array}$

$423 \propto \alpha \quad G \quad M G \quad W$

424 a G MG W

$\begin{array}{llllll}425 & \alpha & g_{S}^{8} & m g & P\end{array}$

426 a $G$ mg W

$\begin{array}{llllll}427 & \alpha & g_{S}^{7} & M G & P\end{array}$

428 a G mg W

$429 \propto$ g $\quad M G \quad P$

430 a G MG W

$431 \alpha$ g $\mathrm{mg} \quad \mathrm{P}$

432 a $\mathrm{g}_{\mathrm{S}}^{12} \mathrm{mg} W$

433 a g MG W

$434 \propto \mathrm{G} \quad \mathrm{mg} \quad \mathrm{P}$

$435 \propto G \quad M G \quad P$
436 a $\mathrm{g}_{\mathrm{S}}^{10} \mathrm{mg} P$

$437 \propto$ G $M G \quad W$

438 a g $M G \quad P$

$439 \propto \quad \mathrm{G} \quad \mathrm{mg} \quad W$

440 a g MG P

$441 \propto \alpha \mathrm{g}_{\mathrm{S}}^{23} \mathrm{MG} \quad \mathrm{P}$

$442 \propto$ G $\mathrm{mg} W$

443 a $G$ mg W

$444 \propto \alpha \quad G \quad M G \quad P$

445 a g mg W

446 a g mg W

$447 \propto$ a $\quad$ G MG $\quad P$

448 a $g_{S}^{25} \mathrm{mg} W$

449 a $G$ mg W

$450 \propto \mathrm{g}_{\mathrm{S}}^{13} \mathrm{MG} \quad \mathrm{P}$

$451 \propto$ a $\quad$ G $\quad P$ 
Table 26-14 (Continued)

\begin{tabular}{|c|c|c|c|}
\hline 452 & ? & $G$ & MG \\
\hline 453 & ? & $\mathrm{g}_{\mathrm{S}}^{12}$ & $m g$ \\
\hline 454 & $?$ & g & MG \\
\hline & ? & $\mathrm{G}$ & $M G$ \\
\hline & a & g & $\mathrm{mg}$ \\
\hline & a & $\mathrm{G}$ & $\mathrm{MG}$ \\
\hline & $\alpha$ & $\mathrm{g}^{12}$ & $M G$ \\
\hline & $\alpha$ & $G$ & \\
\hline
\end{tabular}

The appearance of $\mathrm{g}_{\mathrm{S}}$ cultures is interpreted to indicate that the $\mathrm{G}$ stock (derived from $\mathrm{g}_{\mathrm{S}}$ ) has the ability to convert $\mathrm{g}$ cultures to $g_{S}$; i.e., increases the rate of mutation of $g$ to $G$.

These analyses prove that slow fermenter populations comprise mixtures of original $g$ segregants and $G$ fermenter mutants arising from them. The acquisition of the ability for rapid fermentation by the population is not due to cytoplasmic modifications but to mutation and selection of the $\mathrm{G}$ mutant from the originally $\mathrm{g}$ stock. The fact that the G (original) x G (derived) mating produced no nonfermenters suggests that these genes are at the same locus and that the mutation was a back mutation of $\mathrm{g}$ to $\mathrm{G}$.

\section{HIGH $g$ TO G MUTATION RATE AMONG THE DESCENDENTS OF $\mathrm{g}_{\mathrm{S}}$ PARENTS}

The mating of the derived $\mathrm{G}$ to a stable $\mathrm{g}$ parent cited above indicates that capacity for mutability of $g$ to $G$ is readily transmitted through the derived parent. Since the distinction between $g$ and $\mathrm{g}_{\mathrm{S}}$ phenotypes merely depends upon the chance of mutation occurring in the latter and its failure to occur in the former, an extended analysis was made to determine if the $g$ and $g_{S}$ clones appearing in the same pedigree were distinguishable genotypically. When a mating is made of a $g_{S}$ by a $G$ clone copulations occur between $g$ and $G$ cells, for mutant G cells are only present in the mating tubes with a frequency of $0.5 \times 10^{-7}$. The $g_{S}$ phenotype is only apparent when a test of the segregant is made in galactose broth. 
The original $\mathrm{g}_{\mathrm{S}}$ stock used as a parent in the genetical analysis appeared as a mutant in a pedigree in which regular segregation for the G/g alleles had been clearly established and in which less than one percent of the individual cultures were $\mathrm{g}_{\mathrm{S}}$. Long-term adapting $\left(\mathrm{g}_{\mathrm{S}}\right)$ offspring of the original mutant (grown on glucose) were mated with standard $G$ cultures and produced extensive pedigrees in which the three phenotypes, $G, g$, and $g_{S}$, were distinguished. In heterozygous matings half of the progeny were $G$ while $g_{S}$ and $g$ clones were divided unequally among the remalning individuals; nearly 35 percent of the offspring were $g_{S}$ (Table 26-15).

\section{Table 26-15}

Distribution of 62 asci obtalned from $G$ by $g_{s}$ hybrids. Bach ascus was proved to be a hybrid by use of from 3 to 8 other genetical markers. The parents used for each ating are indicated by the serial numbers heading the columns. The number of asci of each type is indicated in each column and the total in the final column. Only asci producing four viable spores are included. It is significant that most of the non-fermenters arose from the ating of $6145 \times 3403$.

\begin{tabular}{|c|c|c|c|c|c|c|c|c|c|c|c|c|c|c|}
\hline \multicolumn{3}{|c|}{ Type of Ascus } & $\begin{array}{c}5919 \\
x \\
5920\end{array}$ & $\begin{array}{c}5921 \\
x \\
3691\end{array}$ & $\begin{array}{c}2236 \\
x \\
5920\end{array}$ & $\begin{array}{c}5964 \\
x \\
5947\end{array}$ & $\begin{array}{c}3652 \\
x \\
5980\end{array}$ & $\begin{array}{c}3653 \\
x \\
5969\end{array}$ & $\begin{array}{c}5919 \\
x \\
5920\end{array}$ & $\begin{array}{c}5981 \\
x \\
3691\end{array}$ & $\begin{array}{c}5982 \\
x \\
3588\end{array}$ & $\begin{array}{c}6145 \\
x \\
3403\end{array}$ & $\begin{array}{c}3691 \\
x \\
5920\end{array}$ & Total \\
\hline G & $\mathrm{G} \quad \mathrm{g}_{\mathrm{s}}$ & $\mathrm{g}_{\mathrm{S}}$ & 6 & 3 & 1 & 2 & 2 & 1 & 1 & 5 & 6 & 3 & 1 & 31 \\
\hline G & $\mathrm{G} \quad \mathrm{g}_{\mathrm{S}}$ & $g$ & 2 & - & 3 & - & 1 & - & 1 & - & - & 6 & 2 & 15 \\
\hline G & G $g$ & $\mathrm{~g}$ & - & - & 1 & - & 1 & - & - & - & - & 6 & 2 & 10 \\
\hline G & $g_{S} g_{S}$ & $\mathrm{~g}_{\mathrm{g}}$ & 1 & - & 1 & - & - & - & - & - & - & - & - & 2 \\
\hline G & $g_{S} g$ & $\mathrm{~g}$ & - & - & - & - & - & - & - & - & - & 3 & - & 3 \\
\hline G & $g_{S} g_{s}$ & $\mathrm{~g}$ & - & - & - & - & - & - & - & - & - & 1 & - & $\frac{1}{62}$ \\
\hline
\end{tabular}

However, when sibling $\mathrm{g}$ clones derived from this pedigree were mated to $\mathrm{G}$ at least 25 percent of the progeny were $\mathrm{g}_{\mathrm{S}}$ (Table 26-16), suggesting that the $g$ phenotype may be indistinguishable from $g_{S}$ in the same pedigree. Table 3 shows the types of tetrads derived from mating of $\mathrm{G}$ by $\mathrm{g}$ in which the $\mathrm{g}$ cultures were all descendents of $g_{S}$ parents. The considerable number of $g_{S}$ cultures appearing among the offspring indicates that both $G$ and $g$ individuals descended from $g_{S}$ parents transmit the capacity for a high mutation rate of $g$ to $G$. It should be emphasized that in the extensive analyses of $\mathrm{G} / \mathrm{g}$ hybrids previously made in this laboratory, only an extremely small fraction of the progeny were slow 


\section{Table 26-16}

Distribution of 56 asci obtained from $G x$ g hybrids. The $g$ cultures were all descended from $g_{s}$ parents and their $g_{s}$ offspring presumably arose from them. Each ascus was proved to be a hybrid by use of from 3 to 8 other genet ical markers. The parents used for each mating are ind icated by the serial numbers heading the columns. The number of asci of each type is indicated in each column and the total in the final column. Only asci producing four viable spores are included. It is significant that $4 \mathrm{GG}$ $G G$ and $I G G G g$ tetrads occur from the mating $609 x$ 6058. 609 is a $G$ clone from a pedigree in which transformation of $g$ to $G$ occurs frequently (Lindegren, 1949).

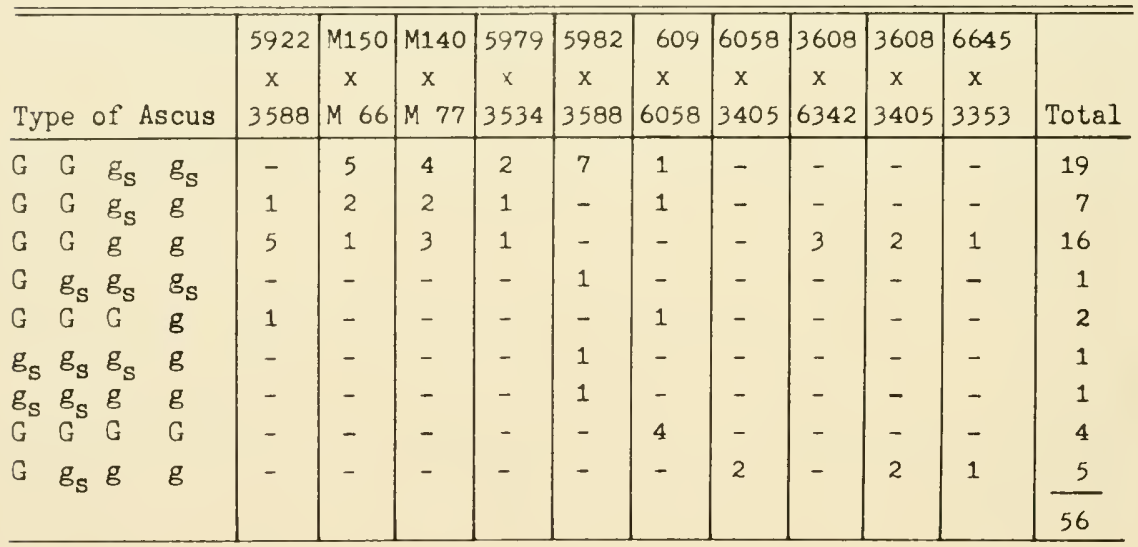

fermenters. Therefore, mutability from $\mathrm{g}$ to $\mathrm{G}$ is a heritable characteristic derived from the original $\mathrm{g}_{\mathrm{S}}$ culture used as a parent in this pedigree.

\section{NON-MENDELIAN SEGREGATIONS}

There were numerous indications of non-Mendelian segregations among these asci. If we characterize $\mathrm{g}$ and $\mathrm{g}_{\mathrm{S}}$ cultures as $\mathrm{g}$ (which they are at the time of segregation) the following tabulation summarizes the irregular segregation of the $\mathrm{G}$ and $\mathrm{g}$ alleles in Tables 26-15 and 26-16.

Table 26-15 Table 26-16

G $g g$
G g g g
G G G
G G $g$ g
$g$ g g g


Asci containing more than two $G$ spores may result either from mutation of $\mathrm{g}$ to $\mathrm{G}$ occurring early in the galactose broth, or directly in the zygote. (It should be recalled that adequate marker genes are present to insure that they are true hybrids.) These considerations show that while the concept of conversion in the heterozygote involves mutation effected by gene-to-gene transfer of gene material, the conversion which produces $\mathrm{G}$ mutants in the $\mathrm{g}_{\mathrm{S}}$ clones involves a long delayed mutation whose original cause may have been a similar transfer of genic material also occurring in the heterozygous condition.

\section{HYBRIDS OF $\mathrm{g} B Y \mathrm{~g}_{\mathrm{S}}$ CLONES}

Table 26-17 shows the results of mating stable $g$ by $g_{S}$ clones. Only five types of asci are possible and the fact that all five types appear indicate that segregations are not involved but that the chance of mutation occurring in the fermentation tube probably determines whether or not a culture is diagnosed as $g_{S^{*}}$. The possibility that the $g \mathrm{~g} g \mathrm{~g}$ and the $g_{S} g_{S} g_{S} g_{S}$ asci could have arisen by illegitimate matings was excluded by the extensive use of other marker genes.

$$
\text { Tabie } 26-17
$$

Frequencies of 5 tetrad types occurring among 22 proven hybrid asci, each obtained by matings of $g x g_{s}$ clones. only these five types are possible, and representatives of each appear.

\begin{tabular}{|c|c|c|c|c|c|c|c|}
\hline \multirow[t]{2}{*}{ Type of Tetrad } & \multicolumn{6}{|c|}{ Matings } & \multirow[t]{2}{*}{ Total } \\
\hline & $\begin{array}{r}\varepsilon \\
608\end{array}$ & $\begin{array}{r}g_{S} \\
X M 120\end{array}$ & $\begin{array}{c}g \\
6580\end{array}$ & $\begin{array}{c}g_{S} \\
\times 6236\end{array}$ & $\begin{array}{c}\mathrm{g} \\
3588\end{array}$ & $\begin{array}{cc} & g_{S} \\
\times \quad 5980\end{array}$ & \\
\hline$g \mathrm{~g} g \mathrm{~g}$ & & 4 & & 1 & & 0 & 5 \\
\hline$g_{S} g g g$ & & 1 & & 2 & & 1 & 4 \\
\hline $\mathrm{g}_{\mathrm{S}} \mathrm{g}_{\mathrm{S}} \mathrm{g} \mathrm{g}$ & & 4 & & 3 & & 1 & 8 \\
\hline$g_{S} g_{S} g_{S} g$ & & 2 & & 0 & & 2 & 4 \\
\hline$g_{S} g_{S} g_{S} g_{S}$ & & 0 & & 0 & & 1 & 1 \\
\hline & & & & & & & 22 \\
\hline
\end{tabular}




\section{EVIDENCE IN WELL-MARKED HYBRIDS THAT CONVERSIONS OF ALLELES OCCUR IN THE HETEROZYGOTE*}

Mundkur and Lindegren's (1949) demonstration that the phenomenon of long-term adaptation to galactose was explicable as the result of mutation of non-fermenter clones $(\mathrm{g})$ to the fermenter allele (G) has raised the question whether the non-Mendelian segregations of galactose and melibiose phenotypes encountered in the above pedigrees were indeed instances of conversion in the heterozygote during melosis, or merely mutations occurring after the spore had germinated. The extra-fermenters in non-Mendelian tetrads described in this chapter ferment as rapidly as the bonafide fermenter segregants and this indicates strongly that they are different from those which ferment "slowly". In the latter case the number of days elapsing before a "slow" clone ferments is very variable, suggesting chance mutation. However, an irregular tetrad is diagnosed as such only if more than the expected number of segregants ferment at the same time, usually within 18 hours; or if greater than the expected number of segregants do not ferment over an extended period of time.

Tetrad analysis involves the transfer of single-spore glucosegrown microcolonies to glucose agar slants. When the four haplophase clones have attained mature growth on the slants they are tested for their mating type specificity and their abilities to ferment various sugars and synthesize vitamins, amino acids and nucleic acid components. This initial growth on glucose may not involve selection of phenotypes, and mutants of recessives to dominant fermenter phenotypes should have equal opportunities for growth. When a hybrid ascus heterozygous for $\mathrm{G} / \mathrm{g}$ and $\mathrm{ME} / \mathrm{me}$ is dissected, one expects two ascospores to be nonfermenters. How ever, if mutation occurred on glucose after these two spores germinated, a segregant culture could comprise mixtures of original $\mathrm{g}$ and mutant $\mathrm{G}$ clones. If this mixed population were used as inoculum in testing for galactose (or melibiose) fermentation the mutant cells would be selected and the culture would ferment. A highly mutable (g to $\mathrm{G}$; me to $\mathrm{ME}$ ) haplophase segregant is thus liable to be diagnosed as an extra-fermenter although the ascospore giving rise to the mutant clones was genotypically $\mathrm{g}$ or me when isolated from the ascus.

Similarly, loss mutations ( $G$ to g; $\mathrm{ME}$ to me) are liable to yield fewer than the expected number (two) of fermenter phenotypes when mature glucose-grown clones are tested for fermentation.

*This section contains material extracted from

Mr. Balaji D. Mundkur's unpublished doctoral dissertation. 
Situations such as these could obscure Mendelian segregations and lead to the incorrect conclusion that gene transformation had occurred. Originally G G $g$ g spore tetrads (where either allele is highly mutable) might be finally diagnosed as G G G G, G G G g, $\mathrm{G} g \mathrm{~g} \mathrm{~g}$, or $\mathrm{g} \mathrm{g} \mathrm{g} \mathrm{g}$.

The decisive criterion for determining whether the non-Mendelian segregations involve gene conversions or mutations during growth on glucose would be, of course, the abilities of the four segregants to ferment at the earliest stage of growth, viz., the spore stage. In the absence of a test sufficiently sensitive to detect fermentation by a single spore, other means must be employed to investigate the problem.

Mundkur (1949) has investigated this problem. He reported experiments in which the fermentative abilities of tetrads derived from "converting" pedigrees were tested under conditions in which their multiplication was restricted and, therefore, before mutations could have obscured the results.

Previous pedigrees in which irregular segregations of galactose and melibiose markers were analyzed (Tables 26-1 and 26-2) were derived from crosses in which culture C1A was used as one of the parents. C1A is an a mating type capable of fermenting sucrose, galactose, melibiose, alpha methyl glucoside and maltose; it is an adenine-independent white culture and is thiamin-, inositol-, and pantothenate-independent, but is pyridoxine-dependent. An adenine-dependent pink haplophase culture was used as a mate for C1A and its pink descendants afforded an added advantage. Two crosses involving C1A and one of its direct descendants (609) were analyzed.

After all the four (or three) spores in a tetrad had germinated to produce microcolonies ranging in population from 100,000 to 500,000 cells per colony, each entire microcolony was transplanted and streaked over a small area on a glucose agar slant. This procedure insures a thorough mixing of mutant fermenter sectors that might have arisen during growth of the microcolony. A small number of cells was then immediately scraped off the slant surface and inoculated into molten $1.75 \%$ nutrient agar at $40^{\circ} \mathrm{C}$. containing $2 \%$ galactose as the carbon source. This molten agar was poured over a layer of solidified non-nutrient $3 \%$ agar in a sterile petri plate. A third layer of non-nutrient agar was poured over the galactose agar after the latter had solidified. This method prevents the fermenter colonies in close proximity from being confluent in growth and also minimizes contaminations. The same procedure was employed in making melibiose pour plates. Fig. 26-1 describes the method diagramatically.

The residual haplophase yeast on the slant was allowed to incubate 24 hours and was then streaked over the entire slant sur- 


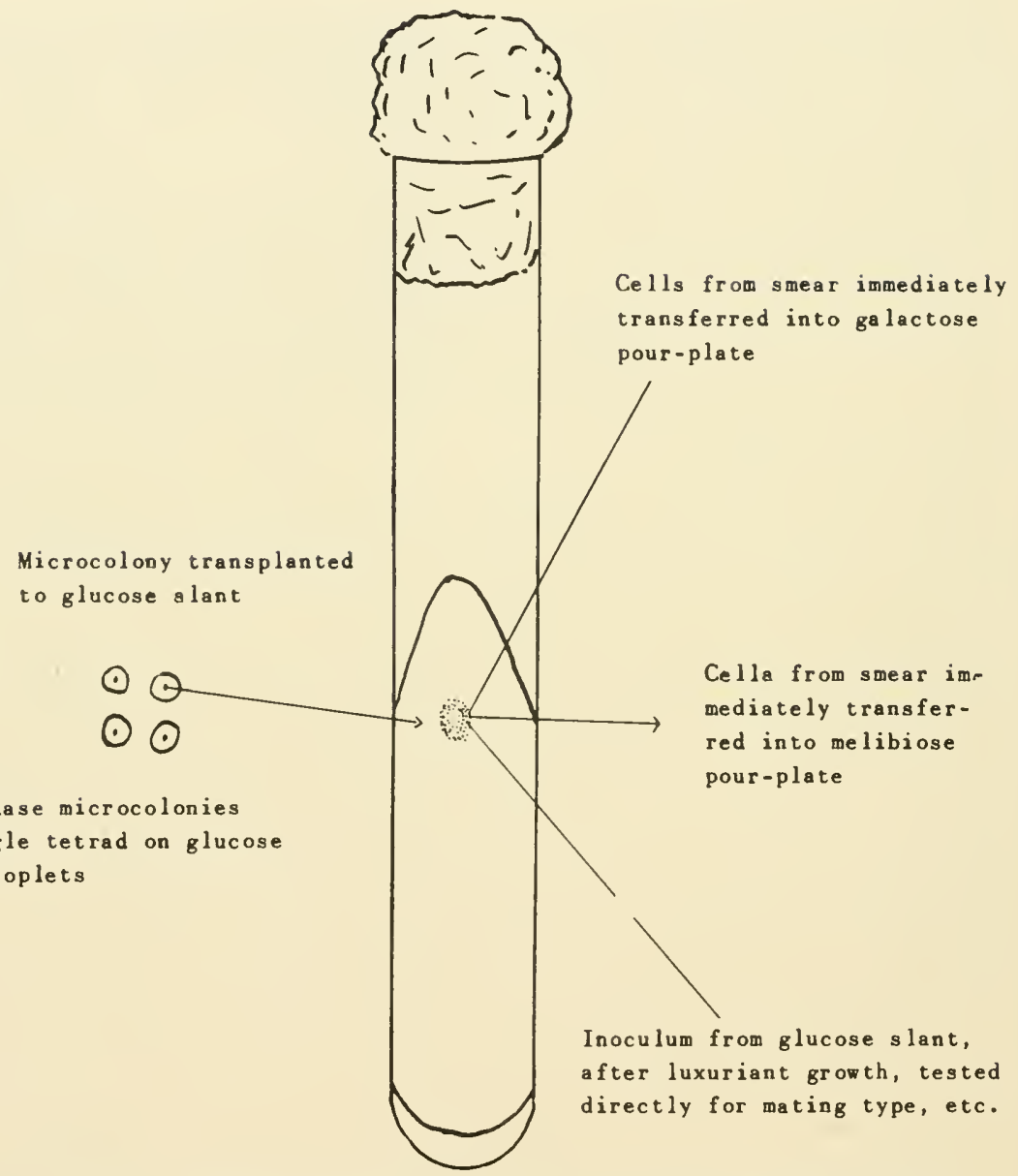

Haplophase microcolonies of single tetrad on glucose agar droplets

Fig. 26-1 Diagram Describing the Procedure Adopted to Minimize mutations in single spore cultures in testing fermentative characteristics of a haplophase segregant.

face to obtain luxuriant growth. Mature inoculum from the glucose slant was subsequently tested for mating type specificity, and fermentative and vitamin-synthesizing abilities. Simultaneous tests for galactose and melibiose fermentation by colonies developing on galactose and melibiose pour plates were conducted.

The widespread disturbances in segregations of fermenter phenotypes (shown in Tables 26-18 and 26-19) are noteworthy, since the stocks to which $\mathrm{C} \perp \mathrm{A}$ and 609 were mated had been carefully selected for regular segregations for the characters. In spite of the extensive non-Mendelian ratios, the truly hybrid nature of the asci analyzed is evident from the regular segregations of at least two marker genes in each ascus. Further support derives from the 
fact that cultures of $\mathrm{C} 1 \mathrm{~A}$ and 609 did not copulate illegitimately and produce ascospores. The possibility that a high rate of mutation of the nonfermenter recessive genes to dominance would explain the irregularities in segregation was excluded by the fact that the haploid clones to which $\mathrm{C} 1 \mathrm{~A}$ and 609 were mated had been derived from pedigrees in which the stability of the recessives was pronounced.

\section{SEGREGATIONS OF GALACTOSE AND MELIBIOSE FERMENTER PHENOTYPES}

It has been pointed out above that the ambiguity in deciding whether the extra-fermenters in G G G g and G G G G (or ME ME $M E$ me and $M E M E M E M E$ ) tetrads originate from gene transformations or from mutation can be resolved by minimizing the chances of mutations in a haplophase segregant. The galactose and melibiose pour plates made with inoculum obtained directly from the microcolonies provided data on this point. Since the mutation rates observed are usually of the order of $1 \times 10^{-7}$ and the number of cells in a microcolony at the time of pouring plates does not exceed 500,000 , it may be assumed that the probability of recovering fermenter mutants is rather remote at this stage of growth.

The colonies in pour plates of both galactose and melibiose agar are principally of three different sizes on the fourth or fifth day after plating. These have been arbitrarily designated as large-, medium- and minute-sized colonies. The large colonies range in diameter from approximately 0.2 to $0.5 \mathrm{~mm}$. in diameter. The minute colonies are visible as specks in the agar.

When a haploid clone is plated out, it may develop into uniformly sized colonies falling in any one of these categories, or a combination of two of these size classes, or it may show no growth at all. In Tables 26-18 and 26-19 when one class alone is indicated for a segregant, it is implied that the colonies in that particular pour-plate are uniformly sized.

The medium- and large-sized colonies are obviously fermenters and consistently corroborate the phenotype of the segregant obtained from the corresponding glucose slant. However, the minute colonies may or may not be fermenters. For instance, in cases where minute and large colonies occur in the same plate, single minute colonies inoculated with a micropipette into sugar broth achieve fermentation and confirm the characteristic of the clone from the corresponding slant. In other cases, however, minute colonies occurring uniformly in a plate were generally found found to be nonfermenters. 
Evidence that the differences in colony sizes result from differing growth rates was obtained from the following experiment: A suspension of a single minute fermenter colony (clone M 533) from a galactose plate was plated out in galactose agar and was found to yield only minute colonies. A galactose pour-plate of a suspension of a single large fermenter colony of the same clone produced both large and minute colonies. Therefore, the minute colonies are slower growing clones descended from the large colonied clones. This explains the occurrence of fermenter colonies of two size classes in the same plate. However, the fact that when only minute colonies occur on a plate, they are generally nonfermenters indicates that the genotype is intrinsic in the spore from which the clone descended.

The uniformity in size of pour-plate colonies in a large number of tetrads, supported by the fact that both large and small colonies were similar in fermentative ability is strong evidence that mutations with regard to fermentative ability occurred rarely, if at all, in the microcolony.

In determining the fermentative abilities of the minute colonies, large numbers of them were inoculated into the same broth tube to establish their phenotype with greater certainty. This is especially important when the corresponding glucose slant clone is a nonfermenter. The use of a large number of minute colonies insures that corroboration of the nonfermenter phenotype from glucose was not coincidental.

On the other hand, the extra-fermenters in a tetrad would have been diagnosed as such if only a single mutation ( $g$ to $G$; me to $M E$ ) occurred on a slant; or, if there were a single fermenter colony amidst the more numerous nonfermenter colonies and this exceptional one were inoculated into sugar broth together with its sister, nonfermenter colonies.

To test for variation among the individual colonies on a plate in the case of tetrads with extra-fermenters, the following experiment was performed: Two representative tetrads having extrafermenters were selected (M532 - M535; M544 - M547) and the clones known to be galactose and melibiose fermenters were plated out in glucose nutrient agar. Ten individual colonies picked at random from each plate were inoculated each into a separate galactose (or melibiose) Durham fermentation tube. All tubes contained gas within 24 hours.

This experiment indicates that individual colony variation with respect to galactose and melibiose fermentations did not occur in the pour-plates; it also confirms that after an originally recessive, nonfermenter clone has acquired the fermentative ability (and thus become the extra-fermenter in the tetrad) this new capacity is not affected by an intervening period of growth on glucose. 
Tetrads M488 - M491 and M500 - M503 (Table 26-18) exemplify a situation one would expect as a corollary to the cases of the extra-fermenter tetrads discussed above. The haplophase parent $\mathrm{C} 1 \mathrm{~A}$ in the crosses analyzed in Table 26-18 is a member of the "converting pedigree" bearing the dominant $\mathrm{G}$ and $\mathrm{ME}$ alleles. The occurrence of extra-fermenters in these crosses has been explained above as being due to a transfer of gene material from the $G$ to the $\mathrm{g}$ (or ME to the me) allele. However, 609 (a parent of M488 M503, Table 26-19) which is also a member of the "converting pedigreen, bore the recessive me allele and was mated to a ME clone (3190) derived from a regularly segregating ( $\mathrm{ME} / \mathrm{me}$ ) pedigree. The fact that tetrads M488 - M491 and M500 - M503 obtained from this cross include more than the 2 expected me clones indicates that the recessive allele in the "converting pedigree", i.e., in 609, can degrade the ME allele of its mate which descended from a pedigree in which the $\mathrm{ME} / \mathrm{me}$ alleles segregate regularly. At the same time an excess of $\mathrm{G}$ phenotypes are produced in the same asci.

The occurrence of 3 nonfermenter segregants in each tetrad is explained on the following basis: During meiosis the me gene from the "converting pedigree" acquires some essential gene component from the homologous dominant allele of its mate (3190). This acquisition is not sufficient to elevate the me allele to functional activity, but the degradation of the ME gene is sufficiently severe to negate its normal function. The single fermenter segregant in each tetrad was presumably not affected or, at least, was apparently not degraded below the critical threshold.

The data presented above eliminate the possibility that nonMendelian tetrads arise from mutations during the growth of originally Mendelian tetrads on glucose. They support the view that irregular segregations of the abilities to ferment galactose and melibiose may originate in the heterozygous condition owing to gene conversions.

The view that non-Mendelian ratios arise from gene conversions in the heterozygote rather than from chance mutations has been confirmed above only with respect to galactose and melibiose fermentations. No attempt was made to verify irregular ratios of other sugar markers (sucrose, alpha methyl glucoside and maltose) using pour-plates of tetrads as checks. The widespread irregular segregations of these markers suggests, however, that conversion of alleles was operative in these cases also.

In addition to irregularities in segregation of the sugar markers, the irregularities in inheritance of adenine-synthesizing ability are pronounced. Several tetrads comprised 4 white progeny out of a pink $x$ white cross but, in some, 2 clones are ad(W) and 2 are $A D(W)$. This fact further confirms the hybridity of the asci analyzed.

Disturbances in mating reactions are also noteworthy. In testing for mating type specificity, a heavy inoculum of each of the four 
Table 26-18

Tetrad Analysts of a "Converting" Pedigree. $C l A$ is the "Converter."

C1A a G ME MG MA AD(W) TH PN IN py x 3349 a $g$ me mg ma ad(P) TH pn in py

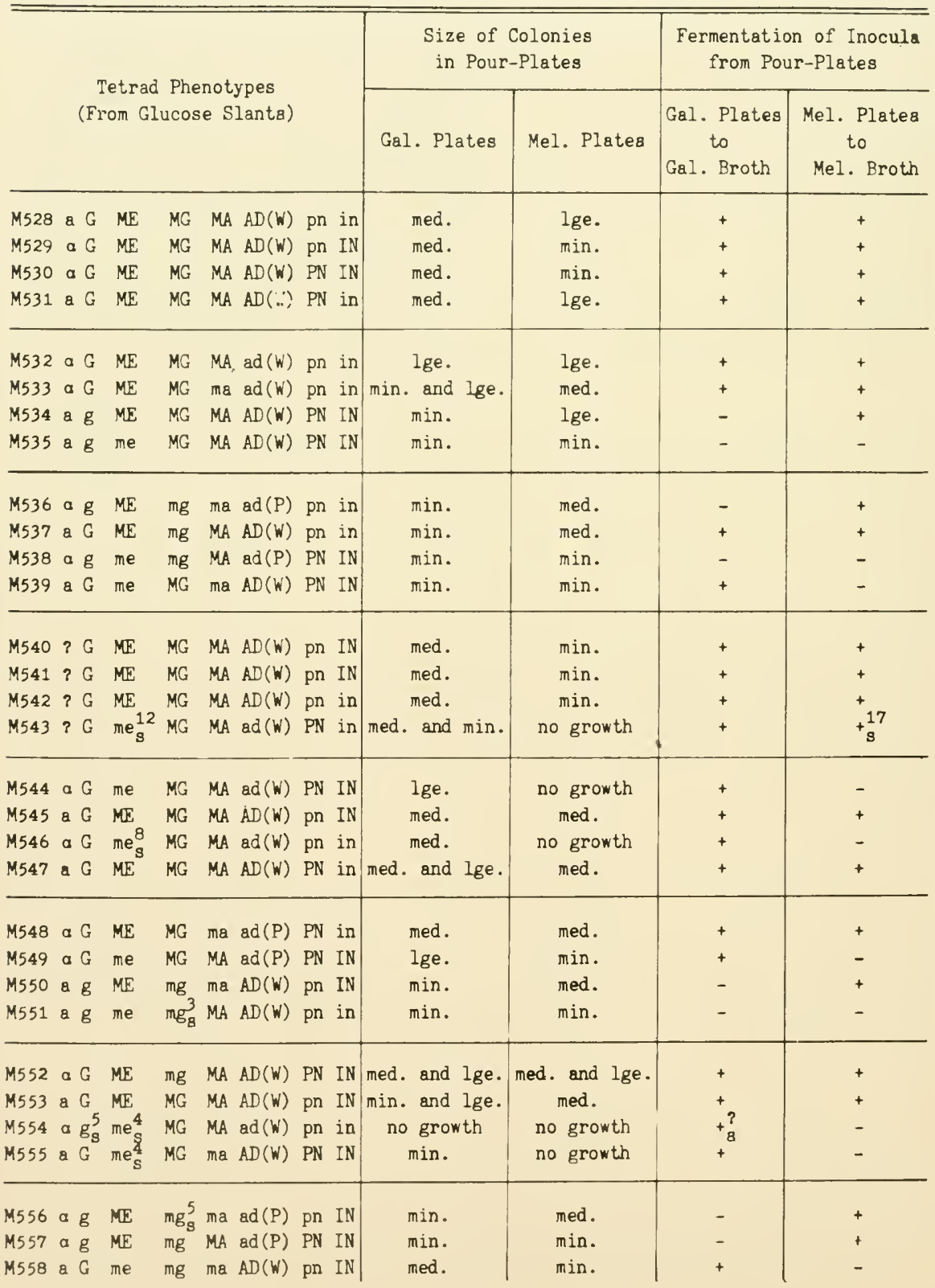


Table 26-18 (Continued)

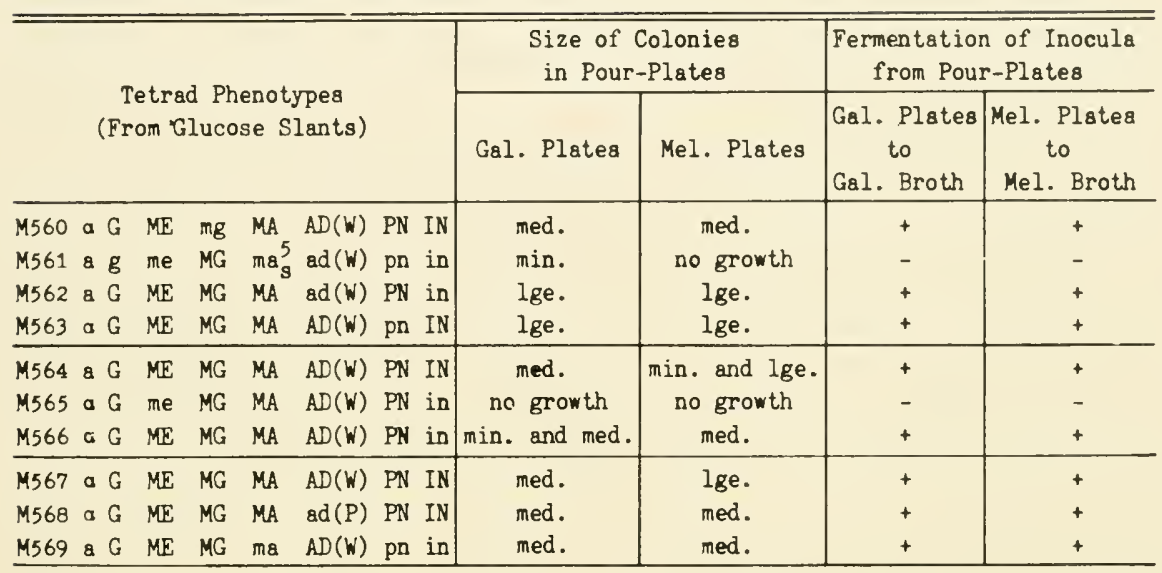


Table 26-19

Tetrad Analysis of a "Converting" Pedigree. 609 is the "Converter."

609 a G me MA AD(W) TH IN py pa $x 3190$ a $g$ ME ma ad(P) th in PY PA

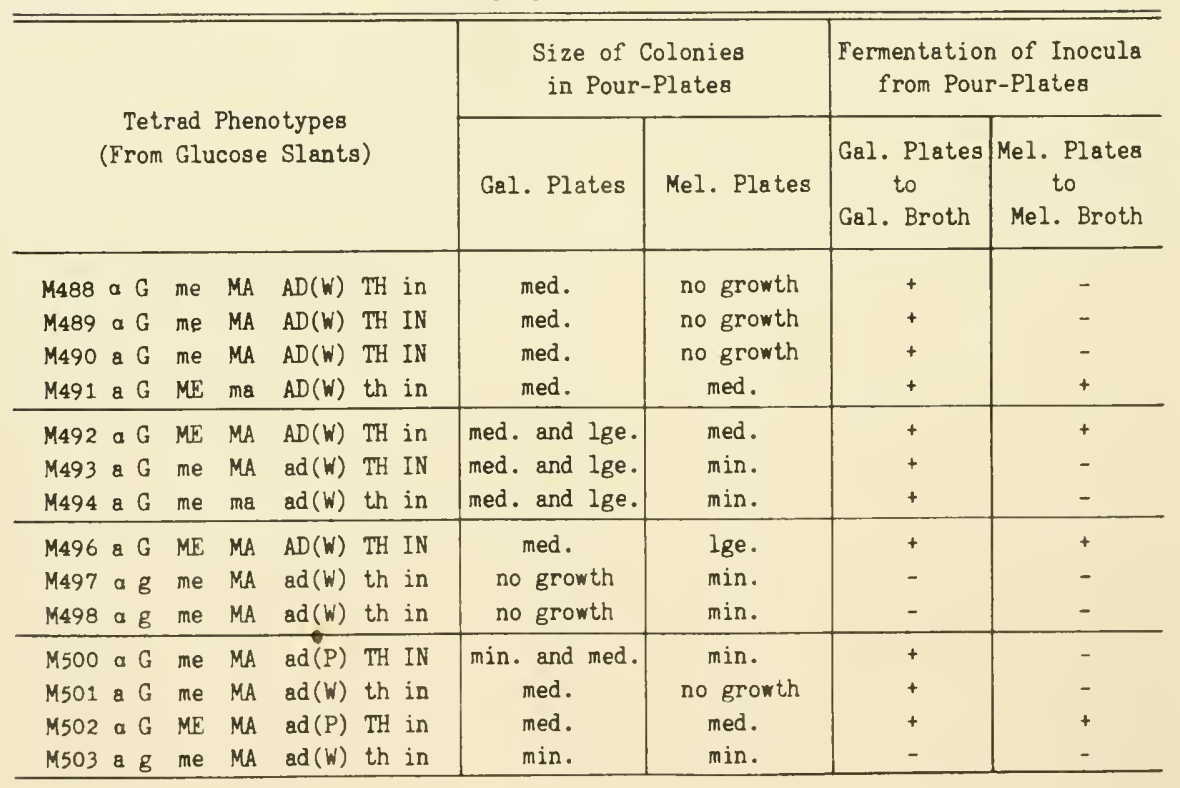


segregants from a tetrad is held in glucose broth together with a standard a clone known to copulate vigorously with other haploids. A positive.mating reaction identifies its mate as a mating type; in the absence of copulations, the mate is diagnosed as a clone of like mating type, i.e., as an a mating type. Copulations are generally effectéd within a few hours.

Mating type specificity segregated $2 \alpha / a \alpha$ in most asci. Occasional disturbances in mating reactions were, however, encountered. For instance, tetrad M528 - M531 (W W W W) mated to the standard $\alpha$ clone but no copulations were observed until the fifth day, while the normal tetrads M536 - M539, and M548 - M551 (2 P: 2 W) copulated vigorously within 12 hours. Other tetrads, none of whose haplophases copulated with the standard a clone even on the fifth day, were subsequently mated with a standard a clone and two of the haplophases copulated vigorously. Still others, M540 M543 (Table 26-18) mated with neither the a nor the standard a clones. Similar instances of W W W W tetrads failing altogether to copulate are cited (chapter 25).

The fact that mating strength is normal in $2 \mathrm{P}: 2 \mathrm{~W}$ asci, but is impaired or completely absent in some $\mathrm{W} \mathrm{W} \mathrm{W} \mathrm{W} \mathrm{asci} \mathrm{(obtained}$ from $\mathrm{P} \times \mathrm{W}$ crosses) suggests that the phenomenon is, in some way, associated with disturbances at the $\mathrm{AD} / \mathrm{ad}$ loci.

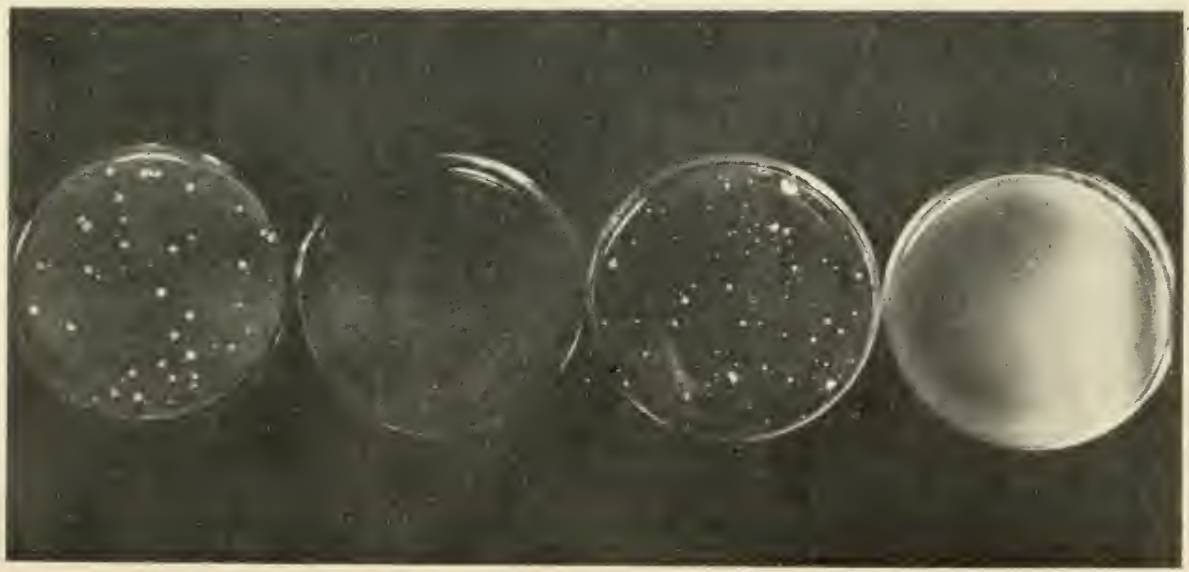

Fig. 26-2 Melibiose Pour-Plates of Tetrad M532. M535. Three plates (left to right) have colonies each of which is individually capable of fermenting melibiose. Inocula de rived from glucose slants of $M 535$ has minyte, nonfermenter colonies and the clone derived from the corresponding slant cannot ferment melibiose. The medium-sized colonies in M535 do not result from crowding. 
Chapter 27

\section{EXAMPLES OF APPARENT CYTOPLASMIC INHERITANCE}

The development of American genetics was most rapid during the first World War. The American School concentrated on the construction of chromosome maps and the precise repeatable type of experimentation which developed with mapping techniques. There was an effective separation from the German workers during the war and the latter, led by Correns, concentrated on the exceptions to Mendelism. This led to two different attitudes toward genetics with the emphasis on exceptions to Mendelism by German workers and a tendency to ignore such exceptions by Americans. Recently in America there has been a recognition of the phenomena described by the German workers and much discussion of cytoplasmic inheritance especially by Sonneborn, and it is noteworthy that his early training did not involve genetic indoctrination as a member or close associate of the Morgan School.

In yeast genetics we encountered many examples of non-Mendelian phenomena and in our early work I interpreted these as involving the hereditary transmission of autonomous cytoplasmic entities. This was a rather difficult thing for me to do because I had been thoroughly indoctrinated in Gene Theory by my long association with Dr. Morgan and the other members of his famous staff. It has been even more difficult to make the reorientation required to interpret these phenomena in terms of the obviously faulty Winkler Konversion hypothesis but tetrad analyses of heterozygous yeasts give this view a new validity, because in this case it is possible to differentiate Konversion from crossing-over. These facts and others have led to the conclusion that most "cytoplasmic" inheritance except that involving chloroplasts, rickettsias and other obvious symbionts or parasites can be explained without assuming the existence of autonomous entities in the cytoplasm. The theory will be presented in the Chapter 28.

\section{SELF-DUPLICATION}

Genes are often described as self-duplicating as if this characteristic distinguished them from other cellular structures. However self-duplication is a general characteristic of many cellular structures such as the plasma membrane, the chondriosomes, and the achromatic apparatus. The entire ceil is a self-duplicating structure, 
no part of which (not even the genes) can be formed except from preexisting precursors originating from the cell nutrients. The failure of any one of a number of cellular components to duplicate itself would result in the failure of the cell and the genes in it to multiply. Not too long ago geneticists found it difficult to understand how the non-genic parts of the chromosome which connected the chromomeres (supposedly genic macromolecules) could duplicate themselves because only the genes, and not the intergenic connections were supposed to have the capacity for self-duplication. Obviously many parts of the cell must have this ability. The difference between the gene and other self-duplicating parts of the cell is that the gene controls the path which self-duplication shall follow. It confers specificity to the enzymes which control the metabolism of the other self-duplicating entities which, like the gene, multiply by accretion of the substances which accumulate in the cell. The gene is also the basis for change, for by mutation it can alter the specificity of the enzyme it produces and thus the direction of its own self-duplication and, as a result, of the self-duplication of other cellular components. All self-duplicating structures in the cell are dependent on the gene, for all control of cellular metabolism resides in the gene. Although many structures besides genes are self-duplicating, none except genes have significance in the alteration of cellular activities or in heredity. All self-duplicating structures provide essential skeletons for their own continued production, but they depend on gene control for the forms which they assume; each permanent structure acts as the "starter" on which its "crystallization" occurs. On this basis, we may define self-duplication as a general biological phenomenon and reserve the adjective "autonomous" for those cellular components which, like the genes and chloroplasts, are capable of directing metabolism and originating changes in cell metabolism by mutation. A rickettsial parasite like the kappa substance would also be considered autonomous in this terminology.

\section{HETEROSIS AND "RUNNING OUT" OF HYBRID VIGOR}

The degeneration or "running out" of hybrids showing heterosis has been one of the principal problems of hybrid vigor (East, 1910). The fact that some degenerated potato hybrids were found to be virusinfected led to a general acceptance of the view that virus infection provided a satisfactory explanation because genetic theory had assumed that drastic alteration of a genotype does not occur during asexual propagation.

Haplophase yeast cultures (isolated in our laboratory in June, 1944) were mated to produce many hybrids. The matings are shown in fig.-10-2. During the ensuing summer individual tubes were selected from the collection stored in the cold room and tested for their 
ability to ferment sucrose on the Warburg apparatus. Only those matings which occurred between unrelated strains were tested; for example, hybrids between $L$ and HD, $L$ and $M, L$ and FLD, $L$ and $\mathrm{D}, \mathrm{L}$ and $\mathrm{B}$; and hybrids produced by mating $(800 \times \mathrm{L}) \times \mathrm{L}$ with $\mathrm{HD}$, $M, F L D, D$, and $B$, as well as some produced by matings of $M \times D$, and $(800 \times L) \times M$ were used in the study. None of the inbred cultures ( $\mathrm{L} \times \mathrm{L}, \mathrm{M} \times \mathrm{M}$, etc.) was tested. A standard baker's yeast which produced around $330 \mathrm{~mm}^{3}$ of $\mathrm{CO}_{2}$ per $\mathrm{mgm}$. of dry weight per hour and consumed about $80 \mathrm{~mm}^{3}$ of $\mathrm{O}_{2}$ per mgm. of dry weight of yeast per hour, was used as a control. The number of $\mathrm{mm}^{3}$ of $\mathrm{CO}_{2}$ produced in the presence of $0_{2}$ per mgm. of dry weight of yeast per hour is designated the $\mathrm{Q}_{\mathrm{CO}}^{\circ}$ (Warburg's notation); the amount of $\mathrm{O}_{2}$ consumed under the same condition is called $Q_{02}$. The ratio of $\mathrm{CO}_{2}$ produced to $\mathrm{O}_{2}$ consumed is called the respiratory quotient, or $\mathrm{R}$. $Q$.

The agar slants on which the hybrids were stored were plated out in petri dishes and single colonies selected and planted in separate tubes of broth. The obviously inferior cultures and the haploids were discarded and the apparently superior diploids were tested in the Warburg apparatus, suspended in 4 per cent sucrose and $\mathbf{M} / 15$ $\mathrm{KH}_{2} \mathrm{PO}_{4}$ solution. The tests made between August 21 and September 5 are shown in Table 27-1. Nine of the hybrids had R. Q's of 1 with $\mathrm{Q}_{\mathrm{C}}^{\mathrm{O}}{ }_{2}$ 's between 313 and 1730 . It is rather unusual to find yeasts which act on external substrate with an $R$. Q. of 1 . Twelve of the hybrids had $\mathrm{Q}_{0} \mathrm{C}_{2}$ 's from 1650 to 8500 . The standard control baker's yeast had a $\mathrm{QCO}_{2}$ of 320 . The metabolic activities of these freshlyformed hybrids were ennrmously higher than normal.

Table 27-1

Heterosis and "Running Out" of Yeast Hybrids

\begin{tabular}{|c|c|c|c|c|c|c|}
\hline Date & of & Expt. & Cultures & No. Tested & \multicolumn{2}{|l|}{${ }^{Q} \mathrm{CO}_{2}$} \\
\hline \multirow{2}{*}{8} & \multirow{2}{*}{$\begin{array}{l}21 \\
\text { to }\end{array}$} & \multirow{2}{*}{44} & $\begin{array}{l}\text { Standard } \\
\text { control }\end{array}$ & 1 & \multicolumn{2}{|l|}{320} \\
\hline & & & Hybrids & 9 & \multirow{2}{*}{$\begin{array}{l}313-1730 \text { (R.Q. } \\
1650-8500\end{array}$} & 1) \\
\hline & 5 & 44 & Hybrids & & & \\
\hline \multirow[t]{2}{*}{10} & \multirow{2}{*}{$\begin{array}{l}6 \\
\text { to }\end{array}$} & 44 & $\begin{array}{l}\text { Standard } \\
\text { control }\end{array}$ & 8 & $158-454$ & \\
\hline & & \multirow{3}{*}{44} & Hybrids & 59 & $218-744$ & \\
\hline \multirow{2}{*}{12} & \multirow{2}{*}{8} & & Hybrid & 1 & 64 (R.Q. & \\
\hline & & & Hybrid & 1 & 337 (R.Q. & \\
\hline
\end{tabular}


After an interval of one month the experiments were continued and more hybrids of the same group were tested. All showed less activity than those which had been tested previously, although the standard control baker's yeast tested had not changed significantly. In eight tests of the standard baker's yeast, the $\mathrm{Q}_{\mathrm{CO}}^{0}$ values ranged from 158 to 454; however, six of the determinations fell between 310 and 375 . Fifty-nine of the hybrids had $\mathrm{Q}_{\mathrm{CO}_{2}}^{0}$ 's of from 218 to 744. The mode was around 475. Two of the hybrids had R. Q.'s of 1 with $\mathrm{Q}_{\mathrm{C}{ }_{2}}^{\mathrm{m}}$ 's of 64 and 337 . It is clear, therefore, that cultures stored for more than four months after the matings were made lost a great deal of the heterosis that characterized the freshly-made hybrids. In one instance, 9 cultures were isolated from the same tube, a single isolate of which had previously shown a $\mathrm{Q}_{\mathrm{C}}^{0}{ }_{2}$ of 7900 . Eight cultures gave $\mathrm{Q}_{\mathrm{C} 0}^{0}$ 's of from 403 to 534 ; one gave a $\mathrm{Q}_{\mathrm{C} 0_{2}}^{0}$ of
64 with an R. Q. of 1 .

A clone originating from a single ascospore is a mixture of haploid cells. I have pointed out that when hybrids between genetically marked parents are made no new mutations are found in the hybrids that could be interpreted as the result of mutations in the haplophase. However, in terms of the fermentative ability of freshly made hybrids there were many differences between diploid hybrids from the same tube. This suggests that there was a rapid diminution in vigor occurring during the time of sampling. Sampling of these cultures was not at random for only superior ones were selected for testing.

Soon after hybridization the superior hybrids are about from ten to twenty times more active metabolically than after they stabilize at their standard level of activity. One culture tested on August 21 showed a $\mathrm{Q}_{\mathrm{C}}^{0}{ }_{2}$ of 7900 and on October the same culture showed a $\mathrm{Q}_{\mathrm{C}}^{0} \mathrm{C}_{2}$ value of 480 . Since these cultures were diploid and no sporuthis period.

Several subsequent attempts were made to reproduce the above experiments without success but the data are published because no flaw has been detectable in the procedures that were employed and the numerous controlled observations of high metabolic activity and subsequent degeneration of the hybrids appears to be completely authentic. Further experiments are being done from time to time in an attempt to duplicate the phenomenon.

\section{THE EFFECT OF NUCLEAR DIVISION WITHOUT CYTOPLASMIC DIVISION}

Winge and Laustsen (1940) described a variation involving change of phenotype without apparent change in genotype. It occurred in the course of inbreeding a culture during which changes in genetic com- 
position would not normally occur. They called it a cytoplasmic effect due to deficiency in an autonomous cytoplasmic apparatus. The yeast produced viable 4-spored asci whose single ascospore cultures were capable in turn of producing viable 4 -spored asci. This is an exception to the otherwise general rule that single ascospore cultures usually produce a high frequency of non-viable spores. Diploids arose from the ascospores in two different ways (fig. 27-1); (a) The spore germinated to produce a bud, and subsequently both the original spore and its daughter cell produced buds. Copulations occurred between the most distantly related cells in the cluster of four and a diploid budded out of each zygote. These diploids had developed from zygotes produced by the fusion of cells whose cytoplasms had undergone complete division before fusion occurred. The spores from the zygotes had a viability of about 70 per cent. (b) Occasional haploid spores
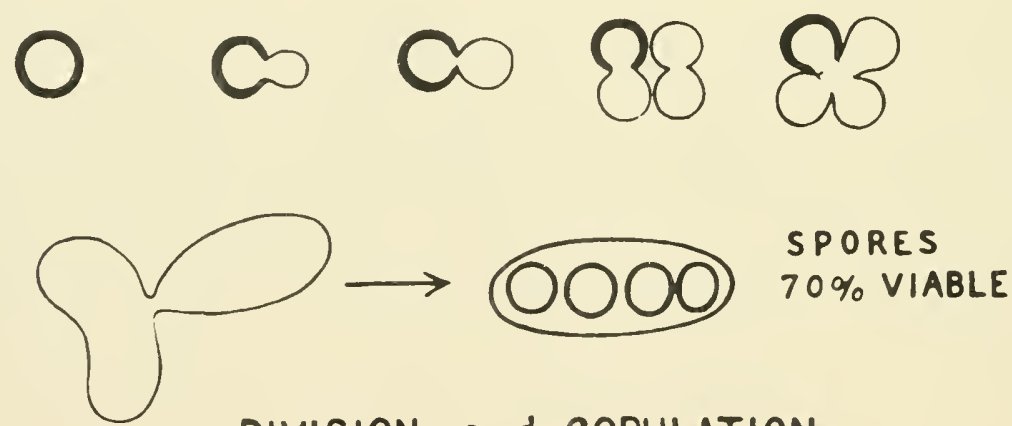

a. DIVISION and COPULATION

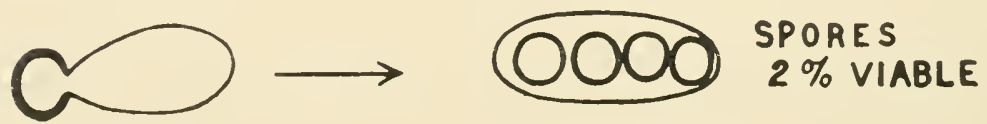

\section{b. DIRECT DIPLOIDIZATION}

Fig. 27-1 The difference between (a) spore viability produced by copulation of two fully grown haploid cells and (b) spore viability of a diploid produced by direct diploidization.

were observed to germinate directly to produce diploid zygotes. It was assumed that the nucleus in the original haploid spore had divided without a concurrent division of the cytoplasm and the two haploid nuclei had fused to produce a diploid nucleus. 
The diploid cells were viable and reproduced vegetatively without any obvious deficiencies. However, when they were induced to sporulate and the spores were isolated, only. about 2 per cent of them were viable. Winge and Laustsen concluded that the low viability of the ascospores from directly diploidized cells arose from the failure of the cytoplasm to divide before copulation. They suggested that the structures in the cytoplasm which fail to divide are chondriosomes or other structures independent of the genome (fig. 27-2).
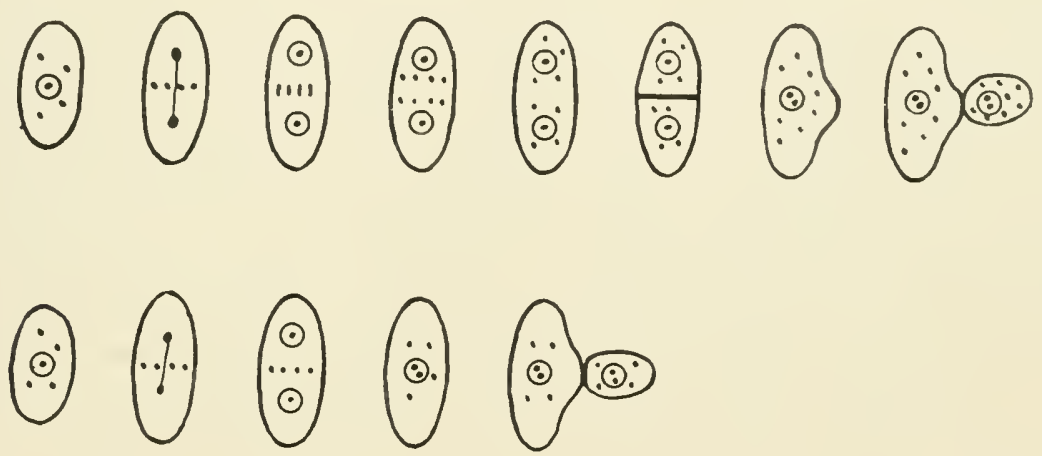

Fig. 27-2 Diagram copied from Wings and Laustsen explaining their hypothesis of degeneration through inbreeding.

\section{ADAPTATION WITHOUT DETECTABLE CHANGE OF GENOME}

Illegitimate diploids are genetically stable forms because they sporulate rarely and if transferred frequently in broth, will not sporulate at all. The failure to sporulate eliminates segregation as a cause of variation. The poorly sporulating illegitimate $R-$ strain diploid described in Chapter 6 can be adapted to corn steep water agar which contains an unknown substance that inhibits yeast growth (Lindegren, 1945).

Malt (M) agar is a relatively complete medium which supports an abundant growth of uniformly large colonies. It contains 10 per cent malt extract, 0.5 per cent dextrose, 0.5 per cent dried yeast, 1 per cent $\mathrm{CaCO}_{3}, 3$ per cent agar. Corn steep water agar (C) contains 0.8 per cent sucrose, 0.7 per cent corn steep water solids, 1 per cent $\mathrm{CaCO}_{3}, 3$ per cent agar. On the first transfer from $\mathrm{M}$ agar to $\mathrm{C}$ agar only a small percentage of cells survives producing 
both large and small colonies, and the variations in colony size on this agar cannot be due to genetic differences because they all a rise from a recent single colony. Fig. 27-3 shows the results of plating serially on $\mathbf{M}$ and $\mathbf{C}$ media.

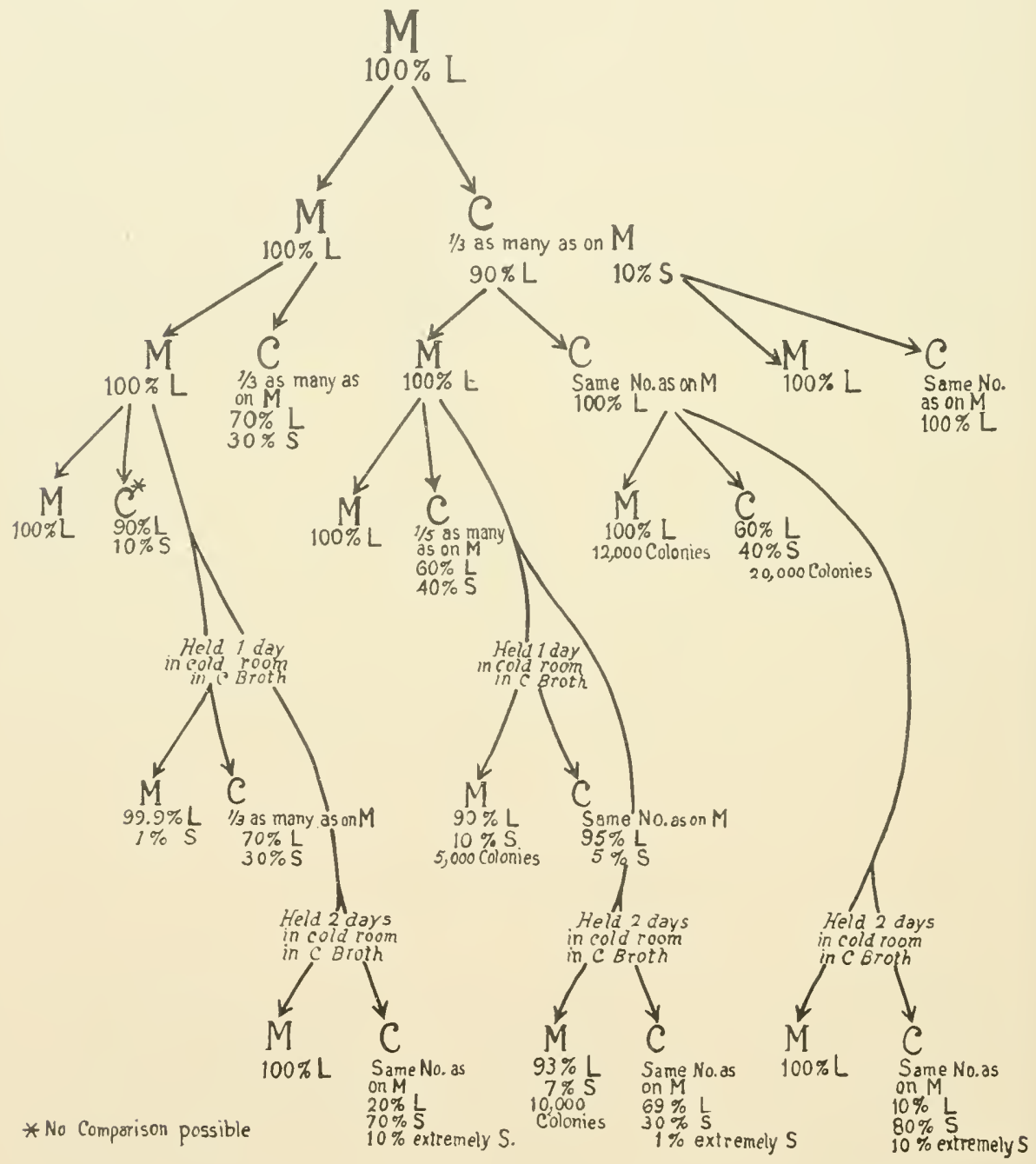

Fig. 27-3 Adaptation of the Illegitimate R-strain grown on malt extract Medium (M) to corn steep water medium (C) as indicated by the number of colonies surviving transfer and size of colonies. ( $L$ = large colonies; $s=$ small colonies

One of the large colonies from $M$ agar was suspended in water, and equal amounts of the suspension planted on $\mathbf{M}$ and $\mathbf{C}$ agar. All the colonies appearing on the $\mathbf{M}$ plate were uniformly large. Only 
about one-third as many colonies appeared on the $\mathrm{C}$ plate, and of these about 90 per cent were large and 10 per cent were small. In all transfers a large number of cells die; from 10 to 30 per cent of the cells transferred from $\mathbf{M}$ to $\mathbf{M}$ usually produce colonies, but the mortality is always greater on the first transfer from $\mathrm{M}$ to $\mathrm{C}$ than on transfers from $\mathbf{M}$ to $\mathbf{M}$. Since the genotype is stabilized, selection must be for some difference, independent of the genotype.

A suspension was made of a large colony growing on a C plate, and equal amounts of the suspension plated on $M$ and $C$ plates. The same number of colonies appeared on both plates and all were full size. This proved that the survivors on the $\mathrm{C}$ plate were adapted to growth on $\mathrm{C}$ agar. A suspension of a small colony gave the same result. The fact that there is no detectable difference between the large and small colonies on $\mathrm{C}$ plates indicates that the small colonies are merely slower in development. If a colony is delayed in development, the staling effect of the more rapidly developing colonies on the medium will prevent it from attaining full normal size.

Equal amounts of a suspension from one of the large colonies on an $\mathrm{M}$ plate (descended from a colony on a $\mathrm{C}$ plate) were spread on $\mathbf{M}$ and $\mathrm{C}$ plates. Only one-fifth as many colonies appeared on the $\mathbf{C}$ plates as on the $\mathbf{M}$ plate and both large and small colonies were found. Therefore, cells growing on a $\mathrm{C}$ plate (which have become adapted to $\mathrm{C}$ agar) lose this adaptation by a single transfer to $\mathbf{M}$ agar. This suggests that the variations have affected a large proportion of the population and have been readily reversed.

It was also possible to adapt the cells to $\mathrm{C}$ agar by holding them in $\mathrm{C}$ broth in the cold room for two days. A large colony from an $M$ plate was suspended in $C$ broth and held two days in a cold room. When samples from this culture were spread on $\mathbf{M}$ and $C$ plates the same number of colonies appeared on both media, although the samples of the untreated culture plated directly from $\mathbf{M}$ plates to both $M$ and $C$ plates showed only one-fifth as many colonies on the $C$ as on the $\mathrm{M}$ plate.

\section{HAPLOPHASE VARIABILITY VS. DIPLOPHASE STABILITY}

Nearly all haplophase yeast cultures, when plated on agar, produce a considerable variety of relatively stable colonial variants. Hybrids are produced by mixing the cells of two such apparently unstable cultures together in a small amount of broth. After copulations occur, diploid cells are produced which subsequently sporulate. Genetical analyses of the hybrid are made by picking and dissecting 4-spored asci at random after sporulation has occurred. One of the puzzling things about this process is the fact that in spite of the apparently considerable mutational potential of the haplophase cultures, the tetrad analyses usually yielded surprisingly regular 
results. This was supposed at first to be the result of statistical sampling; in the enormous population of cells, the chances that a given zygote had been derived from the two preponderant genotypes is presumably very great. However, the demonstration of depletion mutation indicates that many of the variants which appear on the plates are depletion mutations which cannot be transmitted through the zygote. Therefore, the reliability of the method of mass mating is greater than we had at first supposed. As a matter of fact, when the mating types were first discovered, we suspected that it would not be possible to use mass matings for genetical analyses, because the individual cultures appeared to be so "mutable". We thought that it might be preferable to use Winge and Laustsen's original method of spore-to-spore matings and rely on the characteristics of the diplophase for genetical analysis. However, numerous trials have confirmed the reliability of the mass mating method, although at the time, it seemed curious that such apparently unstable gametes could produce such surprisingly stable zygotes.

Each of these examples could be explained by assuming the existence of autonomous cytoplasmic entities. However, an alternative explanation will be presented after a discussion of the plasmagene hypothesis.

\section{THE PLASMAGENE HYPOTHESIS}

Plasmagenes are hypothetical hereditary units supposed to reside in the cytoplasm which perpetuate themselves independently of the genome in cells in which they find a "favorable" environment. They were invented to explain maternal or cytoplasmic inheritance and have been exploited by Sonneborn to explain the "killer" phenomenon in Paramecium and by Spiegelman to explain the mechanism of adaptive enzyme formation in yeasts. They are differentiated from viruses in being transmitted by grafts and by maternal inheritance rather than by infection. Darlington believes that viruses have been evolved from plasmagenes, and that plasmagenes themselves have originated from cytoplasmic proteins which have acquired the property of self-duplication.

\section{A. The Paracrinkle Virus}

Darlington chose as a prime example of a plasmagene the paracrinkle virus which is without symptom in the King Edward potato, but can be transmitted by graft to Arran Victory. The distinguishing characteristic par excellence of plant viruses is the fact that they are not transmitted through the egg. However, when Carson, Howard, Markham, and Smith made hybrids of King Edward ( $q$ ) by flourball $\left(\sigma^{\prime \prime}\right)$ they found the seedlings were not infected with the virus al- 
though the hybrid was capable of infecting scions grafted on to it. Thus, the paracrinkle virus is clearly not a plasmagene but a true virus (having a common characteristic of plant viruses, namely, the inability to pass through the egg), in spite of the fact that it cannot be transmitted except by graft. It is obviously not a plasmagene, since plasmagenes are a priori maternally inherited.

There are many plant viruses which have nearly reached a symbiotic relationship with their hosts or do them so little harm that they persist with little effect on survival of their hosts. Minchin first proposed the view that the chloroplasts are evolved from independent green algae which have become so completely adapted to the cytoplasm of green plants that they are incapable of independent existence, and have in turn made their hosts completely dependent upon them. Woods and duBuy have proposed a theory of the origin of plant viruses which I prefer over one which I had proposed earlier (1938). They have presented abundant evidence indicating that the viruses of green plants are derived from chloroplasts and their arguments based on the wide distribution of viruses and their spread through the plant by cell division rather than invasion support their thesis. The principal advantage of their view over Darlington's, in my opinion, is that it does not require the acquisition of "autonomy" by cytoplasmic proteins. This characteristic seems too complex to be quickly attained and that view is more acceptable to me which makes a virus the product of the longer evolutionary period. At any rate, the paracrinkle virus which Darlington chose as an example has clearly been identified as a true virus and his whole argument was based on the contrary opinion.

One of the phenomena which Darlington claims as support for the plasmagene hypothesis is the maternal inheritance of male sterility in plants, especially in Allium. A male sterile plant is incapable of producing pollen, and therefore, can only be used as a female parent. The male sterile plants are homozygous recessives, ms ms. When such a plant is fertilized by the pollen from a dominant homozygote, MS MS, a hybrid MS ms is produced. The hybrid is male fertile.

The original homozygous recessive was a single unusual plant. Most of the ms ms individuals found in Nature are male fertile. The original $\mathrm{ms} \mathrm{ms}$ male sterile plant was an exception discovered in the field. When reciprocal crosses were made between the heterozygous MS ms hybrid and a male-fertile ms ms plant, the results indicated in fig. 27-4 are obtained. The different results of these reciprocal matings have been considered evidence of the transmission through the cytoplasm of a factor controlling sterility counterbalanced by the ability of the MS gene to produce fertility under any circumstances. However, the fact that both male-fertile and malesterile ms ms plants are known can be interpreted as follows: the original male-sterile $\mathrm{ms} \mathrm{ms}$ plant was infected by a virus which 
Present evidence strongly supports the view that the Kappa substance is a virus which is widely established in the genus because it confers a selective advantage upon the infected individual by causing the death of others competing for the same food. Individuals carrying the $\mathrm{K}$ gene are susceptible to Kappa which the kk individuals are immune. The term "plasmagene" has implications of origin involved in Darlington's definition, and the evidence produced by Sonneborn's study of Kappa does not identify it as a plasmagene nor distinguish it from a virus. The Feulgen-positive structures in the cytoplasm of paramecia carrying Kappa which Dr. Preer exhibited at the A.A.A.S. demonstrations in Washington, 1948, resemble rickettsias closely. I consider this demonstration a confirmation of my earlier views that Kappa is a parasite which gives the infected organisms an advantage by killing their competitors rather than themselves.

\section{Antigenic Plasmagenes in Paramecium}

Sonneborn has described a phenomenon in Paramecium involving maternal inheritance of antigens which he considers similar to the maternal inheritance of Kappa. However, maternal inheritance of antigens in Paramecium appears to be a different phenomenon from the transmission of Kappa substance and more directly related to the mechanism of gene action than the Kappa phenomenon. A Paramecium with an antigenic constitution $A$ can be transformed into one with an antigenic constitution $B$ by treatment with anti $A$ serum. B can in turn be transformed into $C$ by treatment with anti $B$ serum and in this manner a variety of different Paramecia are produced. At least one of the antigens has been demonstrated to be gene-dependent. The variety of different types is produced (according to Sonneborn's hypothesis) by the reduction in the amounts of competing autonomous cytoplasmic plamagenes. He has not distinguished these plasmagenes from the Kappa substance. However, since the Kappa substance is the only one which can be demonstrated by the Feulgen technique it is obviously a unique form and the only thing in common between the antigens and the Kappa substance is the fact that their transmission is controlled to a large extent by maternal inheritance. The exconjugant which originally contained a specific antigenic characteris tic produces offspring with this same antigen.

\section{Adaptive Enzyme Formation}

Spiegelman (1946) uses a variation of the plasmagene hypothesis to explain the phenomenon of adaptative fermentation in yeast. Unfortunately, he uses the same word which Darlington coined and 
defined, and which Sonneborn later used with Darlington's meaning to explain the Kappa phenomenon. However, since neither Darlington's nor Sonneborn's arguments have justified any other terminology than "virus" for the entities with which they were concerned, Spiegelman's use of the word can be considered unique. Spiegelman redefined plasmagenes as autonomous cytoplasmic entities which arise from the nucleus as replicas of the gene and which are cast off into the cytoplasm from the gene itself. He suggests that these hypothetical units can become established in the cytoplasm, especially in the presence of substrate, and multiply in the cytoplasm independently of the gene from which they arose. Darlington's and Sonneborn's viruses (plasmagenes) may or may not have originated from nuclear genes in some earlier evolutionary period, but they do not arise from the nucleus at the present time. Spiegelman's plasmagenes are being continually produced by nuclear genes, although they readily attain autonomy in the presence of substrate and may, according to his hypothesis, persist in the cytoplasm even in the absence of substrate. His hypothesis has its origin in the fact that although a nuclear gene is required to enable a yeast to ferment a specific carbohydrate, once fermentation of the carbohydrate begins, it proceeds as if it were simply a reaction between the caribohydrate and the enzyme, occurring presumably in the cytoplasm. Spiegelman supposes that the adaptation curves indicate that the reaction (which is initiated by a nuclear gene) proceeds as an interaction between the substrate and gene replicaswhich the gene has sent into the cytoplasm. I have developed a different view based on the assumption that the synthesis of a gene-product which confers specificity to a specific fermentative enzyme occurs on the chromosome rather than in the cytoplasm. The gene-product eventually passes into the cytoplasm and makes the enzyme effective. This hypothesis aves not require that gene replicas diffuse into the cytoplasm where they exist as autonomous entities. On this view, the reaction which initiates the process of adaptation, as well as the continued synthesis of the key substance, occurs on the chromosome. The substance which is produced by the chromosome and confers specificity is, by hypothesis, incapable of autonomy.

Spiegelman's version of the plasmagene hypothesis takes its origin from the fact that adaptive enzyme formation follows a sigmoid curve characteristic of an autocatalytic reaction. He reasons that this supports the view that the enzyme $i$ tse $1 f$ must be autonomous. Monod argues against this interpretation. Monod says that the formation of an adaptive enzyme like galactozymase which is involved in the energy processes of the cell might appear to progress by an autocatalytic process simply because the energy which it releases from galactose is the limiting factor in the syn- 
thesis of more enzyme. As more galactose is broken down more energy becomes available for the production of more galactozymase and the synthesis of enzyme takes place at a constantly increasing rate.

Monod follows this destructive criticism of the plasmagene hypothesis with a constructive hypothesis of his own. He proposes a scheme by which an autocatalytic process of enzyme synthesis could occur without the existence in the cytoplasm of autonomous entities. This scheme makes it unnecessary that autonomous gene replicas be released into the cytoplasm. In Monod's view, the gene might simply produce a specific substance B (fig. 27-5) which could unite in the cytoplasm with inactive (presumably protein) units and these when stabilized by the specific substrate (e.g. galactose) could catalyze their own polymerization. This produces an autocatalytic process without the production of an autonomous substance by the gene. Monod's B substance corresponds to the gene-product ribosenucleic acid complex in my scheme of gene action shown in fig. 28-1. It is the small moleculed specificity-conferring substance to which I have previously referred as the substance by which the gene exerts its effects.

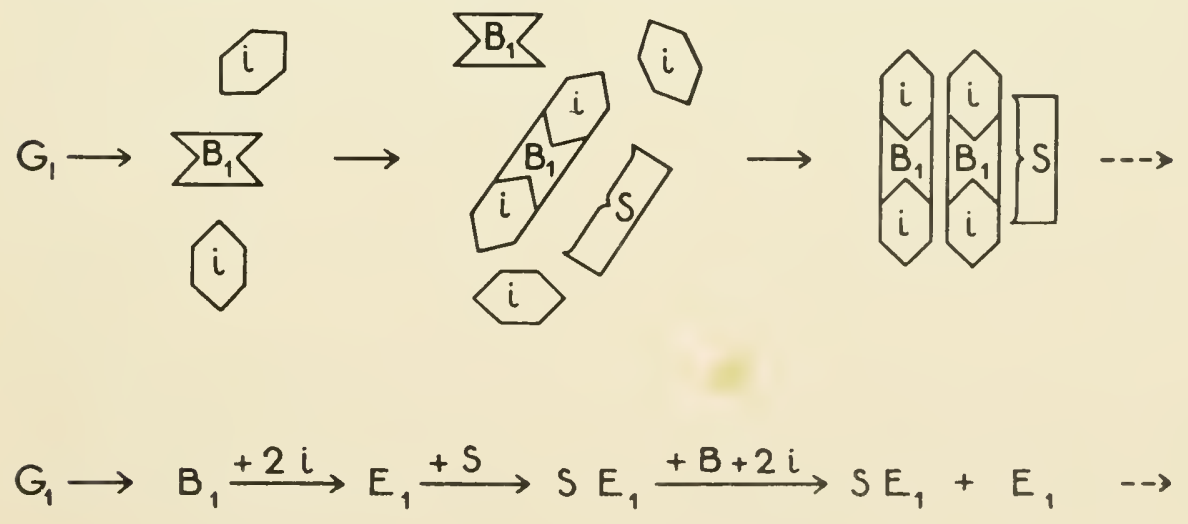

Fig. 27-5 Tentative Scheme of Gene Controlled, Autocatalytic Enzyme Synthesis. $G_{1}=$ gene; $B_{1}=$ "specific building blocks" manufactured by gene; $i=$ non-specific building blocks; $S=$ substrate; $i-B-i$ aggregates = active enzymes. (Copied from Monod.)

My objections to the plasmogene hypothesis have been strongly supported by Marshak's recent work. His demonstration that a nuclear nucleic acid is the precursor for ioth desoxyribose nucleic and ribosenucleic acids proves that both these substances originate from the nucleus and negates the plasmagene hypothesis. 


\section{GENE-DOSAGE AND GENE-PRODUCT}

Conclusive evidence that genes control the composition of the cytoplasm both qualitatively and quantitatively (and, therefore, do not produce autonomous plasmagenes) is derived from studies on the effect.of gene-dosage on phenotype. Mangelsdorf and Fraps (1931) showed that the concentration of carotinoids (and vitamin A) bore a linear relation to the dosage of genes controlling yellow color. Stern (1943) showed that one dose of the $c l$ gene produced a very abnormal wing, two doses a less abnormal wing, and three doses a nearly normal wing. A closer approach to normal was attained with each added gene. The same conclusion can be drawn from both observations: Since quantitative measurement of the phenotype bears a direct relation to gene-dosage, the gene produces a product which is incapable of autonomous multiplication in the cytoplasm. Each molecule of gene-specific substance in the cytoplasm arises from the production of an equivalent (specificity-conferring) molecule by the gene. Therefore, substrate induces the production of an enzyme by acting on the gene and not by a plasmagene-substrate interaction. The gene apparently controls both the quantitative and the qualitative composition of the cytoplasm subject (1) to modifications imposed by changes in gene-product to gene equilibria and (2) to the presence in the environment of substances to which the gene responds adaptively.

\section{DAUERMODIFIKATIONEN AND DEPLETION MUTATIONS}

\section{"Nongenic" Modifications}

Changes occur in cells which are relatively permanent on vegetative reproduction but are incapable of being transmitted through the sexual cycle. These changes correspond to what we have called depletion mutations and are apparently identical with the changes which Jollos called Dauermodifikationen and correspond to what Goldschmidt classified as the phenocopies. In other words, a great variety of different kinds of changes may occur in a cell which cannot be transmitted through the sexual cycle, but which resemble gene mutations phenotypically. Another common characteristic of the depletion mutation, the Dauermodifikation, and the phenocopy is the frequency with which they can be induced, in contrast to the relative rarity of the characteristic gene mutation. The disadvantage of studying this phenomenon in Drosophila is that the only means of propagation of a succeeding generation in Drosophila is by the sexual mechanism and therefore the stability of the variations in vegetative reproduction cannot be tested. As a matter of fact, the view has become prevalent that modifica- 
tions are extraordinarily unstable changes because they are never transmitted to the next sexual generation; they are considered to be simply temporary changes and therefore to be imposed upon the cytoplasm rather than controlled by genes. It is probable, however, from the great stability of similar variations in the vegetative phase in yeast cells that the so-called modifications in Drosophila may be highly stable, like the Dauermodifikationen of Jollos in Paramecium, and that they may be capable of persisting indefinitely throughout the vegetative phases of the cell-cycle, but disappear on sexual reproduction in the flies just as the Dauermodifi kationen of Paramecia disappear on sexual reproduction. Drosophila workers have formed the opinion (probably incorrectly) that modifications are impermanent because Drosophila only reproduce sexually and each new generation is obtained by a sexual fusion, a process which invariably causes the modification to disappear.

Jollos (1913) originally defined Dauermodifikationen as persistent transmissible changes in a cell which disappear slowly on vegetative reproduction and immediately on sexual reproduction. $\mathrm{He}$ insisted on recognition of the distinction between (1) persistent segregating gene mutations. (2) somatic modifications which disappear when the envi ronment changes, and (3) persistent modifications (Dauermodifikationen) which are induced by a change in the environment which are relatively stable on vegetative reproduction but which disappear immediately when outcrossed.

This definition of Dauermodifikationen coincides with what we have called a depletion mutation. Jollos' observations were so difficult to reconcile with classical gene theory that many American geneticists, including myself (1935), interpreted them as indicating that he had been dealing with standard gene mutations which were selected in an adverse environment and eliminated by reduction of the heterozygous diploid nucleus.

However, our observations of depletion mutations and the critical tetrad analysis of the heterozygotes support the validity of Jollos' original observations on Paramecium. Our observations support Jollos', but our interpretation is different. I have designated variations of this type "depletion mutations" to suggest that the effect is genic rather than cytoplasmic as proposed by Jollos.

\section{Cytogenes}

In our earlier papers on non-Mendelian and so-called cytoplasmic inheritance, I coined the word "cytogene" to designate a hypothetical autonomous cytoplasmic entity produced by the chromosome. Subsequent experiments failed to support the view that an autonomous cytoplasmic entity was present in the cell. I attempted to change the definition of the word to conform to these 
new concepts. However, since the word was originally defined to describe an autonomous entity, this was an improper thing to do. It seems best now to discard the word, and this move is even more strongly indicated since several who are conversant with the situation seem to have confused "cytogene" with "plasmagene."

Haplophase Variability and Diplophase Stability in terms of the Plasmagene Hypothesis

Since the depletion mutation cannot pass through the zygote, one might argue correctly, on a formal basis, that it involves a change in the cytoplasm. However, haplophase cultures produce an abundance of different kinds of stable variants, while diplophase cultures are almost completely free from variability. If the variability of the haplophase were due to competition between plasmagenes, a corresponding variability would be expected in the diplophase. The regular uniformity of the diplophase (which contrasts sharply with the "mutability" of the haplophase) supports the view that plasmagenes do not exist. There is no reason why plasmagenes could not be lost as readily in the diplophase as in the haplophase; therefore, the contrast in variability in the two conditions is a strong argument against the plasmagene hypothesis. The uniformity in the diplophase may be due to the insurance provided by the paired condition; if a gene suffers a temporary loss of a gene-component, it is immediately repaired by its allele. 


\section{Chapter 28}

\section{THE BEARING OF THE DATA FROM YEAST GENETICS}

\section{ON THE CURRENT CONCEPT OF THE GENE}

Tetrad anal"ses of hybrid yeasts nave yielded genetical data which are not easily reconciled with current concepts of the gene. These apparent contradictions of the generally accepted theoretical structure have been given critical consideration in attempts to explain them on a conventional basis. Many of the obvious possibilities have been explored and it appears that cytoplasmic inheritance, rapidly mutating genes, modifying genes closely linked to the dominant allele, somatic segregation in the diplophase, changes in ploidy, and several other mechanisms could conceivably be involved. However, each of these explanations becomes extraordinarily complicated or fails to deal adequately with some of the exceptional asci encountered in the pedigrees. The explanation offered below has been chosen because it fits all the cases without any fundamental difficulty except that it requires alteration of our apparently well-established views concerning the stability of the gene. The fact that it is currently preferred does not rule out recourse to a more conventional view at a future time if the data warrant it.

Two new facts have been established by our work in yeast genetics that are difficult to reconcile with current theory. The first is that depletion mutations which occur, apparently spontaneously, during vegetative reproduction and persist as stable variants on continued vegetative reproduction, revert to the original type following sexual reproduction. This phenomenon has been explained by assuming that changes in gene-product to gene equilibria (Chapter 15, and fig. 28-1) may produce stable vegetative variations. This hypothesis states that different stabilized equilibria can exist between gene and gene-product and that a different character may result from each equilibrium.

When an environmental effect changes the equilibrium and a new equilibrium is established a new character appears. On this hypothesis, the variation from pink to white occurs when so much gene-product $\mathrm{X}$ is lost that a crilical threshhold is passed and gene $\mathrm{X}$ can no longer maintain the original concentration of gene-product in the cell. That many characters other than color are affected is shown by the fact that two white variants cannot be inbred. They are sterile when inbred but fertile when outcrossed.

Winge and Laustsen's phenomenon of degeneration through direct diploidization is easily explained by this hypothesis: When the nucleus divides without a concurrent division of the cytoplasm the equilibrium of gene-product to gene is diminished by one-half since two genes are 
present in a gene-product concentration in which only one was previously present. This condition does not influence vegetative multiplication to a pronounced degree, but acts as a relatively effective spore lethal since spores produced by these diploids are poorly viable. Presumably a specific concentration of the gene-product is essential to spore viability. Once the concentration has been lowered it is not easily reestablished. However, this occasionally occurs producing viable spores.

The hypothesis also serves to explain Sonneborn's results in antigenic variation in paramecia. Each antigen is the expression of a specific (gene-product to gene) equilibrium. Removal of the antigen

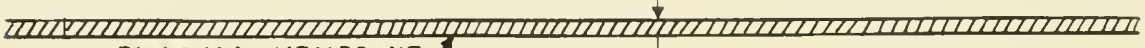
PLASMA MEMBRANE SUBSTRATE

CYTOPLASM

COENZYME

SPECIFIC ENZYME ACTING ON SUBSTRATE

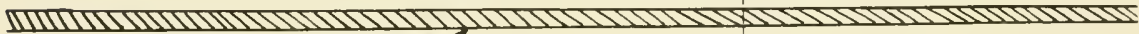
NUCLEAR MEMBRANE

\section{NUCLEOPLASM}

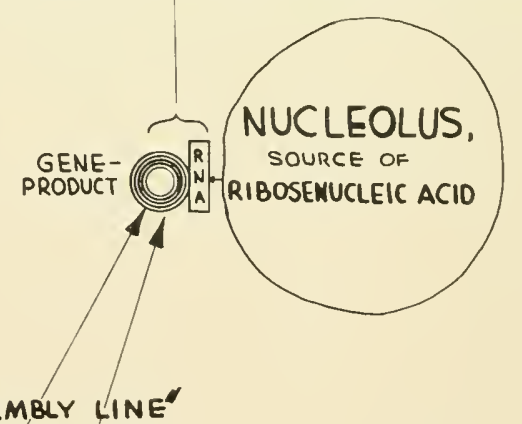

UNIOIRECTIONAL "ASSEMBLY LINE"

SYNTHESIS OF GENE-PRODUCT

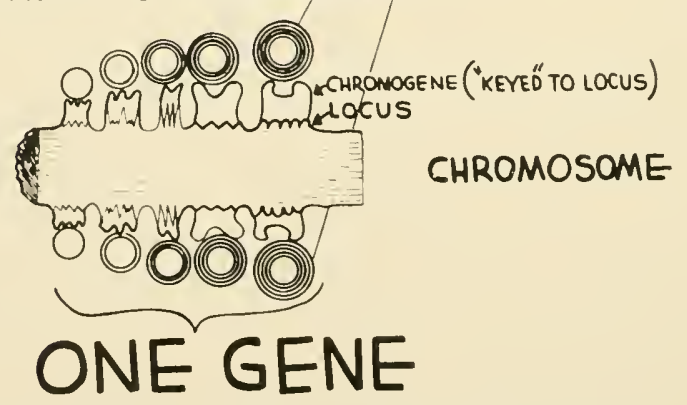

A REGION IN WHICH CROSSING-OVER RARELY OCCURS 
diminishes the concentration of gene-product (the antigen is not necessarily the gene-product) to a point where another equilibrium is established. The genotype always retains the synthetic capacity which makes it potentially capable of reestablishing the first equilibrium under "favorable" environmental conditions.

Adaptive mechanisms also are explicable on the hypothesis which suggests that the presence of a carbohydrate such as galactose may result in the production of galactozymase by increasing the concentration of gene-product to a point where a new equilibrium can be maintained and the presence of galactozymase becomes measurable.

On the same basis ontogeny may involve a series of adaptive reactions each of which results in a shift in equilibrium, conditioned by the changed environment which continued multiplication produces. In adaptive mechanisms substrate is required for the contined production of the enzyme; when the substrate disappears, less enzyme is produced. If the substrate be non-diffusible, a few more steps may be required, but the final result is the same. The fact that an enzyme is produced when the substrate on which it acts appears, explains

Fig. 28-1 A Diagram Illustrating the Proposed Hypothesis of the Nature of the Gene and the Mechanism of Gene Action. The gene is a region along the chromosome where a series of catalysts are arranged in order. Each catalyst is represented many times about the circumference of the chromosome. This view makes the partition of two genes to each cell a relatively certain event as long as a sufficiently large population of catalysts is present at each point on the chromosome. It also makes the delayed expression of phenotypes after irradiation explicable on the assumption that only a small arc of the circle of chromogenes has been affected and that several successive divisions may be required before the affected region is isolated in a single nucleus. The basic chromosome thread provides the locus on which the chromogene, constituting the catalyst, is held in place. Non-Mendelian heredity may result from transfer of chromogenes from active to deficient loci, converting recessive to dominant genes. Conversely dominant genes may be converted to recessive if the chromogenes in a heterozygote are partitioned equally to all loci with the result that an insufficient number is obtained at each locus. The gene product is the result of the orderly action of these enzymes on an initial substance which is probably produced in small amounts in all cells. When substrate appears, it removes enough gene-product to accelerate its production. The gene product is transferred (along with metaphosphate) to the nucleolus. Here it is united with ribosenucleic acid and passed out into the cytoplasm. The gene-product ribosenucleic acid complex corresponds to the B building blocks of Monod (fig. 27-5). In the cytoplasm proteins may unite with the complex to produce the differentiated apoenzyme. Union of apoenzyme, coenzyme and substrate result and the effect of the gene is produced. 
the precise timing of steps in ontogeny, for the cell would respond automatically through substrates which produced changes in geneproduct to gene equilibria, as they occurred. Orderly development would be based on a series of automatically induced environmental reactions.

\section{GENE-TO-GENE TRANSFER OF GENE COMPONENT}

A second fact which must be reconciled to classical genetics is that tetrad analyses of yeasts have revealed non-mendelian ratios which result from the transfer from one chromosome to another of components of the hereditary apparatus. It is suggested that the components are attached to loci along the chromosome by attractive forces. A locus with a maximal attraction for its specific complex is dominant and one with no attraction for it is, recessive. Intermediate reactions produce-irregular or non-mendelian segregations.

\section{THE TRIPARTITE NATURE OF THE GENE}

The new theory proposes that the gene consists of (1) the locus and (2) the chromogene which produces (3) the gene-product. The locus is the place of attachment for the chromogene. Chromogenes may be attached to the keyed skeleton of the chromosome and may be transferred from one locus to its allele on the homologous chromosome, depending on the strength of the attraction of the two allelic loci. Both the locus and the chromogene may be quite complex with many sub-components. The desoxyribosenucleoprotein in the chromosomes studied by Mirsky, the Stedmans, and Stern may comprise the fibrous keyed-skeleton. The concentrated attention on the desoxyribosenucleoprotein has proved very fruitful, but these fibrous structures may merely serve as places of attachment for the chromogenes.

\section{DEGRADATION OF THE GENE}

The theory proposes that the gene may be affected by at least three different degrees of degradation: (1) alteration of the geneproduct to gene equilibrium which can often be repaired in the haplophase, (2) injury or loss of the chromogene which may be repaired in the heterozygote, in the presence of the dominant allele, (3) destruction of the locus which is irreparable. The third type of injury produces "good" genes which segregate well and give regular ratios because activity of the gene ceases and repair is impossible.

\section{MENDELISM AS A SPECIAL CASE OF THE GENERAL THEORY}

Mendel's law is based on the analysis of regularly-segregating hypomorphic, recessive genes which are presumably not affected 
by transforming mechanisms of the type described above. Since transforming mechanisms insuring an excess of wild-type progeny should have considerable survival value, they may be widely distributed in Nature. If this be true, Mendel's law is a special case of a general theory governing the transmission of inherited characters. Many injuries could be immediately "repaired" following copulation with a sufficiently vigorous gamete, especially one which carried a large volume of physiologically normal cytoplasm. The current view that the large volume of cytoplasm in the egg is for nutrition as consumable nutrients may require qualification; the egg cytoplasm may also serve to carry a high concentration of a variety of gene-products which would insure maximal gene-product to gene equilibria. Some of the gene-products may be derived from the normal alleles which were discarded in the polar bodies, but whose gene-products are still represented in the cytoplasm.

\section{NUTRITION AND THE INTEGRITY OF THE GENOME}

The theory throws a new light on the perennial dispute of the respective effects of nutrition and heredity on the characteristics of an individual. Genes controlling hydrolysis or fermentation of sugars may be degraded by poor nutrition to the point where they are unable to produce their respective enzymes. This effect can be produced merely by dissimilating the cells in a substrate deficient in essential nutrients such as phosphate buffer, or culturing them on a synthetic medium capable of promoting poor, rather than vigorous, growth. The degraded cells temporarily lose their fermentative or hydrolytic ability in regard to galactose, melibiose or maltose but regain it after growth in a nutrient capable of promoting vigorous growth even though the specific substrate on which the enzymes act may be absent from the medium. The enzymes acting on these sugars are under gene control indicating that the degrading effect of the poor nutrition acts directly on the gene. This view is confirmed by genetical tetrad analyses which prove that direct interchange of gene material occurred between loci in the heterozygous condition. These conclusions help to reconcile the differences of opinion between practical plant and animal breeders and theoretical geneticists. Mendelian ratios are achieved by theoretical geneticists because they have selected organisms with completely degraded genes which are relatively unaffected by environment and have assumed that environment has a limited ability to alter the gene. Practical breeders, on the contrary, use organisms in which many genes are sensittive to both environment and nutrition, and many of them believe that an organism is fully competent of transmitting its entire complement of hereditary material only if it is nurtured with the best possible nutrients. By the same token, an organism which is genetically weak, but not 
deficient, may transmit competent genes if well nourished but incompetent genes if poorly nourished.

Any environmental change alters the metabolic activity of the cell. If the environmental change is within certain limits the cell adapts to it and the alteration of metabolic activity is corrected by an adaptive counteraction. Similarly if a nutrilite is presented which the cell is unprepared to utilize, the cell adapts its metabolism to the utilization of this material. The sensitivity of the gene to degrading influences suggests that it is easily affected by the environment and that the site at which both degrading and adapting substances or influences finally produce their effects is the locus of the gene. It seems probable that the effect of a gene (in producing a specific enzyme, for example) is the result of a long series of successive reactions, rather than a single step reaction. We conceive of the effect of the environment as also involving a series of steps before the gene is ultimately affected. Therefore, a two-way path to and from the gene is imagined in which effects can be produced by alterations occurring at any one of a number of points.

The so-called environmental modifications (which are supposedly temporary since they are not transmitted to sexual progeny) may be the result of changes made at the locus itself; we have shown that similar changes can be corrected in the sexual cycle. Permanent modifications, which could be corrected by sexual reproduction, would appear to be temporary if each new generation could only be produced by sexual reproduction, as is the case in maize and Drosophila.

\section{LINEAR DIFFERENTIATION ALONG THE CHROMOSOME}

The discussion thus far has considered the chromosome as a structure differentiated in cross-section with the central strand comprising (1) the locus (2) the chromogene which may be transferred from one locus to another and which produces (3) the specific geneproduct. This structure comprises a single gene. I shall now propose that a second degree of complext ty also exists and that single genes, which are identified by their ability to produce a single geneproduct, are multipartite along the long axis of the chromosome as well as in cross-section. What appears to be a Mendelian gene may be a point on the chromosome at which a single step of the synthesis of a gene-product (whose synthesis may require many steps) has been deleted. The synthesis could proceed by a series of reactions controlled by a series of catalysts placed in orderly sequence along the chromosome, which pass the partially completed gene-product from one catalyst to another in assembly-line fashion, until it is completed and leaves the chromosome. A Mendelian gene may correspond to a point along this series at which a specific catalyst has been lost or reduced in quantity. The chromosome is imagined to consist 
of blocks of regions, each of which is devoted to the synthesis of a specific gene-product, some of which may even overlap. If crossovers which tended to break up these specialized regions occurred only rarely there would be little interference with gene action by crossing-over. The deficiency revealed as a Mendelian gene would behave as a single entity on segregation, although it comprised only a single step in a series of reactions.

\section{THE LINEAR CHROMOSOME AS THE SEAT OF ORDERLY REACTIONS}

Some mechanism for carrying out orderly reactions is essential in a cell whose cytoplasm is almost completely fluid. If the chromosome be the seat of all orderly reactions, the necessity for imposing orderliness upon a fluid cytoplasm disappears, and the linear structure of the chromosome takes on deeper significance. According to this view, syntheses requiring a series of steps in which one reaction follows another occur' on the chromosome. When a substance such as galactose appears in the cell, one of these "chain" reactions results in the final production of galactozymase, if the catalysts essential for its completion are all at their proper loci. When the synthesis is in full swing, galactozymase deals with galactose in the cytoplasm, without any necessity for orderly arrangement of the components of the reaction. We may look in the future for short sequences of "genes" along the chromosome which have significance in terms of the reactions that are involved. At the present time, these sequences are not obvious, both because the total number of "genes" is much greater than the standard methods are capable of discovering, and because our knowledge of the chemistry of the synthetic reactions occurring in cells is altogether too inadequate. Present methods probably give lower apparent numbers of "genes" because most segregating irregularities are repaired in the heterozygous condition. Actually, the concept of the "number" of genes may be relatively meaningless and probably only a small fraction of the actual "heterozygosis" is experimentally realized, and the "number" of "genes" may be many hundreds or thousands of times greater than the largest current estimates, for only a few molecules might be sufficient to constitute a given "gene" and blocks of the chromosome may comprise a continuum, with adjacent "genes" occasionally overlapping.

The classical view of the gene was based on (1) the demonstration by genetical experiments that certain characters were inherited as units, and (2) the demonstration of individual chromeres in the chromosomes by cytological methods. The final supposedly "crucial" experiment was the demonstration that the bands in the sailvary chromosomes could be specifically associated with unit characters. However, the view that this constituted a "crucial" experiment is due to 
circuitous reasoning. The one-gene, one-enzyme hypothesis was the outcome of this theoretical background. It seems simpler and more reasonable to consider the filamentous chromosome as a structure on which catalysts are placed in an orderly sequence so that reactions can occur with one step following the other in its proper order. The fact that the nucleus is set off from the rest of the cell in a non-reacting hyaloplasm, with the nucleolus also in this isolated region, makes it possible for the specificities of the enzymes to be determined before they are released into the mixed cytoplasm. The apparent random positions of "genes" along the chromosome is probably due to insufficient data, and further study should reveal sequences along the chromosome, such that one synthetic step follows another in regular order.

According to this view, the chromosomes, like so many other biological structures, do not serve a single purpose, but insure both the precise partitioning of the hereditary apparatus at each cell di vision and the orderly syntheses of cellular enzymes.

\section{LINEAR DIFFERENTIATION AND POSITION EFFECT}

Goldschmidt pointed out that the failure of the classical theory of the gene to account for position effect revealed a fundamental inadequacy of the concept. He argued that the chromosome is not divided into corpusular genes, but is functional along its entire length and that the chromosome operates as a unit and not as a separate series of discrete, independent genes. His proposal that the chromosome rather than independent genes is the functional unit forms the basis for a theory which fits the facts with regard to position effect. The fact that it is possible to demonstrate numerous highly specific loci on the chromosome arranged in linear order is the basis for most of the opposition to Goldschmidt's concept. If the region devoted to the production of a single gene-product were an area through which crossovers seldom occurred and on which a series of transferrable components was located, losses of regions which might produce position effects would occur whenever a slightly unequal exchange took place. The deficiency which might result from exchange could be corrected by an exchange of chromosomecomponents in the heterozygote without involving crossing-over. However, in unequal exchanges involving an inversion between nonhomologous chromosomes, repair would not be so readily achieved. This is the condition most frequently associated with position effect and suggests that the transfer of chromogenes occurs at synapsis. 


\section{BIBLIOGRAPHY}

Allen, C. E., Transactions of the Wisconsin Academy of Sciences, Arts and Letters, 29, 195-202 (1935)

Alexander, J. and Bridges, C. B., Colloidal Chemistry, Theoretical and Applied. The Chemical Catalog Co., (1928)

Andes, J. O., Bull. Torrey Bot. Club., 68, 609-614 (1941)

Auerbach, C., Proc. Roy, Soc. Edinburgh., 62, 211-222 (1945)

Avery, O. T., McLeod, C. M. and McCarty, M. H., Jour. Exp. Med.

79, 137-140 (1944)

Babes, V., Z. Hygiene., 5, 173-190 (1889)

Badian, J., Ark. Mikro., 4, 409-418 (1933)

Badian, J., Bull. Acad. Pol. Sci. Lett. Ser. B. Science N'at., 5, 61-87 (1937)

Baltatu, E., Cent. Bakt. II., 101, $196-225$ (1939)

De Bary, A., Comparative Morphology and Biology of the Fungi,

Mycetozoa and Bacteria, $525 \mathrm{pp}$. The Clarendon Press, Oxford (1887)

Bauch, R., Arch Prot., 70, (1930); 75 (1931)

Bauer, H. and Caspersson, T., Proceedings 8th Int. Cong. Genetics., (1948)

Beadle, G. W., Science in Progress, Yale Univ. Press., 166-196 (1947)

Beadle, G. W., and Coonradt, V. L., Genetics., 29, 291-308 (1944)

Beadle, G. W., and Emerson, S. H., Genetics., 20, 192-206 (1935)

Beadle, G. W. and Tatum, E. L., Proc. Nat. Acad. Sci., 27, 499-506 (1941)

Beijerinck, M. W., Zentr. Bakt. Parasitenk, Infek. I., 16, 49-58 (1894)

Beijerinck, M. W., Zentr. Bakt. Parasitenk, Infek. II., 4, 657-663; $721-730$ (1898)

Belar, K., Die cytologischen Grundlagen der Vererbung. Gebruder Borntraeger, Berlin, $412 \mathrm{pp}$.

Bensuade, M., Thesis (Paris), 1-153 (1918)

Blakeslee, A. J., Proc. Am. Acad. Arts and Sciences., 40, 205-319 (1904)

Boivin, A., Delaunay, A., Vendrely, R., Lehoult, Y., Experentia, 1, $334-335$ (1945)

Bonner, D., Tatum, E. L., and Beadle, G. W., Arch. Biochem., 3, $71-91$ (1943)

Bridges, C. B., Zeitschr. Ind. Abstam. u. Vererb., 60, 207-218 (1932) 
Bridges, C. B., Jour. of Hered., 26, 60-64 (1935)

Brachet, J., Symp. Soc. Exp. Biol., I, 207 (1947)

Brown, J. H., Science., 64, 429-430 (1926)

Brunswik, H., Botan. Abhandl., 5, 1-152 (1924)

Buller, A. H. R., Bot. Rev., 7, 335-431 (1941)

Burgeff, H., Z. I. A. V., 49, 27-94 (1927)

Burkholder, P. R., Am. J. Botany., 30, $206-211$ (1943)

Burkholder, P. R., and Moyer, D., Bull. Torrey Botan. Club., 70, $372-377$ (1943)

Burkholder, P. R., McVeigh, I., and Moyer, D., Jour. Bact., 48, 385-391 (1944)

Callan, Proc. VIII Int. Cong. Genetics (1948)

Carson, G. P., Howard, H. W., Markham, R., Smith, K. M., Nature, 154: 334 (1944)

Caspersson, T., Naturwissenschaften., 24, 108. Chem. Abstr., 30. (1936)

Caspersson, T., and Schultz, Jack., Proc. Nat. Acad. Sci., 26, 507$515(1940)$

Caspersson, T., and Brandt, K., Protoplasma., 35, 507-526, (1941)

Chen, T. T., Jour. Heredity, 31, 175-184 (1940)

Chen, T. T., Jour. Morph., 79, 125-261 (1946)

Chilton, S. J. P., Lucas, G. B., Edgerton, C. W., Am. J. Botany., $32,549-554$ (1945)

Christensen, J. J., Phytopath. Zeits., 4, 129-188 (1931)

Craigie, J. H., Nature., 120, 765-767 (1927)

Darlington, C., Nature., 154, 489 (1944)

Delbruck, M., Cold Spring Harbor Symposia Quant. Biol., 11, 22 (1946)

Derx, H. G., Bull. Soc, Mycol., France., 41, 375-381 (1925)

Demerec, M., and Latarget, R., Cold Spring Harbor Symp. Quant. Biol., 11, 38-50 (1946)

Dickinson, S., Proc. of the Roy. Soc. B., 108, 395-423 (1931)

Dienert, F., Ann. Inst. Pasteur., 14, 139-189 (1900)

Dobzhansky, Th., Zeitschr. Ind. Abstam. u. Vererb., 43, 330-338 (1927)

Dobzhansky, Th., Changes induced by natural selection in wild populations of Drosophila (1946)

Dodge, B. O., J. Agr. Research., 35, 2890305 (1927)

Dodge, B. O., Science., 70, 22 (1929)

Dodge, B. O., Mycologia., 23, 1-50 (1931)

Dodge, B. O., and Seaver, B., Am. Jour. Bot., 25, 156-166 (1938)

East, E. M., Am. Naturalist., 44, 424-430 (1910)

Engel, L., (Thesis)., University of Paris. $59 \mathrm{pp} \mathrm{(1872)}$

Ericksson, J. Ber. d. D. Bot. G., 12, 292-331 (1894)

v. Euler, H., and Janssen, B., Z. physiol. Chem., 169., 226

Goldschmidt, R., Experientia., 2, 1-40 (1946) 
Gorodkowa, A. A., Bull. Hard. Imp. Bot. St. Peters., 8, 163-170 (1908)

Graham, V. R., and Hastings, E. G., Canad. Jour. Res., 19, 251 256 (1941)

Guilliermond, A., I. Recherches cytologiques sur les levures et quelques moisissures a formes levures. Storck. Lyon.

Guilliermond, A., Bull. Soc. Myc. France, 19, 19-31 (1903)

Guilliermond, A., Archiv Protistenk., 19, 289-309 (1910)

Guilliermond, A., Compt. Rend., 150, 835-838 (1910)

Guilliermond, A., Protoplasma., 16, $454-477$ (1932)

Hanna, W. F., Ann. Bot., 39, 436 (1925)

Hansen, E., Compt. Rend. Lab. Carls., 2, 29-85 (1883)

Harder, R. A., Zeit. Bot., 19, 337-407 (1927)

Harper, R. A., Carnegie Inst. Wash. Pub., No. 17, 105 pp., Washington, D. C. (1905)

Henneberg, W., Handbuch der Gurungsbakteriologie. Paul Parey, Berlin, 602 pp. (1926)

Huettig, W., Biol. Zentr., 55, 74-83 (1935)

Huettig, W., Z. f. Bot., 26, (1933)

Janssens, F. A., and Leblanc, A., Le Cellule, 14, 203-239 (1898)

Johnson, T., Cold Spring Harbor Symposia Quant. Biol., 11, 85-93 (1946)

Jollos, V., Arch. f. Protist., 83, $197-219$ (1934)

Kahn, M. C., and Nonidez, Jose., Proc. Soc. Exp. Biol. Med., 30, 577-582 (1933)

Karstrom, J., Ergeb. Enzymforsch., 7, 350-376 (1938)

Kater, J. McA., Biol. Bull., 52, 436-448 (1927)

Keitt, G. W., Langford, M. H., and Shay, J. R., Am. Jour. Bot., 30, 491-500 (1943)

Kluyver, H. J., Biochemische Suikerbepalingen. Delft. (1914)

Kniep, H., Zeit. f. Bot., 9, $81-119$ (1917)

Kruis, K., and Satava, J., O. Vyvoji a kliceni spor jakoz i sexualite kvasinek. Nakl. C., Akad. Praha. 67 pp. (1918)

Lederberg, J., Genetics., 32, 505-525 (1947)

Lederberg, J., and Tatum, E. L., Jour. Biol. Chem., 165, 381-382 (1946)

Legroux, Rene, and Magrou, J., Ann. Inst. Past., 34, 417-431 (1920)

Levan, A., Nature., 158, 626 (1946)

Lindegren, C. C., Bull. of the Torrey Bot. Club., 59, 85-102 (1932)

Lindegren, C. C., Bull. of the Torrey Bot. Club., 59, 119-138 (1932)

Lindegren, C. C., Bull. of the Torrey Bot. Club., 60, 133-154 (1933)

Lindegren, C. C., Am. Jour. Bot., 21, 55-65 (1934)

Lindegren, C. C., Jour. of Genetics., 28, $425-435$ (1934)

Lindegren, C. C., Genetics., 16, 315-320 (1934)

Lindegren, C. C., Zeitschrift fur induktive abstammungs - and Verebungslehre., 68, 331-335 (1935) 
Lindegren, C. C., Zentralblatt fur Bakteriologie, Parasitenkunde und Infektionskrankheiten II., 92, 41-48 (1935)

Lindegren, C. C., Zentralblatt fur Bakteriologie, Parasitenkinde und Infektionskrankheiten II., 93, 113-122 (1935)

Lindegren, C. C., Zentralblatt fur Bakteriologie, Parasitenkinde und Infektionskrankheiten II., 93, 389-400 (1936)

Lindegren, C. C., Jour. of Genetics., 32, 243-256 (1936)

Lindegren, C. C., Jour. of Heredity., 27, 250-259 (1936)

Lindegren, C. C., The American Naturalist., 70, 404-406 (1936)

Lindegren, C. C., and Scott, Mary Allan., La Cellule., 65, 362-371 (1937)

Lindegren, C. C., and Lindegren, Gertrude., Jour. of Heredity., 28, 105-113 (1937)

Lindegren, C. C., and Rumann, Siegfried., Jour. of Genetics., 36, $395-404$ (1938)

Lindegren, C. C., Beanfield, Virginia, and Barber, Roberta., The Botanical Gazette., 100, 592-599 (1939)

Lindegren, C. C., and Lindegren, Gertrude., The Jour. of Heredity., $32,404-440(1941)$

Lindegren, C. C., Iowa State College Jour. of Sci., 45, 371-390 (1942)

Lindegren, C. C., and Lindegren, Gertrude., Genetics., 27, 1-24

(1942)

Lindegren, C. C., Iowa State College Jour. of Sci., 16, 307-318 (1942)

Lindegren, C. C., and Lindegren, Gertrude., Ann. of the Missouri

Botanical Garden., 30, 71-82 (1943)

Lindegren, C. C., and Lindegren, Gertrude., Proc. of the Nat. Acad. of Sci., 29, 306-308 (1943)

Lindegren, C. C., and Lindegren, Gertrude., Ann. of the Missouri Botanical Garden., 30, $453-468$ (1943)

Lindegren, C. C., and Lindegren, Gertrude., Jour. of Bacteriology., 46, 405-419 (1943)

Lindegren, C. C., and Lindegren, Gertrude., The Botanical Gazette, 105, $304-316$ (1944)

Lindegren, C. C., and Hamilton, Elizabeth., The Botanical Gazette., 105, 316-321 (1944)

Lindegren, C. C., and Lindegren, Gertrude., Ann. of the Missouri Botanical Garden., 31, 203-216 (1944)

Lindegren, C. C., Spiegelman, S., and Lindegren, Gertrude., Proceedings of the National Acad. of Sci., 30, 346-352 (1944)

Lindegren, C. C., Wallerstein Communications., 7, 153-168 (1944)

Lindegren, C. C., Spiegelman, S., and Lindegren, Gertrude., Archiv. of Biochem., 6, 185-198 (1945)

Lindegren, C. C., Ann. of the Missouri Botanical Garden., 32, 107123 (1945)

Lindegren, C. C., and Lindegren, Gertrude., Science., 102, 33-34 (1945) 
Lindegren, C. C., Archives of Biochem., 8, 119-134 (1945)

Lindegren, C. C., Mycologia., 37, 767-780 (1945)

Lindegren, C. C., Bacteriological Reviews., 8, 110-170 (1945)

Lindegren, C. C., Proc. of the Nat. Acad. of Sci., 32, 68-70 (1946)

Lindegren, C. C., Archives of Biochem., 9, 353-359 (1946)

Lindegren, C. C., and Lindegren, Gertrude., Cold Spring Harbor

Symp. on Quant. Biol., 11, 115-129 (1947)

Lindegren, C. C., Nature., 159: p. 63 (1947)

Lindegren, C. C., and Raut, Caroline., Ann. of the Missouri Botanical Garden., 34, 75-84 (1947)

Lindegren, C. C., and Raut, Caroline., Ann. of the Missouri Botanical Garden., 34, 85-93 (1947)

Lindegren, C. C., and Lindegren, Gertrude., Ann. of the Missouri Botanical Garden., 34, 95-99 (1947)

Lindegren, C. C., and Lindegren, Gertrude., Proc. of the Nat. Acad. of Sci., 33, 11-314-318 (1947)

Lindegren, C. C., Proc. of the Nat. Acad of Sci., 34, 187-193 (1948)

Lindegren, C. C. Annual Review of Microbiology., 2, 184-218 (1948)

Lindegren, C. C., VII Congress International des Industries Agricoles.

Paris, 8 pp. (1948)

Lindegren, C. C., VIII International Congress of Genetics., Stockholm, (1948)

Lindegren, C. C., and Lindegren, Gertrude., Proc. of the Army Yeast

Symposium, Milwaukee, Wisc., Nov. 8-10 (1948)

Lwoff, A., Sump. on Quant. Biol., 9, 139-153 (1946)

Maneval, W. E., Bot. Gaz., 78, 122-125 (1924)

Mangelsdorf, P. C., and G. S. Fraps, Science, 73 : 241 (1931)

Marshak, A., Records Genetic Soc. Amer., p. 48 (1948)

Mazia, D., Hayashi, T., and Yidowitch, K., Cold Spring Harbor Symp. Quant. Biol., 12, 122-130 (1947)

McAnally, R. A., and Smedley-Maclean, I., Bio-chem. Journal., 29, $1872-1876$ (1935)

McCarty, M., Taylor H. E., Avery, O. T., Cold Spring Harbor Symposia Quant. Biol., 11, 177-183 (1946)

Meissner, R., Zentr. Bakt. Parasitenk. Infek., II, 6, 517-525, 545-554 (1900)

Meyerhof, O., Biochem. Z., 162, 43-86 (1925)

Meyer, A., Mikroskopisches Practicuum. II. 157 pp. Gustav Fischer. Jena. (1903)

Miller, C. P., and Bohnhoff, M., Science, 105, 620-621 (1947)

Minchin, E. A., Amer. Nat., 50, 5-39 and 106-119 (1916)

Monod, J., Growth Symposia, 11, 223 -289 (1947)

Mrak, E. M., Phaff, H. J., and Douglas, H. C., Science, 96, 432 (1942)

Muller, H. J., Proc. Roy. Soc., B., 134, 1-37 (1947)

Nelson, O. E., Stain Tech., 20, 131-132 (1945)

Newton, M., Johnson, T., and Brown, A. M., Sci. Agr., 10, 775-798(193 
Nickerson, W. J., and Thimann, K. V., Amer. Jour. Bot., 28, 617-621 (1941)

Nickerson, W. J., and Thimann, K. V., Amer. Jour. Bot., 30, 94-101 (1943)

Nilsson-Ehle, H., Lunds Universitets àraskrift, p. 15 (1909)

Pisova, Mildred., (Thesis) Karl University, Prague (1930)

Pontecorvo, G., Proc. of the Eighth International Congress of

Genetics (1948)

Quintanilha, A., Boletim Da Sociedade Brotheriana, 13, 425-486 (1939)

Rafalko, J. S., Stain Technology., 21, 91-93 (1946)

Rees, M., Bot. Zeit., 27, 106-118 (1869)

Renaud, Jean., Complis Rendera de l'Acad. Science., 206, 1918-1920 (1938)

Ris, H., and Krause, H., Proc. Nat. Acad. Sc., 31, 321-327 (1945)

Ryan, F. J., Cold Spring Harbor Symposia Quant. Biol., 11, 215-227 (1946)

Sansome, E. R., Demerec, M., and Hollaender, A., Am. J. Botany, $32,218-226$ (1945)

Satava, J., III ${ }^{\mathrm{e}}$ Cong. Internat. Tech. et Chim. des Ind. Agr. Paris.

(Cited from Winge). Chem. Abstr., 29, 2296 (1934)

Schroedinger, E., What is Life? Cambridge (1945)

Schultz, J., Caspersson, T., and Aquilonius, L., Proc. Nat. Acad. Sci., $26,515-523(1940)$

Scott Moncrieff, R., Jour. Genetics., 32, 117 (1936)

de Seyne, M. J., Compt. Rend Acad. Sci., 67, 105-109 (1868)

Shear, C. L., and Dodge, B. O., J. Agr. Research, 34, 1019-1042 (1927)

Skoog, F. K., and Lindegren, C. C., Jour. of Bacteriology, 53, 729742 (1947)

Smedley-Maclean, I., and Hoffert, D., Biochem. J., 17, 720-741 (1923)

Smedley-Naclean, I., and Hoffert, D., Biochem. J., 18, 1273-1278

(1924)

Sonneborn, T. N., Cold Spring Harbor Symposia Quant. Biol., 11,

$236-255(1946)$

Spiegelman, S., Cold Spring Harbor Symposia Quant. Biol., 11, 256$277(1946)$

Spiegelman, S., Reiner, John M., and Morgan, Ida, Arch. Bio., 1, $113-125$ (1947)

Spiegelman, S., and Lindegren, C. C., Annals of the Missouri Botani ical Garden., 31, 219-233 (1944)

Spiegelman, S., Lindegren, C. C., and Hedgecock, L., Proc. Nat. Acad. Sci., 30, $13-23$ (1944)

Spiegelman, S., Lindegren, C. C., and Lindegren, Gertrude., Proc. Nat. Acad. Sci., 31, 95-102 (1945)

Spiegelman, S., and Lindegren, C. C., Jour. of Bacteriology., 49, 257-269 (1945) 
Spiegelman, S., Cold Spring Harbor Symposia on Quant. Biol., 11, 256-277 (1946)

Stadler, L. J., Proc. of the VI International Congress of Genetics., 274-294 (1932)

Stakman, E. C., Kernkamp, M. F., King, T. H., and Martin, W. J., Am. J. Bot., 30, 37-48 (1943)

Stakman, E. C., and Piemeisel, F. J., J. Agric. Res., 10, 429-495 (1917)

Stephenson, M., and Yudkin, J., Biochem. Jour., 31, 506-514., 1311 1315 (1936)

Stern, C., Genetics, 28: 441-475 (1943)

Stern, K., Yale Jour. Biol. Bed., 19, 937-949 (1947)

Sturtevant, A. H., Genetics., 10, 117-147 (1925)

Sylvan, Bengt., Acta. Chir. Scand., Supplement 66, 151 pp (1941)

Vandendries, R., Bull. Soc. R. Bot. Belg., 70, 66-85 (1937)

Vanderlyn, L., Science., 104, 514-515 (1946)

Wager, H., and Peniston, A., Ann. Bot., 24, 45-84 (1910)

Welten, H., Niikrokosmos., 8, 3-5; $41-43$ (1914)

Wiame, J. M., Bull. Soc. Chim. Biol., 28, 552-556 (1946)

Wiame, J. M., Biochemica et Biophysica Acta 1

Wiame, J. M., Fed. Proc. Amer. Soc. Expt. Biol., 6, 302 (1947)

Williams, R. J., Biol. Re., 16, 49-80 (1941)

Winge, O., and Hjort, A., Compt. rend. trav. lab. Carlsberg, ser. physiol., 21, 51-58 (1935)

Winge, O., Compt. rend. trav. lab. Carlsberg, ser. physiol., 21, 77-112 (1935)

Winge, O., and Laustsen, O., Compt. rend. trav. lab. Carlsberg, ser. physiol., 23, 17-39 (1940)

Winkler, H., Biol. Zent , 52, $163-189$ (1932)

Winzler, R. J., Burk, D., and DuVigneau , V., Arch. Biochem., 5, 25 (1944)

Witkin, E. M., Cold Spring Harbor Symposia Quant. Biol., 12, 256 269 (1947)

Woods, M. W., and duBuy, H. G., Science, 102, 591-593 (1945)

Wyss, O., Stone W. S., and Clark, J. B., J. Bact., 54, 767-772 (1947) 




